\title{
Conformational spectroscopy of flexible chain molecules near the folding limit
}

\author{
Dissertation \\ zur Erlangung des mathematisch-naturwissenschaftlichen Doktorgrades \\ "Doctor rerum naturalium" \\ der Georg-August-Universität Göttingen \\ im Promotionsprogramm Chemie \\ der Georg-August-University School of Science (GAUSS) \\ vorgelegt von \\ Sebastian Bocklitz \\ aus Halberstadt
}

Göttingen, 2017 


\section{Betreuungsausschuss:}

Prof. Dr. Martin Suhm

Institut für Physikalische Chemie, Georg-August-Universität Göttingen

Prof. Dr. Götz Eckold

Institut für Physikalische Chemie, Georg-August-Universität Göttingen

\section{Referent:}

Prof. Dr. Martin Suhm,

Institut für Physikalische Chemie, Georg-August-Universität Göttingen

Korreferent:

Prof. Dr. Götz Eckold

Institut für Physikalische Chemie, Georg-August-Universität Göttingen

\section{Weitere Mitglieder der Prüfungskommission:}

Prof. Dr. Ricardo Mata

Institut für Physikalische Chemie, Georg-August-Universität Göttingen

Prof. Dr. Philipp Vana

Institut für Physikalische Chemie, Georg-August-Universität Göttingen

Prof. Dr. Jörg Behler

Institut für Physikalische Chemie, Georg-August-Universität Göttingen

Dr. Sebastian Kruss

Institut für Physikalische Chemie, Georg-August-Universität Göttingen 


\section{ACKNOWLEDGEMENTS}

First of all, I would like to thank my supervisor Prof. Dr. Martin Suhm for his remarkable support, inspiring discussions and constant motivation during my entire scientific career.

I also want to thank my co-referent Prof. Dr. Götz Eckold for his kind support and interesting discussions. Prof. Dr. Ricardo Mata is gratefully acknowledged for valuable assistance in the calculations of longer glyme chain conformations as well as being part of my thesis committee. Prof. Dr. Philipp Vana, Prof. Dr. Jörg Behler and Dr. Sebastian Kruss are gratefully acknowledged for being part of my thesis committee and taking the time to evaluate my work.

I am very thankful to Prof. Dr. Timothy Zwier for hosting me during a research stay in his lab at Purdue University, USA. I would like to express my sincere gratitude to the German-American Fulbright commission who funded this research stay with a Fulbright scholarship. Furthermore, I would like to thank all people who made this research stay an incredible personal and scientific experience. Especially, Daniel Hewett who introduced me to the Zwier lab setups, actively collaborated in a joint project between the Suhm and the Zwier group, proofread major parts of this thesis and was crazy enough to join me in a Spartan race. And Anthony Tomaine who synthesized the phenoxy-substituted glyme analogs and passionately led our intramural soccer team.

Furthermore, I want to thank my German colleagues who were a tremendous support of my work as well. I would like to thank Dr. Katharina Otto for a comprehensive introduction to the OPO system. Furthermore, I would like to thank Felix Maiwald for measuring the liquid glyme samples during the work for his bachelor thesis. Dr. Nils Lüttschwager is acknowledged for his help with the analyses of the elastic moduli, thorough proofreading and valuable scientific discussions. I want to thank Sönke Oswald and Thomas Forsting for thorough proofreading as well as valuable discussions and assistance in the curry-jet development. In general, I want to thank all current and past members of the workgroups of Prof. Suhm and Prof. Zwier for the great working atmosphere.

Most of this research work would have been impossible without the staff of the institute and department workshops. Thus, I would like to acknowledge the support of Volker Meyer, Reinhard Hildebrandt, Andreas Knorr, Annika von Roden and all other workshop co-workers.

Above all, I want to deeply thank my friends, my family and my partner AnnaLena Herzog for their unconditional and loving support at any time. 



\section{Contents}

Chapter 1 - Introduction ........................................................................................................

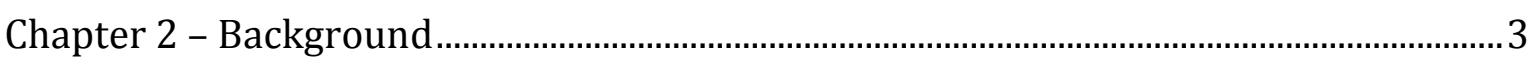

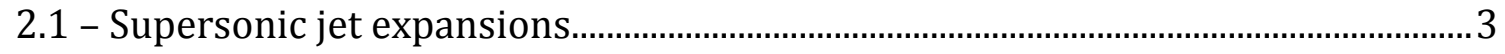

2.2 - UV-UV holeburning spectroscopy .............................................................................

2.3 - Fluorescence-dip infrared spectroscopy..................................................................6

2.4 - Quantum chemical calculations ...............................................................................

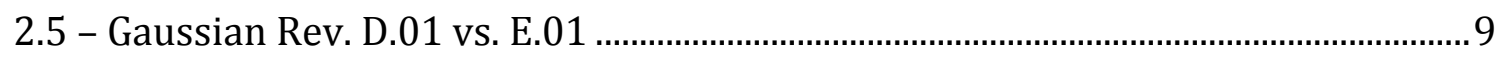

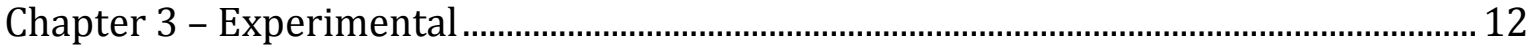

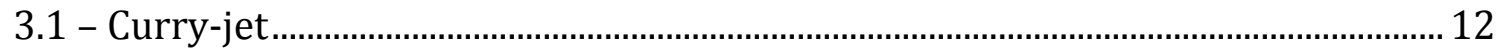

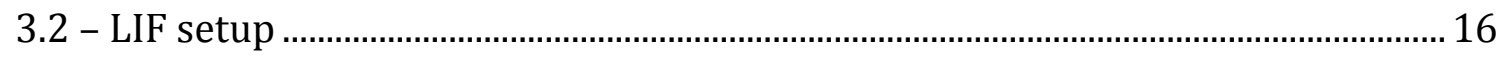

3.3 - IR-Raman double resonance setup...................................................................... 18

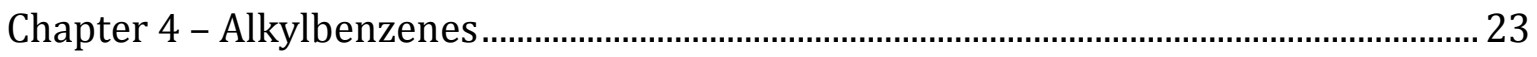

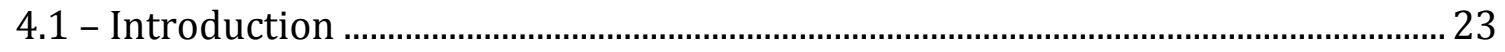

4.2 - Enumeration of alkylbenzenes.................................................................................. 24

4.3 - Quantum chemical calculations ......................................................................... 25

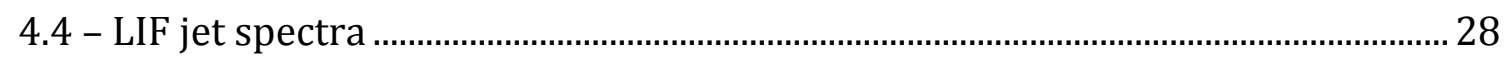

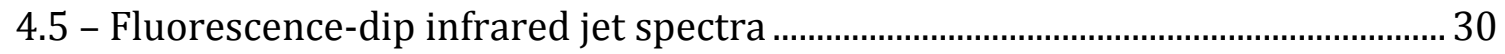

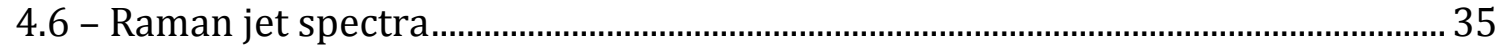

4.7 - Comparison with $n$-alkanes................................................................................. 38

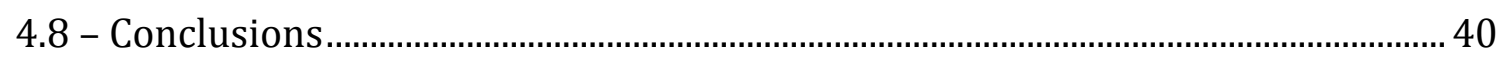

Chapter 5 - 1-Methoxy-2-phenoxyethane ……………………………………………….... 42

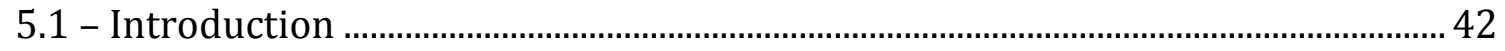

5.2 - Quantum chemical calculations ............................................................................. 42

5.3 - LIF and UV-UV HB spectra ................................................................................ 46

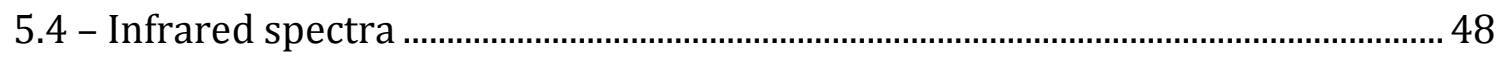

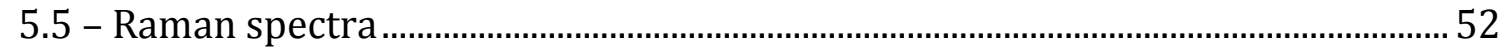

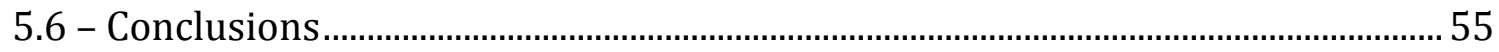

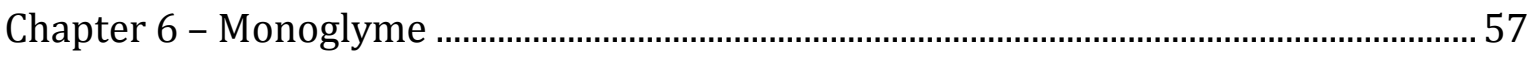

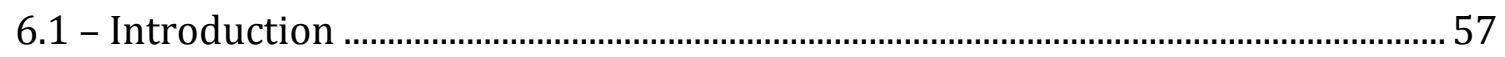

6.2 - Quantum chemical calculations ………………………………………………..... 59

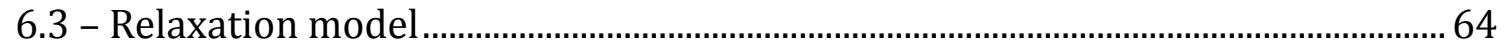

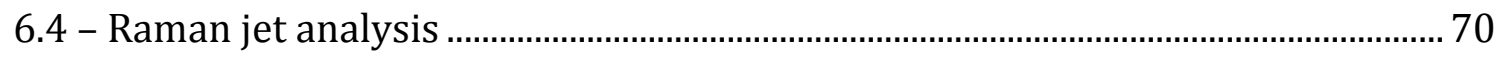

6.5 - Conformation control via experimental parameters............................................... 73 


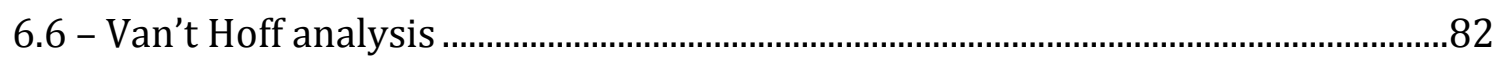

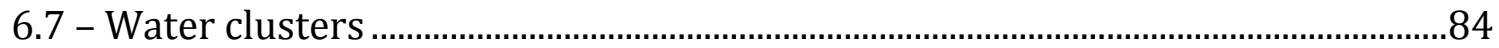

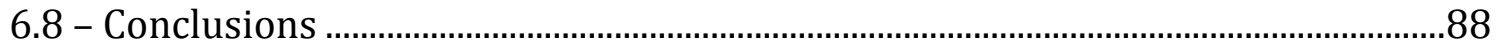

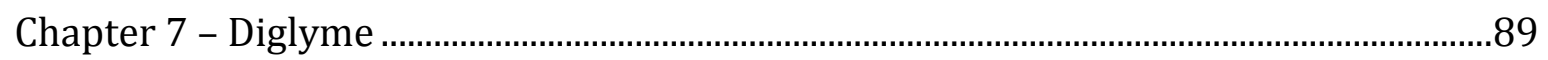

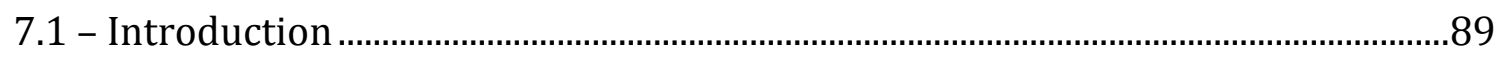

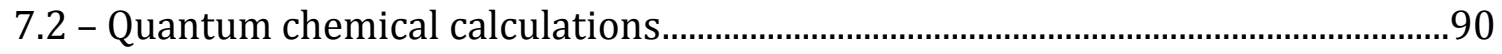

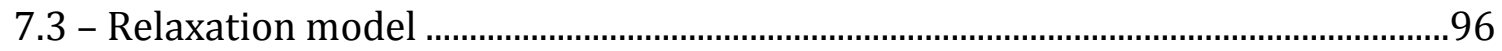

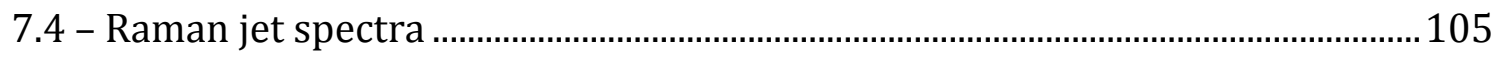

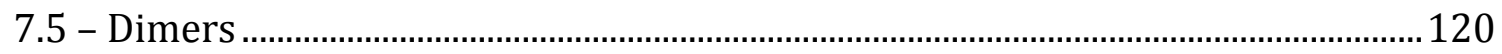

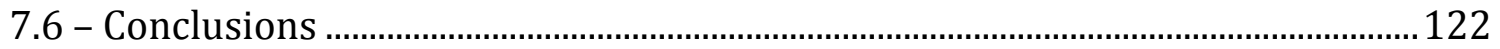

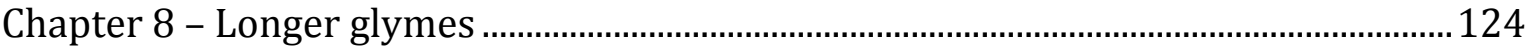

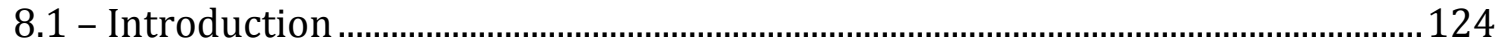

8.2 - Quantum chemical calculations.......................................................................... 124

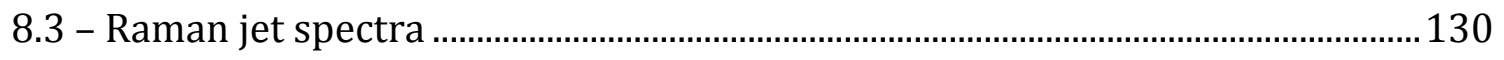

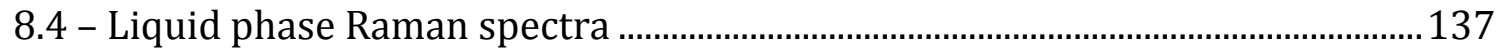

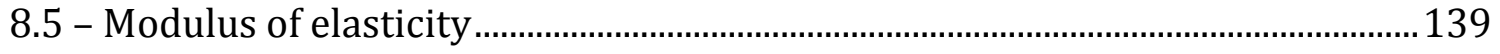

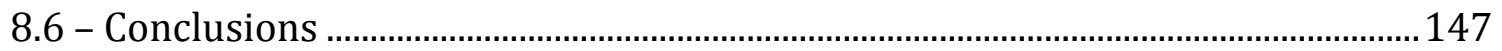

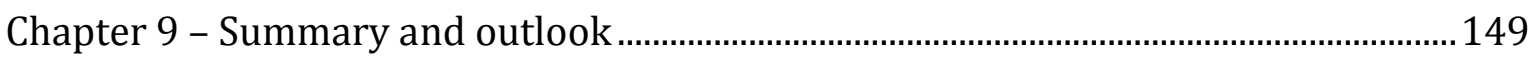

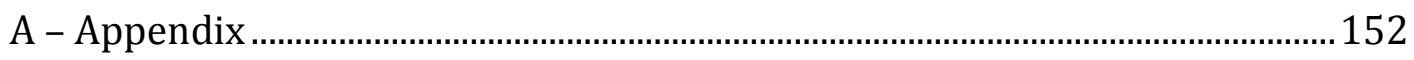

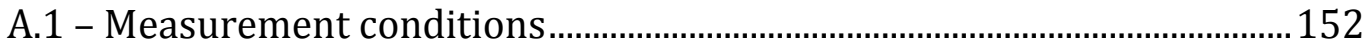

A.2 - Differences in spectral resolutions with changing laser sources in the

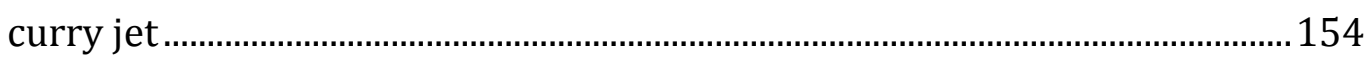

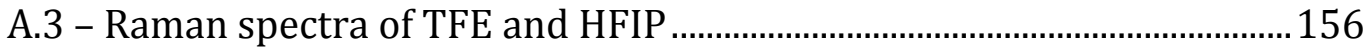

A.4 - Extended results of the conformational search in alkylbenzenes.......158

A.5 - Extended results of the conformational search in diglyme .................... 160

A.6 - Subsidiary $\Delta G^{\mathrm{C}}$ model plots of diglyme ...................................................... 165

A.7 - Further band assignments of diglyme.......................................................... 166

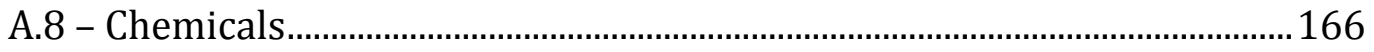

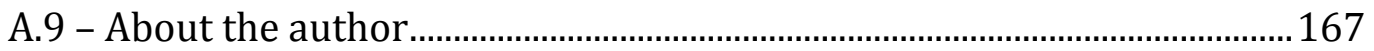

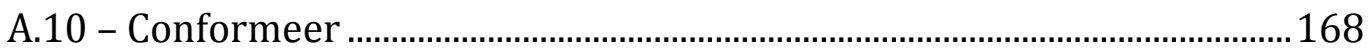

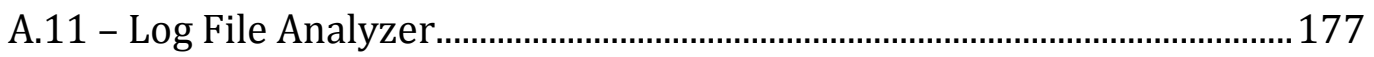




\section{Chapter 1 - Introduction}

The conformation of a molecule which describes the arrangement of atoms around the single bonds determines its "appearance" and is often an important factor for its function. A popular example are proteins which only function correctly if they are present in the appropriate conformation [1], while misfolding may even lead to severe diseases [2]. Thus, understanding conformational preferences and their influencing factors for complex molecular systems is of great importance. The highly competitive conformational landscapes of flexible chain molecules are a difficult challenge even for modern quantum chemistry as several delicate effects, partially counterbalancing each other, play an important role in predicting the correct energy sequence of all conformers. Among stabilizing interactions are London dispersion forces or formation of hydrogen bonds that influence the energetic sequence of molecular conformations $[3,4]$. Thorough experimental investigations of the conformational landscapes of flexible chain molecules provide sensitive and valuable benchmarks for quantum chemistry which aims at the prediction of conformational preferences. Supersonic jet expansion is a preparation technique which has proven particularly helpful for such experimental benchmarks, because it allows to study isolated molecules in the gas phase at low temperatures [5]. The rapid cooling during the expansion lowers the number of conformers with relevant population, if interconversion barriers are sufficiently low [6], and shifts the conformational distribution to the energetically most favorable species. This greatly simplifies the analysis of vibrational spectra which are used to probe jet-cooled gas mixtures. Therefore, the main focus of the present thesis is the spectroscopic investigation of conformational landscapes of jetcooled flexible chain molecules in the gas phase. Additionally, the predictive power of dispersion corrected density functional theory (DFT) approaches [7] and more sophisticated $a b$ initio methods is carefully tested against experimental results.

Oligoglymes or oligoethylenglycol dimethyl ethers $\left(\mathrm{CH}_{3} \mathrm{O}\left(\mathrm{CH}_{2} \mathrm{CH}_{2} \mathrm{O}\right)_{n} \mathrm{CH}_{3}\right)$ are an obvious choice for such a study as they exhibit an unusual degree of chain flexibility. This makes them preferred molecules for a wide range of applications such as cation solvation in battery electrolytes, phase-transfer catalysis or acting as a drug delivery agent $[8,9]$. In this work oligoglymes were investigated by means of Raman spectroscopy, a routine tool for investigations of structural preferences in chain molecules $[10,11]$. The decreasing vapor pressure of longer oligoglymes limits the jetspectroscopical investigations to shorter chains lengths $(n=1-4)$, but the conformational preferences of these smaller chains are already a suitable model for the polymeric system [11-13]. Especially useful for the analysis of Raman spectra and of particular interest for this work are the strongly Raman active and conformationally sensitive longitudinal acoustic modes (LAMs) [13], a specific type of frame vibration where chain segments are displaced in an "accordion-like" fashion. The spectral position of the corresponding vibrational bands depend not only on the conformational arrangement but also on the length of the vibrating segment [14]. An extrapolation of the LAM positions to infinite chain lengths allows deriving a value for the idealized 
elastic modulus of a single polyethylene oxide chain in vacuum at low temperatures [15], analogous to similar investigations for polyethylene [16-19].

Folding tendencies of rather rigid chain molecules can be enhanced by attaching chemical groups that allow for stronger intramolecular interactions. Thus, another part of this thesis investigates folding in $n$-alkylbenzenes and compares the findings to the more rigid counterparts of pure $n$-alkane chains. Here, Raman spectroscopic investigations are augmented by UV and fluorescence-dip IR (FDIR) spectroscopic investigations that allow for a complementary insight regarding the conformational assignments. In contrast to the case of $n$-alkylbenzenes, the addition of a phenyl group to a molecular chain can also lead to an increase of the interconversion barriers of subsequent dihedral conversions. This will be shown through investigations on 1-methoxy-2-phenoxyethane, the phenyl-substituted analog of monoglyme. A combination of laser induced fluorescence (LIF), Raman, UV-UV and single-conformer fluorescence dip IR spectra are used to characterize the conformational landscape of this molecule in supersonic jet expansions to a high extent.

Note that some of the work presented in this thesis was done in cooperation with the research group of Prof. Zwier at Purdue University (USA). A six-month guest stay was funded through a Fulbright scholarship. This made it possible to extend the experimental investigations on the aromatic compounds by fluorescence-based spectroscopic methods.

The outline of this thesis is as follows. In Chapter 2, background information on supersonic jet expansions and the used double resonance techniques will be given. Quantum chemical calculations that are used throughout this work to aid in the spectroscopic assignments, estimate abundances of different conformers and explore interconversion tendencies between them will be outlined there as well. In Chapter 3, the employed experimental setups will be described. An IR-Raman double resonance setup will be described in detail there as well. The alignment routine for the double resonance experiments was improved during this work but did not yet allow for the successful observation of significant double resonance excitation effects.

The subsequent Chapters will focus on the respective investigations of the aforementioned molecules. Each of these Chapters is structured similarly: A short introduction on the investigated molecule, its basic properties and used enumeration/nomenclature will be given. Then, results of quantum chemical calculations will be presented, discussed and compared against jet spectra. Each chapter ends with concluding remarks. Chapter 4 contains the investigations on alkylbenzenes. Results on 1methoxy-2-phenoxyethane are found in Chapter 5. Investigations on mono- and diglyme will be presented in Chapter 6 and Chapter 7, respectively. Results on longer glyme chains will be discussed in Chapter 8 . There, jet spectra will be also compared to spectra of liquid glyme samples and an extrapolation of calculated vibrational frequencies to infinite chain length will be used to derive the elastic modulus of a single polyethylene oxide chain in vacuum at low temperatures. Finally, a summary of the findings of this dissertation is provided in Chapter 9. 


\section{Chapter 2 - Background}

\section{1 - Supersonic jet expansions}

In spectroscopy it is often advantageous to investigate molecules at low temperatures as band shapes become relatively narrow due to rotational cooling which allow for observation of independent conformers or weakly bound complexes. At higher temperatures molecules show a broad variety of properties, such as velocities and conformations which complicate the spectra. One technique that is used to cool molecules or atoms to relatively low temperatures without initializing condensation is expansion in a supersonic jet. A supersonic jet expansion is formed when gas is expanded from high pressures through a small orifice or nozzle, which is larger than the mean free path of the gas-phase molecules, into a vacuum [20]. Therefore, molecules collide at the orifice converting random atomic motions into directed mass flow [21]. This partial redistribution of thermal energy into directed motions leads to an internal cooling of the molecules. Additional collisions in the expanding beam lead to further cooling and further equalized particle velocities. The local speed of sound in expansions decreases proportionally to the square root of the translation temperature ( $\left.T_{\text {trans }}\right)$. Eventually particle velocities exceed the local speed of sound and become supersonic. Jet relaxation effects are limited because the number of collisions decreases further downstream, due to the spreading of the gas beam and equilibration of particle velocities, to a point where no further collisions happen. There the energy redistribution mostly comes to a halt and a "zone of silence" is established. Finally, the lowdensity beam is recompressed due to collisions with the background gas in the chamber, forming a terminal shock wave. Also, lateral shock waves are found at the sides of the expansions, enclosing the cold supersonic core with warmer regions.

Typically, experiments in supersonic jet expansions are carried out in seeded beams in which a small amount of the substance of interest is diluted in a carrier gas, usually a noble gas that acts as the cooling agent. It is advantageous if the carrier gas, like noble gases, has no spectroscopic signature as these could potentially interfere with the substance signals. Concentration and size distribution of the expanding particles can be controlled by the outlet dimensions, seeding fraction in the carrier gas, initial pressure and temperature of the gas. Slit nozzles show narrow velocity distributions along the slit direction which can otherwise also be achieved or amplified by placing a skimmer perpendicular to the direction of the gas flow. If no skimmer is used the expansion is labeled as a "free jet" while others are referred to as "molecular beams".

The separate internal degrees of freedom are cooled differently depending on how efficient energy is removed upon collisions. This non-uniform energy redistribution leads to a non-equilibrium environment in which the translational temperature ( $\left.T_{\text {trans }}\right)$ is usually found to be lower than the rotational temperature $\left(T_{\text {rot }}\right)$. The vibrational temperature $\left(T_{\mathrm{vib}}\right)$ is often found to be even higher because vibrations need more collisions to equilibrate. Moreover, not all vibrations are cooled with equal efficiency and incomplete inter-mode equilibration leads to different final effective tem- 
peratures. In supersonic jet expansions $T_{\text {trans }}$ often reaches the sub-Kelvin regime [22] and was even reported to be $6 \mathrm{mK}$ in jet expansions of helium [23]. Rotational temperatures can be as low as $T_{\text {trans }}$ if the collisions during the expansions are not a limiting factor but small molecules often retain $T_{\text {rot }}$ up to a few tens of Kelvins [24]. A curry-jet ${ }^{\dagger}$ study of $\mathrm{N}_{2}$ found rotational temperatures of $20-80 \mathrm{~K}$ depending on the distance between the probing laser and the nozzle [25]. Conformational interconversions can take place when collisions or formations of short-lived complexes provide enough energy to overcome isomerization barriers. Similar to the vibrational temperatures, these are often found to be higher in temperature than $T_{\text {trans }}$ or $T_{\text {rot. }}$ For example, the gauche-trans isomerization temperature in ethanol was found between 50 and $120 \mathrm{~K}$, depending on the distance between the probing laser and the nozzle, in a previous curry-jet study [26]. As mentioned earlier, when the particle density in the supersonic expansion drops to a certain threshold the interconversions cease and a certain conformational distribution is locked [6,27]. However, studies on trifluoropropanol [28] and $n$-alkanes [18] showed that relevant interconversion motions may be easily frozen during supersonic jet expansions despite ongoing collisions if the barrier is large. In this work interconversion barriers of varying sizes leading to different relaxation behaviors will be of major importance. The cooling performance in jet expansions can be enhanced by the addition of heavier carrier gases [29], but heavier rare gases are more polarizable and tend to form van-der-Waals complexes with the seeded molecules. However, adding a few percent of heavier additives to helium expansion enhances relaxations while limiting cluster formation [19,30].

In principle, jet expansions can be operated pulsed or continuously [31]. Since the position of the terminal shock wave depends on the square root ratio of the stagnation pressure of the gas to the background pressure in the jet chamber, pulsed operation might provide more well-defined expansions and facilitate better cooling if the backing vacuum system is adequately sized and pumped between pulses. Particles with higher velocity components perpendicular to the main direction of propagation are driven towards the outside of the expansion. Thus, the perpendicular translational temperature in the beam center decreases as the beam progresses and since the width of the Maxwell-Boltzmann-distribution decreases with increasing mass, the heavier component in a seeded beam enriches at the expansion core [32]. Large mass disparities in a seeded beam can cause the acceleration of the heavier component to lag behind the lighter one. This velocity slip can be used for isotope separation as demonstrated for the hexafluorides of uranium-235 and uranium-238 [33].

This work will primarily focus on the investigation of relaxation effects at different positions in the supersonic jet expansions. Additionally, effects on the relaxation behavior through altering initial gas pressures and temperatures will be explored and discussed in the upcoming chapters.

† The curry-jet setup will be described in Chapter 3.1. 


\section{2 - UV-UV holeburning spectroscopy}

UV-UV holeburning (UV-UV HB) is a technique that allows recording the electronic excitation spectrum of individual isomers or conformers. The laser excitation scheme for this technique is shown in Figure 2.1. The holeburn laser is fixed on a conformerspecific electronic transition, partially saturating it. Then, the holeburn spectrum is recorded by scanning a time-delayed probe UV laser over the desired UV frequency range. Delay times between lasers should not allow for a full recovery of the ground state population after the holeburn excitation and are typically chosen to be between 50 and $200 \mathrm{~ns}$. Both lasers are spatially overlapped and the holeburn laser is set to operate at half the frequency of the probe laser. Whenever the probe laser hits a transition that shares the same ground state as the holeburn excitation a depletion of the fluorescence signal is detected. The probe spectra with and without the preceding holeburn laser are compared via active baseline subtraction (ABS). Therefore, if the holeburn and probe lasers are resonant with a transition of the same ground state ABS returns a negative value, while it returns zero if the lasers probe transitions belonging to the different ground states. It is worth noting that this technique can be analogously used for ion instead of fluorescence detection. In this case the excitation follows a resonant two-photon ionization (R2PI) scheme where the ions are usually mass-selectively detected.

UV-UV HB allows identifying all vibronic transitions due to a single species from the electronic excitation spectrum [34]. Frequencies and intensities of low-frequency vibronic transitions can be used to assign structures and probe their interactions [35].

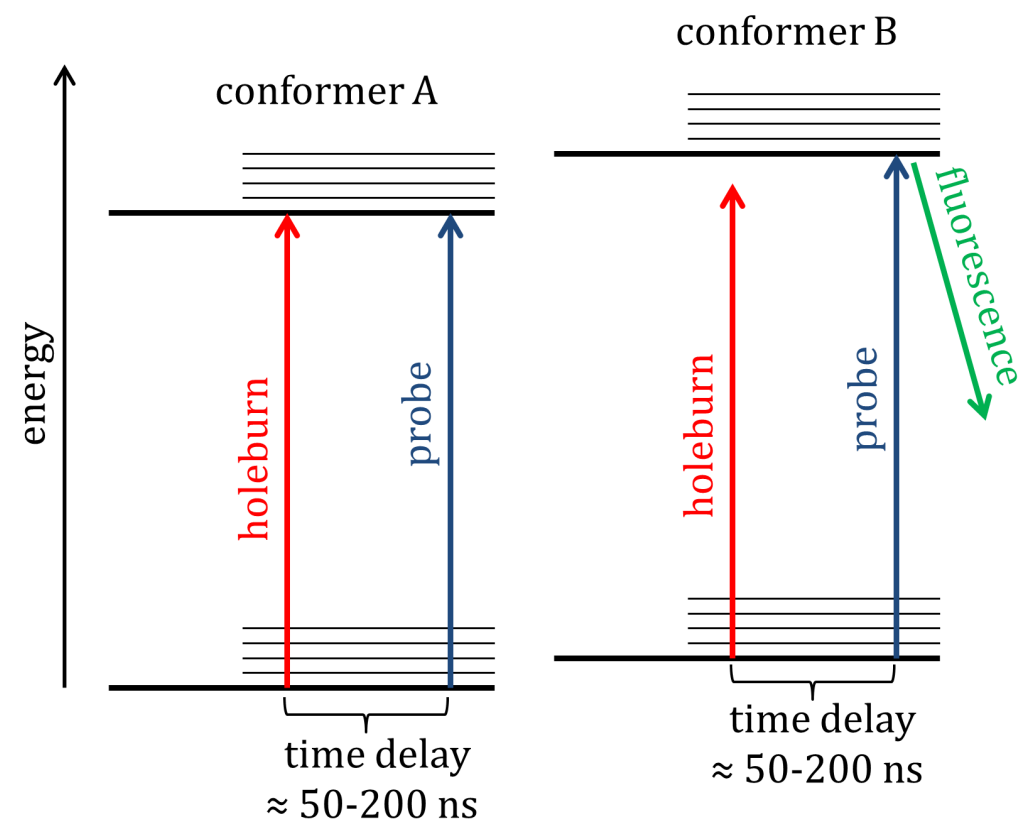

Figure 2.1: UV-UV HB excitation scheme after T. Zwier [34]. Holeburn and probe laser are resonant for conformer A (left side) leading to a depletion of the fluorescence signal, while no depletion is seen in all resonant probe transitions of conformer $B$ (right side). 


\section{3 - Fluorescence-dip infrared spectroscopy}

Fluorescence-dip infrared spectroscopy (FDIRS) allows for the recording of conformation specific IR spectra. The excitation scheme (Figure 2.2) is analogous to the UVUV HB but the holeburn UV laser is replaced with an IR laser. The UV laser is fixed to a desired vibronic transition that is unique to one conformer and both lasers are spatially overlapped with the UV laser temporally delayed by about 50-200 ns. The IR laser is scanned through the desired infrared region while operating at half the frequency of the UV laser. When the IR laser is resonant with a vibrational transition that shares the same electronic ground state as the conformer being probed by the UV laser a depletion in the fluorescence signal is detected. Active baseline subtractions of fluorescence signals with and without preceding IR excitations yield the fluorescencedip infrared spectrum. This technique was first employed by Page et al. on benzene and its dimers [36,37], following the ion-analogue technique known as resonant iondip infrared spectroscopy (RIDIRS).

Switching the timing between the IR and UV pulse so that the UV is preceding the IR excitation allows recording the FDIR spectra in the electronic excited $\mathrm{S}_{1}$ states. If population remains in the $S_{1}$-state the IR laser can promote the excitation of vibrational levels in this excited electronic state. These states have a much smaller fluorescence quantum yield due to non-radiative processes such as the internal conversion (IC) to dark states that ultimately quench the fluorescence signal. A necessary requirement for $\mathrm{S}_{1}$ FDIRS is that the excited state lifetimes are long-lived compared to the lifetimes of the light sources in order to generate sufficient population in $\mathrm{S}_{1}$. In 1994, Huber et. al demonstrated the $S_{1}$-state analogue of FDIRS on propynal [38].
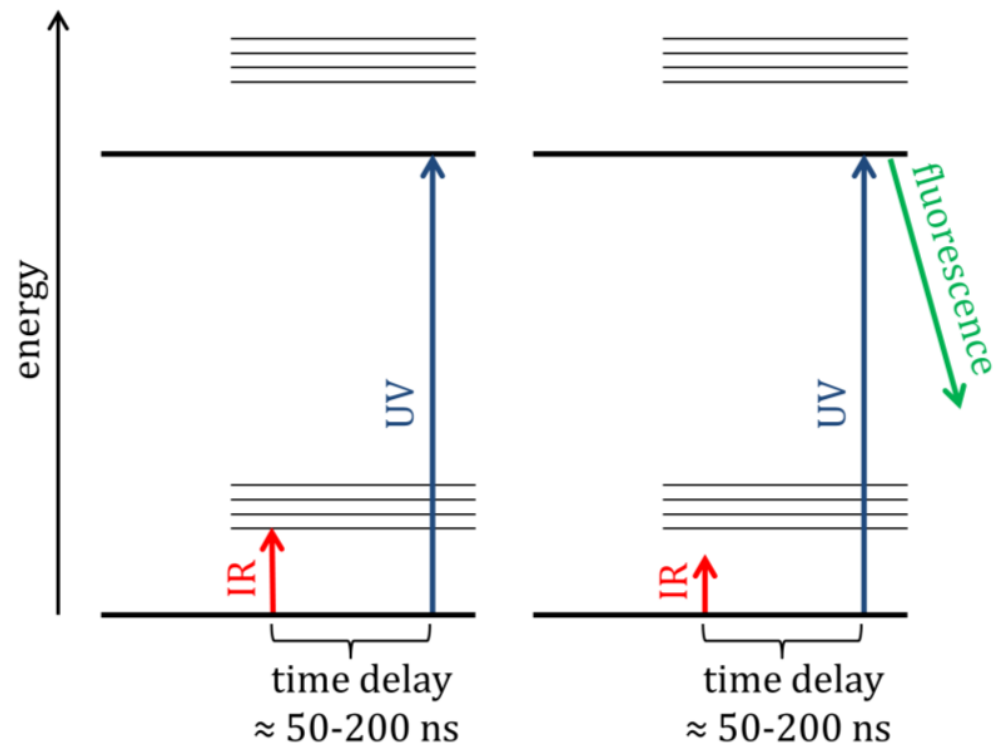

Figure 2.2: FDIRS excitation scheme after T. Zwier [34]. When IR and UV excitations are resonant on a transition belonging to the same ground state a depletion of the fluorescence signal is seen (left side), while no depletion occurs when the IR laser is non-resonant (right side). 


\section{4 - Quantum chemical calculations}

Quantum chemical calculations are of major importance when trying to distinguish between spectral influences of different conformers. Electronic energies, zero-point energies, Gibbs energies, vibrational modes and their spectral strengths all play an important role and can be predicted by quantum chemical calculations. All calculations in this work were performed on the GWDG cluster.

During the early stages of this work calculations were carried out using Gaussian 09 Rev. D.01 [39] which was, by that time, the latest available Gaussian version on the computational cluster. During later stages, Gaussian 09 was updated from Rev. D.01 to E.01 [40] in December 2015 and was made available on the GWDG cluster in April 2016. This update contained an important fix for errors in frequency calculations with Becke-Johnson damping when using Grimme's D3 dispersion [41] which will be explained in detail in Section 2.5. This had a crucial impact on some of the results, especially when calculating Gibbs energies, and made recalculations of almost all previous results in Rev. E.01 necessary.

Additional calculations were carried out using either TURBOMOLE 6.4 [42] or TURBOMOLE 7.0 [43]. Similar to Gaussian, TURBOMOLE got updated on the GWDG cluster during later stages of this work, yet there were found no relevant changes between the two versions regarding basic frequency and energy calculations. Therefore, results obtained with these two versions were equal and no recalculations were necessary.

Starting structures for geometry optimizations were usually generated by self-made scripts that created inputs for all possible backbone dihedral combinations of the investigated molecules under the assumption that only trans $\left(180^{\circ}\right)$ and gauche $\left( \pm 60^{\circ}\right)$ configurations result in stable conformations. In the investigations on alkylbenzenes, starting structures were generated by calculating the conformational equilibrium distributions on a MMFF (Merck Molecular Force Field) [44] using Spartan'08 v.1.2.0 [45]. Since this work focuses on different types and sizes of molecules, the individual processes of generating starting structures and their results will be discussed in the associated chapters.

Most geometry and frequency calculations were carried out using the B3LYP [46] functional. This hybrid functional is widely used and provides accurate predictions of wavenumbers of hydrocarbons at a moderate computational cost level [17]. Additionally, the double-hybrid functional B2PLYP $[47,48]$ was used. This functional contains additional MP2-like perturbation theory that makes it more costly but increases its accuracy [49]. The use of MP2 perturbation theory increases the size of the necessary basis set [50] and limited the use of this functional to smaller molecules. Grimme's latest dispersion correction D3 [7] with Becke-Johnson damping [41] was always added to the DFT functionals and will be abbreviated "2D3" if it only contains 
two-body dispersion corrections or "3D3" when three-body corrections ${ }^{\dagger}$ are added. Second order Møller-Plesset perturbation theory [51] alongside the CCSD(T) method [52-54] are used for single point calculations. These methods contain higher order electron correlations and provide more-accurate electronic energies. The CCSD(T) method is also known as the gold standard of quantum chemistry [55] referring to its very high accuracy. However this high accuracy comes at the drawback of heavily increased computational costs that restrict the use of these methods in this work to single point energy calculations. In general, MP2 and CCSD(T) single point calculations were performed with the resolution of the identity (RI) approximation for twoelectron integrals which reduce the necessary CPU time [56-60].

Paired with all mentioned methods were either the def2TZVP [61] or the augmented correlation-consistent aug-cc-pVXZ (with X = T,Q) [62-64] basis sets. The latter will be shortened to aVXZ and VXZ if diffuse functions were not added.

All geometry optimizations in Gaussian were performed with the Berny algorithm using redundant internal coordinates [65], invoked with the fopt keyword. Furthermore, an ultrafine integration grid and the abovementioned dispersion correction were invoked using the "int=ultrafine" and "empiricaldispersion=gd3bj" keywords, respectively. Other parameters were left to standard values. An scf-convergence of $10^{-8}$ and the $\mathrm{m} 4$ gridsize was used for DFT geometry optimizations in TURBOMOLE, while an scf-convergence of $10^{-10}$ and a density convergence of $10^{-8}$ was used for MP2 and $\operatorname{CCSD}(\mathrm{T})$ single point calculations.

In some cases vibrational perturbation theory to $2^{\text {nd }}$ order (VPT2) $[66,67]$ was used to estimate anharmonic effects on zero-point energies and wavenumbers.

As Raman experiments play a major role in the investigations discussed in this work the calculation of Raman scattering cross sections from quantum chemical results shall be discussed in more detail. Gaussian outputs provide the Raman activity $A_{k}$ and the depolarization ratio $P_{k}$ of the $k$ th vibration while TURBOMOLE outputs provide the related tensor invariants $\alpha_{k}^{\prime}$ and $\gamma_{k}^{\prime}$ of the polarizability tensor. The Raman activity can be linked to its related tensor invariants by the following formula [19].

$$
A_{k}=45 \alpha_{k}^{\prime 2}+7 \gamma_{k}^{\prime 2}
$$

The depolarization ratio of parallel to perpendicular polarized scattered light of the $k$ th vibration at a $90^{\circ}$ scattering geometry can be defined as [68]:

$$
P_{k}=\frac{\|(\mathrm{s})}{\perp(\mathrm{s})}=\frac{3 \gamma_{k}^{\prime 2}}{45{\alpha_{k}^{\prime}}^{2}+4{\gamma_{k}^{\prime}}^{2}} \leq 0.75
$$

Combining Eq. 2.1 and Eq. 2.2 shows that the Raman activity can be split based on its contribution from parallel and perpendicular scattered light.

\footnotetext{
$\dagger$ Note that the addition of three-body dispersion corrections was available in the TURBOMOLE but not the Gaussian program package. 


$$
A_{k}=\underbrace{45{\alpha_{k}^{\prime}}^{2}+4{\gamma_{k}^{\prime}}^{2}}_{\perp(\mathrm{s})}+\underbrace{3 \gamma_{k}^{\prime 2}}_{\|(\mathrm{s})}
$$

Since parallel and perpendicular polarized components of the scattered light are not dispersed with the same efficiency by the grating of the monochromator, the obtained Raman activities need correction [19]. The transmission ratio for perpendicular to parallel polarized scattered light depends on the wavenumber $\tilde{v}_{k}$ of the $k$ th vibration and can be approximated by $1.5+2 \cdot 10^{-4} \mathrm{~cm} \cdot \widetilde{v}_{k}$ in the range of 0 to $3600 \mathrm{~cm}^{-1}$ based on results from N. Lüttschwager with this detection setup [19]. Relative Raman activities $A_{k}^{\text {cor }}$ can then be calculated using the following formula:

$$
A_{k}^{\text {cor }}=45 \alpha_{k}^{\prime 2}+4{\gamma_{k}^{\prime}}^{2}+3 \gamma_{k}^{\prime 2} \cdot\left(1.5+2 \cdot 10^{-4} \mathrm{~cm} \cdot \tilde{v}_{k}\right)^{-1}
$$

Scattered light of totally symmetric vibrations preserves high amounts of the polarization of the incident light resulting in small depolarization ratios $P_{k}$. If the depolarization ratio is zero the corrected and uncorrected Raman activities are the same. When the depolarization ratio is large the corrected Raman activities are smaller than the uncorrected ones.

Raman scattering cross sections were calculated from the following equation [68].

$$
\sigma_{\Phi}^{\prime}=\frac{2 \pi^{2} h}{45 c \tilde{v}_{k}} \cdot \frac{\left(\tilde{v}_{1}-\tilde{v}_{k}\right)^{3} \tilde{v}_{1}}{1-\exp \left(-\frac{h c \tilde{v}_{k}}{k T}\right)} \cdot g_{k} A_{k}^{\text {cor }}
$$

Here $\sigma_{\Phi}^{\prime}$ is the Raman scattering cross section at a $90^{\circ}$ geometry, $\tilde{v}_{1}$ is the wavenumber of the laser, $\tilde{v}_{k}$ is the wavenumber of the $k$ th vibration, $g_{k}$ the degeneracy of this vibration and $A_{k}^{\text {cor }}$ the grating corrected Raman activity of this vibration. A vibrational temperature of $100 \mathrm{~K}$ was assumed in this work.

\section{5 - Gaussian Rev. D.01 vs. E.01}

As mentioned in the previous chapter, the Gaussian version update to E.01 fixed a bug, besides other things that were not crucial for this work, in the implementation of the Becke-Johnson damping when calculating frequencies using the D3 dispersion. In detail, the energy and gradients for the D3 dispersion model with Becke-Johnson damping are calculated correctly but one of the contributions to the Hessian is not included so that frequencies are not correctly calculated [69]. The actual missing term is the second derivative of the dispersion term which is proportional to $r^{-8}$, where $r$ is the distance between particles. Thus, higher-frequency vibrations are almost not affected as this term becomes negligible but there is a significant impact for vibrations in the lower frequency region.

This problem artificially lowers calculated vibrational frequencies obtained in Gaussian 09 Rev.D.01. Test calculations found that for modes above $50 \mathrm{~cm}^{-1}$ the differences between predicted wavenumbers between D.01 and E.01 were small, usually on the order of $1 \mathrm{~cm}^{-1}$. However, for modes below $50 \mathrm{~cm}^{-1}$ the discrepancy between the two versions quickly increased up to $10 \mathrm{~cm}^{-1}$ and more. It could even result in predictions 
of imaginary frequencies in D.01 even though the calculations were performed on relaxed and stable geometries which showed only fairly low real but no imaginary frequencies in E.01.

This becomes especially problematic when calculating Gibbs energies as contributions of low-frequency modes are especially important here. Contributions of each mode that met the requirement of $h c \tilde{v} \ll k T$ can be estimated by the following formula:

$$
\Delta G_{\text {mode }}^{0}=R T \cdot \ln \frac{h c \tilde{v}}{k T}
$$

Not only does this implementation bug lower the Gibbs energies calculated in D.01 as the vibrational modes are falsely shifted to lower frequencies. The Gibbs energy difference between D.01 and E.01 also strongly depends on the wavenumbers $(\tilde{v})$ of the low-frequency modes as it increases exponentially with the reciprocal of the wavenumber. Figure 2.3 shall demonstrate this issue on a series of $n$-alkylbenzenes with increasing number of carbon atoms in the alkyl chain. The geometries of all- $t, g 1$ and $g 1 g 2$ of the shown alkylbenzenes were optimized in Gaussian 09 Rev.D.01 and E.01 at the B3LYP-2D3/def2TZVP level and resulted in equal electronic energies ( $\left.E_{\mathrm{el}}\right)$. Then, the harmonic frequencies were calculated at the same level in both Gaussian versions and the differences in the calculated Gibbs energies between both versions were compared. As the alkyl chain length increases the frequencies of the lowest modes decrease as demonstrated in the progression for the lowest mode $\left(\tilde{v}_{1}^{\mathrm{e}}\right)$ of the three conformers calculated in Gaussian Rev.E.01 (bottom part in Figure 2.3). As anticipated, the Gibbs energy difference between E.01 and D.01 $\left(\Delta \Delta G_{\mathrm{e}-\mathrm{d}}^{0}\right.$, shown in the upper part in Figure 2.3) increases drastically when the wavenumbers of the lowest modes decrease. The difference becomes almost negligible when the lowest vibrational mode exceeds $50 \mathrm{~cm}^{-1}$ but rise to more than $5 \mathrm{~kJ} \cdot \mathrm{mol}^{-1}$ when the lowest vibrational mode is around $20 \mathrm{~cm}^{-1}$ or lower. Moreover, the bug-induced shift to lower frequencies in D.01 is not uniform and can vary from mode to mode and conformer to conformer. Thus, the all- $t$ conformer shows a much larger Gibbs energy discrepancy between E.01 and D.01 than $g 1$ or $g 1 g 2$, even though their lowest vibrational modes are in the same regime. It shall be noted that more modes than just the lowest one, which is shown for demonstration purposes, can have a big influence on the Gibbs energy difference. In essence, all modes that are predicted below $50 \mathrm{~cm}^{-1}$ will contribute to a significant difference in calculated Gibbs energies between D.01 and E.01.

Whenever accurate frequency or Gibbs energy predictions were important all results obtained in Gaussian 09 Rev.D.01 where recalculated in Gaussian 09 Rev.E.01. Differences in zero-point vibrational energies were found to be almost negligible so that a recalculation was not necessary when only electronic or zero-point corrected energies were needed. 


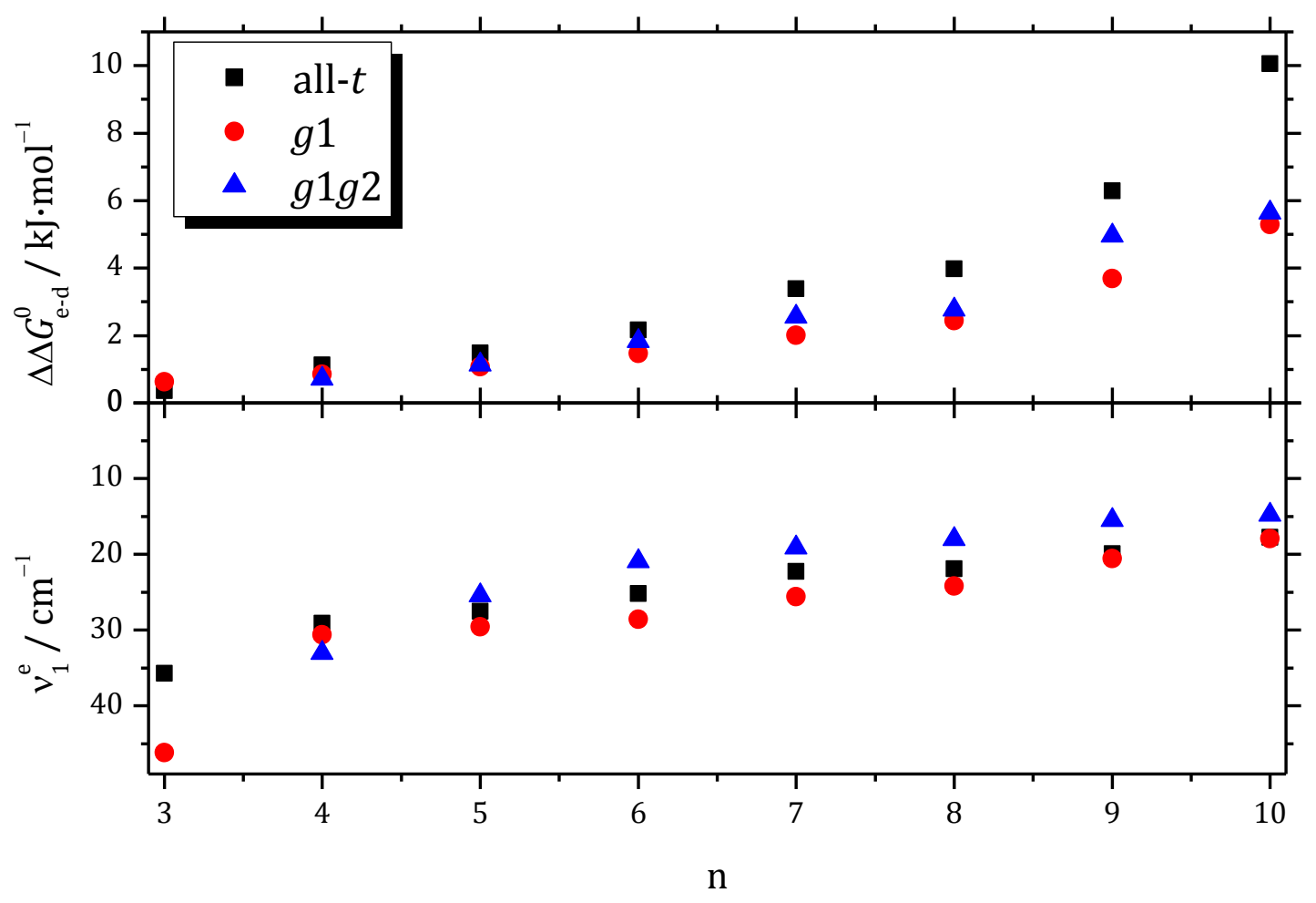

Figure 2.3: Gibbs energy differences $\left(\Delta \Delta G_{\mathrm{e}-\mathrm{d}}^{0}\right)$ between Gaussian Rev.E.01 and D.01 (top part) and the lowest vibrational frequency $\left(\tilde{v}_{1}^{\mathrm{e}}\right)$ predicted in Gaussian Rev.E.01 (bottom part) for all- $t$ (black squares), $g 1$ (red circles) and $g 1 g 2$ (blue triangles) in different $n$-alkylbenzenes with $n$ carbon atoms in the alkyl chain. Geometries of conformers were optimized in both Gaussian versions on the B3LYP2D3/def2TZVP level and showed equal electronic energies. Frequencies were then calculated on the same level in both Gaussian versions. 


\section{Chapter 3 - Experimental}

\section{1 - Curry-jet}

The so called curry-jet combines a locally configured Raman spectrometer with a custom built vacuum chamber in which molecules are cooled rapidly during a supersonic jet expansion. The acronym curry stands for classical unrestricted Raman spectroscopy which renders homage to $\mathrm{C}$. V. Raman by choosing an acronym that is also known as a traditional Indian food. He was the first to experimentally confirm the Raman effect together with Krishnan in 1928 [70]. The first version of this setup was built by Philipp Zielke [71] and was later improved by Tobias Wassermann [68], Zhifeng Xue [72], Nils Lüttschwager [19], Katharina Otto [25], myself and Thomas Forsting [73]. With these improvements the curry-jet became a versatile setup, allowing for investigations of small substances and aggregates as well as larger, nonvolatile molecules. As this work primarily focuses on glymes of different molecular sizes the versatility was used to full extent. The setup was already described in detail in the given references so that it will be outlined here more briefly. A general sketch of the setup is shown in Figure 3.2.

Volatile substances are filled into a glass saturator which can be cooled down to $-35^{\circ} \mathrm{C}$. A carrier gas, typically an inert noble gas, flows through the saturator and picks up some substance. This mixture is temporarily stored in a $4.7 \mathrm{~L}$ Teflon ${ }^{\circledR}$ coated stainless steel reservoir. From there it is admitted through heatable stainless steel tubes to the nozzle and expanded into the vacuum chamber. The curry-jet gas flow is quasi-continuous because the opening time of the reservoir to the saturator feed is controlled by a magnetic valve connected to a pressure gauge which monitors the reservoir pressure. Every time the reservoir pressure decreases below an adjustable threshold the magnetic valve will open for a time period that can be modified between 0.1 to $10 \mathrm{~s}$. If the threshold pressure is not yet reached the magnetic valve will open in subsequent pulses until the desired pressure is reached. This way the initial carrier gas pressure can be set higher than the reservoir pressure, further diluting the substance to reduce cluster formation. Less volatile substances that do not have a sufficient vapor pressure at room temperature are filled into a stainless steel saturator with a Teflon ${ }^{\circledR}$ core that can be heated up to $80^{\circ} \mathrm{C}$ by a PID controller. The substance of interest is applied onto a frit inside of the core to maximize surface interactions. Since the stainless steel reservoir cannot be heated this saturator is installed in the subsequent part that consists of heatable stainless steel tubes. From here on all parts up to the nozzle can be heated to at least $80^{\circ} \mathrm{C}$, thus preventing unwanted condensation of the substance in the setup. Since this design lacks the capability of reducing the gas flow pressure after passing the saturator a lower initial carrier gas pressure is used. Otherwise the stagnation pressure would be too high potentially overloading the vacuum pumps. All experiments in this work were performed with a $4 \times 0.15 \mathrm{~mm}^{2}$ slit nozzle made from stainless steel and painted black for stray light reduction. The vacuum chamber is continuously pumped by two Roots vacuum 
pumps (250 and $\left.500 \mathrm{~m}^{3} \cdot \mathrm{h}^{-1}\right)$ and a rotary vane pump $\left(100 \mathrm{~m}^{3} \cdot \mathrm{h}^{-1}\right)$, typically leading to a background pressure of $\sim 0.8$ mbar during expansions.

The resulting jet expansion is vertically probed by a $532 \mathrm{~nm} \mathrm{cw}$-laser. Due to various problems and breakdowns of some lasers a range of different lasers were used in this work. As light sources either served a Verdi V5, Verdi V18, Verdi G18, Millennia eV 20 or Millennia eV 25. The number behind the model name stands for the maximum output power. The Verdi V5 and V18 are single-mode lasers while the others are multimode lasers. Therefore, the resulting spectral resolutions in the curry-jet setup are not identical. Spectral band widths were found to be increased by approximately $4 \pm 2 \%$ when a multimode instead of a single-mode laser was used (for details see A.2). However, this effect was not too critical as the overall impact on the spectra was rather small as shown for a comparison of 1,2-dimethoxyethane ${ }^{\dagger}$ spectra recorded with the Verdi V-5 and the Millennia eV 25 (Figure 3.1). Moreover, spectra were typically only compared to other spectra that were measured with the same laser source.

The laser beam is focused perpendicular to the expansion by a plano-convex lens with a focal length of $50 \mathrm{~mm}$ and the scattered light is collected at a $90^{\circ}$ angle by a camera objective. The relative position of the nozzle and the camera lens can be controlled with precise motorized actuators. The collimated light is then focused by a collecting lens outside of the vacuum chamber onto the opening slit of a Czerny-Turner monochromator. Before entering the monochromator the light passes a Raman edge filter to suppress the Rayleigh light. The signal is finally detected by a liquid nitrogen cooled CCD camera with $1340 \times 400$ pixels. In order to suppress unwanted stray light an iris diaphragm is mounted onto the plano-convex focus lens and the laser beam path is mostly enclosed by anodized aluminum tubes. All principal components of the setup are listed in Table 3.1.

Increasing alignment stability was achieved through the implementation of a custom built optical cage system (Figure 3.3). This cage system uses four rigid anodized aluminum rods on which stray light protection walls, a lens mount and an iris diaphragm are mounted. The rods are placed on a $60 \times 60 \mathrm{~mm}^{2}$ square so that the cage is compatible with commercially available supplies. The lens mount hosts a plano-convex lens which can be translated by $\pm 2.5 \mathrm{~mm}$ in the $\mathrm{x}$ and $\mathrm{y}$ directions and $\pm 1.5 \mathrm{~mm}$ in the $\mathrm{z}$ direction within the mount. The lens is protected by a retaining ring on each side. The lens mount and its components were obtained commercially as listed in Table 3.2. Additionally, the cage can be mounted with custom built pinholes that aid in the alignment process. Unfortunately the cage system is not suited for measurements at high temperatures (nozzle temperatures $>100^{\circ} \mathrm{C}$ ) as lubricating oil starts to evaporate and condenses on the camera lens. Replacement of the lubricating oil by vacuumresistant oil did not led to an improvement. However, at lower nozzle temperatures the cage system lead to an increase in alignment stability and increased the averagely achieved signal-to-noise ratio.

\footnotetext{
† Band assignments and spectra of 1,2-dimethoxyethane will be discussed in detail in Chapter 6.
} 
The nozzle and detection optics are aligned prior on each measurement day or when the nozzle temperature was modified. For this purpose ambient air is expanded into the vacuum chamber and the Raman signal of the nitrogen vibration is optimized at a nozzle distance of $1 \mathrm{~mm}$ to the probing laser beam. The zero-position of the nozzle is calibrated by placing a neon emission lamp in the optical axis of the camera lens outside of the chamber and moving the nozzle forward until the emission lamp signals decrease to half of their initial values. Typically substance spectra are recorded between one to ten minutes. During these exposure times the CCD camera detects random intense sharp signals that are caused by cosmic rays. Therefore, spectra are recorded four to six times and compared to each other making use of a Matlab script written by Nils Lüttschwager [19] based on an algorithm developed by Philipp Zielke [71] which allows removal of cosmic ray events from the spectra. The neon lamp is placed as described above and its emission spectrum is used for wavelength calibration. All in all, the setup allows measuring spectra with an accuracy of $1 \mathrm{~cm}^{-1}$.

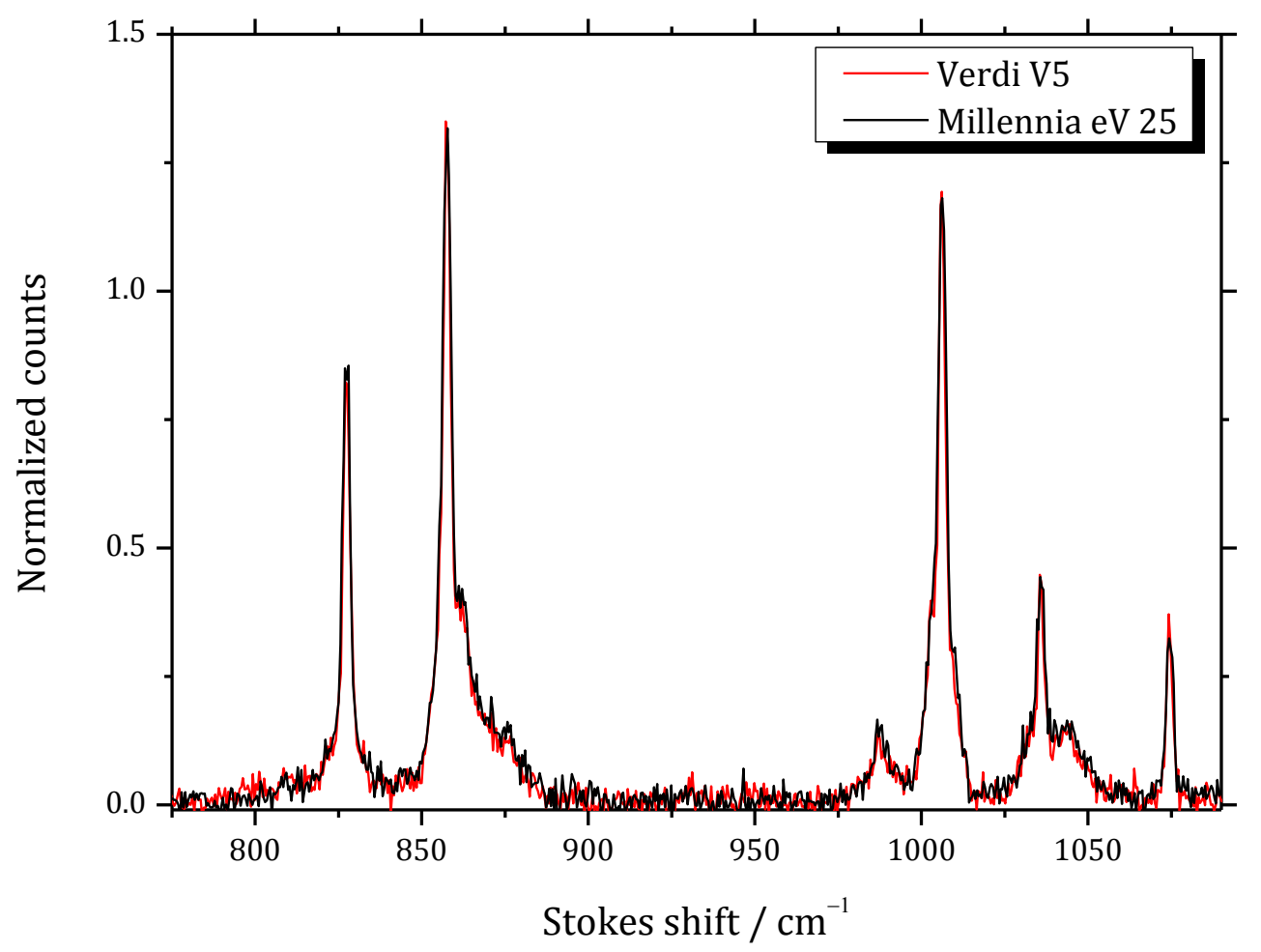

Figure 3.1: Raman jet spectra of 1,2-dimethoxyethane in He at a nozzle distance of $1 \mathrm{~mm}$ and a nozzle temperature of $20^{\circ} \mathrm{C}$. Spectra recorded with the Verdi V5 (red) were accumulated five times for $600 \mathrm{~s}$, while those recorded with the Millennia eV 25 (black) were accumulated five times for $120 \mathrm{~s}$. 


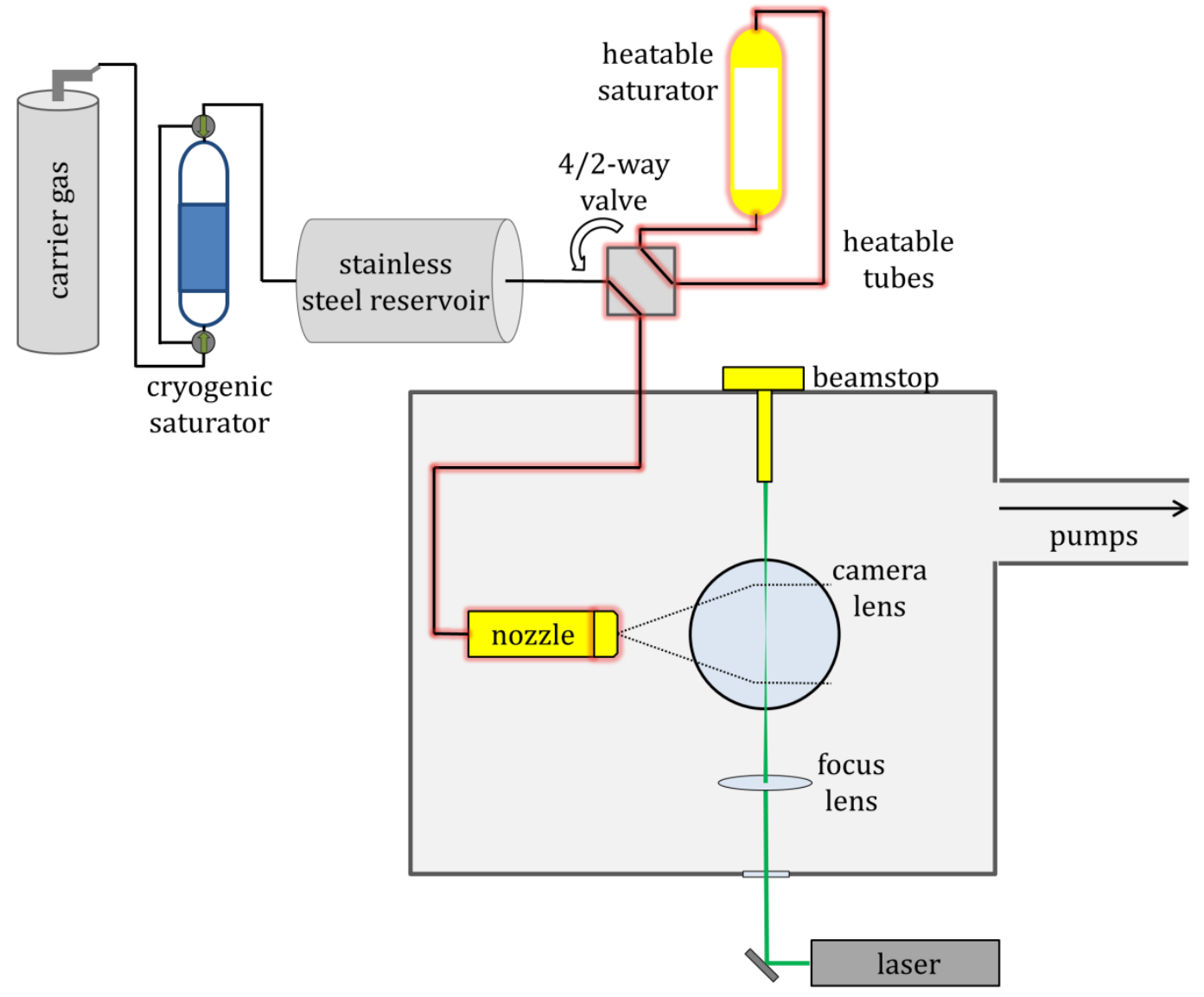

Figure 3.2: Sketch of the curry-jet setup. Heating is provided by resistance heating cables or wires that are wrapped around the components (indicated with red shades).

Table 3.1: Principal components of the curry-jet setup.

\section{Camera lens: Revuenon $f / 1.2,50 \mathrm{~mm}$ focal length}

Rayleigh-filter: LOT-Quantum Design, REFUS532-25 USLR, OD 6.0 (532 nm), $T>90 \%$ for $\lambda>533.7 \mathrm{~nm}$

Monochromator: McPherson Inc. Model 2501, $1 \mathrm{~m}$ focal length, aperture ratio $=8.7$, ruled grating with 1200 groves $\cdot \mathrm{mm}^{-1}$

Detector used for monoglyme measurements (Chapter 6): Princeton Instruments Spec-10-400B, back-illuminated CCD with $1340 \times 400$ pixel (pixel dimensions: $20 \mu \mathrm{m}$ x $20 \mu \mathrm{m})$, cryo-cooled to $-120^{\circ} \mathrm{C}$, quantum efficiency > 90\% (500-700 nm)

Detector used for all other measurements: Princeton Instruments PyLoN400B, backilluminated CCD with 1340 x 400 pixel (pixel dimensions: $20 \mu \mathrm{m} \times 20 \mu \mathrm{m}$ ), cryocooled to $-120^{\circ} \mathrm{C}$, quantum efficiency $>95 \%(600 \mathrm{~nm})$

Laser focus lens: Qioptiq, 312-300-322,ø $=22.4 \mathrm{~mm}, f=50 \mathrm{~mm}$, antireflective coating for visible light (ARB2-Vis)

Roots pumps: Pfeiffer vacuum, WKP 500 AM and WKP $250 \mathrm{AM}, 500$ and $250 \mathrm{~m}^{3} \cdot \mathrm{h}^{-1}$ pump capacity

Rotary vane pump: Dr.-Ing. K. Busch GmbH, UNO $101 \mathrm{~S}, 100 \mathrm{~m}^{3} \cdot \mathrm{h}^{-1}$ pump capacity 

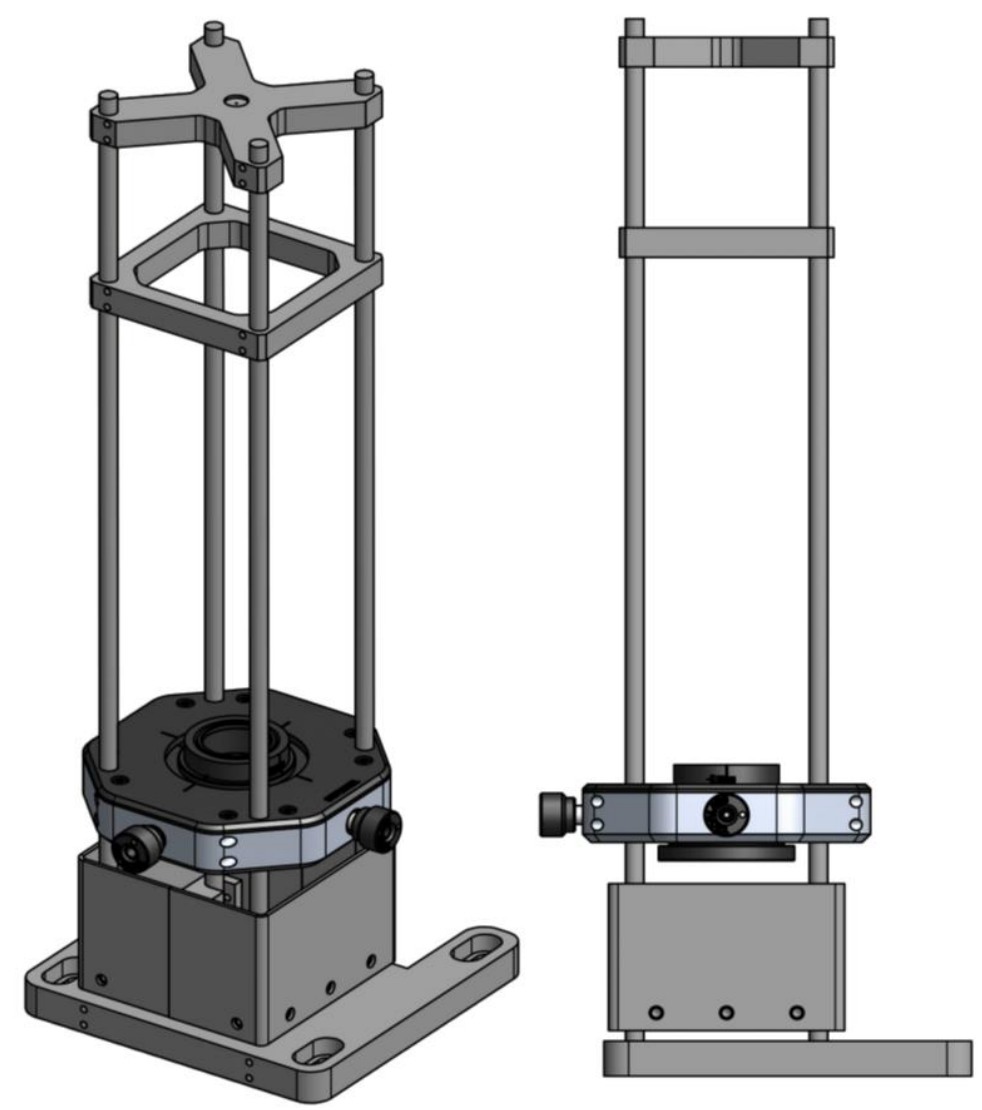

Figure 3.3: Sketch of the custom built optical cage system.

Table 3.2: Lens mount components

Lens mount: Thorlabs, CXYZ1/M, $\pm 2.5 \mathrm{~mm}$ in $\mathrm{x}, \mathrm{y}$ directions and $\pm 1.5 \mathrm{~mm}$ in z direction, compatible with optics up to $10.0 \mathrm{~mm}$ thick and $\varnothing=25.4 \mathrm{~mm}$

Mount lens: Thorlabs, LA4148-YAG, $\varnothing=25.4 \mathrm{~mm}, f=50.2 \mathrm{~mm}$, antireflective coating for visible light

Retaining rings: Thorlabs, SM30RR, $\varnothing=30 \mathrm{~mm}$

\section{2 - LIF setup}

The LIF setup combines a custom built jet chamber with UV and IR laser sources and allows for detection of fluorescence events during supersonic jet expansions. The setup is located in the Zwier group at Purdue University (West Lafayette, USA) and is described in detail in references $[74,75]$ and [76]. Thus, it will be only briefly outlined here.

The substance of interest is applied onto a glass insert filled with glass wool and is placed inside a stainless steel sample holder (Swagelok SS-4F-2, gas filter removed) which can be resistively heated up to $250^{\circ} \mathrm{C}$. A carrier gas, typically helium, flows through the sample holder entraining some substance. The gas mixture is then expanded by a pulsed valve (Parker General, Valve series 9) at a repetition rate of $20 \mathrm{~Hz}$ with a pulse duration of $250 \mu$ s through a small orifice $(\varnothing=800 \mu \mathrm{m}$ ) into a vacuum 16 
forming a supersonic expansion. The chamber is evacuated using a Roots pump (Leybold, model WS 100) backed by two roughing pumps (Sargent-Welch, model 1398). Initial carrier gas pressures were set between 3 and 4 bar for experiments in this work and resulted in chamber pressures between 0.04 and 0.07 mbar. The expansion is probed approximately $1-2 \mathrm{~cm}$ downstream from the nozzle by tunable UV and IR laser sources. The desired UV laser radiation is generated by a frequency doubled tunable dye laser (Lambda-Physik, Scanmate) which is pumped with the third harmonic of a high-powered pulsed Nd:YAG laser (Continuum 660). Coumarin 540A was used as a laser dye throughout this work. Typically UV laser outputs between 3 and $6 \mathrm{~mJ}$ per pulse were achieved. A LaserVision optical parametric converter was used to generate tunable infrared radiation in the $\mathrm{CH}$ stretching region (2800$3100 \mathrm{~cm}^{-1}$ ). For that the $1064 \mathrm{~nm}$ output of a seeded Nd:YAG laser (Continuum 8020) was split using a 30/70 beam splitter. The frequency of the $30 \%$ part was doubled by a potassium titanyl phosphate (KTP) crystal before it passed through two phase matched KTP crystals. This optical parametric oscillator (OPO) stage produces tunable signal $\left(\sim 12500 \mathrm{~cm}^{-1}\right)$ and idler $\left(\sim 6300 \mathrm{~cm}^{-1}\right)$ radiations upon angle tuning of the crystals. The idler of the OPO stage is used to seed a second stage, serving as the signal for a 1064-pumped KTA stage. Then, the OPO idler signal is difference frequency mixed with the $70 \%$ of the YAG fundamental in three KTA crystals. This optical parametric amplifier (OPA) produces an idler beam with an output power between 20 and $40 \mathrm{~mJ}$ per pulse.

The jet chamber contains two baffle arms perpendicular to the direction of the jet expansion flow which allows for counterpropagation of multiple laser sources. Depending on the type of experiment either one UV (LIF), two UV (UV-UV HB) or one UV and one IR laser sources (FDIRS) are used. The resulting fluorescence in these experiments is collected by two spherical mirrors and focused by a plano-convex lens onto a photomultiplier tube (PMT). The output of the PMT is digitized by an oscilloscope that is linked to a PC which stores the total fluorescence signal as a function of excitation wavelength. A sketch of the setup is shown in Figure 3.4. For double resonance experiments the saturation laser was operated at $10 \mathrm{~Hz}$ while the probing laser was operated at $20 \mathrm{~Hz}$. The saturation laser temporally precedes the probe laser by $200 \mathrm{~ns}$. The fluorescence signals from the probe laser with and without the saturation laser present are subtracted on a shot-to-shot basis by using the active baseline subtraction mode of a gated integrator (Stanford Research, SR250). During LIF measurements the UV laser beam was defocused and attenuated by glass plates in order to obtain unsaturated spectra.

RIDIR spectra were recorded in a similar setup that uses a time of flight (TOF) detection scheme. Resulting ions from R2PI processes are accelerated into a field free region of a $1 \mathrm{~m}$ long flight tube. Necessary kinetic energies were provided by a repeller plate and a draw-out grid that are usually operated around $4000 \mathrm{~V}$. Ions then enter a second vacuum chamber where they are detected on a $2.5 \mathrm{~cm}$ micro channel plate (MCP). The source and detection chambers are evacuated by turbomolecular pumps (Pfeifer vacuum, THM 1001 and Pfeifer vacuum, THM 261, respectively) which are 
backed by mechanical roughing pumps resulting in chamber pressures of $10^{-5}$ and $10^{-6}$ mbar, respectively.

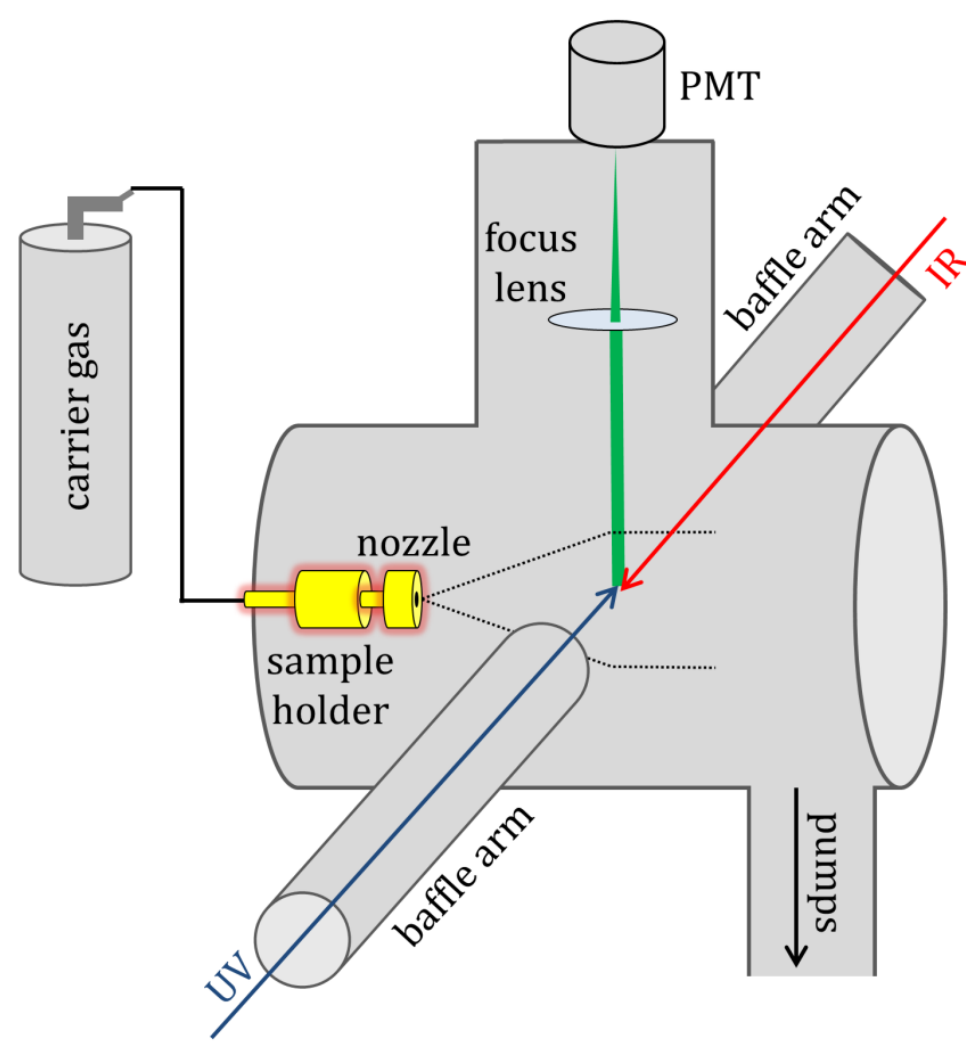

Figure 3.4: Sketch of the LIF setup. Heating is provided by resistance heating cables or wires that are wrapped around the components (indicated with red shades).

\section{3 - IR-Raman double resonance setup}

In an attempt to perform IR-Raman double resonance experiments the curry jet setup (Chapter 3.1) was extended with a tunable cw IR laser (Argos, 2400-BB-30) which was extensively characterized by Katharina Otto [25]. This optical parametric oscillator (OPO) can produce up to $6 \mathrm{~W}$ of infrared light in the wavenumber region between 3125 and $4000 \mathrm{~cm}^{-1}$. The output wavenumber can be modified by adjusting the temperature or position of the periodically poled lithium niobate crystal (PPLN) and test measurements performed by Katharina Otto showed that the resulting IR beam has a diameter of 3-4 mm and a linewidth of $4-6 \mathrm{~cm}^{-1}$ [25]. The Argos OPO is situated at a platform above the jet chamber and can be fed either into the chamber or a FTIR spectrometer through a mirror on a flip mount. IR wavelengths are determined in a modified FTIR spectrometer (Bruker Optik, Vector 22) where the broadband light source was replaced by two mirrors to deflect the IR light to the deuterated triglycine sulfate (DTGS) detector. When the IR light is fed into the jet chamber it is deflected by a fixed and a movable mirror, which is controlled by a precise motorized actuator. Finally, the IR beam is focused by a cylindrical lens (Korth Kristalle, $\mathrm{CaF}_{2}$-IR-lens, plan convex, $22 \mathrm{~mm} \times 22 \mathrm{~mm}, f(193 \mathrm{~nm})=45 \mathrm{~mm})$ onto the cross section between the 
Raman laser and the jet expansion. The position of the cylindrical lens can also be controlled with a precise motorized actuator. The OPO platform is sketched in Figure 3.5, while the beam paths in the jet chamber are shown in Figure 3.6.

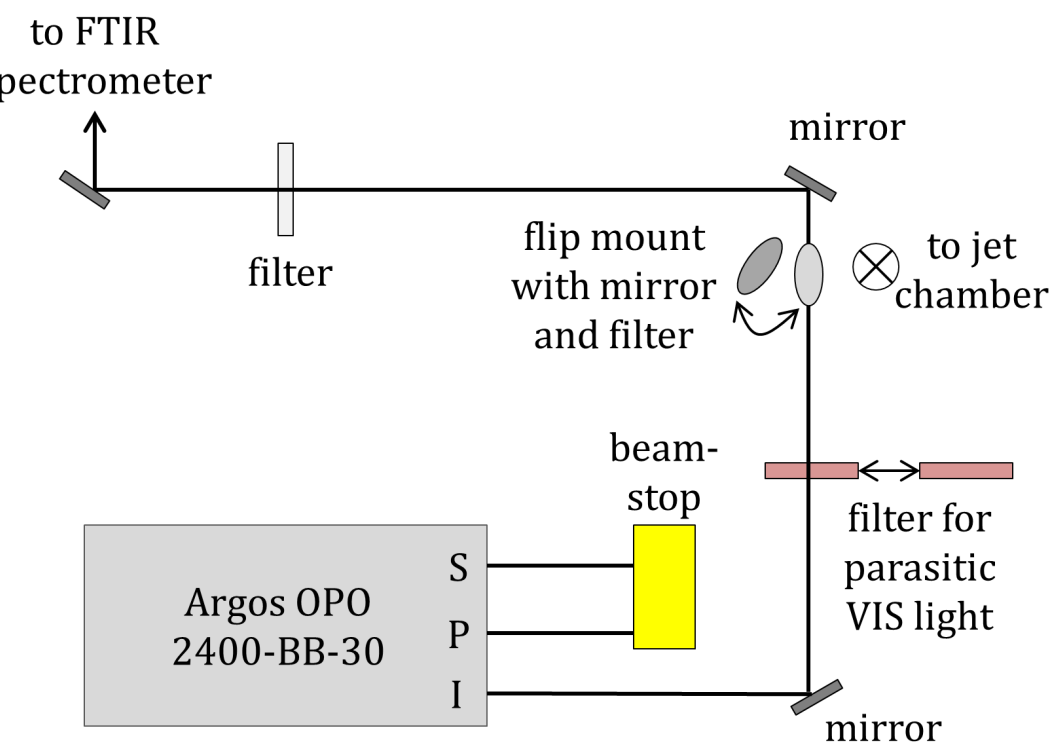

Figure 3.5: Sketch of the OPO platform which is situated above the jet chamber. Signal (S) and pump (P) beams are blocked by a beamstop, while the idler beam is used for IR excitation. The IR source can be fed either into the jet chamber or to a FTIR spectrometer. A flip mounted with a mirror and a filter allows switching between both pathways.

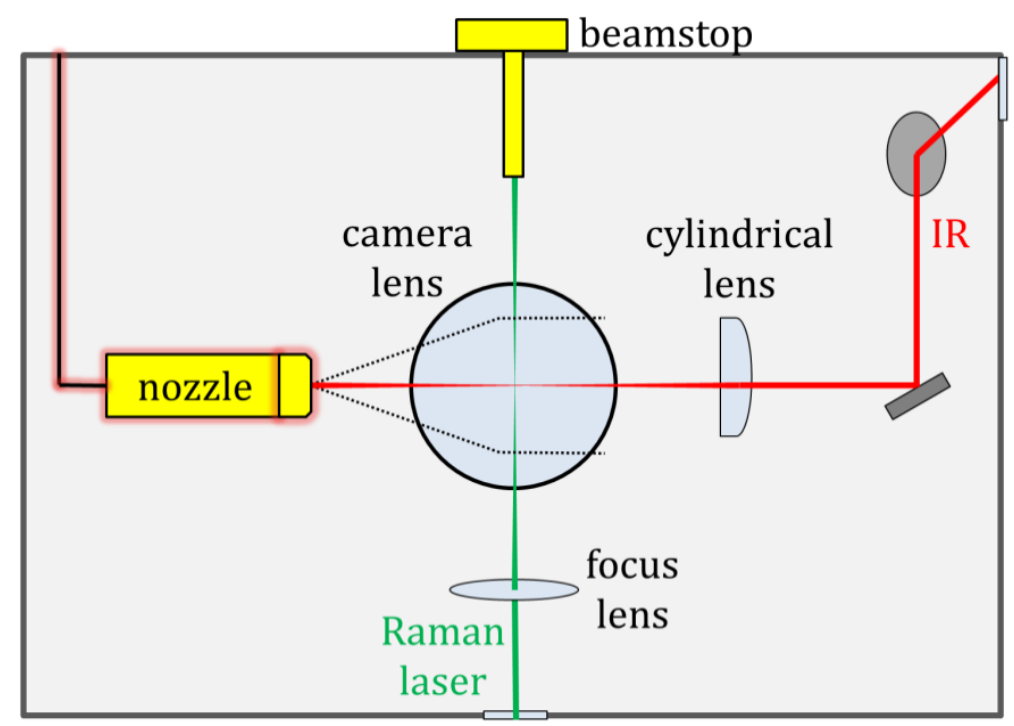

Figure 3.6: IR-Raman double resonance excitation scheme in the curry jet chamber.

The alignment of such a double resonance experiment is not trivial as not only the IR and Raman laser need to share an optimized cross section with the jet expansion but also the position of the camera lens has to be adjusted to the center of the cross section between the lasers and the jet expansion. This is aggravated by the fact that it is not known yet if the anticipated IR excitation effects are larger when the IR laser is focused in the region between the nozzle and the focal point of the Raman laser or if it is more beneficial to overlap the focal points of both lasers. A modified alignment rou- 
tine from the one established by Katharina Otto [25] is described in detail subsequently.

First, the camera lens is adjusted through maximizing the nitrogen vibration in a jet expansion of ambient air at a nozzle distance of $1 \mathrm{~mm}$ to the Raman laser. Then, a custom built attachment made from stainless steel is placed onto the nozzle. This nozzle cap is extended by a thin stainless steel $\operatorname{rod}(3 \mathrm{~mm}, \varnothing=0.75 \mathrm{~mm})$ that is sharpened at the end (Figure 3.7). This attachment extends the nozzle by $5.0 \pm 0.1 \mathrm{~mm}$ in total. The Raman laser power is tuned down to $0.1 \mathrm{~W}$ and the nozzle is moved to a position where the tip of the attachment hits the focused Raman laser beam. This allows adjusting the nozzle center to the Raman focus and the camera lens by monitoring the scattered Rayleigh light from the stainless steel tip. These adjustments are very critical because jet expansion experiments in the curry jet use a slit nozzle which allow for slight mismatches of the vertical position of the camera lens with respect to the expansion center without immediate decrease in Raman signal. This is reflected by the fact that in the usual Raman alignment procedure (Chapter 3.1) the optimal vertical camera lens position is not found at a single point but rather a plateau of points yielding equal Raman intensities. However, in double resonance experiments the vertical position of the camera lens becomes very critical as any non-centered position could mean that an expansion region is probed that might not be strongly affected by IR excitations.

Afterwards the IR beam is aligned in multiple steps. In the first instance the beam is visually prealigned using the parasitic green and red light that the laser produces in a sub-mW regime. During normal measurements this light is suppressed by placing a filter in the beam path (compare Figure 3.5). Afterwards, a small fluorescing plate is pulled over the thin stainless steel rod allowing to detect and align the IR beam as it depletes the fluorescence. This fluorescence depletion can only be seen at low laser powers as higher output powers lead to thermal deactivation of the fluorescence plate. The alignment is then checked and refined at the full output power by visually monitoring the stainless steel tip which starts thermally radiating when the IR beam is properly focused on it. The jet chamber is then evacuated and the alignment of the IR laser is checked and refined again using the glowing tip as an indicator for proper alignment. In vacuum the heating tip cannot dissipate its thermal energy efficiently leading to a rise in its temperature which enhances the intensity of the observed radiation. Therefore, it is easier to monitor the glowing tip in vacuum than in ambient air. Moving the IR focus in the horizontal axes by adjusting the position of the cylindrical lens or the mirror rapidly decreases the intensity of the thermal radiation and thus is anticipated to allow for an alignment of the horizontal IR beam position by $\pm 0.5 \mathrm{~mm}$.

The biggest uncertainty regarding the alignment is the unavoidable movement of the optical components upon evacuation. In tests where the alignment was performed as described and then rechecked after all components were passed through multiple cycles of evacuation and ventilation it was found that the alignment slightly drifts from the optimal settings. Since this occurring drift is likely different in every optical component a readjustment of the camera lens positions through a jet expansion of 
ambient air, as described above, is only performed for its horizontal positions. A readjustment of the vertical positions of the camera lens and the two laser foci would require ventilation of the chamber in order to reinstall the nozzle attachment which caps the nozzle and prevents the formation of jet expansions. Thus, vertical positions cannot be realigned. However, the occurring alignment drifts after passing multiple evacuation cycles were found to be $<0.1 \mathrm{~mm}$ and therefore less than the expected accuracy of the IR beam alignment.

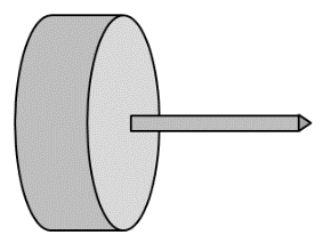

Figure 3.7: Nozzle attachment with a thin stainless steel extension.

As a promising first attempt to establish IR-Raman double resonance experiments in the curry jet the dissociation of weakly-bound molecular complexes through excitation of intramolecular modes was chosen [77]. Comparison of Raman spectra with and without a simultaneous IR excitation will show differences in the associated cluster bands upon partial dissociation. Prime candidates for such investigations should have a strong IR-active band within the OPO laser region $\left(3125-4000 \mathrm{~cm}^{-1}\right)$. Furthermore, the cluster should have a strong Raman band, which can be different from the one that is excited by the IR laser, allowing for a good signal-to-noise ratio. Additionally, the dissociation energy of the investigated cluster needs to be overcome by the IR photon. It is beneficial if the cluster is thermally stable up to $400-500 \mathrm{~K}$ as the nozzle functions as a beam block for the IR light and thus is constantly heated while the IR laser is on. Methanol (MeOH), 2,2,2-trifluoroethanol (TFE) and 1,1,1,3,3,3hexafluoro-2-propanol (HFIP) were chosen for further investigations as promising candidates.

Series of Raman jet spectra under different expansion conditions were taken for each substance in order to determine optimized expansion parameters that allow for large dimer concentrations while showing minimal contributions from trimers and higher oligomers. All these spectra were taken in argon jet expansions because the velocity in these expansions is lower than the velocity in helium expansions, allowing for a longer interaction time between the molecules and the IR excitation laser in later double resonance experiments. The determined optimized expansion parameters for each substance are summarized in Table 3.3.

Table 3.3: Optimized expansion parameters that allow for a maximum dimer concentration of each substance in the curry jet.

\begin{tabular}{|c|c|c|c|c|}
\hline substance & $\boldsymbol{T}_{\text {saturator }} /{ }^{\circ} \mathbf{C}$ & $\boldsymbol{T}_{\text {nozzle }} /{ }^{\circ} \mathbf{C}$ & $\boldsymbol{p}_{\text {carrier gas }} /$ bar & $\boldsymbol{p}_{\text {stagnation }} / \mathbf{b a r}$ \\
\hline $\mathrm{MeOH}$ & -15 & 130 & 1.9 & 0.50 \\
\hline $\mathrm{TFE}$ & -5 & 190 & 2.1 & 0.65 \\
\hline $\mathrm{HFIP}$ & -3 & 150 & 2.1 & 0.50 \\
\hline
\end{tabular}


The anticipated decrease of the $\mathrm{MeOH}$ dimer band upon IR excitation and perfect overlap was estimated to be $>30 \%$ in argon expansions [25], while even larger effects are expected in TFE and HFIP due to their stronger dimer bands in the infrared. Therefore, TFE and HFIP were extensively tested with varying mirror and lens positions in order to alter the IR focus with respect to the focus point of the Raman laser. Unfortunately no significant reproducible changes upon IR excitation were found in the Raman jet spectra (shown in A.3).

One of the largest problems that might prevent the observation of the relatively large anticipated effects is that the cross-section between the IR and the Raman laser is suboptimal. The beam diameter of the focused Raman laser is almost four times smaller than the beam diameter of the focused IR laser [25]. This means that approximately $65 \%$ of the OPO output power is not actually used in the double resonance detection scheme. The cross section was significantly improved by replacing the $f=$ $50 \mathrm{~mm}$ focus lens of the Raman laser with an $f=100 \mathrm{~mm}$ lens. Additionally, a second cylindrical lens (Korth Kristalle, CaF2-IR-lens, plano-convex, $22 \mathrm{~mm} \times 22 \mathrm{~mm}$, $f(193 \mathrm{~nm})=147.51 \mathrm{~mm})$ was used, with its focus plane perpendicular to the other cylindrical lens, in order to further collimate the IR beam. Unfortunately, only a few unsuccessful measurement attempts were possible with these adjustments as several problems with the high powered cw Raman and IR lasers interfered with the continuation of this work.

For further investigations nitrogen complexes with HFIP are a promising candidate as nitrogen clusters are found for two different HFIP conformers in jet expansions, separated by a small interconversion barrier [78]. This would possibly allow investigating isomerization processes induced by IR excitation between both HFIP conformers. Additionally, the associated cluster bands are predicted by quantum chemical methods to be stronger than the HFIP dimer bands [78], allowing for better IR excitation efficiency and Raman detection. A potential drawback is the usually limited abundances of these clusters in supersonic jet expansions. Therefore, future investigations should determine optimal expansion parameters and compare relative abundances between HFIP-nitrogen clusters and HFIP dimers.

Furthermore, the alignment procedure especially for the IR laser in vacuum needs to be improved. In the current state the nozzle attachment can be used in vacuum or ambient air but it blocks the nozzle exit, thus preventing supersonic jet experiments. Therefore, it needs to be removed prior to any measurements. The implementation of a feedback for the IR alignment that can be used in ambient air as well as in vacuum at the full laser power without interfering with the formation of the jet expansion is crucial to the success of the double resonance experiment.

The CCD camera is typically binned over all vertical pixels to ensure a good signal-tonoise ratio. Constraining this binning to only a few hundred pixels in the center of the CCD camera will result in worse signal-to-noise ratios but would allow probing a smaller region of the expansion. If the IR beam is then tightly focused by a spherical lens onto this probed region one could use the full IR output over a small spatial area. 


\section{Chapter 4 - Alkylbenzenes}

In a joint project between the Zwier (Purdue University), Sibert (University of Wisconsin-Madison) and Suhm (Göttingen University) groups UV, IR and Raman jet techniques were combined with density functional and anharmonic local mode theory in order to identify the first folded alkylbenzene. UV and IR spectra of propyl- through decylbenzene were recorded together with Dan Hewett during a Fulbright research stay at the Purdue University. Also, Raman jet spectra of hexyl- through decylbenzene were recorded together with Dan Hewett at Göttingen University. An anharmonic local mode theory developed by Ned Sibert and Daniel Tabor was used to assign and interpret single-conformation IR spectra. This anharmonic local mode theory includes mixing of $\mathrm{CH}$ stretching modes with overtones of scissor/bend fundamentals of the $\mathrm{CH}_{2}$ and $\mathrm{CH}_{3}$ groups in the alkyl chain and is described in detail in [79]. Parts of this work, including calculations, models, plots and conclusions, have been published in [80].

\section{1 - Introduction}

Alkylbenzenes are main components of petroleum-based gasoline and diesel fuels with proportions ranging from $20-30 \%$ of their chemical composition [81]. In these fuels, single- and multiple-chain alkylbenzenes, with chain lengths varying from one to ten or more carbon atoms, are found [82-84]. Recently, decylbenzene was proposed as a surrogate for long-chain alkylbenzenes in diesel fuel [85]. Interestingly, the presence of these long-chain alkyl groups attached to an aromatic ring leads to combustion characteristics that are usually found in aromatics and alkanes separately [85].

Pure alkane chains in this size regime possess complicated conformational energy landscapes with a vast number of different conformers [19]. In alkylbenzenes the presence of the aromatic $\pi$ cloud leads to dispersive interactions between the benzene ring and the alkyl chain $[79,86,87]$. In $n$-alkanes the dihedral angles around each C-C single bond prefer trans $\left(180^{\circ}\right)$ over gauche $\left( \pm 60^{\circ}\right)$ arrangement due to steric effects [88]. Therefore, the all-trans conformation is the global minimum structure up to carbon chain lengths of $17 \pm 1$ [18], with each gauche causing a destabilization of around $2.5 \mathrm{~kJ} \cdot \mathrm{mol}^{-1}[89,90]$. At a critical chain length of $17 \pm 1$ carbon atoms the dispersive interactions between the alkyl chains counteract the steric effects and enable folding motifs, that loop back on themselves, to compete for the global minimum structure [18]. At chain lengths consisting of 20 or more carbon atoms these hairpin structures, containing a sequence of four homochiral gauche defects separated by a single trans dihedral (ggtgg), quickly become the dominant conformational motif [18]. As an example a hairpin structure for octadecane is shown in Figure 4.1. This sequence places two commensurate all-trans portions of the alkane chain in parallel arrangement, as predicted by theory [91]. 
Here, a similar investigation is presented that focuses on the question when the first folded motif that competes energetically with extended chains is found in alkylbenzenes. The presence of the phenyl ring at one end of the alkyl chain provides an electron-rich $\pi$ cloud to interact with the alkyl chain through attractive dispersion forces that are likely to be greater than between two alkyl chain segments [92,93]. For the investigation a combination of complementary experimental techniques, including LIF, UV-IR double resonance and Raman spectroscopies were utilized and combined with harmonic energy calculations and an anharmonic local mode model to assign the main conformers of pentyl- through octylbenzene, and partially assign spectra of decylbenzene. All spectroscopic methods were carried out in jet-cooled samples in the gas phase. In the following section the enumeration of different alkylbenzene conformers will be presented. Subsequently, the quantum chemical calculations will be outlined. Afterwards, the LIF, followed by the single-conformer IR and Raman spectra will be shown and discussed. Eventually, a comparison to the $n$-alkanes is presented, while general conclusions are drawn in the final section.

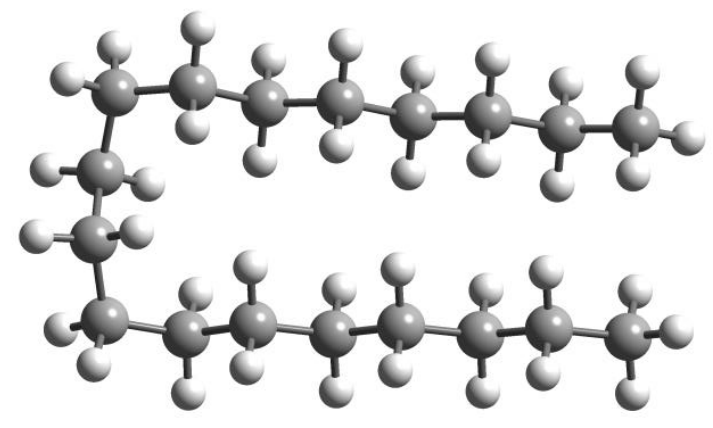

Figure 4.1: Hairpin conformer in octadecane with a ggtgg sequence in the turn.

\section{2 - Enumeration of alkylbenzenes}

Potential energy scans at the B3LYP-2D3/def2TZVP level in Gaussian09 Rev. E.01 revealed that the torsion of the aromatic ring with respect to the alkyl chain has a very low barrier and often prefers a $90^{\circ}$ out-of-plane arrangement (as shown for the all- $t$ conformation of hexylbenzene in Figure 4.2). In fact, all studied conformers were found to be more stable with a relative angle of the aromatic ring and alkyl chain planes close to $90^{\circ}$. The in-plane configurations of all conformers showed very shallow minima as demonstrated in Figure 4.2 and thus can interconvert easily. Therefore, a denotation of the relative angle between the alkyl chain and aromatic ring plane is obsolete. The alkylbenzene conformers will then be designated by their backbone dihedral angles in the alkyl chain starting from $C_{p h}-C_{1}-C_{2}-C_{3}$, where $C_{p h}$ is the carbon atom of the aromatic ring that is connected to the alkyl chain. Dihedral angles of $180^{\circ}$ are denoted as $t$ for trans and angles of $\pm 60^{\circ}$ are designated as $g$ for gauche. If a conformer has multiple gauche kinks of opposite directions then the direction that is found more often will be denoted as $g$ while the opposite directed ones will be denoted $g^{\prime}$. This enumeration is widely used in $n$-alkane chains $[19,94]$. In higher alkylbenzenes the notation of the full alkyl chain becomes quite cumbersome 
and thus only deviations from the all-trans structure will be specified. For unambiguous assignments the location of these deviations will be denoted with a number corresponding to the first carbon atom that is involved in the gauche kink. Numeration for the carbon atoms start with $\mathrm{C}_{\mathrm{ph}}$ and then follow the alkyl chain as shown in Figure 4.3. For example, the $g t$ conformer of butylbenzene will simply be referred to as $g 1$. A fully stretched all-trans chain will be denoted as all- $t$.

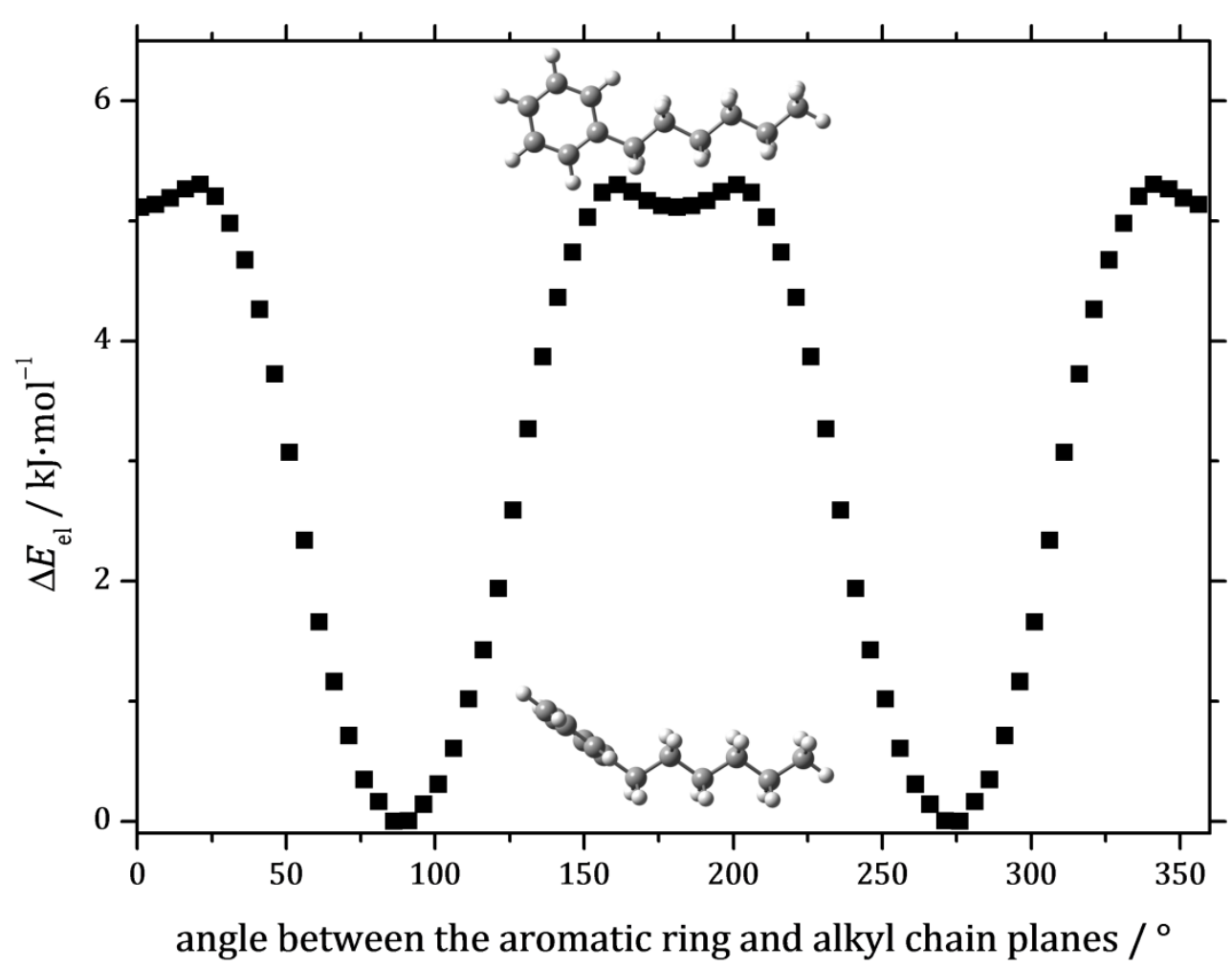

Figure 4.2: Relaxed torsional scan of the angle between the planes of the aromatic ring and the alkyl chain in the all- $t$ conformer of hexylbenzene calculated at the B3LYP-2D3/def2TZVP level in Gaussian 09 Rev. E.01.

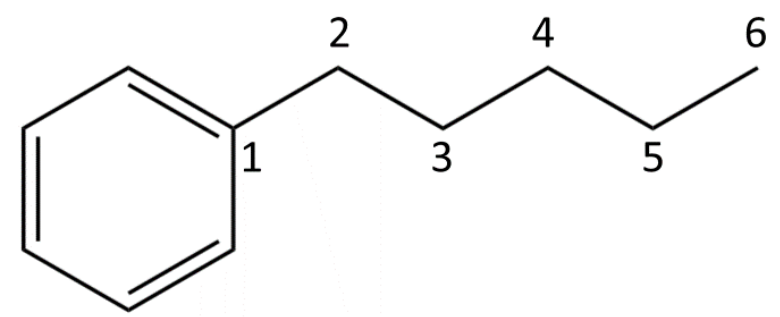

Figure 4.3: The all- $t$ conformer of pentylbenzene with indicated carbon numeration.

\section{3 - Quantum chemical calculations}

As stated in [80] "Conformational equilibrium distributions for the $n$-alkylbenzenes have been calculated on a MMFF (Merck Molecular Force Field) level using Spartan'08 v.1.2.0. All conformers within a threshold of $10 \mathrm{~kJ} \cdot \mathrm{mol}^{-1}$ relative to the global minimum structure were re-optimized and their energies and frequencies have been calculated with the B3LYP-2D3/def2TZVP method in Gaussian 09 Rev. E.01. The 
def2TZVP basis set represents a good compromise between computational cost and reliable results as bigger basis sets were not feasible for $n$-alkylbenzenes with an alkane chain longer than 5 carbon atoms. Previous studies have provided evidence that this level of theory provides good correspondence with experiment in ordering the relative energies of conformers [95]. Harmonic vibrational frequency calculations were carried out on the full series of alkylbenzenes studied at the same level of theory". On the basis of the calculated harmonic frequencies it was possible to assign and interpret the LAM region spectra as this region is very sensitive to the molecules conformation and bands of different conformations are usually well separated. However, for an accurate interpretation of the $\mathrm{CH}$ region anharmonic corrections are necessary as this region shows strong Fermi resonance couplings between the $\mathrm{CH}$ stretch fundamentals and overtones of the scissor and bending motions. The full anharmonic model developed by Ned Sibert and Daniel Tabor shall only be outlined briefly but is described in detail in [79]. Anharmonic couplings between stretches, scissor overtones, and combination states change little with chain length and conformation. Therefore their average values were used for the construction of the Hamiltonian rather than explicitly calculating them for each conformer. The model Hamiltonian was constructed from the Hessian matrix and dipole derivatives of each conformers reoptimized geometry at the B3LYP-2D3/6-311++G(d,p) level.

Results for selected conformers of the harmonic calculations at the B3LYP2D3/def2TZVP level in Gaussian 09 Rev. E01 for butyl- through decylbenzene are shown in Table 4.1 (a more detailed Table is found in A.4). The conformationally density increases drastically from butyl- to hexylbenzene as more possibilities for gauche kinks along the alkyl chain become possible (compare A.4). However certain patterns and trends emerge along the series that will be briefly outlined here and discussed in more detail in consideration of the experiments in the following sections. Afterwards a comparison to the $n$-alkanes is drawn in Section 4.7. The all- $t$ and $g 1$ conformers are very close in energy and compete for the global minimum in butyl- throughout octylbenzene. The third most stable structure in butyl- up to heptylbenzene is $g 1 g 2$ with a predicted energy difference of around $1.2 \mathrm{~kJ} \cdot \mathrm{mol}^{-1}$ above the global minimum, certainly somewhat higher in energy at short alkyl chain lengths. Hexylbenzene is the first molecule with an alkyl chain long enough to support the $g 1 g^{\prime} 4$ motif which is from there on usually found as the forth-most stable structure at around $2.2 \mathrm{~kJ} \cdot \mathrm{mol}^{-1}$ above the global minimum. At higher alkyl chain lengths more folded structures drop in relative energy. Almost all of them follow a motif where the alkyl chain folds back over the aromatic $\pi$ system. Most noticeably, the $g 1 g 3 g 4$ conformer is predicted $1 \mathrm{~kJ} \cdot \mathrm{mol}^{-1}$ less stable in octylbenzene, equally stable in nonylbenzene and almost $1 \mathrm{~kJ} \cdot \mathrm{mol}^{-1}$ more stable in decylbenzene than all- $t$. Similar trends are found for other folded conformers such as $g 1 g 2 g 4 g 5$. 
Table 4.1: Differences in zero-point corrected energies $\left(\Delta E_{0}\right)$, zero-point vibrational energies (ZPVE) and Gibbs energies at $298 \mathrm{~K}\left(\Delta G^{0}\right)$ relative to all- $t$ for selected conformers calculated at the B3LYP2D3/def2TZVP level in Gaussian 09 Rev. E.01. Important conformer families that are observed experimentally were selected. Extended results for all conformers found below a threshold of $5 \mathrm{~kJ} \cdot \mathrm{mol}^{-1}$ are found in A.4.

\begin{tabular}{|c|c|c|c|}
\hline conformer & $\Delta \boldsymbol{E}_{\mathbf{0}} / \mathbf{k J} \cdot \mathbf{m o l}^{-\mathbf{1}}$ & $\Delta \mathbf{Z P V E} / \mathbf{k J} \cdot \mathbf{m o l}^{-1}$ & $\Delta \boldsymbol{G}^{\mathbf{0}} / \mathbf{k J} \cdot \mathbf{m o l}^{-\mathbf{1}}$ \\
\hline \multicolumn{4}{|c|}{ butylbenzene } \\
\hline$g 1$ & -0.20 & 0.27 & -1.38 \\
\hline all- $t$ & 0.00 & 0.00 & 0.00 \\
\hline$g 1 g 2$ & 1.98 & 1.13 & 1.93 \\
\hline$g 2$ & 2.31 & 0.09 & 0.06 \\
\hline \multicolumn{4}{|c|}{ pentylbenzene } \\
\hline$g 1$ & -0.03 & 0.57 & -0.87 \\
\hline all- $t$ & 0.00 & 0.00 & 0.00 \\
\hline$g 1 g 2$ & 1.49 & 1.28 & 1.33 \\
\hline$g 2$ & 2.52 & 0.41 & 0.89 \\
\hline
\end{tabular}

hexylbenzene

\begin{tabular}{|c|c|c|c|}
\hline all- $t$ & 0.00 & 0.00 & 0.00 \\
\hline$g 1$ & 0.01 & 0.70 & -0.72 \\
\hline$g 1 g 2$ & 1.21 & 1.32 & 0.80 \\
\hline$g 1 g^{\prime} 4$ & 2.27 & 1.15 & 1.61 \\
\hline$g 2$ & 2.48 & 0.53 & 0.98 \\
\hline$g 1 g 3 g 4$ & 3.52 & 2.18 & 3.77 \\
\hline
\end{tabular}

heptylbenzene

\begin{tabular}{|c|c|c|c|}
\hline$g 1$ & -0.03 & 0.69 & -0.53 \\
\hline all- $t$ & 0.00 & 0.00 & 0.00 \\
\hline$g 1 g 2$ & 1.14 & 1.38 & 0.95 \\
\hline$g 1 g^{\prime} 4$ & 2.13 & 1.19 & 1.55 \\
\hline$g 1 g 3 g 4$ & 2.85 & 2.33 & 3.41 \\
\hline$g 1 g 2 g 4 g 5$ & 3.48 & 2.98 & 6.00 \\
\hline
\end{tabular}

octylbenzene

\begin{tabular}{|c|c|c|c|}
\hline all- $t$ & 0.00 & 0.00 & 0.00 \\
\hline$g 1$ & 0.02 & 0.75 & -0.32 \\
\hline$g 1 g 3 g 4$ & 0.98 & 3.46 & 4.78 \\
\hline$g 1 g 2$ & 1.17 & 1.47 & 1.18 \\
\hline$g 1 g 2 g 4 g 5$ & 1.81 & 3.26 & 3.46 \\
\hline$g 1 g^{\prime} 4$ & 1.99 & 1.24 & 1.06 \\
\hline
\end{tabular}

nonylbenzene

\begin{tabular}{|c|c|c|c|}
\hline$g 1$ & -0.03 & 0.69 & -0.70 \\
\hline$g 1 g 3 g 4$ & -0.01 & 3.28 & 3.71 \\
\hline all- $t$ & 0.00 & 0.00 & 0.00 \\
\hline$g 1 g 2 g 4 g 5$ & 0.34 & 3.76 & 4.27 \\
\hline$g 1 g 2$ & 1.18 & 1.46 & 0.81 \\
\hline$g 1 g^{\prime} 4$ & 1.89 & 1.19 & 0.88 \\
\hline
\end{tabular}

decylbenzene

\begin{tabular}{|c|c|c|c|}
\hline$g 1 g 3 g 4$ & -0.98 & 3.55 & 3.77 \\
\hline$g 1 g 2 g 4 g 5$ & -0.95 & 3.86 & 3.88 \\
\hline$g 1$ & -0.05 & 0.67 & -0.86 \\
\hline
\end{tabular}




\begin{tabular}{|c|c|c|c|}
\hline conformer & $\boldsymbol{\Delta E}_{\mathbf{0}} / \mathbf{k J} \cdot \mathbf{m o l}^{\mathbf{1}}$ & $\boldsymbol{\Delta} \mathbf{Z P V E} / \mathbf{k J} \cdot \mathbf{m o l}^{\mathbf{- 1}}$ & $\mathbf{\Delta} \boldsymbol{G}^{\mathbf{0}} / \mathbf{k J} \cdot \mathbf{m o l}^{-\mathbf{1}}$ \\
\hline all- $t$ & 0.00 & 0.00 & 0.00 \\
\hline$g 1 g 2$ & 1.15 & 1.45 & 0.65 \\
\hline$g 1 g^{\prime} 4$ & 1.97 & 1.34 & 1.31 \\
\hline
\end{tabular}

\section{4 - LIF jet spectra}

A comparison of the LIF jet spectra of ethyl- through pentylbenzene shows that the $\mathrm{S}_{0}-\mathrm{S}_{1}$ origin transition is especially sensitive to the first dihedral angle in the alkyl chain (Figure 4.4). For example the shift between the all- $t$ and $g 1$ origin in butylbenzene is approximately $62 \mathrm{~cm}^{-1}$, with $g 1$ shifted to lower frequencies due to the interaction of its methylene and methyl groups with the $\pi$ cloud. Gauche defects at other positions along the chain cause much smaller shifts in the UV excitation frequency, thus the $g 1 g 2$ origin transition of butylbenzene is only upshifted by $\sim 1 \mathrm{~cm}^{-1}$ compared to the $g 1$ origin while the $g 2$ origin is $\sim 2 \mathrm{~cm}^{-1}$ lower in frequency than the all- $t$ transition. These types of transitions appear as partially resolved side bands in Figure 4.4, but have been better resolved in earlier studies by Simons et al. [96]. Bands were assigned based on a comparison of their relative intensities and the predicted zeropoint corrected energy differences $\Delta E_{0}$ that were shown in Table 4.1. Due to their importance in the assignments, all calculated conformers below a threshold of $5 \mathrm{~kJ} \cdot \mathrm{mol}^{-1}$ relative to the global minimum structure are shown in energy level diagrams for pentyl- through octylbenzene in Figure 4.5. Since the chain length does not have a critical influence on the position of the $\mathrm{S}_{0}-\mathrm{S}_{1}$ origins, assigned transitions from previous alkylbenzenes with one less carbon atom in the alkyl chain were also used to assign reappearing transitions. In the following section, assignments are then cross-checked based on the comparison of the anharmonic local mode model and the conformationspecific IR spectra.

Figure 4.4 reveals an increasing conformational complexity already at shorter alkyl chains with one, two and four conformers observed and assigned for ethyl-, propyland butylbenzene, respectively. The relative intensities of the $S_{0}-S_{1}$ origins reflect the energy differences calculated at the B3LYP-2D3/def2TZVP level very well. The zeropoint corrected energy differences for the all- $t, g 1, g 1 g 2$ and $g 2$ conformers of butylbenzene are $0.0,0.1,2.1$ and $2.4 \mathrm{~kJ} \cdot \mathrm{mol}^{-1}$, respectively, consistent with the experimental spectrum that shows two equally large (all- $t$ and $g 1$ ) and two weak $(g 1 g 2$ and $g 2)$ transitions. Note that the peak appearing just between the all- $t$ and $g 1$ origins is a vibronic band due to Franck-Condon activity in a low frequency mode of the $g 1$ conformer.

The energy level diagram for pentylbenzene (Figure 4.5) predicts similar populations for all- $t$ and $g 1$ while $g 1 g 2$ is predicted as the third most-stable conformer. This is consistent with the experimental findings (Figure 4.4) which shows two intense bands at approximately the same frequencies where all- $t$ and $g 1$ transitions were found in propyl- and butylbenzene and a third transition with half of the intensity of the larger bands only $5 \mathrm{~cm}^{-1}$ downshifted from the $g 1$ transition, tentatively assigned 
to $g 1 g 2$. The next two conformers in the energy ranking are $g 2$ and $g 3$ with predicted energy differences of 2.5 and $2.6 \mathrm{~kJ} \cdot \mathrm{mol}^{-1}$, respectively. While one would expect their intensities to be smaller than those of the $g 1 g 2$ transition they could still be present in the spectrum. However, based on the butylbenzene results, the origins of these transitions are anticipated to be very close to the all- $t$ origin, since their first dihedrals are in a trans configuration. It is likely that their origins are hidden underneath the all- $t$ contour, a hypothesis that will be tested for in Raman jet experiments, discussed in Section 4.6.

A similar situation is found in hexylbenzene where all-t, $g 1$ and $g 1 g 2$ are predicted to be significantly more stable than others. The LIF spectrum reflects this close similarity as well with two large transitions due to all- $t$ and $g 1$ as well as a weaker transition, just next to $g 1$, tentatively assigned to $g 1 g 2$. Note that hexylbenzene is the first alkylbenzene that can support a $g 1 g 3 g 4$ conformer, with its chain folded back over the ring. Here, this conformer is predicted to be $3.5 \mathrm{~kJ} \cdot \mathrm{mol}^{-1}$ less stable than the all- $t$ minimum and no experimental evidence was found that this conformer is present in jet expansions in hexylbenzene.

The LIF spectrum of heptylbenzene differs in two subtle but important ways. First, the gap between the $g 1$ and $g 1 g 2$ origins has widened and now reveals a new peak between them with roughly one-third of the $g 1 g 2$ intensity. Second, a weak transition at $37497 \mathrm{~cm}^{-1}$ appears approximately downshifted by $15 \mathrm{~cm}^{-1}$ from the $g 1 \mathrm{~g} 2$ origin. Thus, the causer of this band must show stronger interactions with the aromatic $\pi$ cloud than the other conformers. Consulting the energy level diagram of heptylbenzene reveals two reasonable prospects for these new transitions with $g 1 g^{\prime} 4$ being predicted fourth in the energy ranking and $g 1 g 3$ as the next best candidate that should have a transition close to the $g 1$ origin. However, further distinction and confirmation will require the IR and Raman data in the following sections. Note that a slight drop in energy of the $g 1 g 3 g 4$ conformer occurs in the longer heptyl chain but several other folded or partially folded conformers compete with it in energy.

In octylbenzene, the LIF spectrum now more clearly shows the three transitions at the $g 1$ frequency. At lower frequencies several small transitions become visible with one at around $37488 \mathrm{~cm}^{-1}$ that shows nearly equal intensity to the middle peak of the $g 1$ triad. In the next section further evidence will be presented that this transition can be assigned to the $g 1 g 3 g 4$ conformer, consistent with a significant drop in the relative energy of this conformer to $1.0 \mathrm{~kJ} \cdot \mathrm{mol}^{-1}$ which makes it now the third best structure in the predicted energy ranking.

Finally, the LIF spectrum of decylbenzene even though not studied in detail clearly shows an overall increase in the number and intensities of transitions in the lower frequency part, which is a clear indication of the growing presence of more conformers with strong interactions between the alkyl chain and the aromatic $\pi$ cloud. 

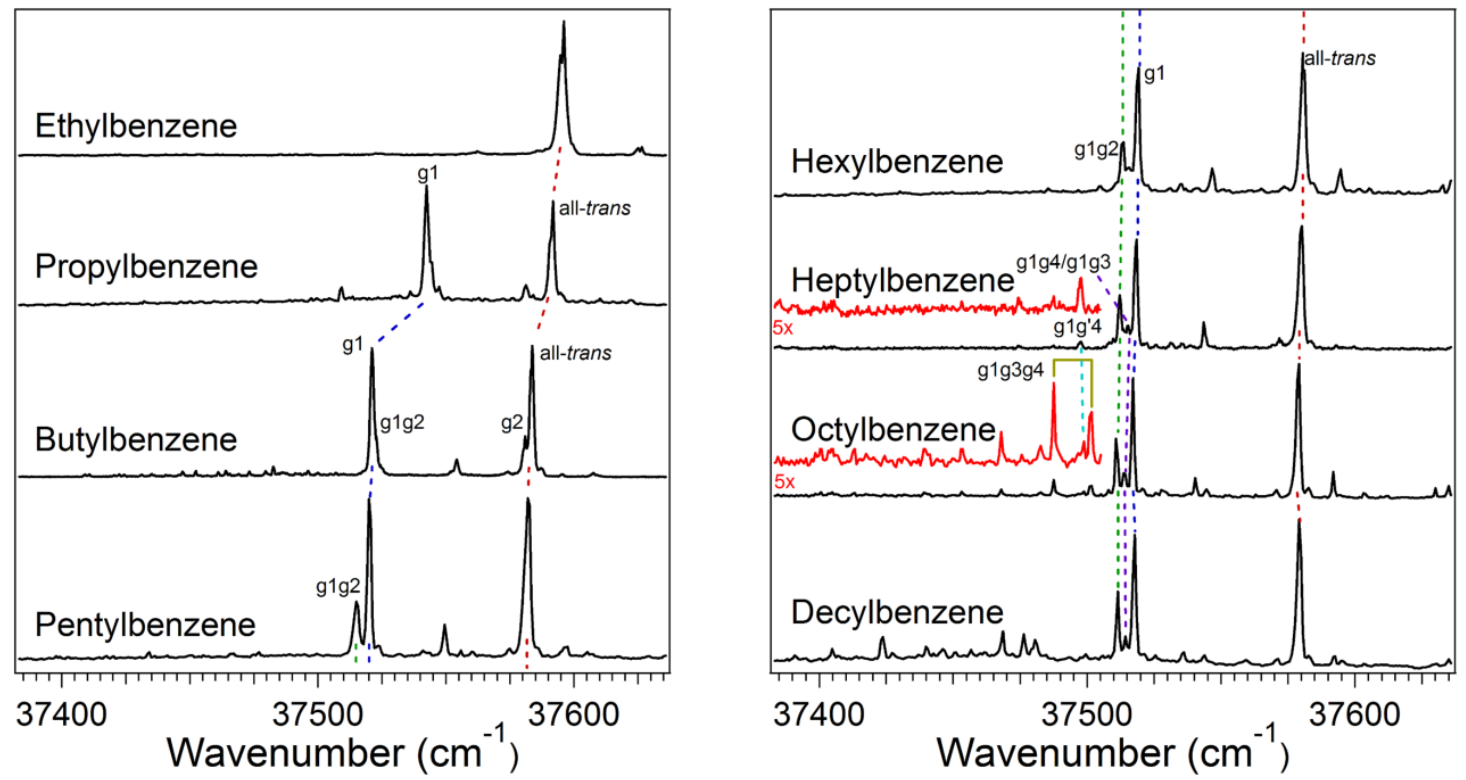

Figure 4.4: LIF excitation spectra for ethyl- through octylbenzene, and decylbenzene. Dashed lines indicate bands with the same assigned structure between molecules. The low frequency regions of heptyl- and octylbenzene are magnified $5 \times$ for clarity (red). The band located between the origins of the $g 1$ and all- $t$ conformer is a vibronic band of the $g 1$ conformer.

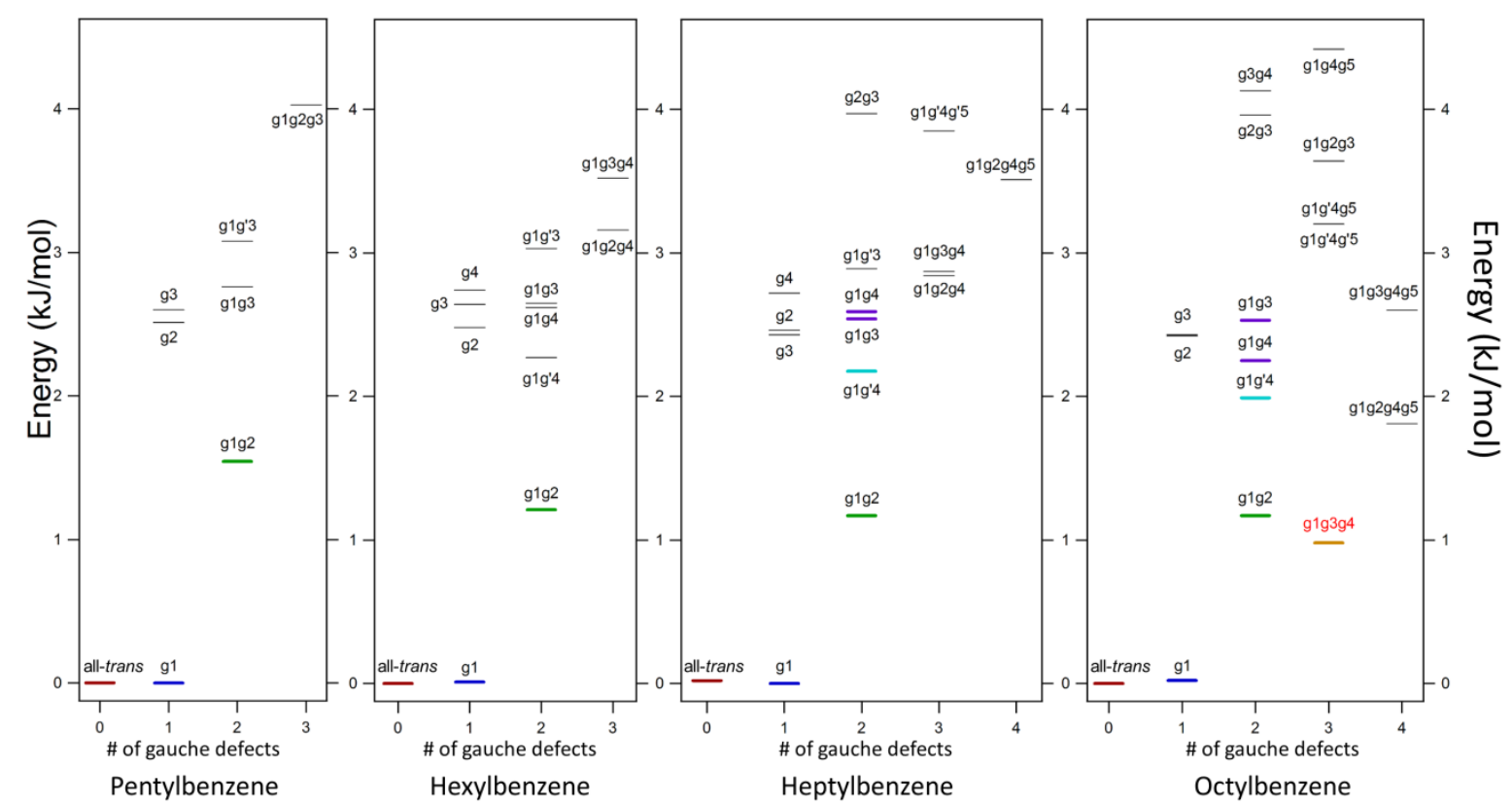

Figure 4.5: Energy level diagrams of pentyl- through octylbenzene. Calculations were done at the B3LYP-2D3/def2TZVP level of theory. The experimentally observed structures are shown with colored markers for clarity. The first observed folded structure, $g 1 g 3 g 4$ in octylbenzene, is shown in red.

\section{5 - Fluorescence-dip infrared jet spectra}

FDIRS spectra of individual conformers in pentyl- through octyl- and decylbenzene were taken in the $\mathrm{CH}$ stretching region from 2800 to $3000 \mathrm{~cm}^{-1}$. Similar to the LIF spectra, the IR spectra evolve with increasing chain length in a systematic way that allows sorting conformers of each alkylbenzene in conformational families that share the same or closely analogous alkyl chain conformations. Thus, spectra were grouped 
by conformational families that reoccur in many of the discussed alkylbenzenes and are shown in Figure 4.7 to 4.10. An optimized structure for every important conformational family in octylbenzene is shown in Figure 4.6. The experimental spectra are compared to the local mode Hamiltonian model described in Section 4.3 and the predicted spectra are shown above the experimental ones. This comparison allows for a careful check of the previously made conformer assignments. Furthermore, the model allows assigning the corresponding vibrational motions to the observed peaks.

Certain features are commonly found in all IR spectra of the measured alkylbenzene conformers. In every spectrum, a doublet in the $2960-2970 \mathrm{~cm}^{-1}$ region is the neardegenerate pair of asymmetric stretch transitions of the methyl group. Furthermore, a weak band at around $2883 \mathrm{~cm}^{-1}$ assigned to the symmetric methyl stretching vibration is common in all spectra. The relative intensity of this motion is decreasing with increasing alkyl chain length for the simple reason that the number of methylene groups is increasing with chain lengths while there is always only one methyl group. The methylene motions are primarily found in the regions of 2850-2890 and 2920$2950 \mathrm{~cm}^{-1}$ with the first region corresponding to the symmetric stretches and Fermi resonances, while the latter contains the asymmetric stretching motions and the higher frequency member of the former Fermi resonances. If the local environment of a methylene group changes, as in a gauche configuration, the local mode site frequencies of each $\mathrm{CH}$ can shift leading to partial localization of the modes. On the other hand, if the environments of all methylene groups are very similar, e.g. in an all- $t$ chain, their site frequencies will be very similar and the coupling between them leads to normal modes that are extended over several methylene groups.

Figure 4.7a and b show the development of the IR spectra of all- $t$ and $g 1$ in pentylthrough octylbenzene, respectively. Both conformers are the two dominant species in all alkylbenzenes that are investigated here and their IR spectra are very similar, a fact that is not surprising considering that a gauche defect at the first position in the alkyl chain is still followed up by a long all-t subchain in higher alkylbenzenes. The asymmetric CH stretching vibrations appear as a doublet at 2963 and $2969 \mathrm{~cm}^{-1}$ in spectra of both conformers. The most noticeable difference between both conformer families is found in the region at $2850-2870 \mathrm{~cm}^{-1}$, where both have a set of two resolved bands whose relative intensity differences are well predicted by the anharmonic model. Also, the $2920-2950 \mathrm{~cm}^{-1}$ region is described with good accuracy by the model. The overall fits are sufficient to give confidence to the assignments of all- $t$ and $g 1$. If small contributions from $g 2$ or $g 3$, that were under suspicion to be hidden underneath the all- $t$ contour in the LIF spectra, are included in the model (blue peaks in Figure 4.7a) the agreement with the experimental spectrum is even better.

The IR spectra of the $g 1 g 2$ conformers (Figure 4.7c) are similar to the all- $t$ and $g 1$ spectra. However, a new band at $2950 \mathrm{~cm}^{-1}$ appears with strong relative intensities in shorter alkyl chains, and somewhat weaker in longer chains, as it would be expected of an isolated methylene transition. Indeed the theoretical model predicts a shift of the first methylene group of the alkyl chain from the region of the asymmetric meth- 
ylene stretching vibrations to the observed frequency. The symmetric stretching vibration of this group is similarly shifted to higher frequencies and is observed as a weak band at $2874 \mathrm{~cm}^{-1}$. The model also predicts this shift correctly but overestimates its relative intensity. These unique features that were correctly predicted by the anharmonic model confirm the assignment of the $g 1 g 2$ conformer.

Heptylbenzene was the shortest molecule in this series with a LIF transition that appeared lower in frequency than the $g 1 g 2$ origin and had sufficient intensity so that its FDIR spectrum could be taken. The $g 1 g^{\prime} 4$ conformer was the leading candidate, being next in the energy ranking after $g 1 g 2$, to explain this transition and a comparison of the spectrum to the anharmonic model of $g 1 g^{\prime} 4$ is presented in Figure 4.8. The spectrum looks similar to those of the previous conformers. However, a buildup of bands in the $2920-2950 \mathrm{~cm}^{-1}$ region is visible which can be a hint that more methylene groups have locally different environments, as would be expected in the $g 1 g^{\prime} 4$ conformation. Also, the symmetric methylene stretches show more individual bands than seen in the spectra of previous conformers. The most significant difference is a new band at $2910 \mathrm{~cm}^{-1}$ that was not present in the spectrum of any other conformer. Moreover, this band is predicted by the anharmonic model only for the $g 1 g^{\prime} 4$ conformer, making it a unique feature of this conformation. The overall agreement of the model and the spectrum is good, despite showing some minor differences in the intensity patterns. This agreement, especially with the correctly predicted unique band in combination with the estimated energy ranking, where $g 1 g^{\prime} 4$ is the next best conformer after all-t, $g 1$ and $g 1 g 2$, fully supports the tentative assignment of the $g 1 g^{\prime} 4$ structure to the transition at $37498 \mathrm{~cm}^{-1}$ in the LIF spectrum of heptylbenzene. The analogous transition in octylbenzene could not be recorded due to its weak intensity.

The fifth unique LIF transition, recorded at $37515 \mathrm{~cm}^{-1}$ (Figure 4.4), is first observed in hexylbenzene but only showed a good-enough separation and sufficient intensity allowing to record a FDIRS spectrum in heptyl- and octylbenzene. The IR spectra of this origin are shown in Figure 4.9 for heptyl- and octylbenzene, respectively. Unfortunately, the IR spectra lack in terms of unique transition that would give confidence in the assignment. Simulations containing equal contributions from $g 1 g 3$ and $g 1 g 4$ result in a reasonable fit. As discussed in the previous section, the LIF transition frequencies as well as the predicted energy ranking of $g 1 g 3$ and $g 1 g 4$ make them reasonable candidates for the explanation of this transition. However, a firm assignment cannot be made.

The LIF spectrum of octylbenzene revealed a unique transition that was shifted to low frequencies, indicating a stronger interaction of the alkyl chain with the aromatic $\pi$ cloud than in any other observed conformer, and appeared at $37488 \mathrm{~cm}^{-1}$. At the same time, the predicted energy ranking shows a significant drop in relative energy of the $g 1 g 3 g 4$ conformer. The IR spectrum of this transition (Figure 4.10) shows several unique features. The number of resolved bands in both the symmetric and asymmetric methylene stretching regions is significantly greater. Additionally, a strong transition is observed at $2950 \mathrm{~cm}^{-1}$ which is correctly predicted by the anharmonic model 
and is associated with adjacent gauche defects as it is indeed present in $g 1 g 3 g 4$. In general the anharmonic model describes the increasing number of transitions very well, especially in the region from $2850-2880 \mathrm{~cm}^{-1}$ where other conformers only had three transitions. The good agreement and reproduction of these unique features gives confidence to the assignment of $g 1 g 3 g 4$. Figure $4.10 \mathrm{~b}$ shows the FDIR spectrum taken at the $37502 \mathrm{~cm}^{-1}$ band in octylbenzene, shown in the inset of Figure 4.4. Its spectrum is identical to that that of the $g 1 g 3 g 4$ origin transition, consistent with it being a vibronic band due to Franck-Condon activity of a low frequency motion of the alkyl chain against the aromatic $\pi$ cloud.

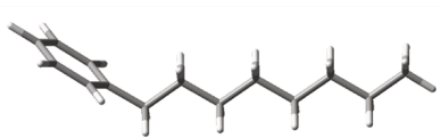

all-trans

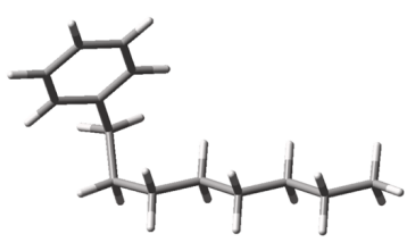

g1g2

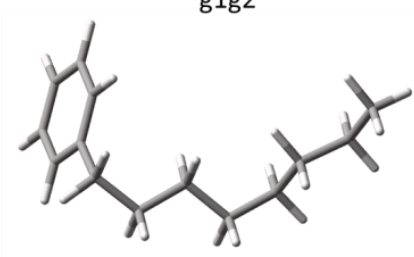

g1g4

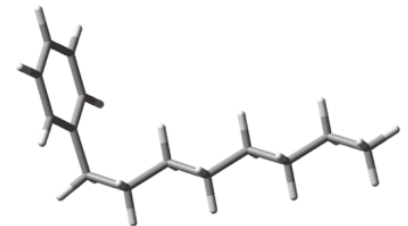

g1

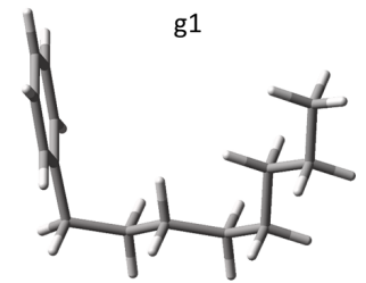

g1g'4

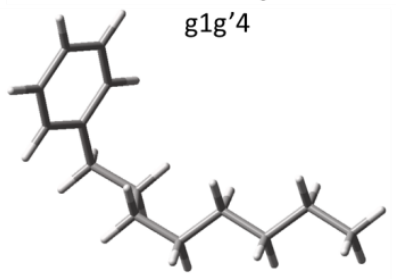

g1g3

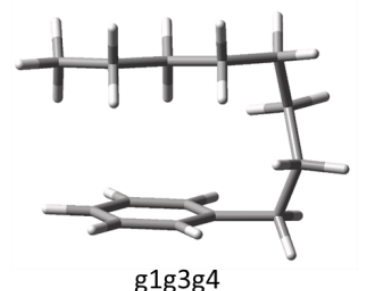

g1g3g4

Figure 4.6: The seven experimentally observed conformations seen in the alkylbenzene series, shown for octylbenzene. 

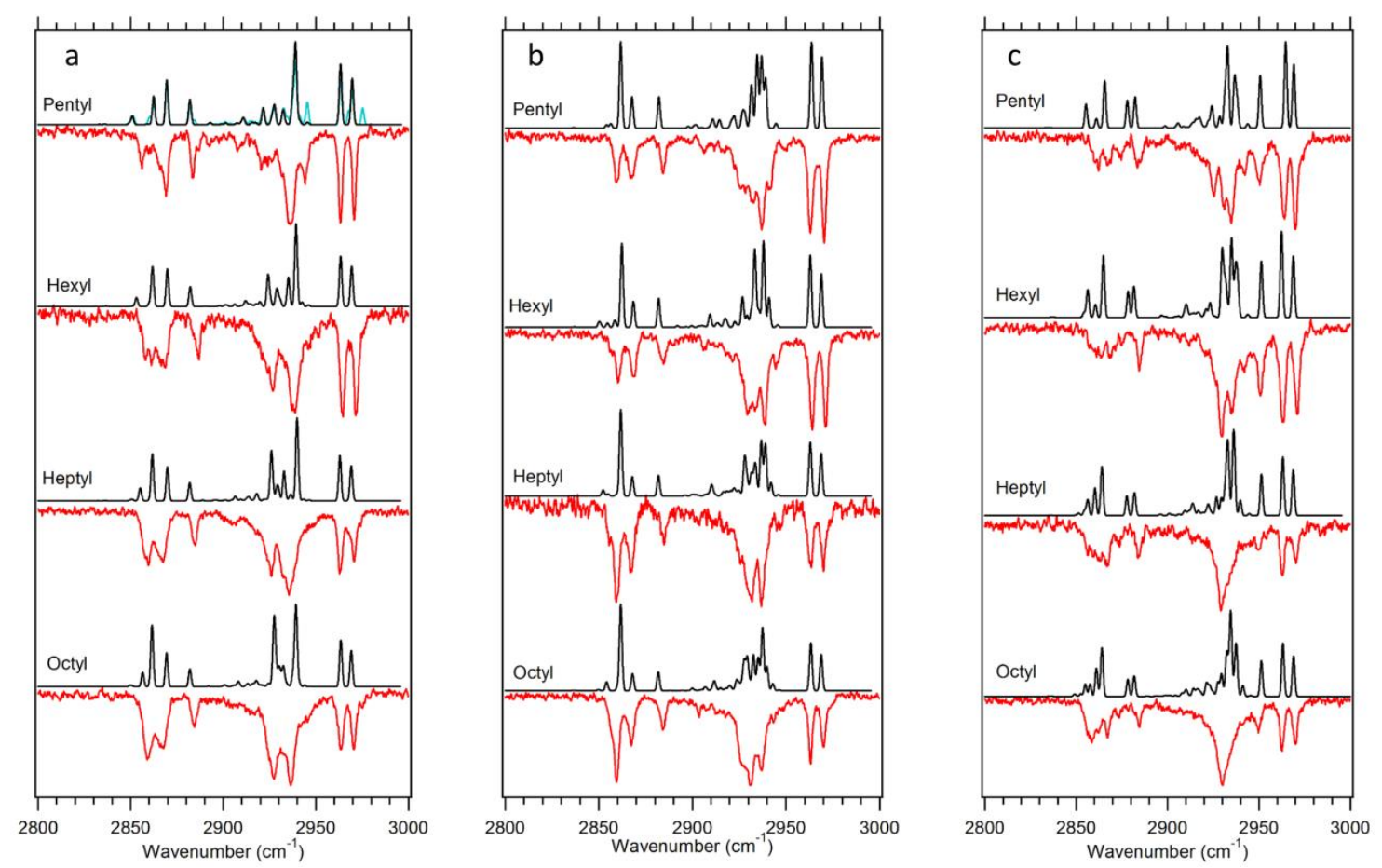

Figure 4.7: Experimental IR spectra of pentyl- through octylbenzene for the all- $t$ (a), $g 1$ (b) and $g 1 g 2$ (c) conformers (red) compared to the calculated spectra of the same conformers using the anharmonic model (black). The blue spectrum on the all- $t$ pentylbenzene trace carries some weight from the $\mathrm{g} 3$ conformer (3:1 all-t-to-g1-ratio).

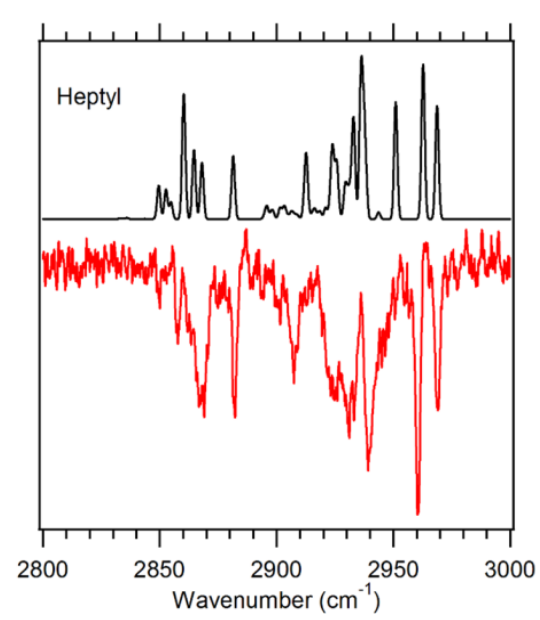

Figure 4.8: Experimental IR spectrum of heptylbenzene taken at $37498 \mathrm{~cm}^{-1}$ (red) in comparison with a theoretical spectrum of the $g 1 g^{\prime} 4$ conformer (black). 


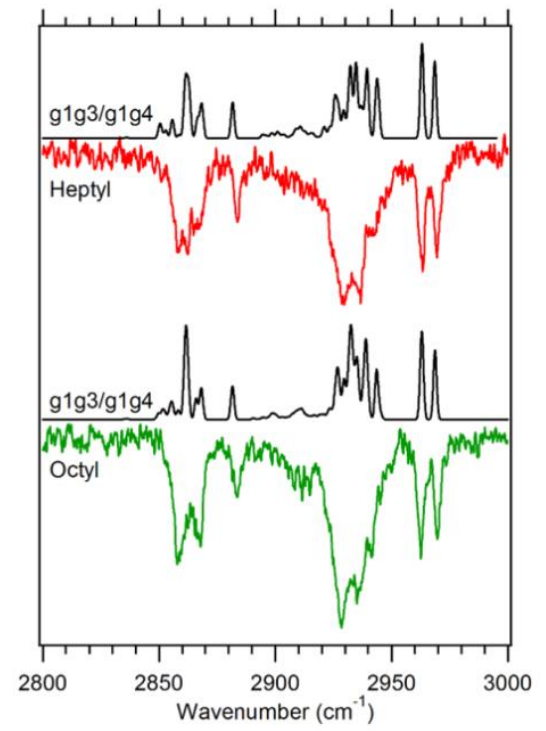

Figure 4.9: Theoretical spectra of a 1:1 ratio of the $g 1 g 3$ and $g 1 g 4$ conformers (black) versus the experimental spectra of heptylbenzene (red) and octylbenzene (green) taken at $37514 \mathrm{~cm}^{-1}$.

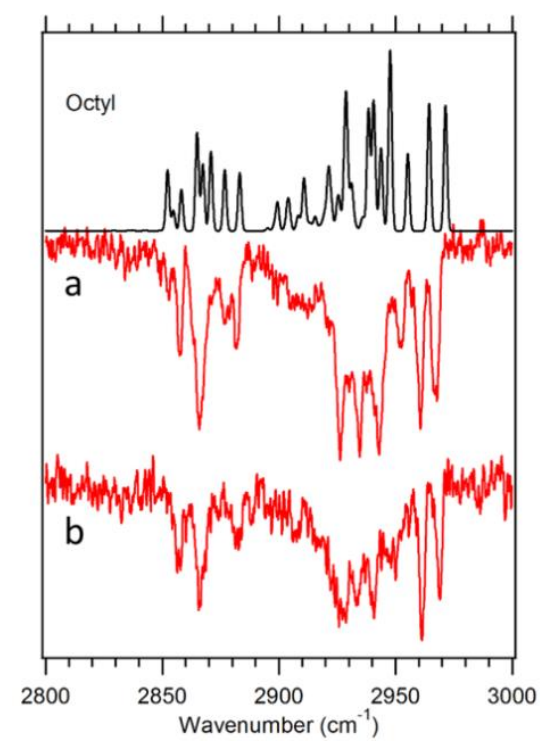

Figure 4.10: Theoretical spectrum of the $g 1 g 3 g 4$ conformer (black) versus the experimental spectra of octylbenzene taken at 37488 (a) and $37502 \mathrm{~cm}^{-1}$ (b).

\section{6 - Raman jet spectra}

Raman spectra of hexyl- through nonylbenzene are shown in Figure 4.11 to 4.13, respectively. Since the Raman technique is not conformation specific all conformers that survive the jet expansion are visible in the spectrum. As the alkylbenzenes, similar to $n$-alkanes, show rather high barriers for dihedral changes along the carbon chain the initial populations, prior to the expansion, are essentially frozen out during the jet expansion. Initial populations of the conformers were calculated based on relative Gibbs energies at the B3LYP-2D3/def2TZVP level (Table 4.1) and were used to weight the predicted Raman scattering cross sections at the same level of theory. The calculated stick spectra for the three major populated conformers (all-t, $g 1$ and $g 1 g 2$ ) 
give remarkably accurate predictions for all observed major bands in hexylbenzene. However, there are a few bands, such as 255, 315 and $485 \mathrm{~cm}^{-1}$ (marked with asterisks in Figure 4.11), that are not accounted for when only considering these three major conformers. Most of these bands can be accounted for when $g 1 g^{\prime} 4, g 2$ and $g 3$ are included in the predictions. Their lower intensities result from the significantly lower population at $298 \mathrm{~K}$. This is in line with the suspected overlap of the LIF transitions of all- $t$ and single gauche conformers which have a gauche kink at other than the first position in the alkyl chain. Therefore, it was not possible to distinguish between these conformers in LIF or FDIR spectra but the Raman experiments confirmed their presence in the jet experiments.

Also, in heptylbenzene these conformers $\left(g 1 g^{\prime} 4, g 2, g 3\right)$ are necessary to describe many bands in the spectrum, e.g. at $245,275,387 \mathrm{~cm}^{-1}$. Some very weak bands, e.g. the band at $288 \mathrm{~cm}^{-1}$, in the spectrum are predicted upon inclusion of the $g 1 \mathrm{~g} 3 \mathrm{~g} 4$ conformer. However, as there are no strong bands to support this assumption it stays uncertain.

Raman spectra of octyl- (Figure 4.12) and nonylbenzene (Figure 4.13) show significantly reduced signal-to-noise ratios due to the large decrease in the vapor pressure of these higher alkylbenzenes. Contributions from all previous mentioned conformers are clearly visible in the spectra. However, the low signal-to-noise ratios make assignments of weak conformers difficult. Weak bands at 271 and $418 \mathrm{~cm}^{-1}$ in octyl- as well as 259 and $389 \mathrm{~cm}^{-1}$ in nonylbenzene might indicate rising contributions from folded conformers like $g 1 g 3 g 4$ or $g 1 g 2 g 4 g 5$.

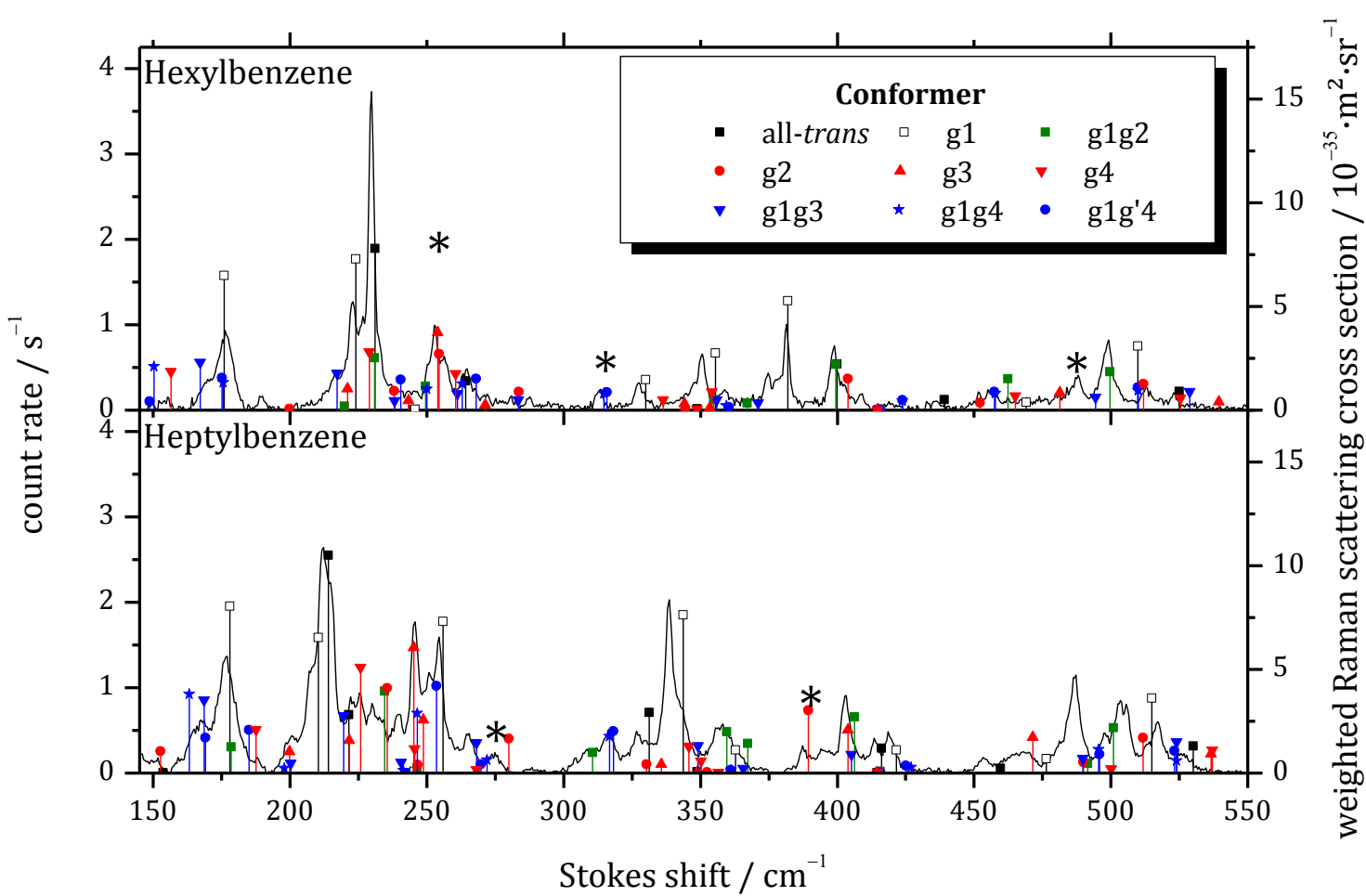

Figure 4.11: Raman jet spectra of hexyl- and heptylbenzene shown with calculated Raman scattering cross sections for the low energy conformations weighted by their estimated relative abundances at 298 K. Bands assigned to conformers not observed in the UV/IR studies are marked with asterisk. 


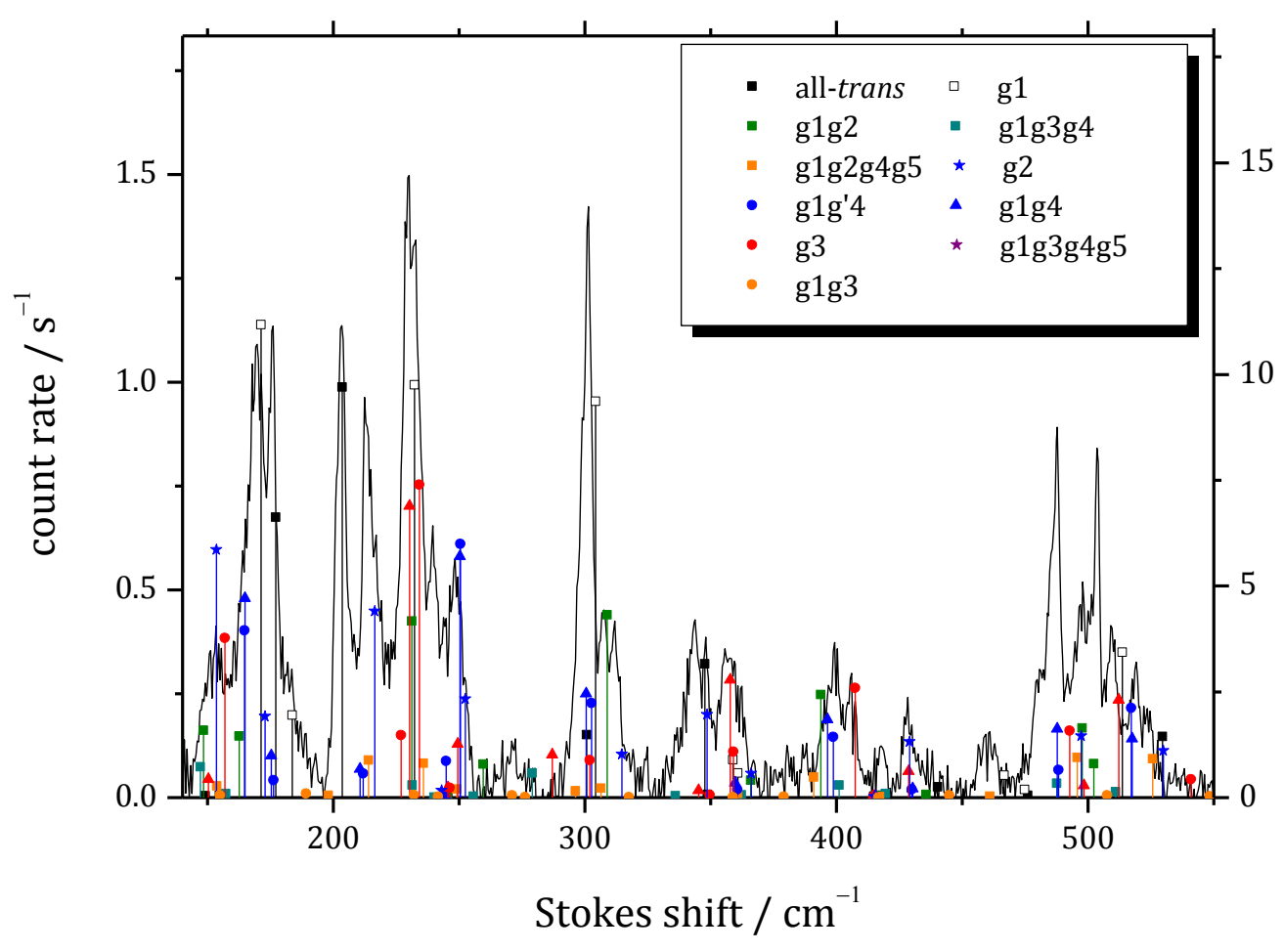

'0

Figure 4.12: Raman jet spectrum of octylbenzene shown with calculated Raman scattering cross sections for the low energy conformations weighted by their relative abundances estimated at $298 \mathrm{~K}$.

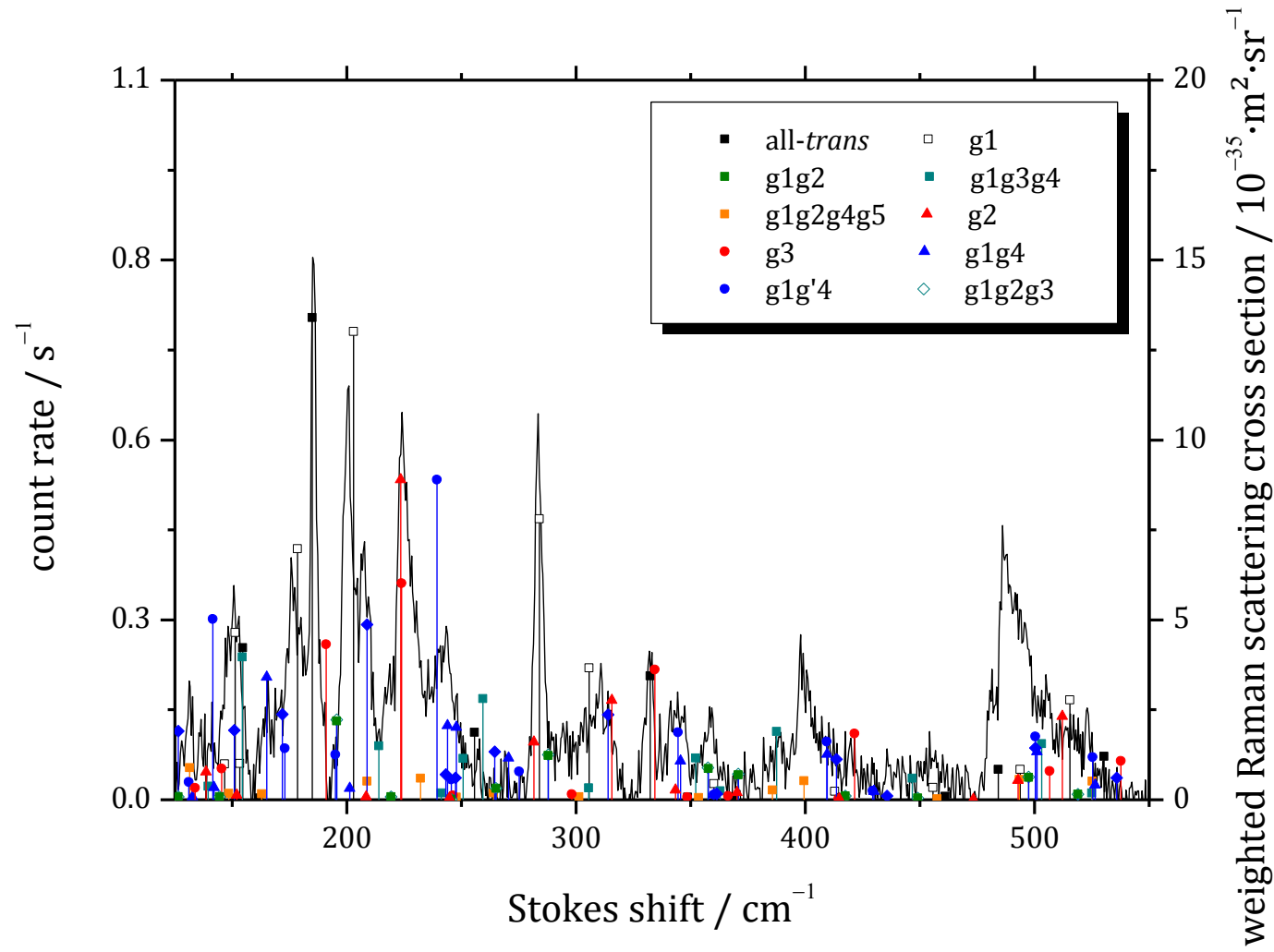

Figure 4.13: Raman jet spectrum of nonylbenzene shown with calculated Raman scattering cross sections for the low energy conformations weighted by their relative abundances estimated at $298 \mathrm{~K}$. 


\section{7 - Comparison with $n$-alkanes}

The LIF spectra proved to be a sensitive indicator of the interaction strength between the alkyl chain and the aromatic $\pi$ cloud. When their interactions are stronger the corresponding LIF transition is shifted to lower frequencies. Thus, out of all conformers all- $t$ has its origin transition at the highest frequency. A gauche kink at the first position of the alkyl chain shifts this transition by approximately $-62 \mathrm{~cm}^{-1}$, almost independent on the alkyl chain length. The LIF transitions of all- $t$ and $g 1$ were almost equally intense, corresponding to very similar populations consistent with the predicted energy ranking at the B3LYP-2D3/def2TZVP level. This is very different in $n$-alkanes where a gauche kink raises the energy of the chain by $\sim 2.5 \mathrm{~kJ} \cdot \mathrm{mol}^{-1}$ [19]. The third methylene group of $g 1$ conformers in alkylbenzenes engages in stabilizing $\mathrm{CH} \cdots \pi$ interactions, as can be seen in Figure 4.6 for octylbenzene, which leads to the observed LIF transition shift and lowers the energy of $g 1$ so that the first gauche defect can occur with no energetic penalty. Gauche defects at other positions in the alkyl chain are energetically more costly. Thus, $g 2, g 3$ etc. are, similar to $n$-alkanes, 2.5 $3.0 \mathrm{~kJ} \cdot \mathrm{mol}^{-1}$ less stable than all-t.

The positive pentane effect describes a stabilization of adjacent gauche dihedrals of the same sign and first appears in pentane. A stabilization of $0.7 \mathrm{~kJ} \cdot \mathrm{mol}^{-1}$ is found in pentane [97]. In line with this the $g 1 g 2$ conformer is found to be the third-lowest structure in the energy rankings of pentyl- through octylbenzene, predicted to be 1.2$1.5 \mathrm{~kJ} \cdot \mathrm{mol}^{-1}$ less stable than all-t. In contrast the $g 1 g^{\prime} 2$ conformer has a predicted energy difference of $5.6 \mathrm{~kJ} \cdot \mathrm{mol}^{-1}$ relative to all- $t$ due to the steric effects between $\mathrm{C}_{\mathrm{i}}$ and $\mathrm{C}_{\mathrm{i}+4}$ when $\mathrm{C}_{\mathrm{i}}$ is the first carbon atom involved in the adjacent gauche defects of opposite signs.

Beginning in hexylbenzene, the $g 1 g^{\prime} 4$ conformer is predicted as the fourth-stable conformer in the energy ranking at a relative energy difference of around $2.3 \mathrm{~kJ} \cdot \mathrm{mol}^{-1}$ above the global minimum. In contrast, the $g 1 g 4$ conformer is predicted slightly higher in energy $\left(2.7 \mathrm{~kJ} \cdot \mathrm{mol}^{-1}\right)$. This slight stabilization of $g 1 g^{\prime} 4$ indicates that the alkyl chain in this conformer can have stronger interactions with the aromatic $\pi$ cloud than $g 1 g 4$, compensating the next-nearest-neighbor gauche defects that usually would favor $g 1 g 4$ (e.g. $g 1 g 3$ is lower in energy than $g 1 g^{\prime} 3$ ).

The $g 1 g 3 g 4$ folding motif was first characterized with measurable intensity in octylbenzene whereas the first folded structural motif, following a ggtgg sequence, in straight alkane chains was found to begin at carbon chain lengths of approximately 17 to 18 atoms [18]. At chain lengths consisting of 20 or more carbon atoms it quickly becomes the dominant conformational motif [18]. In a similar way rising influence of more folded structural conformers was found in higher alkylbenzenes. However, the shortest chain alkylbenzene in which a folded structure is observed is significantly shorter than the first observed structure of the pure alkanes. There are two important main differences between the alkylbenzenes and alkanes that result in earlier folding in the former case. First, the energetic cost for folds starting directly after the phenyl 
ring are reduced by approximately $2.5 \mathrm{~kJ} \cdot \mathrm{mol}^{-1}$. As discussed in Section 4.2 , the preferred dihedral angle of $\mathrm{C}_{\text {ortho }}-\mathrm{C}_{\mathrm{ph}}-\mathrm{C}_{1}-\mathrm{C}_{2}$ is $90^{\circ}$ in the alkylbenzenes and is found in all low-energy conformations. The actual angle between the planes of the aromatic ring and the alkyl chain of the $g 1 g 3 g 4$ conformer in octylbenzene is $67^{\circ}$, so that a more proper labeling in this case might be $g 0 g 1 g 3 g 4$. Thus, it is in effect the same sequence of turns than the one found in $n$-alkanes. Therefore, $g 0$ is in essence not a gauche defect but rather a distinct preference. Furthermore, the first gauche turn in the alkyl chain, labeled as $g 1$, can occur with almost no energetic penalty due to stabilizing interactions of the third methylene group and the aromatic ring. A comparison of torsional scans around the first and second dihedral along the alkyl chain in hexylbenzene (Figure 4.14) demonstrate the energetic advantage of $g 1$ over $g 2$, similar to the advantage of $g 1$ over single gauche $n$-alkane conformers. In sum the conformational energy penalty of this folding motif is reduced by a factor of 2 compared to $n$-alkanes.

Second, in that folding motif the aromatic ring in alkylbenzenes replaces one part of an alkane chain leg in $n$-alkanes. It is less restrictive in its requirements on the turn and provides a wider swath of angles for stabilization of the alkyl chain. Furthermore, the aromatic ring allows for $\mathrm{CH} \cdots \pi$ type interactions that are stronger than those between the pure alkyl chains. These cumulative interactions are especially important when the alkyl chain lengths increase to sizes where they fully extend over the aromatic ring. In hexylbenzene the last carbon atom of the alkyl chain is nearly ringcentered whereas the alkyl chain is fully extended over the ring in octylbenzene, as shown in Figure 4.6. Thus, the energy difference of the $g 1 g 3 g 4$ conformer relative to all- $t$ drops from $3.6 \mathrm{~kJ} \cdot \mathrm{mol}^{-1}$ in hexylbenzene to $2.9 \mathrm{~kJ} \cdot \mathrm{mol}^{-1}$ in heptylbenzene and $1.0 \mathrm{~kJ} \cdot \mathrm{mol}^{-1}$ in octylbenzene.

Even though the $g 1 g 3 g 4$ conformer becomes a competitor for the global minimum structure in nonylbenzene at $0 \mathrm{~K}$ the tight fold leads to an entropic penalty that result in fairly low abundances at $298 \mathrm{~K}$. As seen in Table 4.1, the Gibbs energy difference at $298 \mathrm{~K}$ between $g 1 g 3 g 4$ and all- $t$ is approximately $3.7 \mathrm{~kJ} \cdot \mathrm{mol}^{-1}$, despite showing no significant differences in the zero-point corrected energies. An analogous situation is found in $n$-alkanes [98]. If interconversion barriers, separating the different conformers, are large the pre-expansion populations will be essentially frozen out. Calculations on selected barriers involving isomerizations around single $\mathrm{C}-\mathrm{C}$ bonds, as shown in Figure 4.14, predict barrier heights between 12 and $15 \mathrm{~kJ} \cdot \mathrm{mol}^{-1}$ (around $5 \cdot k T$ ), and are consistent with barriers found in pure alkanes [97]. This makes it a very challenging task to observe these folded structural motifs experimentally as their entropic disadvantage leads to low abundances and thus weak spectral features. 


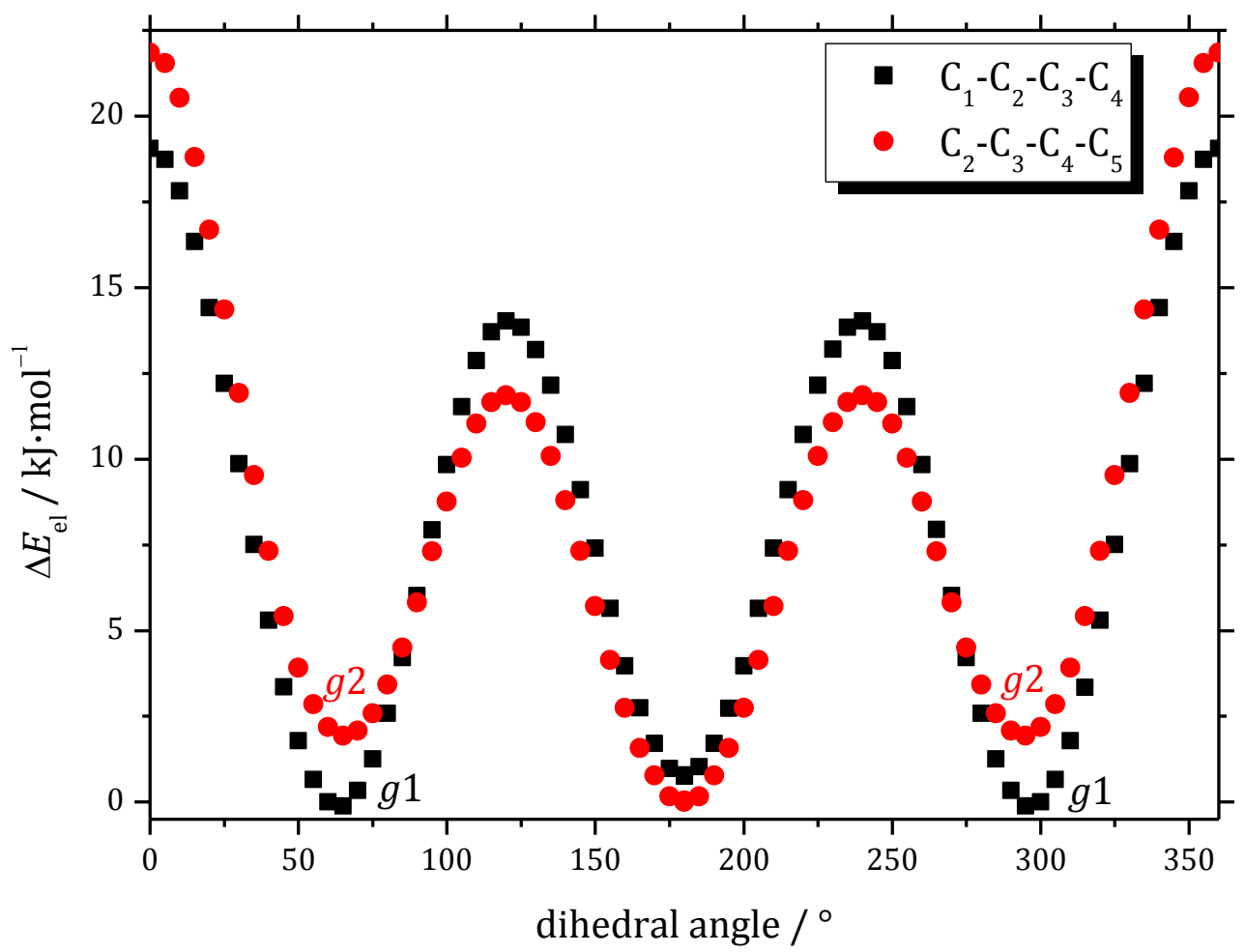

Figure 4.14: Relaxed torsional 1-D scans of the first (black squares) and second (red circles) dihedral along the alkyl chain in hexylbenzene calculated at the B3LYP-2D3/def2TZVP level in Gaussian 09 Rev. E.01. Other dihedrals remained in trans configuration in these calculations.

\section{8 - Conclusions}

Combining electronic frequency shifts, single-conformer IR spectra in the $\mathrm{CH}$ region, dispersion-corrected DFT energies, an anharmonic local mode model and lowfrequency Raman spectra allowed for assigning observed UV transitions to specific conformers in pentyl- through octylbenzene. The alkylbenzenes posed a challenge to apply single-conformation spectroscopy as IR-UV double resonance methods require conformationally unique UV transitions. The UV transitions are sensitive to the first dihedral, but increasingly insensitive to gauche defects at later positions in the alkyl chain which inevitably leads to small contributions of higher-energy conformers in some spectra. The low-frequency Raman spectra allowed for identification of several higher-energy conformers such as $g 2$ or $g 3$ that were hidden in the UV spectra.

The $S_{0}-S_{1}$ origin transition of the first folded structure, in which the chain folds back over the aromatic ring, shifts to lower frequencies compared to the straight all- $t$ chain due to the stronger interactions of the $\pi$ cloud with the alkyl chain. This stronger shift allowed for single-conformer spectroscopy without any interference of other conformers. The infrared spectrum of this transition showed an increasing number of unique bands due to the greater localization of the methylene transitions.

In agreement with the theoretical calculations major contributions from all-t, $g 1$, $g 1 g 2$ were found in all investigated alkylbenzenes, whereas contributions from $g 1 g^{\prime} 4$ were observed from hexyl- to octylbenzene. Additionally, a folded structural motif 
was assigned to the $g 1 g 3 g 4$ conformer which was first observed in octylbenzene. Through the combination of the used complementary experimental techniques and quantum chemical calculations these five different conformers could be assigned with high certainty. 


\section{Chapter 5 - 1-Methoxy-2-phenoxyethane}

\section{1 - Introduction}

1-Methoxy-2-phenoxyethane, also known as (2-methoxy)ethoxybenzene or 2-methoxyethyl phenyl ether, is a molecule that is very rarely investigated. To this date there are no reported safety information or reports on its basic physical properties such as the melting point. In the previous chapter the influence of an aromatic ring on the conformational preferences of alkyl chains was demonstrated. This aromatic influence was also investigated for the simplest oligoglyme chain. As the glyme chain is still rather short 1-methoxy-2-phenoxyethane is anticipated to show similar preferences as 1,2-dimethoxyethane whose conformational preferences are extensively discussed in Chapter 6. Analogously to the alkylbenzenes, the conformers of 1methoxy-2-phenoxyethane will be distinguished by the sequence of backbone dihedrals along the glyme chain starting at $\mathrm{C}_{\mathrm{Ph}}-\mathrm{O}-\mathrm{C}-\mathrm{C}$ where $\mathrm{C}_{\mathrm{Ph}}$ is the aromatic carbon atom that is connected to the glyme chain. Dihedrals are then shortened to $t$ for trans $\left(180^{\circ}\right)$ and $g$ for gauche $\left( \pm 60^{\circ}\right)$. If a conformer has multiple gauche kinks of opposite directions then the direction that is found more often will be denoted as $g$ while the opposite directed ones will be denoted $g$ '.

1-Methoxy-2-phenoxyethane was synthesized by Anthony Tomaine following a similar strategy that was reported for the synthesis of dibenzo-crown ethers [99]. The synthesis is shown in Figure 5.1. The end product was purified by column chromatography and verified through ${ }^{1} \mathrm{H}$ and ${ }^{13} \mathrm{C}$ NMR spectroscopy.

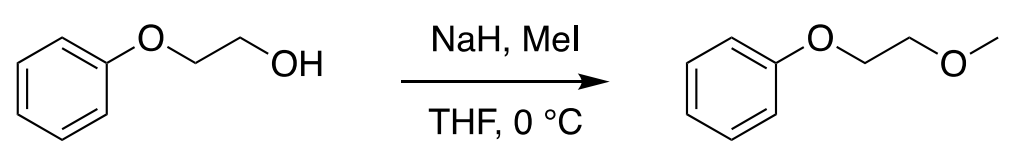

Figure 5.1: Synthesis scheme for 1-methoxy-2-phenoxyethane.

\section{2 - Quantum chemical calculations}

All 14 different combinations of the glyme backbone dihedrals were optimized and their energies and frequencies were calculated with the B3LYP functional paired with the def2TZVP and the aVTZ basis sets in Gaussian 09 Rev. E.01. Zero-point corrected energy differences, Gibbs energies and zero-point vibrational energy differences are found in Table 5.1. The def2TZVP basis set results slightly differ from those obtained with the aVTZ basis set due its stronger intramolecular basis set superposition errors (BSSE). This is especially noticeable for conformers that are bent such as $g g^{\prime} g$ and $g^{\prime} g g$ which show the largest deviations between both basis sets. However, the general agreement between the two basis sets is good as both predict $t t t$ as the global minimum structure and $t t t$, tgt as well as tgg' as the three most stable structures, shown in Figure 5.2, with a larger gap to the following structures. When the electronic energies of the three most-stable structures are evaluated at the MP2/aVTZ level and B3LYP- 
2D3/aVTZ zero-point corrections are added the energy sequence for ttt-tgt-tgg' is predicted to be $0.00-0.65-2.63 \mathrm{~kJ} \cdot \mathrm{mol}^{-1}$. Thus, the B3LYP calculations might overestimate the stability of the $\operatorname{tgg}^{\prime}$ conformer.

All conformers were found to prefer an in-plane configuration between the aromatic ring and the glyme chain reflected by an angle of $0^{\circ}$ for $\mathrm{C}_{\mathrm{ar}}-\mathrm{C}_{\mathrm{ph}}-\mathrm{O}-\mathrm{C}$. In this configuration the oxygen lone pairs can have stronger interactions with the aromatic $\pi$ cloud. Consistently, closely-related molecules that also have an oxygen atom in the benzylic position such as 2-phenoxyethanol or anisole were found to prefer in-plane arrangements between the aromatic ring and the side chain $[100,101]$. In contrast, the alkylbenzenes prefer an out-of-plane arrangement with an angle close to $90^{\circ}$ which minimizes the steric repulsion. Therefore, conformational preferences between 1methoxy-2-phenoxyethane and alkylbenzenes are expected to differ. In the latter, gauche kinks at the first position in the alkyl chain are stabilized due to $\mathrm{CH} \cdots \pi$ interactions and are found to be almost isoenergetic with the stretched all- $t$ conformers, whereas in the former gauche kinks at the first position occur with an energetic penalty of $\sim 4 \mathrm{~kJ} \cdot \mathrm{mol}^{-1}$ as seen in the comparison between $t t t$ and $g t t$ (Table 5.1).

Interconversion barriers between different dihedral configurations along the glyme chain are significantly increased compared to pure glyme chains when they are in close proximity to the aromatic ring. As a comparison in Figure 5.3a shows, the barriers for torsional distortions in the first dihedral along the glyme chain in $t t t$ is raised from $\sim 8 \mathrm{~kJ} \cdot \mathrm{mol}^{-1}$ in 1,2-dimethoxyethane to $\sim 13 \mathrm{~kJ} \cdot \mathrm{mol}^{-1}$ in 1-methoxy-2-phenoxyethane. Dihedral interconversions of the other two positions along the glyme chain show very similar barrier heights than those found in 1,2-dimethoxyethane (Figure $^{2}$ $5.3 \mathrm{~b}$ and 5.4). Barriers for interconversions of the OCCO dihedral are larger $\left(\sim 10 \mathrm{~kJ} \cdot \mathrm{mol}^{-1}\right.$, Figure $\left.5.3 \mathrm{~b}\right)$ than those of the terminal CCOC dihedral $\left(\sim 5 \mathrm{~kJ} \cdot \mathrm{mol}^{-1}\right.$, Figure 5.4). Therefore, $t g g^{\prime}$ can efficiently relax into $t g t$ while an interconversion between $t g t$ and $t t t$ under supersonic jet expansion conditions is unlikely.

An anharmonic local mode model developed by Ned Sibert and Daniel Tabor was used to predict the conformer specific IR spectra of the $\mathrm{CH}$ stretching region. This model was briefly described in Chapter 4.3 and was used analogously here. Coupling constants between $\mathrm{CH}$ stretching fundamentals and overtones of scissor and bending motions were adjusted based on results on 1,2-diphenoxyethane and 1,2diphenylethane [102]. A more detailed description of the anharmonic model is found in [102].

\footnotetext{
₹ Interconversion barriers for dihedral distortions in 1,2-dimethoxyethane are extensively discussed in Chapter 6.3.
} 
Table 5.1: Differences in zero-point corrected energies $\left(\Delta E^{0}\right)$, zero-point vibrational energies ( $\triangle \mathrm{ZPVE}$ ) and Gibbs energies at $298 \mathrm{~K}\left(\Delta G^{0}\right)$ relative to the $t t t$ for all 14 conformers calculated at the B3LYP2D3/aVTZ and B3LYP-2D3/def2TZVP level in Gaussian 09 Rev. E.01.

\begin{tabular}{|c|c|c|c|c|c|c|}
\hline \multirow[b]{2}{*}{ conformer } & \multicolumn{3}{|c|}{ B3LYP-2D3/aVTZ } & \multicolumn{3}{|c|}{ B3LYP-2D3/def2TZVP } \\
\hline & $\begin{array}{c}\Delta E_{0} / \\
\mathbf{k J} \cdot \mathrm{mol}^{-1}\end{array}$ & $\begin{array}{l}\Delta \mathrm{ZPVE} / \\
\mathrm{kJ} \cdot \mathrm{mol}^{-1}\end{array}$ & $\begin{array}{c}\Delta G^{0} / \\
\mathbf{k J} \cdot \mathbf{m o l}^{-1}\end{array}$ & $\begin{array}{c}\Delta E_{0} / \\
\mathrm{kJ} \cdot \mathrm{mol}^{-1}\end{array}$ & $\begin{array}{l}\Delta \mathrm{ZPVE} / \\
\mathrm{kJ} \cdot \mathrm{mol}^{-1}\end{array}$ & $\begin{array}{c}\Delta G^{0} / \\
\mathrm{kJ} \cdot \mathrm{mol}^{-1}\end{array}$ \\
\hline$t t t$ & 0.00 & 0.00 & 0.00 & 0.00 & 0.00 & 0.00 \\
\hline $\operatorname{tgt}$ & 0.48 & 0.00 & -0.96 & 0.50 & -0.04 & -1.09 \\
\hline $\operatorname{tgg}$ & 1.75 & 0.34 & 0.11 & 1.56 & 0.44 & -0.12 \\
\hline$g g^{\prime} t$ & 3.76 & 0.29 & 2.75 & 3.16 & 0.39 & 2.19 \\
\hline$g t t$ & 4.64 & 1.34 & 2.50 & 4.52 & 0.98 & 2.87 \\
\hline$g g t$ & 5.15 & 0.92 & 3.44 & 5.11 & 0.59 & 4.01 \\
\hline $\operatorname{ttg}$ & 5.62 & 0.41 & 3.61 & 5.50 & 0.28 & 2.75 \\
\hline $\operatorname{tgg}$ & 5.67 & 0.76 & 3.02 & 5.37 & 0.42 & 3.25 \\
\hline$g g g^{\prime}$ & 5.80 & 1.37 & 3.59 & 5.60 & 0.99 & 3.85 \\
\hline$g g^{\prime} g$ & 6.16 & 1.27 & 5.57 & 5.47 & 0.96 & 5.48 \\
\hline$g t g^{\prime}$ & 9.93 & 0.76 & 7.56 & 10.35 & 1.24 & 8.37 \\
\hline gtg & 10.04 & 0.87 & 8.00 & 9.86 & 0.99 & 7.93 \\
\hline$g g g$ & 10.50 & 1.43 & 8.58 & 10.42 & 0.99 & 8.93 \\
\hline$g^{\prime} g g$ & 11.38 & 0.57 & 8.85 & 10.76 & 0.18 & 8.44 \\
\hline
\end{tabular}
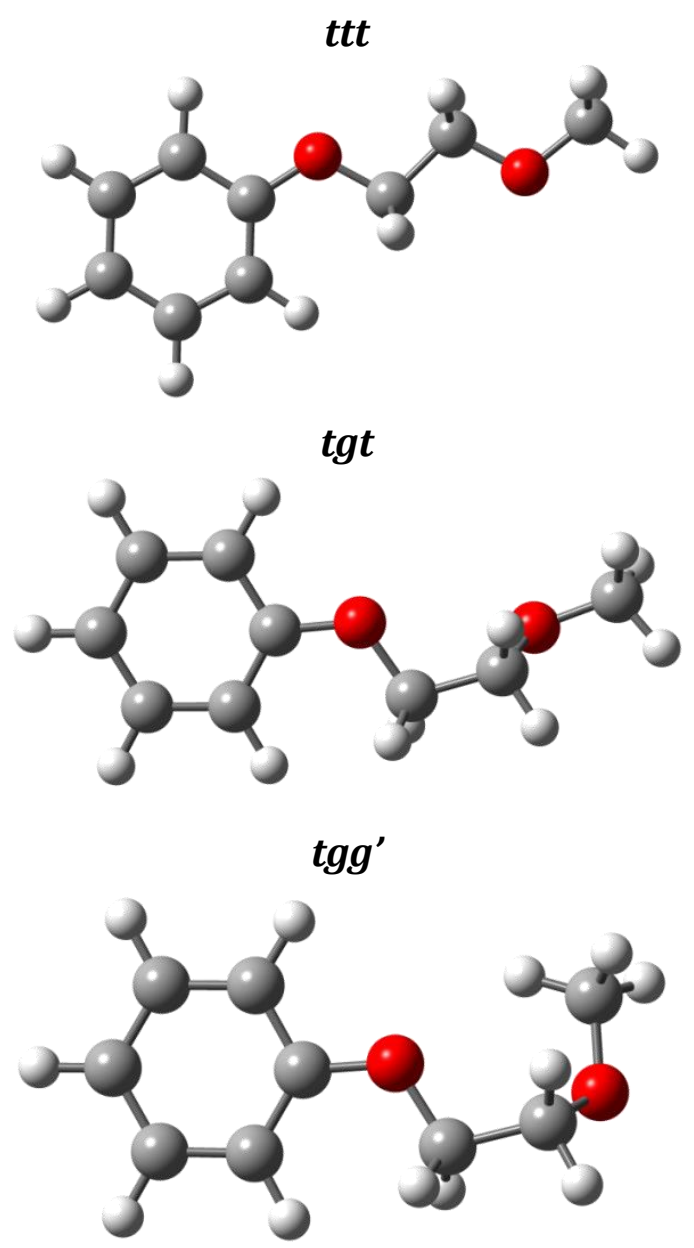

Figure 5.2: Structures of the three lowest conformers in 1-methoxy-2-phenoxyethane. 
a)

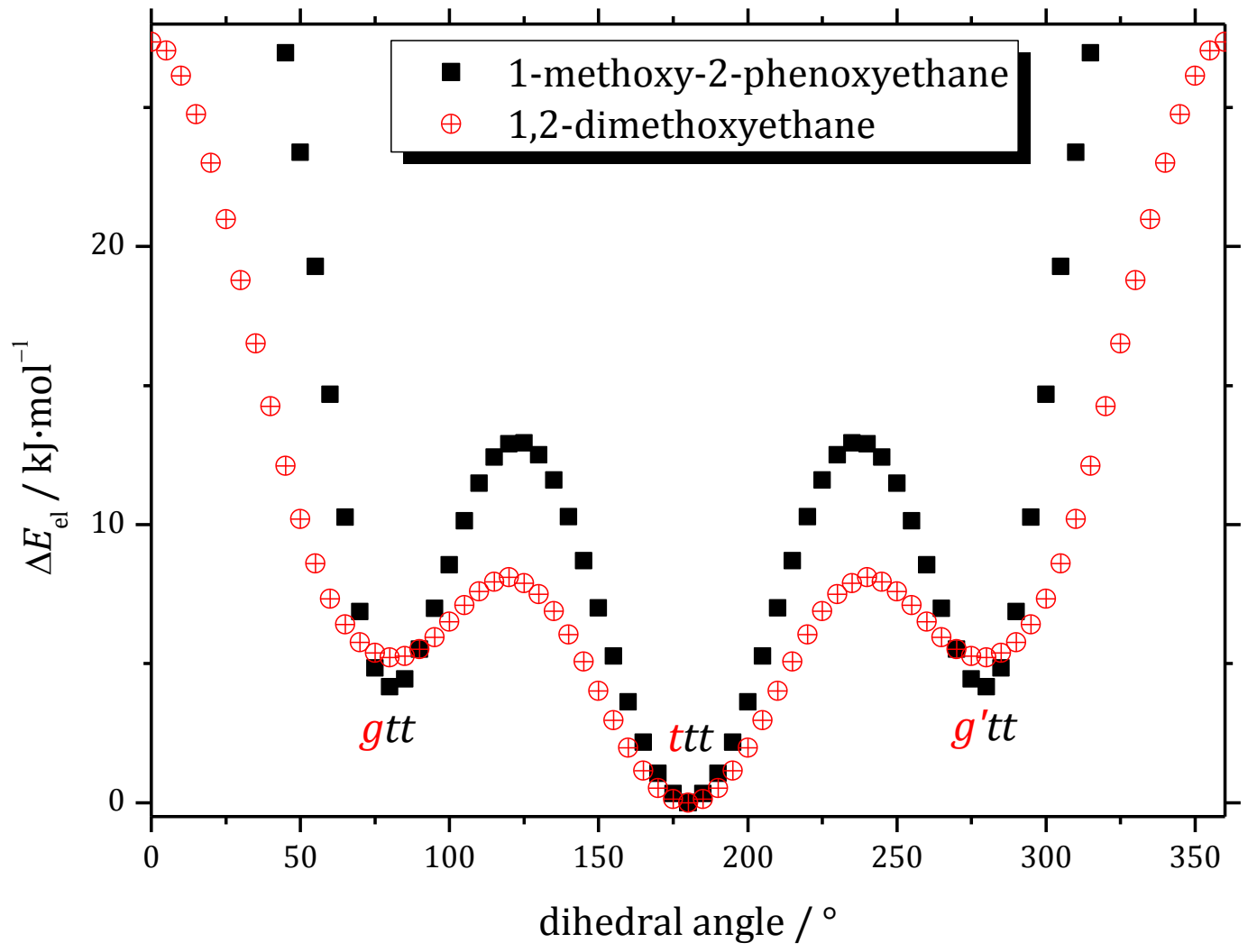

b)

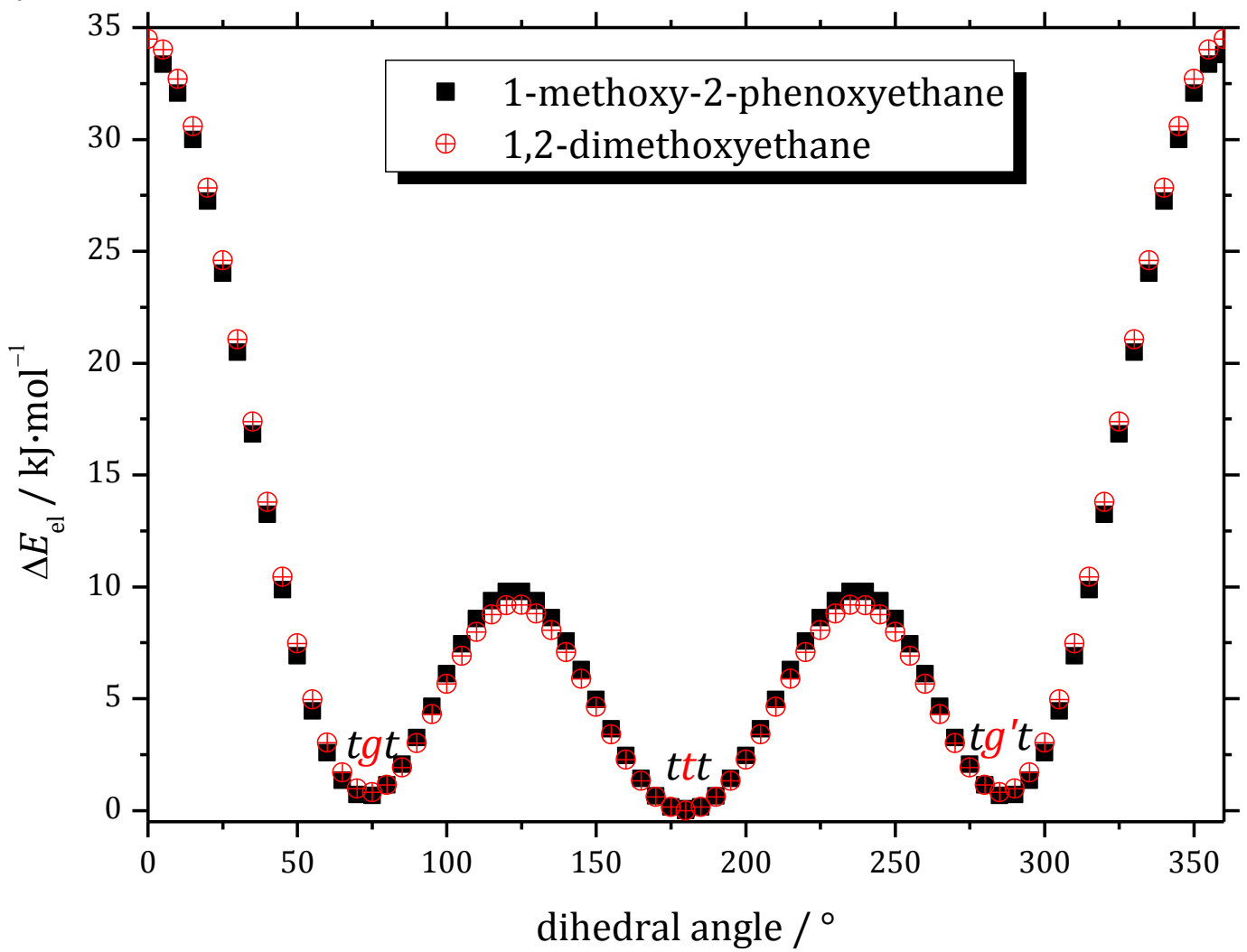

Figure 5.3: Relaxed 1D torsional scans of the first (a) and second (b) backbone dihedral of the ttt conformers in 1-methoxy-2-phenoxyethane (black squares) and 1,2-dimethoxyethane (red circles). Calculations were performed at every $5^{\circ}$ on the B3LYP-2D3/def2TZVP level in Gaussian 09 Rev. E.01. 


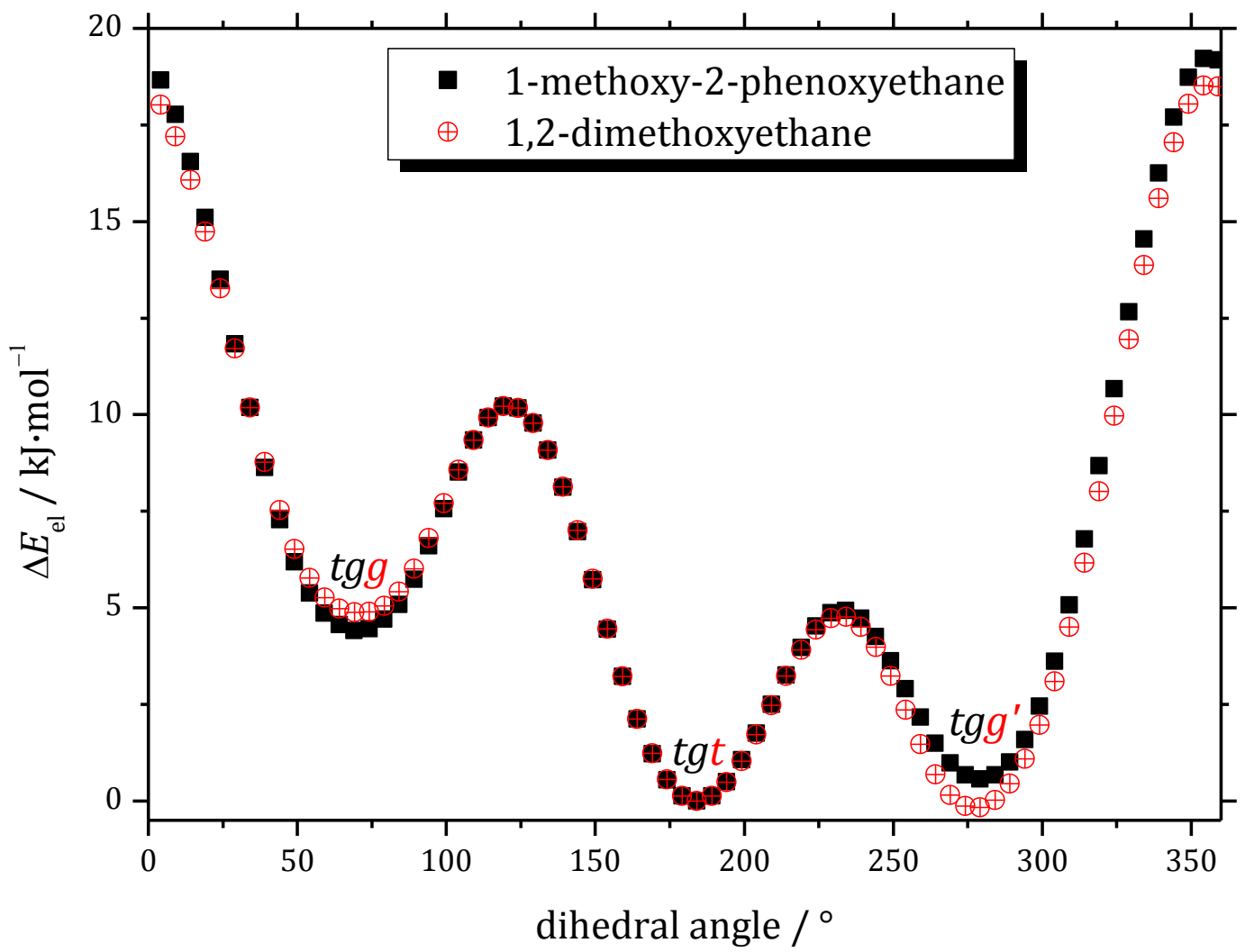

Figure 5.4: Relaxed 1D torsional scans of the third backbone dihedral of the tgt conformers in 1-methoxy-2-phenoxyethane (black squares) and 1,2-dimethoxyethane (red circles). Calculations were performed at every $5^{\circ}$ on the B3LYP-2D3/def2TZVP level in Gaussian 09 Rev. E.01.

\section{3 - LIF and UV-UV HB spectra}

The LIF spectrum of 1-methoxy-2-phenoxyethane (Figure 5.5) shows three unique origin transitions at 36357,36405 and $36450 \mathrm{~cm}^{-1}$ that are assigned to tgt, tgg' and $t t t$, respectively. The origin transition marked with an asterisk is assigned to a water cluster, as will be shown in the following section, that is probably formed with tgt, consistent with findings on water clusters in diphenoxyethane [103]. The $\mathrm{S}_{0}-\mathrm{S}_{1}$ origin transitions of $t g t$ and $t t t$ appear with nearly equal intensity in the spectrum while the tgg' origin is significantly weaker. The assignments are supported by quantum chemical calculations that predict $t t t$, tgt and $t g g^{\prime}$ as the three most-stable conformers with a significant gap to the next conformers. Moreover, $t t t$ and tgt are predicted very close in energy, separated by approximately $0.6 \mathrm{~kJ} \cdot \mathrm{mol}^{-1}$, consistent with the assignment of these conformers to the two strong observed transitions in the spectrum. Furthermore, a jet study on diphenoxyethane found nearly equal tgt and ttt populations with origin transitions at 36432 and $36519 \mathrm{~cm}^{-1}$, respectively [102]. This shift $\left(87 \mathrm{~cm}^{-1}\right)$ is in line with the observed shift between tgt and ttt $\left(93 \mathrm{~cm}^{-1}\right)$ in 1-methoxy-2-phenoxyethane. Predicted shifts, calculated at the B3LYP-2D3/aVTZ level, of the $\mathrm{S}_{0}-\mathrm{S}_{1}$ transitions of $t g t$ and $t g g^{\prime}$ to $t t t$ are 138 and $42 \mathrm{~cm}^{-1}$, respectively, somewhat consistent with the observed shifts of 93 and $45 \mathrm{~cm}^{-1}$. Additionally, the origin transition of $t t t$ is anticipated to be at higher frequencies than other conformers' origins because of weaker interactions between the stretched $t t t$ chain and the 
aromatic ring. Reasonable assignments of the LIF transitions to the three most-stable conformers were possible but will be tested further in the following sections. Interestingly, three conformers with the same dihedral backbone sequence were found to survive jet expansion conditions in the methoxy-substituted analog, 1,2-dimethoxyethane, as will be discussed in Chapter 6 .

A comparison of the LIF and the UV-UV HB spectra (Figure 5.6) taken at the origin transitions of $t g t$, $t g g^{\prime}$ and $t t t$ show that all observed transitions in the LIF spectrum belong to these three conformers. Thus, all other visible bands at higher wavenumbers in Figure 5.5 result from Franck-Condon activity of vibrational modes in these conformers. Due to the low intensity of the origin transition of the water cluster and its close proximity to the $t g g^{\prime}$ origin it was not possible to record its UVHB spectrum.

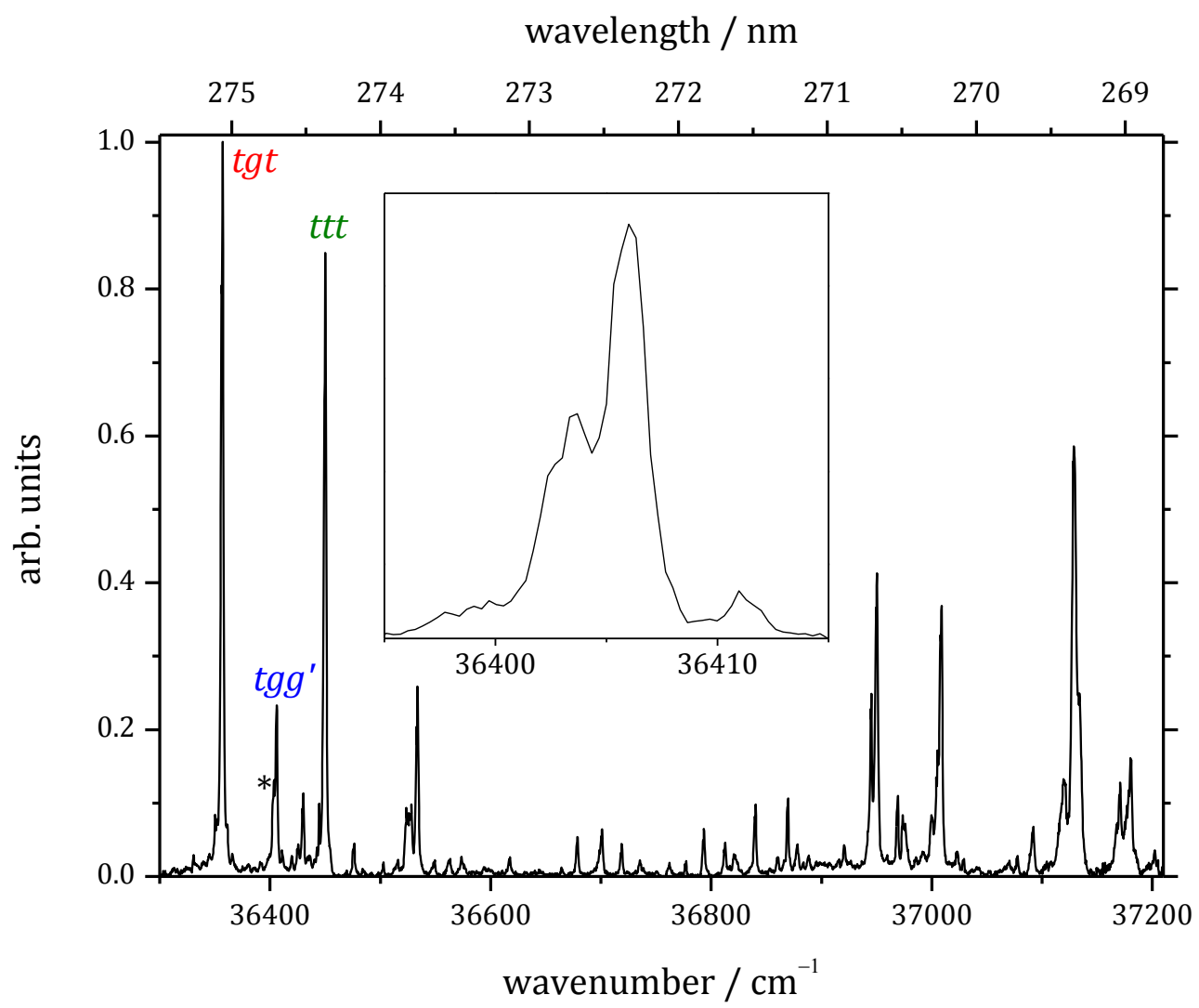

Figure 5.5: LIF excitation spectrum for 1-methoxy-2-phenoxyethane. Three unique $\mathrm{S}_{0}-\mathrm{S}_{1}$ origin transitions are assigned to $t g t, t g g^{\prime}$ and $t t t$ as labeled in the spectrum. An origin transition marked with an asterisk $\left({ }^{*}\right)$ is assigned to a water cluster. The water cluster and $t g g^{\prime}$ origins are magnified in the inset for better visibility. 


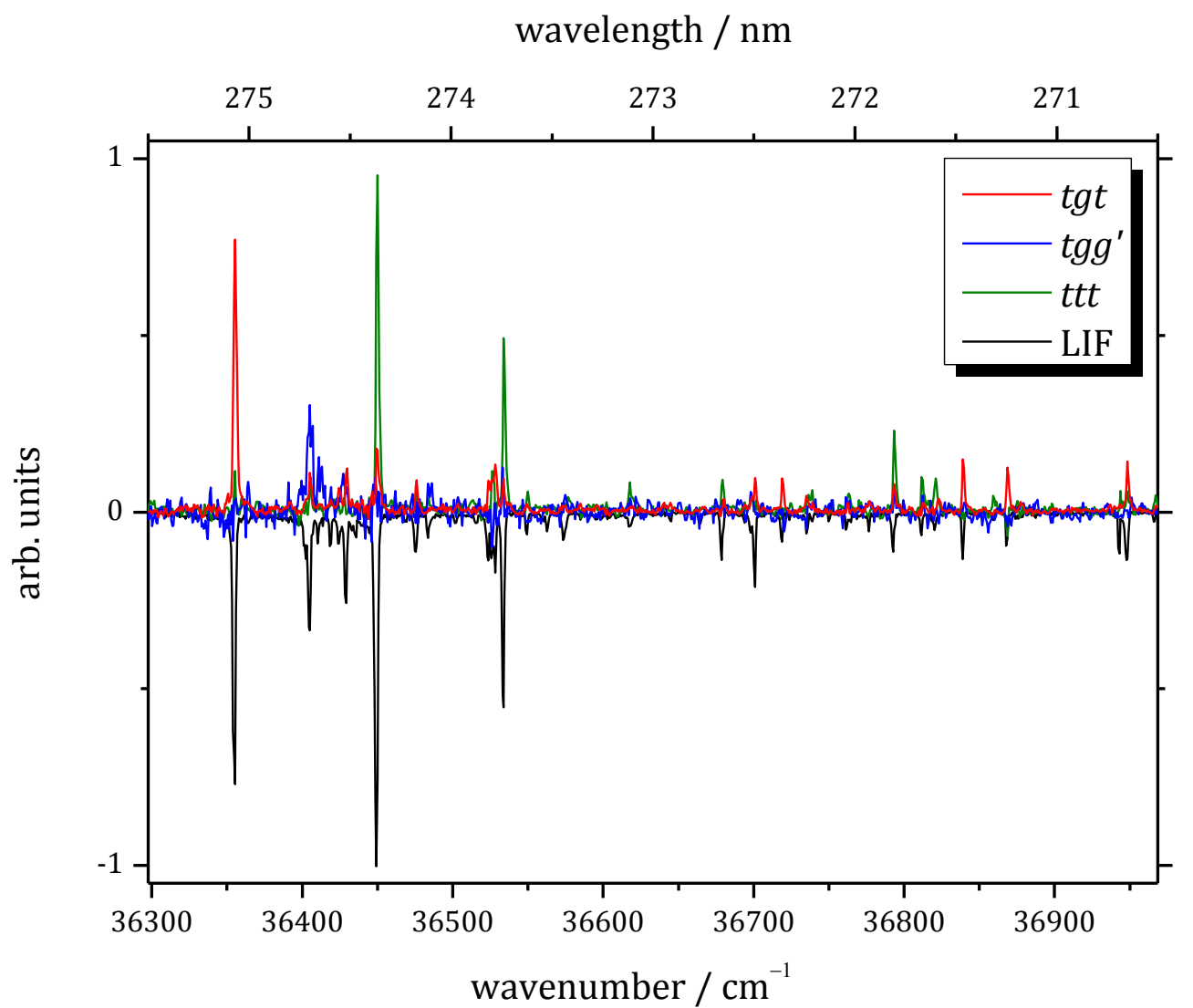

Figure 5.6: UV-UV HB spectra (top trace) at the origin transitions of tgt (red), tgg' (blue) and ttt (green) compared to the LIF spectrum (bottom trace).

\section{4 - Infrared spectra}

The FDIR spectra in the CH stretching region from $2800-3000 \mathrm{~cm}^{-1}$ of $t t t$, tgt and $t g g^{\prime}$ are compared to predicted spectra based on the anharmonic local mode model in Figure 5.7 to Figure 5.9. Various Fermi resonances between scissor overtones with symmetric and asymmetric $\mathrm{CH}$ stretches complicate the spectra. In contrast, the spectrum of $t t$ in 1,2-diphenoxyethane shows only three transitions, due to its high symmetry, that are found to be nearly uncoupled [102]. There, the symmetric $\mathrm{CH}$ stretches are found at the lower frequency end of the spectrum at around $2875 \mathrm{~cm}^{-1}$, while the asymmetric ones are found at approximately $2950 \mathrm{~cm}^{-1}$ and the scissor overtones are found at almost $3000 \mathrm{~cm}^{-1}$. Also in 1-methoxy-2-phenoxyethane these regions show intense bands but the lower symmetry of the molecule and strong Fermi resonances between these bands add to the complexity of the spectra.

The $\mathrm{CH}$ spectra of the three major conformers are easily distinguishable as all of them show unique shifts and intensity patterns. The spectrum of the $t t t$ conformer (Figure 5.7) shows intense bands at 2885, 2933 and $2999 \mathrm{~cm}^{-1}$. All of them are nicely reproduced by the model even though the intensity of the band at $2845 \mathrm{~cm}^{-1}$ is overestimated. The spectrum of the tgt conformer (Figure 5.8) shows intense bands at 2880, 2923 and $3000 \mathrm{~cm}^{-1}$. Nearly all band positions and intensities are reproduced accurately by the model. The spectrum of the $\mathrm{tgg}^{\prime}$ conformer (Figure 5.9) shows intense bands at 2916, 2949 and $2995 \mathrm{~cm}^{-1}$. Here the model shows larger deviations from the 
spectrum, especially in the lower frequency region between 2833 and $2855 \mathrm{~cm}^{-1}$. However, fits of other conformers such as $g g^{\prime} t$ or $g t t$ resulted in a much poorer agreement. In general, the fits reproduce all intense bands with good precision but overestimate or miss some weak bands. This might result from the small database that was used for adjusting coupling constants as mainly 1,2-diphenoxyethane and 1,2-diphenylethane were used for developing the anharmonic model. Results on further extended glyme chains attached to the benzene ring would likely improve the anharmonic model. However, despite these small drawbacks the overall agreement between the spectra and fits is good and sufficient to give confidence in the assignments.

The IR spectrum taken at the origin of the water cluster (asterisk in Figure 5.5) differs from all other spectra as seen in Figure 5.10. Unfortunately this prevents any tentative assignments on the conformation of 1-methoxy-2-phenoxyethane that is involved in this cluster. Furthermore, dispersed fluorescence spectra from all origin transitions did not show significant agreement between the spectrum taken at the water cluster origin and any other conformer. However, a RIDIR spectrum taken in the $\mathrm{OH}$ stretching region from 3550 to $3720 \mathrm{~cm}^{-1}$ and the [1-methoxy-2-phenoxyethane- $\left.\mathrm{H}_{2} \mathrm{O}\right]^{+}$mass channel clearly reveals contributions of water (Figure 5.11). Two bands are found at 3574 and $3709 \mathrm{~cm}^{-1}$ with the latter showing a splitting pattern that is hard to interpret due to the low signal-to-noise ratio. The water complex of 1,2-diphenoxyethane showed two $\mathrm{OH}$ stretch fundamentals at 3597 and a doublet at $3705 / 3707 \mathrm{~cm}^{-1}$ [103]. The first was assigned to the $\mathrm{OH} \cdots \mathrm{O}$ hydrogen bond to one of the phenoxy oxygens while the latter was assigned to weak $\mathrm{OH} \cdots \pi$ interactions [103]. Analogously, the first observed transition in 1-methoxy-2-phenoxyethane is expected to arise from an $\mathrm{OH} \cdots \mathrm{O}$ hydrogen bond to the methoxy oxygen, consistent with the larger shift to lower frequencies when compared to its phenoxy-substituted analog. The second transition arises from $\mathrm{OH} \cdots \pi$ interactions to the aromatic ring and thus is shifted in a very similar way as found in 1,2-diphenoxyethane. 


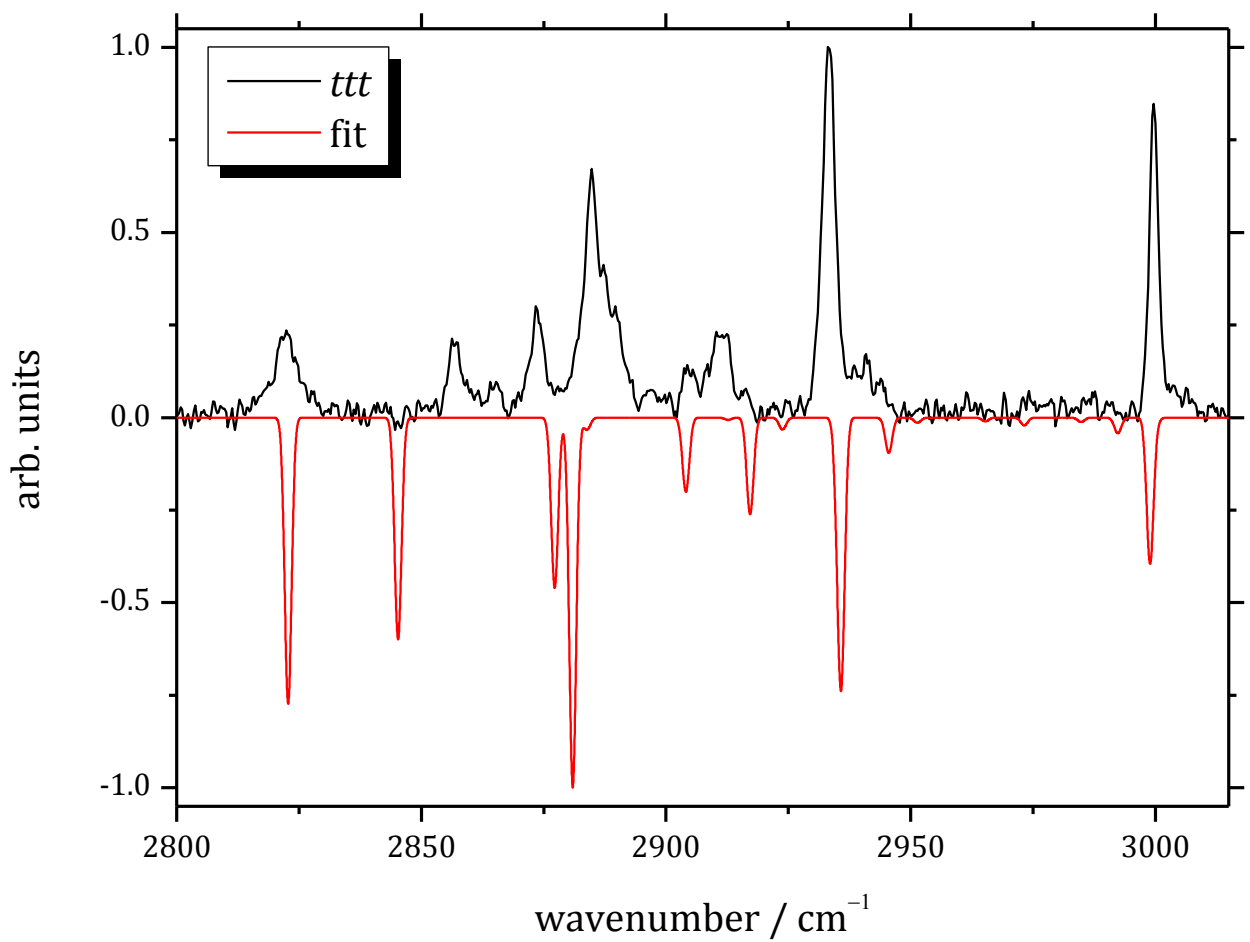

Figure 5.7: Comparison between the experimental (black) and the predicted (red) spectra of $t t t$.

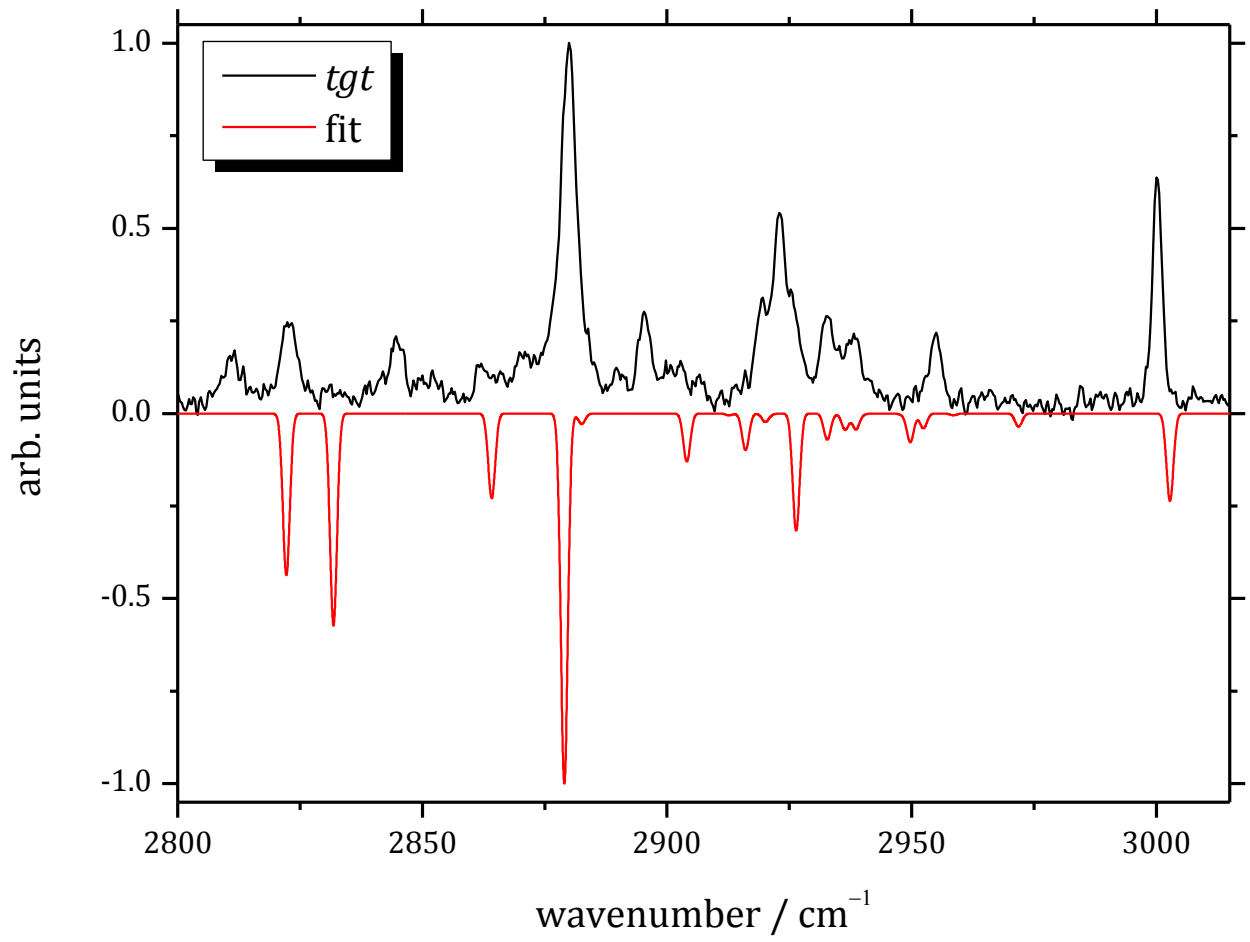

Figure 5.8: Comparison between the experimental (black) and the predicted (red) spectra of tgt. 


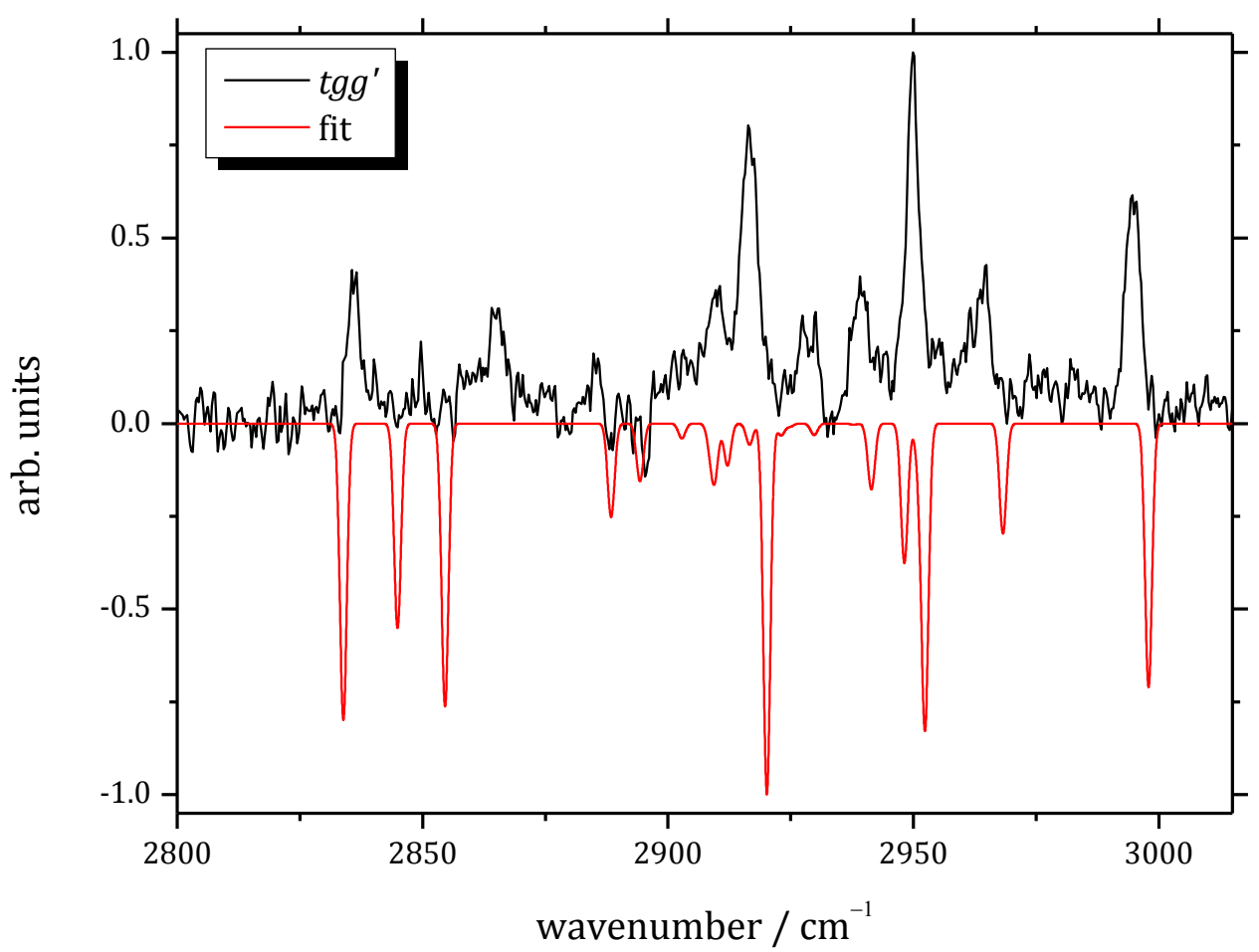

Figure 5.9: Comparison between the experimental (black) and the predicted (red) spectra of tgg' $^{\prime}$.

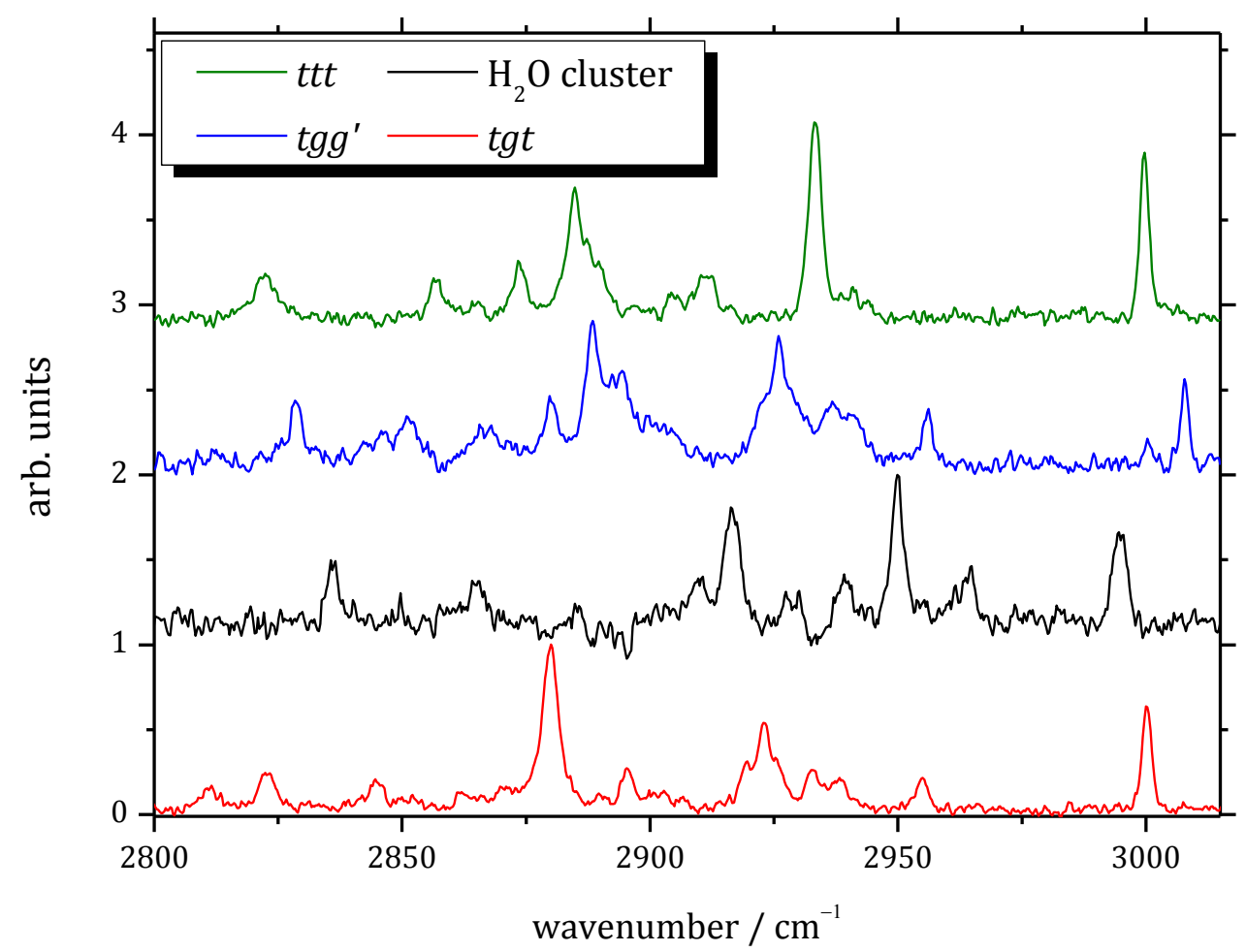

Figure 5.10: Experimental $\mathrm{CH}$ spectra taken at the origin transitions of the three observed conformers and the water cluster band (compare Figure 5.5). 


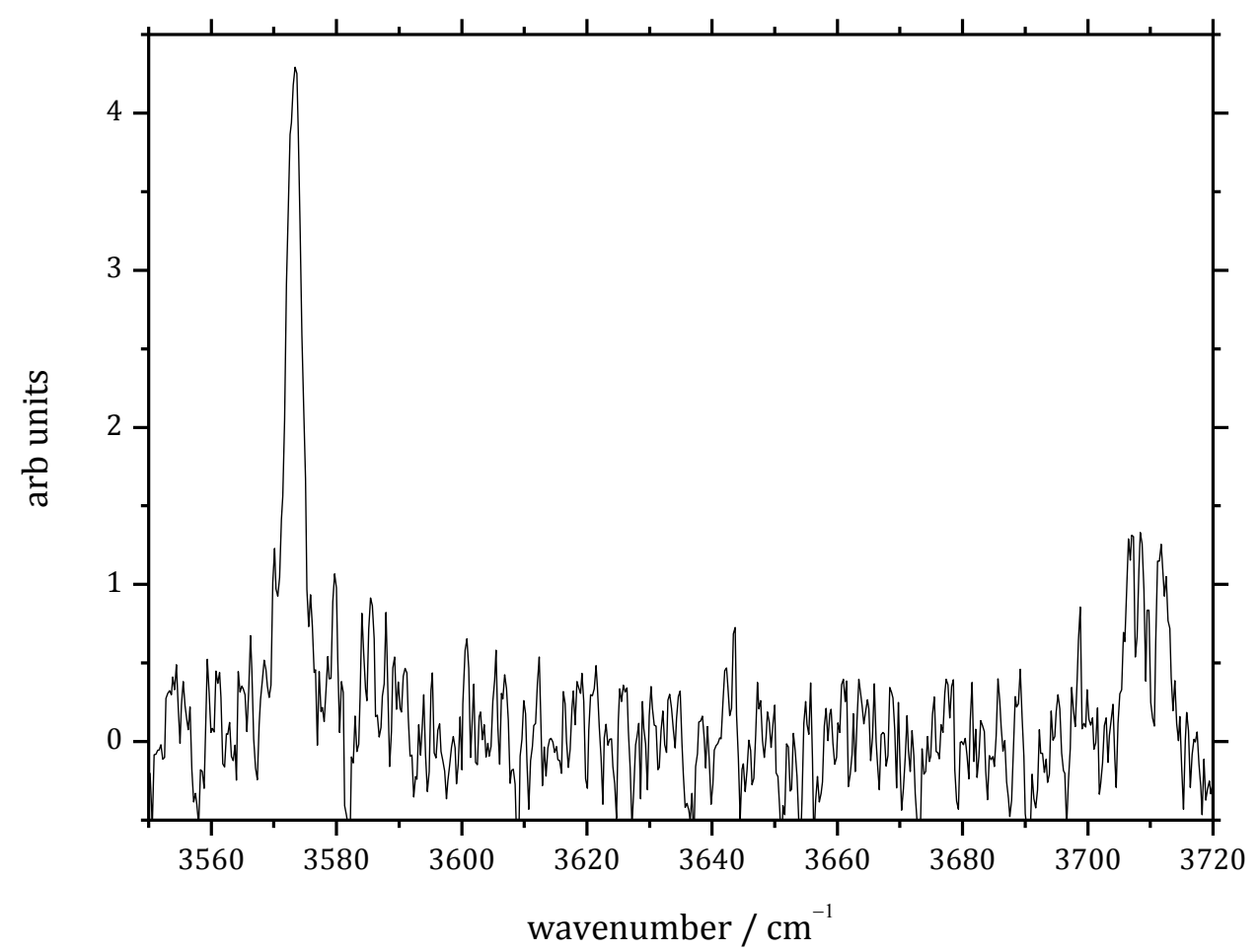

Figure 5.11: RIDIR spectrum of the 1-methoxy-2-phenoxyethane- $\mathrm{H}_{2} \mathrm{O}$ cluster in the $\mathrm{OH}$ stretching region.

\section{5 - Raman spectra}

Similar to the LIF results, Raman jet spectra of 1-methoxy-2-phenoxyethane show major contributions of $t t t$ and tgt (Figure 5.12). However only very small contributions from $t g g^{\prime}$ are found indicating that the relaxation of this conformer is more efficient and rapidly completed in the curry jet expansions. The curry jet uses a slit nozzle which forms a two dimensional expansion zone that allows for more collisions and enhances cooling effects in contrast to three dimensional expansion formed by the cylindrical nozzle used in the LIF setup. The next two conformers in the energy ranking $\left(g g^{\prime} t, g t t\right)$ are predicted to have strong isolated bands around 335 and $540 \mathrm{~cm}^{-1}$, regions where the experimental spectrum shows no signals. Predicted Raman scattering cross sections of $t t t$ and $t g t$ calculated at the B3LYP-2D3/aVTZ level in Gaussian 09 Rev. E.01 show good agreement with the experimental spectrum. The cross sections were weighted with their relative abundances estimated based on Gibbs energy differences at $298 \mathrm{~K}$. This simple approach leads to a reasonable interpretation of the spectrum as all intense bands are accounted for and their intensity patterns are reproduced well. Some smaller unassigned bands are explained upon inclusion of $t g g^{\prime}$. However, the weighted scaling factor for its predicted Raman scattering cross sections needs to be farily low in order to match the observed intensity pattern. Furthermore, some predicted bands of $t g g^{\prime}$ mismatch the observed spectrum making its presence in the jet spectra doubtful. The low frequency region mainly consists of COC torsions $\left(\sim 230-310 \mathrm{~cm}^{-1}\right)$, longitudinal acoustic modes (LAM) $\left(\sim 280-310 \mathrm{~cm}^{-1}\right)$ and ring torsions $\left(\sim 410-430\right.$ and $\left.590-620 \mathrm{~cm}^{-1}\right)$. While the latter are not very conformation-specific, as these modes are mostly localized at the aromatic ring, the longitu- 
dinal acoustic modes proved to be very sensitive to the conformational alignment of the molecule.

The frequency region from $740-1200 \mathrm{~cm}^{-1}$ mostly contains ring torsions and CO stretching vibrations (Figure 5.13). The region between 800 and $835 \mathrm{~cm}^{-1}$ consists of mixing modes between ring torsions and the CO stretching vibration of the oxygen that is directly attached to the aromatic ring. The region between 930 and $990 \mathrm{~cm}^{-1}$ consists of symmetric CO stretching vibrations of the methoxy group, while the asymmetric stretches are found around $1150 \mathrm{~cm}^{-1}$.

Varying experimental parameters such as the nozzle temperature (Figure 5.14) or expanding the molecules with different carrier gases (Figure 5.15) has no influence on the conformational abundances observed in the spectra. Thus, relaxations of 1methoxy-2-phenoxyethane during supersonic jet expansions in the curry jet are completed rapidly. Unfortunately, this hinders the possibility to assess the global minimum structure in jet expansion experiments.

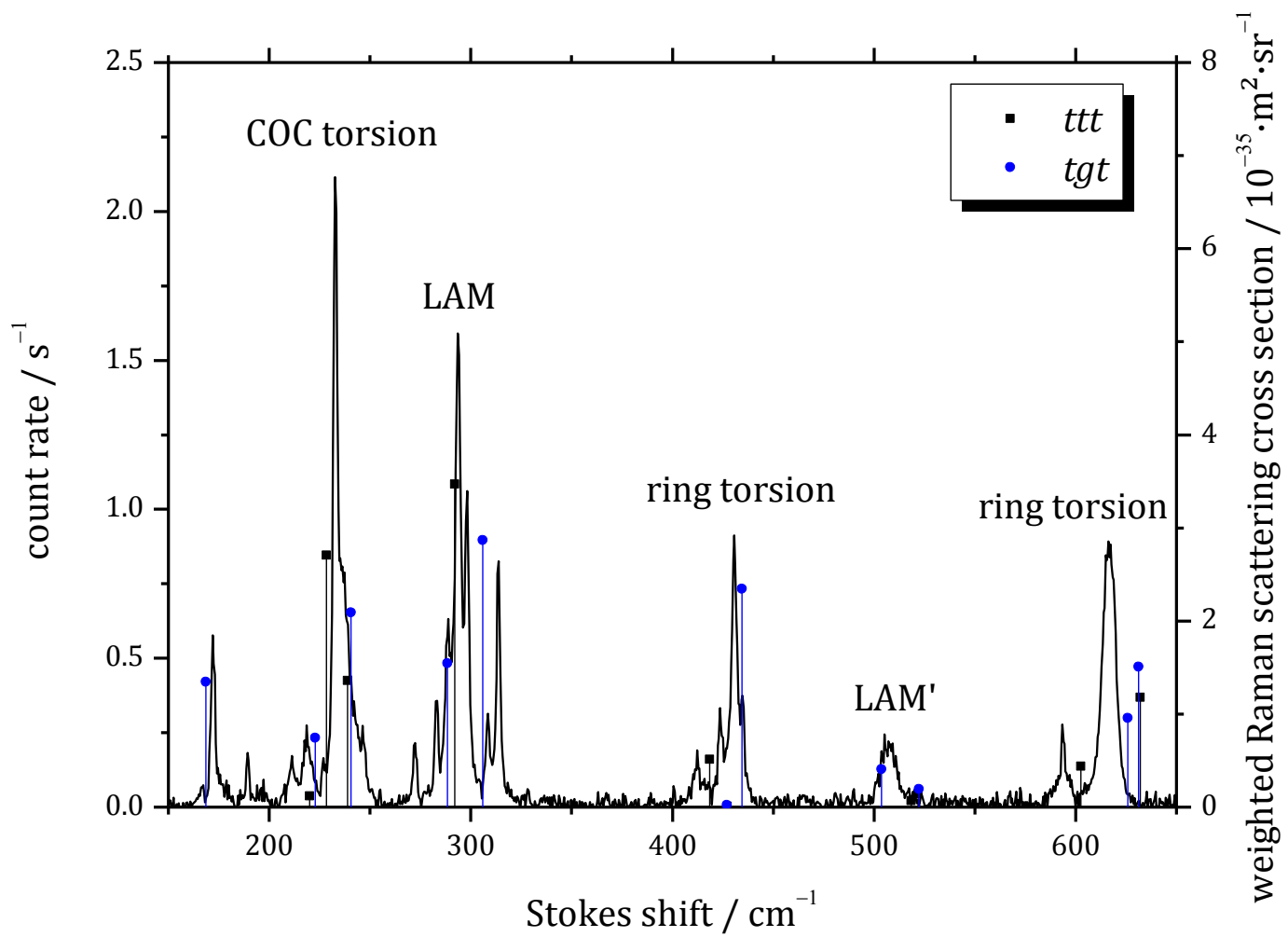

Figure 5.12: Low frequency jet spectrum of 1-methoxy-2-phenoxyethane (measurement conditions can be found in A.1) compared to Raman scattering cross sections calculated at the B3LYP-2D3/aVTZ level and weighted with their relative abundances based on the $\Delta G$ values at $298 \mathrm{~K}$. 


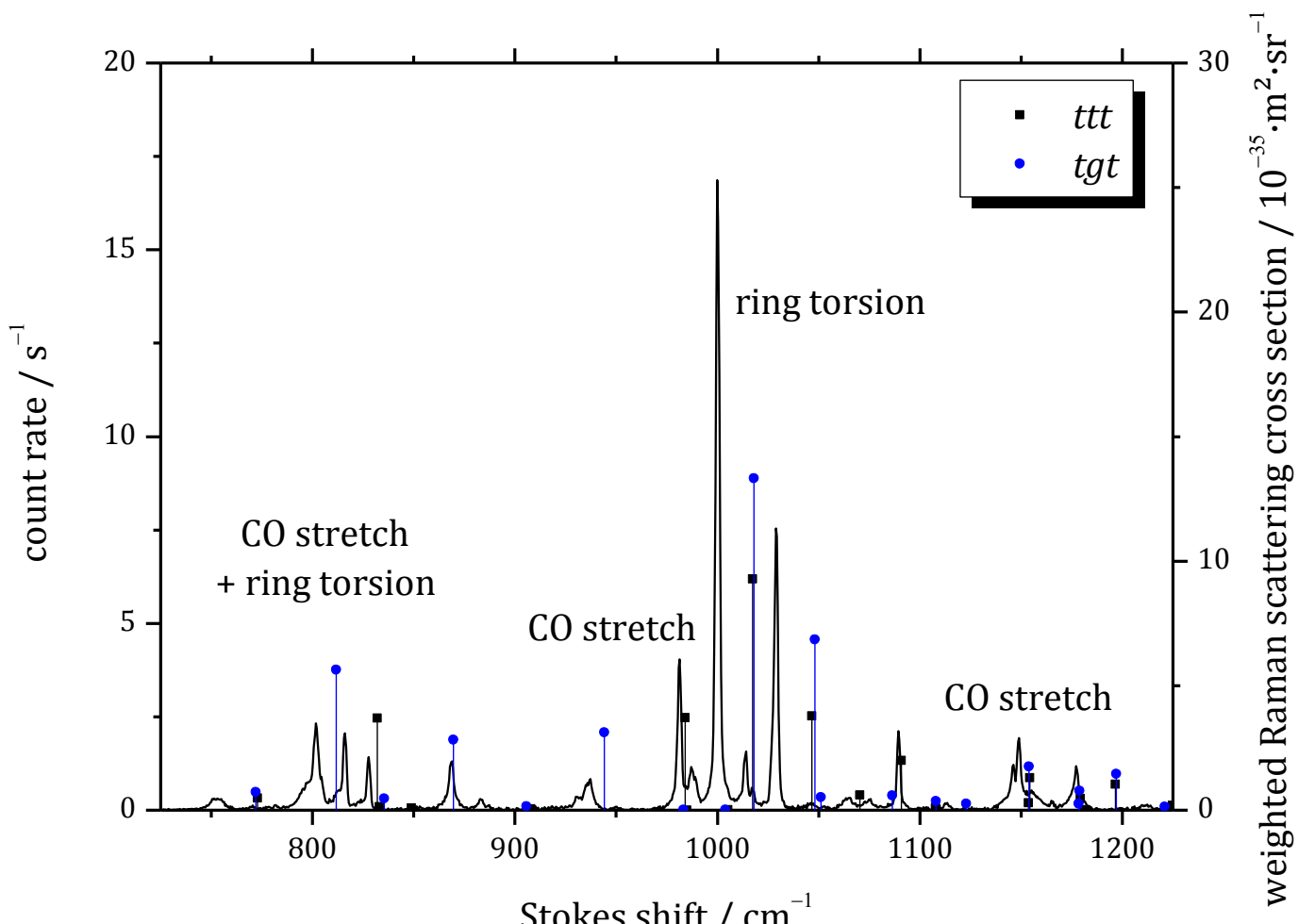

Figure 5.13: Jet spectrum of 1-methoxy-2-phenoxyethane in the region from 725 to $1225 \mathrm{~cm}^{-1}$ (measurement conditions can be found in A.1) compared to Raman scattering cross sections calculated at the B3LYP-2D3/aVTZ level and weighted with their relative abundances based on the $\Delta G$ values at $298 \mathrm{~K}$.

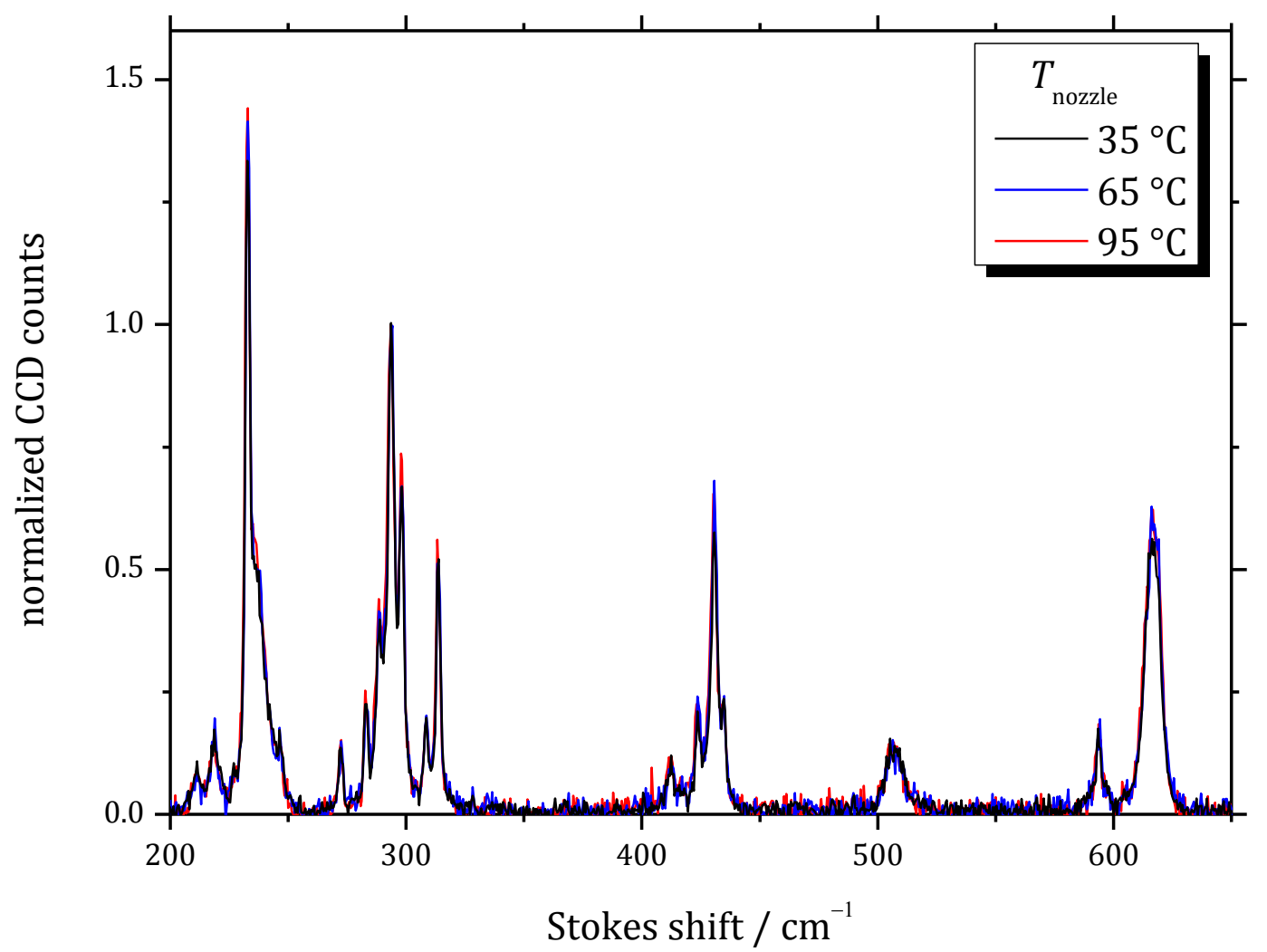

Figure 5.14: Jet spectra of 1-methoxy-2-phenoxyethane in He at nozzle temperatures of 35 (black), 65 (blue) and $95{ }^{\circ} \mathrm{C}$ (red) at $1 \mathrm{~mm}$ nozzle distances in the low frequency region. Spectra were normalized to the $t t t$ LAM. Other measurement parameters are found in A.1 


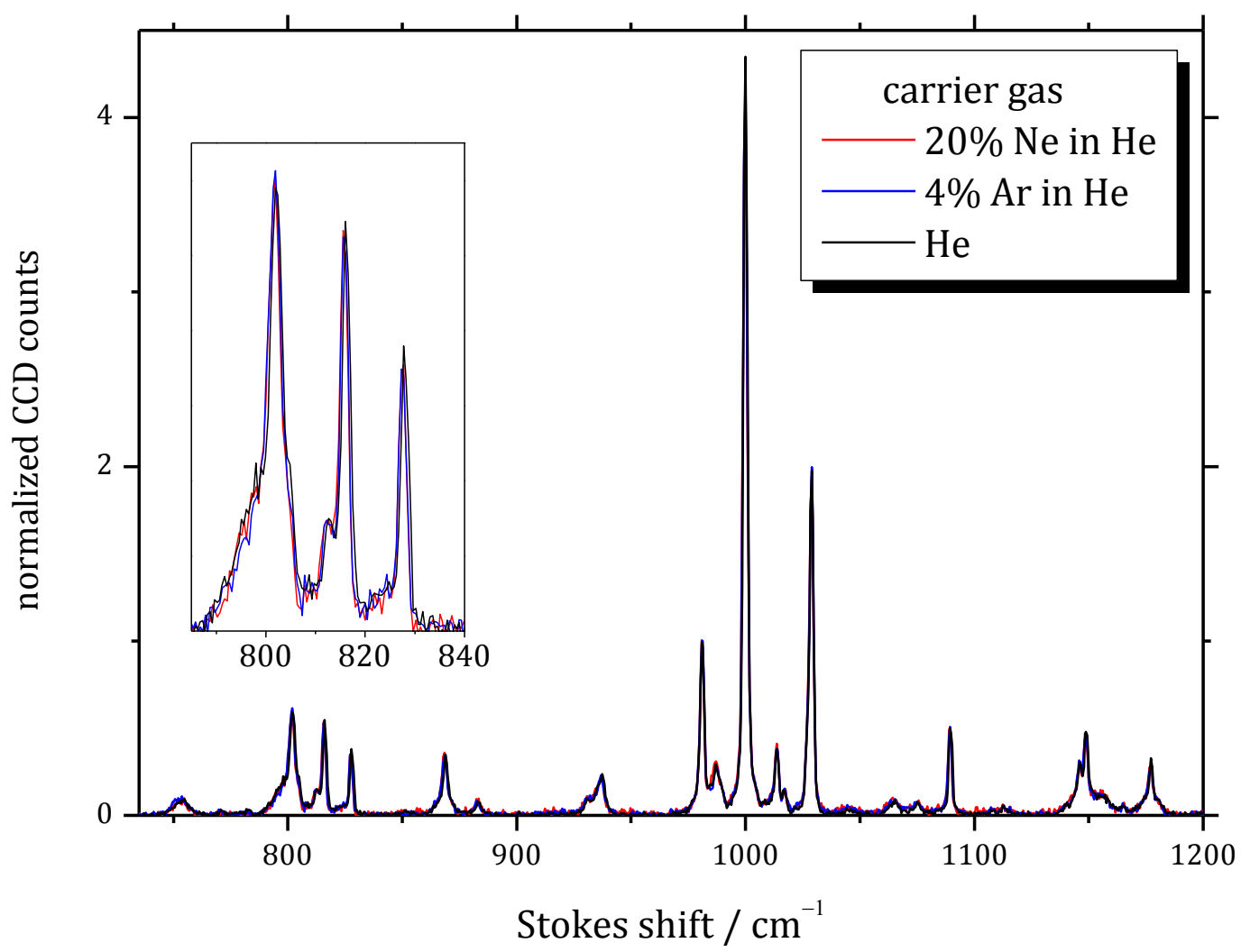

Figure 5.15: Jet spectra of 1-methoxy-2-phenoxyethane in He (black), 20\% $\mathrm{Ne}$ in $\mathrm{He}$ (red) and 4\% $\mathrm{Ar}$ in He (blue) at $1 \mathrm{~mm}$ nozzle distance. Spectra were normalized to the symmetric CO stretching vibration of $t t$ at $981 \mathrm{~cm}^{-1}$. Other measurement parameters are found in A.1

\section{6 - Conclusions}

In summary, a combination of electronic frequency shifts, single-conformer IR spectra in the $\mathrm{CH}$ region, dispersion-corrected DFT energies and an anharmonic local mode model allowed for an assignment of three conformers in 1-methoxy-2phenoxyethane. Contributions of these three conformers were found in all LIF jet spectra and were assigned to $t t t$, tgt and tgg' which are predicted as the three most stable structures in the harmonic calculations.

The observed $\mathrm{S}_{0}-\mathrm{S}_{1}$ origin transitions of tgt and $t g g^{\prime}$ are found at lower frequencies, due to stronger interactions of the $\pi$ cloud with the glyme chain, than the $t t t$ origin. Furthermore, the UV-UV HB spectra confirmed strong Franck-Condon activities, which are seen at higher frequencies in the LIF spectrum, of vibrational modes in every conformer. Conformer specific IR spectra are well reproduced by the anharmonic local mode model and confirm the initial assignments that were made based on a comparison between the LIF spectrum and harmonic DFT as well as TDDFT calculations. Additionally, spectroscopic signs of a 1-methoxy-2-phenoxy-water cluster were found but not further investigated.

Raman spectra in the curry jet only show major contributions of $t t t$ and tgt. The conformationally colder conditions of the slit nozzle expansions in the curry jet might lead to a full relaxation of $\mathrm{tgg}^{\prime}$. Strong Raman bands in the frequency region of 200 to $1200 \mathrm{~cm}^{-1}$ were assigned to their corresponding motions. 
Conformational abundances in the supersonic jet expansions could not be manipulated through variation of the experimental parameters. Therefore, the global minimum structure could not be confirmed experimentally although the LIF intensities indicate that conformational abundances of tgt and ttt are significantly larger than that of tgg'. Moreover, the further cooling in the curry jet expansions lead to an almost full relaxation of tgg', consistent with the prediction that tgt is more stable than tgg'. 


\section{Chapter 6 - Monoglyme}

In this chapter Raman spectroscopic and quantum chemical investigations on the smallest member of the glyme family will be presented. Parts of this work, including calculations, models, plots and conclusions, have been published in [95]. The analysis is partly refined here, to be more consistent with proceedings on other molecules that will be presented in this dissertation.

\section{1 - Introduction}

Monoglyme, also known as 1,2-dimethoxyethane, is chemically very stable as it is resistant to oxidation or reduction processes and can withstand high $\mathrm{pH}$ environments. It is liquid at room temperature with a melting point of $-69^{\circ} \mathrm{C}[104]$ and a boiling point of $85^{\circ} \mathrm{C}$ [105]. Besides its ability to form volatile peroxides it is generally regarded as a relatively safe compound with low acute toxicity. However, experiments on rats revealed that chronic inhalation of monoglyme in high doses can lead to mental and behavioral disorders, bodyweight loss and death [106]. Furthermore, reproductive toxic and teratogenic effects are suspected.

Monoglyme has long been used as a solvent in chemical reactions. For example it is used in the stereoselective synthesis of trans-1,2-disubstituted alkenes [107]. Moreover, it promotes Pausen-Khand reactions [108] and thermally stabilizes catalysts for ethylene polymerizations [109]. Due to its low viscosity, oxidative and thermal stability it is often used as electrolyte component in Li-, Al- and Mg-type batteries [110$113]^{\dagger}$. It enhances the electrochemical performance by decreasing the reductive decomposition of the electrolyte.

Most of these applications utilize the high flexibility of the monoglyme backbone, which can effectively coordinate and chelate many different environments. A fact that is also reflected in the observation that the preferred shape of monoglyme depends on the phase state making it a prototype system for adaptive aggregation [114]. The first IR spectrum of liquid and solid monoglyme was reported in 1967 by Snyder and Zerbi which concluded that crystalline monoglyme consists of tgt chains [115]. A shift in the conformational preferences with dominant tgt contributions in the liquid and solid phases found through a comparison of gaseous, liquid and solid Raman and IR spectra of monoglyme [116] strengthened this conclusion. Additionally, molecular dynamics simulations confirmed dominant tgt contributions in the liquid and solid phase while three conformers $(t t t, t g t, t g g$ ) were found to be very close in energy in the gas phase [117]. Many experimental [13,118-120] and theoretical [121-123] investigations on the prominent water solubility of this molecule revealed a strong preference for the tgt conformer upon interaction with water. Thus, monoglyme is often used as a suitable model compound for the polymeric system in terms of its conformational preferences and solubility $[11,13,124-126]$.

\footnotetext{
† References given display only a selection of publications on that topic.
} 
Surprisingly, the conformational preferences in the gas phase were debated for several decades, citing slight discrepancies between electron diffraction [127], NMR [128] and IR [129,130] studies. Contributions of three conformers (ttt, tgt and tgg' shown in Figure 6.1) were found at low temperatures in matrix isolation [131] and microwave jet [132] studies. The latter was unable to detect the $t t t$ conformer due to its vanishing dipole moment but their supporting calculations indicated that this conformer is the global minimum structure. Interestingly, this study reported a decrease from $\sim 10$ to $\sim 7 \mathrm{~kJ} \cdot \mathrm{mol}^{-1}$ for the methyl torsion barrier in $\operatorname{tgg}^{\prime}$ due to its internal hydrogen bond. Regularly, quantum chemical calculations were consulted to aid in assigning and predicting the best gas phase conformers. Early studies predicted the energy difference between $t t$ and $t g t$ to be quite large [128,129,133-137]. MP2 calculations usually predicted this energy gap to be smaller than $1 \mathrm{~kJ} \cdot \mathrm{mol}^{-1}[132,138-140]$ while different flavors of density functional theory predicted it mostly above $1 \mathrm{~kJ} \cdot \mathrm{mol}^{-1}[102,126,129,141]$. Hartree-Fock methods predicted it far above $1 \mathrm{~kJ} \cdot \mathrm{mol}^{-1}$ $[142,143]$. Every study but one [128] found $t t t$ as the global minimum structure. However, as it is demonstrated in Figure 6.2, the discrepancies between tgg' and tgt

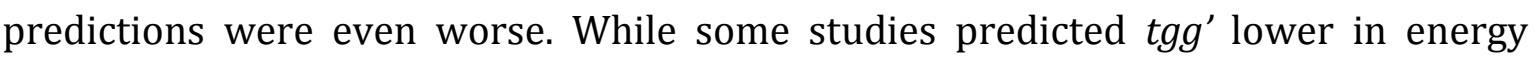
$[102,126,135,141,142]$, most were predicting it to be higher in energy $[132,134,136,138-140,143]$.

It seems timely to revisit this case and apply state-of-the-art quantum chemical methods with larger basis sets than those used in previous studies. Also, dispersion corrections that were missing in previous studies will be added to density functionals. Raman jet spectra of low frequency motions allow observation of isolated and cold molecules which makes the assignment, aided by quantum chemical calculations, more straightforward and reliable. In the following subchapter the quantum chemical calculations will be outlined and their results discussed. Afterwards, a relaxation model will be proposed based on interconversion barriers on the conformational landscape. Then, Raman jet spectra are presented and conformational contributions are assigned based on the quantum chemical calculations. Subsequently, effects of different controllable experimental parameters on the jet spectra are discussed. Afterwards, the energy difference between $t g t$ and $t t t$ will be estimated semiexperimentally and finally cluster tendencies between monoglyme and water will be investigated.
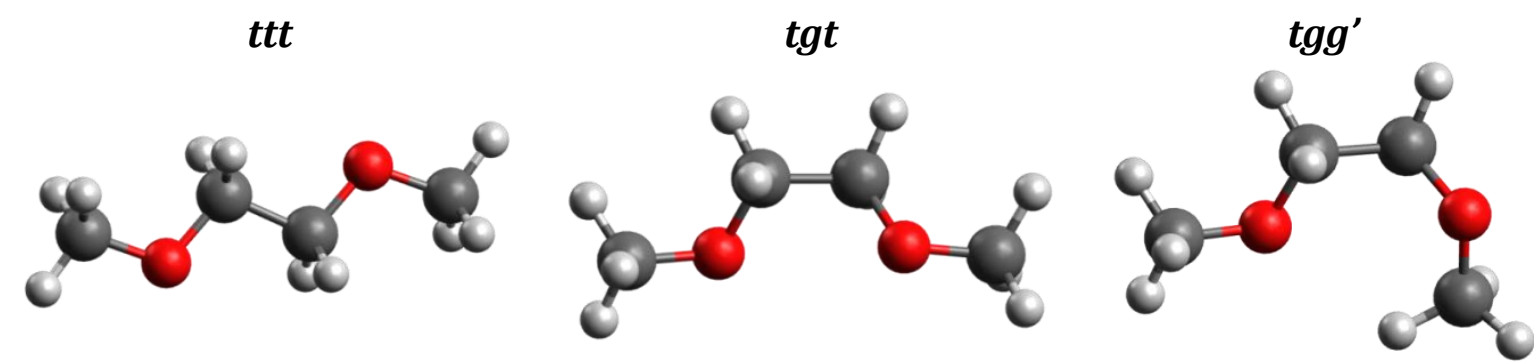

Figure 6.1: Geometries of the three lowest conformers in the monoglyme conformational landscape. 


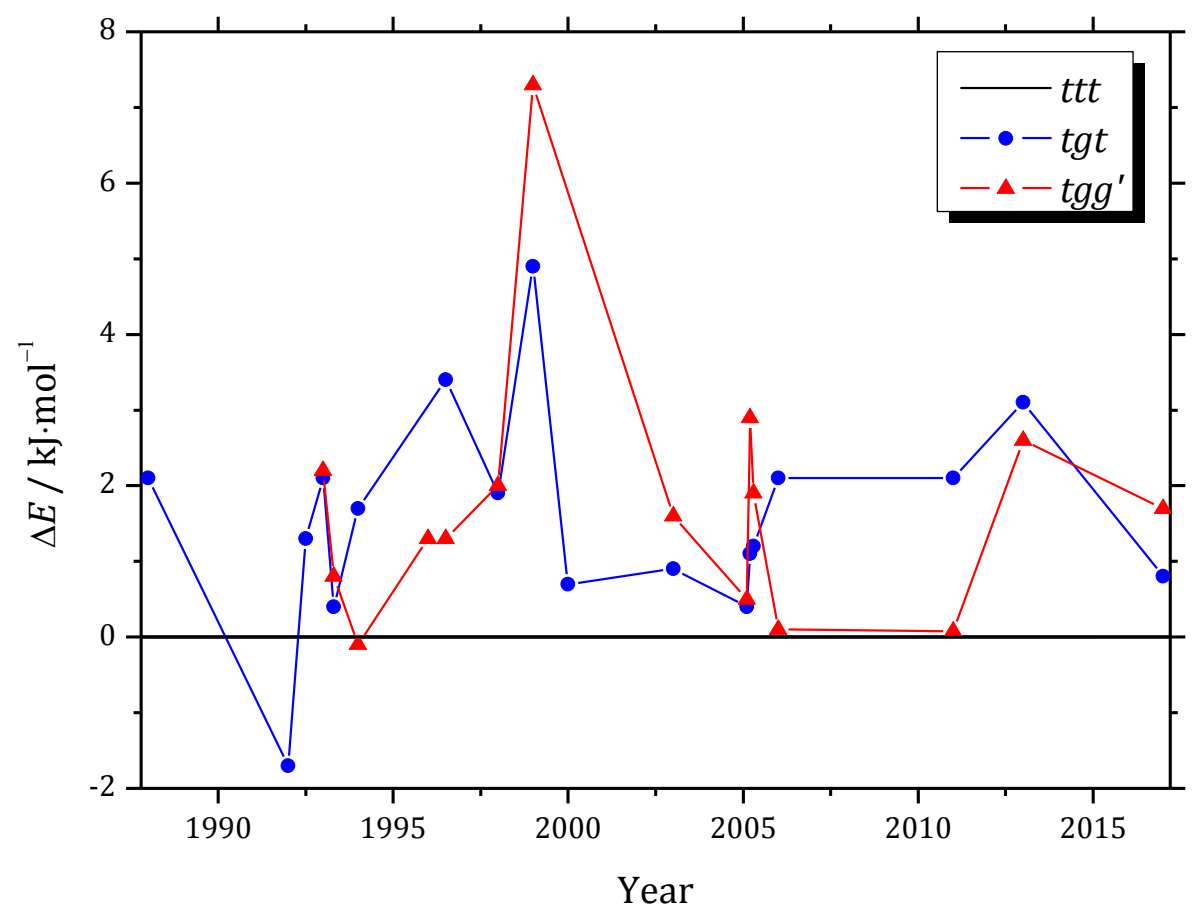

Figure 6.2: Development of the energy differences between the three lowest monoglyme conformations reported by various studies [11,102,126,128,129,132-143].

\section{2 - Quantum chemical calculations}

The three backbone dihedrals of monoglyme (COCC, OCCO, CCOC) result in 27 different combinations when only trans $\left(180^{\circ}\right)$ or gauche $\left( \pm 60^{\circ}\right)$ angles lead to stable configurations. Nine of these 27 combinations are transferable via rotation while all but two $(t t t, g t g$ ) of the remaining structures occur as enantiomeric pairs. Therefore, a total of ten different combinations occur on the conformational landscape of monoglyme. The geometries of all ten conformers were optimized in Gaussian 09 Rev. D.01. For this purpose various basis sets (def2TZVP, VTZ, aVTZ, aVQZ) were used with the MP2 method, the B3LYP or the B2PLYP functional. In order to add threebody dispersion corrections additional optimizations for all conformers were performed in TURBOMOLE 7.0 at the B3LYP-3D3/aVQZ level. Vibrational harmonic frequencies and Raman scattering cross sections were calculated at the appropriate levels of theory and their zero-point energies were added to the appropriate electronic energies. Statistical weights and results of the harmonic calculations are shown in Table 6.1. Remarkably, all methods besides the B3LYP-2D3/VTZ (deviations for tgg' $^{\prime}$ and $g g g^{\prime}$ ) results agree on the ordering of the first eight conformers. Even the energy gaps between the conformers are predicted to be very similar across all methods. The last two conformers ( $g t g^{\prime}$ and $g t g$ ) are predicted to be very close in energy and exchange places depending on the chosen method. While the aVQZ calculations predict $g t g$ ' to be lower in energy than the gtg conformer, other methods alter this sequence. For smaller basis sets like def2TZVP or VTZ larger deviations for some bended structures like $t g g^{\prime}$ are found, most likely caused by intramolecular BSSE effects [140]. In total, the results show a distinct gap between the first three conformers and others in agreement with earlier studies [102,134,143]. 
When replacing the electronic energies of all studied methods with CCSD(T)/aVTZ single point calculations the agreement across all methods is even better (Table 6.2). Only very small differences up to a maximum of $0.26 \mathrm{~kJ} \cdot \mathrm{mol}^{-1}$ can be found between them. The major contributions of these differences result from deviations in the underlying zero-point corrections of the different methods as can be seen in Table 6.5. This shows that the structures that were optimized with different methods and basis sets all converged well into almost identical geometries.

Remarkably, when calculating the electronic energies at this more sophisticated level the energy differences of $t g t$ and $t g g^{\prime}$ relative to $t t t$ both rise by about $\sim 0.5 \mathrm{~kJ} \cdot \mathrm{mol}^{-1}$. When going one step further by replacing the MP2 energies of the CCSD(T)/aVTZ calculations by MP2/aVQZ energies ${ }^{\dagger}$, which will be abbreviated CCSD(T)/aVTQZ, the energy gaps widen once more (Table 6.3). This demonstrates the importance of high level calculations in order to work out the delicate energy differences between the important conformers. When comparing the electronic energies at the $\operatorname{CCSD}(\mathrm{T}) / \mathrm{aVTQZ}$ level for the three most stable conformers optimized at the different DFT and MP2 levels (Table 6.4) one finds that the MP2 optimizations yield the lowest electronic energies followed by the B2PLYP-2D3/aVTZ optimizations. This means that the MP2 optimizations give the best representation of the actual potential minima in this case. The largest parts in the differences between the underlying optimization methods actually come from the MP2 part of the CCSD(T) calculations and therefore it is not too surprising that MP2 optimizations yield the lowest energy. However, when B2PLYP is combined with a rather small basis set like def2TZVP (not shown) the differences in electronic energies rise up to $\sim 0.6 \mathrm{~kJ} \cdot \mathrm{mol}^{-1}$ when compared to the MP2 optimizations. This shows that the B2PLYP method requires large basis sets in order to predict reasonable results. In general, the spread between the electronic energies that are based on different optimization methods is quite large $\left(\sim 0.5 \mathrm{~kJ} \cdot \mathrm{mol}^{-1}\right)$ but the energy differences between the three most stable conformers within each optimization method is well preserved as discussed before. This demonstrates that the DFT methods mismatch intramolecular properties, e.g. bond distances and angles, in a systematic way. Indeed, MP2 optimization for $t t t$ yield longer C-O bond distances $(\sim 0.3 \%)$ and tighter COC angles $(\sim 1.5 \%)$ than the DFT methods.

Anharmonic VPT2 calculations were performed for the three most important species ( $t t t, t g t, t g g$ ) on a B3LYP-2D3/def2TZVP level in Gaussian 09 Rev. D.01. Their relative zero-point vibrational energies are included in Table 6.5. These calculations emphasize an additional rise in the energy gap between $t t t$ and $t g g^{\prime}$ by $\sim 0.1 \mathrm{~kJ} \cdot \mathrm{mol}^{-1}$.

As discussed in Section 6.1, earlier studies struggled to determine the energy sequence of $t t t$, tgt and $t g g^{\prime}$. Inclusion of two-body dispersion correction, preferably even three-body corrections, as well as higher level calculations clearly result in an energy ordering of $t t t<t g t<t g g^{\prime}$. The best estimate that can be made for the energy

\footnotetext{
† Hobza et al. showed that $\operatorname{CCSD}(\mathrm{T})$ energies calculated with a larger basis set can be estimated by combining $\operatorname{CCSD}(\mathrm{T})$ corrections using a smaller basis set and MP2 energies calculated at the larger basis set because the CCSD $(\mathrm{T})$ correction term converges faster than MP2 interactions energies [144]. 
sequence of $t t t$-tgt-tgg' based on all quantum chemical predictions from this work is thus $0.0-1.2-2.0 \mathrm{~kJ} \cdot \mathrm{mol}^{-1}$ with an estimated error of less than $0.3 \mathrm{~kJ} \cdot \mathrm{mol}^{-1}$. 


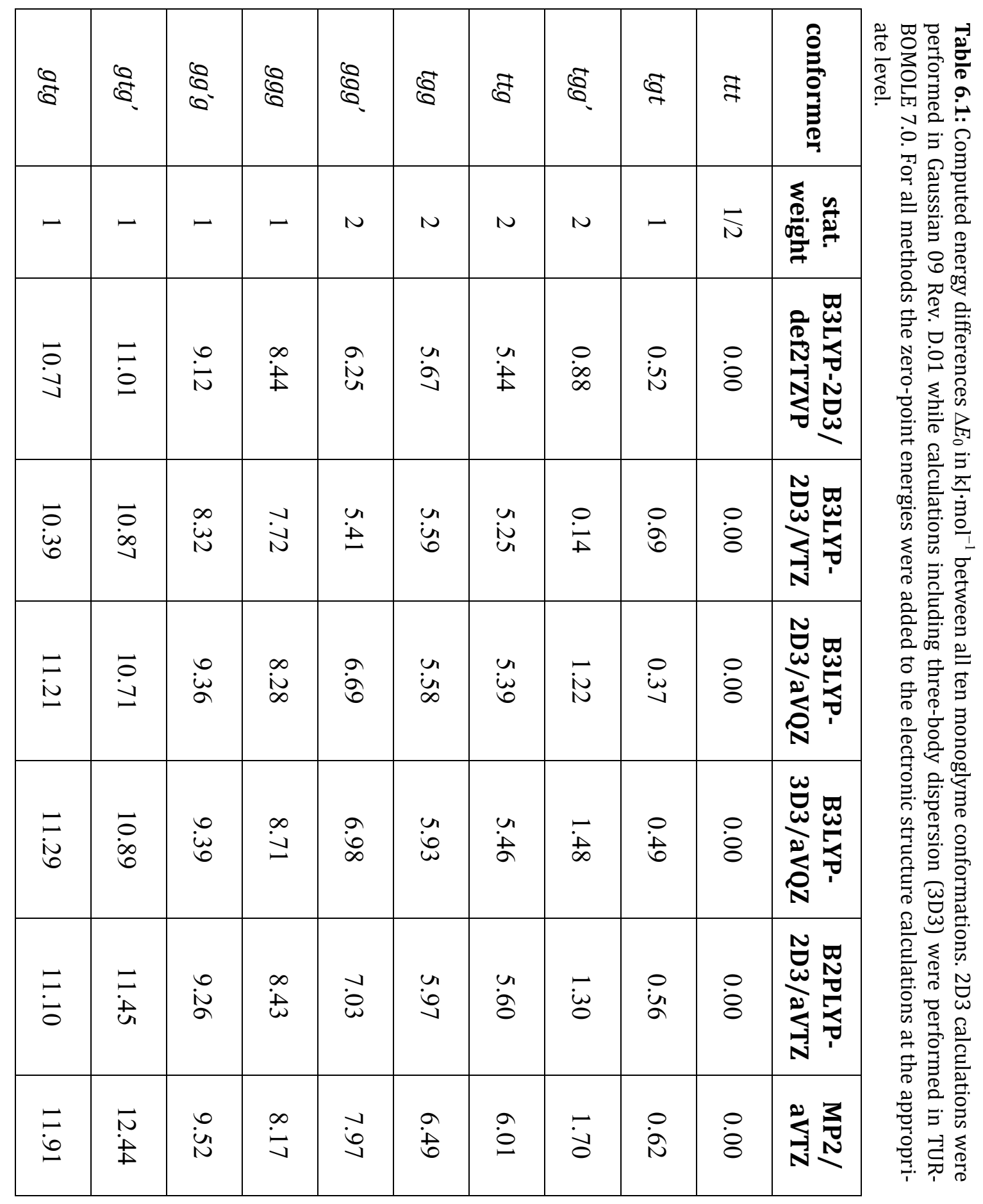


Table 6.2: Energy differences $\Delta E_{0}$ in $\mathrm{kJ} \cdot \mathrm{mol}^{-1}$ between the ten monoglyme conformations at the CCSD(T)/aVTZ level calculated in TURBOMOLE 6.4. Here electronic energies were computed at the CCSD(T) level on structures from previous optimizations (Table 6.1) and their appropriate zero-point corrections were added to the $\operatorname{CCSD}(\mathrm{T}) / \mathrm{aVTZ}$ energies.

\begin{tabular}{|c|c|c|c|c|c|c|c|c|c|c|c|}
\hline $\begin{array}{c}\text { electronic } \\
\text { energy }\end{array}$ & $\begin{array}{c}\text { structure } \\
\text { and } \\
\text { ZPVE }\end{array}$ & $t t t$ & $\operatorname{tg} t$ & $\operatorname{tg} g^{\prime}$ & $\operatorname{ttg}$ & $\operatorname{tgg}$ & $g g g^{\prime}$ & $g g g$ & $\boldsymbol{g} g^{\prime} g$ & $g t g^{\prime}$ & gtg \\
\hline $\begin{array}{c}\mathrm{CCSD}(\mathrm{T}) / \\
\mathrm{aVTZ}\end{array}$ & $\begin{array}{c}\text { B3LYP- } \\
\text { 2D3/ } \\
\text { def2TZVP }\end{array}$ & 0.00 & 1.09 & 1.85 & 5.58 & 6.53 & 7.64 & 8.37 & 9.26 & 11.21 & 11.14 \\
\hline $\begin{array}{c}\mathrm{CCSD}(\mathrm{T}) / \\
\mathrm{aVTZ}\end{array}$ & $\begin{array}{c}\text { B3LYP- } \\
\text { 2D3/ } \\
\text { VTZ }\end{array}$ & 0.00 & 1.07 & 1.83 & 5.51 & 6.32 & 7.56 & 8.15 & 9.11 & 11.58 & 11.02 \\
\hline $\begin{array}{c}\mathrm{CCSD}(\mathrm{T}) / \\
\mathrm{aVTZ}\end{array}$ & $\begin{array}{l}\text { MP2/ } \\
\text { aVTZ }\end{array}$ & 0.00 & 1.10 & 1.76 & 5.51 & 6.68 & 7.64 & 8.32 & 9.07 & 11.51 & 11.02 \\
\hline $\begin{array}{c}\mathrm{CCSD}(\mathrm{T}) / \\
\mathrm{aVTZ}\end{array}$ & $\begin{array}{c}\text { B2PLYP- } \\
\text { 2D3/ } \\
\text { aVTZ }\end{array}$ & 0.00 & 1.09 & 1.87 & 5.60 & 6.54 & 7.69 & 8.29 & 9.16 & 11.41 & 11.14 \\
\hline $\begin{array}{c}\mathrm{CCSD}(\mathrm{T}) / \\
\text { aVTZ }\end{array}$ & $\begin{array}{c}\text { B3LYP- } \\
\text { 3D3/ } \\
\text { aVQZ }\end{array}$ & 0.00 & 1.19 & 2.02 & 5.65 & 6.77 & 7.82 & 8.59 & 9.12 & 11.29 & 11.45 \\
\hline
\end{tabular}

Table 6.3: Energy differences $\Delta E_{0}$ in $\mathrm{kJ} \cdot \mathrm{mol}^{-1}$ for the three most stable monoglyme conformations at the CCSD(T)/aVTQZ level calculated in TURBOMOLE 6.4. Here electronic energies were computed at the CCSD(T)/aVTZ level and enhanced with MP2/aVQZ energies. Structures were used from previous optimizations (Table 6.1) and their appropriate zero-point energies were added to the $\operatorname{CCSD}(\mathrm{T}) / \mathrm{aVTQZ}$ energies.

\begin{tabular}{|c|c|c|c|c|}
\hline electronic energy & $\begin{array}{c}\text { structure and } \\
\text { ZPVE }\end{array}$ & $\boldsymbol{t t t}$ & $\boldsymbol{t} \boldsymbol{t}$ & $\boldsymbol{t g}^{\prime}$ \\
\hline $\begin{array}{c}\text { CCSD(T)/ } \\
\text { aVTQZ }\end{array}$ & $\begin{array}{c}\text { B3LYP-2D3/ } \\
\text { def2TZVP }\end{array}$ & 0.00 & 1.18 & 2.12 \\
\hline $\begin{array}{c}\text { CCSD(T)/ } \\
\text { aVTQZ }\end{array}$ & $\begin{array}{c}\text { B3LYP-2D3/ } \\
\text { VTZ }\end{array}$ & 0.00 & 1.15 & 2.11 \\
\hline $\begin{array}{c}\text { CCSD(T)/ } \\
\text { aVTQZ }\end{array}$ & $\begin{array}{c}\text { MP2/ } \\
\text { aVTZ }\end{array}$ & 0.00 & 1.19 & 2.03 \\
\hline $\begin{array}{c}\text { CCSD(T)/ } \\
\text { aVTQZ }\end{array}$ & $\begin{array}{c}\text { B2PLYP-2D3/ } \\
\text { aVTZ }\end{array}$ & 0.00 & 1.17 & 2.14 \\
\hline $\begin{array}{c}\text { CCSD(T)/ } \\
\text { aVTQZ }\end{array}$ & $\begin{array}{c}\text { B3LYP-3D3/ } \\
\text { aVQZ }\end{array}$ & 0.00 & 1.28 & 2.28 \\
\hline
\end{tabular}


Table 6.4: Differences of electronic energies $\left(\Delta E_{\mathrm{el}}\right)$ in $\mathrm{kJ} \cdot \mathrm{mol}^{-1}$ for the three most stable monoglyme conformations at the $\operatorname{CSD}(\mathrm{T}) / \mathrm{aVTQZ}$ level calculated in TURBOMOLE 6.4. Structures were used from previous optimizations (Table 6.1) as indicated in the first column.

\begin{tabular}{|c|c|c|c|}
\hline structure & $\boldsymbol{t t} \boldsymbol{t}$ & $\boldsymbol{t g} \boldsymbol{t}$ & $\boldsymbol{t g}^{\prime}$ \\
\hline $\begin{array}{c}\text { B3LYP-2D3/ } \\
\text { def2TZVP }\end{array}$ & 0.44 & 1.89 & 2.32 \\
\hline $\begin{array}{c}\text { B3LYP-2D3/ } \\
\text { VTZ }\end{array}$ & 0.40 & 1.85 & 2.26 \\
\hline $\begin{array}{c}\text { MP2/ } \\
\text { aVTZ }\end{array}$ & 0.00 & 1.44 & 1.88 \\
\hline $\begin{array}{c}\text { B2PLYP-2D3/ } \\
\text { aVTZ }\end{array}$ & 0.13 & 1.57 & 2.02 \\
\hline $\begin{array}{c}\text { B3LYP-3D3/ } \\
\text { aVQZ }\end{array}$ & 0.46 & 1.92 & 2.35 \\
\hline
\end{tabular}

Table 6.5: Relative zero-point vibrational energies in $\mathrm{kJ} \cdot \mathrm{mol}^{-1}$ for the ten monoglyme conformations. Frequencies for all methods but the 3D3 one were calculated in Gaussian 09 Rev. D.01. The B3LYP3D3/aVQZ results were obtained using TURBOMOLE 7.0.

\begin{tabular}{|c|c|c|c|c|c|c|c|c|c|c|}
\hline $\mathbf{Z P V E}$ & $\boldsymbol{t t t}$ & $\boldsymbol{t g} \boldsymbol{t}$ & $\boldsymbol{t g} \boldsymbol{g}^{\prime}$ & $\boldsymbol{t t g}$ & $\boldsymbol{t g} \boldsymbol{g}$ & $\boldsymbol{g} \boldsymbol{g} \boldsymbol{g}^{\prime}$ & $\boldsymbol{g g g}$ & $\boldsymbol{g g}^{\prime} \boldsymbol{g}$ & $\boldsymbol{g t g}^{\prime}$ & $\boldsymbol{g} \boldsymbol{g}$ \\
\hline $\begin{array}{c}\text { B3LYP-2D3/ } \\
\text { def2TZVP }\end{array}$ & 0.00 & -0.27 & 0.25 & 0.22 & -0.01 & 0.47 & 1.30 & 0.08 & 0.30 & 0.69 \\
\hline $\begin{array}{c}\text { B3LYP-2D3/ } \\
\text { def2TZVP } \\
\text { anharm. }\end{array}$ & 0.00 & -0.20 & 0.40 & & & & & & & \\
\hline $\begin{array}{c}\text { B3LYP-2D3/ } \\
\text { VTZ }\end{array}$ & 0.00 & -0.29 & 0.25 & 0.25 & -0.06 & 0.48 & 1.26 & 0.43 & 0.25 & 0.61 \\
\hline $\begin{array}{c}\text { B3LYP-2D3/ } \\
\text { aVQZ }\end{array}$ & 0.00 & -0.30 & 0.24 & 0.27 & 0.03 & 0.53 & 1.02 & 0.59 & 0.40 & 0.52 \\
\hline $\begin{array}{c}\text { B3LYP-3D3/ } \\
\text { aVQZ }\end{array}$ & 0.00 & -0.18 & 0.39 & 0.30 & 0.22 & 0.63 & 1.36 & 0.41 & 0.45 & 0.40 \\
\hline $\begin{array}{c}\text { B2PLYP-2D3/ } \\
\text { aVTZ }\end{array}$ & 0.00 & -0.27 & 0.26 & 0.24 & 0.01 & 0.50 & 1.33 & 0.63 & 0.30 & 0.30 \\
\hline $\begin{array}{c}\text { MP2/ } \\
\text { aVTZ }\end{array}$ & 0.00 & -0.24 & 0.15 & 0.16 & 0.20 & 0.50 & 1.52 & 0.43 & 0.20 & 0.62 \\
\hline
\end{tabular}

\section{3 - Relaxation model}

In order to gain more insight into the potential energy surface of monoglyme relaxed 2D torsional scanning calculations around the inner (OCCO) and one outer (CCOC) backbone dihedral were performed at the B3LYP-2D3/def2TZVP level on Gaussian 09 Rev. D.01. Starting from the $t t t$ structure the outer dihedral was scanned in 72 steps of $5^{\circ}$ while the inner dihedral only needed to be scanned in 36 steps $\left(0^{\circ}\right.$ to $\left.180^{\circ}\right)$ due to the inversion symmetry of the 2D surface. This resulted in 2592 single points that were optimized along the surface in each calculation. Another 2D scan was started from an artificial point where both dihedrals were set to $0^{\circ}$ while the third, locked dihedral remained in the trans configuration. Afterwards, results of both scans were compared and at every point the lowest energy of the two was kept. Even though the 
important influence of higher level theories and larger basis sets on the delicate energy differences between the conformers was just illustrated, the demanding computational effort of these scans limited this approach to a DFT functional with a rather small basis set. However, error bars of around $1 \mathrm{~kJ} \cdot \mathrm{mol}^{-1}$ are acceptable for this approach to still end up with a qualitatively correct picture, but in order to tackle this bottleneck a correction surface was calculated at the CCSD(T)/aVTZ level. For this purpose optimized structures from the previous scan were taken at every $60^{\circ}$ steps and energy single point calculations were performed in TURBOMOLE 6.4. Additionally, all local minima on the 2D surface $\left(t t t, t g g^{\prime}, t g t, t g g, t t g\right)$ and the saddle points between them were added, resulting in a total of 61 single points.

The 2D potential energy surface along the inner and one outer dihedral (Figure 6.3a) shows that barriers for interconversions of the inner dihedral are much larger than those for an interconversion around the outer one. This means that tgg' can efficiently relax into $t g t$ while an interconversion between $t g t$ and $t t t$ under supersonic jet expansion conditions is unlikely. Basically, the inner dihedral will stay locked while the outer torsion will relax efficiently during jet expansions, making $t t t$ and tgt very efficient capture basins for all other conformers. Thus, $t t t$ is fed by $t t g, g t g$ and $g t g^{\prime}$ while tgt is fed by tgg, tgg', $g g g, g g g^{\prime}$ and $g g^{\prime} g$. The correction surface (Figure 6.3b) shows that the $t g g^{\prime}$ minimum is predicted too low in energy at the computational level used in Figure 6.3a, but the qualitative picture remains correct. Moreover, an increased instability of the $t g g^{\prime}$ minimum lowers the barrier for the interconversion to tgt even further. This 2D potential energy surface compares well to the original force field map reported by Anderson and Wilson [139].

This situation is fundamentally different from the one found in $n$-alkane chains as a comparison of monoglyme to its hydrocarbon analog $n$-hexane shows (Figure 6.4). While torsions around the inner dihedral show comparably large barriers in both molecules (Figure 6.4a), they are vastly different for torsions around an outer dihedral (Figure 6.4b) due to stereoelectronic effects of the oxygens in monoglyme. Interestingly, the increase of the barrier height in $n$-hexane in the latter case is similar to an increase that was found when one of the methoxy groups was substituted by an aromatic ring (Figure 5.3a).

Figure 6.5 shows the comparison of a gas phase to a jet spectrum. Raman scattering cross sections for the first five conformers were calculated at the B3LYP-3D3/aVQZ level in TURBOMOLE 7.0 and weighted with their relative populations at $300 \mathrm{~K}$ (Figure 6.6). The comparison shows that only ttt, tgt and $t g g^{\prime}$ are populated in the supersonic jet expansion while small contributions of the higher energy conformers can be found in the gas phase spectrum. Additionally, tgg' contributions are much smaller in the jet spectrum indicating a major depopulation due to efficient relaxation. However, the interconversion barrier is still high enough to preserve some population in $t g g^{\prime}$, due to a conformational temperature above the rotational and translational temperature. Comparing the relative populations at different temperatures (Figure 6.6) to the jet spectrum indicates that the effective conformational tempera- 
ture during the jet expansion drops below $100 \mathrm{~K}$. A more detailed analysis of these effects will be presented in Section 6.4.

The relaxation process can be modeled by estimating the population of each conformer at an initial temperature $T_{\mathrm{i}}$ (typically room temperature or corresponding experimental nozzle temperatures). Then, all contributions of higher energy conformers are relaxed to the best structure while keeping the inner (OCCO) dihedral locked. As stated above this means that ttg, gtg and $g t g^{\prime}$ fed ttt while tgg', tgg, ggg, $g g g^{\prime}$ and $g g^{\prime} g$ fed $t g t$. Afterwards, an interconversion between $t g g^{\prime}$ and $t g t$ is calculated at a repopulation temperature $T_{\mathrm{r}}$ which will be derived from the spectra as described in the following subchapter. Small entropy effects beyond a statistical advantage of 2 for $t g g^{\prime}$ over $\operatorname{tg} t$ due to the $C_{2}$ symmetry of $\operatorname{tg} t$ are neglected, because rotational and vibrational temperatures will be far below $T_{\mathrm{i}}$. This way the model allows to predict populations for $t t t$, tgt and $t g g^{\prime}$ that are found in jet expansion experiments. Initial populations were estimated in two different ways. In one, full theoretical standard Gibbs energies $\Delta G^{0}$ within the RRHO approximation were calculated in TURBOMOLE 7.0 at the B3LYP-3D3/aVQZ level of theory. In the other, differences in vibrational and rotational partition functions were neglected so that populations were calculated only based on the symmetry number $\sigma$ and enantiomeric degeneracy $g$ as well as differences in the electronic energies $\left(E_{\mathrm{El}}\right)$ and zero-point vibrational energies (ZPVE) as shown in the following equation.

$$
\Delta G^{\mathrm{C}}=E_{\mathrm{El}}+\mathrm{ZPVE}+k T \cdot \ln \sigma-k T \cdot \ln g
$$

These configurational contributions are expected to dominate over vibrational and rotational corrections and the corresponding Gibbs energies are denoted as $\Delta G^{\mathrm{C}}$. This approach is more simplistic but also more robust as it is independent on falsely predicted low frequency motions which are often problematic in the harmonic approximation. 
a)

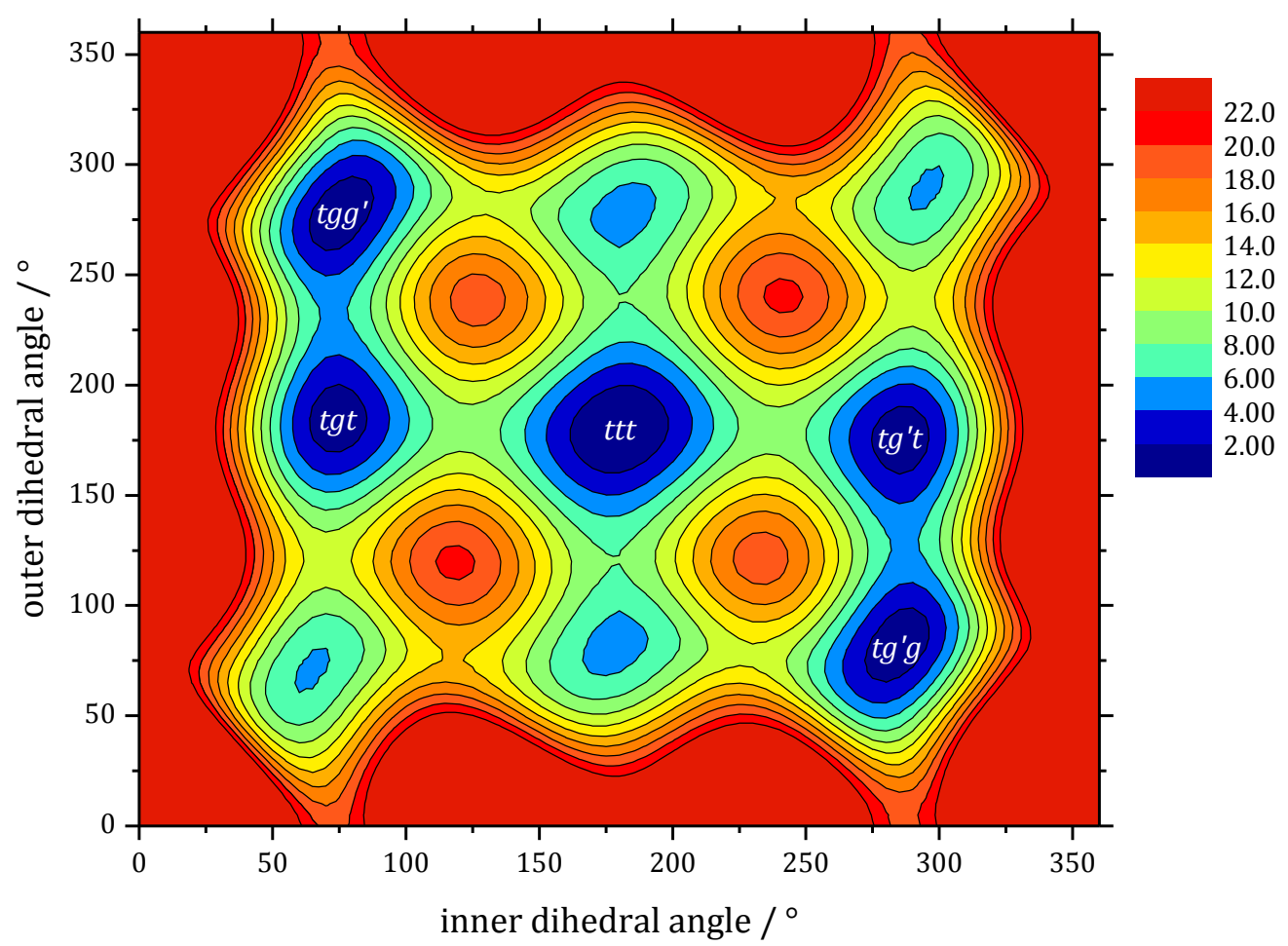

b)

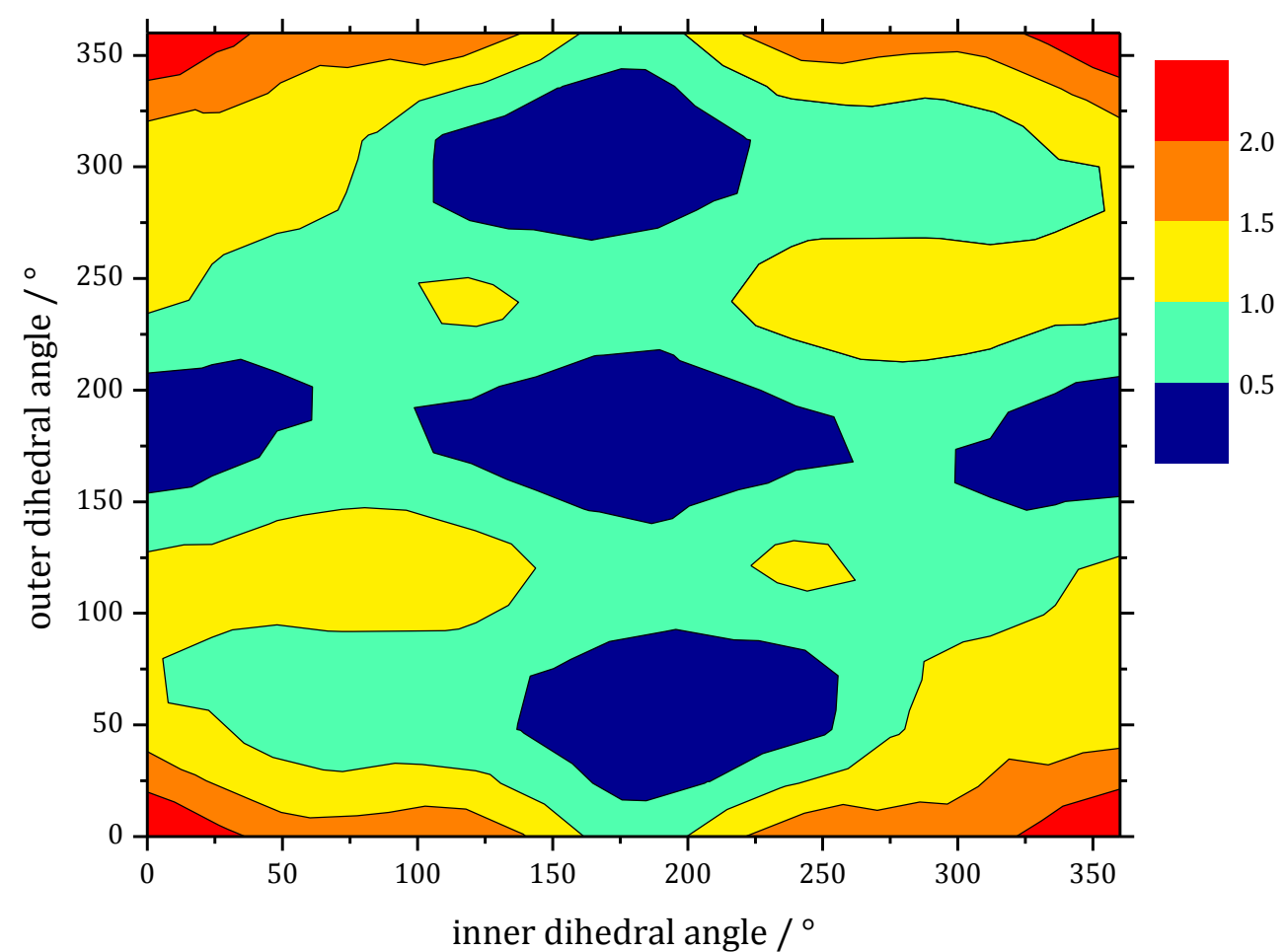

Figure 6.3: a) Relaxed 2D potential energy surface of the inner (OCCO) and one outer (CCOC) backbone dihedral (the other outer dihedral remained in trans configuration) of monoglyme with equidistant $2 \mathrm{~kJ} \cdot \mathrm{mol}^{-1}$ contour lines. Calculations were performed at every $5^{\circ}$ at the B3LYP-2D3/def2TZVP level in Gaussian 09 Rev. D.01.

b) Correction surface for the relaxed 2D potential energy surface (a) calculated at the CCSD(T)/aVTZ level in TURBOMOLE 6.4 with $0.5 \mathrm{~kJ} \cdot \mathrm{mol}^{-1}$ contour lines. CCSD(T) single point corrections were calculated on a $60^{\circ}$ grid from relaxed B3LYP-2D3/def2TZVP calculations. Also, all local minima and saddle points between them were added to the grid. 
a)

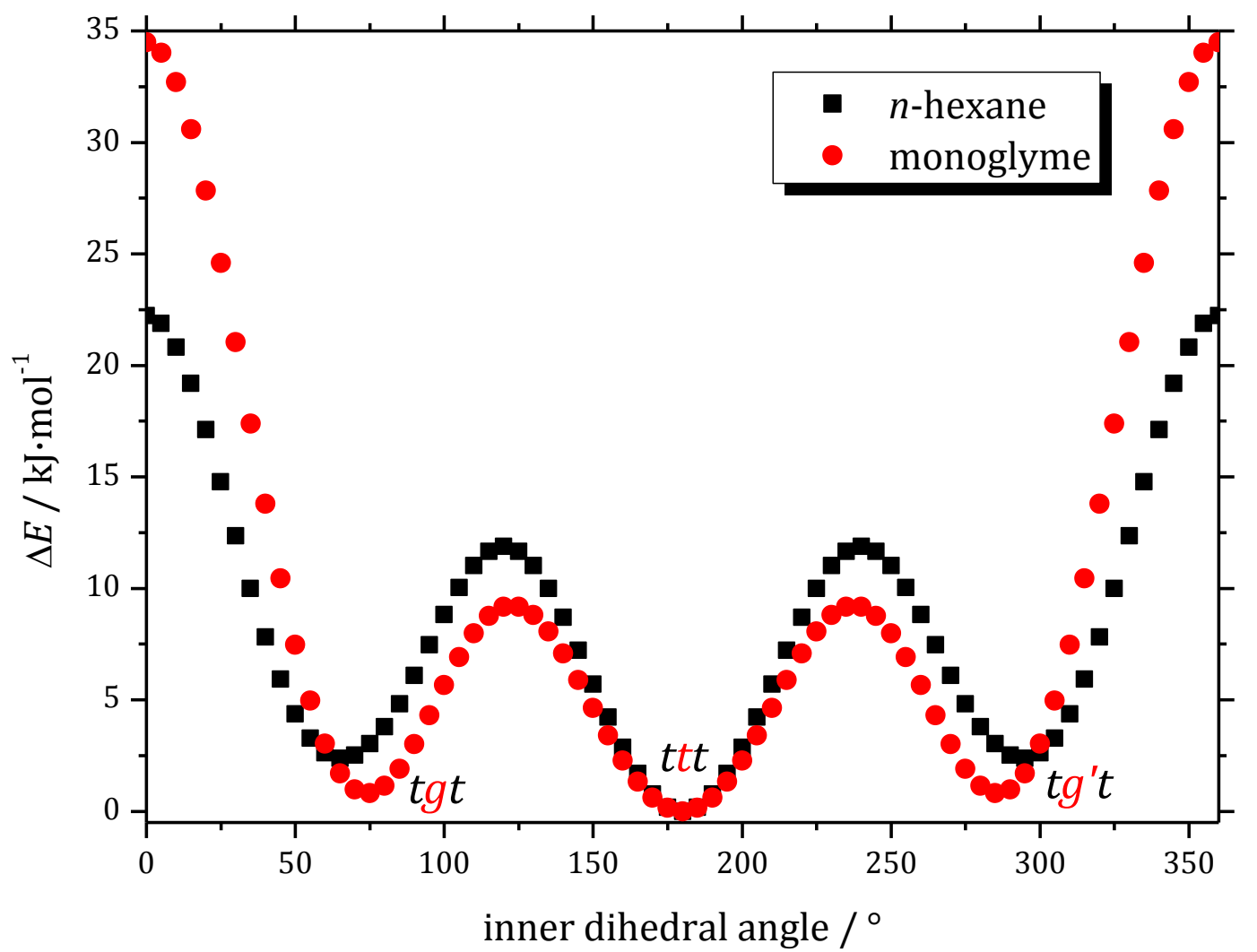

b)

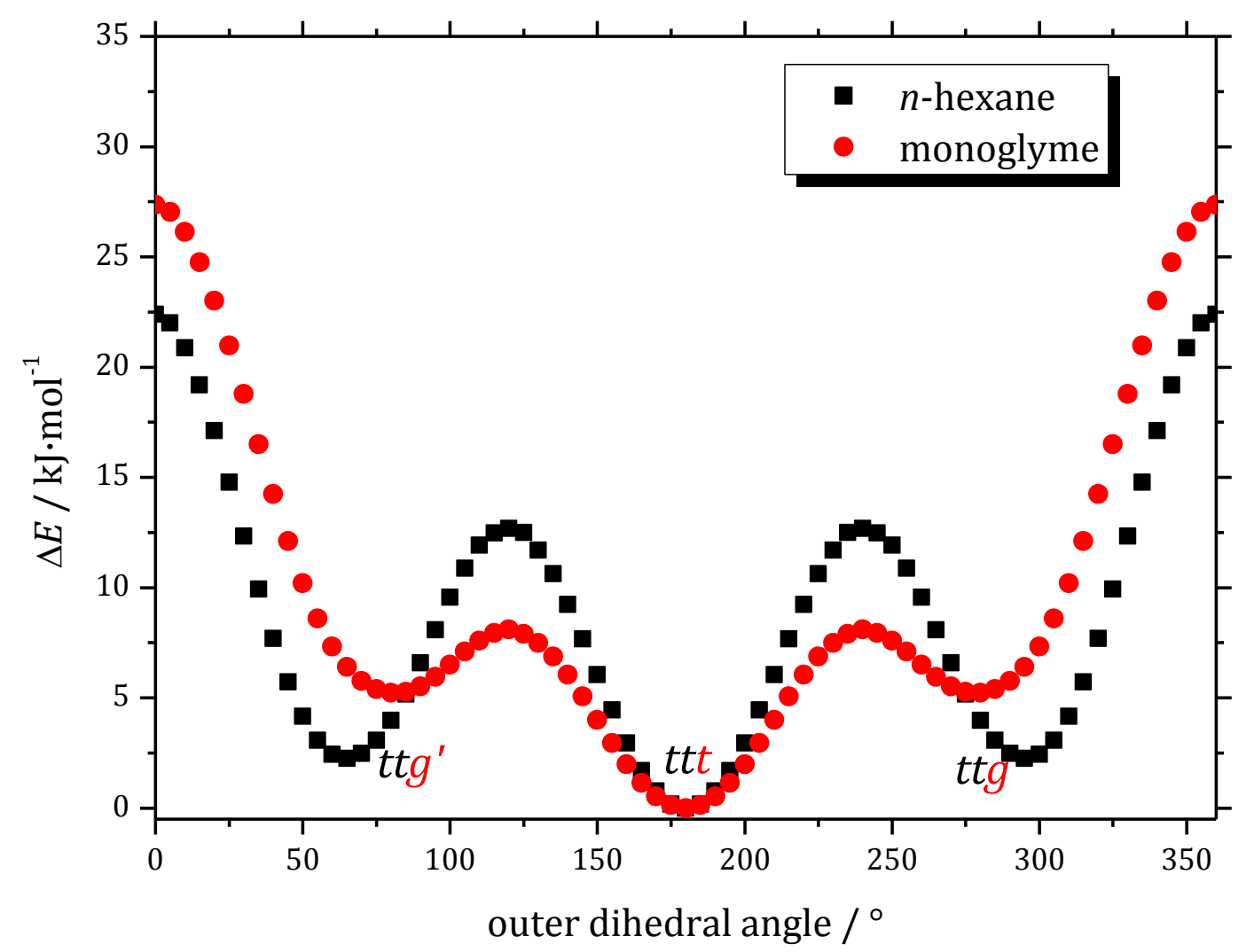

Figure 6.4: Relaxed torsional 1D-scans of the a) inner and b) one outer backbone dihedral of $n$-hexane and monoglyme calculated at the B3LYP-2D3/def2TZVP level in Gaussian 09 Rev. E.01. The comparison shows that interconversion barriers for the inner dihedral are similar in both molecules while the situation is much different for the outer torsion. Monoglyme shows a much smaller barrier allowing efficient relaxations around this dihedral during supersonic jet expansions. 


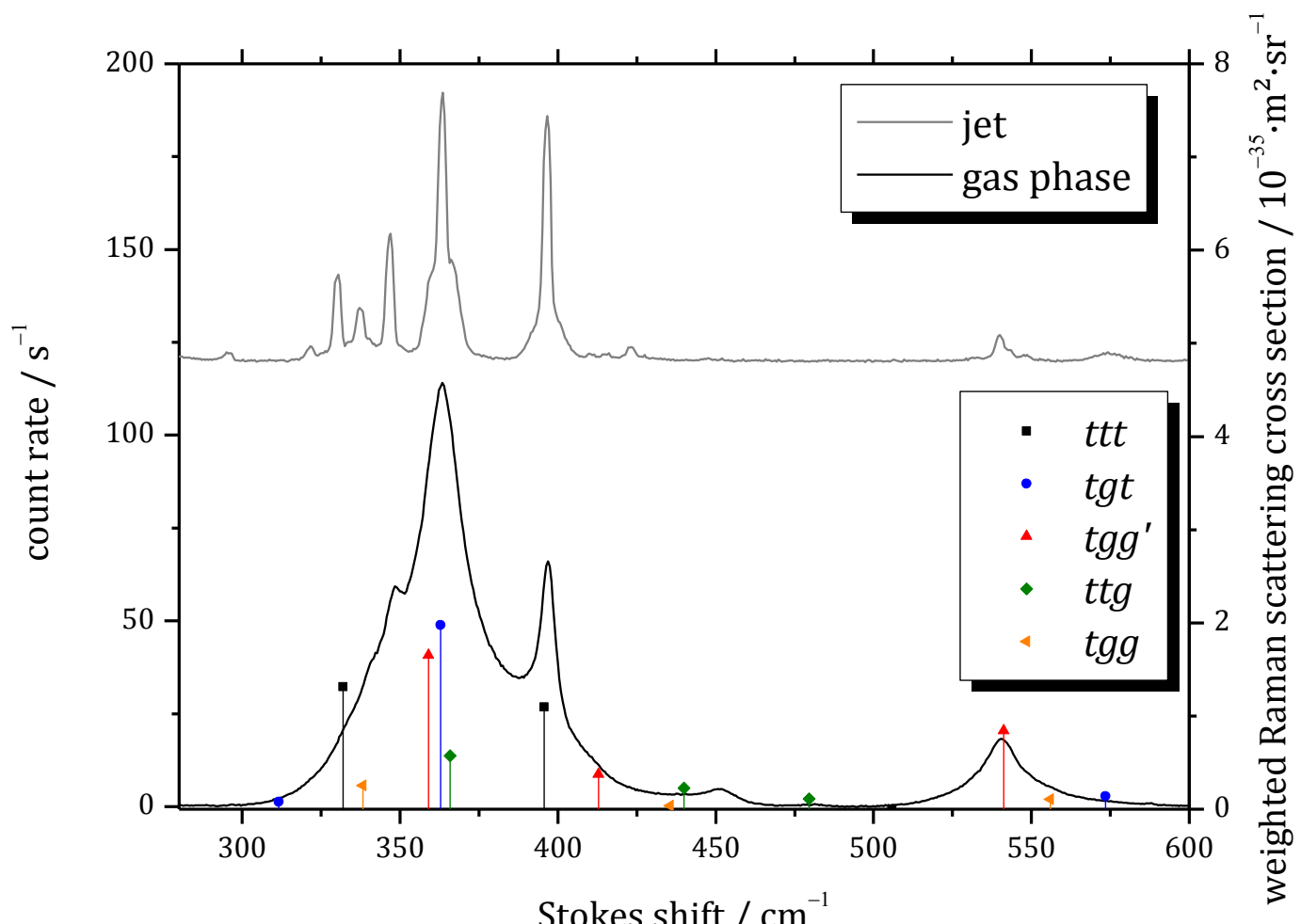

Figure 6.5: Comparison of a jet and a gas phase spectrum of monoglyme in the LAM region (measurement conditions can be found in A.1). Raman scattering cross sections for the best five conformers were calculated at the B3LYP-3D3/aVQZ level in TURBOMOLE 7.0 and weighted with their relative abundances based on the $\Delta G^{\mathrm{C}}$ values at $300 \mathrm{~K}$ (Figure 6.6).

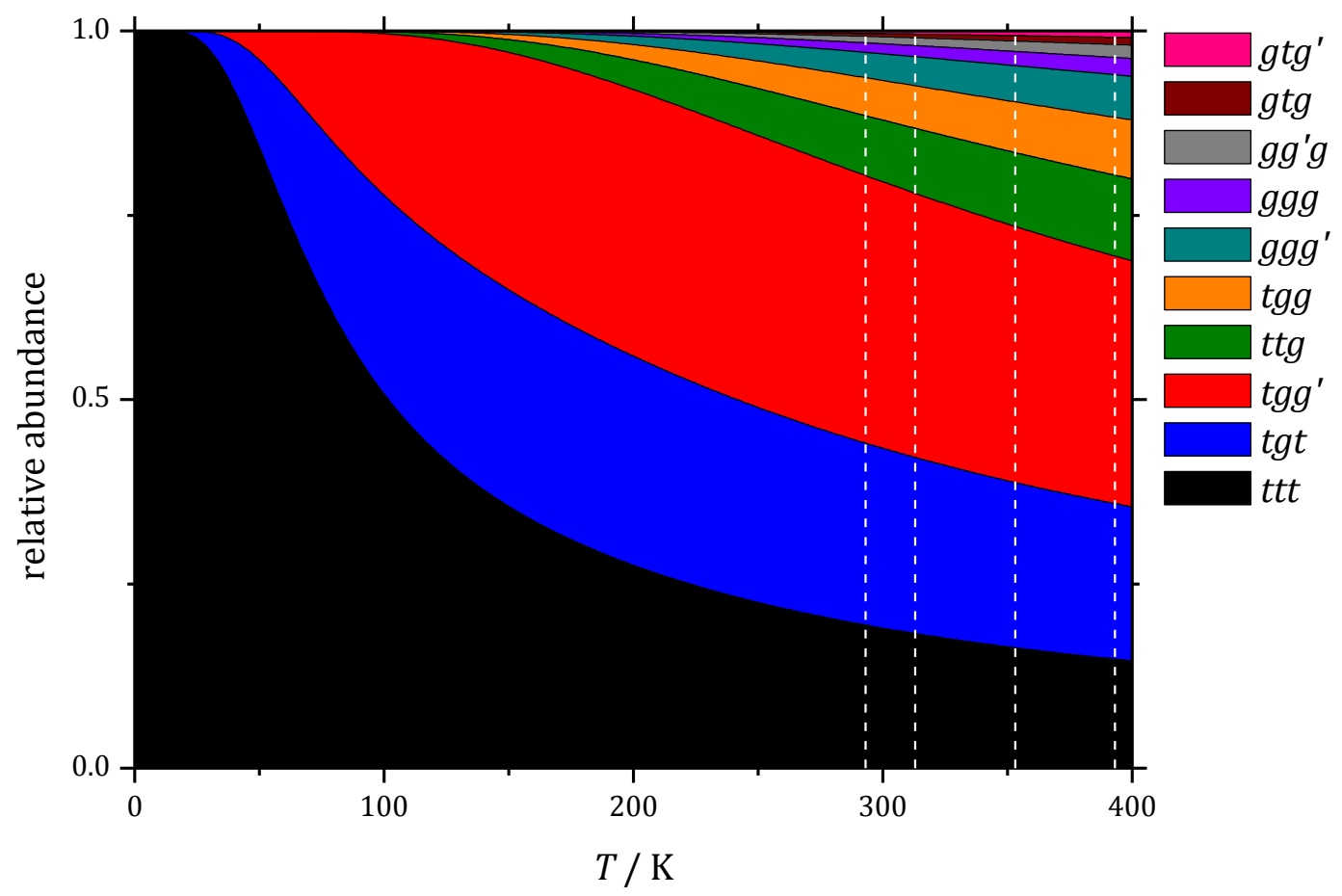

Figure 6.6: Relative abundances of the ten monoglyme conformers between 0 and $400 \mathrm{~K}$. Populations were estimated using the $\Delta G^{\mathrm{C}}$ model (Eq. 6.1) with zero-point corrections evaluated at the B3LYP3D3/aVQZ level and electronic energies at the CCSD(T)/aVTZ level. White dotted lines mark the nozzle temperatures used in the experiments discussed in the next chapter. 


\section{4 - Raman jet analysis}

Figure 6.7 shows an overview Raman spectrum of monoglyme which can be divided into three spectral regions: the low frequency region (up to $\sim 600 \mathrm{~cm}^{-1}$ ), which contains mostly skeletal vibrations such as the longitudinal acoustic modes (LAM), followed by the $\mathrm{CC} / \mathrm{CO}$ stretching region $\left(\sim 750-1500 \mathrm{~cm}^{-1}\right)$ and the intense $\mathrm{CH}$ stretching manifold ( $\sim 2700-3100 \mathrm{~cm}^{-1}$ ). Since these Raman experiments lack conformational resolution the analysis will focus on the first two spectral regions as they include more conformationally sensitive vibrations [13] than the latter region. In the $\mathrm{CH}$ stretching region bands overlap heavily, making discrete assignments impossible. Moreover, this region is heavily plagued by Fermi resonances that are not accounted for in harmonic calculations[79].

Figure 6.8 shows the comparison of a monoglyme jet spectrum in the LAM/CO/CC range and calculated Raman scattering cross sections at the B3LYP-3D3/aVQZ level for the lowest three conformers weighted with a ttt:tgt:tgg' abundance ratio of 7:8:5. The relevant fundamental vibrations for this work, disregarding other skeleton and $\mathrm{CH}$ amplitudes, are the $\mathrm{CO}$ and $\mathrm{CC}$ stretching modes (shortened to $\mathrm{CO}, \mathrm{CC}$ or, in the case of a mixture, to $\mathrm{CO} / \mathrm{CC}$ ). A preceding abbreviation denotes the symmetry character of the corresponding stretching mode with an $\mathrm{s}$ in case of symmetric motions and a for asymmetric motions. Relevant vibrations are listed in Table 6.6 but a more detailed mode assignment can be found in earlier work $[11,136]$. As can be seen, the predicted wavenumbers match the spectrum very well especially in the lower frequency range. In the CO/CC stretching region the harmonic predictions are slightly upshifted which is to be expected without any anharmonic corrections. Although not every band can be assigned by harmonic quantum chemical predictions (e.g. the complex band structure between 320 and $350 \mathrm{~cm}^{-1}$ ) no evidence for significant contributions of other conformers was found in the jet spectra as shown in Figure 6.5. In general the predicted intensity patterns for $t t t$ and $t g t$ match the spectra well besides the $\mathrm{LAM}_{\text {tgt }}$ and the COC bending motion of $t t t$ at $330 \mathrm{~cm}^{-1}$ (which was not labeled in Figure 6.8 as it will not be used for an analysis) which are overestimated. The intensity pattern of $t g g^{\prime}$ does not match the spectrum in a uniform way and especially bands with low intensities show stronger overestimations. Yet, the agreement between predictions and the jet spectrum are quite good and allow for further analysis.

Integrals of the most isolated bands of each conformer were compared to their predicted Raman band strengths $\left(\sigma_{\Phi}^{\prime}\right)$ to determine the relative populations in every spectrum. For $t t t$ the LAM and CC, for tgt the LAM and sCO+aCO and for tgg' the LAM' and SCO vibrations were chosen. These bands were fitted with Lorentzian-Gaussian cross product functions. In case relevant bands were partly overlapping with other, for this analysis, irrelevant bands (e.g. the tgt LAM shows a certain overlap with the $\operatorname{tg} g^{\prime}$ LAM as well as the tgt sCO+aCO with the aCO of $t g g$ ') then multiple bands were fitted and only the integral of the relevant band fit was picked. As a self-consistency check abundances were calculated separately for different spectral regions. This means that relative populations were calculated either by only comparing LAM bands 
with each other or through a comparison of the CO/CC stretching motions. An example for the calculation of the relative abundance of the $t t t$ conformer in the LAM region is given in Eq. 6.2. Relative tgt and $t g g^{\prime}$ fractions are determined analogously. Exchanging the LAM values in Eq. 6.2 by the corresponding CO/CC vibrations mentioned above yields the formula for the $\mathrm{CO} / \mathrm{CC}$ region.

$$
x_{t t t}=\frac{\frac{\int \mathrm{LAM}_{t t t}}{\sigma_{\Phi}^{\prime}\left(\mathrm{LAM}_{t t t}\right)}}{\frac{\int \mathrm{LAM}_{t t t}}{\sigma_{\Phi}^{\prime}\left(\mathrm{LAM}_{t t t}\right)}+\frac{\int \mathrm{LAM}_{t g t}}{\sigma_{\Phi}^{\prime}\left(\mathrm{LAM}_{t g t}\right)}+\frac{\int \mathrm{LAM}_{t g g^{\prime}}^{\prime}}{\sigma_{\Phi}^{\prime}\left(\mathrm{LAM}_{t g g^{\prime}}^{\prime}\right)}}
$$

The repopulation temperature $T_{\mathrm{r}}$ is calculated for the LAM region following Eq. 6.3. Again, $T_{\mathrm{r}}$ was determined separately for the LAM as well as the CO/CC region. An energy difference $\Delta E_{\text {tgg'tgt }}$ of $800 \mathrm{~J} \cdot \mathrm{mol}^{-1}$, as it was extrapolated in Section 6.2 , is assumed.

$$
T_{\mathrm{r}}=\frac{\Delta E_{t g g^{\prime}-t g t}}{R \cdot \ln \left(\frac{2 \cdot \int \mathrm{LAM}_{t g t} \cdot \sigma_{\Phi}^{\prime}\left(\mathrm{LAM}_{t g g^{\prime}}\right)}{\int \mathrm{LAM}_{t g g^{\prime}}^{\prime} \cdot \sigma_{\Phi}^{\prime}\left(\mathrm{LAM}_{t g t}\right)}\right)}
$$

In the next section spectral changes induced by changing the carrier gas, stagnation pressure, nozzle temperatures or distances will be discussed. Relative abundances and repopulation temperatures were determined for each spectrum as described and are summarized in Table 6.7 at the end of the following section. The comparison of repopulation temperatures and abundances of the two different spectral regions will reveal the relative error bars of this method. 


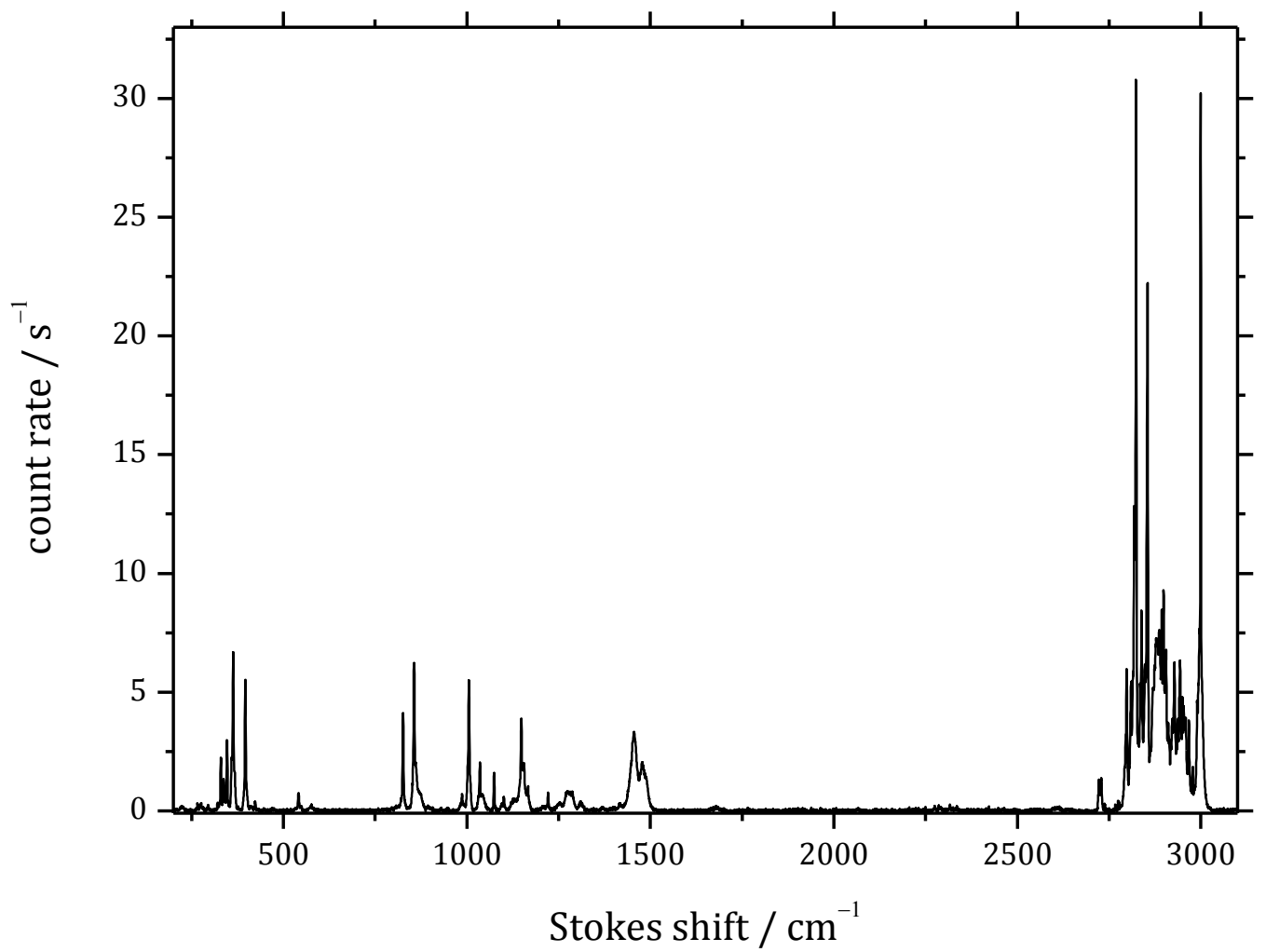

Figure 6.7: Survey $25 \mathrm{~W}$ Raman jet spectrum of monoglyme. Measurement conditions can be found in A.1.

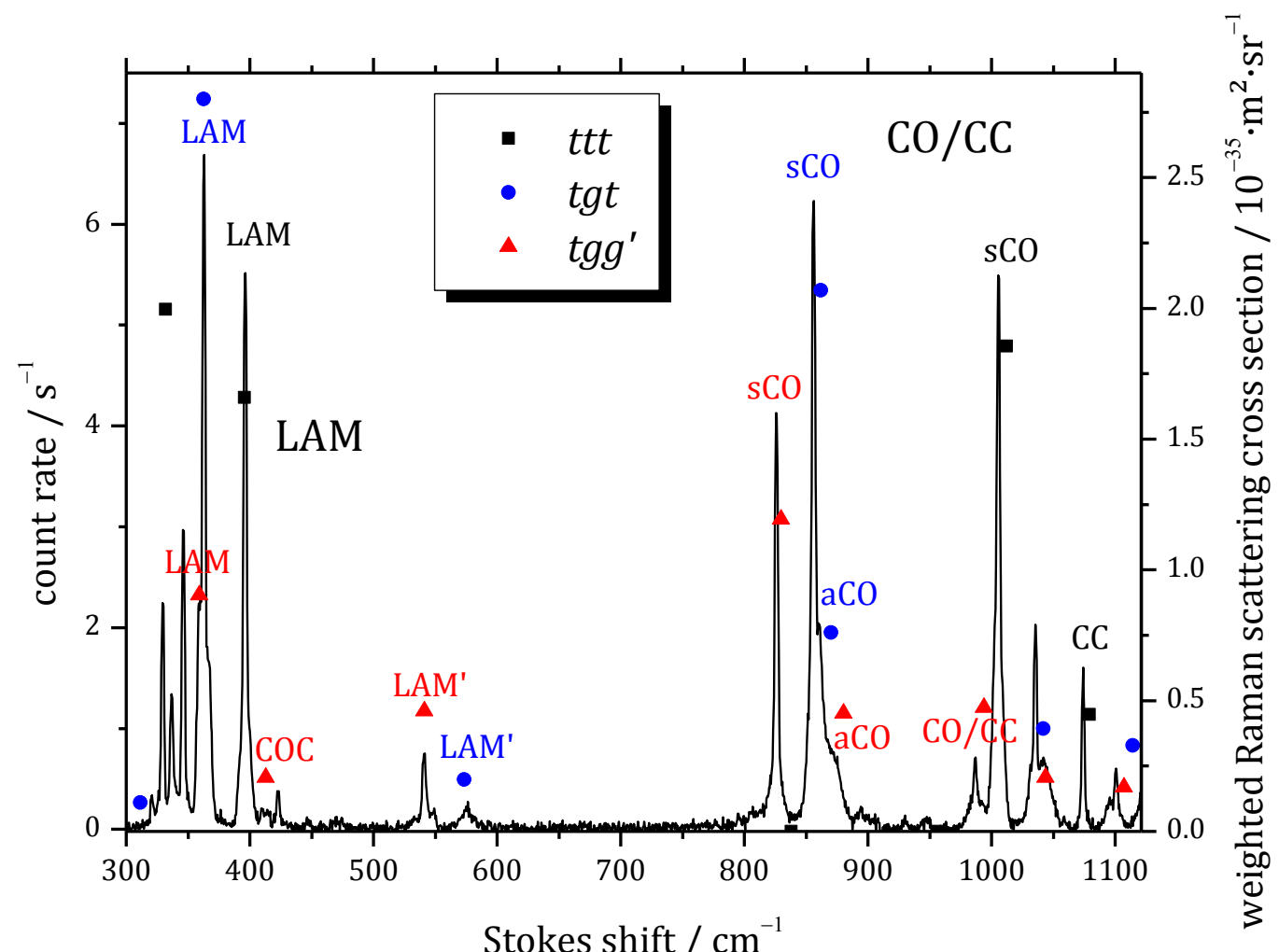

Figure 6.8: $25 \mathrm{~W}$ jet spectrum of monoglyme in the LAM/CO/CC range (measurement conditions can be found in A.1) compared to Raman scattering cross sections for the three lowest conformations calculated at the B3LYP-3D3/aVQZ level and weighted with a ttt:tgt:tgg' abundance ratio of 7:8:5. 
Table 6.6: Assignment of the relevant vibrations and comparison of their experimental and theoretical wavenumbers predicted at the B3LYP-3D3/aVQZ level in TURBOMOLE 7.0. Denotation of s for symmetrical and a for asymmetric is used in front of the corresponding mode if applicable.

\begin{tabular}{|c|c|c|c|}
\hline$\tilde{\boldsymbol{v}}_{\mathrm{exp}} / \mathbf{c m}^{-1}$ & $\tilde{\boldsymbol{v}}_{\mathrm{th}} / \mathbf{c m}^{-1}$ & mode & conformer \\
\hline 359 & 359 & LAM & $\operatorname{tgg} g^{\prime}$ \\
\hline 363 & 363 & LAM & $\operatorname{tg} t$ \\
\hline 396 & 396 & LAM & $t t t$ \\
\hline 410 & 413 & LAM & $\operatorname{tgg}{ }^{\prime}$ \\
\hline 540 & 541 & LAM' & $\operatorname{tgg}{ }^{\prime}$ \\
\hline 574 & 573 & LAM' $^{\prime}$ & $\operatorname{tgt}$ \\
\hline 828 & 830 & sCO & $\operatorname{tgg} g^{\prime}$ \\
\hline 858 & 862 & sCO & $\operatorname{tg} t$ \\
\hline 863 & 870 & $\mathrm{aCO}$ & $\operatorname{tg} t$ \\
\hline 874 & 880 & $\mathrm{aCO}$ & $\operatorname{tg} g^{\prime}$ \\
\hline 987 & 994 & $\mathrm{CO} / \mathrm{CC}$ & $\operatorname{tg} g^{\prime}$ \\
\hline 1006 & 1012 & $\mathrm{sCO}$ & $t t t$ \\
\hline 1074 & 1079 & $\mathrm{CC}$ & $t t t$ \\
\hline
\end{tabular}

\section{5 - Conformation control via experimental parameters}

The relative populations of the three observed conformers can be manipulated and controlled to a certain extent by switching carrier gases, varying the stagnation pressure, nozzle temperature or distance. Due to the changing conditions all following spectra were scaled to the integrals of the isolated CC or LAM band of the ttt conformer. This makes relative population changes between different experimental conditions directly visible. In order to minimize spectral changes due to differences in alignment or repositioning of the setup each series of tunable experimental parameters was measured consecutively on the same day. Note that some of the experiments were performed with a $25 \mathrm{~W}$ laser while others used a $5 \mathrm{~W}$ laser source. Spectra recorded with a $5 \mathrm{~W}$ laser show a decrease in the count rate by roughly a factor of 5 as signal intensity scales linearly with the power. However, other scattering processes in the jet chamber do not and lead to a significant decrease of the signal-to-noise ratio in $5 \mathrm{~W}$ spectra.

When switching the carrier gas from helium to argon a huge increase of the tgt and a likewise decrease of the $t g g^{\prime}$ population (Figure 6.9a) is observed. This clearly supports the derived relaxation model with a preferred interconversion from tgg' to tgt. When only mixing $20 \%$ argon to helium a somewhat intermediate state between those of the pure carrier gases is achieved. As Table 6.7 reveals the tgt gain is almost exclusively at the cost of $t g g^{\prime}$ population and is comparably strong in the LAM region (Figure 6.9b). However, the tgt LAM reveals some cluster effects as can be seen in the increase of the higher wavenumber wing in the argon spectrum. Quantum chemical calculations at the B3LYP-2D3/aVTZ level in Gaussian 09 Rev. D.01 revealed that tgttgt dimers are at least $1 \mathrm{~kJ} \cdot \mathrm{mol}^{-1}$ more stable than other examined combinations (10). 
They yield a dissociation energy of $26 \mathrm{~kJ} \cdot \mathrm{mol}^{-1}$ which is large compared to a vaporization enthalpy of $36.6 \mathrm{~kJ} \cdot \mathrm{mol}^{-1}[145,146]$, showing that this dimer is very stable. This effect might also be superimposed by additional cluster formation with argon. The cluster effects in argon expansions cannot be entirely separated from conformational interconversions so that evaluations in pure argon have to be taken with care. The cluster effects are less pronounced in the argon-helium mixture which shows an increase of the major tgt LAM and only slighter increases in the higher wavenumber shoulder. The lower wavenumber shoulder vanishes due to the decrease of $\operatorname{tg} g^{\prime}$. In combination with the drastic changes in the $\mathrm{CO} / \mathrm{CC}$ region an interconversion between tgt and tgg' is confirmed even with slightly interfering cluster effects. Their derived repopulation temperatures and relative abundances (Table 6.7) compare well with each other predicting a drop of $T_{\mathrm{r}}$ from 67 to 41 to $30 \mathrm{~K}^{\dagger}$ when going from helium to $20 \%$ argon in helium to pure argon expansions.

Variation in the stagnation pressure of argon expansions prove that the shape of the tgt LAM is much more susceptible to cluster effects (Figure 6.10b) than the CO band (Figure 6.10a). As can be seen, the higher wavenumber flank of the tgt LAM increases drastically at higher stagnation pressures while the CO band shows a much smaller band-shape-change. The $t t t$ bands show no signs of clustering which is expected as this conformer does not have a permanent dipole moment leading to only weak electrostatic interactions. The tgg' population is so low in argon expansions that it cannot be examined regarding its potential cluster formations. However, the previous carrier gas series showed no signs of clustering for the $t g g^{\prime}$ conformation either. Earlier studies investigating the aggregation of monoglyme in the liquid and solid phases found strong preferences for the tgt conformation $[11,116,117,147]$, which is in line with the observed cluster formation of $\operatorname{tg} t$ in the jet expansion.

When varying the nozzle temperature in pure helium expansions the effects of conformational interconversions are less pronounced yet visible (Figure 6.11a). The tgg' population increases with higher nozzle temperatures at the cost of tgt. Therefore, this population transfer is expected to be less efficient than the other way around due to a higher barrier as tgt is slightly more stable than tgg'. As seen in Table 6.7 the relative tgt population decreases by roughly 5\% when going from 293 to 393 K. Similar effects are found in the LAM region (Figure 6.11b). Again, small clustering effects are visible in the helium spectra as the higher wavenumber flank of the tgt LAM decreases at higher nozzle temperatures. However, clustering effects in helium are comparably small so they can be neglected.

Nozzle distance series in helium (Figure 6.12a) and argon (Figure 6.12b) show that the conformational interconversions continue from the nozzle exit to at least $1.5 \mathrm{~mm}$ downstream. The repopulation temperature drops from 69 to 67 to $66 \mathrm{~K}$ in helium and from 34 to 29 to $26 \mathrm{~K}$ in argon for nozzle distances of $0.5,1.0$ and $1.5 \mathrm{~mm}$, respectively. The first drop is larger than the second one indicating that fewer relaxations happen further downstream. In an attempt to minimize cluster formation while still

† Values from both spectral regions were averaged. 
maintaining a fairly low expansion temperature a mixing bottle with $0.2 \%$ monoglyme in helium was prepared and co-expanded with argon. Under these conditions (Figure 6.13) the conversions come to a halt after a nozzle distance of $1.5 \mathrm{~mm}$ as no further spectral changes are visible at larger distances. These expansion conditions show very similar repopulation temperatures than the ones found in pure argon expansions (Table 6.7), demonstrating that the goal of reaching a fairly low expansion temperature was achieved.

In general, strong relaxation effects between tgg' and tgt are found in all spectra. Some conditions facilitate the formation of clusters but the overall effects are too strong to be solely based on cluster formation. Moreover, population increases of $\operatorname{tgt}$ are always concomitant with a decrease of the $t g g^{\prime}$ population by the same amount. Molar fractions of $t t t$, $t g t$ and $t g g^{\prime}$ (Eq. 6.2) as well as the estimated repopulation temperatures (Eq. 6.3) are summarized in Table 6.7 for all shown spectra. The jet spectra contain approximately $0.05 \%$ monoglyme with $t t$ abundances of around $30-40 \%$ and tgt/tgg' ratios between 15/1 and 2/1, depending on expansion conditions. The corresponding repopulation temperatures are around $30 \mathrm{~K}$ when $\mathrm{tg}$ t/tgg' ratios are close to $15 / 1$, while they are around $75 \mathrm{~K}$ for ratios of $2 / 1$. 
a)

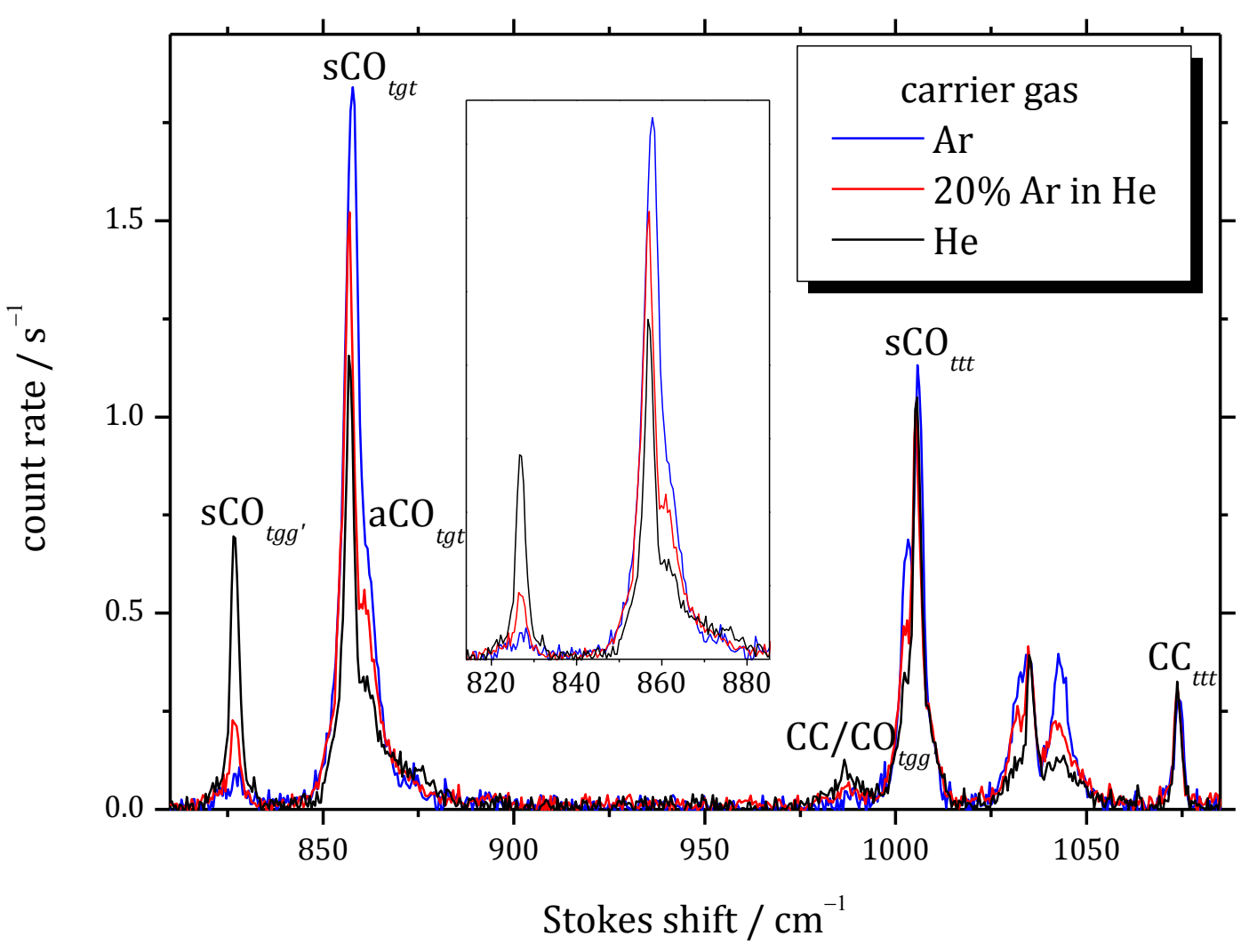

b)

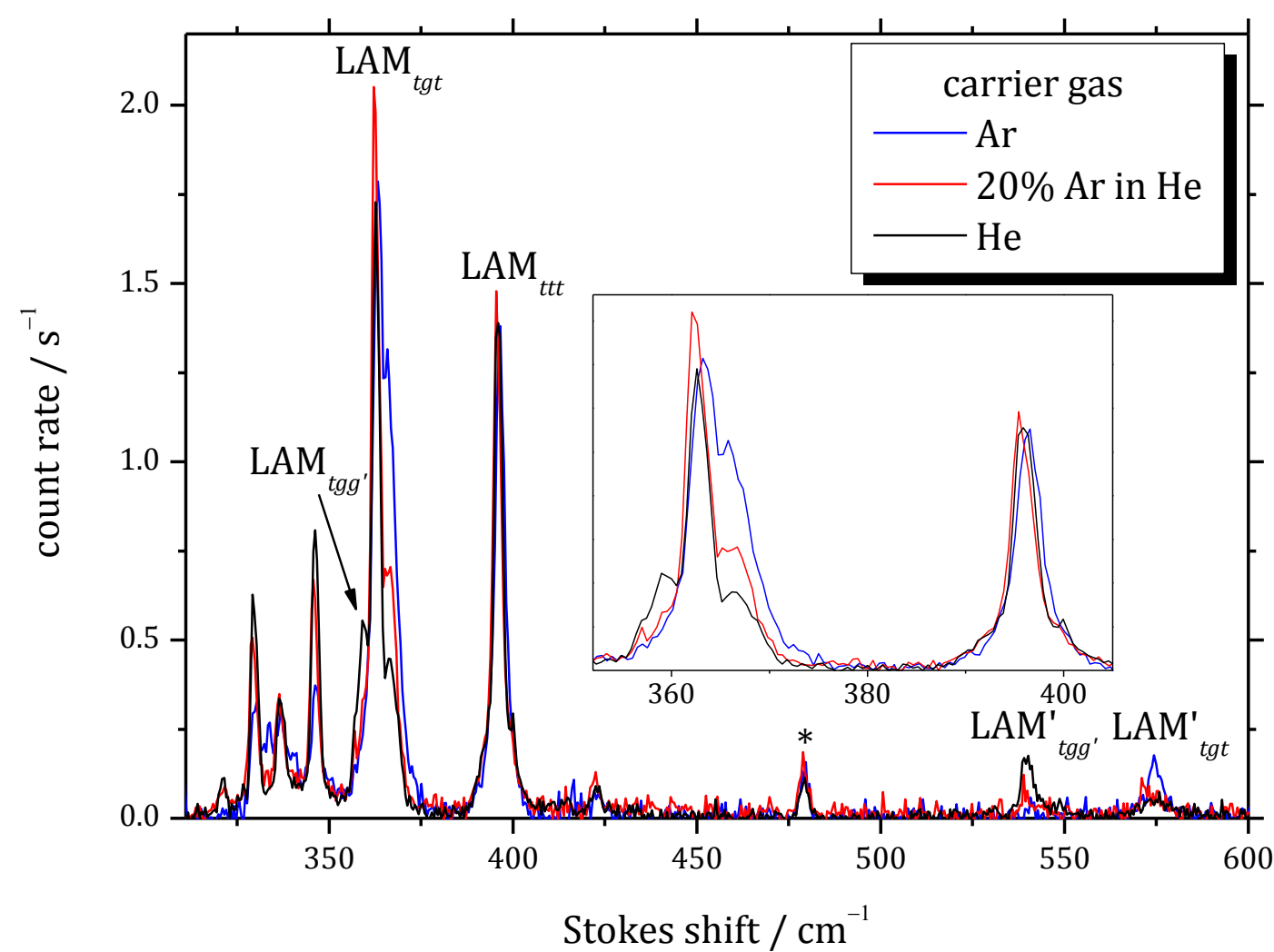

Figure 6.9: $5 \mathrm{~W}$ jet expansion spectra of monoglyme in He (black), 20\% $\mathrm{Ar}$ in $\mathrm{He}$ (red) and $\mathrm{Ar}$ (blue) at $1 \mathrm{~mm}$ nozzle distance in the CO/CC (a) and LAM (b) region. Spectra were scaled to equal $\mathrm{CC}_{t t t}(\mathrm{a})$ and LAM $_{t t t}$ integrals in (b). Count rates refer to the He spectra. The CO and LAM insets illustrate the relaxation differences. An impurity $\left({ }^{*}\right)$ at $478 \mathrm{~cm}^{-1}$ became visible at low laser powers. Other measurement parameters can be found in A.1. 
a)

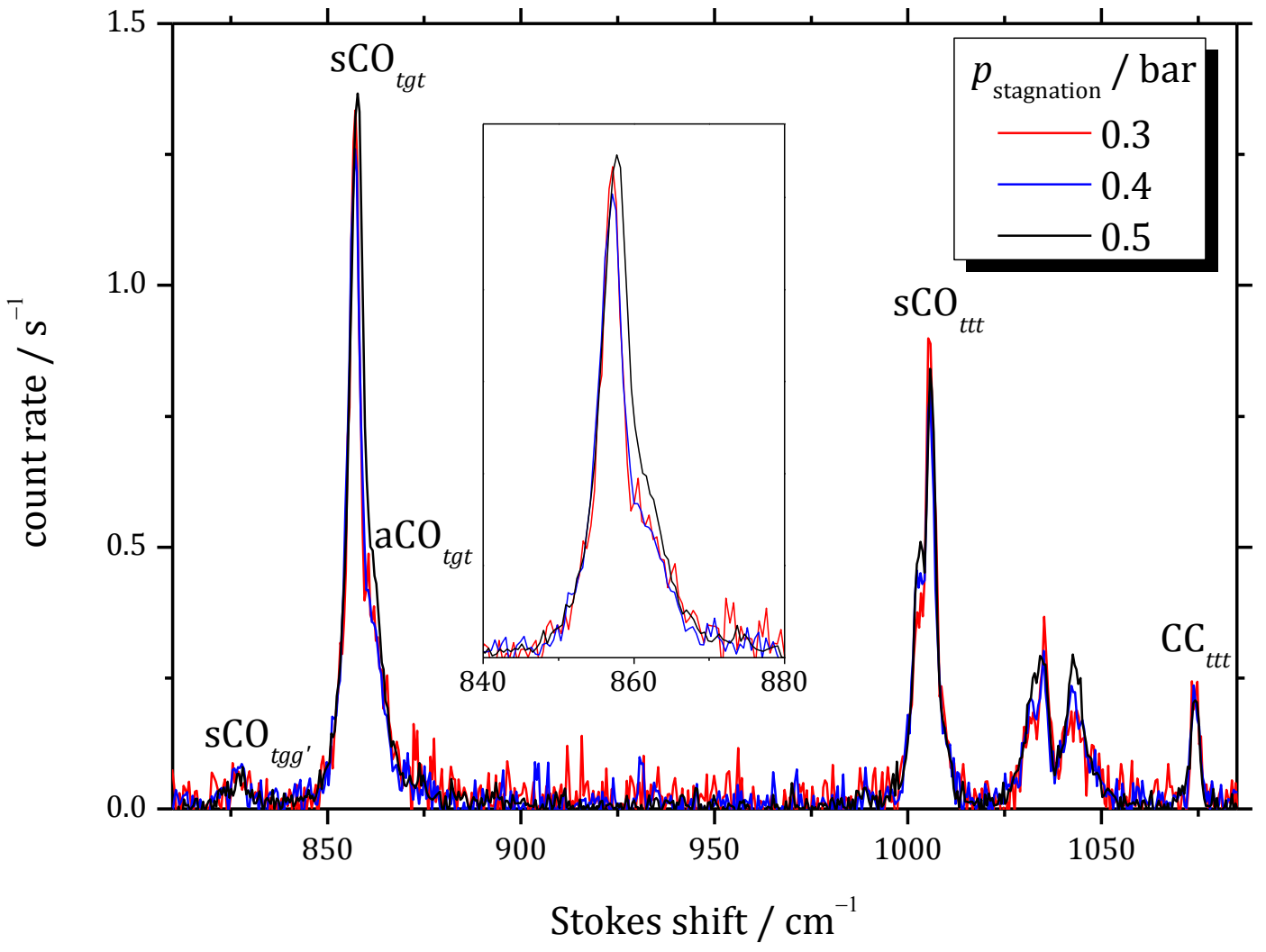

b)

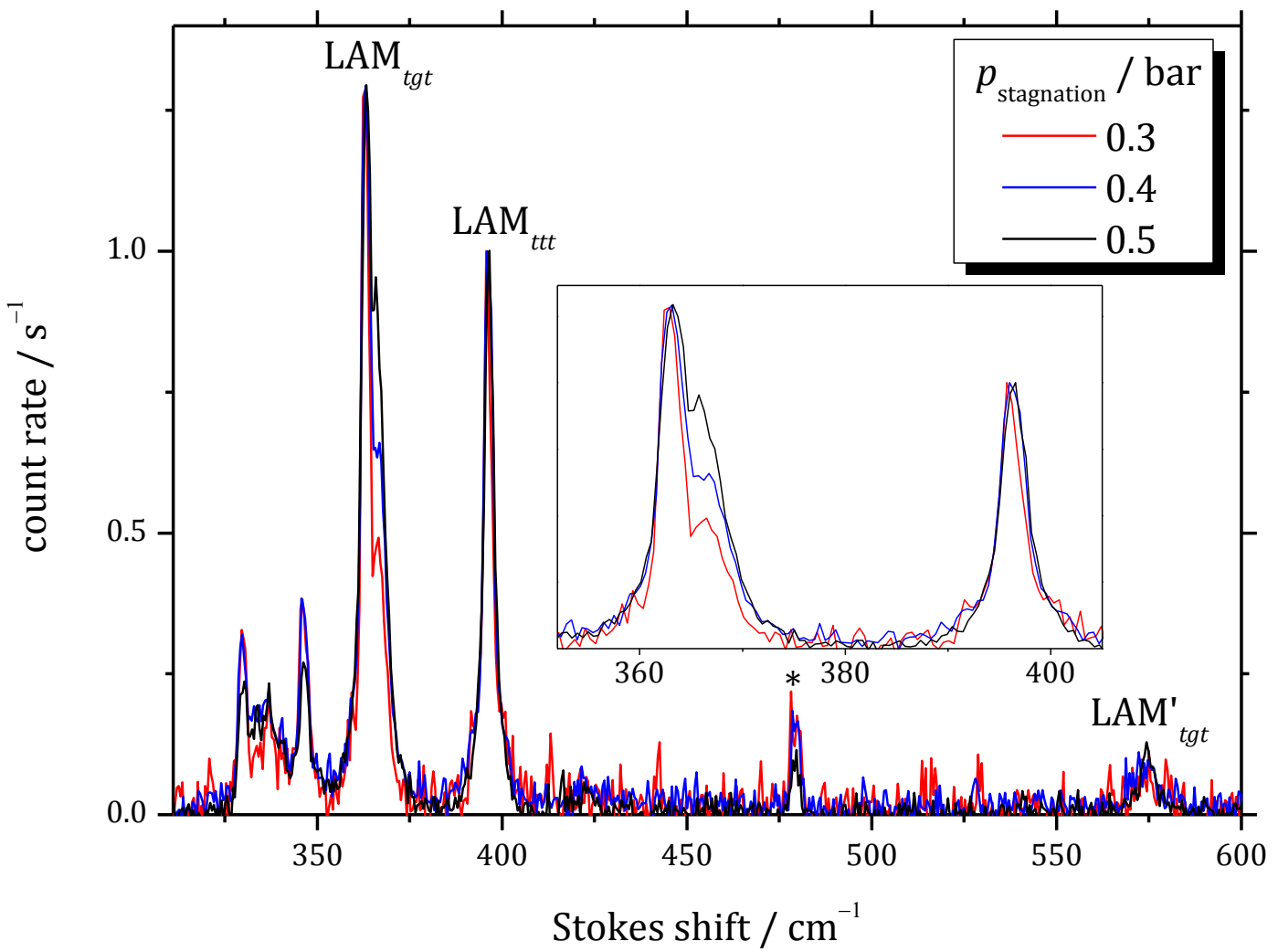

Figure 6.10: $5 \mathrm{~W}$ jet spectra of monoglyme in Ar at a stagnation pressure of 0.3 (red), 0.4 (blue) and 0.5 (black) bar at $1 \mathrm{~mm}$ nozzle distance in the CO/CC (a) and LAM (b) region. Spectra were scaled to the $\mathrm{CC}_{t t t}$ (a) and $\mathrm{LAM}_{t t t}$ integrals (b). Count rates refer to the 0.5 bar spectra. The CO and LAM insets reveal differences in the cluster tendencies of the corresponding tgt bands. An impurity $(*)$ at $478 \mathrm{~cm}^{-1}$ became visible at low laser powers. Other measurement parameters can be found in A.1. 
a)

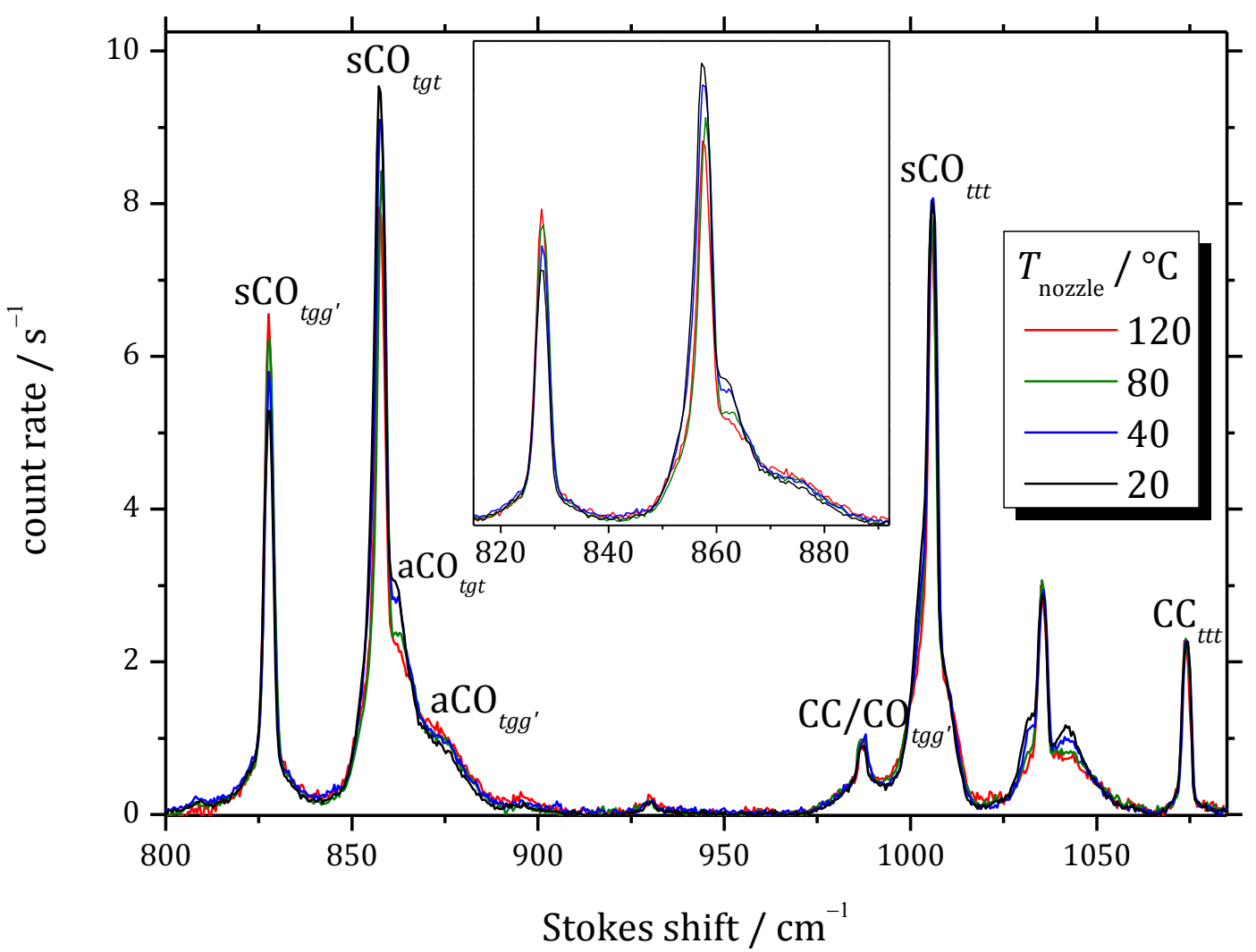

b)

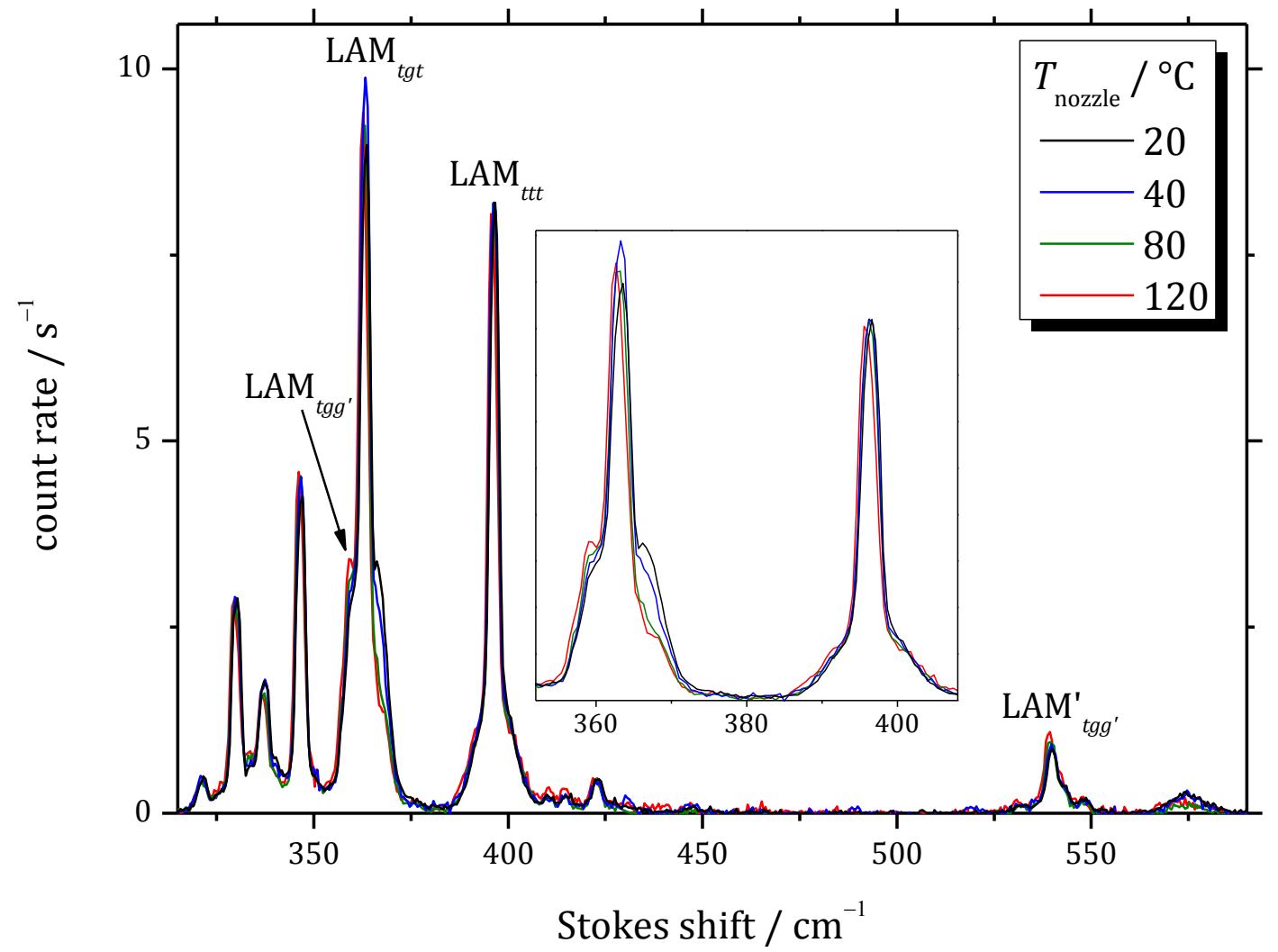

Figure 6.11: $25 \mathrm{~W}$ jet spectra of monoglyme in He at nozzle temperatures of 20 (black), 40 (blue), 80 (green) and $120^{\circ} \mathrm{C}$ (red) at $1 \mathrm{~mm}$ nozzle distance in the CO/CC (a) and LAM (b) region. Spectra were scaled to the $\mathrm{CC}_{t t t}$ (a) and $\mathrm{LAM}_{t t t}$ integrals (b). Count rates refer to the $20^{\circ} \mathrm{C}$ spectra. The CO and LAM insets show the relaxation of $t t g^{\prime}$ to $t g t$. Other measurement parameters can be found in A.1. 
a)

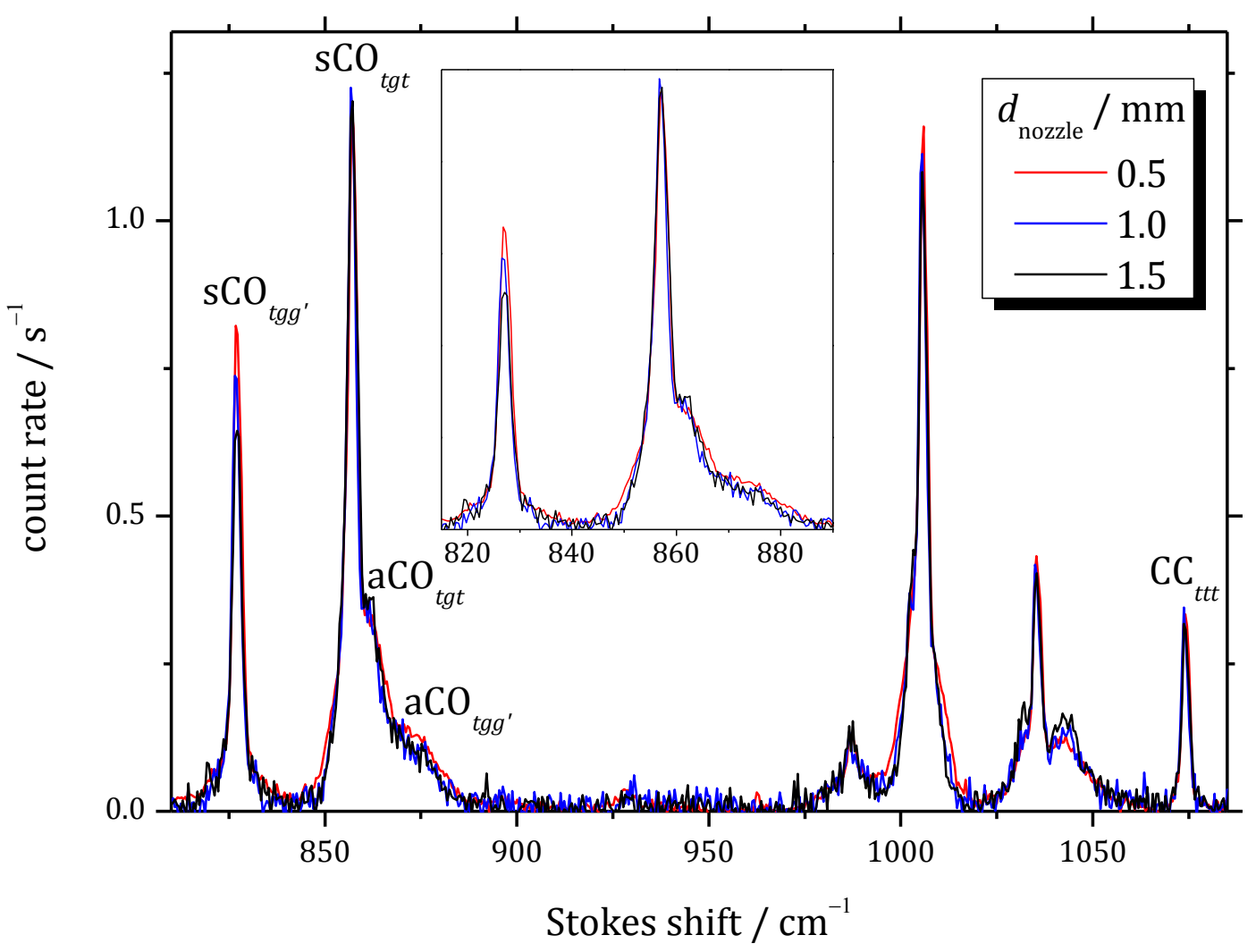

b)

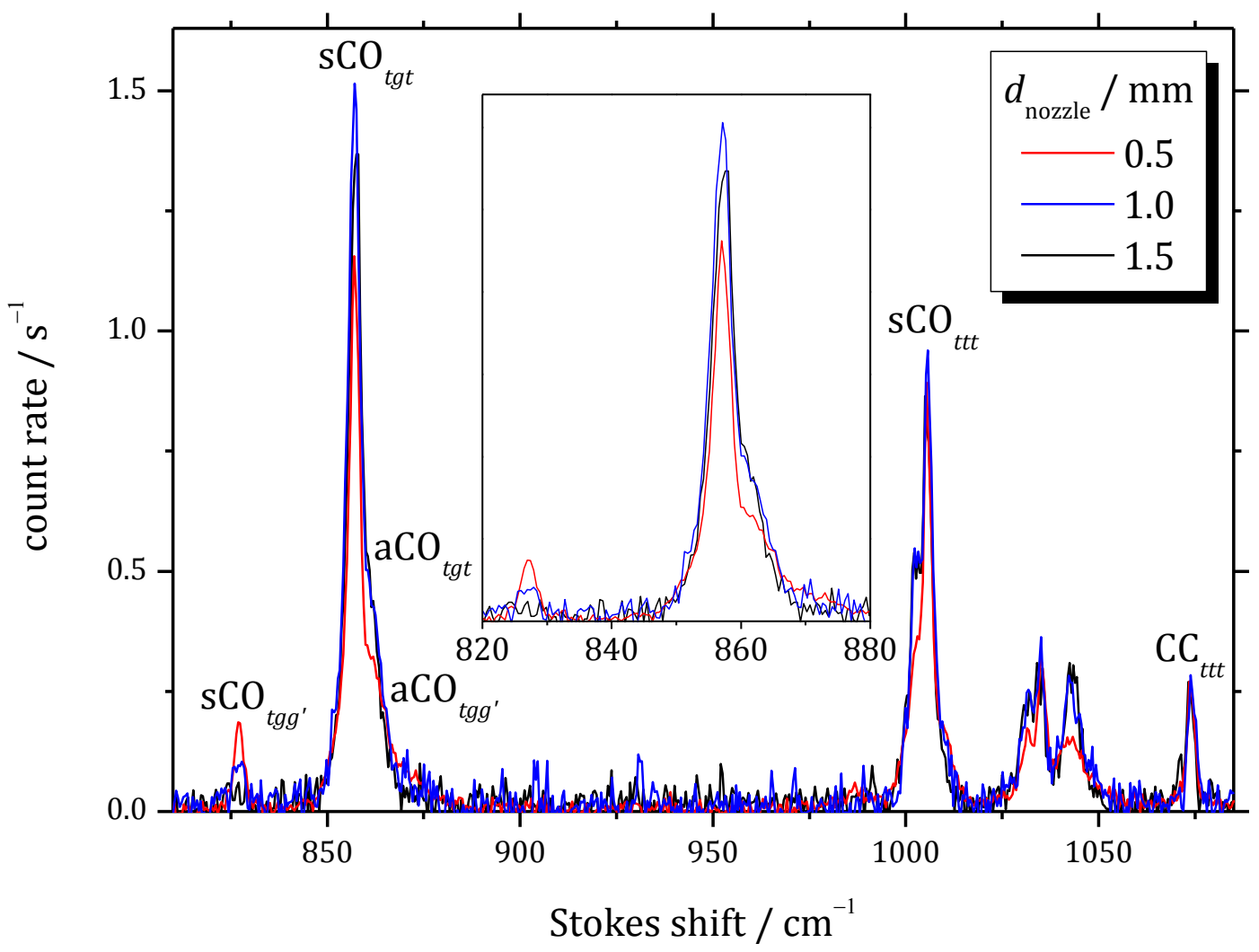

Figure 6.12: $5 \mathrm{~W}$ jet spectra of monoglyme in $\mathrm{He}(\mathrm{a})$ and $\mathrm{Ar}(\mathrm{b})$ at nozzle distances of 0.5 (red), 1.0 (blue) and $1.5 \mathrm{~mm}$ (black) in the $\mathrm{CO} / \mathrm{CC}$ region. Spectra were scaled to the $\mathrm{CC}_{t t t}$ integrals. Count rates refer to the $1 \mathrm{~mm}$ spectra. The CO and LAM insets show the continuous relaxation of $t$ tg' to tgt. Other measurement parameters can be found in A.1. 


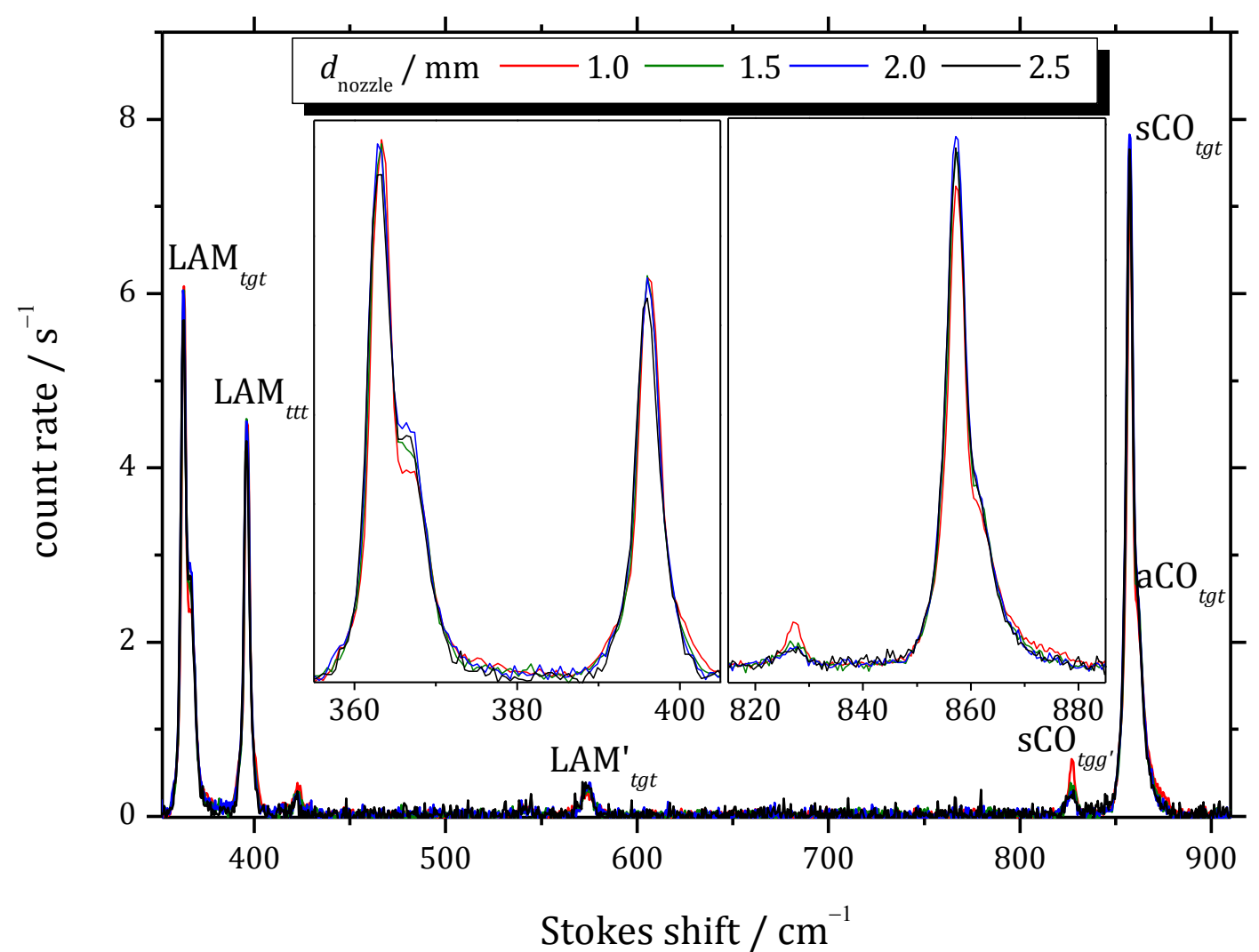

Figure 6.13: $25 \mathrm{~W}$ jet spectrum obtained under cold expansion conditions through premixing $0.2 \%$ monoglyme in He and adding $60 \%$ Ar. Spectra were scaled to the LAM $_{t t t}$ integrals. Count rates refer to the $1 \mathrm{~mm}$ spectrum. The $\mathrm{CO}$ and LAM insets reveal that relaxations come to a halt after $1.5 \mathrm{~mm}$. Other measurement parameters can be found in A.1. 
Table 6.7: Molar fractions of $t t t$, tgt and $t g g^{\prime}$ (Eq. 6.2) as well as the repopulation temperature ( $T_{\mathrm{r}}$ ) between tgt and tgg' (Eq. 6.3) derived from the Raman jet spectra shown in Figure 6.9-13. Color coding is used for orientation purposes and defines the different series of experimental parameters that are also presented in the separate figures.

\begin{tabular}{|c|c|c|c|c|c|c|c|c|}
\hline figure & carrier gas & $\begin{array}{c}T_{\text {Nozzle }} \\
/ \mathbf{K}\end{array}$ & $\begin{array}{c}p_{\text {Stagnation }} \\
/ \text { bar }\end{array}$ & $\begin{array}{l}d_{\text {Nozzle }} \\
/ \text { mm }\end{array}$ & $x_{t t t}$ & $x_{t g t}$ & $x_{t g g^{\prime}}$ & $T_{\mathbf{r}} / \mathbf{K}$ \\
\hline \multirow{3}{*}{$6.9 a$} & $\mathrm{He}$ & \multirow{3}{*}{293} & \multirow{3}{*}{0.5} & \multirow{3}{*}{1.0} & 0.30 & 0.48 & 0.22 & 66 \\
\hline & $\begin{array}{c}20 \% \mathrm{Ar} \\
\text { in } \mathrm{He} \\
\end{array}$ & & & & 0.31 & 0.60 & 0.09 & 37 \\
\hline & $\mathrm{Ar}$ & & & & 0.28 & 0.67 & 0.05 & 29 \\
\hline \multirow{3}{*}{$6.9 \mathrm{~b}$} & $\mathrm{He}$ & \multirow{3}{*}{293} & \multirow{3}{*}{0.5} & \multirow{3}{*}{1.0} & 0.40 & 0.41 & 0.19 & 68 \\
\hline & $\begin{array}{c}20 \% \mathrm{Ar} \\
\text { in } \mathrm{He}\end{array}$ & & & & 0.40 & 0.48 & 0.12 & 45 \\
\hline & $\mathrm{Ar}$ & & & & 0.39 & 0.57 & 0.04 & 30 \\
\hline \multirow{3}{*}{$6.10 \mathrm{a}$} & \multirow{3}{*}{$\mathrm{Ar}$} & \multirow{3}{*}{293} & 0.3 & \multirow{3}{*}{1.0} & 0.31 & 0.62 & 0.07 & 33 \\
\hline & & & 0.4 & & 0.31 & 0.63 & 0.07 & 33 \\
\hline & & & 0.5 & & 0.27 & 0.68 & 0.05 & 29 \\
\hline \multirow{3}{*}{$6.10 \mathrm{~b}$} & \multirow{3}{*}{$\mathrm{Ar}$} & \multirow{3}{*}{293} & 0.3 & \multirow{3}{*}{1.0} & 0.46 & 0.49 & 0.05 & 33 \\
\hline & & & 0.4 & & 0.44 & 0.52 & 0.04 & 32 \\
\hline & & & 0.5 & & 0.42 & 0.55 & 0.03 & 30 \\
\hline \multirow{4}{*}{$6.11 \mathrm{a}$} & \multirow{4}{*}{$\mathrm{He}$} & 293 & \multirow{4}{*}{0.5} & \multirow{4}{*}{1.0} & 0.26 & 0.51 & 0.23 & 63 \\
\hline & & 313 & & & 0.26 & 0.50 & 0.24 & 67 \\
\hline & & 353 & & & 0.27 & 0.47 & 0.26 & 73 \\
\hline & & 393 & & & 0.27 & 0.46 & 0.27 & 76 \\
\hline \multirow{4}{*}{$6.11 b$} & \multirow{4}{*}{$\mathrm{He}$} & 293 & \multirow{4}{*}{0.5} & \multirow{4}{*}{1.0} & 0.41 & 0.43 & 0.16 & 56 \\
\hline & & 313 & & & 0.41 & 0.43 & 0.16 & 57 \\
\hline & & 353 & & & 0.42 & 0.41 & 0.17 & 62 \\
\hline & & 393 & & & 0.41 & 0.39 & 0.20 & 70 \\
\hline \multirow{3}{*}{$6.12 \mathrm{a}$} & \multirow{3}{*}{$\mathrm{He}$} & \multirow{3}{*}{293} & \multirow{3}{*}{0.5} & 0.5 & 0.27 & 0.48 & 0.25 & 69 \\
\hline & & & & 1.0 & 0.29 & 0.49 & 0.22 & 66 \\
\hline & & & & 1.5 & 0.29 & 0.49 & 0.22 & 65 \\
\hline \multirow{3}{*}{$6.12 \mathrm{~b}$} & & & & 0.5 & 0.37 & 0.56 & 0.07 & 34 \\
\hline & $\mathrm{Ar}$ & 293 & 0.4 & 1.0 & 0.33 & 0.63 & 0.05 & 29 \\
\hline & & & & 1.5 & 0.35 & 0.62 & 0.03 & 26 \\
\hline & & & & 1.0 & 0.34 & 0.61 & 0.06 & 32 \\
\hline 612 & $60 \% \mathrm{Ar}$ & 202 & 05 & 1.5 & 0.33 & 0.63 & 0.04 & 28 \\
\hline 0.10 & in $\mathrm{He}^{\dagger}$ & J & 0.0 & 2.0 & 0.32 & 0.64 & 0.04 & 27 \\
\hline & & & & 2.5 & 0.32 & 0.64 & 0.04 & 27 \\
\hline
\end{tabular}

${ }^{\dagger}$ In order to reduce cluster formation, which commonly occurs in argon expansions, $0.2 \%$ monoglyme and helium were added in a mixing bottle and co-expanded with $60 \%$ argon. 


\section{6 - Van't Hoff analysis}

Spectra at different nozzle temperatures in helium, as shown in Figure 6.11, are suitable for a van't Hoff analysis of the tgt and $t t t$ bands. These spectra were recorded with a $25 \mathrm{~W}$ laser source resulting in a good signal-to-noise ratio and show less pronounced cluster formation than other carrier gases. Therefore, the band pairs $\mathrm{LAM}_{t g t} / \mathrm{LAM}_{t t t}$ and $\mathrm{CO}_{t g t} / \mathrm{CC}_{t t t}$ were chosen and the logarithm of their integrated intensity ratio was plotted against the inverse temperature (Figure 6.14). The slopes of the raw data imply an enthalpy difference of $0.5\left(\mathrm{CO} / \mathrm{CC}\right.$ region) and $0.8 \mathrm{~kJ} \cdot \mathrm{mol}^{-1}$ (LAM region) in favor of tgt. The differences might mostly result from different amounts of band contaminations in the different spectral regions. That tgt wins in this analysis is not surprising as one could clearly see a decrease of $t g t$ relative to $t t t$ bands at higher nozzle temperatures. However, this contradicts the presented quantum chemical calculations (Section 6.2) that predict a preference of $t t t$ by $1.2 \mathrm{~kJ} \cdot \mathrm{mol}^{-1}$ at $0 \mathrm{~K}$ and $0.8 \mathrm{~kJ} \cdot \mathrm{mol}^{-1}$ at $300 \mathrm{~K}$. This contradiction is understood when considering that tgt profits from relaxation effects more than $t t t$. First because it is directly fed by the only other conformer surviving a jet expansion (tgg') and second because the combined statistical weights of the conformers feeding tgt are two times larger than those feeding $t t t$. These relaxations have to be considered and are mimicked as described in the relaxation model in Section 6.3. As stated there, initial populations were estimated for all ten conformers at the corresponding nozzle temperatures. Then, all populations from conformers with an inner (OCCO) dihedral in trans configuration $(t t g, g t g, g t g$ ) are relaxed into $t t t$, while all conformers with a gauche configuration $\left(t g g^{\prime}, t g g, g g g, g g g^{\prime}, g g^{\prime} g\right.$ ) are relaxed to $t g t$. Finally, $t g g^{\prime}$ is populated in a Boltzmann equilibrium with $\mathrm{tgt}$ at the corresponding repopulation temperatures (Table 6.7) assuming an energy difference of $800 \mathrm{~J} \cdot \mathrm{mol}^{-1}$ between both. When initial populations were calculated based on full theoretical Gibbs energies $\left(\Delta G^{0}\right)$ the slopes invert and yield an enthalpy difference of 0.9 (LAM region) and $1.1 \mathrm{~kJ} \cdot \mathrm{mol}^{-1}$ (CO/CC region) in favor of $t t t$. Also when the mostly configurational Gibbs energies $\left(\Delta G^{\mathrm{C}}\right)$ were used to estimate initial populations an inversion of the slopes yielding enthalpy differences of 0.7 and $1.0 \mathrm{~kJ} \cdot \mathrm{mol}^{-1}$, respectively, are seen. The differences between all values must be seen as a realistic error estimation for the enthalpy difference.

The same analysis with slight differences in the underlying quantum chemical calculations was reported in [95] and is shown in Figure 6.15. There, B3LYP2D3/def2TZVP calculations were used for predicting the Raman scattering cross sections while Gibbs and zero-point energies were calculated at the B2PLYP-2D3/aVTZ level. Corrected enthalpy differences were obtained in the range of 0.4 to $1.7 \mathrm{~kJ} \cdot \mathrm{mol}^{-1}$. This shows that the analysis and the relaxation model are very robust and still lead to similar predictions although different quantum chemical methods were used. The lower fluctuations of the analysis presented in Figure 6.14 may be a result of the larger basis set and addition of three-body dispersion corrections, resulting in a higher reliability of the quantum chemical predictions. Therefore, a predicted enthalpy difference of $0.9 \pm 0.3 \mathrm{~kJ} \cdot \mathrm{mol}^{-1}$ in favor of $t t t$ in the temperature range of $300-400 \mathrm{~K}$ is 
reasonable. It should be emphasized that the energy difference between $\operatorname{tg} t$ and $t t t$ at $0 \mathrm{~K} \mathrm{did} \mathrm{not} \mathrm{enter} \mathrm{the} \mathrm{model} \mathrm{at} \mathrm{any} \mathrm{point} \mathrm{and} \mathrm{thus} \mathrm{can} \mathrm{be} \mathrm{used} \mathrm{as} \mathrm{a} \mathrm{consistency} \mathrm{check.}$ The best theoretical value for this difference is $1.2 \mathrm{~kJ} \cdot \mathrm{mol}^{-1}$ at $0 \mathrm{~K}$ as discussed in Section 6.2 . This value is lowered to $0.8 \mathrm{~kJ} \cdot \mathrm{mol}^{-1}$ when going to the $300-400 \mathrm{~K}$ range and therefore it is in line with the semiexperimental result from this analysis. The comparison of the $1 / T=0$ intercepts of the van't Hoff plots with their predicted Raman scattering ratios (shown as star symbols in Figure 6.14) serves as a further consistency check. Various different quantum chemical methods were used (compare Section 6.2) and are shown without further coding as they all are very close together. As can be seen the van't Hoff plot intercepts show good agreement with their predicted intercepts. This comparison suggests that the LAM plot might be seen as a lower bound of the enthalpy difference between $t g t$ and $t t t$.

This result is also roughly consistent with an earlier IR study in the gas phase [129] that found an experimental energy difference $\Delta E_{t g g^{\prime}-t t t}$ of $1.3 \pm 0.2 \mathrm{~kJ} \cdot \mathrm{mol}^{-1}$ and stated that the difference between $t g t$ and $t g g^{\prime}$ should be $\ll 1 \mathrm{~kJ} \cdot \mathrm{mol}^{-1}$. Furthermore, it agrees well with an unpublished infrared matrix isolation value of $0.7 \mathrm{~kJ} \cdot \mathrm{mol}^{-1} \mathrm{re}$ ported in [11].

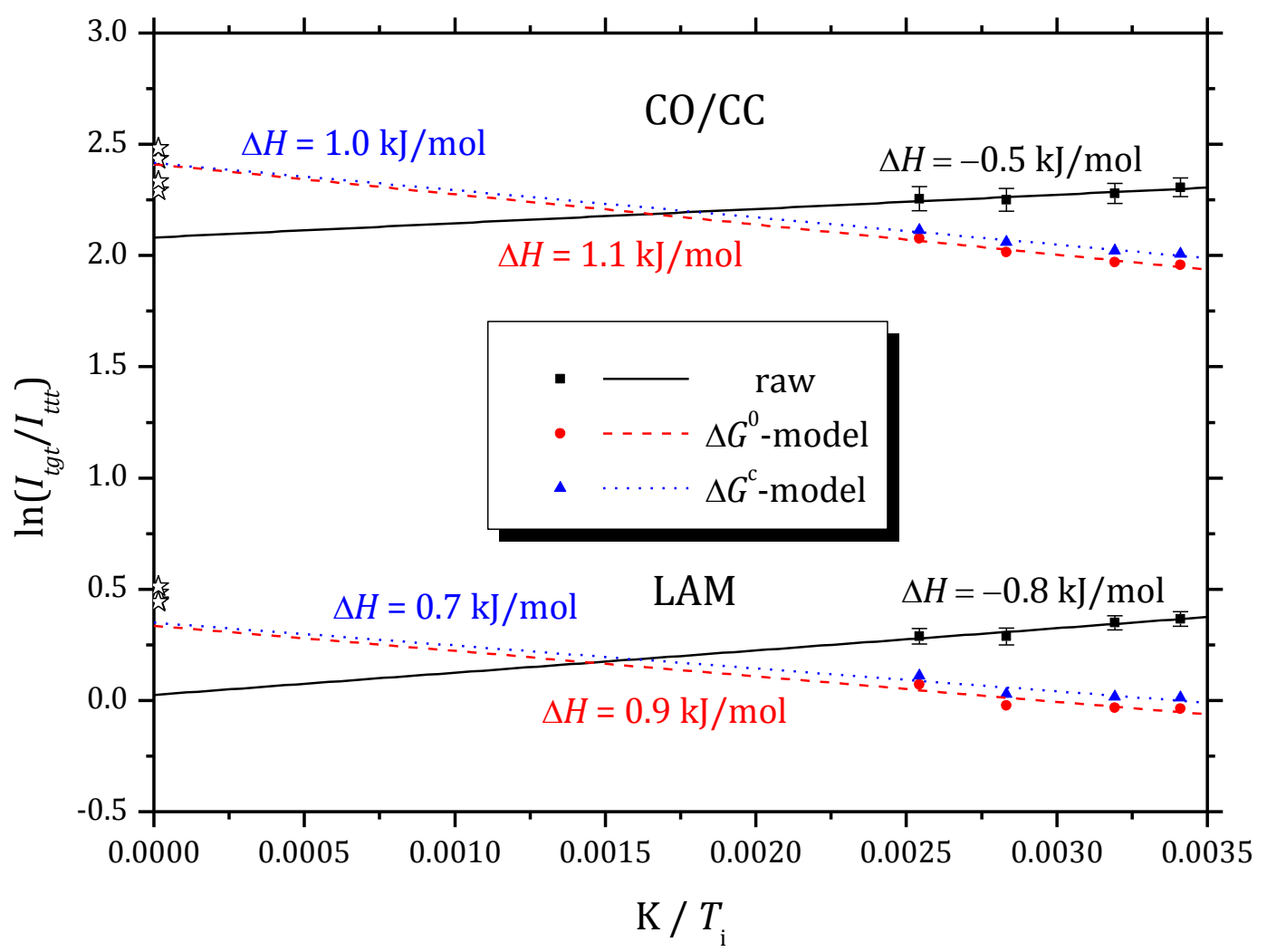

Figure 6.14: Logarithm of $t g t / t t$ intensity ratios for the CO/CC and LAM region as a function of inverse temperature. The raw data is shown in black, while corrections based on the $\Delta G^{0}$ model are shown in red dashes and corrections based on the $\Delta G^{\mathrm{C}}$ model are shown in blue dots. Predicted ordinate intercepts based on various DFT methods are shown as star symbols. Corrections are needed to invert the slopes of the van't Hoff plots and yield enthalpy differences between tgt and ttt that are consistent with high level quantum chemical calculations. 


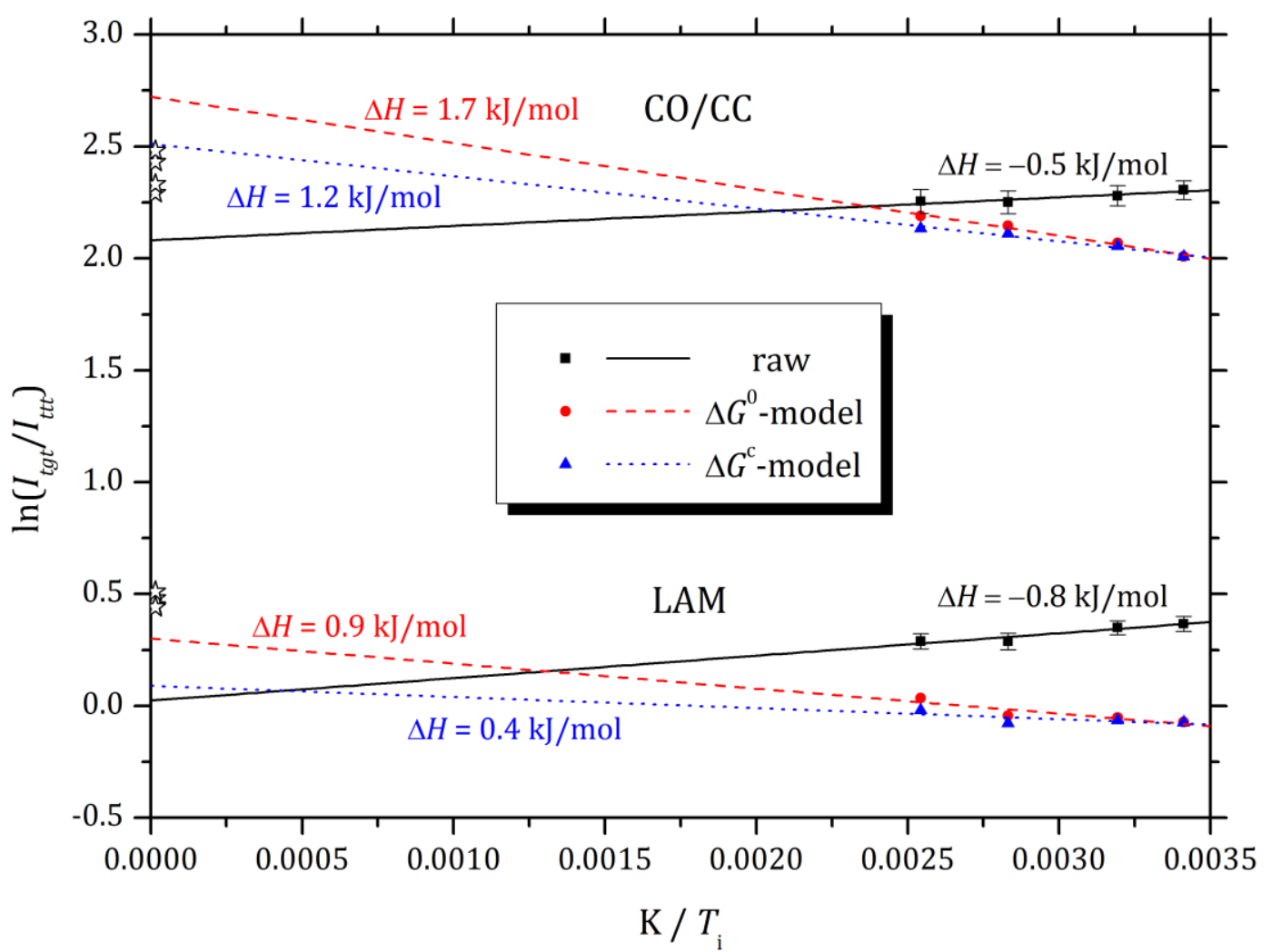

Figure 6.15: Resulting van't Hoff plots with same experimental raw data but slightly different quantum chemical corrections to the $\Delta G^{0}$ and $\Delta G^{\mathrm{C}}$ model (see text for details) as reported in [95].

\section{7 - Water clusters}

Interactions between monoglyme and water change the conformational preferences quite drastically. Numerous experimental [13,118-120] and theoretical [117,121123] studies investigated interactions between them. All of them find a strong preference of the tgt conformer in aqueous solutions. One study suggests that the excellent water solubility of monoglyme is based on the very low free energy of transfer from the pure liquid to the water phase of the tgt conformer [13]. Hints for a link between the water solubility and the tgt conformation can even be found in longer glymes up to PEO [148-151]. Early on it was suggested that in aqueous solution the $(\operatorname{tg} t)_{\mathrm{n}}$ conformer for PEO systems is able to form networks similar to those of bulk water as the oxygen distances are similar to those found in an ice structure [148]. A similar conformational preference was found in jet studies on 1,2-diphenoxyethane, where water clusters were only found with the tgt conformer [152].

The objective of this cluster study was to experimentally and theoretically confirm the preferred interaction of water with $\mathrm{tg}$. For this purpose, a complete examination and assignment of all possible cluster structures is not necessary and was therefore not carried out.

Input geometries were generated by combining the ten monoglyme conformers with one water molecule in a chemically intuitive way. These inputs were optimized and their frequencies calculated on the B3LYP-2D3/aVTZ level in Gaussian 09 Rev. D.01. 
At this level the ttt-tgt-tgg' energy sequence changed from 0-0.4-1.2 in the monomer to $3.9-0-6.8 \mathrm{~kJ} \cdot \mathrm{mol}^{-1}$ for the $1: 1$ cluster. This demonstrates the drastic effects upon hydration that should easily be observable in jet expansion spectra. An optimized structure for the $\mathrm{C}_{2}$ symmetric $t g t$-water cluster is shown in Figure 6.16. As seen, tgt forms two equivalent hydrogen bonds with water that are slightly longer than the one found in the water dimer [153]. Adding more water molecules might enable a competition for the hydrogen bond acceptors of monoglyme, allowing different network structures to be formed. However, exploratory calculations showed that the strong interactions in the 1:1 complex compel the next water molecule to form hydrogen bonds solely with the other water molecule instead of competing for the acceptor sites of monoglyme.

In order to enable a good comparison between expansion conditions that only contain monoglyme and those with additional water content, both cryo-saturators of the curry-jet were utilized and each substance was filled in one saturator. Spectra containing both substances and those only containing monoglyme were taken with the same expansion parameters. For the pure spectra the water saturator was bypassed but its lines were still used for co-addition of the carrier gas to keep its pressure nearly constant. Upon addition of water in the jet expansion the tgt bands increase drastically as shown in Figure 6.17. Cluster formation is especially visible for the upshifted shoulder of the tgt LAM that also showed the largest cluster effects in the jet spectra shown in Section 6.5. This is consistent with quantum chemical calculations that predict an upshift of $6 \mathrm{~cm}^{-1}$ of the tgt LAM upon water cluster formation that is completed rapidly as no significant further effects are seen at larger nozzle distances (Figure 6.18). Bands assigned to $t g g^{\prime}$ and $t t t$ show almost no effects which clearly demonstrates that water prefers to interact with the tgt conformer.

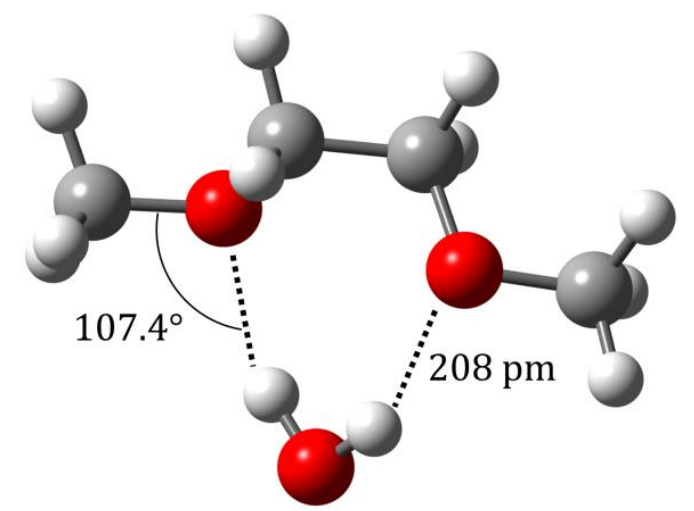

Figure 6.16: Geometry of the tgt-water cluster at the B3LYP-2D3/aVTZ level. 
a)

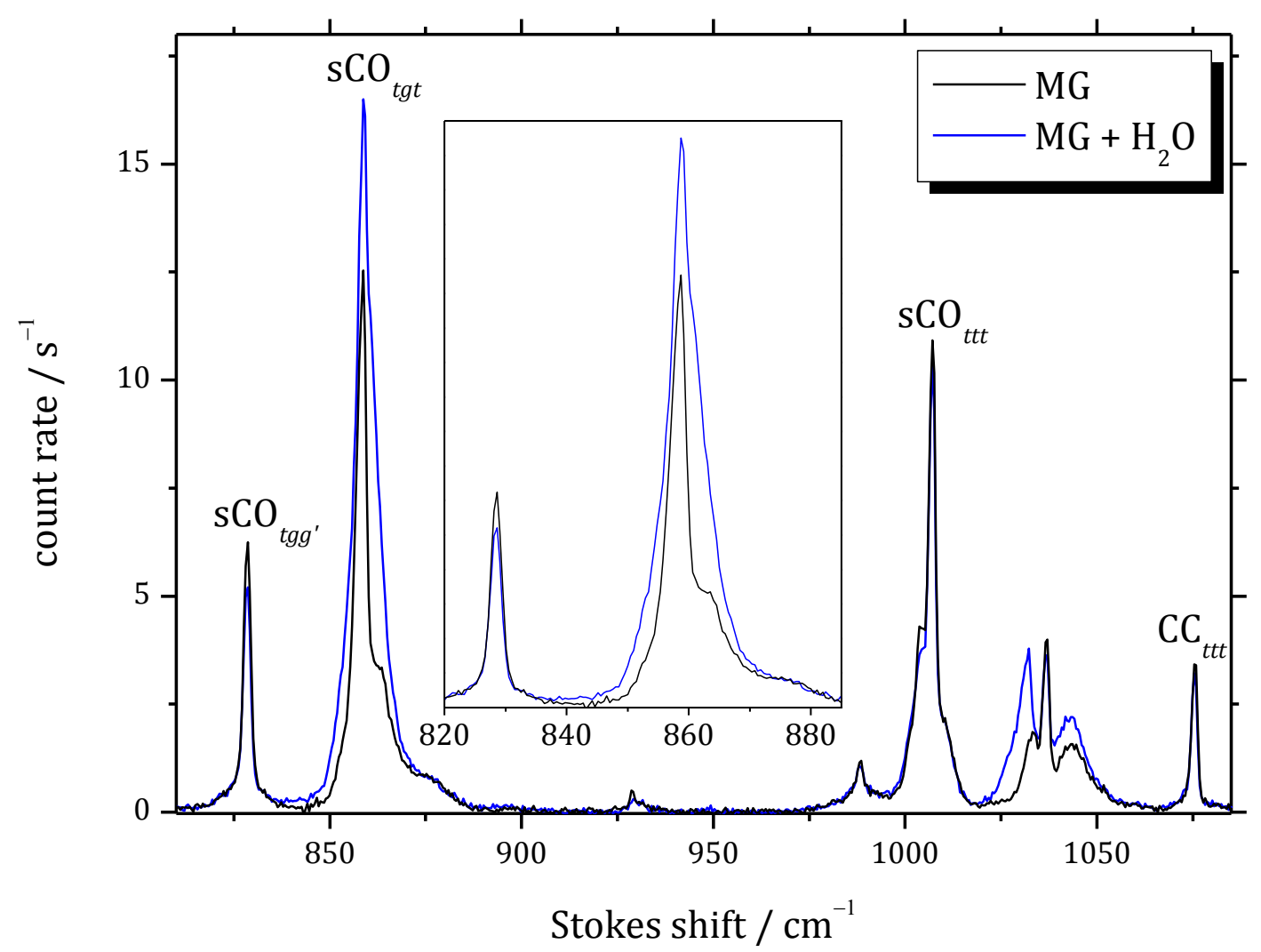

b)

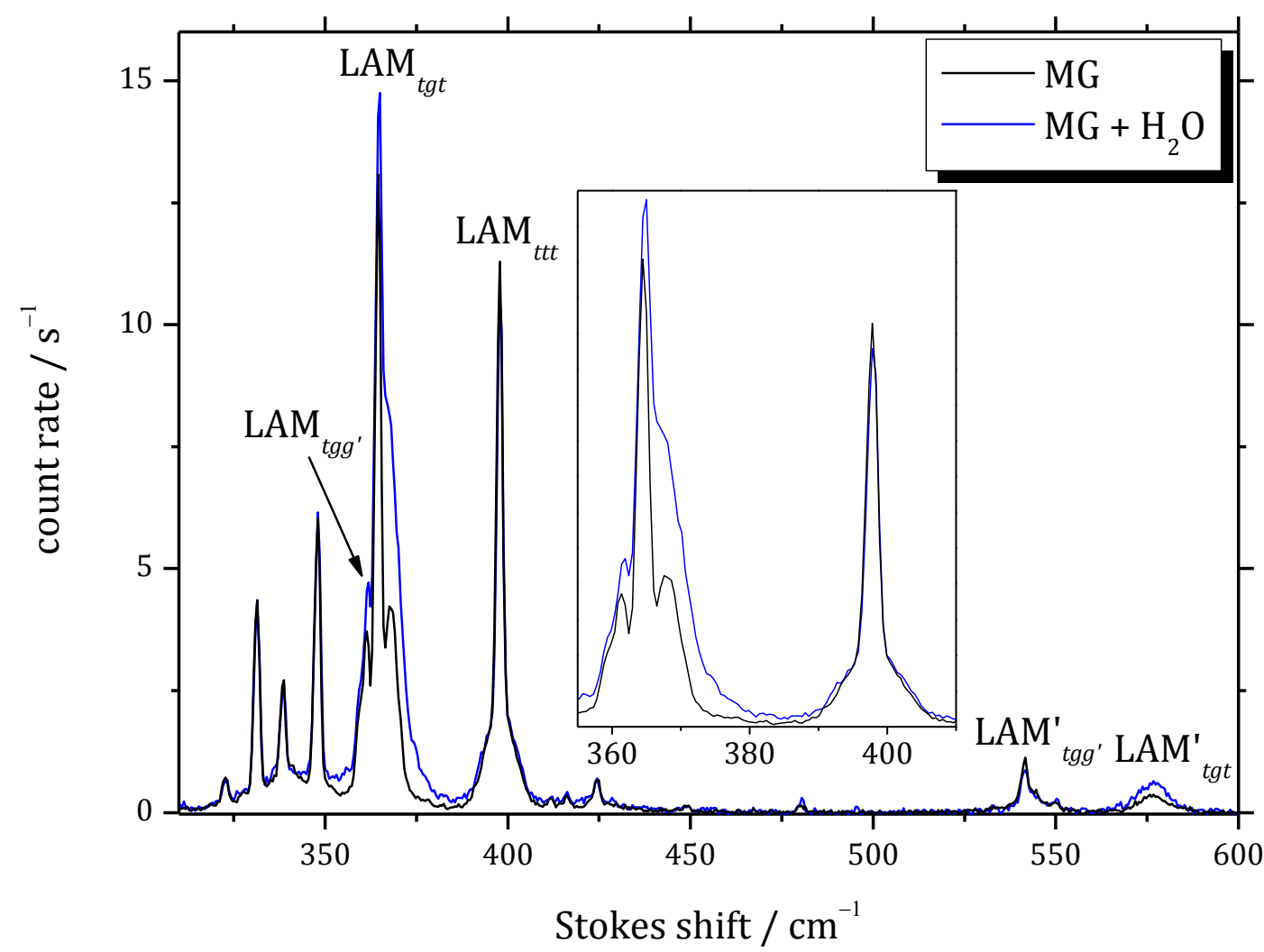

Figure 6.17: Comparison between jet spectra of monoglyme (black) with monoglyme and water (blue) at $1 \mathrm{~mm}$ nozzle distance in the CO/CC (a) and LAM (b) region. Spectra were scaled to equal CCttt (a) and LAMttt (b) integrals. Count rates refer to the pure monoglyme spectrum. The CO and LAM insets show the strong relative increase of the tgt bands upon hydration. Other measurement parameters are found in A.1. 
a)

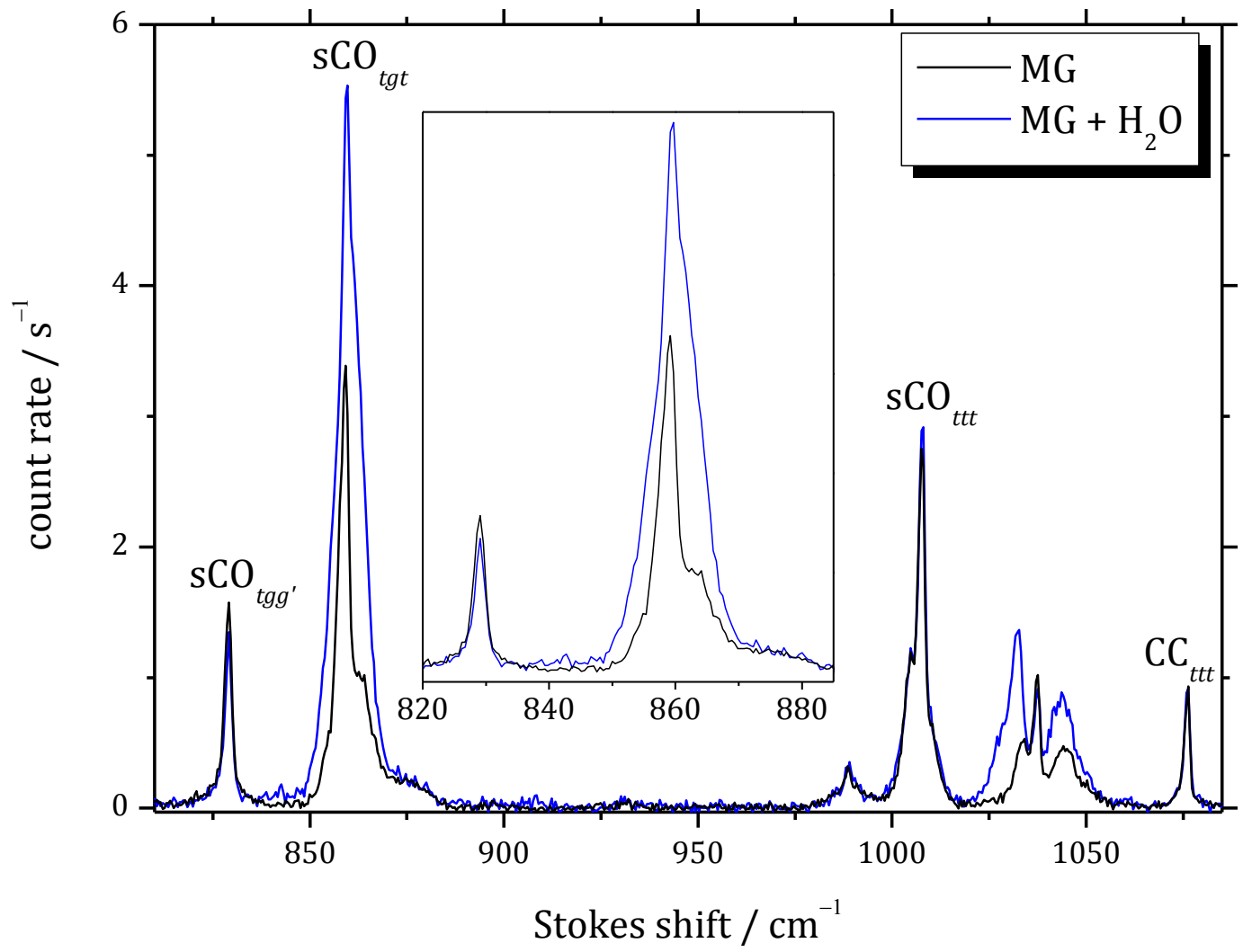

b)

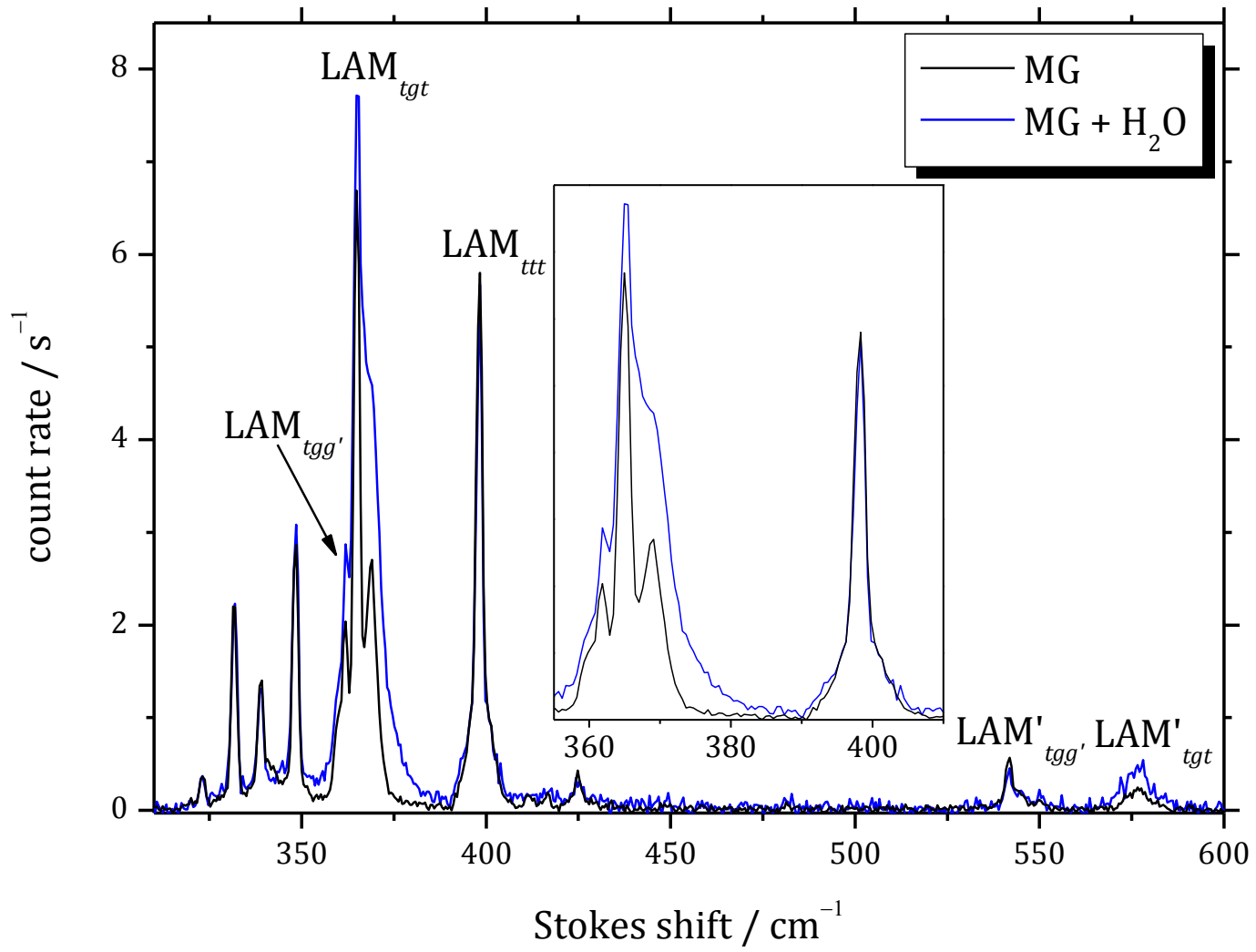

Figure 6.18: Comparison between jet spectra of monoglyme (black) with monoglyme and water (blue) at $2 \mathrm{~mm}$ nozzle distance in the CO/CC (a) and LAM (b) region. Spectra were scaled to equal CCttt (a) and LAMttt (b) integrals. Count rates refer to the pure monoglyme spectrum. No significant changes are visible when comparing these spectra to Figure 6.17, suggesting that cluster formation between tgt and water is completed rapidly during jet expansions. Other measurement parameters are found in A.1 


\section{8 - Conclusions}

In summary, extensive quantum chemical calculations for the ten monoglyme conformers result in a consimilar picture where $t t t$, tgt and $t g g^{\prime}$ are predicted to be significantly more stable than others. Coinciding, Raman spectra under supersonic jet expansion conditions only show contributions of these three conformers. When calculating the electronic energies of all conformers at the CCSD(T)-aVTZ level a remarkable agreement across all different used geometry-optimization-methods is found. The best estimate that is made for the energy sequence of ttt-tgt-tgg' based on all quantum chemical predictions is $0.0-1.2-2.0 \mathrm{~kJ} \cdot \mathrm{mol}^{-1}$ with an estimated error of less than $0.3 \mathrm{~kJ} \cdot \mathrm{mol}^{-1}$. Based on calculated 2D potential energy surface scans a relaxation model for jet expansion conditions was developed that freezes out interconversions of the central torsional (OCCO) angle at the initial temperature while the outer torsional (COCC) angles form a sub-100 K Boltzmann equilibrium. This model allows for an interpretation of the Raman jet spectra in a consistent way with high level quantum chemical calculations. The combined theoretical and experimental analysis of the conformational distributions concludes that the $t t$ conformation is the global minimum structure, followed by the entropically favored $\operatorname{tg} t$ and the entropically even more favorable tgg'. Earlier reports on tgg' being energetically below tgt can be ruled out by experiments and theory. A semiexperimental estimation for the energy difference of tgt and $t t t$ yields $0.9 \mathrm{~kJ} \cdot \mathrm{mol}^{-1}$, consistent with high level quantum chemical calculations.

The phenyl-substituted analog 1,2-diphenoxyethane was predicted to have a wider spacing of the low-lying conformations (separation of $t t t$ and $t g t$ by $2-3 \mathrm{~kJ} \cdot \mathrm{mol}^{-1}$ ) [102], although that prediction is based on quantum chemical calculations on a moderate level that could be improved. However, nearly equal populations of $t t t$ and $t g t$ were obtained in an infrared population transfer spectroscopy experiment. This fits the monoglyme findings with high barriers of OCCO torsional interconversions that are not overcome in jet expansions. Additionally, LIF and Raman jet spectra on 1-methoxy-2-phenoxyethane (Chapter 5) show major contributions of $t$ tt and tgt. No relaxation effects were found in the Raman spectra upon changing experimental parameters in the curry jet expansion, consistent with the high interconversion barrier of the OCCO torsion separating $t t t$ and tgt. In contrast to findings in monoglyme, no significant contributions of $t g g^{\prime}$ were found in Raman jet spectra which might be explained by the lower interconversion barrier between tgg' $^{\prime}$ and $\operatorname{tgt}$ in 1-methoxy-2phenoxyethane (Figure 5.4). Additionally, calculations of the electronic energies at the MP2/aVTZ level in 1-methoxy-2-phenoxyethane showed that the separation between tgt and tgg' might be larger than in monoglyme.

A study of the monoglyme-water-clusters under jet expansion conditions reveals a strong preference of the tgt conformer, consistent with earlier reports on monoglyme in aqueous solutions. Additional quantum chemical calculations support the adaptive aggregation of monoglyme and predict the 1:1 tgt-water cluster to be $\sim 4 \mathrm{~kJ} \cdot \mathrm{mol}^{-1}$ more stable than the water cluster formed with $t t t$. 


\section{Chapter 7 - Diglyme}

\section{1 - Introduction}

Diglyme, also known as diethylene glycol dimethyl ether is, like most glymes, chemically very stable. It is resistant to oxidation and reduction processes, can withstand high $\mathrm{pH}$ environments and is miscible with water as well as organic solvents. Diglyme melts at $-64{ }^{\circ} \mathrm{C}$ [154] and boils under standard pressure at $162{ }^{\circ} \mathrm{C}$ [155] making it liquid at room temperature. Despite being able to form volatile peroxides it is generally regarded as a relatively safe compound with low acute toxicity. However, Kennedy Jr. and co-workers revealed in their studies on rats that diglyme can cause developmental damage [156] as well as cellular injuries [157] during chronic inhalation. In 2014 the European Commission added diglyme to the list of substances of very high concern (SVHC) due to its reproductive toxicity [158] and beginning August 2017 [159] the import and usage of diglyme in the European Union will require authorization from the European Chemicals Agency (ECHA).

Despite these drawbacks diglyme is still widely used in many different applications due to its beneficial physical properties. The diglyme backbone contains three oxygen atoms that can coordinate and chelate reactive species or metals to enhance their reactivity. Thus it has long been used as a solvent in chemical reactions such as the syntheses of dialkylboranes [160] and trialkylboranes [161]. Additionally, Zultanski and $\mathrm{Fu}$ reported in 2013 that $\mathrm{Ni}$-catalyzed Suzuki arylation of unactivated tertiary alkyl bromides is only possible when diglyme coordinates the catalyst [162]. Recently diglyme received a lot of attention as a prime solvent for Li- [163-168], Mg- [169,170] and Al-type [171] batteries ${ }^{\dagger}$. In these studies diglyme was found to enhance electron transfer processes through solvating and chelating high ion concentrations, increasing thermal and oxidative stabilities and having a high dielectric constant and low viscosity. It is also used as a model to understand conductivity processes in PEObased electrolytes as it is still of manageable size for modern quantum chemical computations $[172,173]$. Furthermore, Compel et al. found that diglyme mediates the selfassembly of gold nanoclusters [174]. Adding diglyme as oxygenate component in diesel fuels increases the effective thermal efficiency [175] and reduces soot as well as carbon monoxide emissions [176].

These widespread applications utilize the high flexibility of the diglyme backbone, which can effectively coordinate and chelate many different environments. Therefore, it is crucial to understand the molecule, its potential energy surface and dynamics in detail. The first vibrational analysis of diglyme in the liquid state was reported in 1964 by Machida and Miyazawa [147], followed by a report on the infrared spectrum in the crystalline state by Matsuura et al. in 1973 [177]. Later, ab initio investigations revealed a variety of stable conformers close in energy [172,178-181]. These studies find the all-trans structure as the global minimum usually followed closely by tgtttt and tgttgt. Combining spectroscopic and theoretical studies led to the conclusion that

\footnotetext{
† References given display only a selection of publications on that topic.
} 
likely the tgttgt conformation is present in the solid phase, while various conformations were vaguely assigned in the liquid phase $[172,179,182]$. When adding alkali

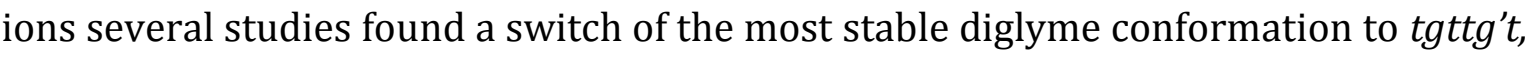
a geometry that can coordinate to these ions in a crown-ether type motif $[166,168,173,183]$.

The competitive conformational landscape of diglyme with very subtle energy differences makes it reasonable to revisit this case and apply state-of-the-art quantum chemical methods with larger basis sets than used in previous studies [172,179-181]. Also, dispersion corrections that were missing in these studies will be added to density functional theories. In contrast to the condensed phase studies $[147,172,177,179,182]$ Raman jet spectra of low frequency motions allow the observation of isolated and cold molecules which makes the assignment aid by quantum chemical calculations more straightforward and reliable. In this chapter the conformational search as well as the quantum chemical calculations will be outlined. Then, a relaxation model will be proposed based on barriers of interconversions on the conformational landscape. Afterwards, Raman jet spectra are interpreted with the help of quantum chemical predictions and lastly cluster tendencies and effects are presented.

\section{2 - Quantum chemical calculations}

A self-made program named Conformeer has been used to generate Gaussian inputs for all possible diglyme conformations assuming that only gauche $\left( \pm 60^{\circ}\right)$ or trans $\left(180^{\circ}\right)$ backbone dihedrals will result in stable conformations. Note that slightly adjusted dihedrals of $\pm 75^{\circ}$ for gauche have been used here based on averaged results from preliminary calculations. Also, the program disregards enantiomeric and rotational pairs. Enantiomeric pairs have inverted signs for every gauche dihedral along the chain. Since the Hamiltonian is inversion-symmetric both enantiomers exhibit the same electronic energy ${ }^{\dagger}$, thus making it unnecessary to calculate both enantiomers. However, the enantiomeric partner still has to be considered when it comes to statistical weight factors. Rotational pairs have their dihedral sequence reversed and are simply disregarded. The full source code of this program can be found in A.10.

The program created 196 input structures of which three had to be disregarded because they represented structures that were folding into themselves $\left(g g^{\prime} g g^{\prime} g g^{\prime}\right.$, $g g g^{\prime} g g^{\prime} g$ \& $t g g^{\prime} g g^{\prime} g$ ). The geometries of the remaining 193 structures were optimized in Gaussian 09 Rev. D.01 using the B3LYP-2D3/def2TZVP method. Afterwards harmonic frequencies were calculated with the same functional and basis set. The output files of the geometry optimizations were checked with the Conformeer program for a change in their backbone dihedral sequence during the optimization. Hereby 26 structures were found that converted into a different conformation. All of these structures converted around the first or last dihedral angle indicating a very small inter-

\footnotetext{
${ }^{\dagger}$ Enantiomers do not have the same electronic energy when parity violations are considered [184], yet the effect is so weak that it can be easily neglected for the purpose of this work. 
conversion barrier and emphasizing a high flexibility of the molecule around these. In general, this approach allowed for an extensive overview of the conformational landscape. In agreement with previous studies [172,179-181] many conformers were found very close in energy as already 23 structures were identified to be within a $5 \mathrm{~kJ} \cdot \mathrm{mol}^{-1}$ threshold relative to the global minimum structure. Surprisingly, the global minimum was predicted in a tgttg'g conformation which other studies had not considered thus far. The full table containing all calculated conformers can be found in A.5. Note that small imaginary frequencies $\left(>-10 \mathrm{~cm}^{-1}\right)$ were found in six cases that are caused by a bug in the implementation of the Becke-Johnson damping function when calculating frequencies in Gaussian 09 Rev. D.01 (see Chapter 2.5). Frequencies of these structures calculated in Gaussian 09 Rev. E.01 show no imaginary frequencies. However, one structure $\left(t g g^{\prime} g^{\prime} g g^{\prime}\right)$ showed a large imaginary frequency indicating that it is actually not a local minimum structure and rather a transition state. Because of this Becke-Johnson damping bug all frequency calculations but the initial B3LYP-2D3/def2TZVP scan of all 193 conformers were redone in Gaussian 09 Rev. E.01.

The structures of the lowest four conformers, tgttg'g, tttttt, tgtttt, tgttgt, are shown in Figure 7.1. As mentioned earlier, to get the correct order and energy gaps for such subtle differences one has to apply larger basis sets and state-of-the-art quantum chemical methods.
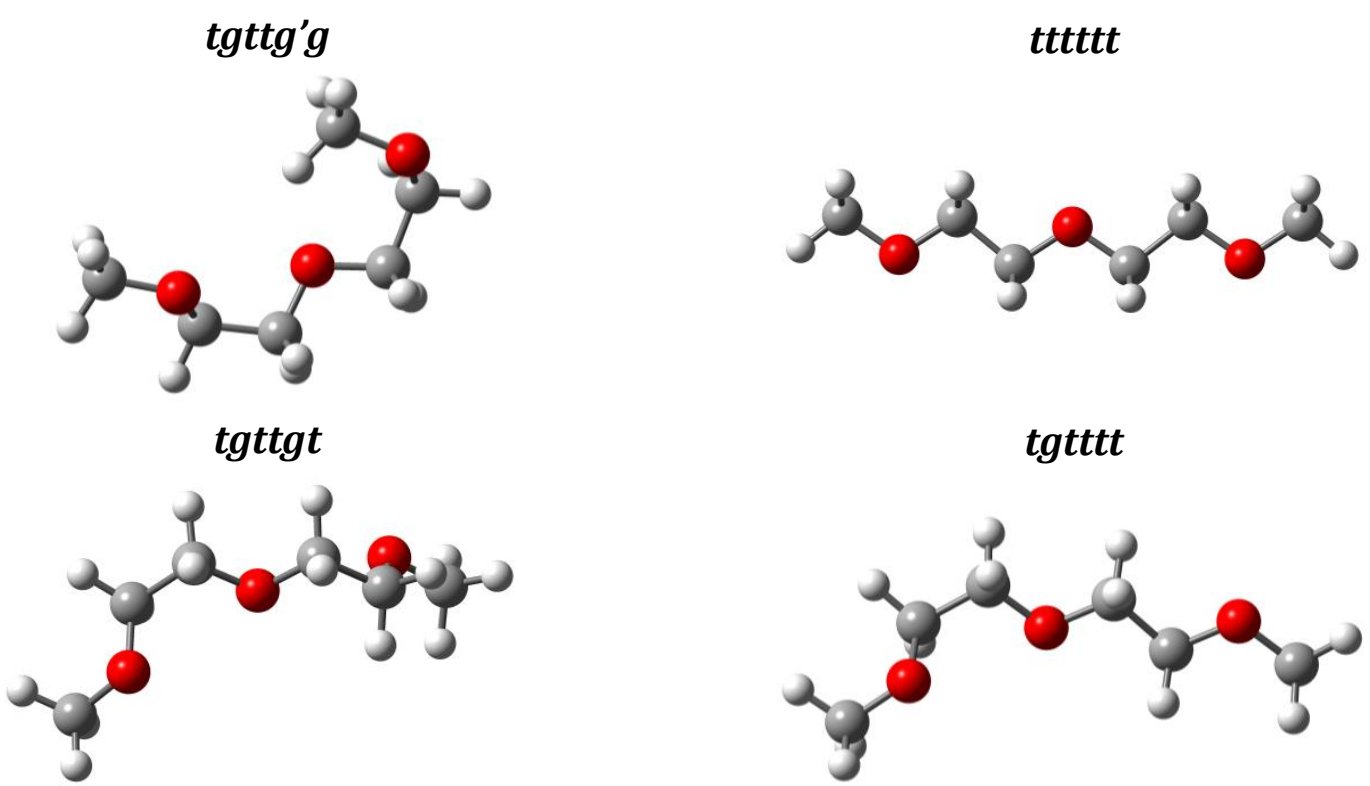

Figure 7.1: Geometries of the four lowest conformers in the diglyme conformational landscape.

All conformers that were found below a threshold of $5 \mathrm{~kJ} \cdot \mathrm{mol}^{-1}$ (23) were reoptimized and their frequencies were calculated at the B3LYP-2D3/aVTZ level of theory. This allowed narrowing down the conformers once more as now all conformers below a $4 \mathrm{~kJ} \cdot \mathrm{mol}^{-1}$ threshold (17) were chosen for further optimizations utilizing the B2PLYP or B3LYP functional along with the aVTZ or aVQZ basis sets and D3 disper- 
sion correction. Because of the high demand of resources of the B2PLYP method it could only be used with the smaller aVTZ basis set. Vibrational harmonic frequencies and Raman scattering cross sections were, for the same reason, calculated with the B3LYP functional. Thus, zero-point vibrational energies from B3LYP-2D3/aVQZ were added to the electronic energies of B2PLYP-2D3/aVTZ calculations, which will be abbreviated B2PLYP-2D3/aVT(Q)Z in this chapter. For all B3LYP results, zero-point energies were always added to the electronic energies at the appropriate level of theory. The results are shown in Table 7.1. Note that in order to enable better comparability conformers were always sorted based on the $\operatorname{CSSD}(\mathrm{T})$ results which are found in Table 7.2. As can be seen all methods agree reasonably well with each other and predict tgttg'g as the global minimum structure. The smaller def2TZVP basis set shows larger deviations for conformers with adjacent gauche kinks of different sign which is a clear indication of intramolecular BSSE. Still this basis set seems to be a good strategic method for narrowing down the search for the most stable structures. However, employing bigger basis sets is essential in order to work out the delicate energy differences between the best structures. Switching to the B2PLYP functional spreads out the energies of these conformers a bit more than using the B3LYP functional. This is likely an artefact of the more basis-set hungry B2PLYP method where larger basis sets exceeded the computation cluster resources.

Table 7.2 shows additional optimization and harmonic frequency calculations that have been carried out using TURBOMOLE 7.0. This allowed investigating differences in the implementation of the B3LYP functional between Gaussian 09 and TURBOMOLE 7.0. B3LYP-2D3/aVQZ calculations showed small differences ranging from 0.03 up to $0.10 \mathrm{~kJ} \cdot \mathrm{mol}^{-1}$ between both programs which is well within the expected accuracy of these types of calculations especially when it cannot be ensured that convergence criteria as well as integration grids are the same as both programs use their own independent subset of commands to adjust them. Therefore, differences were found to be negligible and are not explicitly shown in Table 7.2. Another benefit of using the TURBOMOLE software package is the implementation of the three-body AxilrodTeller-Muto dispersion term which can be actively calculated by adding "ABC" after the \$disp3 command in the control file of the input. When Axilrod-Teller-Muto dispersion terms are included and added to the D3 dispersion it will be indicated by the abbreviation 3D3. When regarding 3D3 corrections the all-trans structure drops in energy more than the others resulting in it being predicted as the global minimum. This leads to the assumption that the pairwise dispersion interactions, which are larger in folded structures, require correction in the dispersion corrected B3LYP methods.

Additionally, $\operatorname{CCSD}(\mathrm{T})$ and MP2 single point calculations were carried out in TURBOMOLE utilizing the RI-approximation. Here MP2/aVQZ energies were further enhanced with $\operatorname{CCSD}(\mathrm{T}) / \mathrm{aVTZ}$ calculations which will be abbreviated $\operatorname{CCSD}(\mathrm{T}) / \mathrm{aVTQZ}$ in this chapter. Converged structures and zero-point energies were used from the TURBOMOLE B3LYP-3D3/aVQZ calculations. Further calculations with the converged Gaussian 09 B2PLYP-2D3/aVTZ structures showed almost identical results with max- 
imal differences of $0.04 \mathrm{~kJ} \cdot \mathrm{mol}^{-1}$ illustrating that the converged structures are very similar. When going from the B3LYP functional to the MP2 level the energies of the primarily unfolded tttttt and tgtttt conformers drop compared to the more folded structures so that $t t t t t$ is predicted as the distinct global minimum. Raising the computational level to $\operatorname{CCSD}(\mathrm{T})$ further enhances this trend whereas $t t t t t t$ drops even more strongly in energy than tgtttt. A comparison of the computed energy differences for the 17 leading conformations at different levels of theory is shown in Figure 7.2. Relative zero-point vibrational energies obtained with the different B3LYP basis sets and their appropriate converged structures are shown Table 7.3. A maximum basis set deviation of \pm 0.37 and an average deviation of $\pm 0.13 \mathrm{~kJ} \cdot \mathrm{mol}^{-1}$ is found among the conformers. Thus, the stabilization trend of the ttttt conformer is unlikely to results from ZPVE differences. Especially since the relative ZPVE differences rather decreases with larger basis sets.

Table 7.1: Computed Gaussian 09 Rev. E.01 energy differences $\Delta E_{0}$ in $\mathrm{kJ} \cdot \mathrm{mol}^{-1}$ between the 17 leading conformations of diglyme. Note that aVT(Q)Z here means that the electronic structure calculations have been performed on a B2PLYP-2D3/aVTZ level whereas the zero-point energy correction was carried out using B3LYP2D3/aVQZ. For all other methods the zero-point energies were added to the electronic structure calculations at the appropriate level. Conformers were sorted based on CCSD(T) results which are found in Table 7.2.

\begin{tabular}{|c|c|c|c|c|c|}
\hline conformer & $\begin{array}{c}\text { statistical } \\
\text { weight }\end{array}$ & $\begin{array}{c}\text { B3LYP-2D3/ } \\
\text { def2TZVP }\end{array}$ & $\begin{array}{c}\text { B3LYP-2D3 / } \\
\text { aVTZ }\end{array}$ & $\begin{array}{c}\text { B3LYP-2D3/ } \\
\text { aVQZ }\end{array}$ & $\begin{array}{c}\text { B2PLYP-2D3/ } \\
\text { aVT(Q)Z }\end{array}$ \\
\hline$t t t t t t$ & $1 / 2$ & 0.00 & 0.00 & 0.00 & 0.00 \\
\hline $\operatorname{tgtttt}$ & 2 & 0.01 & -0.04 & -0.08 & 0.03 \\
\hline $\operatorname{tgttg}^{\prime} g$ & 2 & -0.83 & -0.24 & -0.17 & -0.34 \\
\hline $\operatorname{tgttg} t$ & 1 & 0.16 & 0.00 & -0.01 & 0.18 \\
\hline $\operatorname{tgg}^{\prime} g^{\prime} g t$ & 1 & 0.05 & 0.77 & 0.93 & 0.65 \\
\hline $\operatorname{tg} \operatorname{tg}^{\prime} g t$ & 2 & 0.18 & 0.40 & 0.44 & 0.56 \\
\hline$t t t t g g^{\prime}$ & 2 & 0.67 & 0.95 & 0.98 & 0.98 \\
\hline $\operatorname{tttgg} t^{\prime}$ & 2 & 0.83 & 1.21 & 1.23 & 1.25 \\
\hline $\operatorname{tgtgg}^{\prime} t$ & 2 & 0.68 & 0.96 & 1.03 & 1.09 \\
\hline $\operatorname{tg} \operatorname{ttg} g^{\prime}$ & 2 & 0.79 & 0.89 & 0.97 & 1.00 \\
\hline $\operatorname{tgttg}^{\prime} t$ & 2 & 1.99 & 1.87 & 1.85 & 2.17 \\
\hline $\operatorname{tgg}^{\prime} \operatorname{tg} g^{\prime}$ & 2 & 1.22 & 1.81 & 1.88 & 1.91 \\
\hline $\operatorname{tgg}^{\prime} \operatorname{tg}^{\prime} t$ & 2 & 1.81 & 2.90 & 3.12 & 2.14 \\
\hline $\operatorname{tg}^{\prime} g \operatorname{tgg}$ & 2 & 1.28 & 1.91 & 2.02 & 1.95 \\
\hline$g g^{\prime} t t g^{\prime} g$ & 1 & 1.60 & 2.03 & 2.16 & 1.95 \\
\hline$g g^{\prime} t t g g^{\prime}$ & 2 & 2.61 & 2.93 & 3.07 & 2.59 \\
\hline $\operatorname{ttg} g^{\prime} g t$ & 2 & 3.04 & 3.65 & 3.78 & 3.32 \\
\hline
\end{tabular}


Table 7.2: Computed TURBOMOLE 7.0 energy differences $\Delta E_{0}$ in $\mathrm{kJ} \cdot \mathrm{mol}^{-1}$ between the 17 leading conformations of diglyme. Note that aVTQZ here means that the MP2 energy has been calculated on a MP2/aVQZ level while the aVTZ basis was used for CCSD(T) calculations. Converged structures and zero-point energies from the B3LYP-3D3/aVQZ level were used for the MP2 and CCSD(T) calculations.

\begin{tabular}{|c|c|c|c|}
\hline conformer & $\begin{array}{c}\text { B3LYP-3D3/ } \\
\text { aVQZ }\end{array}$ & $\begin{array}{l}\text { MP2/ } \\
\text { aVQZ }\end{array}$ & $\begin{array}{c}\text { CCSD(T)/ } \\
\text { aVTQZ }\end{array}$ \\
\hline$t t t t t t$ & 0.00 & 0.00 & 0.00 \\
\hline $\operatorname{tgtttt}$ & 0.20 & 0.34 & 0.89 \\
\hline $\operatorname{tgttg}^{\prime} g$ & 0.21 & 0.58 & 1.38 \\
\hline $\operatorname{tgttg} t$ & 0.23 & 0.50 & 1.57 \\
\hline $\operatorname{tgg}^{\prime} g^{\prime} g t$ & 1.15 & 1.53 & 2.02 \\
\hline $\operatorname{tg} \operatorname{tg}^{\prime} g t$ & 0.59 & 1.39 & 2.05 \\
\hline ttttgg' & 1.31 & 1.95 & 2.10 \\
\hline $\operatorname{tttgg} t$ & 1.32 & 1.94 & 2.10 \\
\hline tgtgg't & 1.05 & 1.75 & 2.47 \\
\hline $\operatorname{tgttg} g^{\prime}$ & 1.22 & 1.91 & 2.60 \\
\hline $\operatorname{tgttg}^{\prime} t$ & 2.06 & 2.57 & 3.52 \\
\hline $\operatorname{tgg}^{\prime} \operatorname{tgg}^{\prime}$ & 2.17 & 3.45 & 3.70 \\
\hline $\operatorname{tgg}^{\prime} \operatorname{tg}^{\prime} t$ & 3.26 & 3.17 & 3.81 \\
\hline $\operatorname{tg}^{\prime} g \operatorname{tgg} g^{\prime}$ & 2.25 & 3.42 & 3.96 \\
\hline$g g^{\prime} t t g^{\prime} g$ & 2.28 & 3.36 & 4.12 \\
\hline$g g^{\prime} t \operatorname{tg} g^{\prime}$ & 3.08 & 4.15 & 4.37 \\
\hline $\operatorname{ttg} g^{\prime} g t$ & 4.24 & 4.85 & 4.97 \\
\hline
\end{tabular}

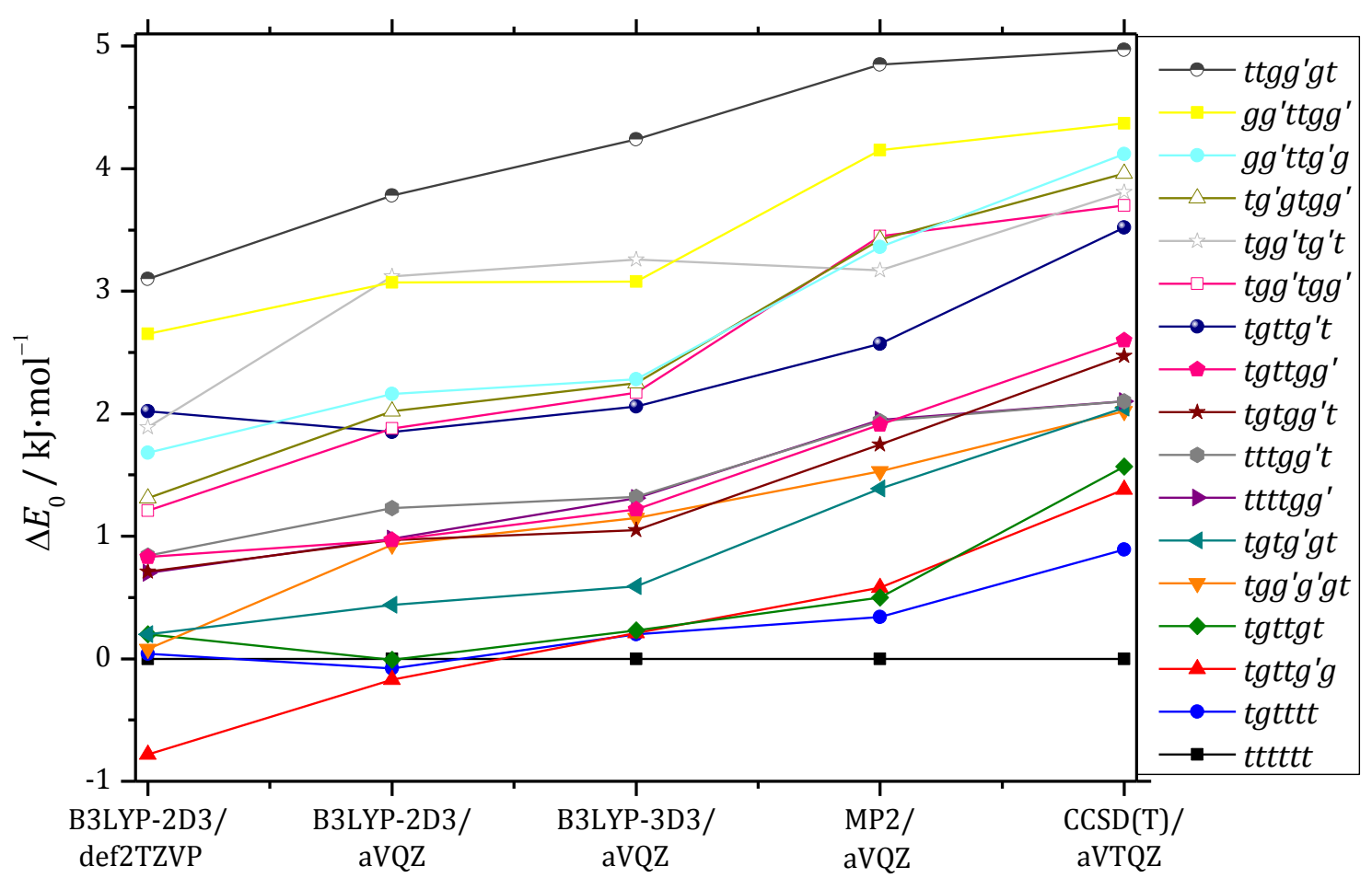

Figure 7.2: Comparison of computed energy differences $\Delta E_{0}$ for the 17 leading conformations of diglyme at different levels of theory. 
Table 7.3: Relative zero-point vibrational energies in $\mathrm{kJ} \cdot \mathrm{mol}^{-1}$ obtained with the converged structures at the appropriate level of theory between the 17 leading conformations in diglyme.

\begin{tabular}{|c|c|c|c|}
\hline conformer & $\begin{array}{l}\text { B3LYP-2D3/ } \\
\text { def2TZVP }\end{array}$ & $\begin{array}{c}\text { B3LYP-2D3/ } \\
\text { aVQZ }\end{array}$ & $\begin{array}{c}\text { B3LYP-3D3/ } \\
\text { aVQZ }\end{array}$ \\
\hline tttttt & 0.00 & 0.00 & 0.00 \\
\hline tgtttt & -0.19 & -0.25 & -0.02 \\
\hline $\operatorname{tgttg}^{\prime} g$ & 0.57 & 0.48 & 0.49 \\
\hline $\operatorname{tgttg} t$ & -0.30 & -0.40 & -0.23 \\
\hline $\operatorname{tg} g^{\prime} g^{\prime} g t$ & 1.20 & 1.06 & 0.99 \\
\hline $\operatorname{tg} \operatorname{tg} g t$ & 0.47 & 0.35 & 0.32 \\
\hline ttttgg' & 0.36 & 0.32 & 0.45 \\
\hline $\operatorname{tttg} g^{\prime} t$ & 0.56 & 0.47 & 0.35 \\
\hline $\operatorname{tgtgg}^{\prime} t$ & 0.57 & 0.47 & 0.24 \\
\hline $\operatorname{tgttg} g^{\prime}$ & 0.32 & 0.21 & 0.23 \\
\hline $\operatorname{tgttg}^{\prime} t$ & -0.35 & -0.43 & -0.33 \\
\hline $\operatorname{tgg}^{\prime} \operatorname{tgg}^{\prime}$ & 0.88 & 0.77 & 0.74 \\
\hline $\operatorname{tgg}^{\prime} \operatorname{tg}^{\prime} t$ & 1.52 & 1.32 & 0.95 \\
\hline $\operatorname{tg}^{\prime} g \operatorname{tg} g^{\prime}$ & 1.06 & 0.95 & 0.74 \\
\hline$g g^{\prime} t t g^{\prime} g$ & 1.17 & 1.04 & 0.66 \\
\hline$g g^{\prime} t t g g^{\prime}$ & 1.14 & 1.08 & 0.92 \\
\hline $\operatorname{ttg} g^{\prime} g t$ & 0.98 & 0.87 & 1.01 \\
\hline
\end{tabular}

In order to draw a comparison between the diglyme and monoglyme results it is beneficial to use the same reference point. The most reasonable reference point is the alltrans conformer, especially since it is predicted to be the global minimum structure in both cases according to the highest level of theory used in this work. Also, these types of structures are the least affected by interactions between the chain ends such as dispersion interactions so that it poses the fairest direct comparison between both molecules. Table 7.4 shows very similar stabilization of the all-trans conformer by increasing the computational method to the $\operatorname{CCSD}(\mathrm{T})$ level. Furthermore, this stabilization energy seems to be almost additive in a way that the combined stabilization of two monoglyme fragments yields the total stabilization of the resulting diglyme conformer. For example the tgt conformer is destabilized by $0.68 \mathrm{~kJ} \cdot \mathrm{mol}^{-1}$ relative to $t t t$. Therefore, two combined tgt fragments should give a total relative destabilization of $1.36 \mathrm{~kJ} \cdot \mathrm{mol}^{-1}$ and indeed a destabilization of $1.34 \mathrm{~kJ} \cdot \mathrm{mol}^{-1}$ was found in tgttgt. Note that the computational level of the zero-point vibrational energies is different in both cases. While frequencies of monoglyme conformers were obtained on a B2PLYP/aVTZ level they were only calculated on a B3LYP/aVQZ level in diglyme. 
Table 7.4: Comparison of energy differences $\Delta E_{0}$ in $\mathrm{kJ} \cdot \mathrm{mol}^{-1}$ obtained using B3LYP-3D3/aVQZ and $\operatorname{CCSD}(\mathrm{T}) / \mathrm{aVTQZ}$ (see Table 7.2) as well as the relative differences $\left(\Delta \Delta E_{0}\right)$ between these two levels in a) monoglyme and $b$ ) diglyme. Note that aVTQZ here means that the MP2/aVQZ single point energies were enhanced by $\operatorname{CCSD}(\mathrm{T}) / \mathrm{aVTZ}$ energies. Zero-point energies at the B3LYP-3D3/aVQZ level were added to $\operatorname{CCSD}(\mathrm{T})$ single point energies.

a)

Monoglyme

\begin{tabular}{|c|c|c|c|}
\hline conformer & $\begin{array}{c}\text { B3LYP-3D3/ } \\
\text { aVQZ }\end{array}$ & $\begin{array}{c}\text { CCSD(T)/ } \\
\text { aVTQZ }\end{array}$ & $\begin{array}{c}\Delta \text { (CCSD(T)- } \\
\text { B3LYP) }\end{array}$ \\
\hline$t t t$ & 0.00 & 0.00 & 0.00 \\
\hline$t g t$ & 0.49 & 1.17 & 0.68 \\
\hline$t g g^{\prime}$ & 1.48 & 2.15 & 0.67 \\
\hline
\end{tabular}

b)

\begin{tabular}{|c|c|c|c|}
\hline conformer & $\begin{array}{c}\text { B3LYP-3D3/ } \\
\text { aVQZ }\end{array}$ & $\begin{array}{c}\text { CCSD(T)/ } \\
\text { aVTQZ }\end{array}$ & $\begin{array}{c}\Delta \text { (CCSD(T)- } \\
\text { B3LYP) }\end{array}$ \\
\hline$t t t t t$ & 0.00 & 0.00 & 0.00 \\
\hline$t_{\text {tgtttt }}$ & 0.20 & 0.89 & 0.69 \\
\hline $\operatorname{tgttg}^{\prime} g$ & 0.21 & 1.38 & 1.17 \\
\hline tgttgt & 0.23 & 1.57 & 1.34 \\
\hline
\end{tabular}

\section{3 - Relaxation model}

As pointed out in the beginning of this chapter diglyme exhibits a very flexible backbone with six dihedrals, resulting in many stable conformations that are closely connected on certain trajectories along the potential energy surface. Potential energy scans of the rotation around different dihedrals shall be discussed in this chapter to reveal interconversion tendencies. For that purpose torsional scanning calculations have been performed on the B3LYP-2D3/def2TZVP level on Gaussian 09 Rev. D.01. As pointed out in Chapter 2.5, the Becke-Johnson damping bug in Rev. D.01 only affects frequency calculations. Since these torsional scans did not include vibrational calculations it was not necessary to redo them in Rev. E.01. Even though the important influence of large basis sets and high level theories on the delicate energy differences between the conformers was just illustrated, the demanding computational effort of these scans limited this approach to this rather small basis set. Moreover, these subtle energy differences do not play an important role in this case as error bars of a few $\mathrm{kJ} \cdot \mathrm{mol}^{-1}$ are acceptable to still end up with a qualitatively correct picture. The potential energy was typically scanned over 72 steps of $5^{\circ}$. The molecule turned out to be so flexible that all other backbone dihedrals, besides the one that was being scanned, had to be frozen. If the other dihedrals were not frozen the structure would tend to convert into a different one along the scanning trajectory resulting in a different structure and energy after a full $360^{\circ}$ turn. Locking out most dihedrals from optimizations raised the question which input structure to use for a scan, as this creates a bias which is illustrated in Figure 7.3. Due to that it is impossible to obtain the correct structures for all minima along a trajectory. However, because of only subtle changes in the remaining structure it is still possible to obtain a semi-quantitative and qualitative comparison between the barriers. Since these calculations were lacking zero96 
point vibrational energies it seemed to be a good compromise to use the structure with the lowest zero-point vibrational energy along the trajectory minima as an input to compensate for the missing ZPVE differences. All in all this approach only allowed determining barrier heights with an estimated uncertainty of $\pm 2 \mathrm{~kJ} \cdot \mathrm{mol}^{-1}$.

In principle the backbone dihedrals of diglyme can be broken down into three different categories:

1) Dihedrals containing a terminal methoxy group of the molecule - here called outer dihedrals

2) Dihedrals containing two oxygen atoms of the molecule - here called inner subunit dihedrals

3) Dihedrals containing a single oxygen atom and no terminal methoxy group - here called inner dihedrals

When taking a closer look at the actual barrier heights, as shown in Figure 7.4, it becomes clear that two of the three categories are essentially in the same energy range while the third one is much higher in energy. The same trend was observed in monoglyme (see Chapter 6.3) which is unsurprising since the diglyme molecule can be understood as two assembled monoglyme subunits. It further confirms the results of the ab initio study by Sutjianto and Curtiss [180]. The inner subunit dihedral contains two oxygen atoms and when rotating this dihedral these atoms get into an eclipsed conformation which is sterically and electronically unfavorable.

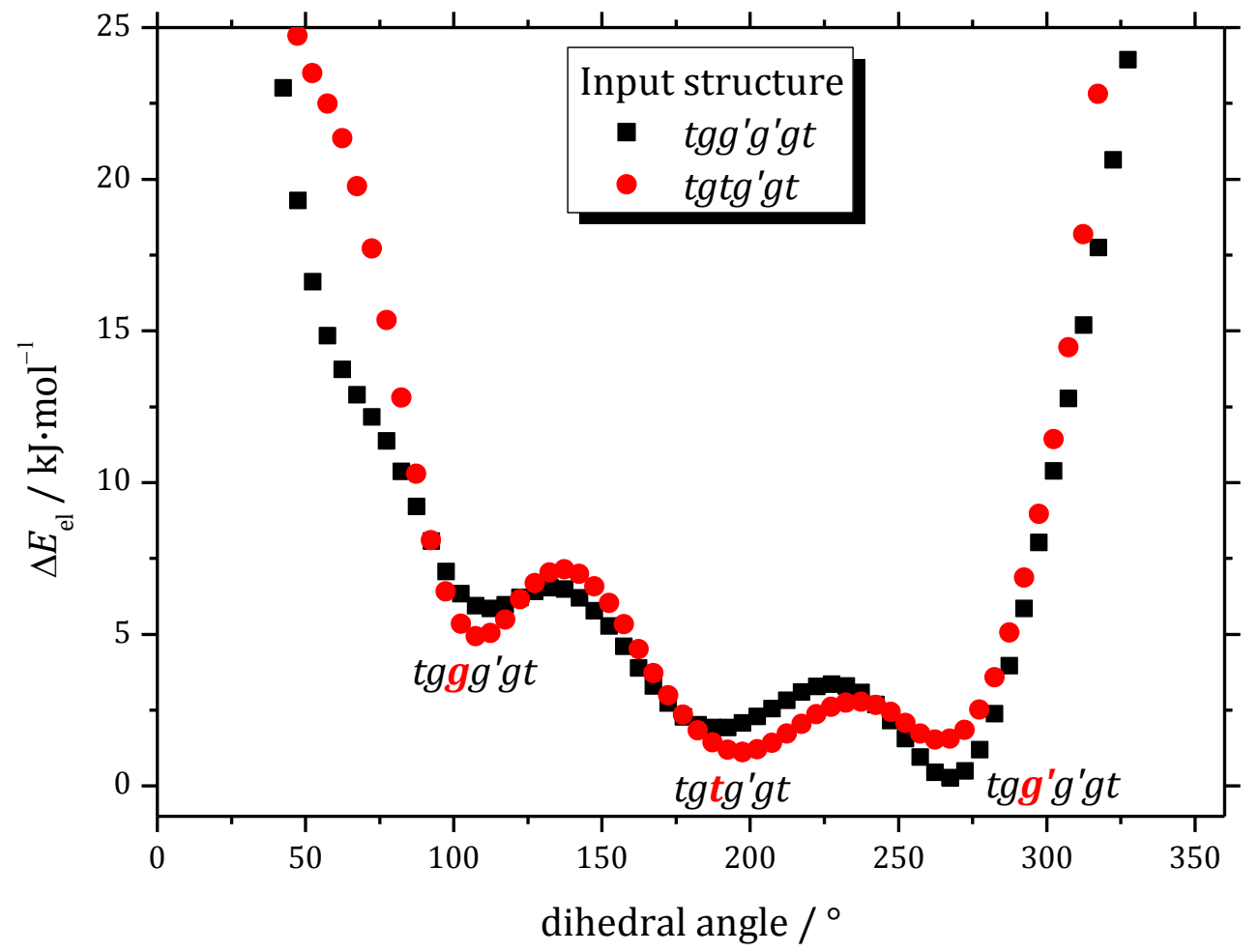

Figure 7.3: Torsional 1D-scans of the third backbone dihedral in diglyme with two different starting structures. 


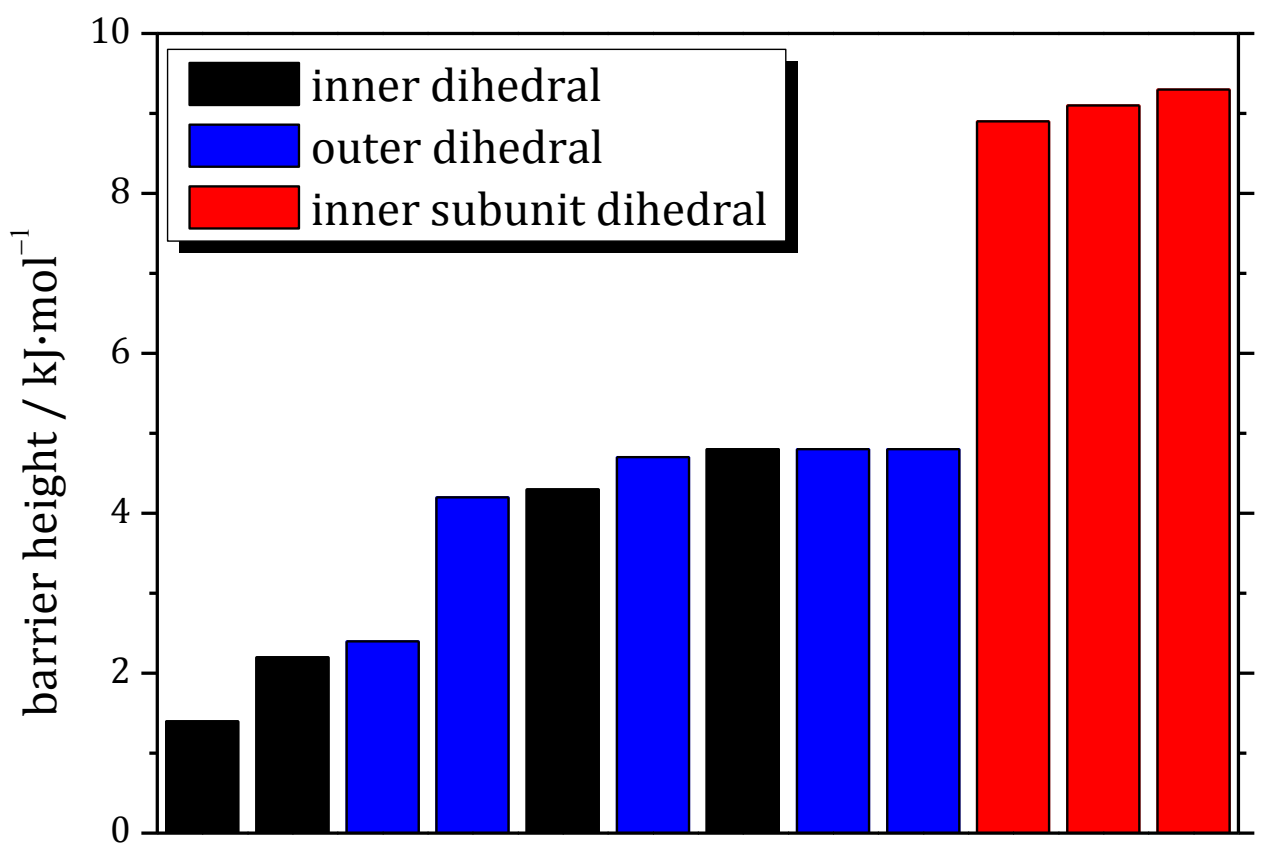

Figure 7.4: Barrier heights for the most probable interconversions between the ten most-stable conformers. Colors indicate the position of the changing dihedral.

Multiple pathways for the conversion between the ten most stable conformers were analyzed in order to reveal the most probable ones. Subsequently these paths shall be discussed.

\section{Interconversion between the four lowest structures}

The four lowest conformers in the energy ranking (compare Table 7.1) represent all different combinations of inner subunit dihedrals. As discussed before the barrier of interconversion of these dihedrals is relatively high, at around $9 \mathrm{~kJ} \cdot \mathrm{mol}^{-1}$ as shown in Figure 7.5 a) \& b), and is unlikely to be overcome in supersonic jet expansions $[6,19]$. Therefore, these structures embody well isolated families that are expected to coexist in jet expansions.

a)

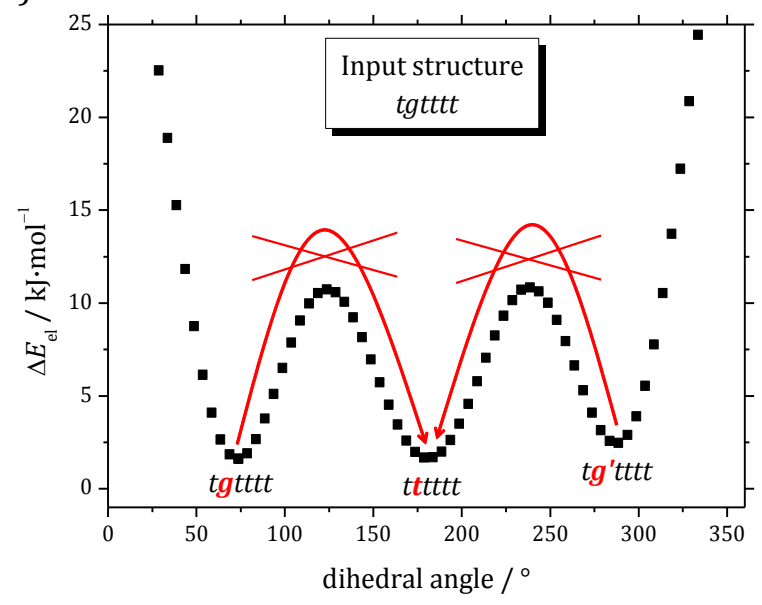

b)

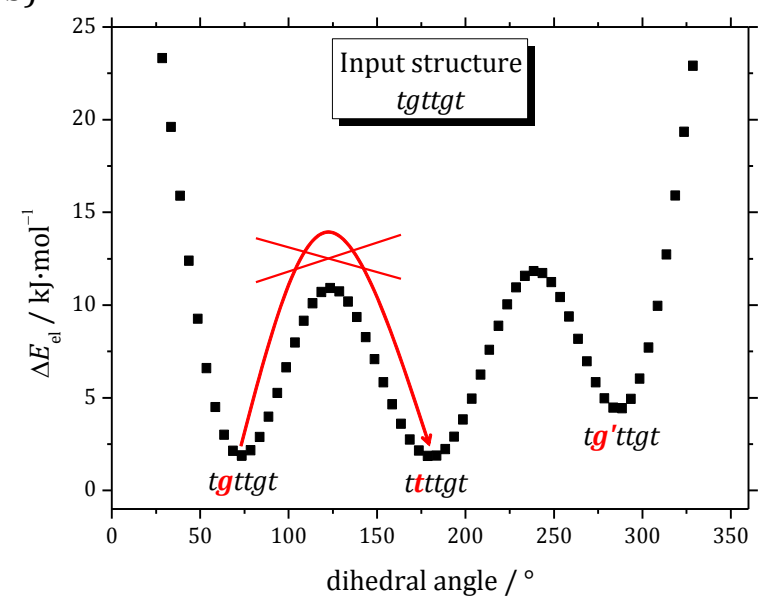

Figure 7.5: Torsional 1D-scans of the inner subunit dihedrals of a) tgttttt and b) tgttgt. 


\section{Relaxation of the two next higher structures into tgttgt}

By surpassing a $4.8 \mathrm{~kJ} \cdot \mathrm{mol}^{-1}$ barrier the sixth best structure $\mathrm{tg}^{\prime} g^{\prime} g t$ can relax into tgttgt (Figure 7.6a). These barrier heights can be accessible in supersonic jet expansions and were overcome in the monoglyme expansions. The minima were calculated to be nearly equal in energy leaving this conversion with a very small thermodynamic driving force. As mentioned above, these scans lack the consideration of zero-point vibrational energies, therefore minima spacing's are distorted in this case. Table 7.1 shows that while the thermodynamic driving force is larger than illustrated in Figure 7.6a it is still fairly small in general.

The fifth best structure $t g g^{\prime} g$ 'gt can relax very efficiently into $\operatorname{tg}^{\prime} g$ 'gt as these minima are only separated by a $1.4 \mathrm{~kJ} \cdot \mathrm{mol}^{-1}$ barrier (Figure $7.6 \mathrm{~b}$ ). This, in turn, relaxes to tgttgt (Figure 7.6a).

a)

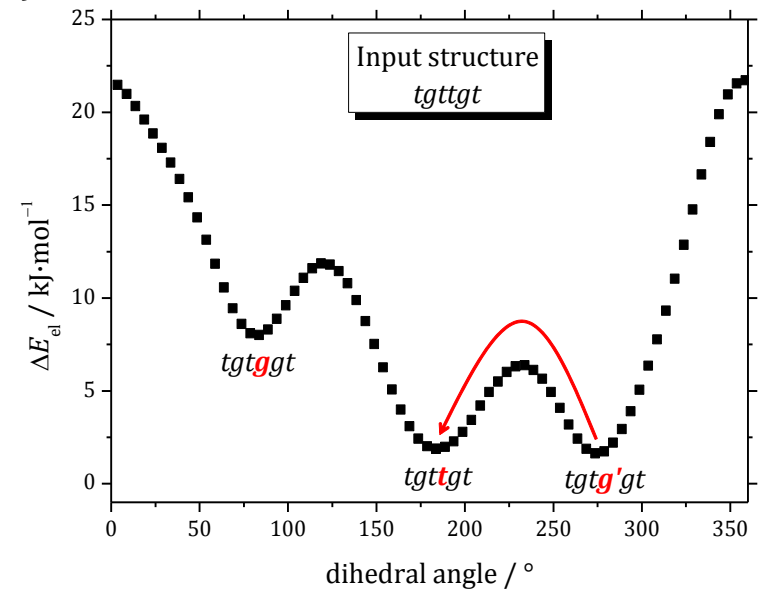

b)

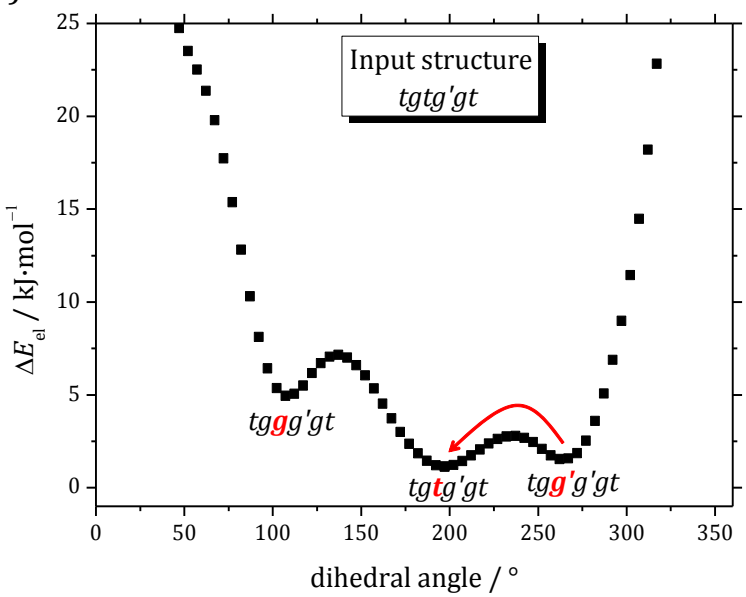

Figure 7.6: Torsional 1D-scans of the inner dihedral of a) tgttgt and b) $\operatorname{tgtg}^{\prime} g t$.

\section{Relaxation of the seventh \& eighth conformer into tgtttt}

The scan of the outer dihedral (Figure $7.7 \mathrm{a}$ ) shows a $4.8 \mathrm{~kJ} \cdot \mathrm{mol}^{-1}$ barrier between the seventh best structure ttttgg' and tttgt. The scan of the inner dihedral (Figure 7.7b) shows very similar potential energies. Here, the relaxation path between the eighth best conformer tttgg't and tttgt shows a $4.3 \mathrm{~kJ} \cdot \mathrm{mol}^{-1}$ barrier.

a)

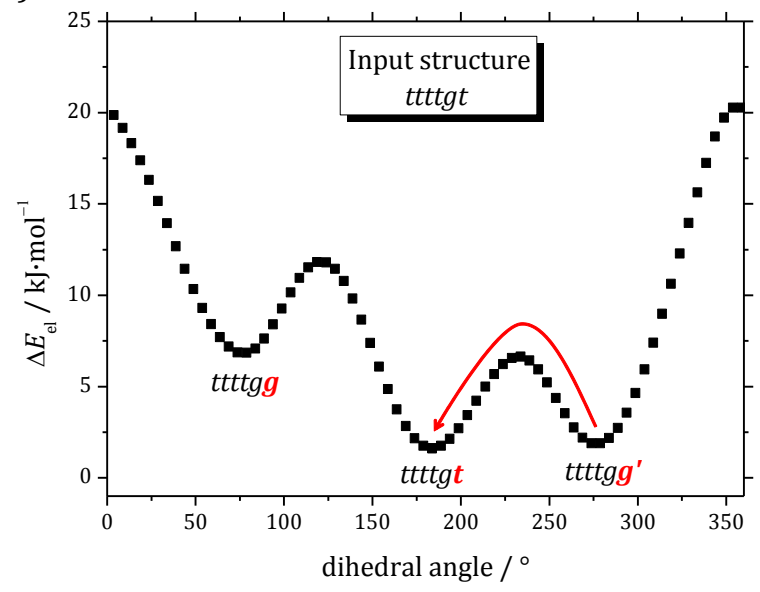

b)

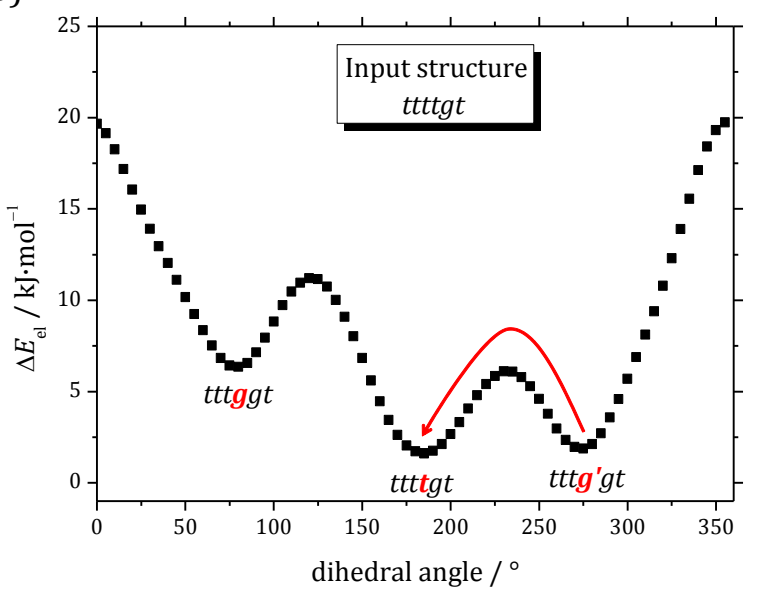

Figure 7.7: Torsional 1D-scan of the outer (a) and inner (b) dihedral of ttttgt. 


\section{Relaxation of the ninth conformer into $\operatorname{tgttg}^{\prime} g$}

The ninth conformer tgtgg't needs to rotate around the outer dihedral (Figure 7.8a) as well as the inner dihedral (Figure 7.8b) in order to relax into the $\operatorname{tgttg}^{\prime} g$ structure. The first dihedral change leads to the energetically unfavored $\operatorname{tgtgg}^{\prime} g$ which is an intermediate state as this undergoes another dihedral change to relax to the favored $\operatorname{tgttg}^{\prime} g$ conformer. Both rotations show very small barriers. The latter represents the only exception from the abovementioned input structure rule as the $\operatorname{tgttg}^{\prime} g$ conformer shows the lowest zero-point vibrational energy along this path. However, when calculating the torsion starting from this structure $\operatorname{tg}^{\prime} g g^{\prime} g$ is not found as a minimum at all. Even when the $\operatorname{tgtg}^{\prime} g^{\prime} g$ structure is used as a starting point this minimum is missing in the scan. Using the tgtgg'g structure as an input was the only possibility to get all three minima along this path as the residual dihedral changes are quite large because the structure is very bent in general. This illustrates the importance of dispersion forces in these folded structures as without dispersion interactions the residual structure would mainly stay unchanged. However, the absence of the tgtgg'g minimum when starting from the other two minimum structures indicates a small barrier. But as can be seen the $\operatorname{tgttg}^{\prime} g$ minimum is predicted way too high in energy as it should be the global minimum structure in these types of calculations (see Table 7.1).

a)

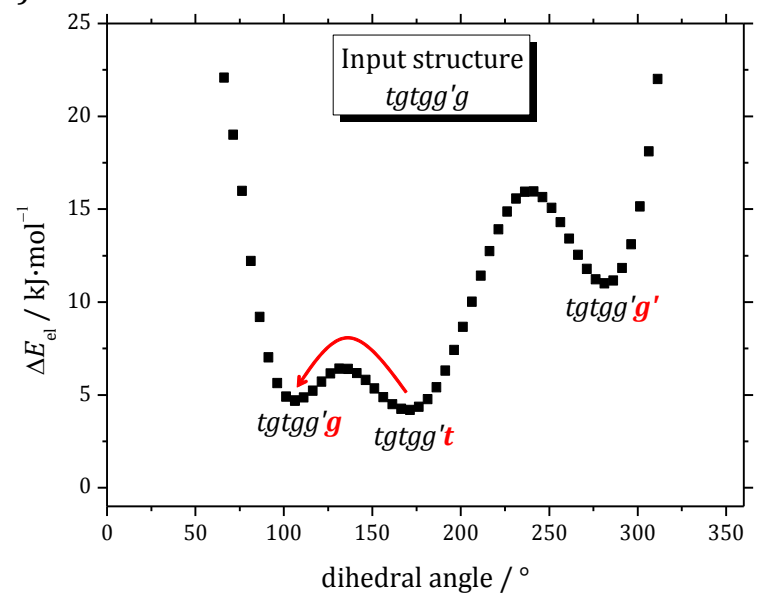

b)

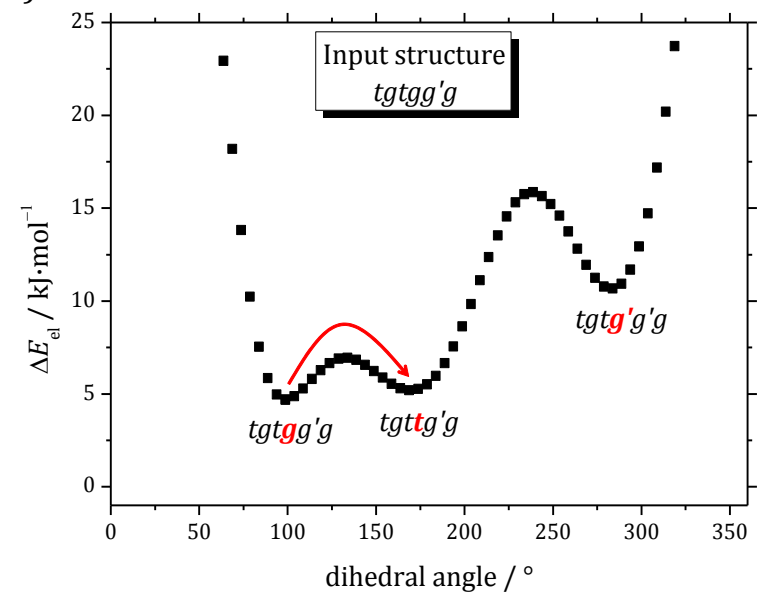

Figure 7.8: Torsional 1D-scans of the a) outer dihedral and b) inner dihedral of tgtgg'g. 


\section{Relaxation of the tenth conformer into tgttgt}

The relaxation of $\operatorname{tgttgg}$ ' into $\operatorname{tgttg} t$ shows a $4.7 \mathrm{~kJ} \cdot \mathrm{mol}^{-1}$ barrier (Figure 7.9 ) and is in line with other barrier heights found.

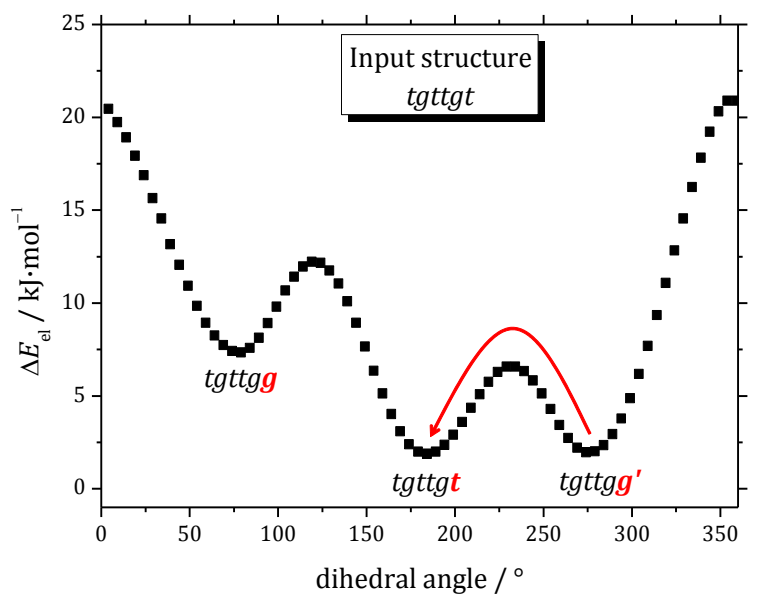

Figure 7.9: Torsional 1D-scan of the inner dihedral of ttttgt.

These calculations emphasize that every conformer can be grouped into a family based on its inner subunit dihedrals. The four family origins (tttttt, tgtttt, $\operatorname{tgttg}^{\prime} g$, tgttgt) act as very efficient capture basins for all other conformers undergoing supersonic jet expansions. Barriers along their paths to the origin are typically in the range of $\sim 5 \mathrm{~kJ} \cdot \mathrm{mol}^{-1}$. When a conformer is already strongly bent in multiple consecutive dihedrals then this stress seems to lower the barriers to around $2 \mathrm{~kJ} \cdot \mathrm{mol}^{-1}$. An energy level diagram, based on energy differences obtained at the $\operatorname{CCSD}(\mathrm{T}) / \mathrm{aVTQZ}$ level, where the ten most-stable conformers are grouped into their appropriate families is shown in Figure 7.10. This illustrates that not all family origin conformers gain equally from relaxation processes. As can be seen ttttt does not gain any population from the next nine conformations whereas the other low energy conformers profit from relaxations. In fact the next best structure in the tttttt family is gtttt with a predicted CCSD(T)/aVTQZ energy difference of $5.6 \mathrm{~kJ} \cdot \mathrm{mol}^{-1}$ including zero-point corrections at the B3LYP-3D3/aVQZ level of theory.

The total relaxation process during supersonic jet expansions can be modeled and understood in three steps. In the first step the population of every conformer is estimated at an initial temperature $T_{\mathrm{i}}$ of $298 \mathrm{~K}$, which corresponds to the nozzle temperature for most of the experiments. For this first step two different approaches seem reasonable. In one, the full theoretical standard Gibbs energies $\Delta G^{0}$ within the RRHO approximation are used to calculate equilibrium compositions. The other approach neglects differences in vibrational and rotational partition functions, so that populations are calculated only based on zero-point corrected energy differences $\Delta E_{0}$, symmetry number $\sigma$ and enantiomeric degeneracy $g$ as shown in the following equation.

$$
\Delta G^{\mathrm{C}}=E_{\mathrm{El}}+\mathrm{ZPVE}+k T \cdot \ln \sigma-k T \cdot \ln g
$$

These configurational contributions are expected to dominate over vibrational and rotational corrections and the corresponding Gibbs energies are denoted as $\Delta G^{\mathrm{C}}$. Even though this approach is more simplistic it is also more robust as $\Delta G^{0}$ values are highly 
dependent on the low frequency motions, whose absolute values can easily deviate between different quantum chemical methods and are anyway problematic in the harmonic approximation. The second step includes a full hypothetical relaxation of all conversions barriers below $5 \mathrm{~kJ} \cdot \mathrm{mol}^{-1}$, so that only the four family origins are now populated. In a third and final step, conformers within each family are repopulated with an effective temperature $T_{\mathrm{r}}$ of $100 \mathrm{~K}$. In essence, this relaxation model contains two conformational freezing temperatures $-298 \mathrm{~K}$ for the dihedrals containing two 0 atoms and $100 \mathrm{~K}$ for the dihedrals containing one $\mathrm{O}$ atom in the central bond.

Gibbs free energies were calculated in Gaussian 09 Rev. E.01 on the B3LYP-2D3/aVQZ and in TURBOMOLE 7.0 on the B3LYP-3D3/aVQZ level of theory. The comparison between these two methods will give an impression of the relative error of the $\Delta G^{0}$ model as each program implemented the DFT functional slightly different and the 3D3 method includes the addition of three-body dispersion. Zero-point and thermal corrections from the $\Delta G^{\mathrm{C}}$ and $\Delta G^{0}$ models were then added to electronic energies at the $\operatorname{CCSD}(\mathrm{T}) / \mathrm{aVTQZ}$ level calculated with converged structures from B3LYP3D3/aVQZ (Table 7.2). Resulting Gibbs energies at 298 and $100 \mathrm{~K}$ are shown in Table 7.5. The three step relaxation process is shown in Figure 7.11a for the $\Delta G^{\mathrm{C}}$ model. Relaxed populations based on the $\Delta G^{0}$ model calculated at the B3LYP-3D3/aVQZ level are shown in Figure 7.11b while those calculated at the B3LYP-2D3/aVQZ level are shown in Figure 7.11c. As can be seen, the differences between the two B3LYP methods result in average population deviations of around 1-2\% with a maximum difference in the tgttt conformer population of 7.1\%. The comparison of either $\Delta G^{0}$ model to the $\Delta G^{\mathrm{C}}$ model show similar population differences with smaller deviations when comparing the $\Delta G^{\mathrm{C}}$ to the $\Delta G^{0}$ model based on the B3LYP-3D3/aVQZ calculations. This analysis verifies that the main contributions are indeed captured in the $\Delta G^{\mathrm{C}}$ model and that this robust approach leads to reasonable results. Furthermore, this approach will be the only reasonable one for longer glymes as Gibbs energy calculations with large basis sets will not be feasible for much larger molecules. When switching to smaller basis sets the relative error in full theoretical Gibbs energies raises drastically as the description of low frequency motions is rather poor with these basis sets.

As will be seen in the following subchapter, this analysis helps evaluating the spectra of diglyme significantly. Conversely, the spectra will be used to assess how realistic the relaxation model and its assumptions are. For clarity reasons spectra will only be compared to the $\Delta G^{\mathrm{C}}$ and the $\Delta G^{0}$ model based on the B3LYP-3D3/aVQZ calculations. Both $\Delta G^{0}$ models lead to very similar qualitative trends and are basically indistinguishable considering the error bars of this analysis. Thus, from here on the $\Delta G^{0}$ model based on the B3LYP-3D3/aVQZ calculations will only be referred to as the $\Delta G^{0}$ model. 


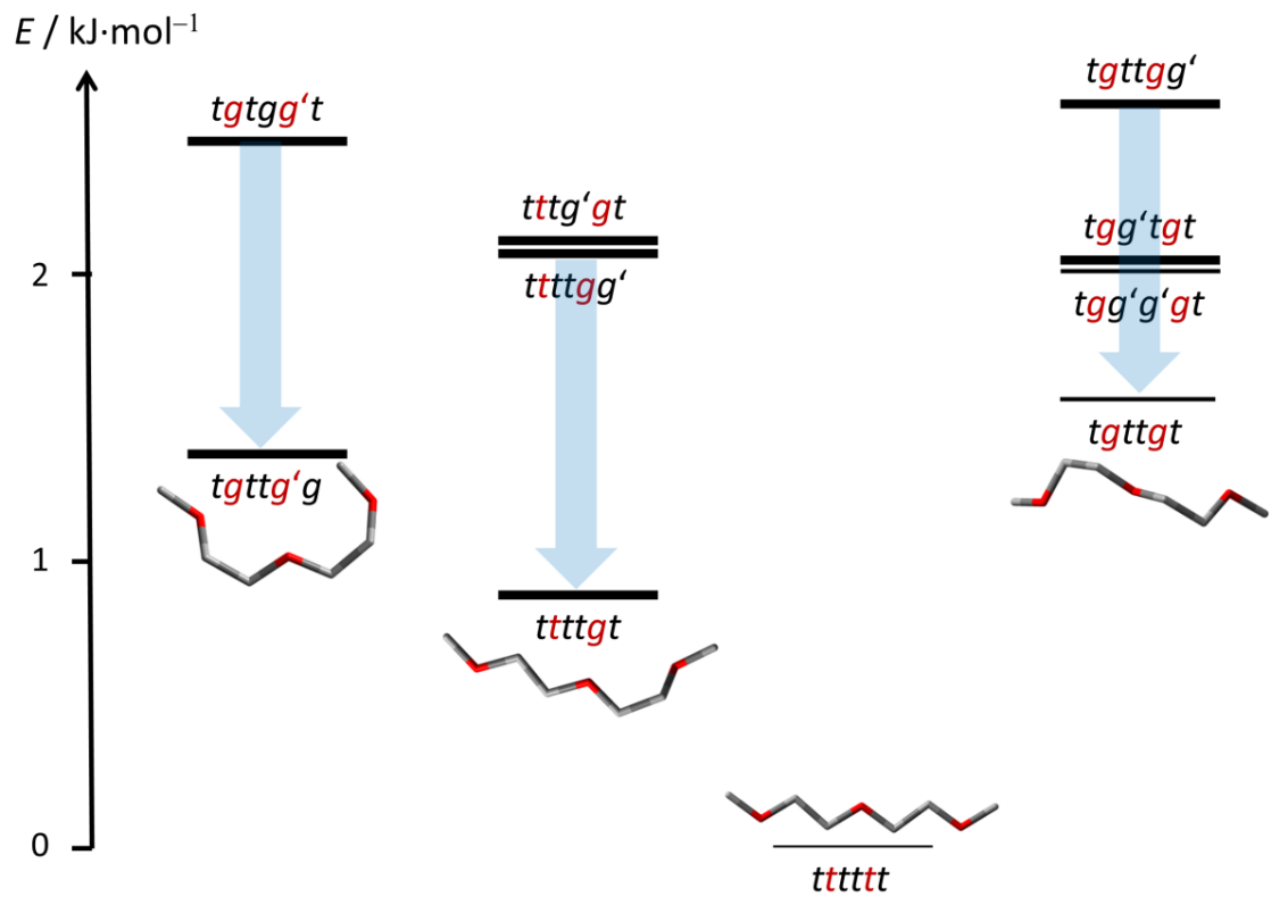

Figure 7.10: Energy level diagram for the ten most-stable diglyme conformers grouped into their respective inner subunit dihedral (marked in red) families. The thickness of each energy level displays the statistical weight of the given conformer. Energy differences are based on CCSD(T)/aVTQZ calculations (Table 7.2).

Table 7.5: Full theoretical relative Gibbs energies $\Delta G^{0}$ and configurational Gibbs energies $\Delta G^{\mathrm{C}}$ at 100 and $298 \mathrm{~K}$ for the 17 leading conformers of diglyme. The full theoretical thermal corrections including zero-point energies to the Gibbs free energies were calculated in Gaussian 09 Rev. E.01 at the B3LYP2D3/aVQZ and in TURBOMOLE 7.0 at the B3LYP-3D3/aVQZ level of theory. Thermal corrections from the $\Delta G^{0}$ and $\Delta G^{\mathrm{C}}$ model were added to electronic energies at the $\operatorname{CCSD}(\mathrm{T}) / \mathrm{aVTQZ}$ level calculated with converged structures from B3LYP-3D3/aVQZ (Table 7.2). Zero-point corrections at the B3LYP$3 \mathrm{D} 3 / \mathrm{aVQZ}$ level were included in the $\Delta G^{\mathrm{C}}$ values.

\begin{tabular}{|c|c|c|c|c|c|c|}
\hline \multicolumn{3}{|c|}{ 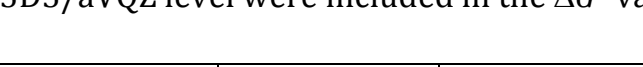 } & \multicolumn{2}{|c|}{ B3LYP-3D3/aVQZ } & \multicolumn{2}{|c|}{ B3LYP-2D3/aVQZ } \\
\hline conformer & $\begin{array}{c}\Delta G^{\mathrm{C}} \\
100 \mathrm{~K}\end{array}$ & $\begin{array}{c}\Delta G^{\mathrm{C}} \\
298 \mathrm{~K}\end{array}$ & $\begin{array}{c}\Delta G^{0} \\
100 \mathrm{~K}\end{array}$ & $\begin{array}{c}\Delta G^{0} \\
298 \mathrm{~K}\end{array}$ & $\begin{array}{c}\Delta G^{0} \\
100 \mathrm{~K}\end{array}$ & $\begin{array}{c}\Delta G^{0} \\
298 \mathrm{~K}\end{array}$ \\
\hline$t t t t t$ & 0.00 & 0.00 & 0.00 & 0.00 & 0.00 & 0.00 \\
\hline $\operatorname{tg} t t t t$ & -0.26 & -2.54 & -0.35 & -2.20 & -0.74 & -3.12 \\
\hline $\operatorname{tgttg}^{\prime} g$ & 0.23 & -2.05 & 0.08 & -1.30 & -0.19 & -1.94 \\
\hline $\operatorname{tgttg} t$ & 0.99 & -0.15 & 0.85 & 0.49 & 0.57 & -0.18 \\
\hline $\operatorname{tgg} g^{\prime} g t$ & 1.45 & 0.31 & 1.56 & 2.17 & 1.56 & 2.05 \\
\hline $\operatorname{tg} \operatorname{tg} g t$ & 0.89 & -1.39 & 0.58 & -1.17 & 0.75 & -0.70 \\
\hline ttttgg' & 0.95 & -1.34 & 0.83 & -1.11 & 0.60 & -1.67 \\
\hline tttgg't & 0.95 & -1.33 & 0.61 & -1.92 & 0.81 & -1.34 \\
\hline $\operatorname{tg} \operatorname{tg} g^{\prime} t$ & 1.31 & -0.97 & 0.73 & -1.55 & 1.26 & -0.30 \\
\hline $\operatorname{tg} t \operatorname{tgg}$ & 1.45 & -0.83 & 1.22 & -0.55 & 1.17 & -0.72 \\
\hline $\operatorname{tg}^{\prime} t g^{\prime} t$ & 2.45 & 0.16 & 2.28 & 0.73 & 2.12 & 0.38 \\
\hline $\operatorname{tgg}^{\prime} \operatorname{tg} g^{\prime}$ & 2.52 & 0.24 & 2.15 & 0.10 & 2.03 & 0.02 \\
\hline $\operatorname{tgg}^{\prime} \operatorname{tg}^{\prime} t$ & 2.66 & 0.38 & 3.07 & 3.29 & 3.62 & 4.50 \\
\hline $\operatorname{tg}^{\prime} g \operatorname{tgg} g^{\prime}$ & 2.59 & 0.31 & 1.98 & -0.44 & 2.42 & 0.57 \\
\hline$g g^{\prime} t t g^{\prime} g$ & 3.10 & 1.96 & 2.77 & 1.78 & 3.41 & 3.15 \\
\hline$g g^{\prime} \operatorname{ttg} g^{\prime}$ & 3.21 & 0.93 & 3.21 & 2.12 & 3.49 & 2.73 \\
\hline$t \operatorname{tg} g^{\prime} g t$ & 3.82 & 1.54 & 3.93 & 3.05 & 3.69 & 2.51 \\
\hline
\end{tabular}


a)

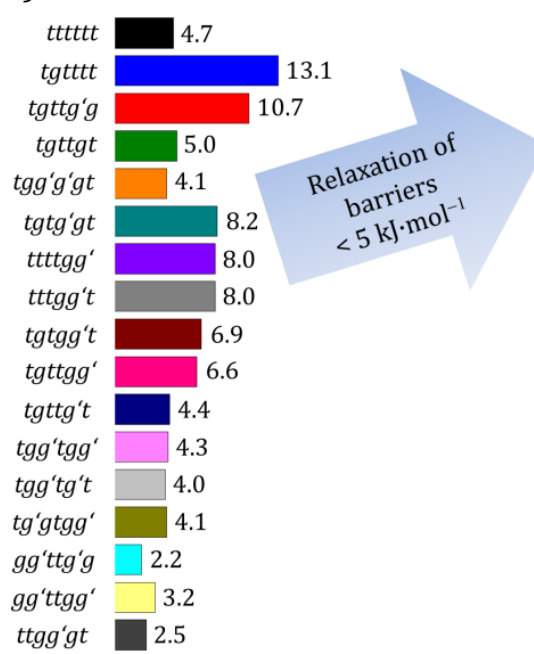

\section{$\Delta G^{\mathrm{C}}$ model}

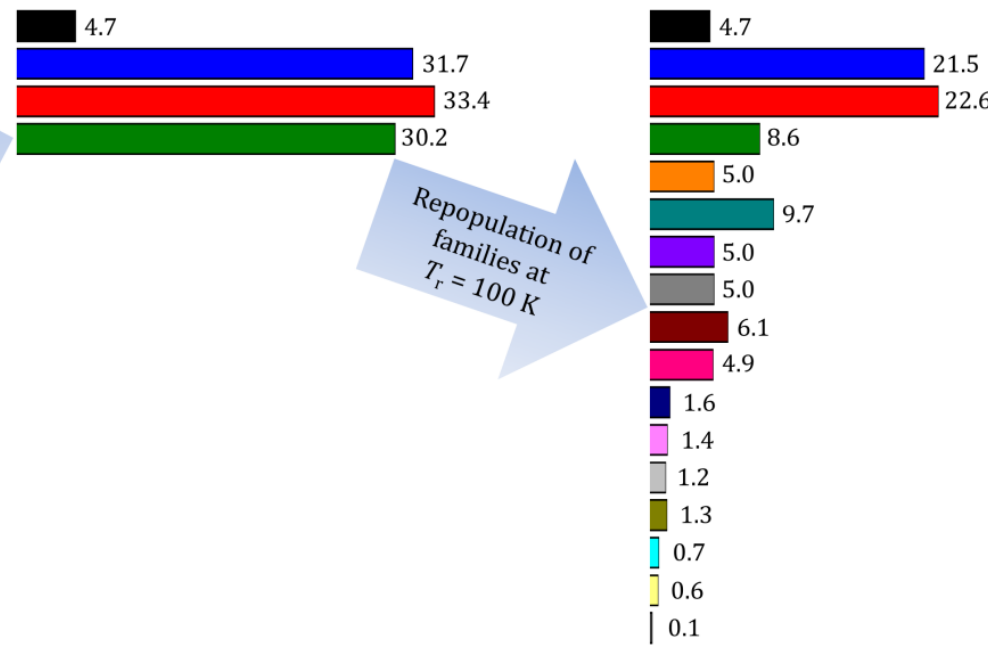

b)

$\Delta G^{0}$ model - B3LYP-3D3/aVQZ
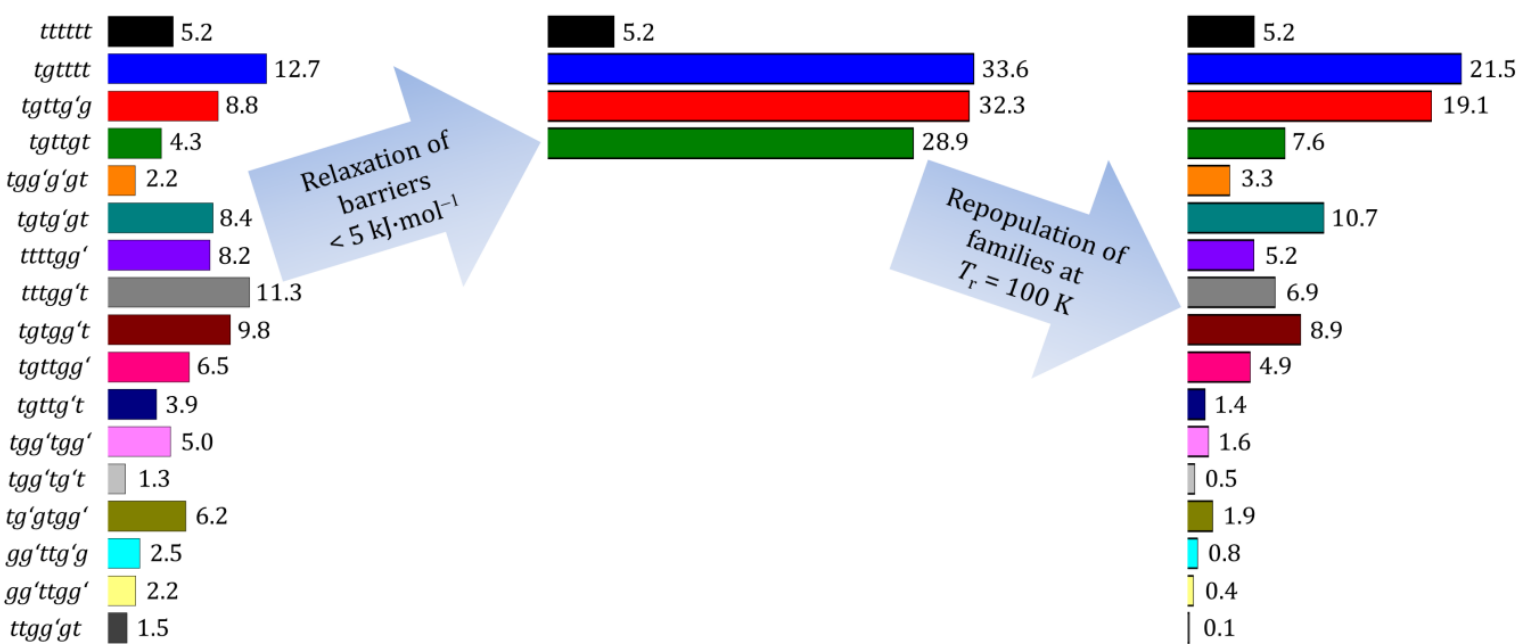

c)

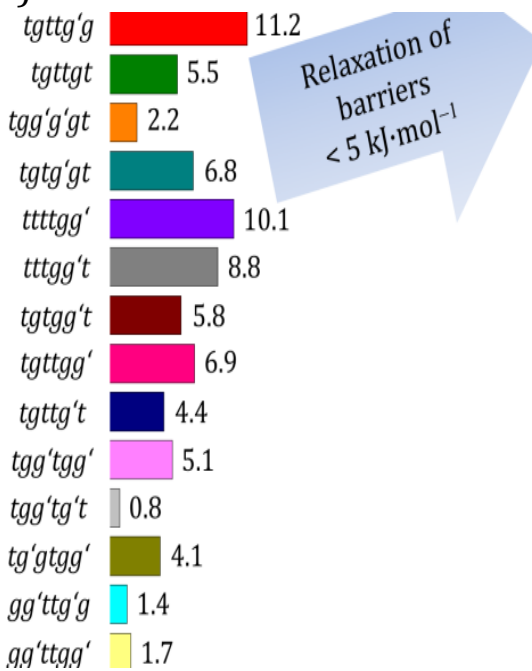

$\Delta G^{0}$ model - B3LYP-2D3/aVQZ
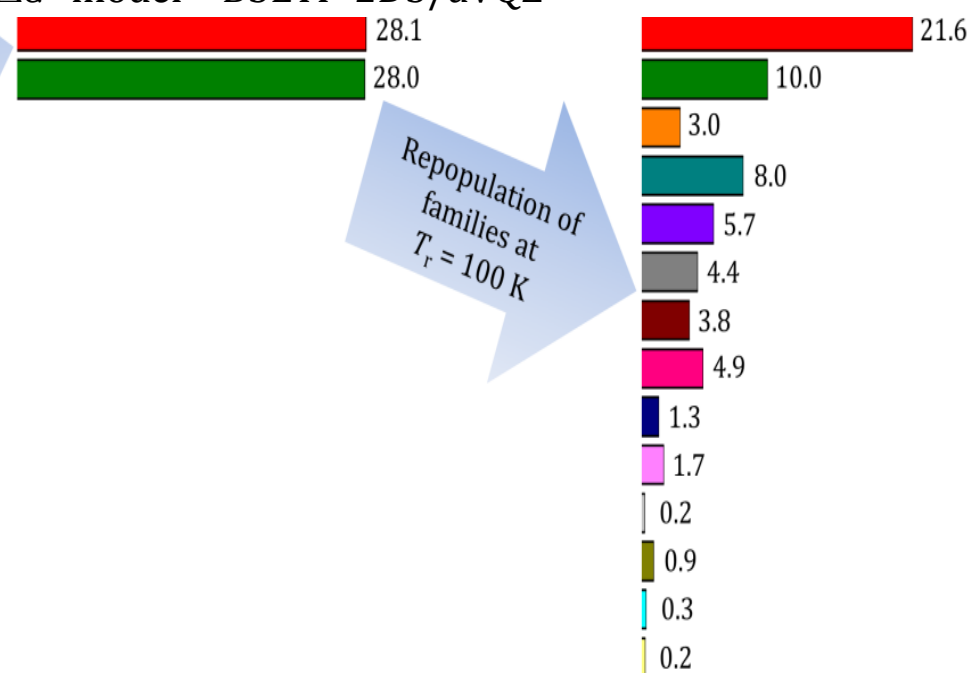

Figure 7.11: Relaxed populations of the 17 most-stable conformers of diglyme during the three steps of the jet relaxation model. Calculated populations in a) are based on configurational relative Gibbs energies $\Delta G^{\mathrm{C}}$. Populations based on full theoretical Gibbs energies $\Delta G^{0}$ at the B3LYP-3D3/aVQZ level are shown in b) while those at the B3LYP-2D3/aVQZ level are shown in c). The used relative Gibbs energies are found in Table 7.5. 


\section{4 - Raman jet spectra}

Figure 7.12 shows the survey Raman spectrum of diglyme which can be divided into three spectral regions. The low frequency region (up to $\sim 600 \mathrm{~cm}^{-1}$ ) which contains mostly skeletal vibrations such as the longitudinal acoustic modes (LAM), followed by the CC/CO stretching region $\left(\sim 750-1500 \mathrm{~cm}^{-1}\right)$ and the intense $\mathrm{CH}$ stretching region $\left(\sim 2750-3000 \mathrm{~cm}^{-1}\right)$. The low frequency region is very helpful because the skeletal vibrations are usually very sensitive to the conformational alignment of the molecule, typically resulting in larger shifts between similar types of vibrations of different conformers than in other spectral regions. In the latter two regions bands overlap heavily and make discrete assignments impossible in the absence of conformational resolution [80], so this work focusses on the examination of the low frequency region in diglyme. Raman scattering cross sections predicted by quantum chemical calculations aid in the assignment of the spectrum.

Figure 7.13 shows a comparison of the experimental diglyme spectrum and the calculated Raman scattering cross sections at the B3LYP-3D3/aVQZ level of the ten lowest conformers weighted with their respective statistical weights (Table 7.1). In general, all signals assigned to one of the four family origins are clearly visible in the spectrum. The other conformers are also present but definitely less visible than predicted by their weighted cross sections. Additional conformers could only be partially assigned to very weak signals, suggesting they are almost not present in the expansion. All strong signals in the low frequency region are assigned to LAM-type motions. Remarkably tgtttt is the only conformer with two strong signals in that region which is a result of the LAM mixing with another vibration. Since the two bands are not resolved in the spectra and mode mixing ratios are likely miscalculated by harmonic calculations these two bands can be rather seen as one large band. In all following spectra the Raman scattering cross section of the weaker of these two is therefore added to the stronger band. Furthermore the band at $274 \mathrm{~cm}^{-1}$, next to the all-trans LAM, sticks out as the only strong band without a reasonable assignment by all ten conformers. Anharmonic calculations on the B3LYP-2D3/def2TZVP level show a possible Fermi resonance between the all-trans fundamental LAM motion and an overtone of one of the transversal acoustic modes (TAM). This gives a reasonable explanation for this strong band as well as the small shift to lower wavenumbers of the experimental LAM position compared to its theoretical prediction.

Although the majority of the bands in the experimental spectrum can be understood and assigned, certain overestimations of band strengths of the higher energy conformers are especially visible in the frequency range from $400-600 \mathrm{~cm}^{-1}$. This is not surprising as the weighting of these scattering cross sections with only the respective statistical weights of each conformer neglects any energy and non-configurational entropy differences between them. A more realistic prediction can be made by following the three steps of the derived relaxation model (see Chapter 7.3). The effects of each step on the predicted Raman scattering cross sections shall be briefly discussed and compared to the spectrum for the $\Delta G^{0}$ model calculated at the B3LYP-3D3/aVQZ 
level of theory. The corresponding plots for the $\Delta G^{\mathrm{C}}$ approach can be found in the A.6 as their influence on the spectra are very similar which is also demonstrated in the population changes in Figure 7.11. The final result of each approach is then compared to the spectrum and discussed.

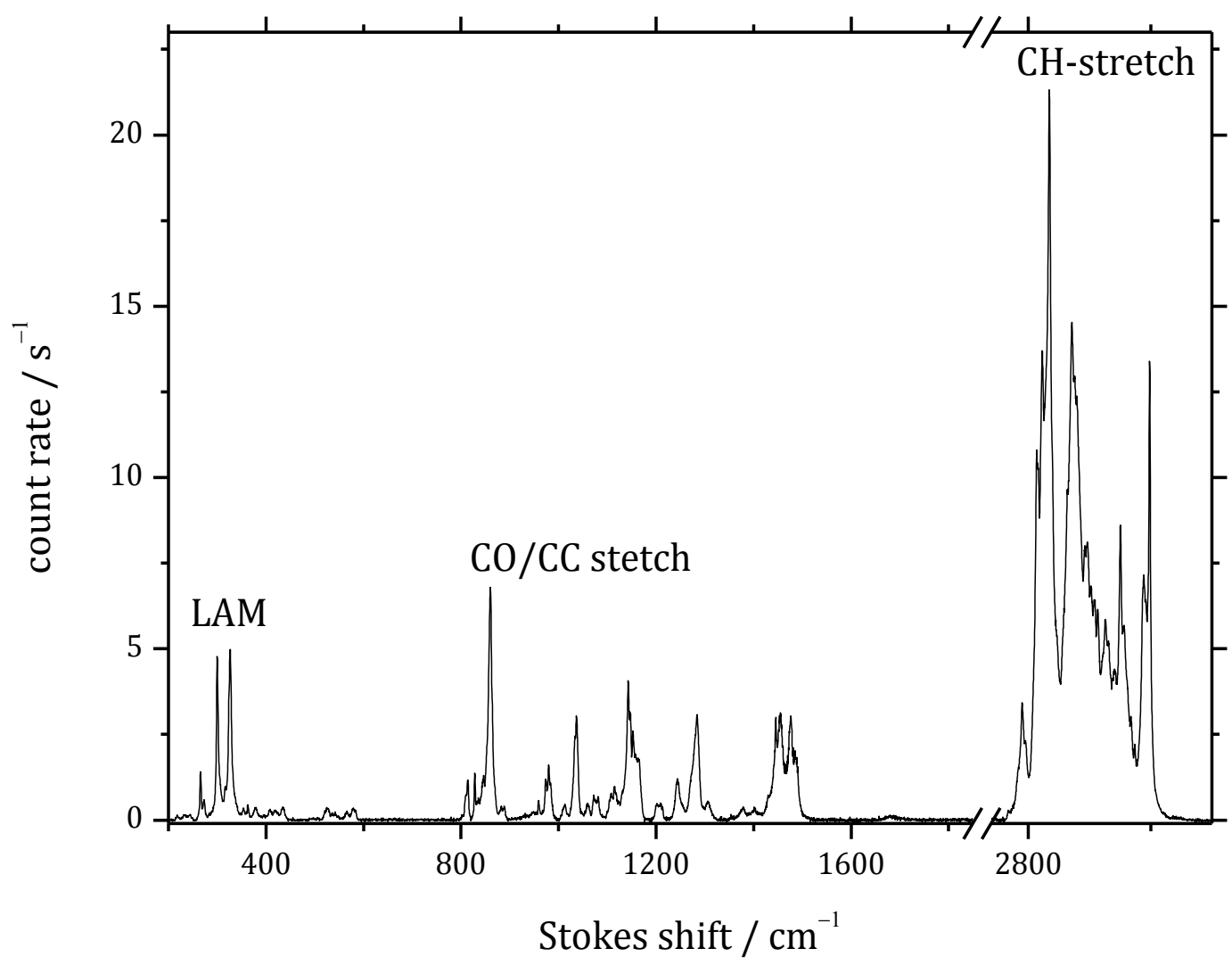

Figure 7.12: Survey Raman jet spectrum of diglyme. Measurement conditions can be found in A.1. 


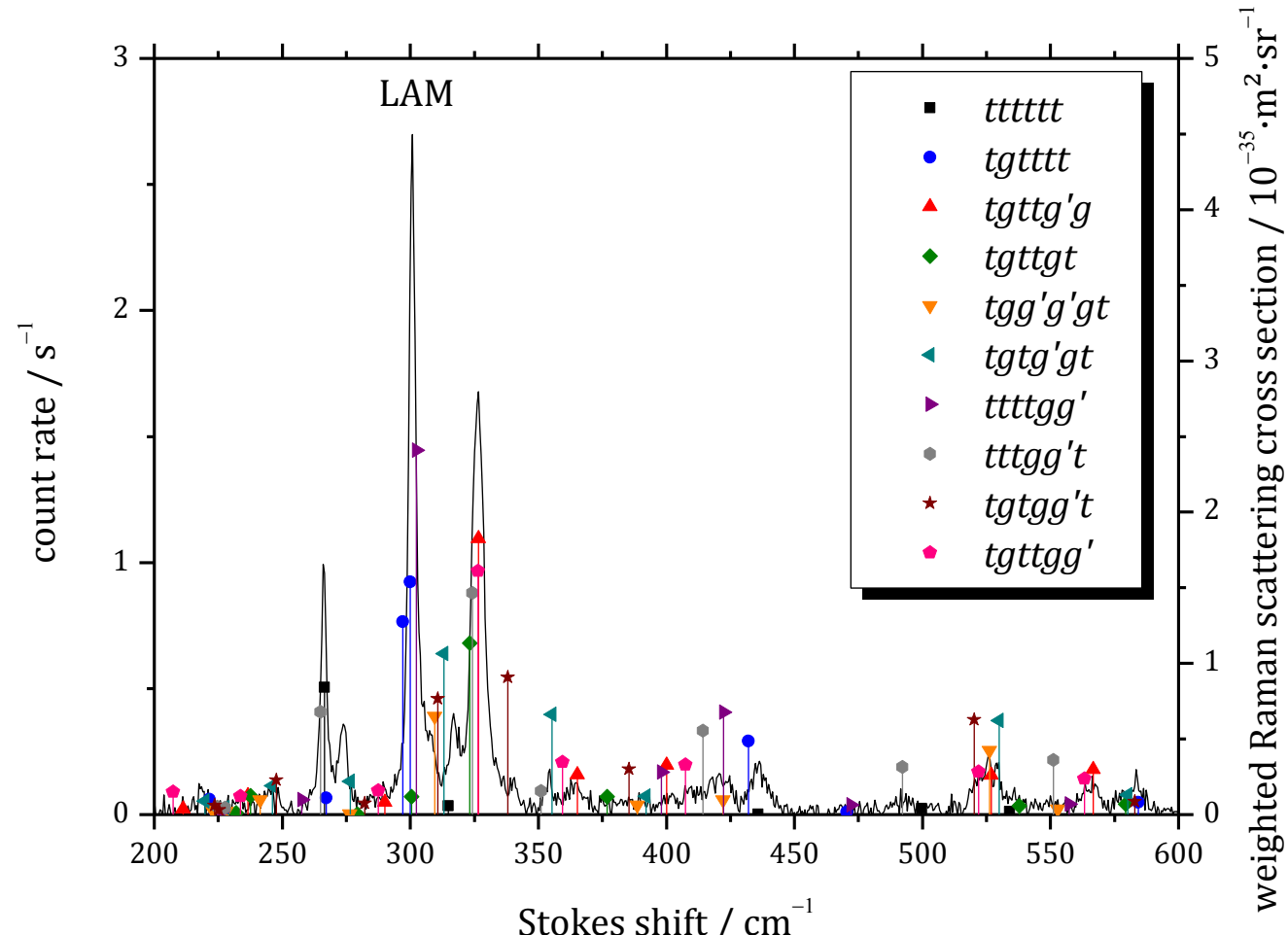

Figure 7.13: Low frequency jet spectrum of diglyme (measurement conditions can be found in A.1) compared to Raman scattering cross sections calculated at the B3LYP-3D3/aVQZ level and weighted with their respective statistical weights (see Table 7.1).

Figure 7.14 shows the effect on the Raman scattering cross sections for the first step of the relaxation model, where they were weighted with the relative abundances based on $\Delta G^{0}$ values at $298 \mathrm{~K}$ (Table 7.5). As can be seen the theoretical predictions of higher energy conformers in this frequency range now fit a bit better to the spectrum yet still remain somewhat over predicted. Additionally, the tgtttt LAM at $300 \mathrm{~cm}^{-1}$ and the $\operatorname{tgttg}^{\prime} g$ LAM at $326 \mathrm{~cm}^{-1}$ are predicted too weak. This supports the assumption of further relaxations between the conformers as shown in the aforementioned subchapter. Figure 7.15 shows a comparison of the same spectrum to the calculated Raman scattering cross sections weighted with their relative abundances after a relaxation of all barriers below $5 \mathrm{~kJ} \cdot \mathrm{mol}^{-1}$ as shown in the second step of Figure $7.11 \mathrm{~b}$. It becomes obvious that this weighting is predicting most tgttg'g and tgttgt bands too strong, while those assigned to the ttttt conformer are predicted too weak. Furthermore, many weaker bands are not explained with this simulation which clearly shows that the relaxation will only be partially completed. The third step of the relaxation model, where a repopulation within each conformer family at a temperature $T_{\mathrm{r}}$ of $100 \mathrm{~K}$ is estimated, is shown in Figure 7.16 and as can be seen it indeed seems to be the most realistic prediction of all presented simulations for the experimental spectrum.

In order to quantitatively verify how well these simulations fit to the experimental spectrum the three strong LAM bands at 266, 300 and $326 \mathrm{~cm}^{-1}$ as well as the band at $526 \mathrm{~cm}^{-1}$ were fitted with Gaussian-Lorentzian cross product functions and their relative integrals were compared to the relative sum of the Raman scattering cross sec- 
tions of the underlying conformers of each band ${ }^{\dagger}$. Final populations and sums of Raman scattering cross sections were calculated at 298, 150, 100 and $0 \mathrm{~K}$. These bands were chosen for verification because the strong LAM bands are assigned to the family origin conformers while the band at $526 \mathrm{~cm}^{-1}$ shows major contributions from various higher energy conformers. The results are shown in Table 7.6. As expected, the relative contribution of the Raman scattering cross section sum at $526 \mathrm{~cm}^{-1}$ increases with rising $T_{\mathrm{r}}$. The same holds true for the cross section sum of the tttttt LAM at $266 \mathrm{~cm}^{-1}$ while the cross sections sums of the other two LAM bands decrease with increasing temperature. This analysis fully supports the qualitative comparison between the spectrum and the simulations that was just drawn. It illustrates once again that the higher energy conformers are over predicted at $298 \mathrm{~K}$ and predicted far too

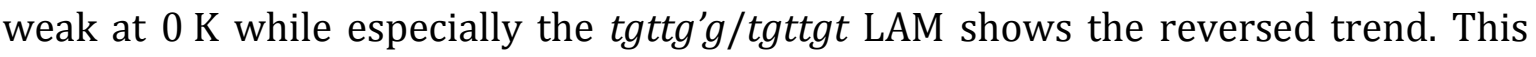
analysis does not allow judging whether 150 or $100 \mathrm{~K}$ is a more realistic repopulation temperature as both temperatures allow for a good fit of the spectrum and show rather similar deviations from the experimental bands. However, since this evaluation has been performed with a helium jet spectrum the lower repopulation temperature seems more reasonable as addition of heavier noble gas atoms to the carrier gas, as will be discussed later in this chapter, will enhance cooling effects during the supersonic jet expansions. Furthermore, this evaluation allowed determining scaling factors for the ordinates of the plots shown in Figure 7.14-17. The ratio between the integral of the four experimental bands to the total Raman scattering cross section sum of these bands was kept constant so that the plotted comparison is not additionally distorted through changes in the relative ordinate scaling.

A more direct simulation of the spectrum is achieved by representing the weighted Raman scattering cross sections with Gaussian functions of the following form:

$$
f_{\text {Gaussian }}(\tilde{v})=\sigma_{\mathrm{v}}^{\prime} \cdot \exp \left(-\frac{\ln 16 \cdot\left(\tilde{v}-\tilde{v}_{\mathrm{v}}\right)^{2}}{\mathrm{FWHM}^{2}}\right)
$$

Here, $\sigma_{\mathrm{v}}^{\prime}$ is the weighted Raman scattering cross section and $\tilde{v}_{\mathrm{v}}$ the wavenumber of a specific vibration. The factor $\ln (16)$ ensures that FWHM corresponds to the full width at half maximum of the simulated band. A comparison between the spectrum and simulations of the 17 lowest diglyme conformers using a FWHM of $4 \mathrm{~cm}^{-1}$ for all bands and weighted Raman scattering cross sections at repopulation temperatures of 298,0 and $100 \mathrm{~K}$, representing the three steps of the relaxation model, respectively, is shown in Figure 7.18. Thus, Figure 7.18 offers a different visual representation of the simulated spectral changes at the three repopulation temperatures used in the relaxation model. Therefore, it follows the same arguments that were just described when the spectrum was compared to the weighted scattering cross sections at these temperatures.

Even though the outlined relaxation model is based on a series of rigorous assumptions and some tunable parameters, as for example the temperature for the initial populations as well as the temperature for the repopulation within each conformer

${ }^{\dagger}$ Assignments of the conformers to the used bands are found in A.7. 
family, it still gives a reasonable and realistic interpretation of the experimental data. The two different approaches $\left(\Delta G^{\mathrm{C}}\right.$ and $\left.\Delta G^{0}\right)$ are shown in Figure 7.16-17, respectively. Through the comparison to the experiment it cannot be determined which approach gives the most realistic results as their differences are too small. In general it can be concluded that the outlined relaxation model leads to realistic simulations of the experimental spectrum and that both approaches work comparably well here. For longer chain molecules the $\Delta G^{0}$ approach is likely to fail due to high inaccuracies in the low frequency motions as was shown for $n$-alkanes by Nils Lüttschwager [19].

Note that the change in relative intensities between Figure 7.12 and Figure 7.13-18 is due to a change in the saturator temperature and stagnation pressure. The influences on the spectra and possible formations of clusters will be discussed Chapter 7.5.

Table 7.6: Comparison between relative integrals of the Lorentzian-Gaussian cross product functions of the experimental strong LAM bands as well as the band at $526 \mathrm{~cm}^{-1}$ and the relative sum of the Raman scattering cross sections $\left(\sigma^{\prime}\right)$ of all underlying conformers of each band (assignments are found in A.7). Relative populations at the indicated temperatures were calculated based on the $\Delta G^{0}$ model in a) and on the $\Delta G^{\mathrm{C}}$ model in b).

a)

\begin{tabular}{|c|c|c|c|c|c|}
\hline $\begin{array}{l}\tilde{v}_{\exp } \\
/ \mathrm{cm}^{-1}\end{array}$ & $\int_{\exp }^{\mathrm{rel}}$ band $/ \%$ & $\sum_{298 \mathrm{~K}}^{\mathrm{rel}} \sigma^{\prime} / \%$ & $\sum_{150 \mathrm{~K}}^{\mathrm{rel}} \sigma^{\prime} / \%$ & $\sum_{100 \mathrm{~K}}^{\mathrm{rel}} \sigma^{\prime} / \%$ & $\sum_{0 \mathrm{~K}}^{\mathrm{rel}} \sigma^{\prime} / \%$ \\
\hline 266 & 13.2 & 16.7 & 14.6 & 12.7 & 8.3 \\
\hline 300 & 42.8 & 34.9 & 37.1 & 38.6 & 39.6 \\
\hline 326 & 33.6 & 34.5 & 34.6 & 36.7 & 48.6 \\
\hline 526 & 10.4 & 13.9 & 13.7 & 12.0 & 3.5 \\
\hline
\end{tabular}

b) $\Delta G^{\mathrm{C}}$ model

\begin{tabular}{|c|c|c|c|c|c|}
\hline $\begin{array}{l}\tilde{v}_{\exp } \\
/ \mathrm{cm}^{-1}\end{array}$ & $\int_{\exp }^{\mathrm{rel}}$ band $/ \%$ & $\sum_{298 \mathrm{~K}}^{\mathrm{rel}} \sigma^{\prime} / \%$ & $\sum_{150 \mathrm{~K}}^{\mathrm{rel}} \sigma^{\prime} / \%$ & $\sum_{100 \mathrm{~K}}^{\mathrm{rel}} \sigma^{\prime} / \%$ & $\sum_{0 \mathrm{~K}}^{\mathrm{rel}} \sigma^{\prime} / \%$ \\
\hline 266 & 13.2 & 14.5 & 12.4 & 11.0 & 7.6 \\
\hline 300 & 42.8 & 35.7 & 37.2 & 37.9 & 37.9 \\
\hline 326 & 33.6 & 34.8 & 37.0 & 39.1 & 50.9 \\
\hline 526 & 10.4 & 15.0 & 13.4 & 12.0 & 3.6 \\
\hline
\end{tabular}




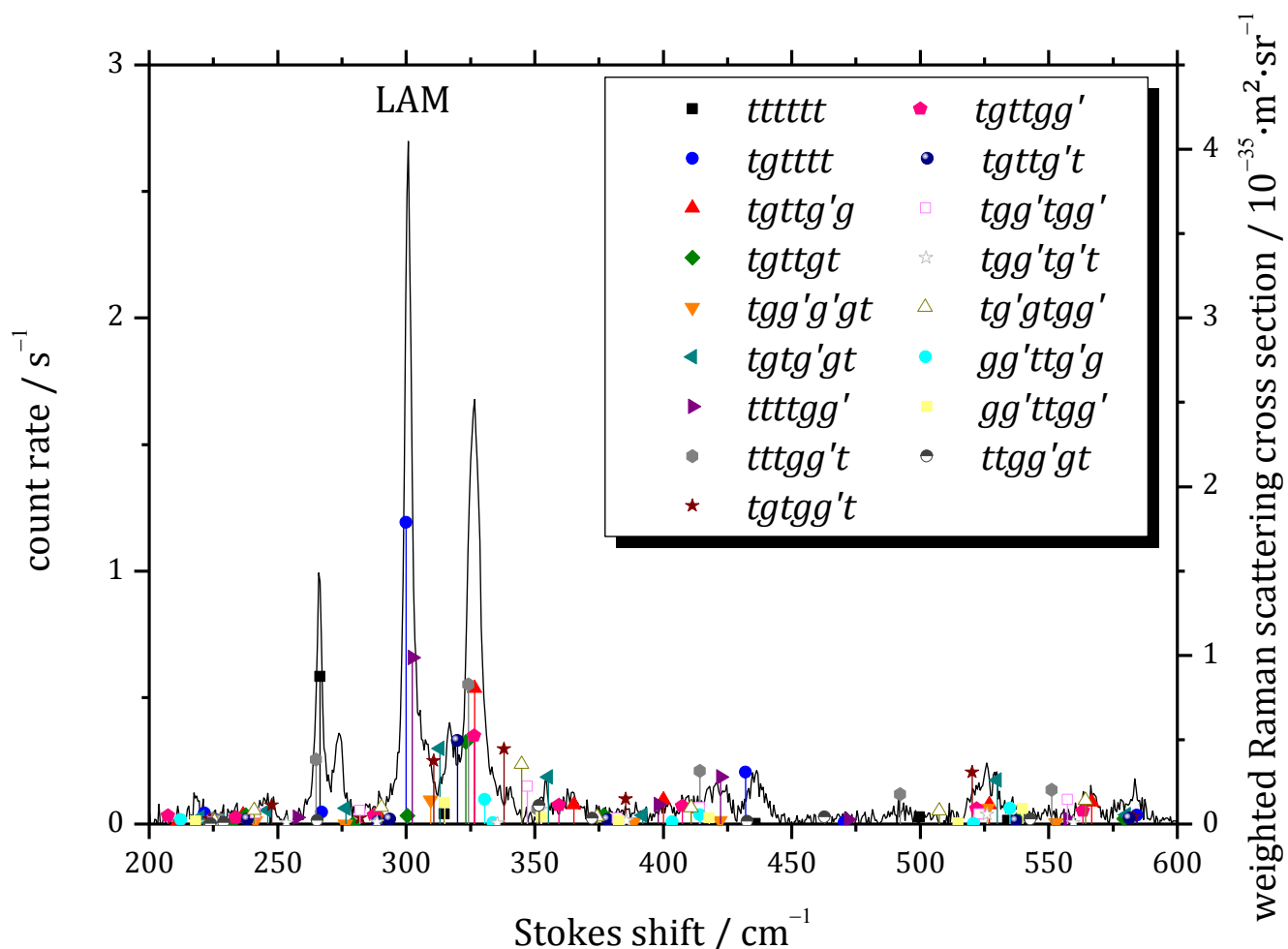

Figure 7.14: Low frequency jet spectrum of diglyme (measurement conditions can be found in A.1) compared to Raman scattering cross sections calculated at the B3LYP-3D3/aVQZ level and weighted with their relative abundances based on the $\Delta G^{0}$ values at $298 \mathrm{~K}$ (Table 7.5). This comparison mimics the first step of the relaxation process shown in Figure 7.11b.

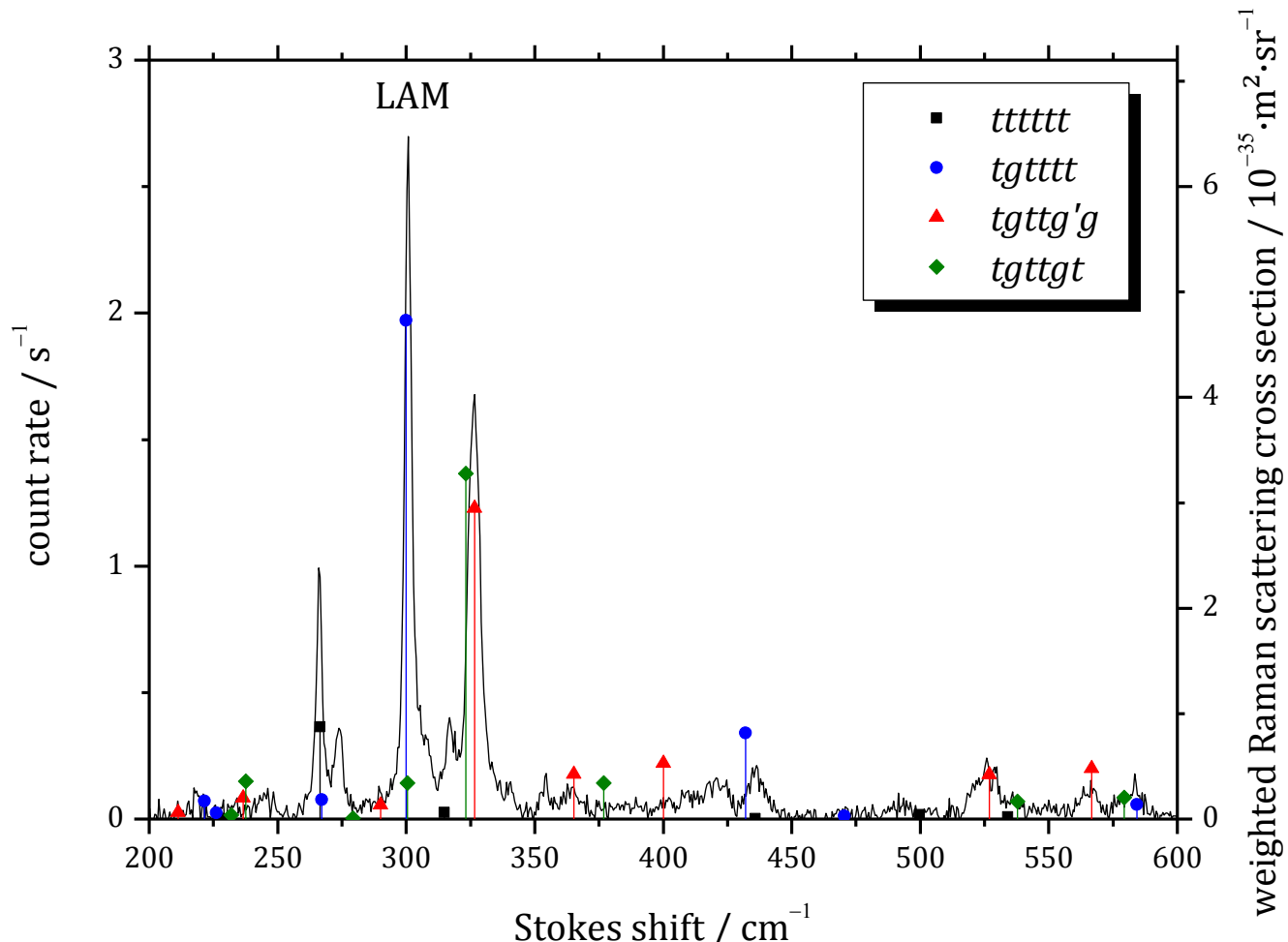

Figure 7.15: Low frequency jet spectrum of diglyme (measurement conditions can be found in A.1) compared to Raman scattering cross sections calculated at the B3LYP-3D3/aVQZ level and weighted with their relative abundances after a full relaxation across all barriers below $5 \mathrm{~kJ} \cdot \mathrm{mol}^{-1}$ within the $\Delta G^{0}$ approach. This comparison mimics the second step of the relaxation process shown in Figure $7.11 \mathrm{~b}$. 


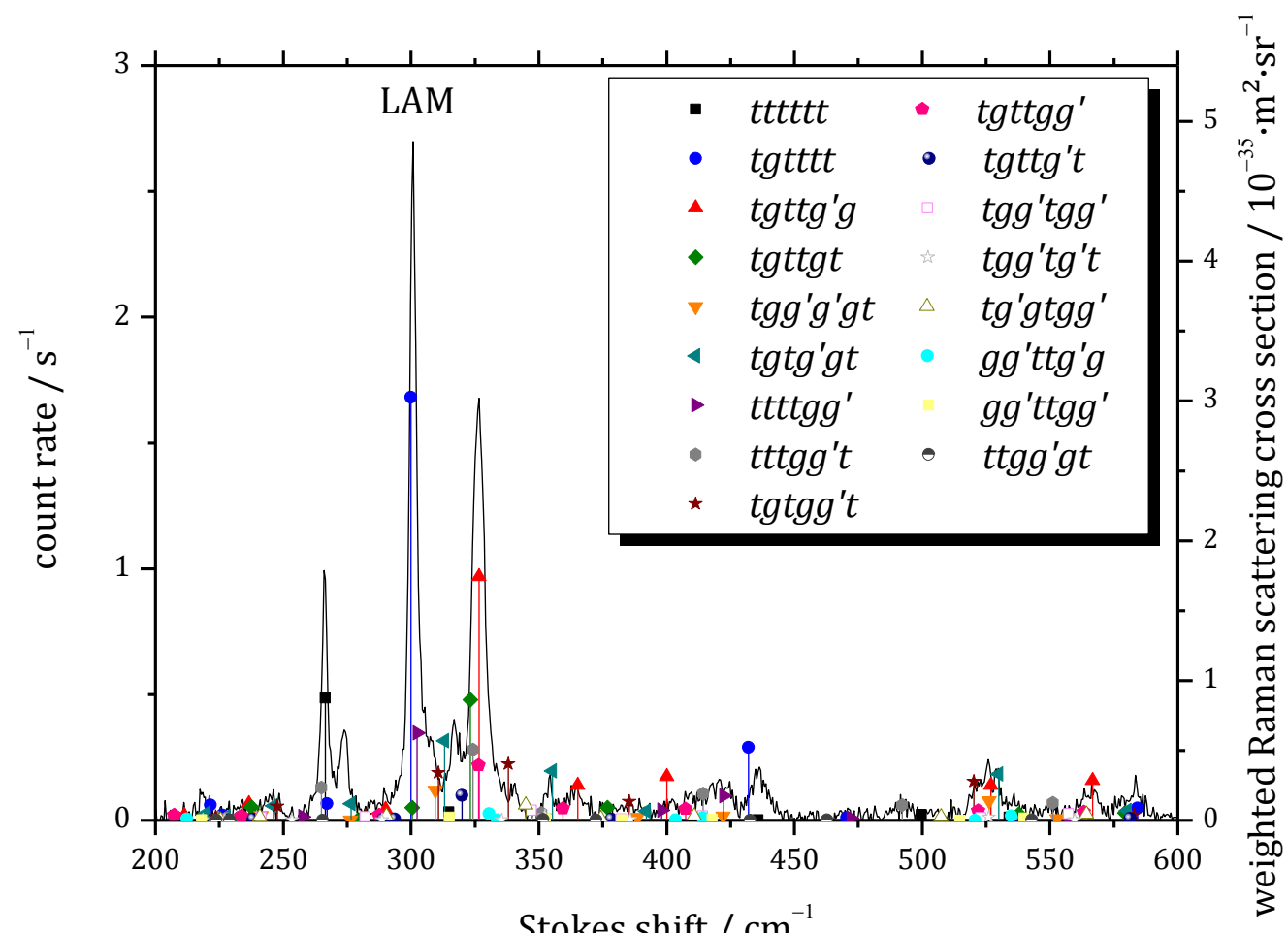

Figure 7.16: Low frequency jet spectrum of diglyme (measurement conditions can be found in A.1) compared to Raman scattering cross sections calculated at the B3LYP-3D3/aVQZ level and weighted with their relative abundances after a repopulation of the conformer families at $T_{\mathrm{r}}$ of $100 \mathrm{~K}$ within the $\Delta \boldsymbol{G}^{\mathbf{0}}$ approach. This comparison mimics the third step of the relaxation process shown in Figure 7.11b.

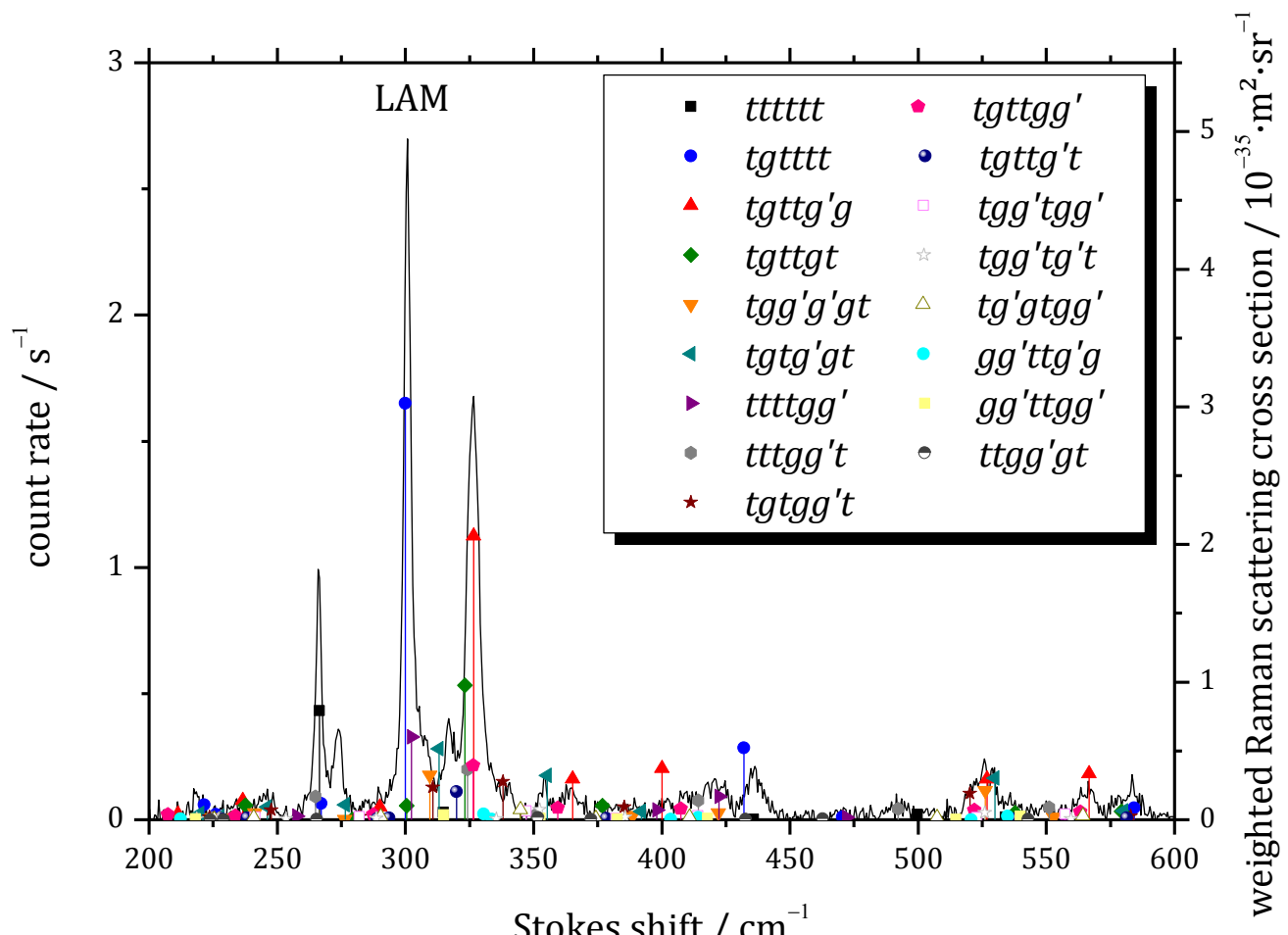

Figure 7.17: Low frequency jet spectrum of diglyme (measurement conditions can be found in A.1) compared to Raman scattering cross sections calculated at the B3LYP-3D3/aVQZ level and weighted with their relative abundances after a repopulation of the conformer families at $T_{\mathrm{r}}$ of $100 \mathrm{~K}$ within the $\Delta \boldsymbol{G}^{\mathrm{C}}$ approach. This comparison mimics the third step of the relaxation process shown in Figure 7.11a. 


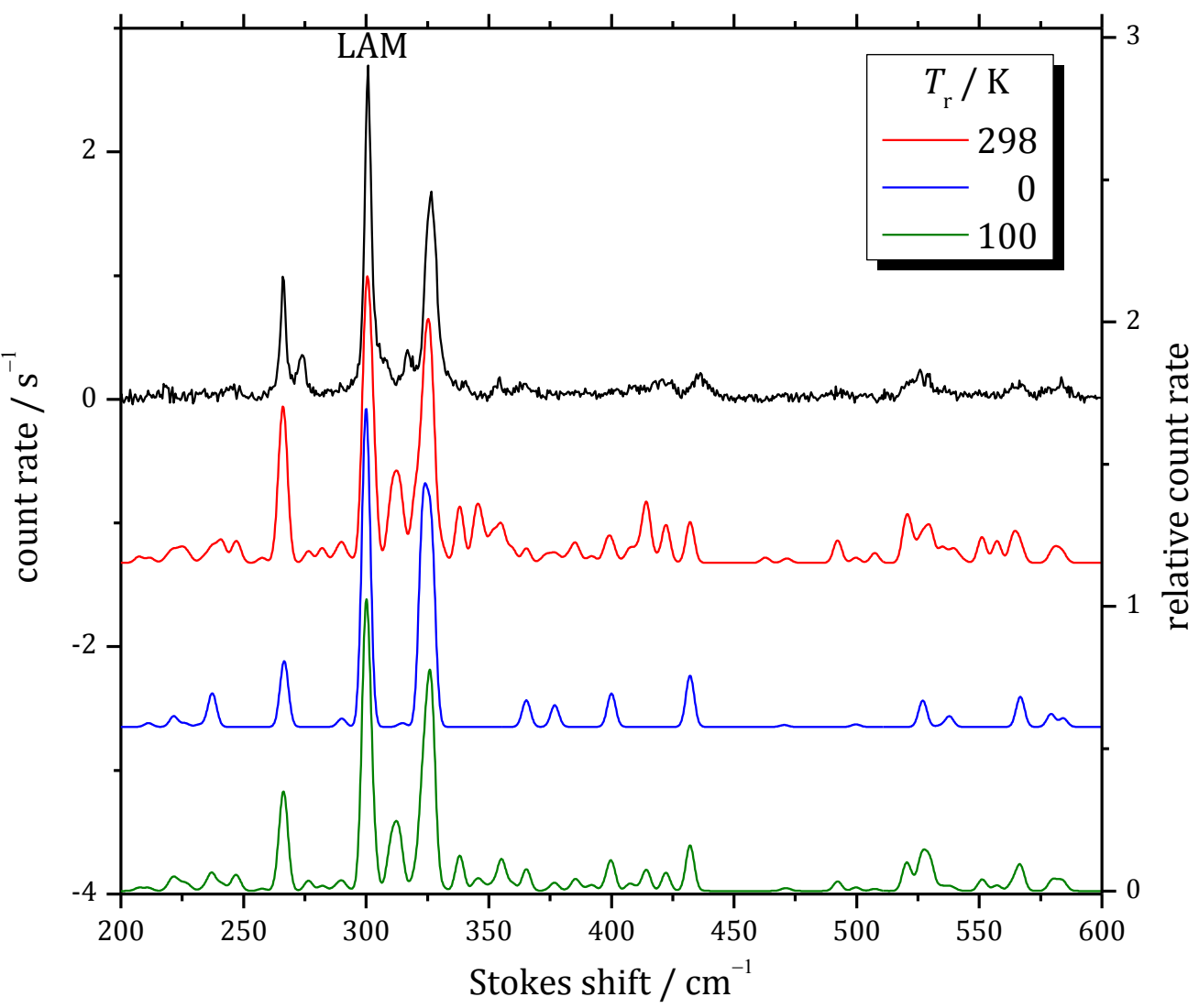

Figure 7.18: Low frequency jet spectrum of diglyme (black) compared to a simulated spectra consisting of Gaussian functions (Eq. 7.2) using a FWHM of $4 \mathrm{~cm}^{-1}$ for all band and the weighted Raman scattering cross sections of the 17 lowest conformation at $T_{\mathrm{r}}$ of 298 (red), 0 (blue) and $100 \mathrm{~K}$ (green) within the $\Delta \boldsymbol{G}^{0}$ approach. Simulated spectra were normalized to equal integrals of the tgtttt LAM $\left(300 \mathrm{~cm}^{-1}\right)$.

Additional measurements with varying nozzle distances, temperatures and different carrier gases were carried out to gain further insights in the relaxation dynamics and energetic sequence of the diglyme conformers. Due to the changing conditions all following spectra in this section were normalized to the rather isolated LAM of the tttttt conformer. This makes relative population changes between different experimental conditions directly visible. In order to minimize spectral changes due to differences in alignment or repositioning of the setup each series of tunable experimental parameters is measured consecutively on the same day. Hereafter, influences on the spectra in expansions at different nozzle temperatures, measurements with varying carrier gases as well as changing nozzle distances will be discussed.

Helium jet expansions at different nozzle temperatures (Figure 7.19) do not show any significant changes besides the band at $273 \mathrm{~cm}^{-1}$, which gains intensity with rising temperature and is slightly shifted to lower wavenumbers. This phenomenon is explained with the contribution of higher energy conformers. Weak signals of $\operatorname{tg} \operatorname{tg}^{\prime} g t$ and $t g g^{\prime} g$ 'gt are predicted in that region (Figure 7.13) but the band at $525 \mathrm{~cm}^{-1}$, predicted with larger Raman scattering cross sections of the same conformers, shows weaker increases. This leads to the assumption that conformers that rank even higher in energy and thus were not considered in the relaxation model have an influence here. A very likely candidate is the gttttt conformer which has a predicted strong LAM 112 
band slightly upshifted by $13 \mathrm{~cm}^{-1}$ of the $t t t t t$ LAM band. A similar yet much weaker gain was found in a nozzle temperature series where the substance was expanded in a helium jet expansion doped with $5 \%$ argon (Figure 7.20 ). This is within expectations as the addition of the heavier argon atoms increases the collisional cooling during the jet expansion and leads to lower effective temperatures, therefore partly counterbalancing the increasing nozzle temperatures. These systematic gains support the assumption of a slight increase in population of higher energy conformers with increasing nozzle temperature. Additionally, a loss in intensity of the $\operatorname{tg}^{\prime} t g$ 'g/tgttgt LAM at $326 \mathrm{~cm}^{-1}$ is seen when nozzle temperatures are increased above room temperature in mixed argon-helium expansions. As Figure 7.15 and 7.16 show, this band is most sensitive to $T_{\mathrm{r}}$. Therefore, the increase at $20^{\circ} \mathrm{C}$ nozzle temperature might be explained by a lower $T_{\mathrm{r}}$, i.e. better cooling across the low barriers. However this should give a more continuous trend. The most likely interpretation of this sudden behavior is the formation and prevention of argon-diglyme clusters. These clusters would prefer more folded conformers as they can solvate an argon atom more efficiently than the open forms. Calculations on a B3LYP-2D3/aVTZ level predicted argon clusters with tgttgt, $\operatorname{tgttg}^{\prime} g$ and $\operatorname{tgtttt}$ conformers to be approximately $2 \mathrm{~kJ} \cdot \mathrm{mol}^{-1}$, which corresponds to roughly $40 \%$ of their dissociation energies, more stable than clusters with ttttt. With increasing nozzle temperatures cluster formation is greatly reduced and hence only the LAM bands of folded conformers lose intensity.

A significant decrease in the tgttt band at $300 \mathrm{~cm}^{-1}$ is observed when a gas mixture of $20 \%$ neon in helium instead of pure helium is used (Figure 7.21), indicating a population transfer of tgtttt to tttttt. As seen in Section 7.3 these inner subunit dihedral changes have much larger barriers and can only be slightly facilitated by addition of heavier atoms into the expansion gas. A population transfer from $\operatorname{tgttg}^{\prime} g$ and $t g t t g t$ to $t t t t t$ is less likely as it needs to overcome the large inner subunit dihedral barriers twice. Seeding the helium backing gas with a heavier atom, such as argon, is expected to further enhance the cooling effects observed in the neon/helium mixture increasing population transfer to the global minimum structure. However, due to the abovementioned diglyme-argon cluster formations the order of the most stable conformers is scrambled in favor of the folded $\operatorname{tg}_{\mathrm{ttg}} \mathrm{g}$ and $\mathrm{tg} t \mathrm{tg} t$ conformations explaining the observed increase in intensity of the $\operatorname{tgttg}^{\prime} g / \operatorname{tg} t \operatorname{tg} t$ LAM. When comparing the different carrier gases at a larger nozzle distance of $2 \mathrm{~mm}$ (Figure 7.22) the same trends can be observed. While the mixed neon-helium expansion shows a certain population transfer from tgtttt to $t t t t t$, the mixed argon-helium expansion shows growing cluster effects rendering a direct comparison impossible.

Likewise a nozzle distance series in helium shows no significant changes at all (Figure 7.23) while expansions in helium doped with $20 \%$ neon show a significant decrease in the tgtttt band with increasing nozzle distances (Figure 7.24).

Since most of the observed spectral changes are small they have to be carefully distinguished from changes induced by differences in alignments of the setup, thermal fluctuations and post-processing steps like baseline subtraction and normalization of the spectra. As stated earlier, to minimize changes due to thermal fluctuations, align- 
ment or repositioning of the setup each series of tunable experimental parameters was measured consecutively on the same day without any changes in the alignment. This allows comparing spectra with high precision. The largest error source remains in the post-processing steps. Figure 7.25 gives an example of the deviations due to different baseline subtraction and normalization. Figure 7.25a shows the carrier gas series at $1 \mathrm{~mm}$ nozzle distance that was already shown in Figure 7.21. There, all spectra were normalized to the peak maximum of the LAM of the ttttt conformer. Figure $7.25 \mathrm{~b}$ shows the same series where all spectra were now normalized to the integral of the ttttt LAM, which was fitted by a Lorentzian curve. The same comparison is then shown in Figure 7.26a and b for the nozzle distance series in helium where no significant effects were visible. This shall demonstrate that the observed spectral changes even though mostly weak are still real effects. 


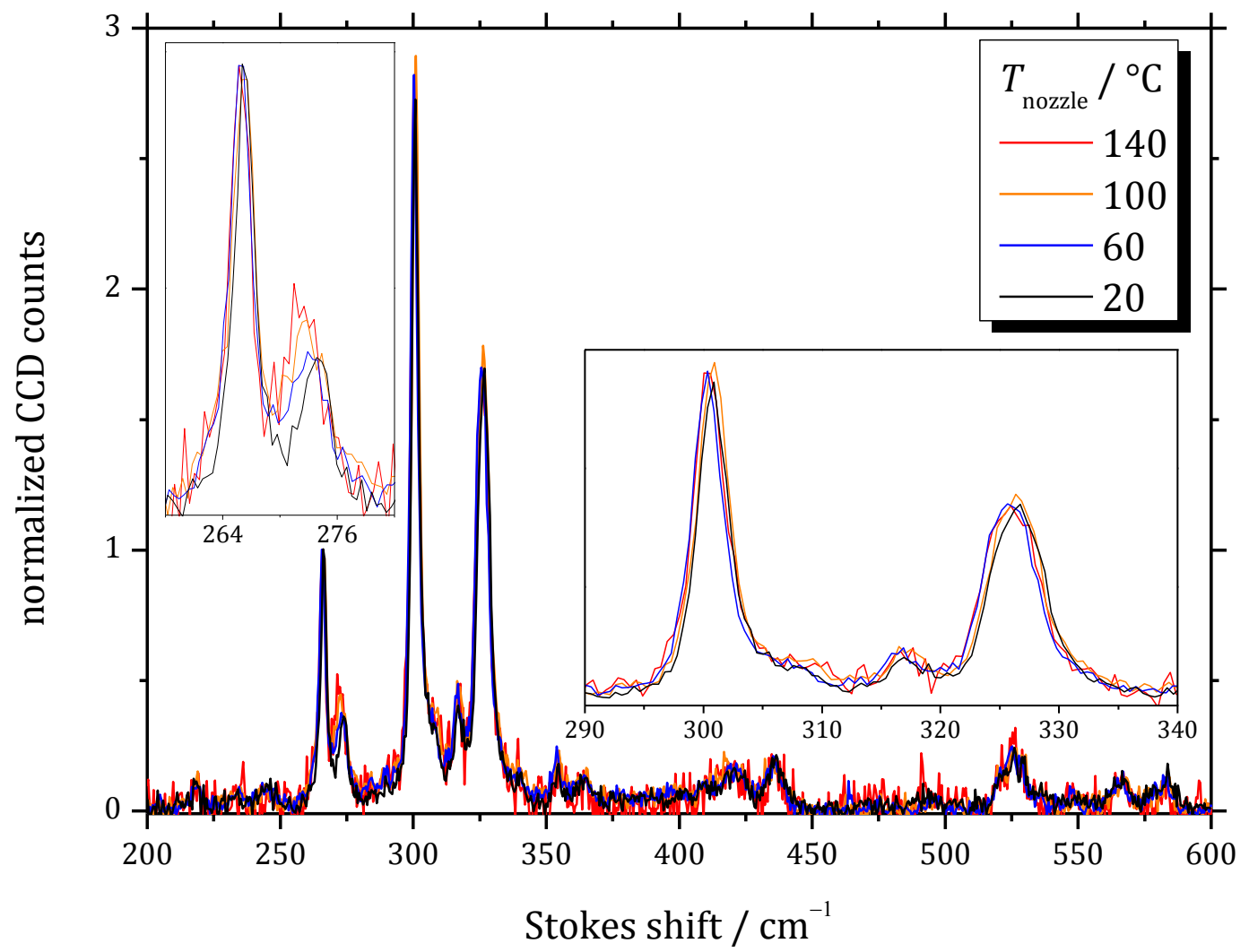

Figure 7.19: Helium jet expansions of diglyme at 20 (black), 60 (blue), 100 (orange) and $140{ }^{\circ} \mathrm{C}$ (red) nozzle temperature. Spectra were normalized to the maximum of the LAM $(t t t t t t)$. Other measurement parameters can be found in A.1.

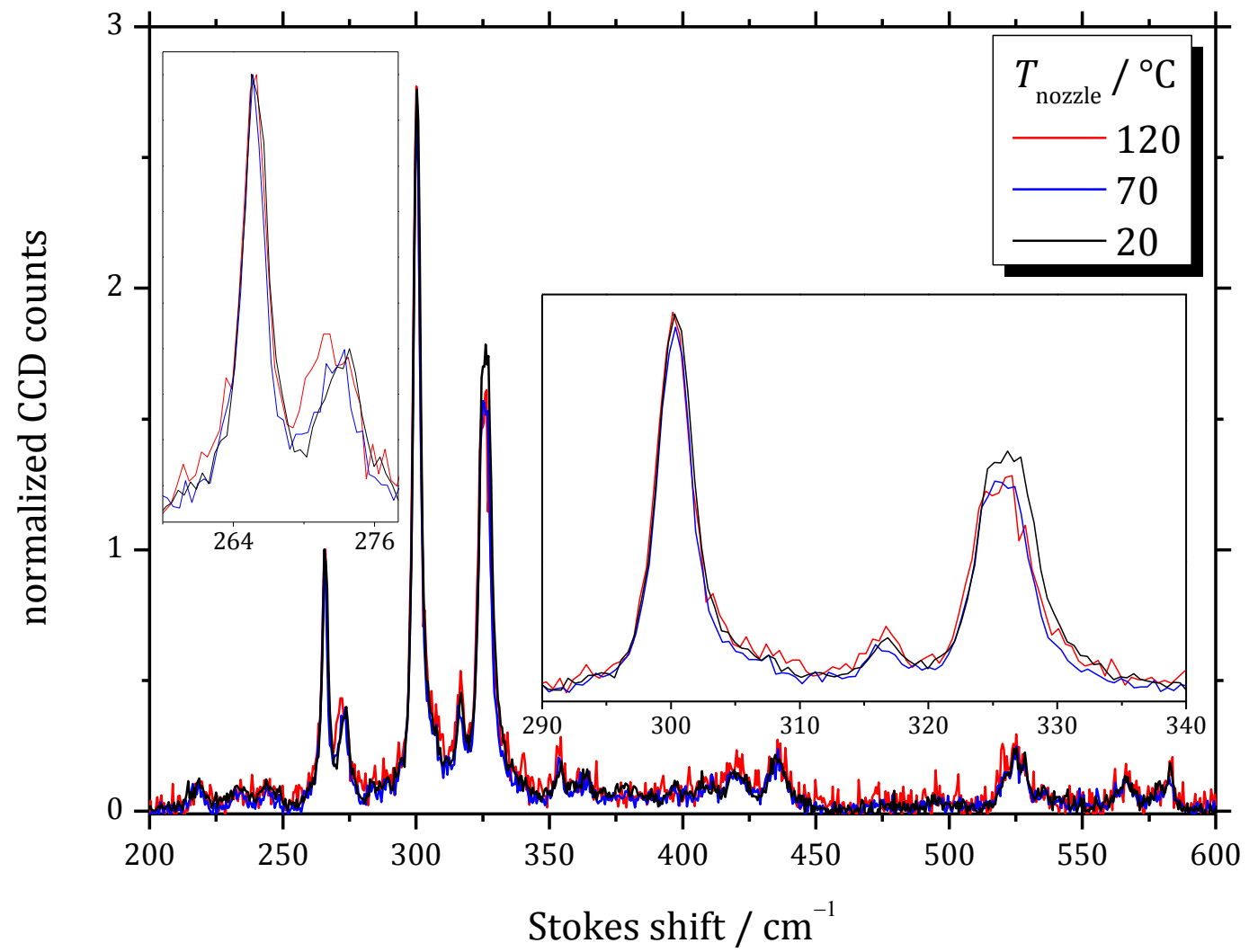

Figure 7.20: Jet expansions of diglyme in a mixture of $5 \%$ Ar in He at 20 (black), 70 (blue) and $120{ }^{\circ} \mathrm{C}$ (red) nozzle temperature. Spectra were normalized to the maximum of the all-trans LAM. Other measurement parameters can be found in A.1. 


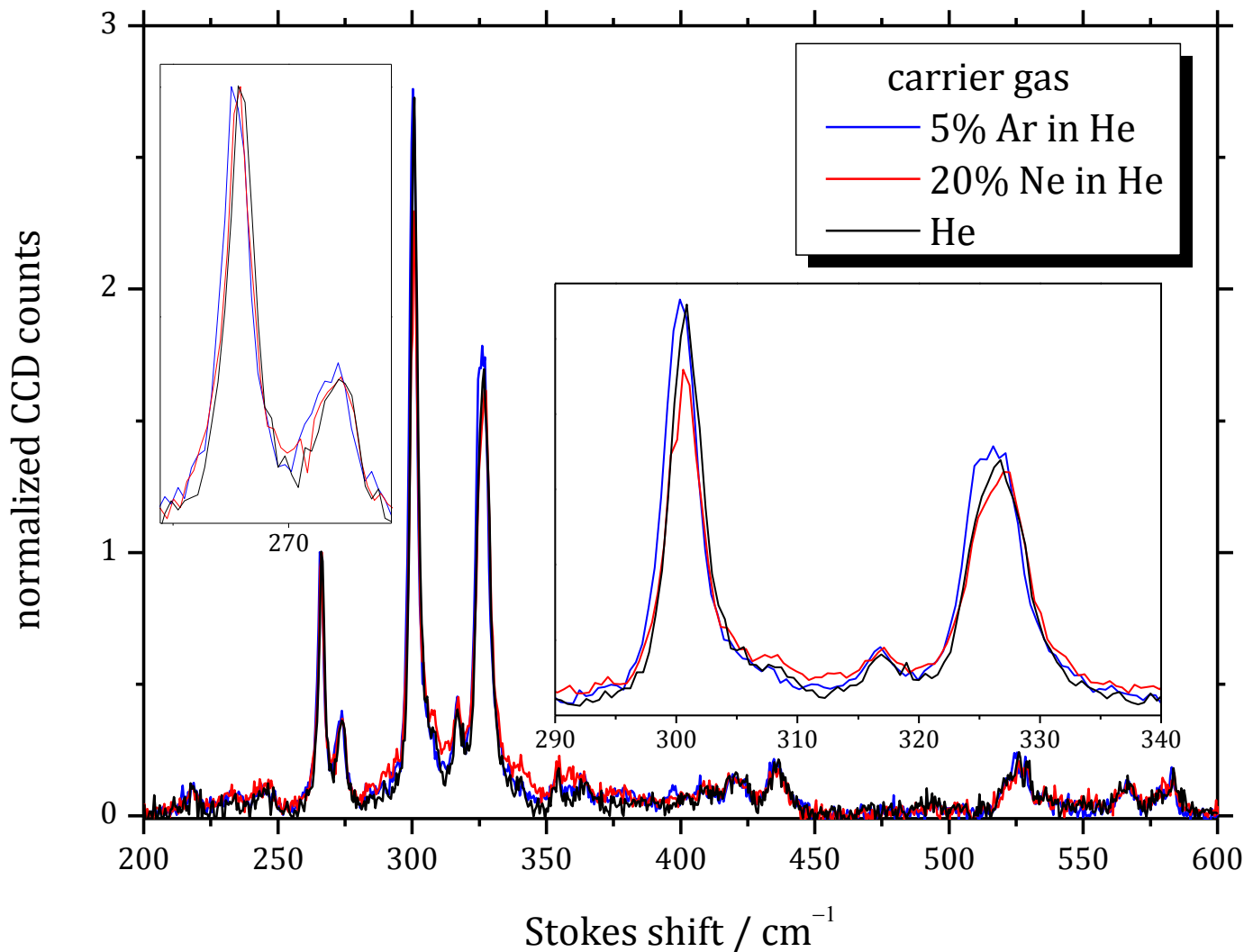

Figure 7.21: Jet expansions of diglyme in He (black), 20\% $\mathrm{Ne}$ in $\mathrm{He}$ (red) and $5 \% \mathrm{Ar}$ in $\mathrm{He}$ (blue) at $1 \mathrm{~mm}$ nozzle distance. Spectra were normalized to the maximum of the all-trans LAM. Other measurement parameters can be found in A.1.

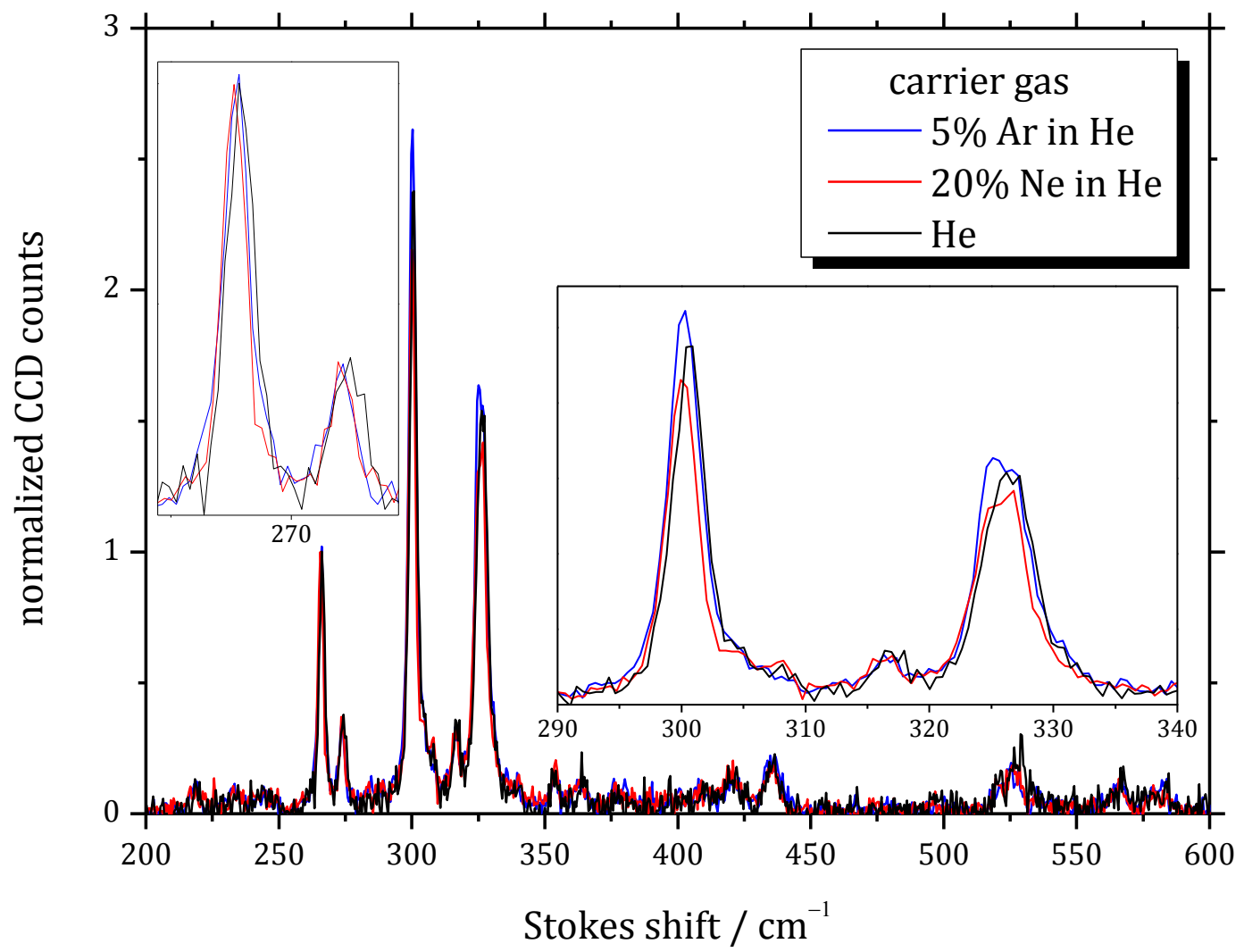

Figure 7.22: Jet expansions of diglyme in He (black), 20\% $\mathrm{Ne}$ in He (red) and 5\% Ar in He (blue) at $2 \mathrm{~mm}$ nozzle distance. Spectra were normalized to the maximum of the all-trans LAM. Other measurement parameters can be found in A.1. 


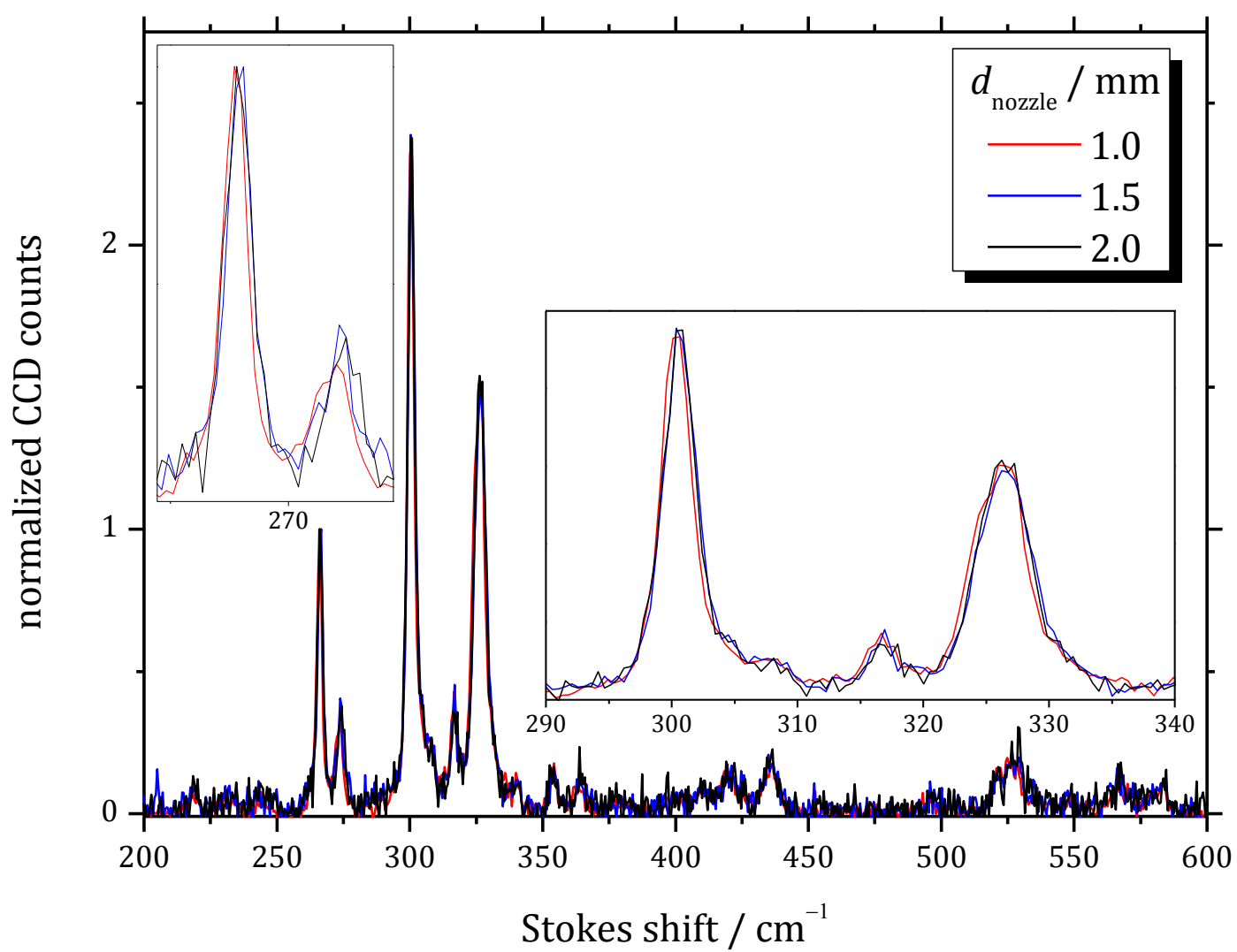

Figure 7.23: Jet expansions of diglyme in He at 1.0 (red), 1.5 (blue) and $2.0 \mathrm{~mm}$ (black) nozzle distance. Spectra were normalized to the maximum of the all-trans LAM. Other measurement parameters can be found in A.1.

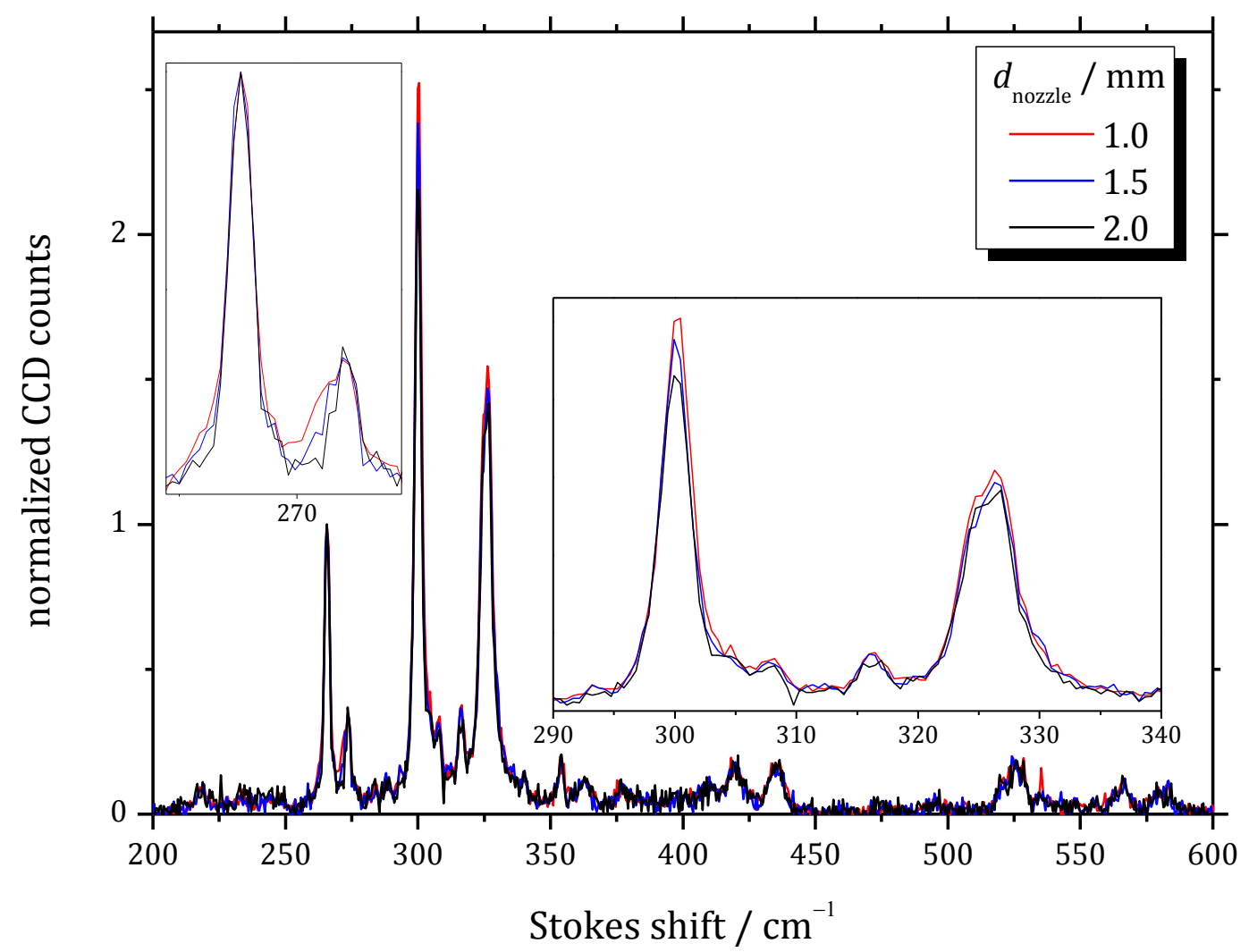

Figure 7.24: Jet expansions of diglyme in $20 \% \mathrm{Ne}$ in $\mathrm{He}$ at 1.0 (red), 1.5 (blue) and $2.0 \mathrm{~mm}$ (black) nozzle distance. Spectra were normalized to the maximum of the all-trans LAM. Other measurement parameters can be found in A.1. 
a)

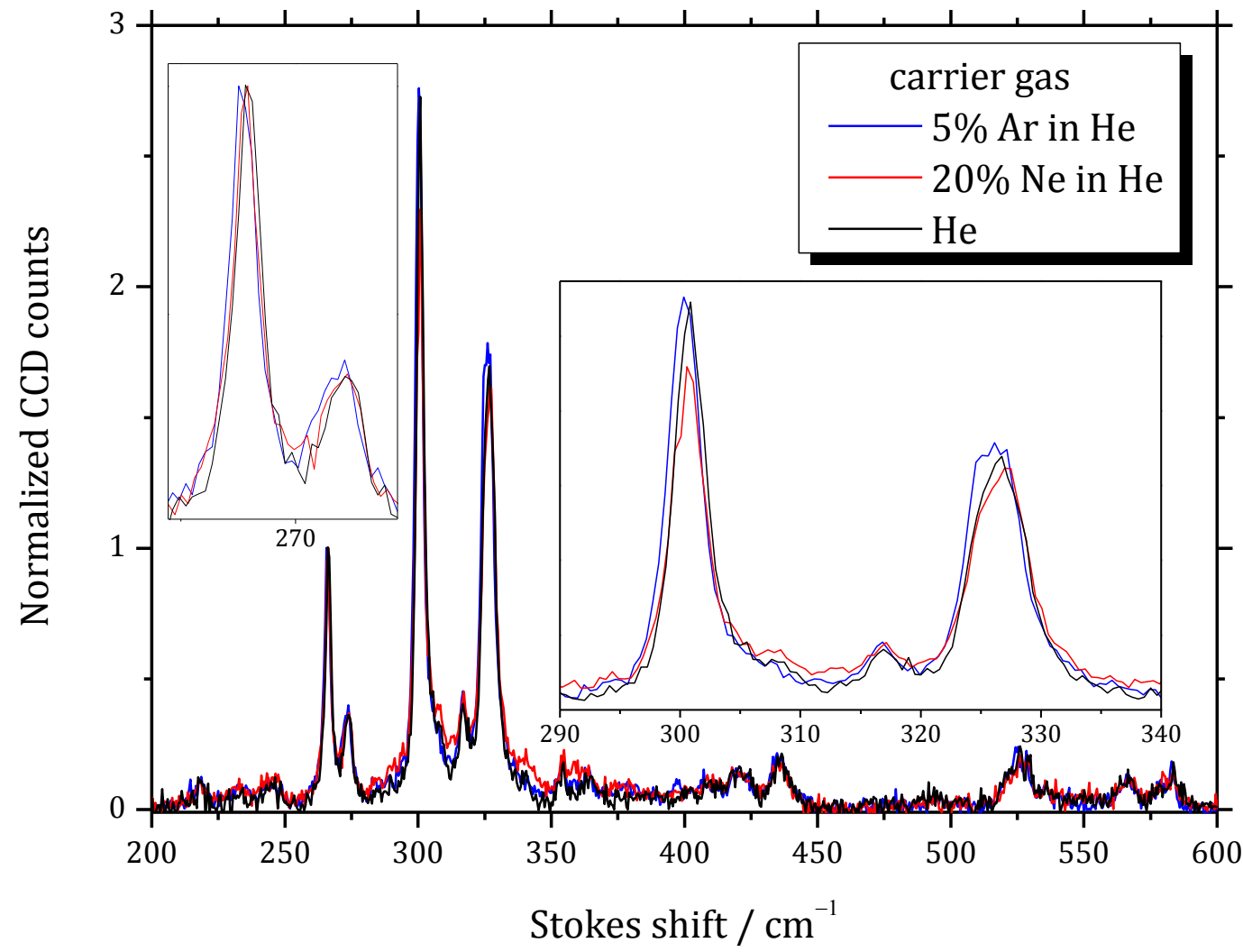

b)

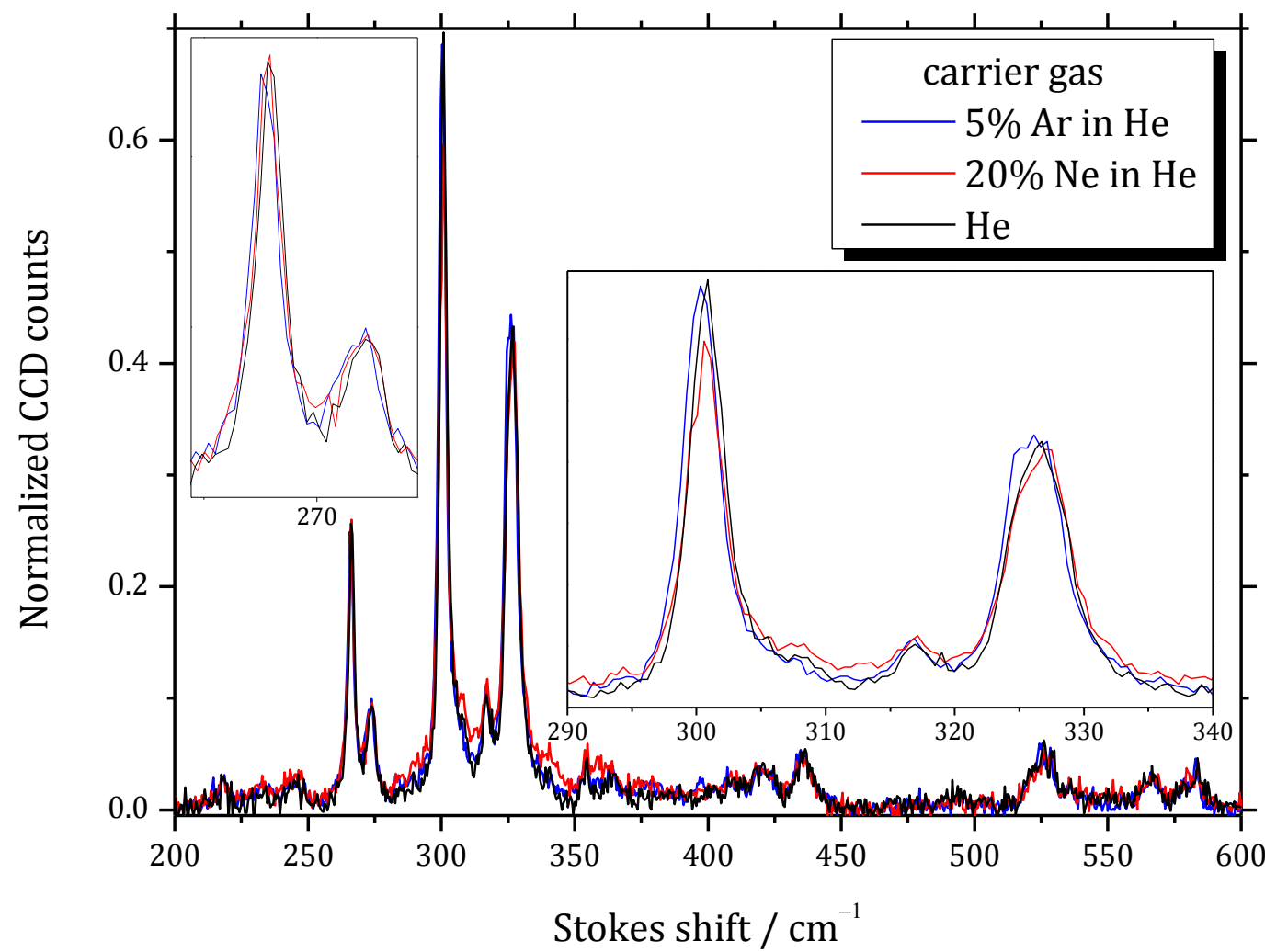

Figure 7.25: Comparison of jet expansions of diglyme in He (black), $20 \% \mathrm{Ne}$ in $\mathrm{He}$ (red) and $5 \% \mathrm{Ar}$ in He (blue) at $1 \mathrm{~mm}$ nozzle distance with different normalizations. Spectra in a) were normalized to the maximum of the all-trans LAM while those in b) were normalized to the integral of the alltrans LAM, which was fitted with a Lorentzian profile. Other measurement parameters can be found in A.1. 
a)

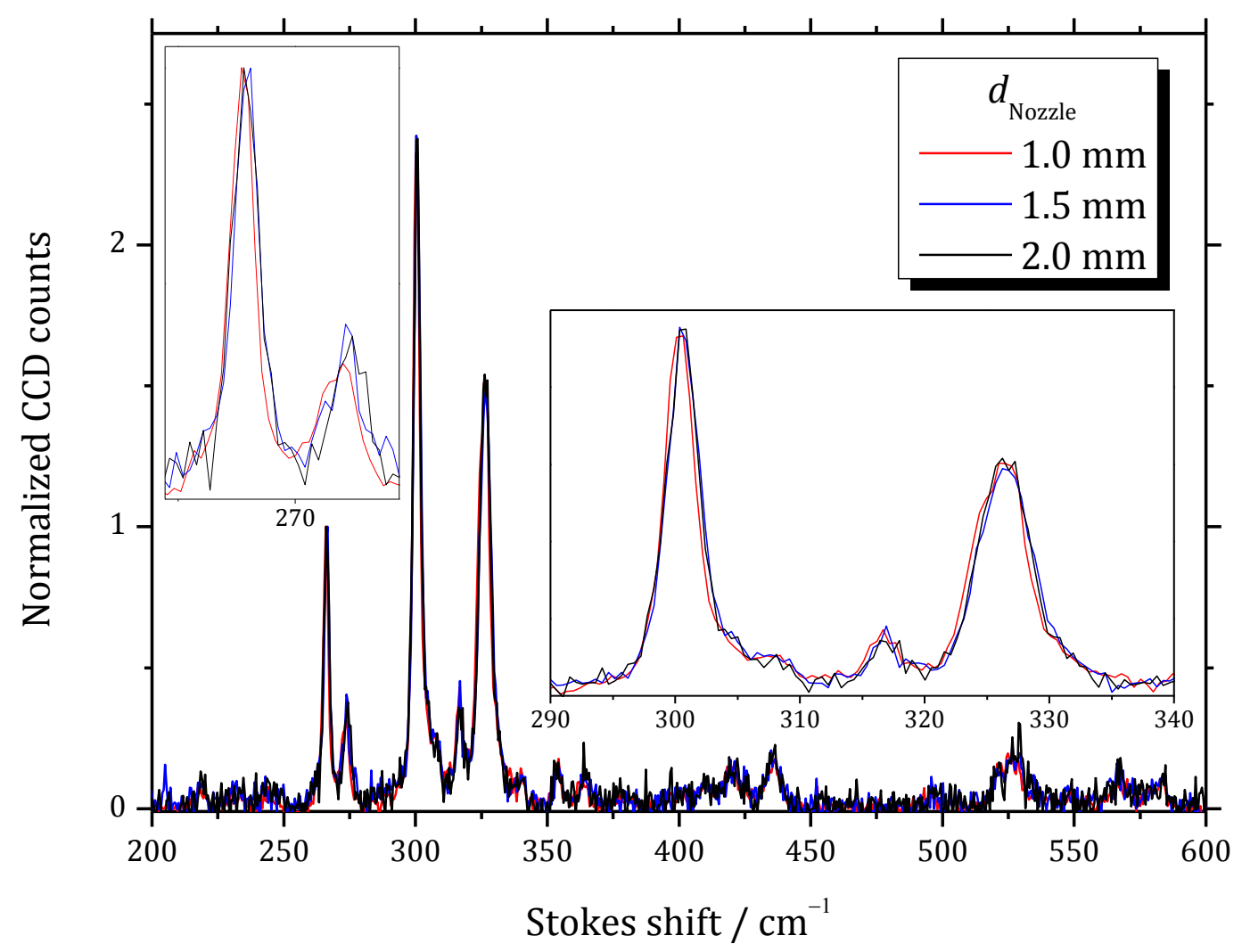

b)

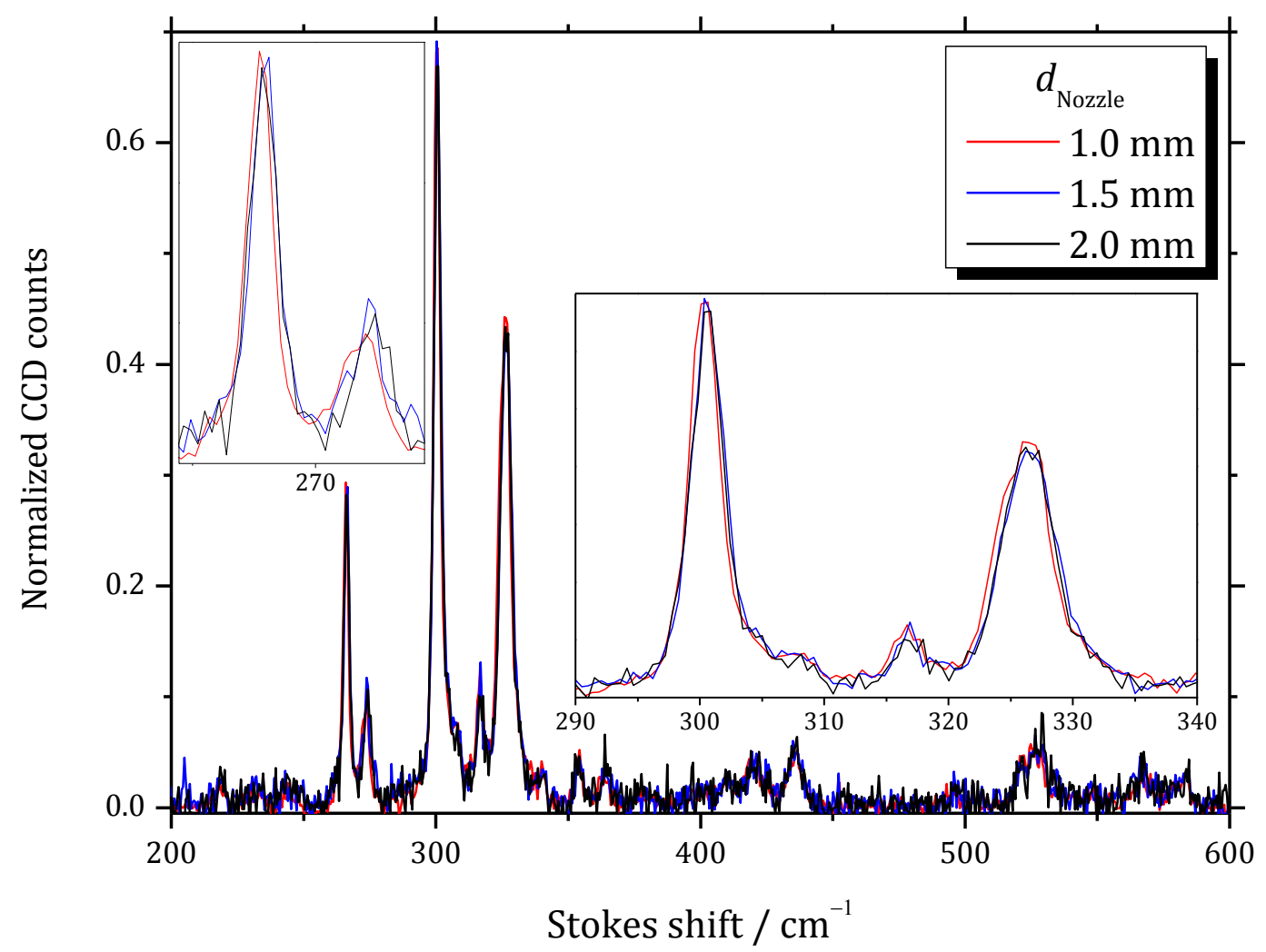

Figure 7.26: Comparison of jet expansions of diglyme in He at 1.0 (red), 1.5 (blue) and $2.0 \mathrm{~mm}$ (black) nozzle distance with different normalizations. Spectra in a) were normalized to the maximum of the all-trans LAM while those in b) were normalized to the integral of the all-trans LAM, which was fitted with a Lorentzian profile. Other measurement parameters can be found in A.1. 


\section{5 - Dimers}

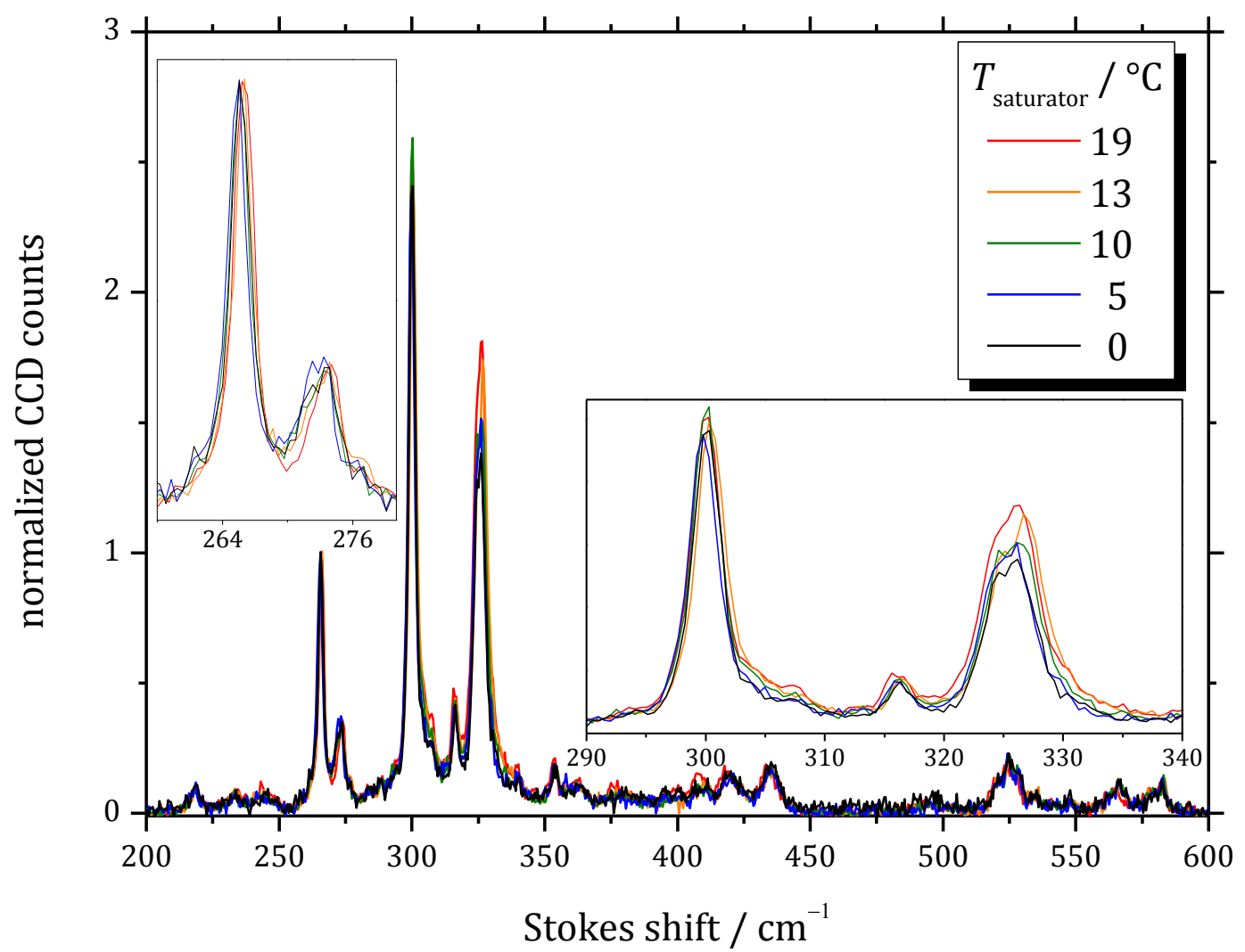

Figure 7.27: Jet expansions of diglyme in He through a nozzle at room temperature at different saturator temperatures. Spectra were normalized to the maximum of the all-trans LAM. Other measurement parameters can be found in A.1.

The saturator temperature gives control over the substance to carrier gas ratio. Thus, the temperature can be modified to give a good qualitative impression on selfclustering in the jet spectra. Figure 7.27 shows the helium jet spectra of diglyme at different saturator temperatures. The spectrum does not show significant changes with increasing temperatures except for the LAM band of the $\operatorname{tg}^{t} \operatorname{tg}^{\prime} g$ and $\operatorname{tgttg} t$ conformers $\left(327 \mathrm{~cm}^{-1}\right)$. This band is increasing in intensity and width, providing clear evidence for cluster formation in this region. Its band maximum is also slightly shifted to higher wavenumbers as the saturator temperature is increased.

Diglyme shows a strong tendency to form clusters, a fact illustrated by the following estimation. A saturator temperature of $19{ }^{\circ} \mathrm{C}$ corresponds to an equilibrium vapor pressure of 2.2 mbar while a temperature of $0{ }^{\circ} \mathrm{C}$ results in 0.5 mbart. This vapor pressure is then mixed with 1.9 bar of carrier gas, indicating that in a per mille mixture diglyme already shows significant cluster effects. Usually an estimated mixing ratio of $\sim 1 \%$ substance to carrier gas is necessary to show significant cluster effects in the curry jet spectra for more volatile compounds as seen in the case of methanol clusters [186] or mixed methanol-water clusters [187].

\footnotetext{
† These calculations were performed using the Antoine equation with the A,B \& C constants taken from Ref. [185]. It is questionable whether equilibrium conditions are fulfilled during the gas pulses in the saturator but since these calculations only serve as a rough estimation it shall be tolerated.
} 
The objective of this cluster study was not to give a complete examination and assignment of all possible cluster structures; instead the goal was to find a reasonable dimer structure that potentially explains the spectral changes. Dimers have been calculated using Gaussian 09 Rev. D.01 and the B3LYP-2D3/def2TZVP method. Input geometries were generated by adding all possible combinations of the six lowest monomer conformations in a chemically intuitive way. Additional inputs were generated by calculating the conformational equilibrium distribution on a MMFF level using Spartan'08. A total of 28 different inputs were optimized and their harmonic frequencies and Raman scattering cross sections were calculated.

Through this search it became obvious that it is especially beneficial if both monomer fragments have some gauche kinks of the same orientation in their backbones as this gives a very good possibility to fold both fragments around each other. The best structure that was found during the dimer search is depicted in Figure 7.28. This dimer contains the tgtttt and tgttgt monomers and was predicted to be at least $5 \mathrm{~kJ} \cdot \mathrm{mol}^{-1}$ more stable than any other calculated dimer structure. Here the tgttgt monomer folds around the all-trans part of the tgtttt monomer, forming three weak hydrogen bonds. The gauche kink of the tgtttt part is then orientated to the side of the tgttgt unit forming another hydrogen bond. The calculated dissociation energy of this dimer yields $41.3 \mathrm{~kJ} \cdot \mathrm{mol}^{-1}$, a rather large value when compared to the enthalpy of vaporization of diglyme of $45.4 \mathrm{~kJ} \cdot \mathrm{mol}^{-1}$ found by Li et al. [188] or $46.4 \mathrm{~kJ} \cdot \mathrm{mol}^{-1}$ when extrapolated from the Antoine parameters [185]. The predicted dissociation energy greatly profits from a large basis set superposition error of the small def2TZVP basis and therefore displays an upper threshold for this value. However, it still supports the idea of the strong dimerization propensities in diglyme.
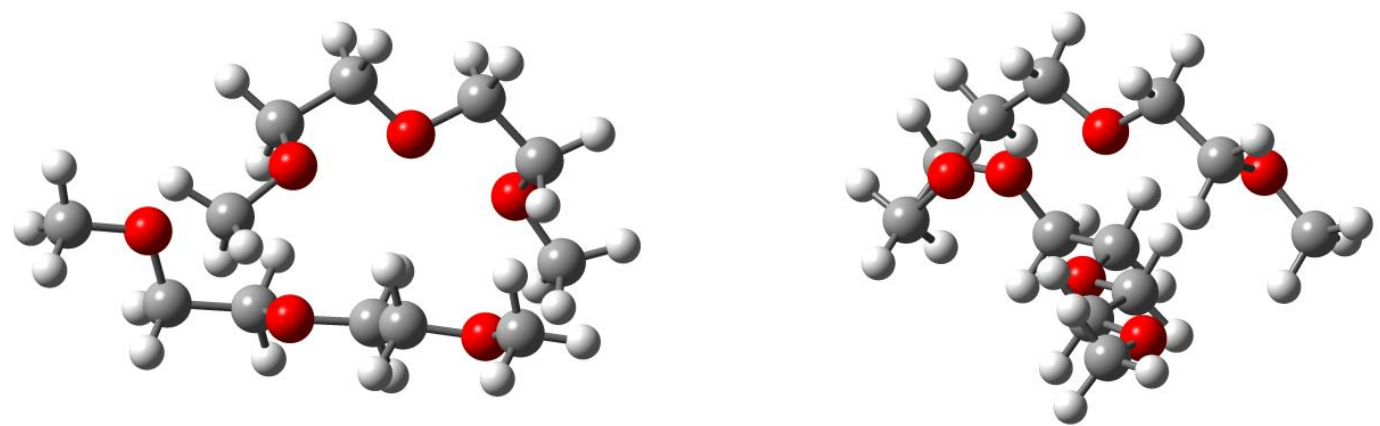

Figure 7.28: Geometry from two different perspectives of the most stable dimer found $(\operatorname{tgtttt}+\operatorname{tgttg} t)$.

Figure 7.29 shows a Raman jet spectrum of diglyme and the calculated scattering cross sections of the four important monomers weighted with their statistical weights so that they can be better compared to the added cross sections of the tgtttt + tgttgt dimer. Indeed a strong band, which corresponds to the LAM motion of the tgttgt fragment, is found slightly upshifted to the tgttg'g and tgttgt monomer LAMs, potentially explaining the significant change seen in Figure 7.27. Furthermore, the perturbed LAM of the tgtttt is also slightly upshifted in the dimer which explains the asymmetric increase of the tgtttt LAM wing with higher saturator temperatures as seen in Figure 7.27. 
All in all it can be concluded that a reasonable dimer structure, which can explain the spectral changes, could be found. Diglyme shows a stronger tendency to form oligomers than monoglyme, which is consistent with the higher flexibility, lower volatility and the increase of hydrogen bond donors and acceptor possibilities in diglyme. Thus, it is expected that the self-aggregation tendency in longer glymes will increase with chain length.

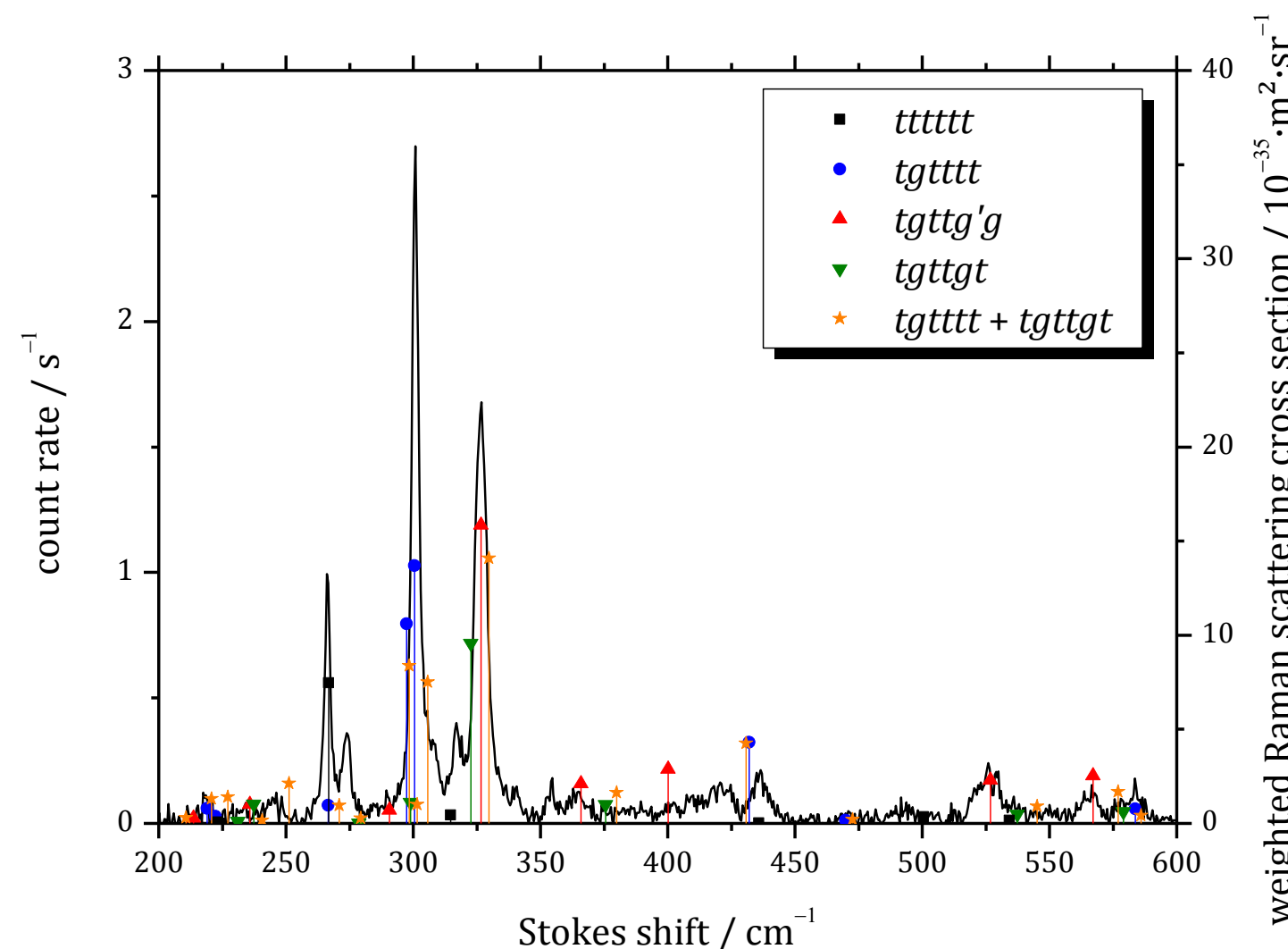

Figure 7.29: Low frequency jet spectrum of diglyme compared to calculated Raman scattering cross sections for the four best monomers and the tgtttt + tgttgt dimer weighted with their respective statistical weights. Other measurement conditions can be found in A.1.

\section{6 - Conclusions}

In summary, an extensive exploration of the conformational landscape led to the contribution of 17 conformers to the relaxation model. Consistent with findings in monoglyme, all inner subunit dihedral angles (OCCO) are kinetically isolated by large interconversion barriers leading to four different isolated conformational families. Within the relaxation model abundances of conformers in each family are estimated at an effective repopulation temperature of $100 \mathrm{~K}$. This model allows for a reasonable simulation and interpretation of the Raman jet spectra. Relaxation processes during supersonic jet expansions in helium are completed rapidly and show no further changes with increasing nozzle distances or temperature. However, upon addition of heavier atoms such as neon the high interconversion barriers are partially overcome and slight population shifts become observable. All observed experimental population shifts show a decrease of other populations in favor of ttttt. Therefore, experiment and theory beyond B3LYP-2D3/aVQZ calculations agree in predicting tttttt 
slightly below other conformers as the global minimum structure. This shows that higher level calculations such as MP2 and $\operatorname{CCSD}(\mathrm{T})$ are necessary to work out the delicate energy differences between the different conformers as the B3LYP-2D3 calculations predict $\operatorname{tg} t \mathrm{tg}^{\prime} g$ as the global minimum structure. Mixed helium/argon expansions show evidence of diglyme-argon cluster formation which need to be carefully separated from conformational interconversions of the monomer.

The investigation on monoglyme showed that $t t t$ is the distinct global minimum and even though diglyme also prefers the stretched state at low temperatures in the gas phase it might be the last oligoglyme with this preference. Increasing chain lengths lead to further possibilities for intramolecular interactions which favor folded over stretched conformers. The next chapter shall highlight conformational preferences in longer glyme chains.

A study of diglyme clusters under jet conditions shows a significant change in the band shape of the tgtttt LAM indicating that this monomer is involved in the formed clusters. Consistently, the most stable dimer structure that was found at the B3LYP2D3/def2TZVP level contains tgttttt and tgttgt conformers. In this structure the tgttgt monomer folds around the all-trans part of the tgtttt monomer, forming three weak hydrogen bonds and the gauche kink of the tgtttt part is orientated to the side of the tgttgt unit forming another hydrogen bond. 


\section{Chapter 8 - Longer glymes}

\section{1 - Introduction}

Extension of the glyme chain leads to a polymeric chain known as poly(ethylene glycol) dimethyl ether. These polymers are usually abbreviated PEG at molar masses between 200 and $25000 \mathrm{~g} \cdot \mathrm{mol}^{-1}$ and PEO (short for polyethylene oxide) at molar masses $\geq 25000 \mathrm{~g} \cdot \mathrm{mol}^{-1}$. However, this nomenclature is somewhat arbitrary and the boundary varies for different scientific fields. PEG polymers are readily available in a large number of different molar masses and their physical properties, such as the melting point, slightly vary at different chain lengths [189]. Crystal structures of polyethylene oxides determined by x-ray diffraction show helical structures that consist of dihedral tgt-sequences [190-192]. Most of the beneficial properties of these flexible polymer chains that are utilized in a wide range of applications were already highlighted for the shorter glyme chains. In summary, some important properties of finite glyme chains include complete miscibility with water, low viscosity, low acute toxicity, high chemical and thermal stability [8]. An overview of the wide range of different applications including cation solvation in battery electrolytes, phase-transfer catalysis, enhancing organic or catalytic reactions through templating or solvating reactants or acting as drug delivery agent can be found in References $[8,9]$.

The decreasing vapor pressure of longer glymes makes it substantially harder to reach sufficient gas phase concentrations that allow recording Raman jet spectra. Therefore, this work focuses on the characterization of tri- and tetraglyme that have recorded melting points of -45 and $-30{ }^{\circ} \mathrm{C}$, respectively [193]. First, quantum chemical calculations on selected conformers are utilized to predict energy differences between folded and stretched conformers. Then, Raman jet spectra are discussed and used to evaluate the predicted conformational preferences. Jet relaxation effects observed in the Raman spectra are outlined and a comparison to spectra of liquid samples is drawn. Eventually, the predicted LAM wavenumber positions of stretched glyme conformations are extrapolated to infinite chain lengths in order to derive the elastic modulus of idealized glyme chains in vacuum at low temperatures. This may then be compared to the elastic modulus of real high molecular weight PEO at low temperatures, if available.

\section{2 - Quantum chemical calculations}

The number of different trans-gauche combinations in the dihedral backbone increases rapidly with increasing glyme chain lengths. When rotational isomers and enantiomeric pairs are negelected the number of different combinations can be calculated by $3^{n}$ where $n$ is the number of different backbone dihedrals. This number increaes from 27 in monoglyme over 729 in diglyme to 19683 in triglyme. Therefore, an extensive investigation on the whole conformational landscape is outside the scope of 
this work. However, some conformational preferences in tri- and tetraglyme can still be explored through calculations of selected structures. Six triglyme (Figure 8.1) and four tetraglyme structures (Figure 8.2) were selected that include stable monoglyme subunits. Geometries of the selected structures were optimized at the B3LYP2D3/def2TZVP, B3LYP-2D3/aVTZ and B3LYP-3D3/aVTZ levels of approximation and their harmonic frequencies were calculated with the same functional and basis sets afterwards. Calculations including three-body dispersion corrections (3D3) were performed in TURBOMOLE 7.0 while others were carried out in Gaussian 09 Rev. E.01. Additionally, MP2/aVTZ single point energies were calculated for all optimized structures in TURBOMOLE 7.0. First, the triglyme results will be presented followed by the discussion of the results for tetraglyme. This exploration shall help answering the question if diglyme is the last oligoglyme that prefers a stretched conformation at low temperatures in the gas phase. However, it is not sufficient to characterize the global minimum structure in tri- or tetraglyme.

Zero-point corrected energy differences calculated at the three DFT levels (Table 8.1) predict the same ordering for the six selected conformers. As anticipated from results on mono- and diglyme, the def2TZVP basis set shows a larger intramolecular BSSE and therefore predicts folded structures lower in energy than larger basis sets. Out of the selected six structures tgttg'ttgg', which resembles an open-chain crown ether type motif, is predicted to be the most stable one. The predicted zero-point corrected energy difference between this folded structure and the stretched tttttttt decreases from 2.1 to $1.5 \mathrm{~kJ} \cdot \mathrm{mol}^{-1}$ upon inclusion of three-body dispersion corrections. When calculating the electronic energies at the MP2/aVTZ level (Table 8.3) the different DFT optimizations are found to be in good agreement. A maximum deviation of $0.45 \mathrm{~kJ} \cdot \mathrm{mol}^{-1}$ is found between the different methods although the largest part of this deviation comes from discrepancies in the underlying zero-point vibrational energies (Table 8.2). This shows that the structures optimized with different methods all converged well into almost identical geometries. The evaluation of the electronic energies at the MP2/aVTZ level increases the gap between tgttg'ttgg' and tttttttt again to $\sim 2 \mathrm{~kJ} \cdot \mathrm{mol}^{-1}$. Interestingly, this reverses the trend that was found in diglyme where the evaluation of the electronic energies at the MP2 level increases the gap between tttttt and the best folded structure $t g t t g^{\prime} g$ by $\sim 0.5 \mathrm{~kJ} \cdot \mathrm{mol}^{-1}$ in favor of the all- $t$ conformer. Based on the very limited sample size it is unlikely that tgttg'ttgg' represents the global minimum structure. However, as this conformer already has a distinct energy lead over the all-trans structure it can be concluded that quantum chemical calculations predict that diglyme is indeed the last oligoglyme with a stretched global minimum structure in the gas phase. Experimental evidence that is consistent with these findings will be presented in the next section.

The energetic penalty of a single gauche kink in a COCC dihedral in the stretched glyme chain stays almost independent on the chain length as it is found to be $\sim 6 \mathrm{~kJ} \cdot \mathrm{mol}^{-1}$ in triglyme and $\sim 5.5 \mathrm{~kJ} \cdot \mathrm{mol}^{-1}$ in monoglyme (see Chapter 6.2 ). Relaxed 1D torsional scanning calculations of ttttttt at the B3LYP-2D3/def2TZVP level show that the interconversion barriers around OCCO dihedral angles in triglyme are around 
$10 \mathrm{~kJ} \cdot \mathrm{mol}^{-1}$ while those around COCC/CCOC dihedrals are significantly lower. This is consistent with findings in mono- and diglyme and approves that the classification into conformational families based on their OCCO dihedral angles is still valid for longer glymes. Relaxation effects are expected to occur mainly within these conformational families.

The general trend of further stabilization of folded conformers over stretched structures continues when going to tetraglyme as the two DFT methods with the aVTZ basis set now predict an energy difference of $\sim 4 \mathrm{~kJ} \cdot \mathrm{mol}^{-1}$ between $\operatorname{tg}^{\prime} \operatorname{tg}^{\prime} t t^{\prime} t \operatorname{tg}^{\prime} g$ and ttttttttttt (Table 8.4). The discrepancy of the def2TZVP results grow in tetraglyme which shows that these smaller basis set are critical for larger molecules but are often the last resort when computational times become too expensive. However, when calculating the electronic energies at the MP2/aVTZ level (Table 8.6) results between the different DFT methods show a good agreement. Therefore, even smaller basis sets might be used for optimizations if electronic energies are carried out on a more sophisticated level with a larger basis set. Similar to the triglyme results, the largest deviation in the MP2 energies arises from the underlying zero-point vibrational energies (Table 8.5) and is found to be $0.45 \mathrm{~kJ} \cdot \mathrm{mol}^{-1}$ between the different DFT structures. The evaluation of the electronic energies at the MP2/aVTZ level increases the gap between $t^{\prime} t t g^{\prime} t t g t t g ' g$ and $t t t t t t t t t t t$ to $\sim 5.5 \mathrm{~kJ} \cdot \mathrm{mol}^{-1}$. The helical arrangement of the tgttgttgttgt structure which is the predominant conformational arrangement in the solid phase $[190,191]$ can be nicely seen in the tetraglyme structure (Figure 8.2).

Figure 8.3 shows a comparison of the energies between conformers that consist of consecutive equal monoglyme subunits $(t t t)_{n},(\operatorname{tg} t)_{n}$ and $(\operatorname{tgg})_{n}$ for mono- through tetraglyme $(n=1-4)$. For this comparison the geometries of these structures were optimized at the B3LYP-3D3/aVTZ level and electronic energies of the optimized structures were calculated at the MP2/aVTZ level in TURBOMOLE 7.0.The energy difference between $(t g t)_{n}$ and $(t t t)_{n}$ glyme chains is decreasing further with increasing glyme chain length. In tetraglyme $(n=4)$ both structures are found to be essentially isoenergetic. In contrast, a comparison between $(\operatorname{tgg})_{n}$ and $(t t t)_{n}$ segments shows the reversed trend, thus the $(t g g)_{n}$ energies are increasing compared to the $(t t t)_{n}$ segments at longer chain lengths. This trend is further enhanced when zero-point corrections at the B3LYP-3D3/aVTZ level are added. 


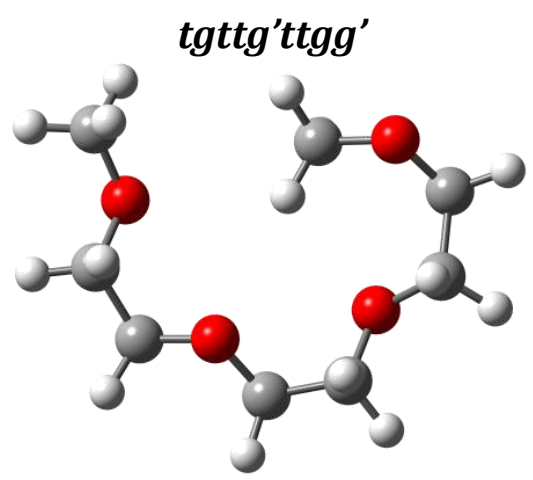

tgttgttgt

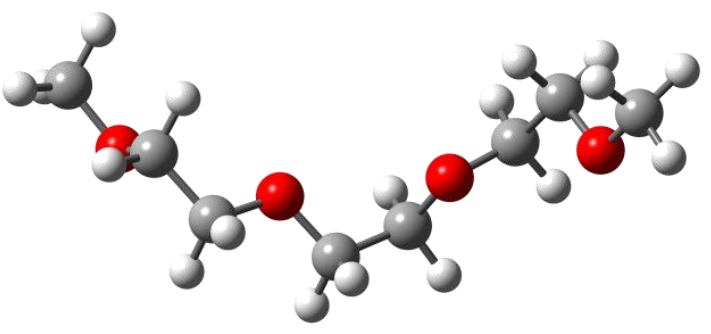

tgttg'ttgt

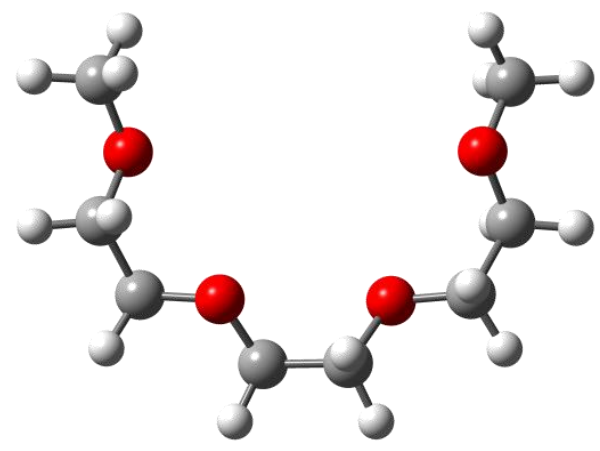

tttttttt

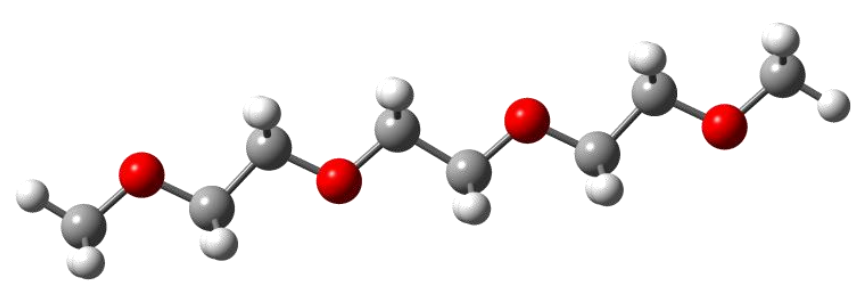

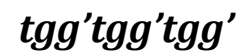

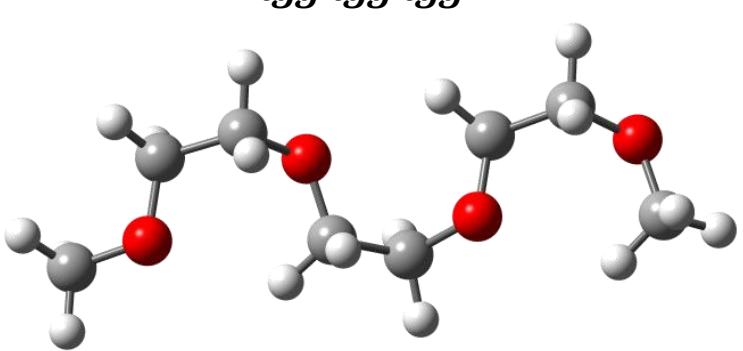

ttgtttttt

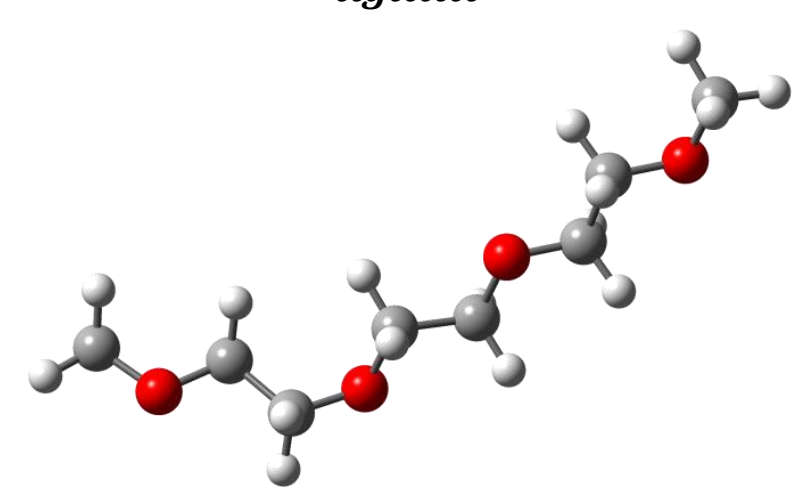

Figure 8.1: Structures of the six selected triglyme conformers.

Table 8.1: Computed energy differences $\Delta E_{0}$ in $\mathrm{kJ} \cdot \mathrm{mol}^{-1}$ between six selected triglyme conformations. 2D3 calculations were performed in Gaussian 09 Rev. E.01 while calculations including three-body dispersion (3D3) were performed in TURBOMOLE 7.0. For all methods the zero-point energies were added to the electronic structure calculations at the appropriate level.

\begin{tabular}{|c|c|c|c|c|}
\hline conformer & $\begin{array}{c}\text { stat. } \\
\text { weight }\end{array}$ & $\begin{array}{c}\text { B3LYP-2D3/ } \\
\text { def2TZVP }\end{array}$ & $\begin{array}{c}\text { B3LYP-2D3/ } \\
\text { aVTZ }\end{array}$ & $\begin{array}{c}\text { B3LYP-3D3/ } \\
\text { aVTZ }\end{array}$ \\
\hline tgttg'ttgg' $^{\prime}$ & 2 & 0.00 & 0.00 & 0.00 \\
\hline$t_{t t t t t t t}$ & $1 / 2$ & 3.28 & 2.12 & 1.51 \\
\hline${ }_{\text {tgttgttgt }}$ & 1 & 3.47 & 2.13 & 1.70 \\
\hline$t_{\text {tgg'tgg'tgg' }}^{\prime}$ & 2 & 4.94 & 4.73 & 4.17 \\
\hline tgttg'ttgt $^{\prime}$ & 2 & 7.13 & 5.74 & 5.45 \\
\hline$t_{t g t t t t t t}$ & 2 & 8.58 & 7.44 & 6.62 \\
\hline
\end{tabular}


Table 8.2: Relative zero-point vibrational energies in $\mathrm{kJ} \cdot \mathrm{mol}^{-1}$ for six selected triglyme conformations. Frequencies for all methods but the 3D3 one were calculated in Gaussian 09 Rev. E.01. The B3LYP-3D3/aVTZ results were obtained using TURBOMOLE 7.0.

\begin{tabular}{|c|c|c|c|c|c|c|}
\hline ZPVE & $\operatorname{tgttg}^{\prime} \operatorname{tgg} g^{\prime}$ & ttttttttt & tgttgttgt & $\operatorname{tgg}^{\prime} \operatorname{tgg} g^{\prime} \operatorname{tgg}{ }^{\prime}$ & tgttg'ttgt & ttgtttttt \\
\hline $\begin{array}{c}\text { B3LYP-2D3/ } \\
\text { def2TZVP }\end{array}$ & 0.00 & -1.35 & -1.22 & 0.69 & -1.52 & -0.52 \\
\hline $\begin{array}{c}\text { B3LYP-2D3/ } \\
\text { aVTZ }\end{array}$ & 0.00 & -1.25 & -1.19 & 0.73 & -1.54 & -0.37 \\
\hline $\begin{array}{c}\text { B3LYP-3D3/ } \\
\text { aVTZ }\end{array}$ & 0.00 & -1.02 & -1.04 & 0.34 & -1.37 & -0.53 \\
\hline
\end{tabular}

Table 8.3: Energy differences $\Delta E_{0}$ in $\mathrm{kJ} \cdot \mathrm{mol}^{-1}$ between six selected triglyme conformations at the MP2/aVTZ level calculated in TURBOMOLE 7.0. Here electronic energies were computed at the MP2 level on structures from DFT optimizations (Table 8.1) and their appropriate zero-point corrections were added to the MP2/aVTZ energies.

\begin{tabular}{|c|c|c|c|c|c|c|c|}
\hline $\begin{array}{c}\text { electronic } \\
\text { energy }\end{array}$ & $\begin{array}{c}\text { structure } \\
\text { and } \\
\text { ZPVE }\end{array}$ & tgttg'ttgg $^{\prime}$ & ttttttttt & tgttgttgt & $\operatorname{tgg}^{\prime} \operatorname{tgg}{ }^{\prime} \operatorname{tgg}{ }^{\prime}$ & tgttg'ttgt & ttgtttttt \\
\hline MP2/aVTZ & $\begin{array}{c}\text { B3LYP- } \\
\text { 2D3/ } \\
\text { def2TZVP }\end{array}$ & 0.00 & 2.01 & 2.48 & 6.32 & 6.60 & 7.99 \\
\hline MP2/aVTZ & $\begin{array}{c}\text { B3LYP- } \\
\text { 2D3/ } \\
\text { aVTZ }\end{array}$ & 0.00 & 2.03 & 2.52 & 6.37 & 6.58 & 8.06 \\
\hline MP2/aVTZ & $\begin{array}{c}\text { B3LYP- } \\
\text { 3D3/ } \\
\text { aVTZ }\end{array}$ & 0.00 & 2.32 & 2.59 & 5.92 & 6.59 & 7.90 \\
\hline
\end{tabular}




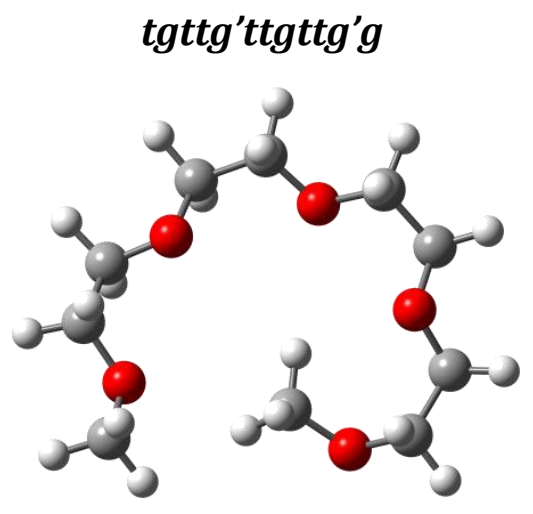

tgttgttgttgt

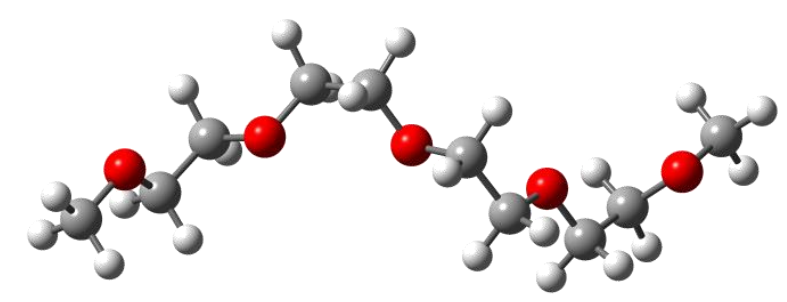

tttttttttt

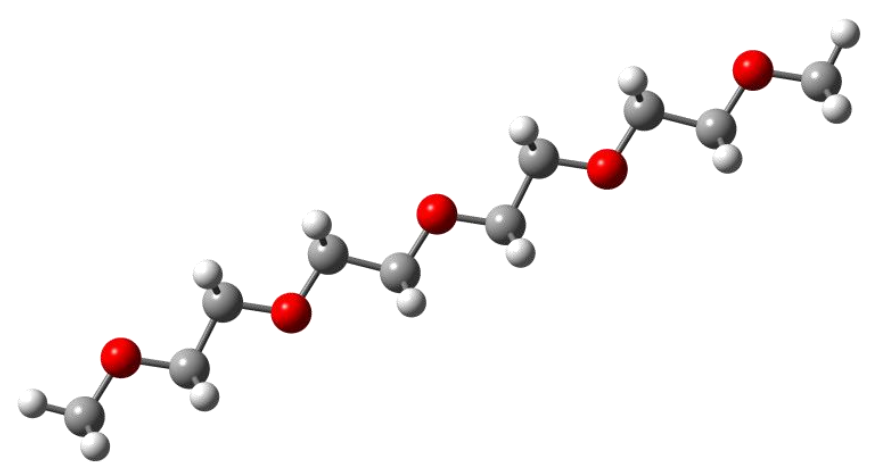

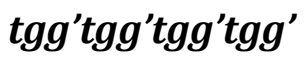

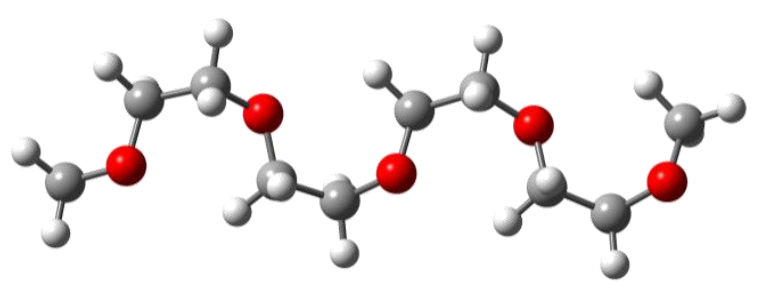

Figure 8.2: Structures of the four selected tetraglyme conformers.

Table 8.4: Computed energy differences $\Delta E_{0}$ in $\mathrm{kJ} \cdot \mathrm{mol}^{-1}$ between four selected tetraglyme conformations. 2D3 calculations were performed in Gaussian 09 Rev. E.01 while calculations including threebody dispersion (3D3) were performed in TURBOMOLE 7.0. For all methods the zero-point energies were added to the electronic structure calculations at the appropriate level.

\begin{tabular}{|c|c|c|c|c|}
\hline conformer & $\begin{array}{c}\text { stat. } \\
\text { weight }\end{array}$ & $\begin{array}{l}\text { B3LYP-2D3/ } \\
\text { def2TZVP }\end{array}$ & $\begin{array}{c}\text { B3LYP-2D3/ } \\
\text { aVTZ }\end{array}$ & $\begin{array}{c}\text { B3LYP-3D3/ } \\
\text { aVTZ }\end{array}$ \\
\hline $\operatorname{tg} t t g^{\prime} t \operatorname{tg} t \operatorname{tg} g$ & 2 & 0.00 & 0.00 & 0.00 \\
\hline $\operatorname{tg} t \operatorname{tgttg} t \operatorname{tg} t$ & 1 & 5.84 & 4.05 & 3.97 \\
\hline$t t t t t t t t t t t t$ & $1 / 2$ & 5.95 & 4.31 & 4.15 \\
\hline $\operatorname{tgg}^{\prime} \operatorname{tgg}^{\prime} \operatorname{tg} g^{\prime} \operatorname{tgg}^{\prime}$ & 2 & 7.02 & 6.62 & 7.02 \\
\hline
\end{tabular}

Table 8.5: Relative zero-point vibrational energies in $\mathrm{kJ} \cdot \mathrm{mol}^{-1}$ for four selected tetraglyme conformations. Frequencies for all methods but the 3D3 one were calculated in Gaussian 09 Rev. E.01. The B3LYP-3D3/aVTZ results were obtained using TURBOMOLE 7.0.

\begin{tabular}{|c|c|c|c|c|}
\hline ZPVE & tgttg'ttgttg'g & tgttgttgttgt & tttttttttttt & $\operatorname{tgg}^{\prime} \operatorname{tg} g^{\prime} \operatorname{tg} g^{\prime} \operatorname{tg} g^{\prime}$ \\
\hline $\begin{array}{c}\text { B3LYP- } \\
\text { 2D3/ } \\
\text { def2TZVP }\end{array}$ & 0.00 & -1.16 & -1.24 & 0.74 \\
\hline $\begin{array}{c}\text { B3LYP- } \\
\text { 2D3/ } \\
\text { aVTZ }\end{array}$ & 0.00 & -1.31 & -1.33 & 0.53 \\
\hline $\begin{array}{c}\text { B3LYP- } \\
\text { 3D3/ } \\
\text { aVTZ }\end{array}$ & 0.00 & -1.44 & -1.20 & 0.59 \\
\hline
\end{tabular}


Table 8.6: Energy differences $\Delta E_{0}$ in $\mathrm{kJ} \cdot \mathrm{mol}^{-1}$ between four selected tetraglyme conformations at the MP2/aVTZ level calculated in TURBOMOLE 7.0. Here electronic energies were computed at the MP2 level on structures from DFT optimizations (Table 8.4) and their appropriate zero-point corrections were added to the MP2/aVTZ energies.

\begin{tabular}{|c|c|c|c|c|c|}
\hline $\begin{array}{l}\text { electronic } \\
\text { energy }\end{array}$ & $\begin{array}{c}\text { structure } \\
\text { and } \\
\text { ZPVE }\end{array}$ & tgttg'ttgttg'g & tgttgttgttgt & ttttttttttt & $\operatorname{tgg}^{\prime} \operatorname{tgg}^{\prime} \operatorname{tgg} g^{\prime} \operatorname{tgg}$ \\
\hline MP2/aVTZ & $\begin{array}{c}\text { B3LYP- } \\
\text { 2D3/ } \\
\text { def2TZVP }\end{array}$ & 0.00 & 5.65 & 5.50 & 9.71 \\
\hline MP2/aVTZ & $\begin{array}{c}\text { B3LYP- } \\
\text { 2D3/ } \\
\text { aVTZ }\end{array}$ & 0.00 & 5.35 & 5.56 & 9.56 \\
\hline MP2/aVTZ & $\begin{array}{c}\text { B3LYP- } \\
\text { 3D3/ } \\
\text { aVTZ }\end{array}$ & 0.00 & 5.46 & 5.47 & 9.63 \\
\hline
\end{tabular}

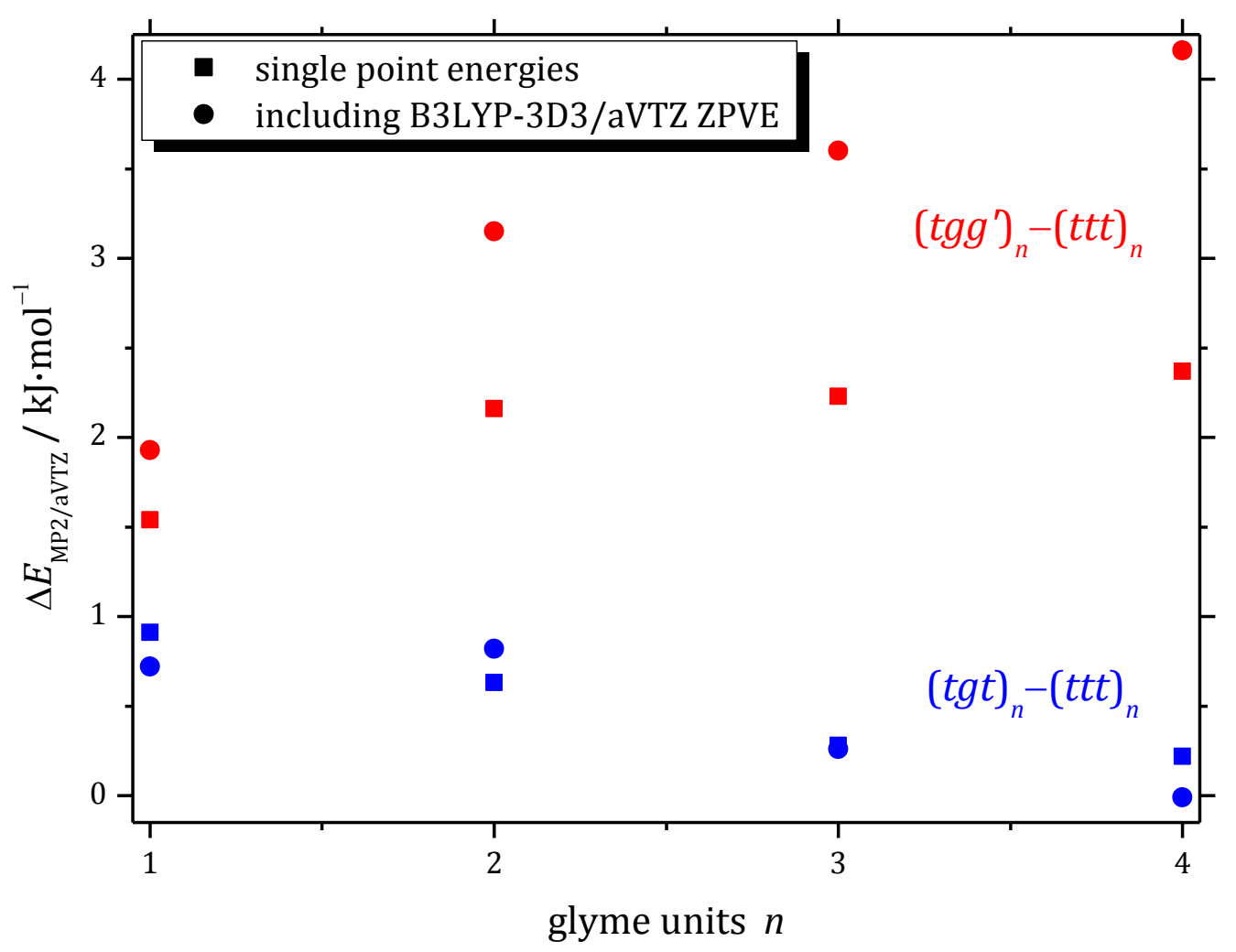

Figure 8.3: Energy differences of $(\operatorname{tg} t)_{n}$ (blue) and $(\operatorname{tgg})_{n}$ (red) compared to $(t t t)_{n}$ for mono- through tetraglyme. Single point energies were calculated at the MP2/aVTZ level from structures optimized at the B3LYP-3D3/aVTZ level. All calculations were carried out in TURBOMOLE 7.0. Square symbols show the differences in electronic energy of the MP2/aVTZ calculations, while circle symbols include zero-point corrections from the B3LYP-3D3/aVTZ calculations.

\section{3 - Raman jet spectra}

Figure 8.4 shows Raman jet spectra of mono- through tetraglyme in the low frequency region. For better comparability all spectra were scaled to equal integrated intensities over the shown frequency range. For orientation purposes calculated Raman scattering cross sections for all-ttt and all-tgt conformations at the B3LYP-3D3/aVTZ 
level are added to the plot and were scaled to equal sums over the shown spectral range for each glyme. The decreasing vapor pressure of tri- and tetraglyme, which is only partially counterbalanced by increasing saturator temperatures and CCD acquisition times, leads to a significant reduction in the signal-to-noise ratios in these spectra. Contributions of all-ttt and all-tgt are clearly visible in jet spectra of mono- and diglyme but become doubtful in triglyme. As a consequence of increasing stabilization of folded conformers over stretched structures the all-ttt abundance decreases with increasing glyme chain lengths and furthermore tetraglyme spectra do not show any evidence for presence of the all-ttt structure in jet expansions. Moreover, a broad multi gauche D-LAM band (LAM indicating conformational disorder [10,194,195]) becomes more prominent around $\sim 250-360 \mathrm{~cm}^{-1}$ in the tri- and tetraglyme spectra. Somewhat sharp and intense signals grow from the D-LAM background and might allow for an assignment of particular conformers even though the spectral congestion and increasing band overlaps will render specific assignments more challenging. The increasing intensity of the broad D-LAM band indicates the growing number of accessible multi gauche conformers at higher chain lengths. However, when going from trito tetraglyme the D-LAM band structure slightly refocuses indicating that the local environment of single dihedrals becomes more similar again. Initially the spectral main area shifts to lower wavenumbers when going from mono- to diglyme as the LAM band positions depend linearly on the inverse of the chain length'. However, this shift comes to a halt in tri- and tetraglyme as the D-LAM, which is mostly insensitive to the chain length, is becoming more prominent. This combined with the missing contributions of all-ttt in tetraglyme clearly reflects that folded conformers are more favored than stretched structures in jet expansions of tri- and tetraglyme.

† The dependency of the LAM band positions on the chain length are discussed in the following subchapter. 


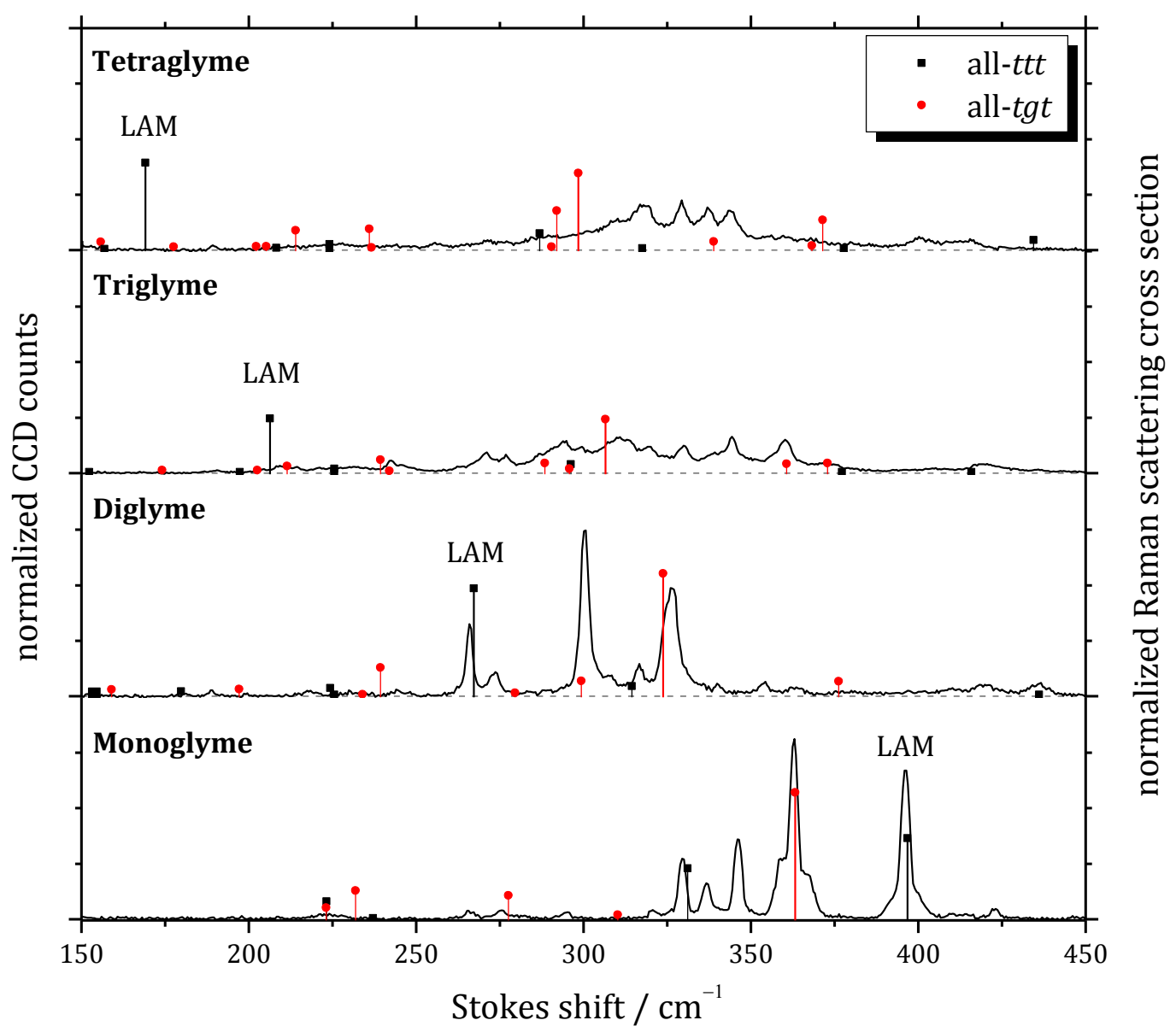

Figure 8.4: Low frequency Raman jet spectra of mono- through tetraglyme in He at a nozzle distance of $1 \mathrm{~mm}$ (further measurement conditions can be found in A.1). Spectra were scaled to equal integrated intensities from 150 to $450 \mathrm{~cm}^{-1}$. Raman scattering cross sections calculated at the B3LYP3D3/aVTZ level are added for orientation purposes and were scaled to equal sums over all cross sections from 150 to $450 \mathrm{~cm}^{-1}$ for each glyme.

Contributions of the most stable conformers cannot be assigned in the recorded Raman jet spectra of tri- and tetraglyme as the conformational landscapes are mostly left unexplored. However, relaxation effects during supersonic jet expansions shall be discussed briefly here as they provide valuable experimental benchmarks for future theoretical investigations on the conformational landscapes of these molecules. Triglyme spectra were normalized to the peak maximum at $360 \mathrm{~cm}^{-1}$ while tetraglyme spectra were normalized to the peak maximum of the band at $329 \mathrm{~cm}^{-1}$.

Jet expansion spectra of triglyme utilizing admixture of heavier atoms to the carrier gas do not differ significantly from pure helium expansions (Figure 8.5). Most noticeably the bands at around $\sim 311 \mathrm{~cm}^{-1}$ change their relative intensities in the different carrier gas environments. Pure helium expansions show a stronger peak at $311 \mathrm{~cm}^{-1}$ with a small shoulder at $313 \mathrm{~cm}^{-1}$, while expansions with admixture of $4 \%$ argon show two equally strong peaks at 310 and $312 \mathrm{~cm}^{-1}$. Additionally, mixed neon-helium expansions show a stronger peak at $309 \mathrm{~cm}^{-1}$ with a small shoulder at $313 \mathrm{~cm}^{-1}$. Besides that the only other noticeable difference in the spectra is a decreasing intensity of the band at $319 \mathrm{~cm}^{-1}$ in mixed argon-helium expansions. 
Helium jet expansions at different nozzle distances (Figure 8.6) show a significant increase of the background at shorter nozzle distances. This reflects that contributions of multi gauche conformers, that give rise to the broad D-LAM background, are more prominent at shorter nozzle distances and relax to some degree further downstream. Additionally, the bands around $311 \mathrm{~cm}^{-1}$ change their relative intensities. At a nozzle distance of $1.0 \mathrm{~mm}$ a stronger band at $311 \mathrm{~cm}^{-1}$ and a shoulder at $313 \mathrm{~cm}^{-1}$ is seen, while at 1.5 and $2.0 \mathrm{~mm}$ two equally strong bands at 310 and $313 \mathrm{~cm}^{-1}$ are visible.

Likewise an increase of the nozzle temperature (Figure 8.7) leads to a significant increase in the D-LAM background which complicates a comparison of the spectrum recorded at the highest nozzle temperature to the others. The most noticeable effects are seen in the changes of the relative intensities of the bands around $311 \mathrm{~cm}^{-1}$. At lower nozzle temperatures the stronger band at $310 \mathrm{~cm}^{-1}$ is accompanied by a smaller shoulder at $313 \mathrm{~cm}^{-1}$, while at the highest nozzle temperature two equally intense peaks are seen at 310 and $312 \mathrm{~cm}^{-1}$.

In general, only weakly pronounced relaxation effects are seen in jet expansions of triglyme. The spectral changes hint that contributions to the shoulder at $313 \mathrm{~cm}^{-1}$ might arise from conformers that profit from relaxation effects as it increases with intensity at larger nozzle distances. Additionally, conformers that are ranked higher in energy might contribute to a band just slightly down shifted from it as a band at $312 \mathrm{~cm}^{-1}$ becomes more prominent at high nozzle temperatures. However, these anticipated conformational contributions are highly speculative and need to be verified with the aid of quantum chemical predictions.

Variation of the carrier gas mixtures in jet expansions of tetraglyme (Figure 8.8) lead to some more pronounced relaxation effects. The band at $309 \mathrm{~cm}^{-1}$ clearly increases in intensity when neon is added to helium expansions. The decreasing intensity of other bands in mixed neon-helium expansions is explained by the decrease of the broad D-LAM background due to the increased collisional cooling during these expansions. In contrast, variation of the nozzle distance in mixed neon-helium expansions (Figure 8.9) show that the band at $309 \mathrm{~cm}^{-1}$ increases in intensity at shorter nozzle distances, while no pronounced relaxation effects are found for this band when a nozzle distance series is carried out in helium expansions (Figure 8.10). The broad D-LAM background significantly increases at short nozzle distances but stays constant after $1.5 \mathrm{~mm}$. Furthermore, the band at $345 \mathrm{~cm}^{-1}$ seems to decrease in intensity with decreasing nozzle distances but this assessment is strongly perturbed by the broad background in the spectrum at $0.5 \mathrm{~mm}$.

Similar to the triglyme spectra, jet expansions of tetraglyme do not show significant relaxation effects except for the band at $309 \mathrm{~cm}^{-1}$ that shows changes upon addition of neon to the expansion gas. This is reminiscent of an observation in diglyme expansions where large interconversion barriers were partially overcome upon addition of neon to the helium expansions. Therefore, this band is an interesting candidate to probe for with quantum chemical predictions. 


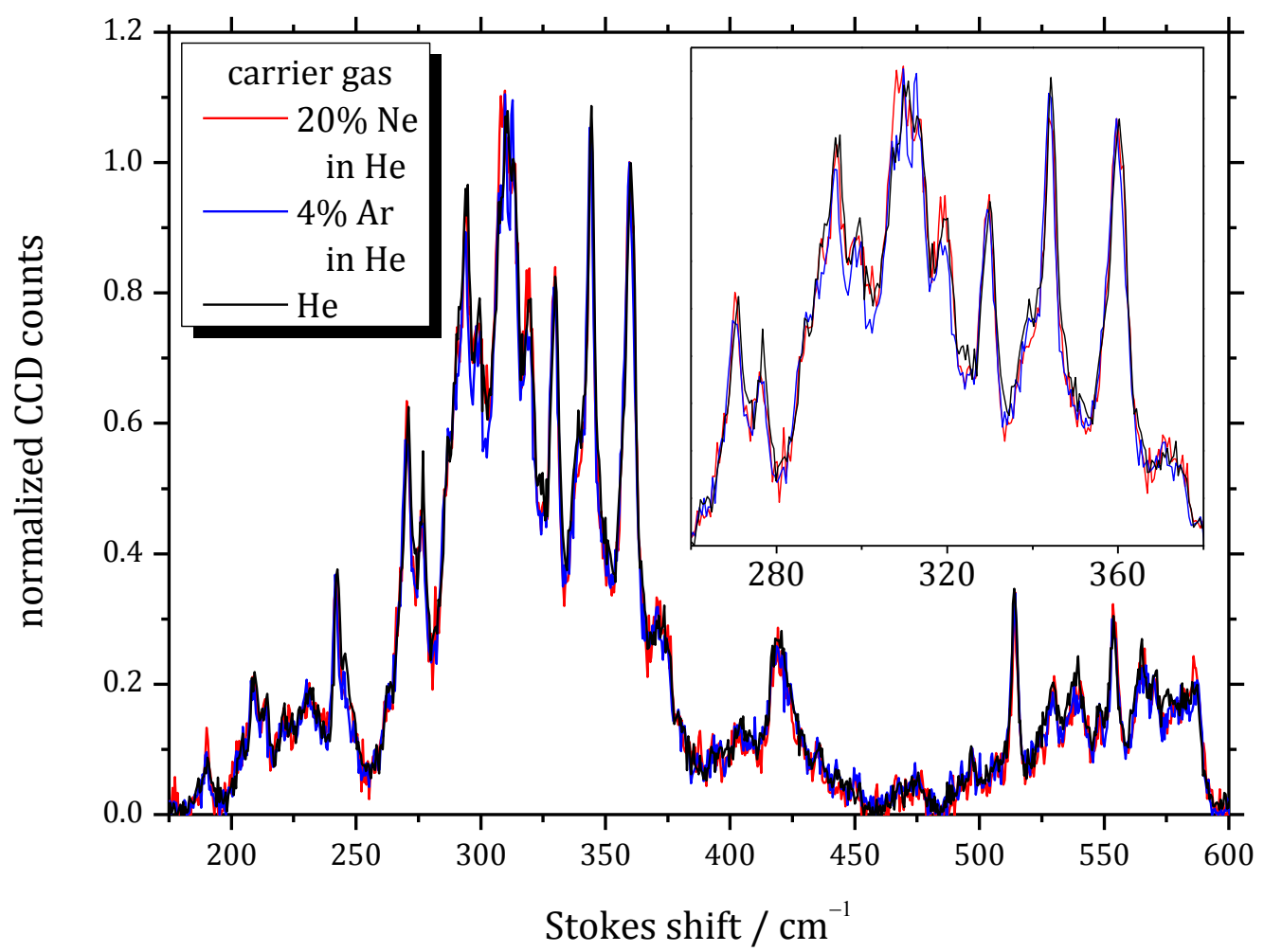

Figure 8.5: Jet expansions of triglyme in He (black), $4 \% \mathrm{Ar}$ in $\mathrm{He}$ (blue) and $20 \% \mathrm{Ne}$ in $\mathrm{He}$ (red) at $1 \mathrm{~mm}$ nozzle distance. Spectra were normalized to the maximum of the band at $360 \mathrm{~cm}^{-1}$. Other measurement parameters can be found in A.1.

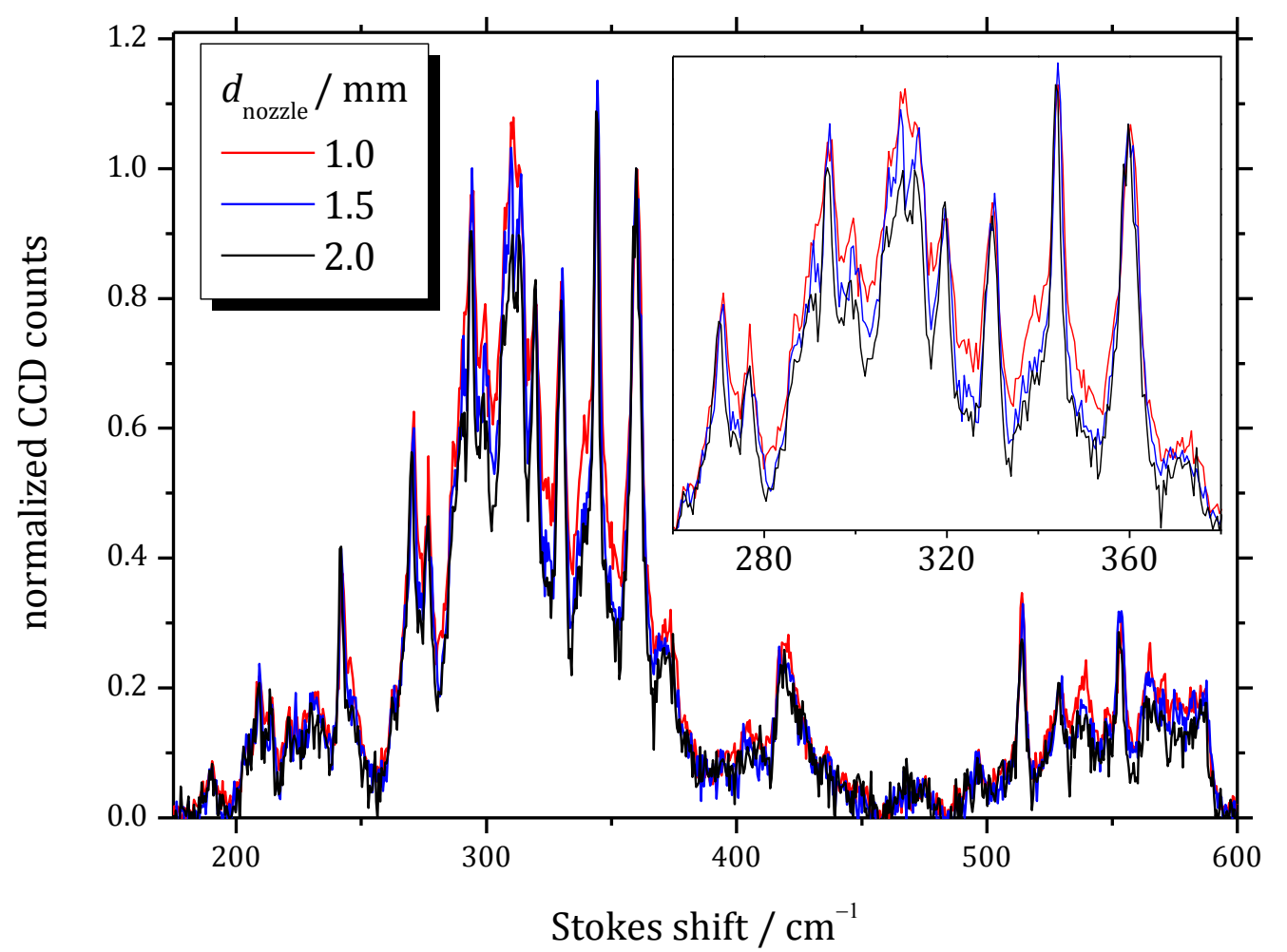

Figure 8.6: Jet expansions of triglyme in He at nozzle distances of 1.0 (red), 1.5 (blue) and $2.0 \mathrm{~mm}$ (black). Spectra were normalized to the maximum of the band at $360 \mathrm{~cm}^{-1}$. Other measurement parameters can be found in A.1. 


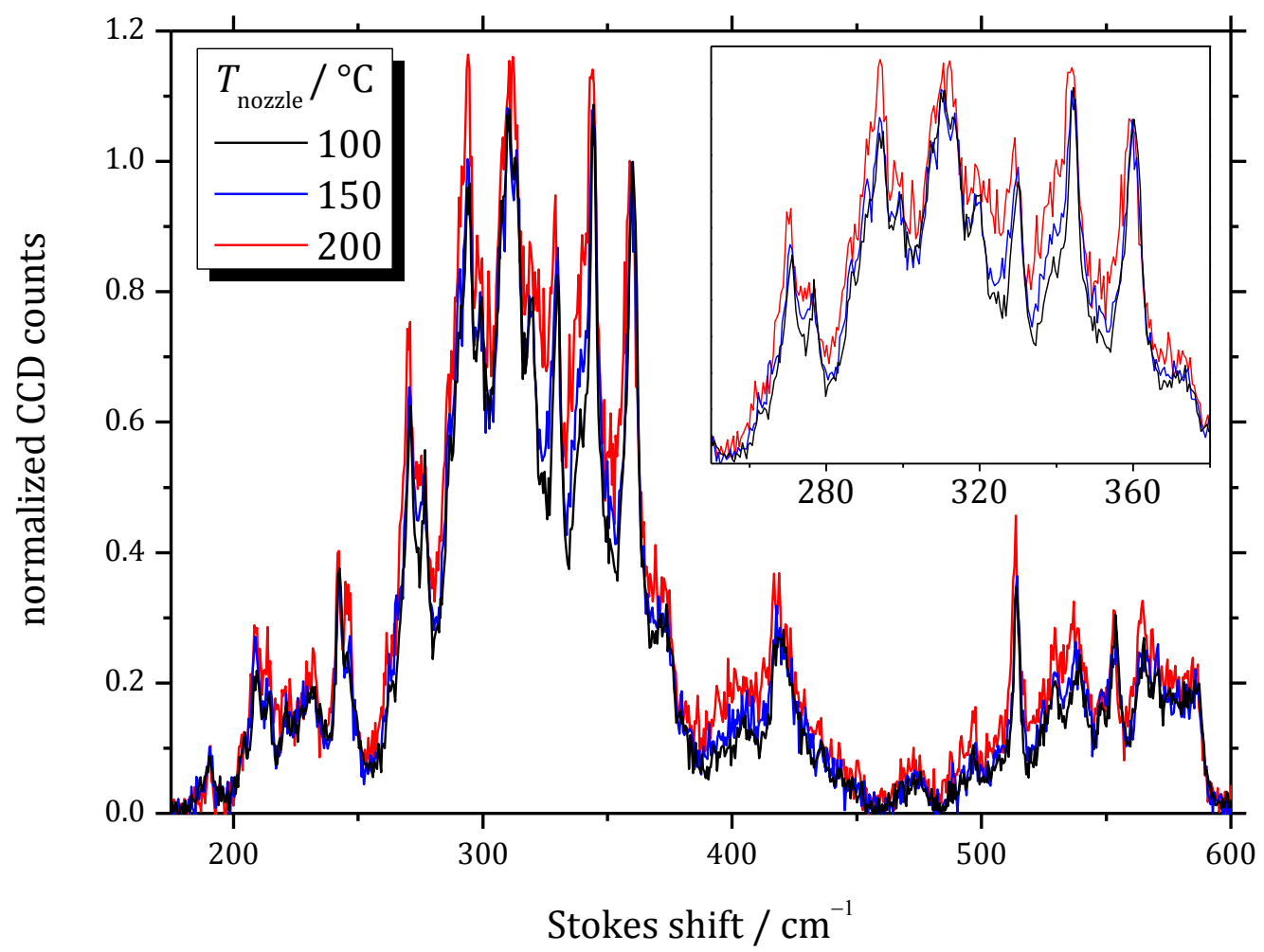

Figure 8.7: Jet expansions of triglyme in He at 100 (black), 150 (blue) and $200{ }^{\circ} \mathrm{C}$ (red) nozzle temperature. Spectra were normalized to the maximum of the band at $360 \mathrm{~cm}^{-1}$. Other measurement parameters can be found in A.1.

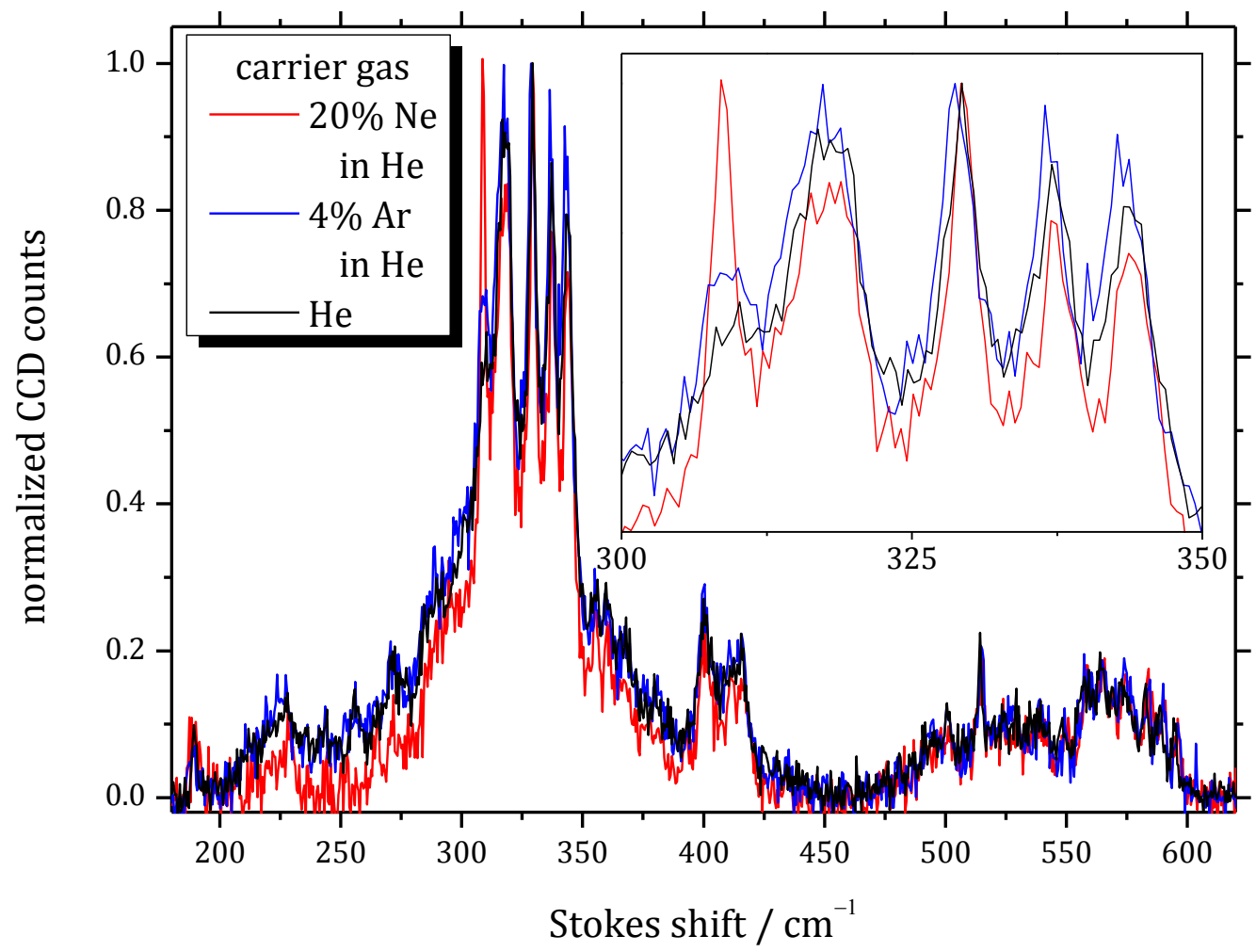

Figure 8.8: Jet expansions of tetraglyme in He (black), $4 \% \mathrm{Ar}$ in $\mathrm{He}$ (blue) and $20 \% \mathrm{Ne}$ in $\mathrm{He}$ (red) at $1 \mathrm{~mm}$ nozzle distance. Spectra were normalized to the maximum of the band at $329 \mathrm{~cm}^{-1}$. Other measurement parameters can be found in A.1. 


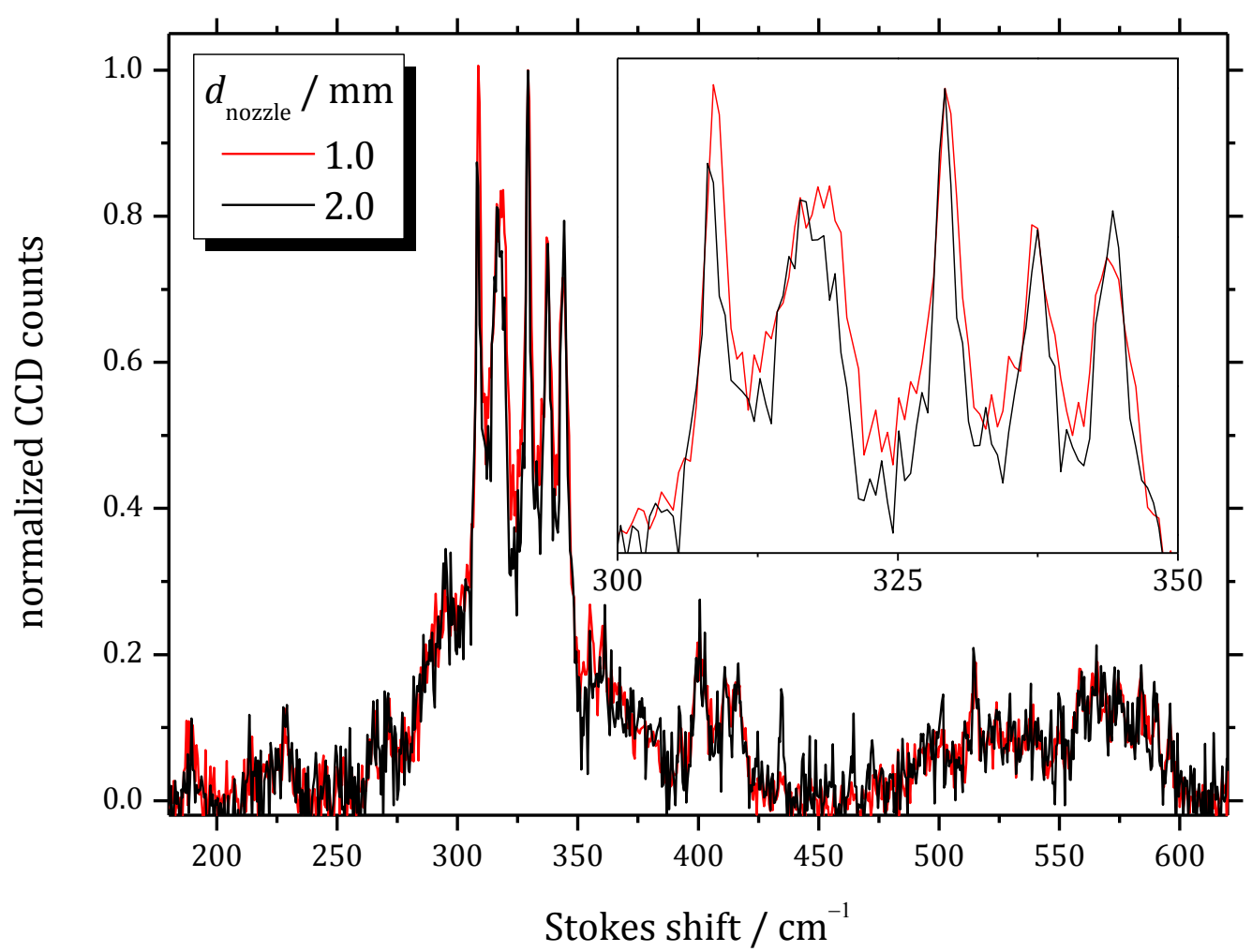

Figure 8.9: Jet expansions of tetraglyme in $20 \% \mathrm{Ne}$ in He at nozzle distances of 1.0 (red) and $2.0 \mathrm{~mm}$ (black). Spectra were normalized to the maximum of the band at $329 \mathrm{~cm}^{-1}$. Other measurement parameters can be found in A.1

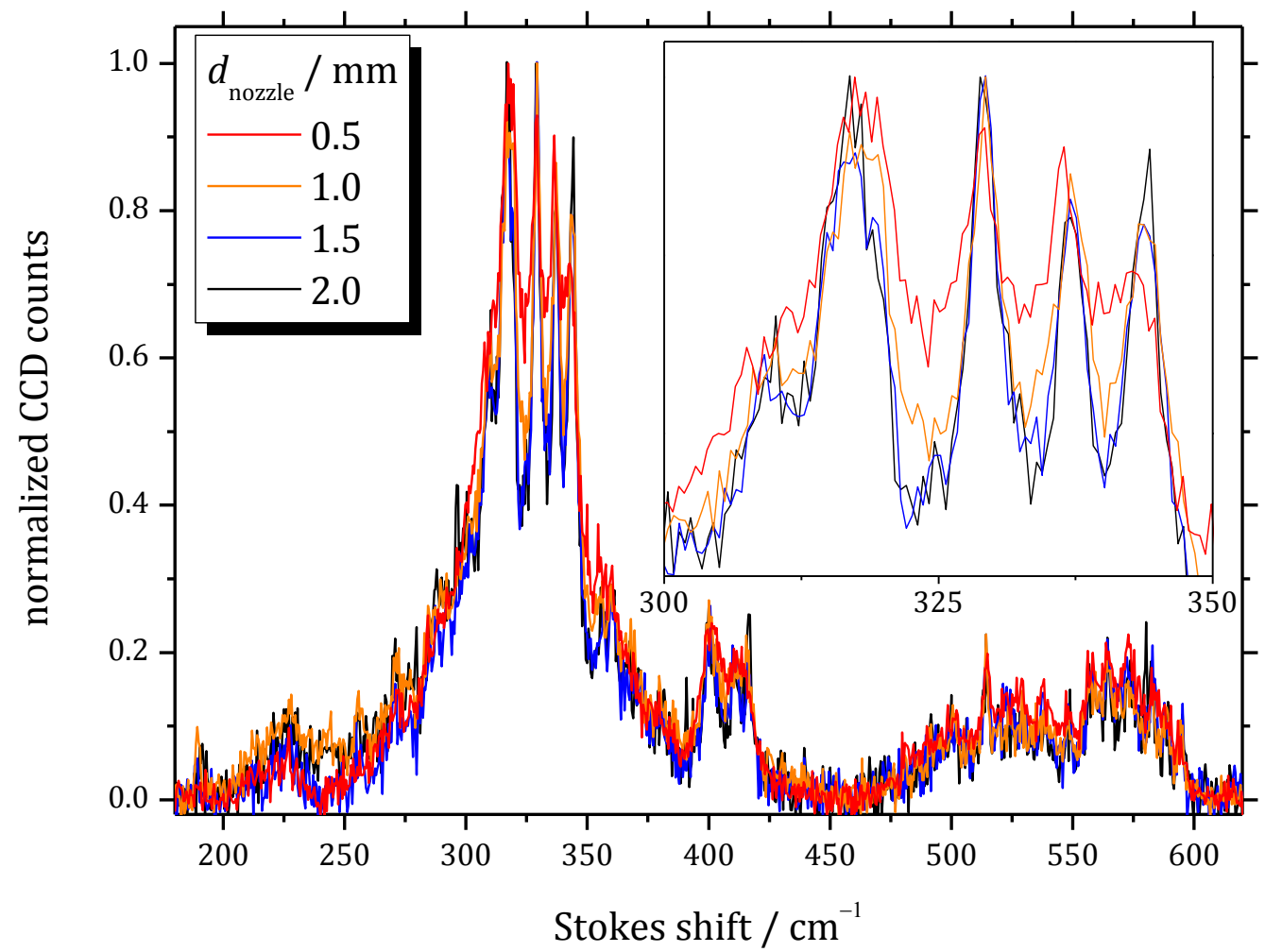

Figure 8.10: Jet expansions of tetraglyme in He at 0.5 (red), 1.0 (orange), 1.5 (blue) and $2.0 \mathrm{~mm}$ (black) nozzle distance. Spectra were normalized to the maximum of the band at $329 \mathrm{~cm}^{-1}$. Other measurement parameters can be found in A.1. 


\section{4 - Liquid phase Raman spectra}

Raman spectra of liquid samples of mono- trough tetraglyme and poly(ethylene glycol) dimethyl ether (PEG) with an average molecular weight of $500 \mathrm{~g} \cdot \mathrm{mol}^{-1}$ were recorded using a commercially available Raman microscope (WITec, alpha300R). Figure 8.11 shows a comparison of their Raman spectra. In order to enable a good comparability all spectra were normalized to equal integrals over the $\mathrm{CH}$-stretching region assuming that the laser probes equal amounts of $\mathrm{CH}$ stretches in all samples. Unsurprisingly, spectra of longer glyme chains are very similar to the spectrum of the polymer sample. Larger deviations are found in spectra of monoglyme because the end group vibrations play a more important role. Therefore, the $\mathrm{CH}$-stretching region shows more structure in mono- and diglyme than it does in spectra of longer glymes. The observed vibrations are assigned to their corresponding motions in detail in References $[116,147,196]$ and thus shall only be discussed briefly here.

The wavenumber region between 150 and $1750 \mathrm{~cm}^{-1}$ (Figure 8.12) shows an increase in intensity with increasing glyme chain lengths for vibrations that correspond to $\mathrm{CH}_{2}$ motions, such as the band at $1285 \mathrm{~cm}^{-1}\left(\mathrm{CH}_{2}\right.$ twisting motion). Additionally, the relative intensities of the bands at 1450 and $1474 \mathrm{~cm}^{-1}$ change with increasing chain lengths. The former corresponds to $\mathrm{CH}_{3}$ deformation while the latter is assigned to a $\mathrm{CH}_{2}$ scissoring motion, consistent with a significant increase of the former band in monoglyme. Contribution of the tgt structure is mainly found in monoglyme spectra and thus bands that are assigned to tgt show a significant increase in intensity, e.g. bands at 360 and $848 \mathrm{~cm}^{-1}$. At larger chain lengths the conformational diversity increases giving rise to the broad D-LAM band at $\sim 295 \mathrm{~cm}^{-1}$.

Consistently, a comparison of the liquid phase and jet spectra of mono- through tetraglyme in the LAM region (Figure 8.13) shows that the liquid phase spectra match the jet spectra reasonably well at short chain lengths as both show major contributions of the same structures (all-tgt, all-ttt) but become very broad and rather unspecific (D-LAM) at longer chain lengths. Moreover, the spectral center is slightly downshifted in liquid spectra of tri- and tetraglyme compared to the corresponding jet spectra which might indicate that the major contributing conformers are different in both phases. Thus, randomly coiled conformers in the liquid phase possess longer consecutive sections of stretched backbone dihedrals than those in supersonic jet expansions. 


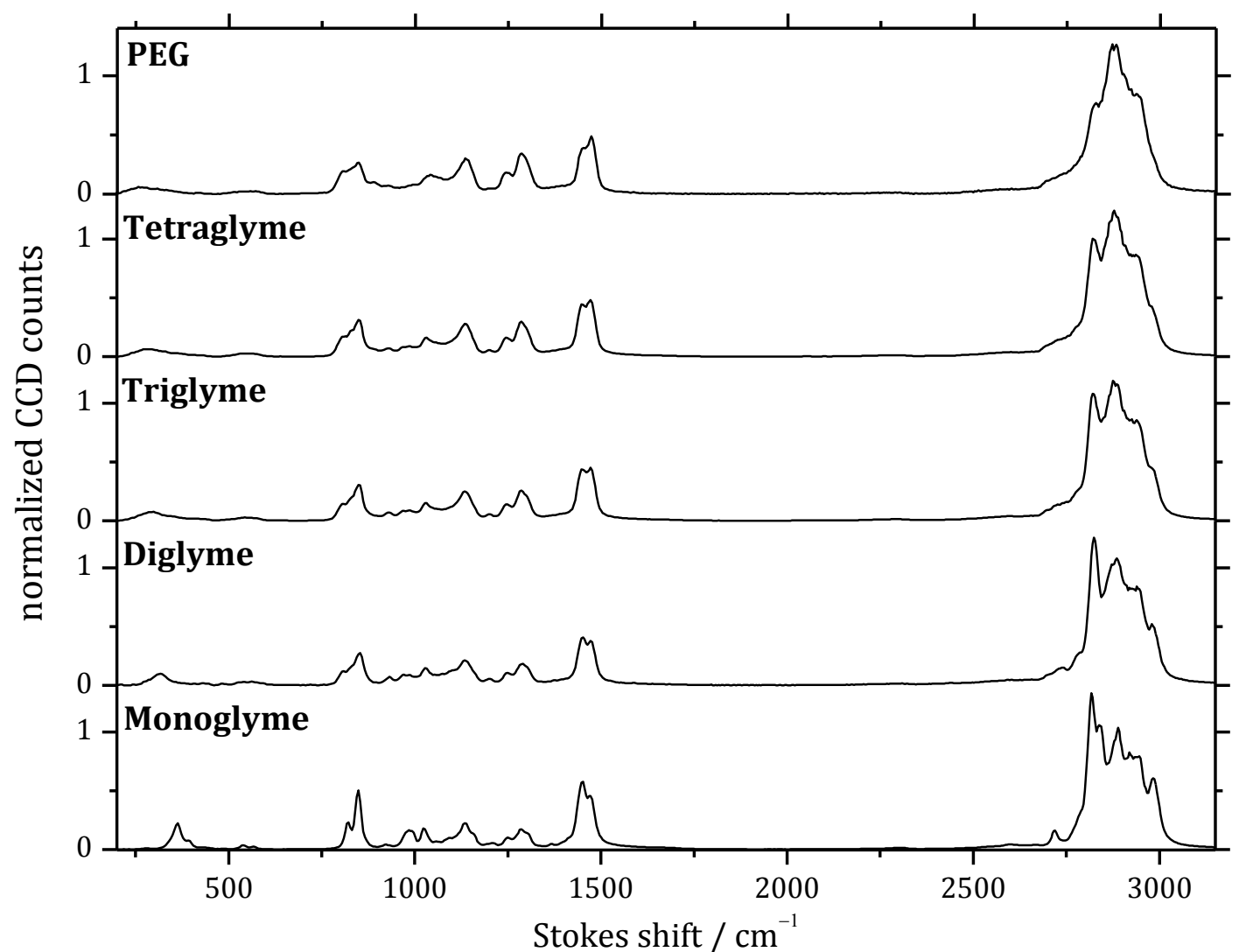

Figure 8.11: Raman spectra of liquid samples of mono- through tetraglyme and poly(ethylene glycol) dimethyl ether (PEG) with an average molecular weight of $500 \mathrm{~g} \cdot \mathrm{mol}^{-1}$. Spectra were normalized to equal integrals over the $\mathrm{CH}$ stretching region assuming that the laser probes equal amounts of $\mathrm{CH}$ stretches in each sample.

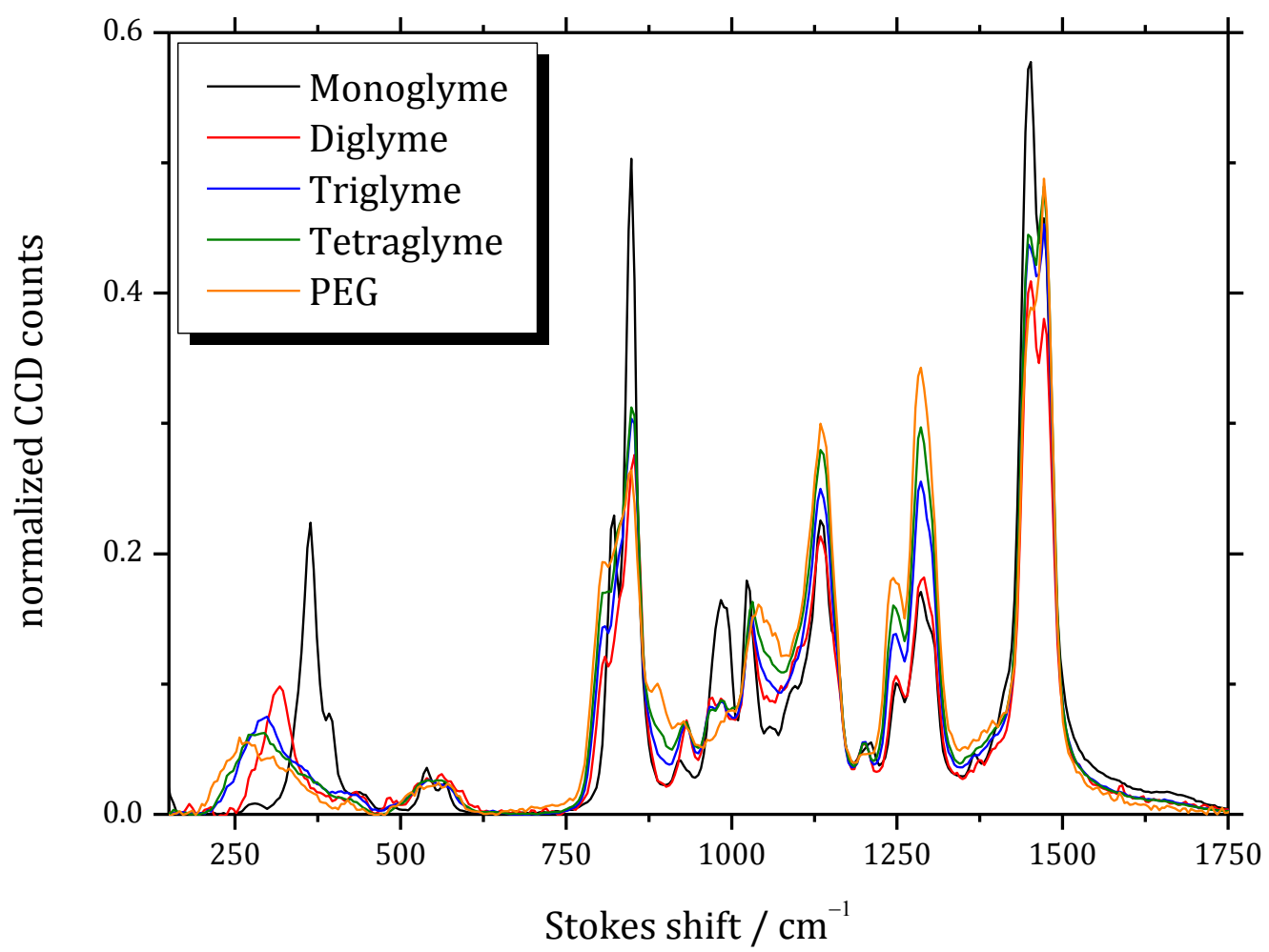

Figure 8.12: Raman spectra of liquid samples of mono- through tetraglyme and poly(ethylene glycol) dimethyl ether (PEG) with an average molecular weight of $500 \mathrm{~g} \cdot \mathrm{mol}^{-1}$ in the wavenumber region between 150 and $1750 \mathrm{~cm}^{-1}$. Spectra were normalized to equal integrals over the $\mathrm{CH}$ stretching region (not shown) assuming that the laser probes equal amounts of $\mathrm{CH}$ stretches in each sample. 


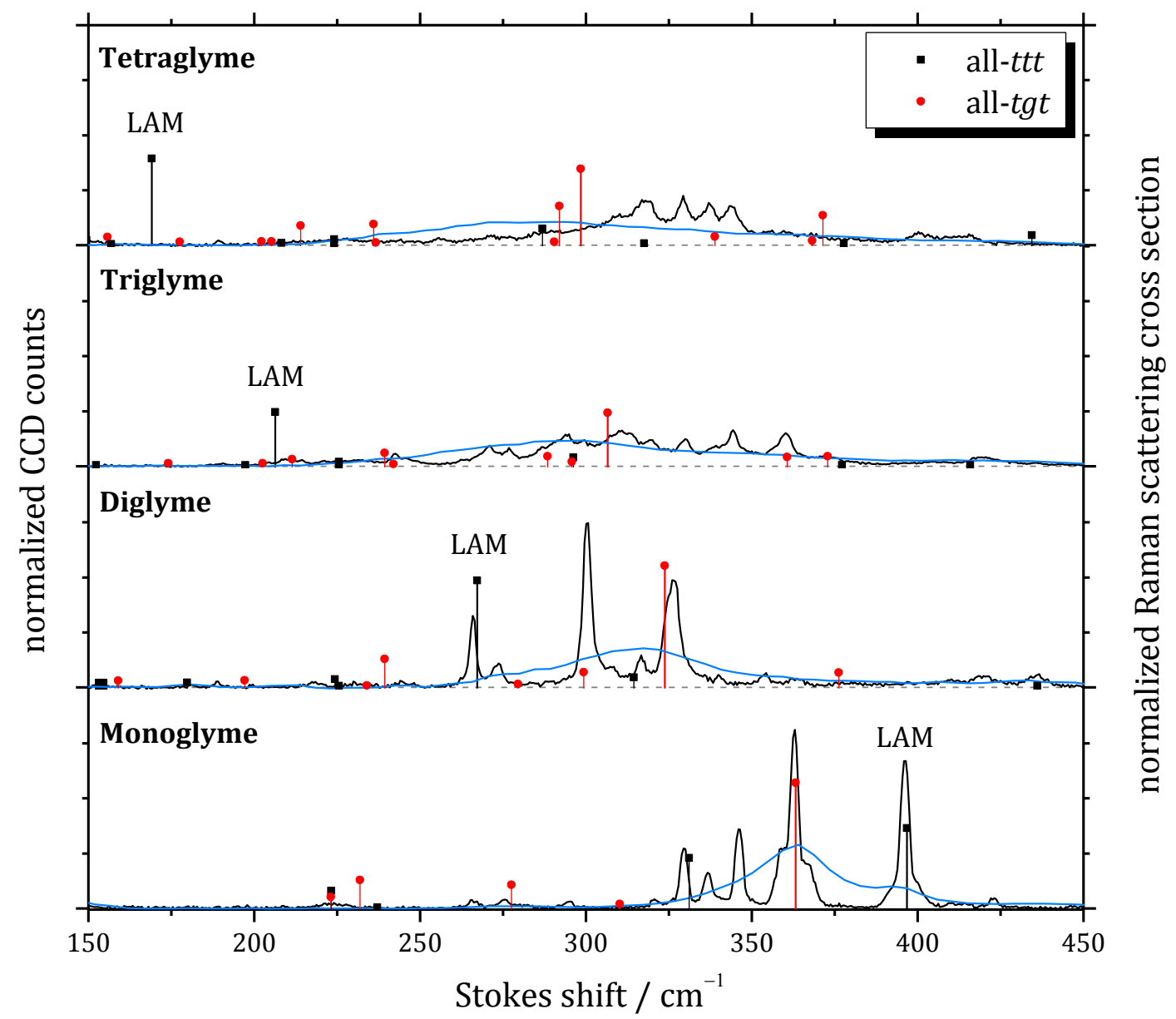

Figure 8.13: Comparison between Raman spectra of mono- through tetraglyme in supersonic jet expansions (black) in He at $1 \mathrm{~mm}$ nozzle distance and the liquid phase (blue). All spectra were scaled to equal integrated intensities from 150 to $450 \mathrm{~cm}^{-1}$. Raman scattering cross sections calculated at the B3LYP-3D3/aVTZ level are added for orientation purposes and were scaled to equal sums over all cross sections from 150 to $450 \mathrm{~cm}^{-1}$ for each glyme.

\section{5 - Modulus of elasticity}

Previously it was shown for alkane chains of finite lengths that the wavenumber position of the LAM bands can be used in order to extrapolate the elastic or Young's modulus of an infinite, stretched, single polymer strand $[14,17,18]$. In contrast to bulk measurements, extrapolating the elastic modulus from jet spectroscopic data allows deriving its value free from intermolecular interactions. The elastic modulus $(E)$ is a measure for the stiffness of a material and is defined as the ratio between stress (force per area, $F / A$ ) and strain (proportional deformation, $\Delta l / I$ ).

$$
E=\frac{\text { stress }}{\text { strain }}=\frac{F / A}{\Delta l / l}
$$

The connection between this macroscopic property and spectroscopic data is made by the fact that the longitudinal modes of extended chain molecules can be understood as vibrations of homogenous rods in the limit of long chain lengths [197]. The 
length of the molecular rod is linked to the molecular chain length $a^{\dagger}$, e.g. $a=6$ for mono- and $a=9$ for diglyme, by:

$$
l=a d+c
$$

Where $d$ is the segment length, related to $\mathrm{C}-\mathrm{O}$ and C-C bond lengths and angles in the case of glyme chains, and the constant $c$ accounts for terminal chain atoms which become negligible at long chain lengths. The wavenumber position $\tilde{v}$ of longitudinal acoustic modes can be expressed as:

$$
\tilde{v}=\frac{m}{2 c_{0} l} \sqrt{\frac{E}{\rho}}
$$

Where $\rho$ is the density of the material, $c_{0}$ is the speed of light in vacuum and $m$ refers to the number of nodes of the LAM. The rod length $l$ can be replaced by ad omitting the constant $c$ in the long chain length limit.

$$
\tilde{v}=\frac{1}{a} \cdot \frac{m}{2 c_{0} d} \sqrt{\frac{E}{\rho}}
$$

This shows that a plot of the LAM wavenumber against the inverse of the chain length yields a linear relation when the segment separation $d$ and the density $\rho$ are constant. The slope of this plot is defined as:

$$
S=\frac{m}{2 c_{0} d} \sqrt{\frac{E}{\rho}}
$$

And the elastic modulus is obtained for $m=1$ through:

$$
E=4 \rho\left(c_{0} d S\right)^{2}
$$

In order to reach the homogenous rod limit spectroscopic data at long chain lengths are needed to extrapolate to an infinite chain length. Unfortunately, the jet spectra of glyme chains only allow determining LAM band positions for mono- and diglyme perhaps tentatively triglyme. Therefore, LAM-1* band positions are predicted by quantum chemical calculations for the all-ttt conformation of mono- through pentadecaglyme and compared to the limited experimental data set. LAM band positions were calculated at the B3LYP-2D3/def2TZVP and HF/STO-3G level in Gaussian 09 Rev. E.01. The obtained values are listed in Table 8.7. In some cases $(n=4,5)$ intense mode mixing of the LAM-1 and transverse acoustic modes (TAM) of the same symmetry involving several nodes was found. Recovering the deperturbed frequencies was accomplished by a first-order perturbation approach $[17,18,198]$ in the form of:

$$
\Delta \tilde{v}_{0}=\Delta \tilde{v} \cdot \frac{R-1}{R+1}
$$

\footnotetext{
† The molecular chain length (heavy atoms in the molecular backbone) is abbreviated $a$ in order to avoid confusion with the number of glyme units that was defined as $n$. The relationship between $a$ and $n$ is: $a=3 \cdot n+3$

* LAM-1 refers to the longitudinal acoustic mode with one node $(m=1)$. 
Here $\Delta \tilde{v}_{0}$ refers to the deperturbed wavenumber difference between the LAM and the TAM, while $\Delta \tilde{v}$ refers to the perturbed wavenumber difference and $R$ stands for the ratio of the Raman scattering cross sections $\left(R=\sigma_{\mathrm{LAM}}^{\prime} / \sigma_{\mathrm{TAM}}^{\prime}\right)$. Figure 8.14 shows the effect of the perturbation and illustrates the necessity of deperturbation to recover the linear relationship between the LAM/TAM wavenumber and the inverse chain length. For hexaglyme $(n=6)$ a smaller perturbation is still noticeable, but recovering the deperturbed frequencies following Eq. 8.7 leads to an overcompensation of this effect. Hence, the deperturbation was omitted and only performed for tetra- and pentaglyme $(n=4,5)$. For other glymes the band position differences between the LAM and the TAM wavenumbers are too large to allow for noticeable perturbations.

Table 8.7: Experimental and predicted LAM-1 wavenumbers calculated the B3LYP-2D3/def2TZVP and HF/STO-3G level in Gaussian 09 Rev. E.01 for $(t t t)_{n}$ with $n=1-15$ glyme units (mono- through pentadecaglyme). Deperturbation of the LAM-1 position was performed for $n=4,5$. Perturbed positions are listed in parentheses.

\begin{tabular}{|c|c|c|c|}
\hline \multirow[b]{2}{*}{$n$} & \multicolumn{2}{|c|}{ predicted LAM-1 wavenumber $/ \mathrm{cm}^{-1}$} & \multirow{2}{*}{$\begin{array}{l}\text { experimental LAM-1 } \\
\text { wavenumber } / \mathrm{cm}^{-1}\end{array}$} \\
\hline & $\begin{array}{c}\text { B3LYP- } \\
\text { 2D3/def2TZVP }\end{array}$ & & \\
\hline 1 & 396.7 & 422.7 & 397 \\
\hline 2 & 267.2 & 295.5 & 266 \\
\hline 3 & 206.2 & 226.8 & 209 \\
\hline 4 & $164.9(169.1)$ & $182.6(186.4)$ & \\
\hline 5 & $139.7(131.6)$ & $154.2(143.6)$ & \\
\hline 6 & 116.7 & 127.4 & \\
\hline 7 & 103.2 & 112.1 & \\
\hline 8 & 91.3 & 100.1 & \\
\hline 9 & 82.7 & 90.6 & \\
\hline 10 & 75.5 & 82.4 & \\
\hline 11 & 69.1 & 75.7 & \\
\hline 12 & 63.9 & 70.0 & \\
\hline 13 & 59.4 & 64.9 & \\
\hline 14 & 55.4 & 60.7 & \\
\hline 15 & 52.0 & 57.0 & \\
\hline
\end{tabular}




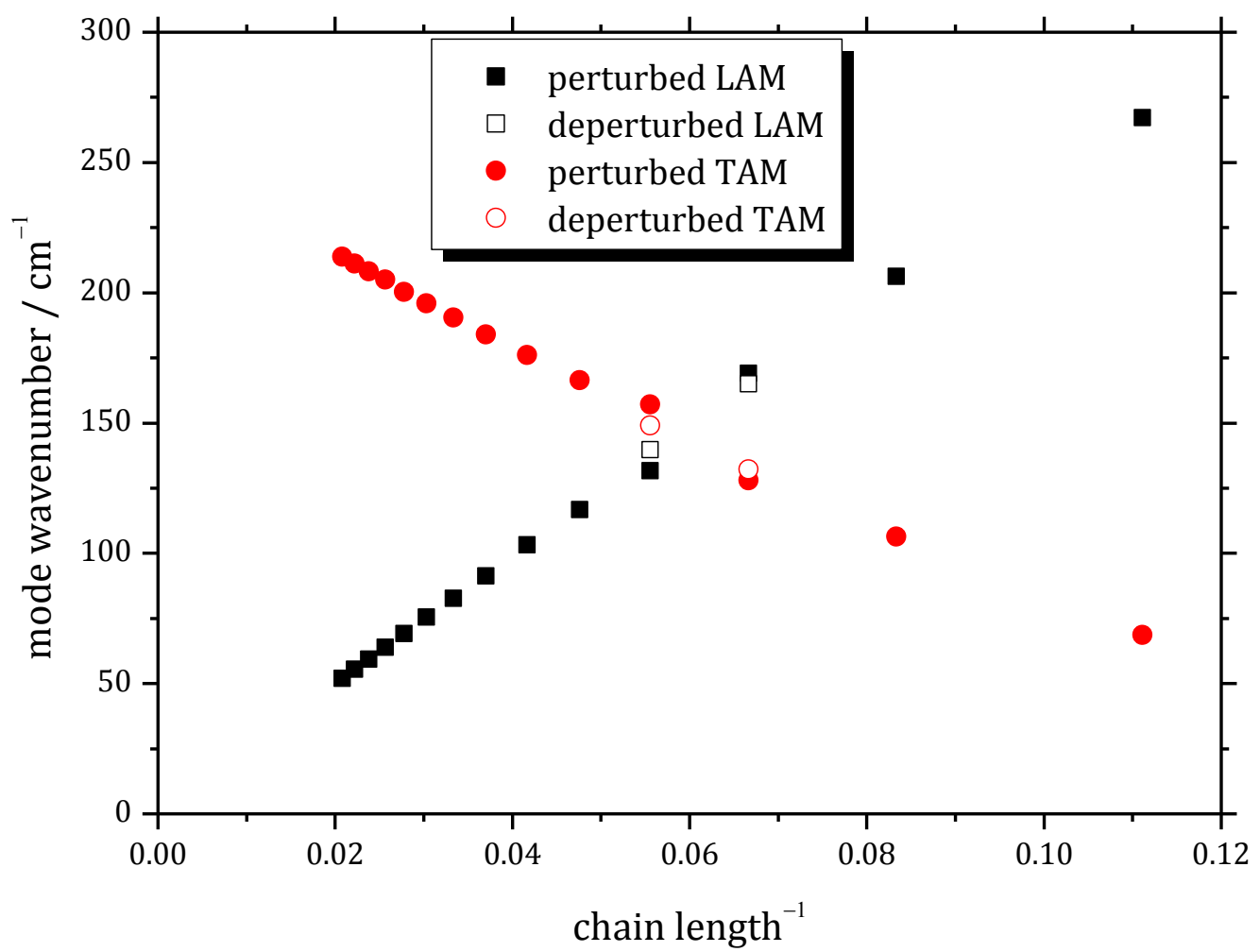

Figure 8.14: Predicted band positions of the LAM (black squares) and TAM (red circles) calculated at the B3LYP-2D3/def2TZVP level in Gaussian 09 Rev. E.01 for $(t t t)_{n}$ with $n=1-15$ glyme units (monothrough pentadecaglyme) plotted against the inverse chain length. Open symbols display the deperturbed band positions after corrections following Eq. 8.7.

A plot of the LAM-1 wavenumbers predicted at the B3LYP-2D3/def2TZVP level against the inverse of the chain length is shown in Figure 8.15. The linear relationship is fulfilled well for glyme chains containing three and more glyme units $(a \geq 12)$ as a linear fit (blue line, forced intercept at 0 ) of this data set shows. Results for mono- and diglyme slightly deviate from the linear relationship because of their short chain lengths that do not fulfill the homogenous rod approximation. Experimentally observed LAM-1 positions for mono-, di- and triglyme agree well with the predicted positions. Also, LAM positions calculated at the B3LYP-2D3/aVTZ level for $n=1-5$ show good agreement with the def2TZVP results. Analogously, the LAM-1 positions were deperturbed for $n=4,5$ (Eq. 8.7). Due to the increased computation cost this basis set was only carried out for mono- to pentaglyme. However, due to the good agreement between def2TZVP, aVTZ and experimental results it can be concluded that the evaluation with the def2TZVP basis set leads to fairly accurate results that should only deviate slightly with larger basis sets. Addition of three-body dispersion corrections for $n=1-5$ had no significant influence on the slope $\mathrm{S}$ of the linear fit and were thus omitted. A linear fit of similar quality is achieved when using the HF/STO-3G data for $n=3$ 15 (Figure 8.16). However, the Hartree-Fock calculations predict the LAM-1 positions systematically too high leading to a larger slope $S$. 


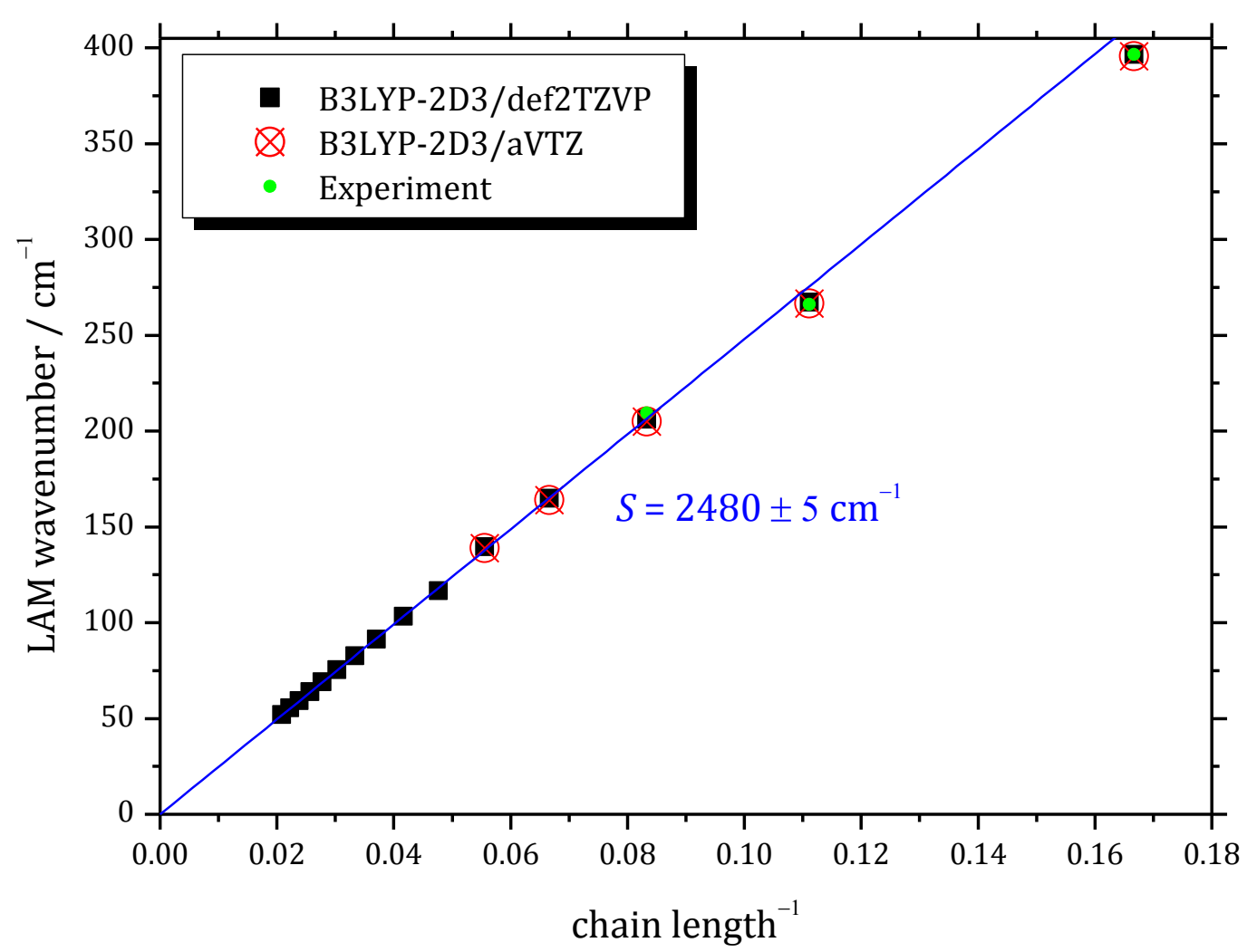

Figure 8.15: LAM-1 band positions predicted by B3LYP-2D3/def2TZVP calculations (black squares) of the all-ttt conformations. The linear fit (forced intercept at 0) shown in blue only uses LAM wavenumbers for glymes with $n=3-15$. Obtained experimental band positions are indicated with green circles and predicted band positions calculated at the B3LYP-3D3/aVTZ level are shown as crossed red circles.

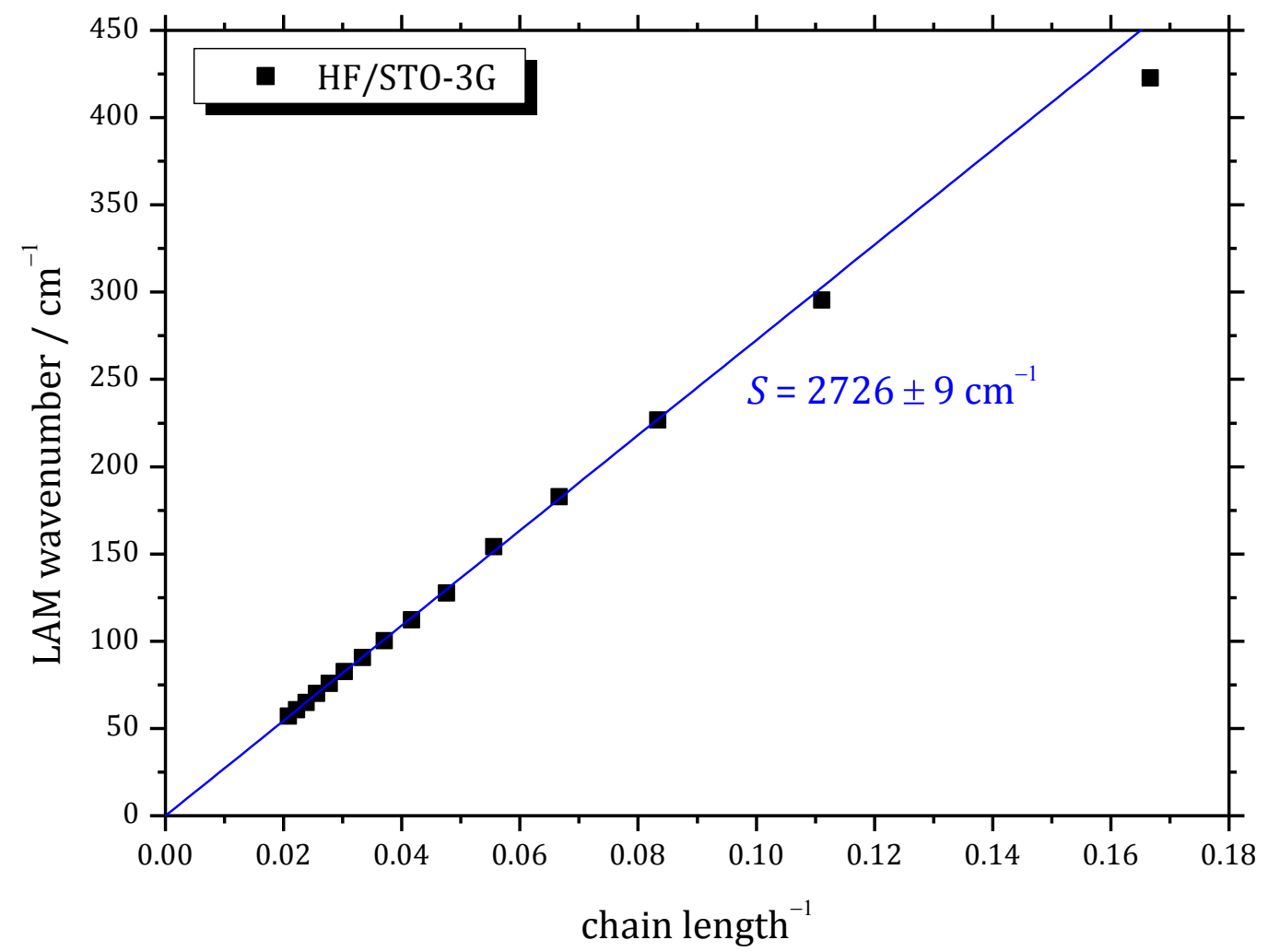

Figure 8.16: LAM-1 band positions predicted by HF/STO-3G calculations (black squares) of the all-ttt conformations against the inverse chain length. The linear fit (forced intercept at 0 ) shown in blue only uses LAM wavenumbers for glymes with $n=3-15$. 
A similar evaluation was done for the $(\operatorname{tg} t)_{n}$ structures which are the dominant structural motif in solid phases of PEO $[190,191]$. Due to the decreased symmetry and increased computational time compared to $(t t t)_{n}$ structures calculations for $(\operatorname{tg} t)_{n}$ were only carried out on the far-reaching HF/STO-3G level for $n=1-13$. The situation of $(\operatorname{tg} t)_{n}$ conformations is somewhat special as they form helical arrangements that need a certain number of glyme units to complete a full helix turn (compare Figure 8.2). At long chain lengths the $(\operatorname{tg} t)_{n}$ conformation can be envisioned as a helical spring that has two different elongation possibilities. The first is analogous to the elongation of stretched $(t t t)_{n}$ chains and involves simultaneous bending of COC angles leading to a similar longitudinal band wavenumber progression with chain length. The second elongation possibility arises from tilting of the dihedral backbone angles leading to a chain length dependent band wavenumber progression in the optical branch. The obtained band positions at the HF/STO-3G level are shown in Table 8.8. Deperturbation (Eq. 8.7) was performed for the LAM of $n=9$. Figure 8.17 shows the predicted band positions for $n=1-13$. The optical and acoustic branches can be easily separated in the infinite chain length limit. While the acoustic branch approaches a wavenumber of $0 \mathrm{~cm}^{-1}$ the optical branch converges to a value of $\approx 295 \mathrm{~cm}^{-1}$ (see Table 8.8). For chain lengths containing four and more glyme units a linear relationship for the LAM is fulfilled well as a fit (blue line, forced intercept at 0 ) of this data set shows. However, the slope $S$ of this linear regression is almost four times smaller than the slope of the extrapolated $(t t t)_{n}$ data set. Thus, the helical $(\operatorname{tg} t)_{n}$ arrangement is more soft than the rather stiff $(t t t)_{n}$ chain.

Table 8.8: Predicted LAM-1 wavenumbers calculated at the B3LYP-2D3/def2TZVP and HF/STO-3G level in Gaussian 09 Rev. E.01 for $(\operatorname{tg} t)_{n}$ with $n=1-13$ glyme units (mono- through tridecaglyme). Deperturbation of the LAM- 1 position was performed for $n=9$. Perturbed positions are listed in brackets.

\begin{tabular}{|c|c|c|}
\hline$n$ & $\begin{array}{c}\text { predicted LAM-1 wavenumber } \\
/ \mathrm{cm}^{-1} \\
\text { HF/STO-3G }\end{array}$ & $\begin{array}{c}\text { predicted optical mode wave- } \\
\text { number } / \mathrm{cm}^{-1} \\
\text { HF } / \text { STO-3G }\end{array}$ \\
\hline 1 & 84.4 & 419.6 \\
\hline 2 & 73.0 & 368.8 \\
\hline 3 & 56.5 & 335.8 \\
\hline 4 & 49.9 & 316.4 \\
\hline 5 & 38.7 & 310.8 \\
\hline 6 & 32.6 & 306.4 \\
\hline 7 & 29.3 & 303.0 \\
\hline 8 & 25.3 & 300.6 \\
\hline 9 & $21.6(21.1)$ & 298.8 \\
\hline 10 & 21.1 & 297.4 \\
\hline 11 & 18.7 & 296.5 \\
\hline 12 & 16.3 & 295.7 \\
\hline 13 & 16.2 & 295.0 \\
\hline
\end{tabular}




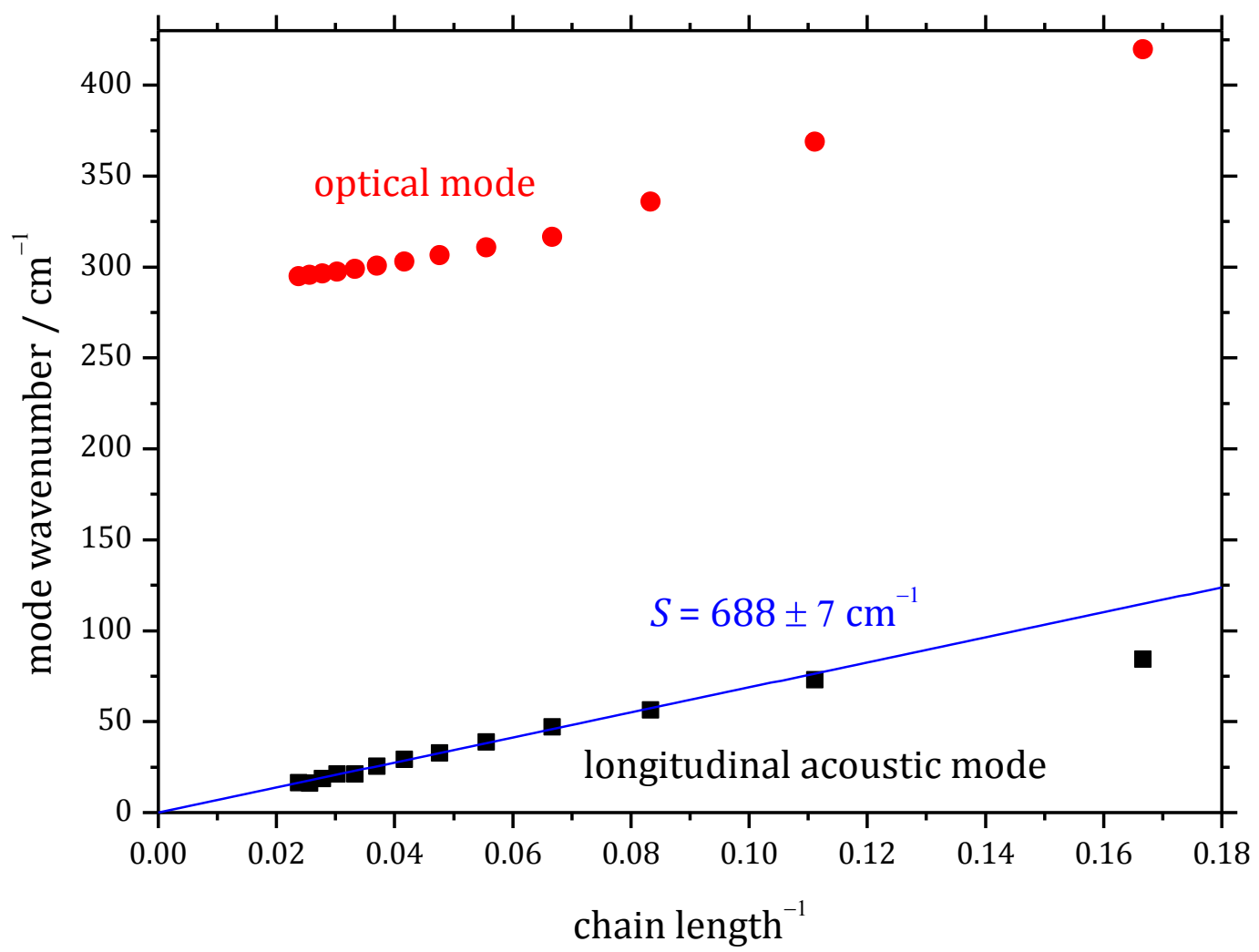

Figure 8.17: Band positions of the LAM-1 (black) and optical mode (red) predicted by HF/STO-3G calculations of the all-tgt conformations against the inverse chain length. The linear fit (forced intercept at 0 ) shown in blue only uses LAM wavenumbers for glymes with $n=4-13$.

The slopes of the linear fits can be used to estimate the elastic modulus of a single isolated polyglyme chain in vacuum at low temperatures. Calculations of the elastic moduli require the segment separation $d$ and the density $\rho$ (Eq. 8.6) which can be obtained from crystal structure data. For a better comparison and impression on the accuracy of this method the segment separation is also derived from the appropriate quantum chemical calculations. As mentioned above, crystal structures of PEO are only available for the helical $(\operatorname{tg} t)_{n}$ arrangement. The experimental segment separation $d$ is obtained from crystal structure data reported in Reference [191], in good agreement with earlier reported data [190], yielding $0.093 \mathrm{~nm}$. A PEO density $\rho$ of $1.23 \mathrm{~kg} \cdot \mathrm{dm}^{-3}$ is used in agreement with References $[190,200]$. The extrapolated $(t t t)_{n}$ slope cannot be used to estimate an elastic modulus in a meaningful way as the theoretical chain density and segment separation is certainly different from the helical $(\operatorname{tg} t)_{n}$ arrangement. However, for completeness elastic moduli of $(t t t)_{n}$ chains are estimated with the available $(\operatorname{tg} t)_{n}$ density and a hypothetical density of $1.06 \mathrm{~kg} \cdot \mathrm{dm}^{-3}$ arising from the crystal density of polyethylene $\left(1.008 \mathrm{~kg} \cdot \mathrm{dm}^{-3}\right)$ [19] but considering the higher mass of an oxygen atom over a methylene unit. The segment chain length for $(t t t)_{n}$ chains is only derived from quantum chemical calculations. Parameters and results for the calculations of the elastic moduli are summarized in Table 8.9.

As anticipated from the different slopes $S$, obtained elastic moduli for the $(t t t)_{n}$ conformations are larger than those for the $(\operatorname{tg} t)_{n}$ conformation. DFT and HF studies were found to overestimate the predicted elastic moduli in case of polyethylene (PE) 
[19]. Therefore, the obtained results are rather upper limits for the elastic moduli of isolated PEO chains. The derived elastic modulus for the $(\operatorname{tg} t)_{n}$ conformation is somewhat larger than results obtained measuring the variations in the lattice plane distances using x-ray diffraction yielding an elastic modulus of $10 \mathrm{GPa}$ [201]. A study measuring the force displacement curves using atomic force microscopy (AFM) finds an average elastic modulus of $7 \mathrm{GPa}$ for various sized PEO fibres [202]. Additionally, a study deriving the elastic modulus in an analogous way to the presented results of this study, by measuring the Raman spectra and determining the LAM-1 positions at different PEO chain lengths, finds an elastic modulus of $13 \mathrm{GPa}$ [15] after correction for lateral interchain interactions. Also, an early force field study derives an elastic modulus of $13 \mathrm{GPa}$ [199]. Even though most of these reported values were derived from semicrystalline samples rather than isolated and pure PEO chains the agreement to the obtained value in this study is good. Especially considering that the derived elastic modulus from HF/STO-3G predictions is an upper threshold as a comparison to the B3LYP-2D3/def2TZVP method for $(t t t)_{n}$ shows. Therefore, the obtained elastic modulus represents an idealized value for isolated chains in vacuum at low temperatures.

A study of the LAM-1 positions in Raman jet spectra of alkanes finds a similar slope of $2295 \mathrm{~cm}^{-1}$ for stretched finite alkane chains $[18,19]$ than the $(t t t)_{n}$ slope of PEO derived in this study. The determined elastic moduli are similar but 22-74 GPa larger in case of PEO due to the larger crystal densities and slope derived from B3LYP2D3/def2TZVP calculations. The HF calculations show a larger deviation due to the systematic overestimation of the LAM-1 wavenumbers. However, a very good agreement between the B3LYP-2D3/def2TZVP, B3LYP-2D3/aVTZ and experimental LAM-1 positions (Figure 8.15) suggest that the elastic modulus of PE should rather be compared to the B3LYP-2D3/def2TZVP results for PEO. Thus, the stretched $(t t t)_{n}$ conformation of an isolated PEO chain is comparably stiff than a stretched polyethylene (PE) chain in the vacuum limit. Consistently, the COC bending in dimethylether [203] and CCC bending in $n$-propane [204] are found to have similar force constants. The lower elastic modulus in PEO over PE chains, e.g. reported in [201], is therefore mostly attributed by the conformational arrangement of $(\operatorname{tg} t)_{n}$ helices in the former molecule. 
Table 8.9: Used parameters and results for the calculations of the elastic moduli.

\begin{tabular}{|c|c|c|c|c|c|c|}
\hline polymer & calculation & conformation & $\begin{array}{c}S / \\
\mathrm{cm}^{-1}\end{array}$ & $d / \mathrm{nm}$ & $\begin{array}{c}\rho / \\
\mathrm{kg} \cdot \mathrm{dm}^{-3}\end{array}$ & $\begin{array}{c}E / \\
\text { GPa }\end{array}$ \\
\hline \multirow{5}{*}{ PEO } & $\begin{array}{c}\text { B3LYP-2D3/ } \\
\text { def2TZVP }\end{array}$ & $(t t t)_{n}$ & 2480 & $0.118^{\mathrm{a}}$ & $1.23^{b}$ & 378 \\
\hline & $\begin{array}{c}\text { B3LYP-2D3/ } \\
\text { def2TZVP }\end{array}$ & $(t t t)_{n}$ & 2480 & $0.118^{\mathrm{a}}$ & $1.06^{c}$ & 326 \\
\hline & HF/STO-3G & $(t t t)_{n}$ & 2726 & $0.120^{\mathrm{a}}$ & $1.23^{b}$ & 471 \\
\hline & HF/STO-3G & $(\operatorname{tg} t)_{n}$ & 684 & $0.093^{\mathrm{d}}$ & $1.23^{\mathrm{b}}$ & 18.1 \\
\hline & HF/STO-3G & $(\operatorname{tg} t)_{n}$ & 684 & $0.097^{\mathrm{a}}$ & $1.23^{b}$ & 19.7 \\
\hline PE & - & $(t t t)_{n}$ & 2295 & $0.127^{\mathrm{e}}$ & $1.01^{\mathrm{e}}$ & 304 \\
\hline \multicolumn{7}{|c|}{$\begin{array}{l}\text { a) Segment separations derived from appropriate calculations (see column calculation). } \\
\text { b) PEO crystal density from Ref. [190,200]. } \\
\text { c) Hypothetical density arising from the crystal density of polyethylene (1.008) [19] but } \\
\text { considering the higher mass of an oxygen atom over a methylene unit. } \\
\text { d) Segment separation from Ref. [190,191]. } \\
\text { e) Polyethylene values from Ref. [19]. }\end{array}$} \\
\hline
\end{tabular}

\section{6 - Conclusions}

In summary, quantum chemical calculations of selected conformers in tri- and tetraglyme confirm the energetic advantage of folded conformers over stretched structures in the gas phase at low temperatures. The energy difference between the all-ttt conformer and a folded structure that resembles an open-chain crown-ether type motif increases from $\sim 2.0$ to $\sim 5.5 \mathrm{~kJ} \cdot \mathrm{mol}^{-1}$ in favor of the folded structure when going from tri- to tetraglyme. Raman jet spectra of tri- and tetraglyme only show a few weak relaxation effects upon variation of the carrier gas, nozzle distance or temperature. However, some of these weak effects might help in later evaluations through quantum chemistry. A comparison of the jet spectra of mono- through tetraglyme in the LAM region shows that the spectral main area initially shifts to lower wavenumbers when going from mono- to diglyme due to the distinct presence of the all-ttt and all-tgt LAM's but stays rather constant in tri- and tetraglyme as the chain length insensitive D-LAM becomes more prominent, consistent with the theoretical predictions that favor folded over stretched structures in these molecules. Likewise, spectra of liquid glyme samples show increasing contributions of several multi gauche conformers, giving rise to a broad D-LAM band at $\sim 275-300 \mathrm{~cm}^{-1}$, and decreasing contributions of all-ttt and all-tgt at longer chain length. Quantum chemical investigations on the LAM band position extrapolated to infinite chain length show a linear dependency on the inverse of the chain length for $(t t t)_{n}$ and $(\operatorname{tg} t)_{n}$ conformations. The extrapolation allowed determining idealized elastic moduli of isolated infinite $(t t t)_{n}$ and $(t g t)_{n}$ glyme chains in vacuum at low temperatures. A comparison to derived elastic moduli in the vacuum limit for stretched polyethylene chains showed that the resulting lower elastic modulus of PEO over PE is mostly attributed 
by the conformational helical $(\operatorname{tg} t)_{n}$ arrangement in PEO. An idealized stretched $(t t t)_{n}$ PEO chain shows a similar stiffness than a stretched PE chain. 


\section{Chapter 9 - Summary and outlook}

The focus of this thesis was on the investigation of conformational preferences of flexible chain molecules at low temperatures. Throughout this work jet expansion experiments allowed exploring isolated molecules almost free of interactions with a molecular environment by means of spectroscopic techniques. Quantum chemical calculations were consulted to aid spectroscopic assignments, estimate abundances of different conformers and explore interconversion tendencies between them.

The key findings are: Substitution of a terminal methyl group in $n$-alkanes with a phenyl ring leads to an enhanced folding of the attached alkane chain (Chapter 4). For a series of $n$-alkylbenzenes (ethyl- through octyl- and decylbenzene) assignments of the all-t, $g 1$ and $g 1 g 2$ conformer were made to LIF spectra (Chapter 4.4). Additional contributions from $g 1 g^{\prime} 4$ were observed in spectra of hexyl- to octylbenzene. All assignments were confirmed by comparisons of conformer-specific FDIRS spectra to an anharmonic local mode model (Chapter 4.5). The first folded structural motif ggtgg where the alkyl chain folds back over the aromatic $\pi$ system was observed in $n$-octylbenzene (see Figure 4.6). Thus, the shortest chain alkylbenzene in which a folded structure is observed is significantly shorter than in case of pure $n$-alkanes in which first indications of a structural motif ggtgg were found to begin at a chain length of 18 carbon atoms $[18,19]$. Two important factors were identified leading to an earlier folding in the former case. First, a gauche configuration directly after the phenyl ring is actually preferred over a trans configuration. Furthermore, the first gauche turn in the alkyl chain, labeled as $g 1$, occurs with virtually no energetic penalty due to stabilizing $\mathrm{CH} \cdots \pi$ interactions of the third methylene group with the aromatic ring. Therefore, the energetic cost of the folding sequence is reduced by a factor of 2 in alkylbenzenes compared to $n$-alkanes. Second, the aromatic ring replaces the neighboring alkane subchain in folded $n$-alkane conformers. The aromatic ring allows for $\mathrm{CH} \cdots \pi$ type interactions that are stronger than those between the pure alkyl chains. Furthermore, it is less restrictive in its requirements on the turn and provides a wider swath of angles for stabilization of the alkyl chain. These cumulative interactions are especially important when the alkyl chain length increases to sizes where it can fully extend over the aromatic ring like in octylbenzene.

Substitution of every third methylene group with an oxygen atom in polyethylene chains leads to the flexible polyethylene oxides, also called polyglymes. The simplest building block of poly(ethylene glycol) dimethyl ethers is monoglyme which was extensively characterized in Chapter 6. Raman jet spectra of monoglyme showed contributions of three conformers ( $t t t, t g t, t g g$ ). A model for conformational relaxation in jet expansions, based on calculated 2D potential energy surface scans, was developed (Chapter 6.3) and allowed for an interpretation of the Raman jet spectra in a consistent way with high level quantum chemical calculations. In essence, the model freezes out interconversions around the inner (OCCO) dihedral angle at the gas temperature prior to the expansion while the outer torsional (COCC) angles form a sub$100 \mathrm{~K}$ Boltzmann equilibrium. The combined theoretical and experimental analysis of 
the conformational distributions concluded that the ttt conformation is the global minimum structure, followed by tgt and $t g g^{\prime}$. Moreover, a semiexperimental energy difference between $t g t$ and $t t t$ was estimated to be $0.9 \mathrm{~kJ} \cdot \mathrm{mol}^{-1}$ (Chapter 6.6), which is consistent with high level quantum chemical calculations.

Substitution of one of the terminal methyl groups in monoglyme with a phenyl group yields 1-methoxy-2-phenoxyethane (Chapter 5). LIF and Raman jet spectra showed major contributions of $t t t$ and tgt in this molecule while small contributions of $t g g^{\prime}$ were found only in the LIF spectra. The more pronounced conversion from tgg' to tgt, following the derived relaxation model for monoglyme, was partly explained by quantum chemical calculations that yield a slightly lower interconversion barrier between both conformers (Chapter 5.2). The substitution with a phenyl group leads to a significant increase, similar to the one found in a comparison of monoglyme and $n$-hexane (Figure 6.4), for the interconversion barrier of the subsequent dihedral.

An extensive exploration of the conformational landscape in diglyme was presented in Chapter 7. The relaxation model, which was formulated based on findings in monoglyme, was successfully extended and allowed for an interpretation of the Raman jet spectra. Experiment and theory beyond B3LYP-2D3/aVQZ calculations agreed in predicting $t t t t t$ as the global minimum structure with only a slight energetic advantage. Studies on diglyme clusters (Chapter 7.5) and mixed cluster of monoglyme and water (Chapter 6.7) revealed that the conformational preferences quickly shift to $(\operatorname{tg} t)_{n}$ structures that allow for better electrostatic interactions than the stretched all-trans chains.

In longer glyme chains (Chapter 8) folded conformers become more stable than stretched structures. A combined theoretical and experimental exploration in tri- and tetraglyme indicates that diglyme is the last oligoglyme with a stretched global minimum structure at low temperatures in the gas phase. Comparison of Raman spectra of liquid glyme samples (Figure 8.11) showed that spectra for long chain polyglymes do not differ significantly from spectra of di-, tri- and tetraglyme indicating that the dihedral angles are mainly randomly oriented in the liquid phase. Differences in Raman spectra of tetraglyme in supersonic jet expansions and the liquid phase (Figure 8.13) underlined conformational differences in both phases. Extrapolation to infinite chain length of quantum chemically predicted LAM-1 band positions allowed determining the idealized elastic modulus of $(t t t)_{n}$ and $(\operatorname{tg} t)_{n}$ polyethylene oxide chains in vacuum at low temperatures. A comparison to derived elastic moduli in the vacuum limit for stretched polyethylene chains showed that the resulting lower elastic modulus of polyethylene oxide is mostly attributed to the conformational helical $(\operatorname{tg} t)_{n}$ arrangements. The derived upper threshold for the elastic modulus of cold, interaction-free polyethylene oxide is approximately $20 \mathrm{GPa}$.

The experimental results on tri- and tetraglyme need to be extended with an in-depth investigation on the conformational landscapes. The vastly increasing number of possible conformers makes a systematic analysis for longer glyme chains very challenging. Clever strategic approaches need to be applied to find and investigate the most 
stable conformers. Neural networks, genetic algorithms, Monte-Carlo and molecular dynamics simulations are often used for this kind of problems [205]. The derived glyme relaxation model helps developing a sensible strategic approach to search for the important conformers which possibly allow interpreting the Raman jet spectra.

Monoglyme-water clusters show interesting effects that were only explored briefly in this work (Chapter 6.7). Raman jet studies could be extended to further investigate and characterize the clusters and explore the competition of multiple water molecules to the hydrogen bond acceptors of monoglyme. Moreover, this cluster is still a rather small system allowing for application of a broad range of state-of-the-art quantum chemical treatments. 


\section{A - Appendix}

\section{A.1 - Measurement conditions}

Table A.1.1: Measurement conditions for Raman jet spectra of alkylbenzenes (Chapter 4).

\begin{tabular}{|c|c|c|c|c|c|c|c|}
\hline figure & $\begin{array}{c}\boldsymbol{P}_{\text {laser }} \\
/ \mathbf{W}\end{array}$ & $\begin{array}{c}\boldsymbol{T}_{\text {saturator }} \\
/{ }^{\circ} \mathbf{C}\end{array}$ & $\begin{array}{c}\boldsymbol{T}_{\text {nozzle }} \\
/{ }^{\circ} \mathbf{C}\end{array}$ & $\begin{array}{c}\boldsymbol{d}_{\text {nozzle }} \\
/ \mathbf{m m}\end{array}$ & carrier gas & $\begin{array}{c}\boldsymbol{p}_{\text {stagnation }} \\
/ \mathbf{b a r}\end{array}$ & $\begin{array}{c}\boldsymbol{t}_{\text {integration }} \\
/ \mathbf{m i n}\end{array}$ \\
\hline 4.11 & 20 & $\begin{array}{c}43 \text { for } \\
\text { hexyl } \\
60 \text { for } \\
\text { heptyl }\end{array}$ & $\begin{array}{c}100 \text { for } \\
\text { hexyl; } \\
150 \text { for } \\
\text { heptyl }\end{array}$ & 1 & He & 0.5 & $10(5 \mathrm{x})$ \\
\hline 4.12 & 20 & 65 & 150 & 1 & $\mathrm{He}$ & 0.5 & $10(6 \mathrm{x})$ \\
\hline 4.13 & 20 & 80 & 150 & 1 & $\mathrm{He}$ & 0.5 & $12(6 \mathrm{x})$ \\
\hline
\end{tabular}

Table A.1.2: Measurement conditions for Raman jet spectra of 1-methoxy-2-phenoxyethane (Chapter 5).

\begin{tabular}{|c|c|c|c|c|c|c|c|}
\hline figure & $\begin{array}{c}\boldsymbol{P}_{\text {laser }} \\
/ \mathbf{W}\end{array}$ & $\begin{array}{c}\boldsymbol{T}_{\text {saturator }} \\
/{ }^{\circ} \mathbf{C}\end{array}$ & $\begin{array}{c}\boldsymbol{T}_{\text {nozzle }} \\
/{ }^{\circ} \mathbf{C}\end{array}$ & $\begin{array}{c}\boldsymbol{d}_{\text {nozzle }} \\
/ \mathbf{~ m m}\end{array}$ & carrier gas & $\begin{array}{c}\boldsymbol{p}_{\text {stagnation }} \\
/ \mathbf{b a r}\end{array}$ & $\begin{array}{c}\boldsymbol{t}_{\text {integration }} \\
/ \mathbf{m i n}\end{array}$ \\
\hline $5.11-12$ & 20 & $25^{\dagger}$ & 40 & 1 & $\mathrm{He}$ & 0.5 & $7(5 \mathrm{x})$ \\
\hline 5.13 & 20 & 25 & see Fig. & 1 & $\mathrm{He}$ & 0.5 & $7(5 \mathrm{x})$ \\
\hline 5.14 & 20 & 25 & 40 & 1 & see Fig. & 0.5 & $7(5 \mathrm{x})$ \\
\hline
\end{tabular}

Table A.1.3: Measurement conditions for Raman jet spectra of monoglyme (Chapter 6).

\begin{tabular}{|c|c|c|c|c|c|c|c|}
\hline figure & $\begin{array}{c}P_{\text {laser }} \\
/ \mathrm{W}\end{array}$ & $\begin{array}{c}T_{\text {saturator }} \\
/^{\circ} \mathrm{C}\end{array}$ & $\begin{array}{c}T_{\text {nozzle }} \\
/{ }^{\circ} \mathrm{C} \\
\end{array}$ & $\begin{array}{l}d_{\text {nozzle }} \\
/ \mathbf{m m} \\
\end{array}$ & carrier gas & $\begin{array}{c}p_{\text {stagnation }} \\
/ \text { bar }\end{array}$ & $\begin{array}{c}t_{\text {integration }} \\
/ \text { min }\end{array}$ \\
\hline 6.5 jet & 18 & -34 & RT & 1 & $\mathrm{He}$ & 0.5 & $5(5 x)$ \\
\hline $\begin{array}{l}6.5 \text { gas } \\
\text { phase }\end{array}$ & 18 & - & - & - & - & $5 \mathrm{mbar}$ & $5(5 x)$ \\
\hline $6.7-8$ & 25 & -35 & RT & 1 & $\mathrm{He}$ & 0.5 & $10(5 x)$ \\
\hline 6.9 & 5 & -35 & RT & 1 & see Fig. & 0.5 & $10(5 x)$ \\
\hline 6.10 & 5 & -35 & RT & 1 & $\mathrm{Ar}$ & see Fig. & $10(5 x)$ \\
\hline 6.11 & 25 & -35 & see Fig. & 1 & $\mathrm{He}$ & 0.5 & $5(5 x)$ \\
\hline 6.12 & 5 & -35 & RT & see Fig. & $\begin{array}{l}\text { a) } \mathrm{He} \\
\text { b) } \mathrm{Ar}\end{array}$ & 0.5 & $10(5 x)$ \\
\hline 6.13 & 25 & - & RT & see Fig. & $\begin{array}{c}0.2 \% \\
\text { monoglyme } \\
\text { premixed in } \\
\text { He and added } \\
\text { by } 60 \% \mathrm{Ar}\end{array}$ & 0.5 & $10(5 x)$ \\
\hline 6.17 & 20 & $\begin{array}{l}\text { MG: }-35 \\
\mathrm{H}_{2} \mathrm{O}: 15\end{array}$ & RT & 1 & $\mathrm{He}$ & 0.7 & $5(5 x)$ \\
\hline 6.18 & 20 & $\begin{array}{l}\mathrm{MG}:-35 \\
\mathrm{H}_{2} \mathrm{O}: 15\end{array}$ & RT & 2 & $\mathrm{He}$ & 0.7 & $5(5 x)$ \\
\hline
\end{tabular}

\footnotetext{
$\dagger$ In order to avoid unwanted condensation in the curry-jet lines this substance was used with the heatable saturator (and lines).
} 
Table A.1.4: Measurement conditions for Raman jet spectra of diglyme (Chapter 7).

\begin{tabular}{|c|c|c|c|c|c|c|c|}
\hline figure & $\begin{array}{c}P_{\text {laser }} \\
\text { / W }\end{array}$ & $\begin{array}{c}T_{\text {saturator }} \\
\quad /{ }^{\circ} \mathrm{C}\end{array}$ & $\begin{array}{c}T_{\text {nozzle }} \\
/^{\circ} \mathrm{C}\end{array}$ & $\begin{array}{l}d_{\text {nozzle }} \\
/ \text { mm }\end{array}$ & carrier gas & $\begin{array}{c}p_{\text {stagnation }} \\
/ \text { bar }\end{array}$ & $\begin{array}{c}t_{\text {integration }} \\
/ \text { min }\end{array}$ \\
\hline $\begin{array}{c}\text { Figure } \\
7.12\end{array}$ & 18 & RT & RT & 1 & $\mathrm{He}$ & 0.5 & $7(5 x)$ \\
\hline $7.13-18$ & 25 & 10 & RT & 1 & $\mathrm{He}$ & 0.4 & $8(5 x)$ \\
\hline 7.19 & 25 & 10 & see Fig. & 1 & $\mathrm{He}$ & 0.4 & $8(5 x)$ \\
\hline 7.20 & 25 & 10 & see Fig. & 1 & $5 \% \mathrm{Ar}$ in $\mathrm{He}$ & 0.4 & $8(5 x)$ \\
\hline 7.21 & 25 & 10 & RT & 1 & see Fig. & 0.4 & $8(5 x)$ \\
\hline 7.22 & 25 & 10 & RT & 2 & see Fig. & 0.4 & $8(5 x)$ \\
\hline 7.23 & 25 & 10 & RT & see Fig. & $\mathrm{He}$ & 0.4 & $8(5 x)$ \\
\hline 7.24 & 20 & 10 & $\mathrm{RT}$ & see Fig. & $20 \% \mathrm{Ne}$ in $\mathrm{He}$ & 0.4 & $8(5 x)$ \\
\hline 7.25 & 25 & 10 & RT & 1 & see Fig. & 0.4 & $8(5 x)$ \\
\hline 7.26 & 25 & 10 & RT & see Fig. & $\mathrm{He}$ & 0.4 & $8(5 x)$ \\
\hline 7.27 & 25 & see Fig. & RT & 1 & $\mathrm{He}$ & 0.5 & $\begin{array}{c}\text { for low } T_{\text {sat: }} \text { : } \\
10(5 \mathrm{x}) \\
\text { for high } T_{\text {sat }} \text { : } \\
7(5 \mathrm{x})\end{array}$ \\
\hline 7.29 & 25 & 10 & RT & 1 & $\mathrm{He}$ & 0.5 & $8(5 x)$ \\
\hline
\end{tabular}

Table A.1.5: Measurement conditions for Raman jet spectra of longer glymes (Chapter 8).

\begin{tabular}{|c|c|c|c|c|c|c|c|}
\hline figure & $\begin{array}{c}P_{\text {laser }} \\
/ \text { W }\end{array}$ & $\begin{array}{c}T_{\text {saturator }} \\
\quad /{ }^{\circ} \mathrm{C} \\
\end{array}$ & $\begin{array}{l}T_{\text {nozzle }} \\
/{ }^{\circ} \mathrm{C}\end{array}$ & $\begin{array}{l}d_{\text {nozzle }} \\
/ \mathbf{m m}\end{array}$ & carrier gas & $\begin{array}{c}p_{\text {stagnation }} \\
\text { / bar }\end{array}$ & $\begin{array}{c}t_{\text {integration }} \\
/ \text { min }\end{array}$ \\
\hline $\begin{array}{c}8.4 \\
\text { monoglyme }\end{array}$ & 25 & -35 & RT & 1 & $\mathrm{He}$ & 0.5 & $10(5 x)$ \\
\hline $\begin{array}{c}8.4 \\
\text { diglyme }\end{array}$ & 25 & 10 & RT & 1 & $\mathrm{He}$ & 0.4 & $8(5 x)$ \\
\hline $\begin{array}{c}8.4 \\
\text { triglyme }\end{array}$ & 20 & 50 & 100 & 1 & $\mathrm{He}$ & 0.5 & $10(6 x)$ \\
\hline $\begin{array}{c}8.4 \\
\text { tetraglyme }\end{array}$ & 20 & 70 & 120 & 1 & $\mathrm{He}$ & 0.5 & $10(6 x)$ \\
\hline 8.5 & 20 & 50 & 100 & 1 & see Fig. & 0.5 & $10(6 x)$ \\
\hline 8.6 & 20 & 50 & 100 & see Fig. & $\mathrm{He}$ & 0.5 & $10(6 x)$ \\
\hline 8.7 & 20 & 50 & see Fig. & 1 & $\mathrm{He}$ & 0.5 & $10(6 x)$ \\
\hline 8.8 & 20 & 70 & 120 & 1 & see Fig. & 0.5 & $10(5 x)$ \\
\hline 8.9 & 20 & 70 & 120 & see Fig. & $\begin{array}{c}20 \% \mathrm{Ne} \text { in } \\
\mathrm{He}\end{array}$ & 0.5 & $10(5 x)$ \\
\hline 8.10 & 20 & 70 & 120 & see Fig. & $\mathrm{He}$ & 0.5 & $10(5 x)$ \\
\hline
\end{tabular}




\section{A.2 - Differences in spectral resolutions with changing laser sources in the curry jet}

During this work a Verdi V5, Verdi V18, Verdi G18, Millennia eV 20 and Millennia eV 25 were used as laser light sources for Raman jet experiments. The Verdi V5 and V18 are single-mode lasers while the others are multimode lasers. Differences in the resulting spectral resolutions were probed for by fitting 13 rotational S-branch lines of the nitrogen fundamental in ambient air between 2339 and $2435 \mathrm{~cm}^{-1}$ with Gaussian functions (Figure A.2.1). The FWHM of the 13 Gaussian fits were compared between all used laser sources and the resulting relative average deviation from the Verdi V5 fits was used as a reference. The error of this evaluation was estimated by repeating the procedure several times for the same laser source and was conservatively estimated to be $\sim 2 \%$.

No significant deviations in the resulting band widths between the Verdi V5 and Verdi V18 fits were found. The following relative band width increases were found when compared to the Verdi V5 fits.

Millennia eV 20: $3 \pm 2 \%$

Millennia eV 25: 4 $\pm 2 \%$

Verdi G18: $4 \pm 2 \%$

Therefore, a slightly significant increase of the resulting band widths was found when one of the multimode instead of a single-mode lasers was used. However, this effect did not impact any of the analyses of this work. A comparison of the Raman spectra of the rotational S-branch between 2330 and $2349 \mathrm{~cm}^{-1}$ of the nitrogen fundamental of ambient air between the Verdi V5 and Verdi G18 is shown in Figure A.2.2. 


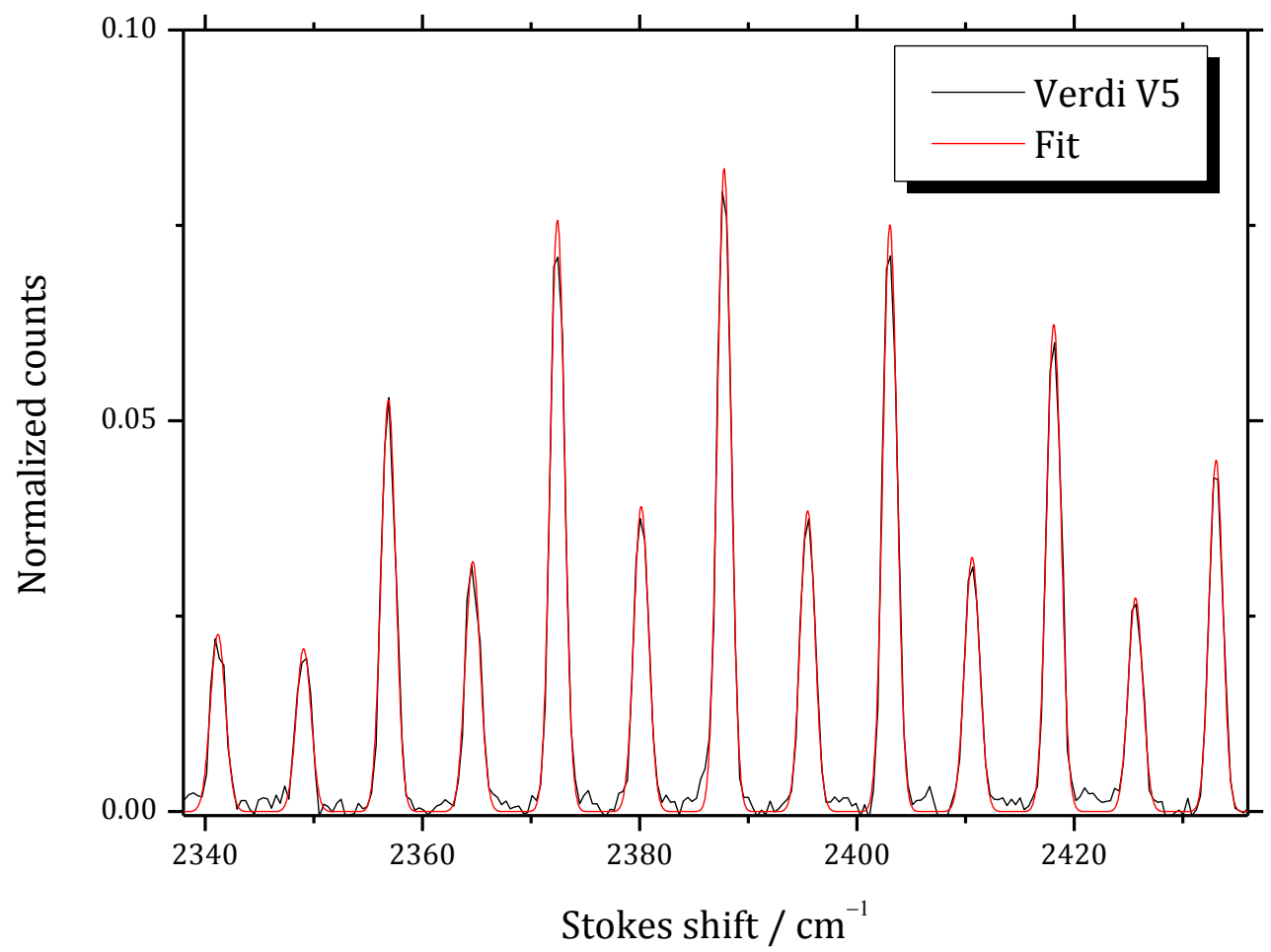

Figure A.2.9.1: Raman spectrum of the S-branch between 2339 and $2436 \mathrm{~cm}^{-1}$ of the nitrogen fundamental in ambient air measured with the Verdi V5 (black) and the the cumulative Gaussian fit (red).

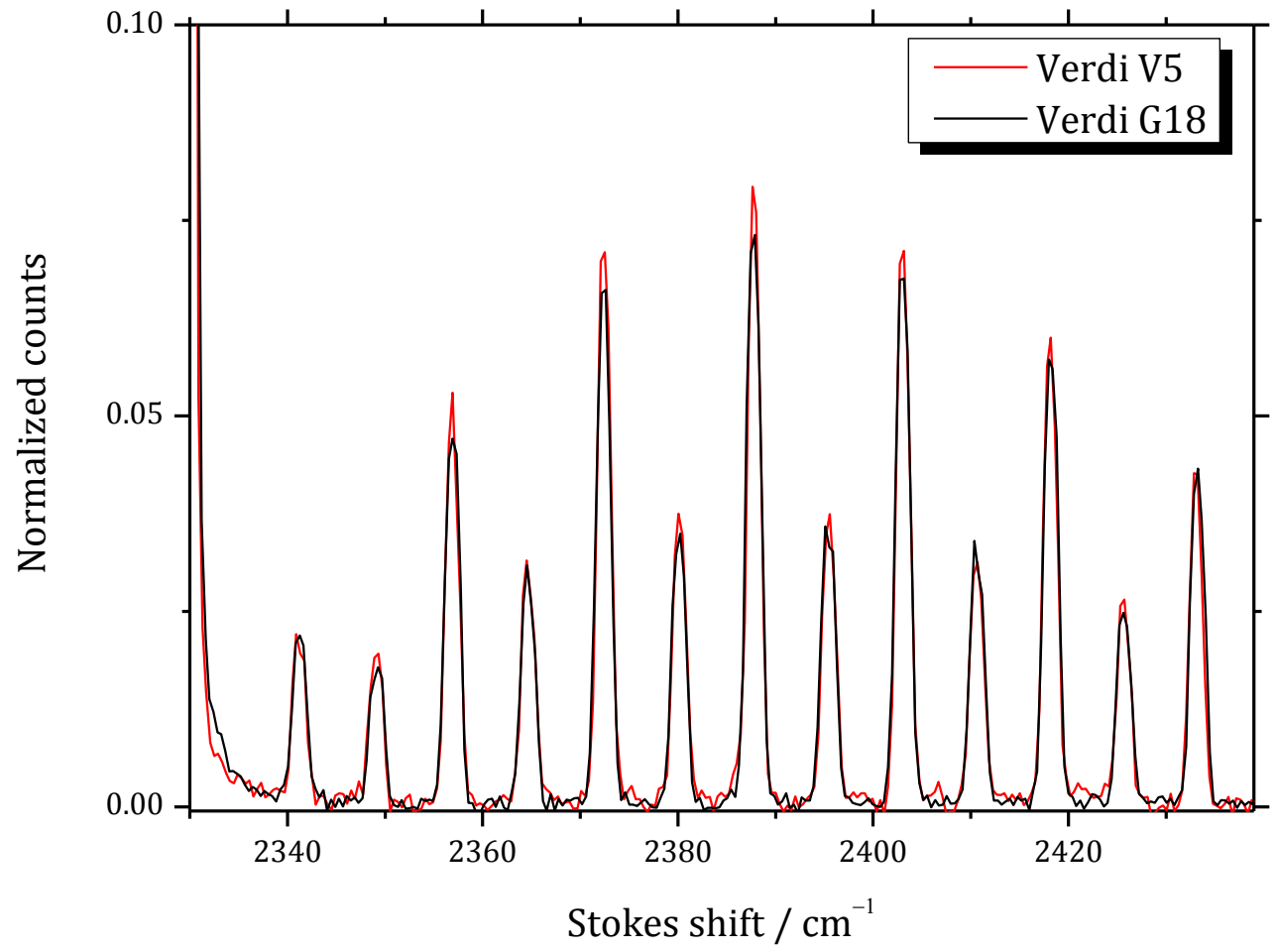

Figure A.2.2: Raman spectra of the S-branch between 2330 and $2439 \mathrm{~cm}^{-1}$ of the nitrogen fundamental in ambient air measured with the Verdi V5 (red) and the Verdi G18 (black). 


\section{A.3 - Raman spectra of TFE and HFIP}

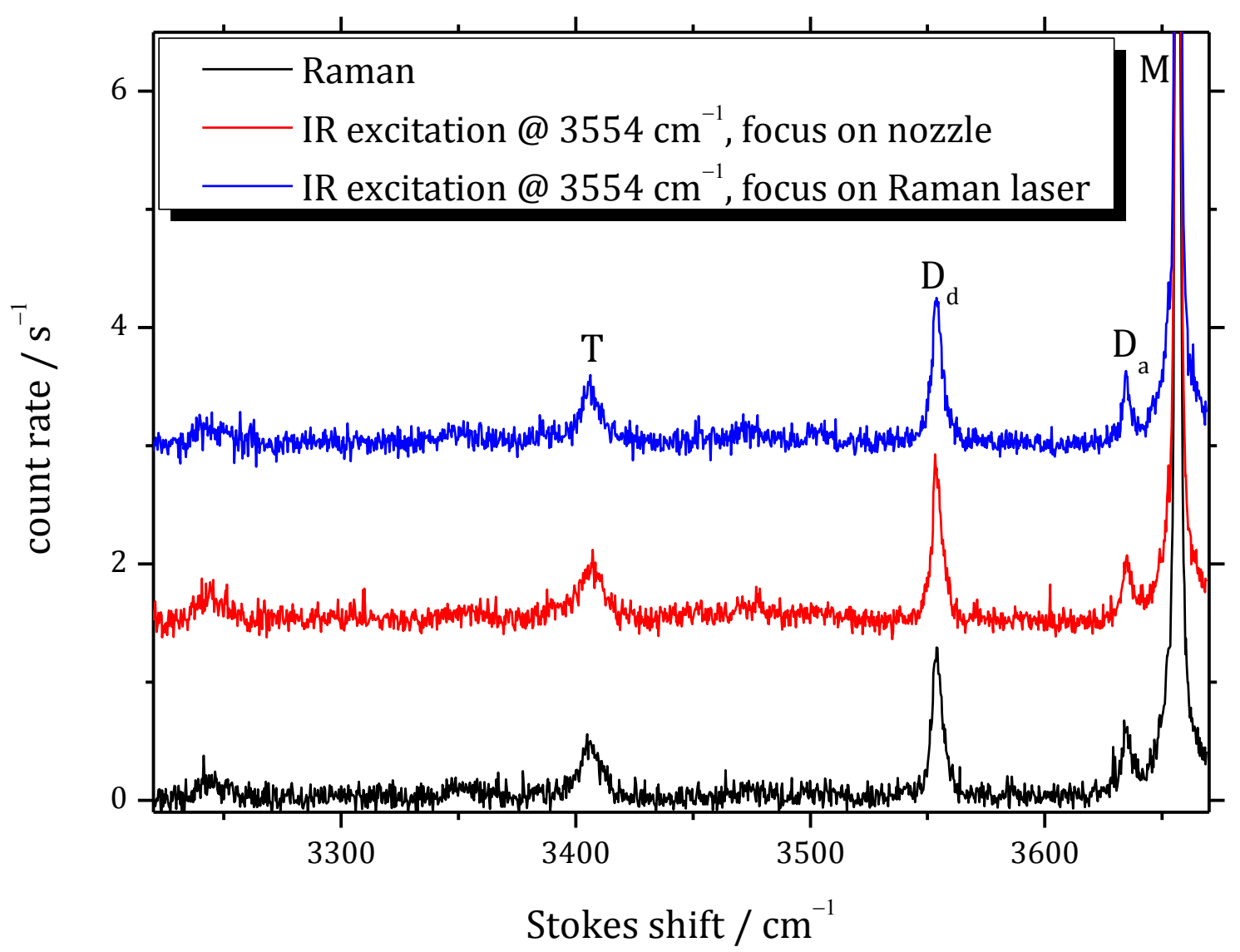

Figure A.3.1: $\mathrm{OH}$-stretching Raman jet spectra of 2,2,2,-trifluorethanol (TFE) in Ar. IR-Raman double resonance spectra with IR excitation of the $3554 \mathrm{~cm}^{-1}$ band are vertically shifted. Assignments were made in comparison with Reference [206] and allowed for assignments of the monomer (M), dimer acceptor $\left(\mathrm{D}_{\mathrm{a}}\right)$, dimer donor $\left(\mathrm{D}_{\mathrm{d}}\right)$ and a broader trimer $(\mathrm{T})$ band.

Measurement conditions: $T_{\text {saturator }}=-5^{\circ} \mathrm{C}, d_{\text {nozzle }}=1 \mathrm{~mm}, p_{\text {stagnation }}=0.65 \mathrm{bar}, t_{\text {integration }}=100 \mathrm{~s}(5 \mathrm{x}), P_{\text {laser }}$ $=25 \mathrm{~W}$. 


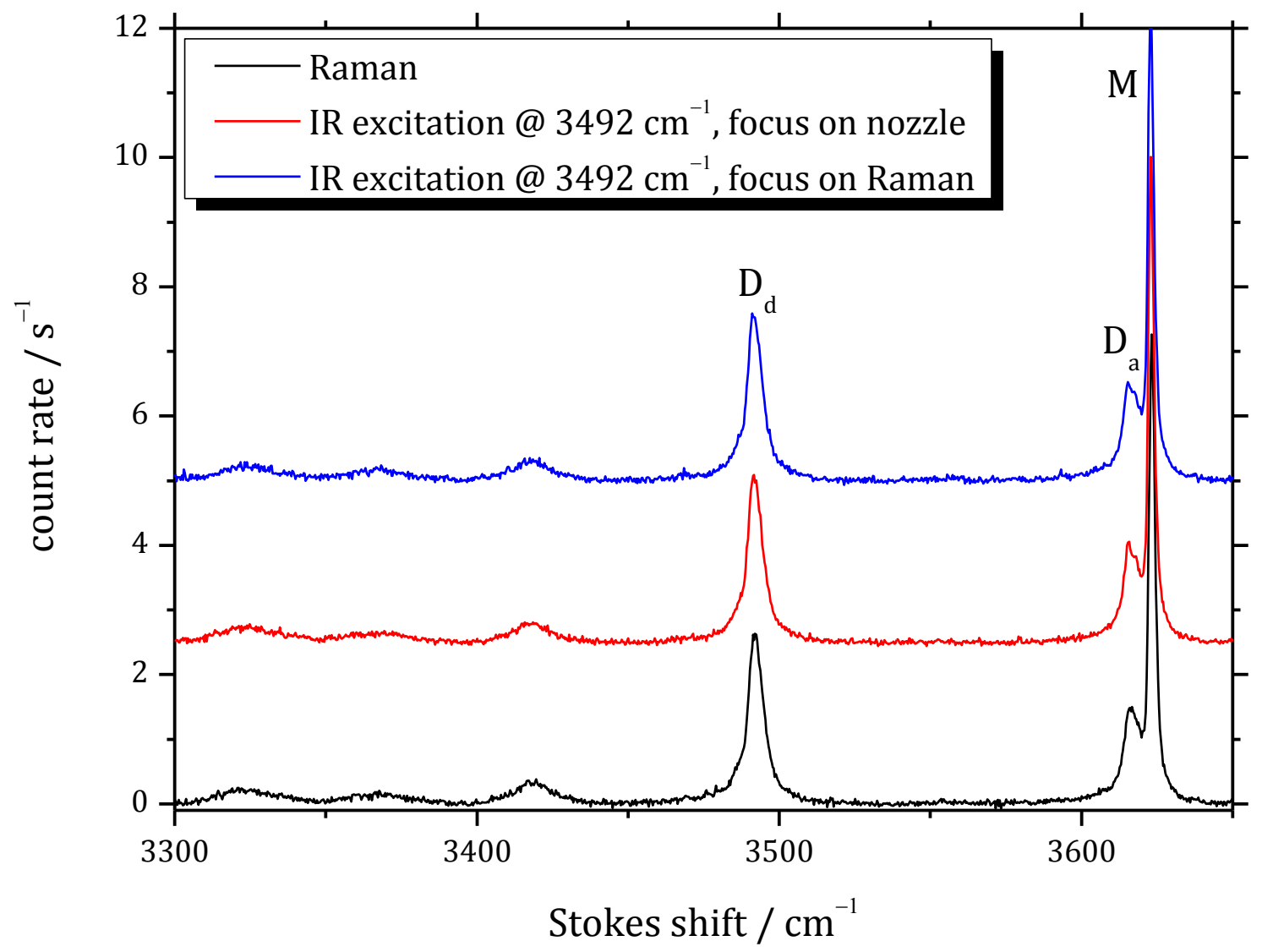

Figure A.3.2: $\mathrm{OH}$-stretching Raman jet spectra of 1,1,1,3,3-hexafluoro-2-propanol (HFIP) in Ar. IRRaman double resonance spectra with IR excitation of the $3492 \mathrm{~cm}^{-1}$ band are vertically shifted. Assignments were made in comparison with Reference [78] and allowed for assignments of the monomer $(M)$, dimer acceptor $\left(D_{a}\right)$, dimer donor $\left(D_{d}\right)$ band.

Measurement conditions: $T_{\text {saturator }}=-3{ }^{\circ} \mathrm{C}, d_{\text {nozzle }}=1 \mathrm{~mm}, p_{\text {stagnation }}=0.5$ bar, $t_{\text {integration }}=600 \mathrm{~s}(5 \mathrm{x}), P_{\text {laser }}=$ $25 \mathrm{~W}$. 


\section{A.4 - Extended results of the conformational search in alkylben- zenes}

Table A.4.1: Extension of Table 4.1. Differences in zero-point corrected energies ( $\left.\Delta E_{0}\right)$, zero-point vibrational energies (ZPVE) and Gibbs energies at $298 \mathrm{~K}\left(\Delta G^{0}\right)$ relative to all- $t$ for all conformers found below a threshold of $5 \mathrm{~kJ} \cdot \mathrm{mol}^{-1}$ in butyl- trough decylbenzene calculated at the B3LYP2D3/def2TZVP level in Gaussian 09 Rev. E.01. Note that not every possible conformer was calculated for higher alkylbenzenes but rather representatives for the important conformer families.

\begin{tabular}{|c|c|c|c|}
\hline conformer & $\Delta E_{0} / \mathbf{k J} \cdot \mathbf{m o l}^{-1}$ & $\Delta \mathrm{ZPVE} / \mathrm{kJ} \cdot \mathrm{mol}^{-1}$ & $\Delta G^{0} / \mathrm{kJ} \cdot \mathrm{mol}^{-1}$ \\
\hline \multicolumn{4}{|c|}{ butylbenzene } \\
\hline$g 1$ & -0.20 & 0.27 & -1.38 \\
\hline all- $t$ & 0.00 & 0.00 & 0.00 \\
\hline$g 1 g 2$ & 1.98 & 1.13 & 1.93 \\
\hline$g 2$ & 2.31 & 0.09 & 0.06 \\
\hline \multicolumn{4}{|c|}{ pentylbenzene } \\
\hline$g 1$ & -0.03 & 0.57 & -0.87 \\
\hline all- $t$ & 0.00 & 0.00 & 0.00 \\
\hline$g 1 g 2$ & 1.49 & 1.28 & 1.33 \\
\hline$g 2$ & 2.52 & 0.41 & 0.89 \\
\hline$g 3$ & 2.61 & 0.51 & 0.99 \\
\hline$g 1 g 3$ & 2.73 & 1.12 & 2.07 \\
\hline$g 1 g^{\prime} 3$ & 3.05 & 1.08 & 2.56 \\
\hline$g 1 g 2 g 3$ & 3.99 & 1.81 & 4.18 \\
\hline$g 2 g 3$ & 4.37 & 1.27 & 3.53 \\
\hline \multicolumn{4}{|c|}{ hexylbenzene } \\
\hline all- $t$ & 0.00 & 0.00 & 0.00 \\
\hline$g 1$ & 0.01 & 0.70 & -0.72 \\
\hline$g 1 g 2$ & 1.21 & 1.32 & 0.80 \\
\hline$g 1 g^{\prime} 4$ & 2.27 & 1.15 & 1.61 \\
\hline$g 2$ & 2.48 & 0.53 & 0.98 \\
\hline$g 1 g 4$ & 2.62 & 1.19 & 1.90 \\
\hline$g 3$ & 2.64 & 0.57 & 1.07 \\
\hline$g 1 g 3$ & 2.65 & 1.14 & 1.74 \\
\hline$g 4$ & 2.74 & 0.50 & 1.07 \\
\hline$g 1 g^{\prime} 3$ & 3.03 & 1.11 & 2.41 \\
\hline$g 1 g 2 g 4$ & 3.16 & 1.91 & 2.70 \\
\hline$g 1 g 3 g 4$ & 3.52 & 2.18 & 3.77 \\
\hline$g 1 g^{\prime} 2$ & 4.61 & 1.21 & 4.93 \\
\hline$g 1 g 2 g 3$ & 4.83 & 1.31 & 4.54 \\
\hline \multicolumn{4}{|c|}{ heptylbenzene } \\
\hline$g 1$ & -0.03 & 0.69 & -0.53 \\
\hline all- $t$ & 0.00 & 0.00 & 0.00 \\
\hline$g 1 g 2$ & 1.14 & 1.38 & 0.95 \\
\hline$g 1 g^{\prime} 4$ & 2.13 & 1.19 & 1.55 \\
\hline$g 3$ & 2.40 & 0.51 & 0.34 \\
\hline$g 2$ & 2.43 & 0.49 & 1.04 \\
\hline$g 1 g 3$ & 2.51 & 1.18 & 1.70 \\
\hline
\end{tabular}


A.4 - Extended results of the conformational search in alkylbenzenes

\begin{tabular}{|c|c|c|c|}
\hline conformer & $\Delta E_{0} / \mathrm{kJ} \cdot \mathrm{mol}^{-1}$ & $\Delta \mathrm{ZPVE} / \mathrm{kJ} \cdot \mathrm{mol}^{-1}$ & $\Delta G^{0} / \mathrm{kJ} \cdot \mathrm{mol}^{-1}$ \\
\hline$g 1 g 4$ & 2.56 & 1.22 & 1.99 \\
\hline$g 4$ & 2.69 & 0.49 & 1.18 \\
\hline$g 1 g 2 g 4$ & 2.81 & 1.96 & 2.36 \\
\hline$g 1 g 3 g 4$ & 2.85 & 2.33 & 3.41 \\
\hline$g 1 g^{\prime} 3$ & 2.87 & 1.08 & 2.38 \\
\hline$g 1 g 2 g 4 g 5$ & 3.48 & 2.98 & 6.00 \\
\hline$g 1 g^{\prime} 4 g^{\prime} 5$ & 3.82 & 2.10 & 4.17 \\
\hline$g 2 g 3$ & 3.94 & 1.43 & 3.24 \\
\hline$g 1 g 2 g 3$ & 4.64 & 1.29 & 12.74 \\
\hline \multicolumn{4}{|c|}{ octylbenzene } \\
\hline all- $t$ & 0.00 & 0.00 & 0.00 \\
\hline$g 1$ & 0.02 & 0.75 & -0.32 \\
\hline$g 1 g 3 g 4$ & 0.98 & 3.46 & 4.78 \\
\hline$g 1 g 2$ & 1.17 & 1.47 & 1.18 \\
\hline$g 1 g 2 g 4 g 5$ & 1.81 & 3.26 & 3.46 \\
\hline$g 1 g^{\prime} 4$ & 1.99 & 1.24 & 1.06 \\
\hline$g 1 g 4$ & 2.25 & 1.07 & 0.93 \\
\hline$g 2$ & 2.42 & 0.48 & 0.73 \\
\hline$g 3$ & 2.43 & 0.57 & 0.66 \\
\hline$g 1 g 3$ & 2.53 & 1.20 & 1.28 \\
\hline$g 1 g 3 g 4 g 5$ & 2.60 & 3.95 & 7.23 \\
\hline$g 1 g^{\prime} 4 g^{\prime} 5$ & 3.20 & 2.23 & 3.09 \\
\hline$g 1 g 2 g 3$ & 3.64 & 2.10 & 4.05 \\
\hline$g 2 g 3$ & 3.96 & 1.52 & 3.33 \\
\hline$g 3 g 4$ & 4.13 & 1.50 & 3.41 \\
\hline$g 1 g 4 g 5$ & 4.42 & 2.17 & 4.74 \\
\hline$g 1 g 5 g 6$ & 4.56 & 1.97 & 4.08 \\
\hline$g 1 g^{\prime} 3 g^{\prime} 4$ & 4.62 & 1.99 & 4.73 \\
\hline$g 5 g 6$ & 4.85 & 1.44 & 4.15 \\
\hline \multicolumn{4}{|c|}{ nonylbenzene } \\
\hline$g 1$ & -0.03 & 0.69 & -0.70 \\
\hline$g 1 g 3 g 4$ & -0.01 & 3.28 & 3.71 \\
\hline all- $t$ & 0.00 & 0.00 & 0.00 \\
\hline$g 1 g 2 g 4 g 5$ & 0.34 & 3.76 & 4.27 \\
\hline$g 1 g 2$ & 1.18 & 1.46 & 0.81 \\
\hline$g 1 g^{\prime} 4$ & 1.89 & 1.19 & 0.88 \\
\hline$g^{\prime} 1 g 4 g 5$ & 2.00 & 3.25 & 5.22 \\
\hline$g 2$ & 2.38 & 0.45 & 0.74 \\
\hline$g 1 g 4$ & 2.48 & 1.35 & 2.15 \\
\hline$g 1 g 3$ & 2.54 & 1.25 & 1.58 \\
\hline$g 3$ & 2.66 & 0.59 & 0.88 \\
\hline$g 1 g 2 g 4 g 5 g 6$ & 2.82 & 4.42 & 7.95 \\
\hline$g 1 g 2 g 3$ & 3.66 & 2.12 & 3.93 \\
\hline$g 1 g 2 g 7$ & 3.83 & 1.86 & 3.29 \\
\hline$g 3 g 4$ & 4.14 & 1.59 & 3.36 \\
\hline$g 5 g 6$ & 4.62 & 2.00 & 4.32 \\
\hline
\end{tabular}


decylbenzene

\begin{tabular}{|c|c|c|c|}
\hline conformer & $\Delta E_{0} / \mathrm{kJ} \cdot \mathrm{mol}^{-1}$ & $\Delta \mathrm{ZPVE} / \mathrm{kJ} \cdot \mathrm{mol}^{-1}$ & $\Delta G^{0} / \mathrm{kJ} \cdot \mathrm{mol}^{-1}$ \\
\hline$g 1 g 3 g 4$ & -0.98 & 3.55 & 3.77 \\
\hline$g 1 g 2 g 4 g 5$ & -0.95 & 3.86 & 3.88 \\
\hline$g 1$ & -0.05 & 0.67 & -0.86 \\
\hline all- $t$ & 0.00 & 0.00 & 0.00 \\
\hline$g^{\prime} 1 g 4 g 5$ & 0.35 & 3.87 & 6.72 \\
\hline$g 1 g 2$ & 1.15 & 1.45 & 0.65 \\
\hline$g 1 g^{\prime} 4$ & 1.97 & 1.34 & 1.31 \\
\hline$g 1 g 4$ & 2.30 & 1.19 & 1.28 \\
\hline$g 2$ & 2.40 & 0.48 & 0.82 \\
\hline$g 3$ & 2.43 & 0.61 & 0.72 \\
\hline$g 1 g 5$ & 2.50 & 1.19 & 1.62 \\
\hline$g 1 g 3$ & 2.50 & 1.22 & 1.51 \\
\hline$g 4$ & 2.60 & 0.59 & 1.06 \\
\hline$g 7$ & 2.64 & 0.43 & 0.39 \\
\hline$g 6$ & 2.65 & 0.41 & 0.44 \\
\hline$g 5$ & 2.68 & 0.69 & 1.32 \\
\hline$g 8$ & 2.75 & 0.49 & 1.07 \\
\hline$g 1 g 2 g 3$ & 3.66 & 2.15 & 3.92 \\
\hline$g 2 g 3$ & 3.88 & 1.51 & 2.71 \\
\hline$g 1 g 5 g 6$ & 4.14 & 2.25 & 4.16 \\
\hline$g 1 g 4 g 5$ & 4.15 & 2.25 & 4.79 \\
\hline$g 5 g 6$ & 4.46 & 1.61 & 3.96 \\
\hline
\end{tabular}

\section{A.5 - Extended results of the conformational search in diglyme}

Table A.5.1: Obtained results from geometry optimizations of all generated diglyme structures (A.10), neglecting those that converted into other structures during the geometry optimization, at the B3LYP2D3/def2TZVP level carried out in Gaussian 09 Rev. D.01.

\begin{tabular}{|c|c|c|c|c|}
\hline conformer & $\begin{array}{c}\text { electronic ener- } \\
\text { gy } / E_{h}\end{array}$ & $\begin{array}{c}\text { ZPVE } \\
/ E_{h}\end{array}$ & $\begin{array}{c}\Delta E_{0} / \\
\mathrm{kJ} \cdot \mathrm{mol}^{-1}\end{array}$ & $\begin{array}{l}\text { lowest vibrational } \\
\text { frequency } / \mathrm{cm}^{-1}\end{array}$ \\
\hline $\operatorname{tgttg}^{\prime} g$ & -462.908549 & 0.202275 & 0.00 & 32.6 \\
\hline$t t t t t t$ & -462.908015 & 0.202038 & 0.78 & 31.4 \\
\hline ttttgt & -462.907938 & 0.201975 & 0.82 & 32.4 \\
\hline $\operatorname{tg} g^{\prime} g^{\prime} g t$ & -462.908447 & 0.202498 & 0.85 & 22.6 \\
\hline $\operatorname{tgttg} t$ & -462.90784 & 0.201935 & 0.97 & 30.0 \\
\hline $\operatorname{tgtg} g t$ & -462.908126 & 0.202224 & 0.98 & 17.1 \\
\hline$t t t t g g^{\prime}$ & -462.907896 & 0.202185 & 1.48 & 36.2 \\
\hline $\operatorname{tgtgg}^{\prime} t$ & -462.907973 & 0.202267 & 1.49 & 13.5 \\
\hline $\operatorname{tgttg} g^{\prime}$ & -462.907834 & 0.202171 & 1.60 & 25.3 \\
\hline $\operatorname{ttg} g^{\prime} t$ & -462.907915 & 0.202257 & 1.62 & 25.3 \\
\hline $\operatorname{tgg}^{\prime} \operatorname{tgg}{ }^{\prime}$ & -462.907885 & 0.202369 & 1.99 & 6.9 \\
\hline $\operatorname{tgg}^{\prime} \operatorname{tg}^{\prime} g$ & -462.907927 & 0.202449 & 2.09 & 10.2 \\
\hline$g g^{\prime} \operatorname{tg} g^{\prime} g$ & -462.907849 & 0.202512 & 2.46 & 32.4 \\
\hline
\end{tabular}


A.5 - Extended results of the conformational search in diglyme

\begin{tabular}{|c|c|c|c|c|}
\hline conformer & $\begin{array}{c}\text { electronic ener- } \\
\text { gy / } E_{\mathbf{h}}\end{array}$ & $\begin{array}{l}\text { ZPVE } \\
/ E_{\mathrm{h}}\end{array}$ & $\begin{array}{c}\Delta E_{0} / \\
\mathrm{kJ} \cdot \mathrm{mol}^{-1}\end{array}$ & $\begin{array}{l}\text { lowest vibrational } \\
\text { frequency } / \mathrm{cm}^{-1}\end{array}$ \\
\hline $\operatorname{tgg}^{\prime} \operatorname{tg}^{\prime} t$ & -462.907903 & 0.202646 & 2.67 & 50.1 \\
\hline $\operatorname{tgttg}^{\prime} t$ & -462.907122 & 0.201916 & 2.80 & 34.0 \\
\hline$g g^{\prime} \operatorname{ttg} g^{\prime}$ & -462.907457 & 0.202489 & 3.43 & 27.1 \\
\hline $\operatorname{tggtgg}$ & -462.907402 & 0.202599 & 3.86 & 28.1 \\
\hline $\operatorname{ttg} g^{\prime} g t$ & -462.907229 & 0.202431 & 3.88 & 36.6 \\
\hline $\operatorname{tg} g^{\prime} g ' g g$ & -462.907706 & 0.202931 & 3.94 & 30.7 \\
\hline $\operatorname{tgg}^{\prime} \operatorname{tg}^{\prime} g^{\prime}$ & -462.907442 & 0.202734 & 4.11 & 19.8 \\
\hline $\operatorname{tgtggg}$ & -462.907212 & 0.202545 & 4.22 & 28.3 \\
\hline ggttg'g & -462.907104 & 0.202549 & 4.51 & 19.4 \\
\hline $\operatorname{tg} g g^{\prime} g t$ & -462.906751 & 0.202248 & 4.65 & 32.5 \\
\hline $\operatorname{tg}^{\prime} g g g^{\prime}$ & -462.906824 & 0.20254 & 5.22 & 22.3 \\
\hline $\operatorname{tg} \operatorname{tg} g^{\prime} g$ & -462.906768 & 0.20249 & 5.24 & 43.5 \\
\hline $\operatorname{ttggg}^{\prime} t$ & -462.906611 & 0.202449 & 5.54 & 31.4 \\
\hline tttggt & -462.906415 & 0.202295 & 5.66 & 23.4 \\
\hline $\operatorname{ttg} \operatorname{tg} t$ & -462.906409 & 0.202289 & 5.66 & 15.0 \\
\hline tttgtt & -462.90629 & 0.202205 & 5.75 & 25.9 \\
\hline $\operatorname{tgggg}^{\prime} t$ & -462.906394 & 0.202334 & 5.81 & 18.8 \\
\hline $\operatorname{tgtg} g g$ & -462.906359 & 0.202331 & 5.90 & 22.7 \\
\hline $\operatorname{tg}{ }^{\prime} \operatorname{tgg}$ & -462.906419 & 0.202397 & 5.91 & 22.3 \\
\hline ttttgg & -462.906184 & 0.202178 & 5.96 & 21.3 \\
\hline $\operatorname{tg}^{\prime} g^{\prime} g^{\prime} t$ & -462.906136 & 0.202188 & 6.11 & 23.5 \\
\hline $\operatorname{tgtttg}$ & -462.906083 & 0.202143 & 6.13 & 28.9 \\
\hline tttttg & -462.906083 & 0.202153 & 6.15 & 25.8 \\
\hline $\operatorname{tgg}^{\prime} t \operatorname{tg}$ & -462.906346 & 0.202445 & 6.23 & 25.8 \\
\hline $\operatorname{ttg} t g^{\prime} t$ & -462.906016 & 0.202188 & 6.42 & 17.0 \\
\hline ttgtgg & -462.906261 & 0.202461 & 6.50 & 15.7 \\
\hline $\operatorname{tgg}^{\prime} \operatorname{tg}^{\prime}$ & -462.906166 & 0.202385 & 6.55 & 22.0 \\
\hline $\operatorname{tgttgg}$ & -462.905931 & 0.202153 & 6.56 & 23.6 \\
\hline$t t t g g g^{\prime}$ & -462.906273 & 0.202526 & 6.64 & 34.2 \\
\hline ggttgg' & -462.906053 & 0.202307 & 6.64 & 19.2 \\
\hline $\operatorname{tg} t t t g^{\prime}$ & -462.905933 & 0.20219 & 6.65 & 27.7 \\
\hline $\operatorname{tggtg} g$ & -462.906107 & 0.202386 & 6.70 & 16.8 \\
\hline gtttgg' & -462.905961 & 0.202292 & 6.84 & 27.4 \\
\hline$t t t g g^{\prime} g^{\prime}$ & -462.906122 & 0.202469 & 6.88 & 27.5 \\
\hline $\operatorname{ttg} \operatorname{tg} g$ & -462.90594 & 0.202325 & 6.98 & 9.5 \\
\hline gtttg'g & -462.905889 & 0.202279 & 7.00 & 25.0 \\
\hline $\operatorname{tg} g^{\prime} g^{\prime} g^{\prime} g$ & -462.906343 & 0.202738 & 7.01 & 25.7 \\
\hline $\operatorname{tgtggt}$ & -462.905617 & 0.202081 & 7.19 & -9.2 \\
\hline $\operatorname{tg} g^{\prime} g^{\prime} g g^{\prime}$ & -462.905819 & 0.202294 & 7.22 & -68.4 \\
\hline $\operatorname{tg} g^{\prime} g \operatorname{tg}$ & -462.906243 & 0.202736 & 7.27 & 38.9 \\
\hline$g g g^{\prime} \operatorname{tg} g^{\prime}$ & -462.906064 & 0.202576 & 7.32 & 15.4 \\
\hline $\operatorname{tgtg}^{\prime} g^{\prime} g$ & -462.905885 & 0.202418 & 7.37 & 9.5 \\
\hline $\operatorname{tg} t g^{\prime} g^{\prime}$ & -462.905848 & 0.202391 & 7.40 & 23.9 \\
\hline$g g^{\prime} \operatorname{tg}^{\prime} g^{\prime} g$ & -462.906256 & 0.20282 & 7.45 & 38.1 \\
\hline
\end{tabular}




\begin{tabular}{|c|c|c|c|c|}
\hline conformer & $\begin{array}{c}\text { electronic ener- } \\
\text { gy / } E_{\mathrm{h}}\end{array}$ & $\begin{array}{l}\text { ZPVE } \\
/ E_{\mathrm{h}}\end{array}$ & $\begin{array}{c}\Delta E_{0} / \\
\mathrm{kJ} \cdot \mathrm{mol}^{-1}\end{array}$ & $\begin{array}{l}\text { lowest vibrational } \\
\text { frequency } / \mathrm{cm}^{-1}\end{array}$ \\
\hline $\operatorname{tg} g^{\prime} g^{\prime} g^{\prime} g^{\prime}$ & -462.90646 & 0.203151 & 7.79 & 45.4 \\
\hline $\operatorname{tgtg} g^{\prime} g^{\prime}$ & -462.905621 & 0.202365 & 7.92 & 21.9 \\
\hline$g g^{\prime} \operatorname{tg} g g^{\prime}$ & -462.905865 & 0.202681 & 8.12 & 22.7 \\
\hline $\operatorname{tg}^{\prime} \operatorname{tg}^{\prime} g^{\prime} g^{\prime}$ & -462.905725 & 0.202597 & 8.26 & 23.1 \\
\hline$g g^{\prime} \operatorname{tg} g$ & -462.905761 & 0.202663 & 8.34 & 17.8 \\
\hline tttggg & -462.905652 & 0.202613 & 8.49 & 27.9 \\
\hline ggtggg' & -462.905624 & 0.202671 & 8.72 & 31.6 \\
\hline gggtgg' & -462.905914 & 0.202998 & 8.82 & 27.0 \\
\hline ttggtt & -462.905304 & 0.202402 & 8.86 & 29.2 \\
\hline $\operatorname{ttgg}^{\prime} g^{\prime} t$ & -462.905498 & 0.202629 & 8.94 & 40.4 \\
\hline $\operatorname{tgg}^{\prime} g \operatorname{tg}^{\prime}$ & -462.905332 & 0.202576 & 9.24 & 31.3 \\
\hline gggtg'g & -462.90554 & 0.202788 & 9.25 & 22.0 \\
\hline tgg'ggg & -462.905516 & 0.20278 & 9.29 & 18.9 \\
\hline $\operatorname{ttg} g^{\prime} g g$ & -462.905294 & 0.202658 & 9.55 & 40.3 \\
\hline $\operatorname{tg} g g^{\prime} g^{\prime} g$ & -462.905151 & 0.202528 & 9.59 & 11.3 \\
\hline $\operatorname{tgg}^{\prime} g^{\prime} \operatorname{tg}^{\prime}$ & -462.905063 & 0.202528 & 9.82 & 19.1 \\
\hline$g g g^{\prime} g g^{\prime} g$ & -462.905732 & 0.203236 & 9.92 & 35.3 \\
\hline $\operatorname{ggtgg}^{\prime} g^{\prime}$ & -462.905177 & 0.20278 & 10.18 & 24.0 \\
\hline ttgggt & -462.904605 & 0.202233 & 10.25 & 28.6 \\
\hline $\operatorname{ttgg}^{\prime} t t$ & -462.904813 & 0.202461 & 10.30 & 17.9 \\
\hline $\operatorname{tgtggg}$ & -462.904755 & 0.202581 & 10.77 & 26.3 \\
\hline $\operatorname{tg} g^{\prime} g^{\prime} \operatorname{tg}$ & -462.904657 & 0.202485 & 10.77 & 32.5 \\
\hline ttgtgg & -462.904426 & 0.202306 & 10.91 & 16.5 \\
\hline ttgggg' & -462.904627 & 0.202524 & 10.95 & 27.9 \\
\hline $\operatorname{tg} g g^{\prime} g g$ & -462.904448 & 0.202369 & 11.02 & 25.0 \\
\hline gttttg & -462.90412 & 0.202068 & 11.09 & 4.0 \\
\hline ttgttg' & -462.904235 & 0.202219 & 11.18 & -14.8 \\
\hline $\operatorname{ttg} g g^{\prime} g$ & -462.904401 & 0.20239 & 11.19 & 21.9 \\
\hline ttgttg & -462.904401 & 0.202392 & 11.20 & 17.5 \\
\hline tggttg' & -462.904514 & 0.202512 & 11.22 & 22.9 \\
\hline gttg'gg & -462.904581 & 0.202591 & 11.25 & 26.2 \\
\hline $\operatorname{tggttg}$ & -462.904415 & 0.202428 & 11.26 & 20.7 \\
\hline $\operatorname{ttggg}^{\prime} g^{\prime}$ & -462.904442 & 0.202472 & 11.30 & 23.4 \\
\hline $\operatorname{tg} \operatorname{tg} \operatorname{tg}^{\prime}$ & -462.904294 & 0.202336 & 11.33 & 6.1 \\
\hline ggtg'gg & -462.904434 & 0.202478 & 11.34 & 21.4 \\
\hline $\operatorname{ttg}^{\prime} g^{\prime}$ & -462.904223 & 0.202281 & 11.38 & -0.2 \\
\hline tttgtg & -462.904211 & 0.202281 & 11.41 & 8.3 \\
\hline $\operatorname{ttg} g^{\prime} g^{\prime} g^{\prime}$ & -462.904657 & 0.202752 & 11.47 & 47.8 \\
\hline $\operatorname{gtttg}^{\prime} g^{\prime}$ & -462.904128 & 0.202235 & 11.50 & 17.2 \\
\hline gttttg' & -462.90407 & 0.202191 & 11.54 & 15.4 \\
\hline tttgtg' & -462.904213 & 0.202371 & 11.64 & 24.2 \\
\hline$g g g^{\prime} g g g^{\prime}$ & -462.904476 & 0.202648 & 11.68 & 28.4 \\
\hline tgtg'tg & -462.904053 & 0.202231 & 11.69 & 17.8 \\
\hline $\operatorname{tggtg}^{\prime} g^{\prime}$ & -462.904141 & 0.202331 & 11.72 & 20.7 \\
\hline
\end{tabular}


A.5 - Extended results of the conformational search in diglyme

\begin{tabular}{|c|c|c|c|c|}
\hline conformer & $\begin{array}{c}\text { electronic ener- } \\
\text { gy / } E_{\mathrm{h}} \\
\end{array}$ & $\begin{array}{c}\text { ZPVE } \\
/ E_{\mathrm{h}} \\
\end{array}$ & $\begin{array}{c}\Delta E_{0} / \\
\mathbf{k J} \cdot \mathrm{mol}^{-1}\end{array}$ & $\begin{array}{l}\text { lowest vibrational } \\
\text { frequency } / \mathrm{cm}^{-1}\end{array}$ \\
\hline gtttgg & -462.904199 & 0.202389 & 11.72 & 26.3 \\
\hline $\operatorname{gttg}^{\prime} g^{\prime} g$ & -462.904247 & 0.202448 & 11.75 & 11.1 \\
\hline ggttgg & -462.903877 & 0.202171 & 11.99 & 21.3 \\
\hline$g \operatorname{tg}^{\prime} \operatorname{tg}^{\prime} g$ & -462.904122 & 0.20246 & 12.11 & 13.0 \\
\hline $\operatorname{tg}^{\prime} \operatorname{tg}^{\prime}$ & -462.903926 & 0.20227 & 12.13 & 6.3 \\
\hline ggtggg & -462.904846 & 0.203195 & 12.14 & 29.0 \\
\hline $\operatorname{tg} g g g^{\prime} g^{\prime}$ & -462.904429 & 0.202792 & 12.18 & 36.4 \\
\hline $\operatorname{gttgg}^{\prime} g^{\prime}$ & -462.904118 & 0.202502 & 12.23 & 15.7 \\
\hline gttggg & -462.904314 & 0.202709 & 12.26 & 19.2 \\
\hline tggtgg & -462.904293 & 0.202702 & 12.30 & 22.3 \\
\hline $\operatorname{tg} g g^{\prime} g^{\prime} t$ & -462.903997 & 0.202418 & 12.33 & 34.4 \\
\hline gtgtgg' & -462.904137 & 0.202584 & 12.40 & 17.2 \\
\hline $\operatorname{ttg} g^{\prime} g^{\prime} g$ & -462.904 & 0.202474 & 12.47 & 16.2 \\
\hline $\operatorname{tggggg}$ & -462.903858 & 0.202345 & 12.50 & 22.9 \\
\hline ggtg'g'g & -462.904048 & 0.202573 & 12.60 & 22.9 \\
\hline$g g g g^{\prime} g^{\prime} g$ & -462.904493 & 0.203034 & 12.64 & 50.9 \\
\hline $\operatorname{tggggt}$ & -462.90363 & 0.202173 & 12.65 & 26.2 \\
\hline $\operatorname{gtg}^{\prime} \operatorname{tgg}{ }^{\prime}$ & -462.903886 & 0.202457 & 12.72 & 21.6 \\
\hline gtgtg'g & -462.903837 & 0.202436 & 12.80 & -2.4 \\
\hline$g g g g g^{\prime} g^{\prime}$ & -462.9044 & 0.203041 & 12.90 & 36.7 \\
\hline $\operatorname{ggttg}^{\prime} g^{\prime}$ & -462.903706 & 0.202374 & 12.98 & 2.9 \\
\hline$g g^{\prime} g^{\prime} g^{\prime} g^{\prime} g$ & -462.903965 & 0.202649 & 13.02 & 29.3 \\
\hline$g g g^{\prime} g^{\prime} g^{\prime} g$ & -462.904042 & 0.202795 & 13.20 & 16.9 \\
\hline$g^{\prime t t g} g^{\prime} g^{\prime}$ & -462.904008 & 0.202778 & 13.24 & 27.3 \\
\hline $\operatorname{tggg}^{\prime} g^{\prime} g^{\prime}$ & -462.904021 & 0.202863 & 13.43 & 30.5 \\
\hline$g \operatorname{tg} g^{\prime} g g$ & -462.903936 & 0.202853 & 13.63 & 40.0 \\
\hline$g^{g t g} g^{\prime} g^{\prime}$ & -462.903842 & 0.202782 & 13.69 & 31.3 \\
\hline $\operatorname{tggg} \operatorname{tg}$ & -462.903457 & 0.202524 & 14.03 & 29.6 \\
\hline gttggg & -462.903672 & 0.202786 & 14.15 & 29.4 \\
\hline $\operatorname{tggg}^{\prime} \operatorname{tg}^{\prime}$ & -462.903372 & 0.202567 & 14.36 & 26.5 \\
\hline ttggtg' & -462.903238 & 0.20244 & 14.38 & 25.7 \\
\hline $\operatorname{tg} g g g g$ & -462.903703 & 0.202924 & 14.43 & 38.0 \\
\hline ttggtg & -462.903239 & 0.202479 & 14.48 & 26.5 \\
\hline ttgggg & -462.903198 & 0.202473 & 14.57 & 20.2 \\
\hline$g^{t g} g g^{\prime} g^{\prime}$ & -462.903244 & 0.202687 & 15.01 & 39.4 \\
\hline $\operatorname{ttg} g^{\prime} \operatorname{tg}$ & -462.902853 & 0.202464 & 15.45 & 24.9 \\
\hline $\operatorname{ttg} \operatorname{tg}^{\prime}$ & -462.902874 & 0.202487 & 15.46 & 29.7 \\
\hline$g g g g^{\prime} g g$ & -462.90294 & 0.202581 & 15.53 & -10.1 \\
\hline $\operatorname{tgggtg}^{\prime}$ & -462.902622 & 0.202303 & 15.64 & 29.7 \\
\hline tgggtg & -462.902444 & 0.202333 & 16.18 & 29.3 \\
\hline gggggg & -462.90344 & 0.203352 & 16.24 & 45.4 \\
\hline$g g g g g g^{\prime}$ & -462.903079 & 0.20305 & 16.40 & -6.6 \\
\hline$g^{\operatorname{tg}} \operatorname{tg}^{\prime} g^{\prime}$ & -462.902455 & 0.20244 & 16.43 & 19.5 \\
\hline$g \operatorname{tg}^{\prime} g$ gg & -462.902623 & 0.202611 & 16.44 & 23.7 \\
\hline
\end{tabular}




\begin{tabular}{|c|c|c|c|c|}
\hline conformer & $\begin{array}{c}\text { electronic ener- } \\
\text { gy / } E_{\mathrm{h}}\end{array}$ & $\begin{array}{l}\text { ZPVE } \\
/ E_{h}\end{array}$ & $\begin{array}{c}\Delta E_{0} / \\
\mathrm{kJ} \cdot \mathrm{mol}^{-1}\end{array}$ & $\begin{array}{l}\text { lowest vibrational } \\
\text { frequency } / \mathrm{cm}^{-1}\end{array}$ \\
\hline$g \operatorname{tg} g^{\prime} g^{\prime} g$ & -462.902621 & 0.202621 & 16.47 & 34.6 \\
\hline gtgggg' & -462.902486 & 0.202489 & 16.48 & 25.2 \\
\hline gttgtg' & -462.902312 & 0.202402 & 16.71 & 17.1 \\
\hline$g^{t t g} \operatorname{tg}$ & -462.90223 & 0.202408 & 16.94 & 18.0 \\
\hline$g \operatorname{tg}^{\prime} \operatorname{tgg}$ & -462.902291 & 0.202471 & 16.95 & 17.9 \\
\hline$g^{\prime t g} \operatorname{tg}^{\prime}$ & -462.902172 & 0.202439 & 17.18 & 10.7 \\
\hline gtgtgg & -462.902293 & 0.202567 & 17.19 & 17.2 \\
\hline gttgtg & -462.902224 & 0.202502 & 17.20 & 18.6 \\
\hline$g \operatorname{tgg} g^{\prime} g^{\prime}$ & -462.902701 & 0.202991 & 17.24 & 32.8 \\
\hline $\operatorname{gtgtg}^{\prime} g^{\prime}$ & -462.902022 & 0.202372 & 17.39 & 6.3 \\
\hline $\operatorname{gtggg}^{\prime} g^{\prime}$ & -462.902707 & 0.203088 & 17.47 & 16.5 \\
\hline$g \operatorname{tg}^{\prime} g g g$ & -462.902535 & 0.202962 & 17.59 & 33.1 \\
\hline$g^{t g} g g g^{\prime}$ & -462.90201 & 0.202652 & 18.16 & 25.7 \\
\hline $\operatorname{gtgg}^{\prime} g^{\prime} g$ & -462.901848 & 0.202732 & 18.80 & 26.5 \\
\hline$g g g g^{\prime} g^{\prime} g^{\prime}$ & -462.901799 & 0.202827 & 19.17 & 23.6 \\
\hline$g^{t g} g^{\prime} g^{\prime} g^{\prime}$ & -462.901333 & 0.202592 & 19.78 & 16.6 \\
\hline gtggtg & -462.901267 & 0.202537 & 19.81 & 17.7 \\
\hline $\operatorname{gtg}^{\prime} g^{\prime} t g$ & -462.901182 & 0.202485 & 19.90 & 25.0 \\
\hline gtgggg & -462.901157 & 0.202687 & 20.49 & 23.9 \\
\hline gtggtg & -462.901129 & 0.202711 & 20.63 & 26.9 \\
\hline $\operatorname{gtgg}^{\prime} \operatorname{tg}$ & -462.900904 & 0.20253 & 20.74 & 28.1 \\
\hline$g^{\prime} g^{\prime} \operatorname{tg}^{\prime}$ & -462.900725 & 0.202578 & 21.34 & 27.9 \\
\hline
\end{tabular}




\section{A.6 - Subsidiary $\Delta G^{\mathrm{C}}$ model plots of diglyme}

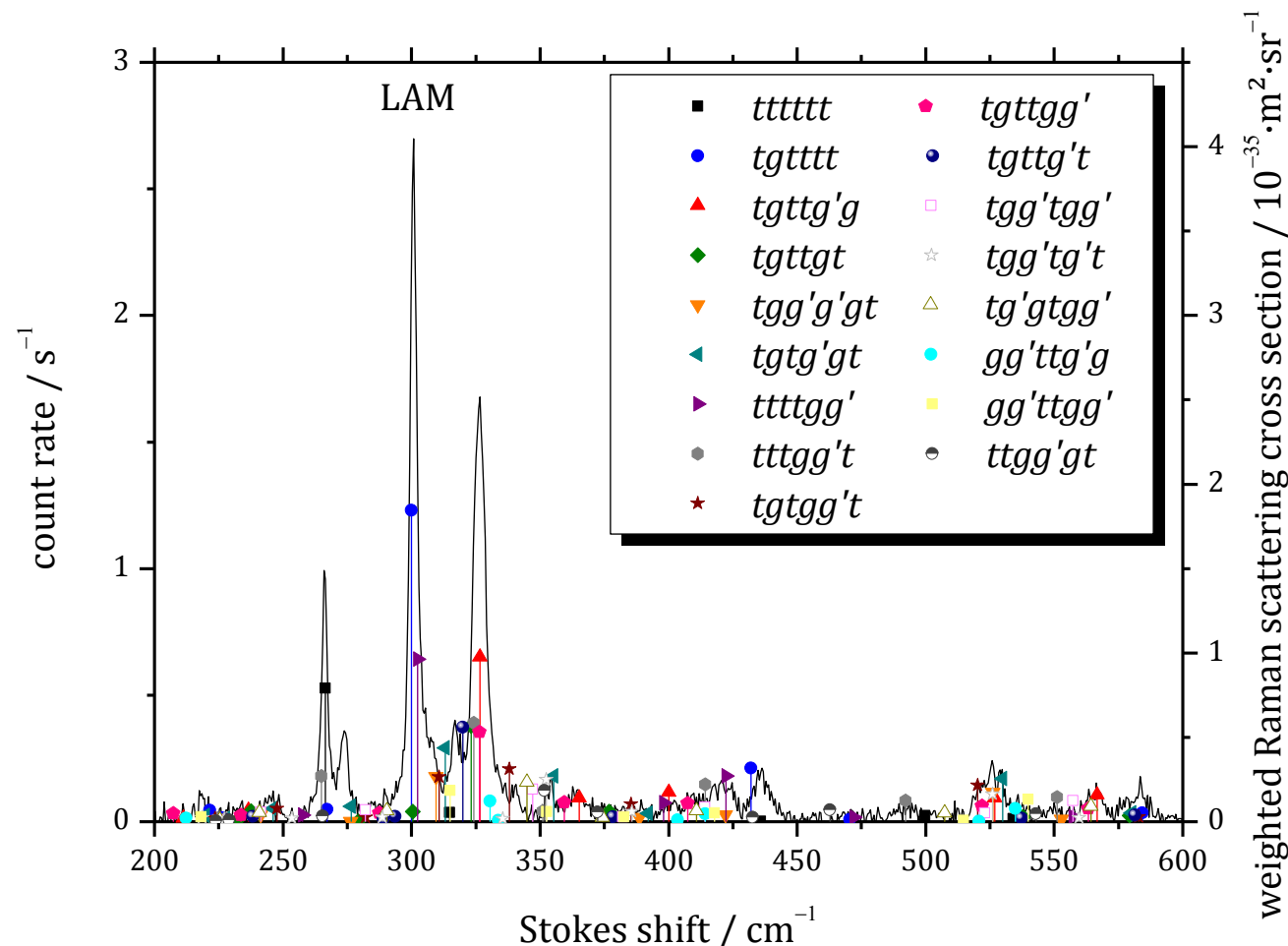

Figure A.6.1: Low frequency jet spectrum of diglyme (measurement conditions can be found in A.1) compared to Raman scattering cross sections calculated at the B3LYP-3D3/aVQZ level and weighted with their relative abundances based on the $\Delta G^{\mathrm{C}}$ values at $298 \mathrm{~K}$ (Table 7.5). This comparison mimics the first step of the relaxation process shown in Figure 7.11a.

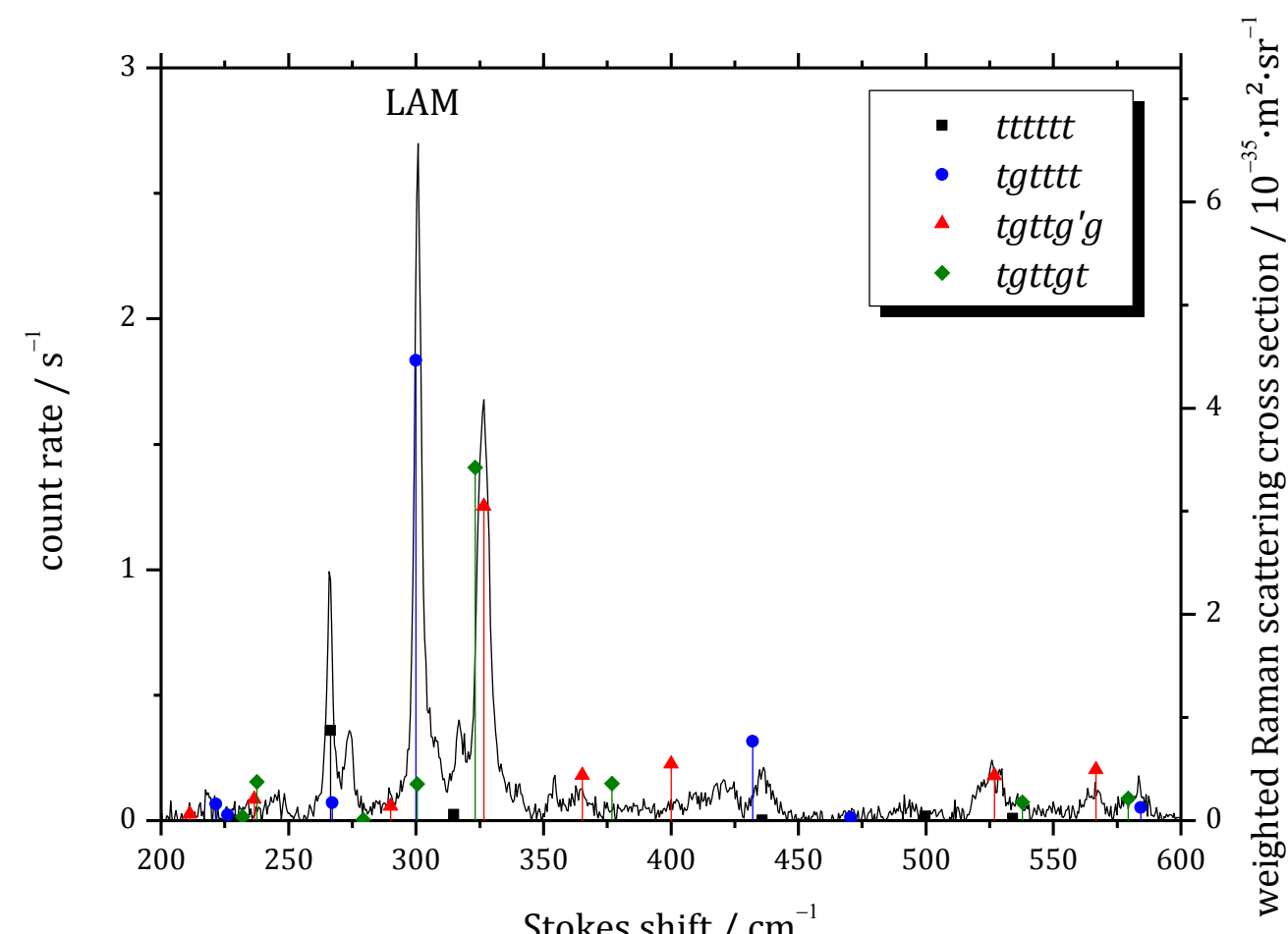

Figure A.6.2: Low frequency jet spectrum of diglyme (measurement conditions can be found in A.1) compared to Raman scattering cross sections calculated at the B3LYP-3D3/aVQZ level and weighted with their relative abundances after a full relaxation across all barriers below $5 \mathrm{~kJ} \cdot \mathrm{mol}^{-1}$ within the $\Delta G^{\mathrm{C}}$ approach. This comparison mimics the second step of the relaxation process shown in Figure 7.11a. 


\section{A.7 - Further band assignments of diglyme}

\begin{tabular}{|c|c|}
\hline$\tilde{v}_{\text {exp }} / \mathrm{cm}^{-1}$ & conformers \\
\hline 266 & tttttt, tgtttt, tttgg't, ttgg'gt \\
\hline 300 & tgtttt, tgttgt, ttttgg' \\
\hline 326 & $\operatorname{tgttg}^{\prime} g, \operatorname{tgttg} t, \operatorname{tttgg}^{\prime} t, \operatorname{tg}^{\prime} \operatorname{tgg}^{\prime}, g^{\prime} \operatorname{ttg}^{\prime} g$ \\
\hline 526 & 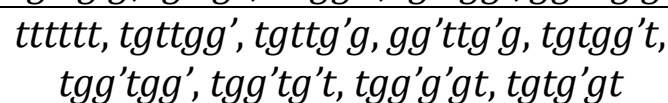 \\
\hline
\end{tabular}

\section{A.8 - Chemicals}

\begin{tabular}{|c|c|c|c|}
\hline compound & CAS & purity / \% & supplier \\
\hline$n$-pentylbenzene & $538-68-1$ & 99 & Sigma Aldrich \\
\hline$n$-hexylbenzene & $1077-16-3$ & 98 & Sigma Aldrich \\
\hline$n$-heptylbenzene & $1078-71-3$ & 98 & Alfa Aesar \\
\hline$n$-octylbenzene & $2189-60-8$ & 99 & Alfa Aesar \\
\hline$n$-nonylbenzene & $1081-77-2$ & 97 & Alfa Aesar \\
\hline n-decylbenzene & $107-72-3$ & 99 & Alfa Aesar \\
\hline 1,2-dimethoxyethane & $110-71-4$ & 99 & TCI Chemicals \\
\hline $\begin{array}{c}\text { (diethylene glycol) di- } \\
\text { methyl ether }\end{array}$ & $111-96-6$ & 99 & TCI Chemicals \\
\hline $\begin{array}{c}\text { (triethylene glycol) di- } \\
\text { methyl ether }\end{array}$ & $112-49-2$ & 99 & abcr GmbH \\
\hline $\begin{array}{c}\text { (tetraethylene glycol) di- } \\
\text { methyl ether }\end{array}$ & $109-17-1$ & 98 & TCI Chemicals \\
\hline $\begin{array}{c}\text { (polyethylene glycol) di- } \\
\text { methyl ether } \\
M_{\mathrm{n}} \sim 500\end{array}$ & $24991-55-7$ & & Sigma Aldrich \\
\hline $\begin{array}{c}\text { 1,1,1,3,3,3-hexafluor-2- } \\
\text { propanol }\end{array}$ & $920-66-1$ & 98 & TCI Chemicals \\
\hline 2,2,2-trifluorethanol & $75-89-8$ & 99 & TCI Chemicals \\
\hline helium & 7440-59-7 & 99.996 & Linde \\
\hline argon & $7440-37-1$ & 99.999 & Alphagaz \\
\hline neon & $7440-01-9$ & 99.999 & Linde \\
\hline
\end{tabular}




\section{A.9 - About the author}

Sebastian Bocklitz, * May 24, 1988, Halberstadt, Germany

\section{Curriculum Vitae}

2007 Abitur (general higher education), Jacobson Gymnasium Seesen

2008-2011 Bachelor of Science in Chemistry at the Georg-August-Universität Göttingen, bachelor thesis: "Charakterisierung von Phasenwechselmaterialien als thermische Speicher"

2011-2013 Master of Science in Chemistry at the Georg-August-Universität Göttingen, master thesis: „Conformational analysis of $n$-alkanes in cryosolutions"

04/2013$09 / 2013$

Erasmus stay at the Universiteit Antwerpen, Belgium

2013-2017 Promotion (Ph. D. candidate), Georg-August-Universität Göttingen, supervisor: Prof. Dr. Martin Suhm

11/2012- Student employee at H.M. Heizkörper GmbH \& Co. KG., Dingelstädt, 03/2013 Germany

05/2015- Guest stay in the research group of Prof. Dr. Zwier at the Purdue Uni10/2015 versity, West Lafayette, USA

\section{Publications}

- S. Bocklitz, M. A. Suhm, Constraining the conformational landscape of a polyether building block by Raman jet spectroscopy, Z. Phys. Chem. 2015, 229, 1625-1648.

- D. Zhang, S. Bocklitz. T. S. Zwier, Broadband Microwave Spectroscopy of Prototypical Amino Alcohols and Polyamines: Competition between H-Bonded Cycles and Chains, J. Phys. Chem. A 2016, 120, 55-67.

- D. P. Tabor, D. M. Hewett, S. Bocklitz, J. A. Korn, A. J. Tomaine, A. K. Gosh, T. S. Zwier, E. L. Sibert, Anharmonic modeling of the conformation-specific IR spectra of ethyl, $n$-propyl, and $n$-butylbenzene, J. Chem. Phys. 2016, 144, 224310.

- D. M. Hewett, S. Bocklitz, D. P. Tabor, E. L. Sibert, M. A. Suhm, T. S. Zwier, Identifying the first folded alkylbenzene via ultraviolet, infrared, and Raman spectroscopy of pentylbenzene through decylbenzene, Chem. Sci. 2017, 8, 53055318.

- S. Bocklitz, M. A. Suhm, Polymer segments at the folding limit: Raman scattering for the diglyme benchmark, ChemPhysChem 2017, 18, 3570-3575. 


\section{A.10 - Conformeer}

The Conformeer program was used to generate Gaussian inputs for diglyme geometries of all possible combinations of $t, g$ and $g^{\prime}$ backbone dihedrals. In a first step, the program calculates the conformational tree and disregards enantiomeric pairs as well as rotational isomers. Afterwards, a z-matrix and appropriate Gaussian input files are created for every remaining conformer. In principle the program can be adapted to generate inputs for oligoglymes of any desired length if the generation of the z-matrix is adjusted accordingly. The graphical user interface (GUI) is shown in Figure A.10.1. The source code of the program is written in Visual Basic and is listed subsequently.

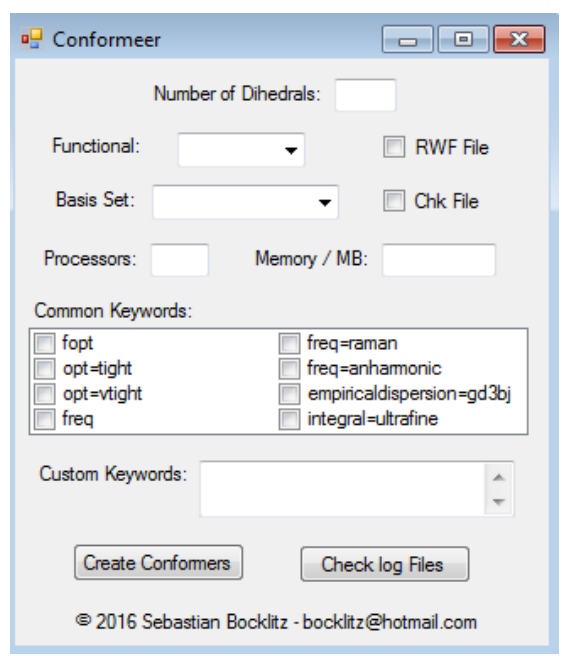

Figure A.10.1: Graphical user interface of the Conformeer program.

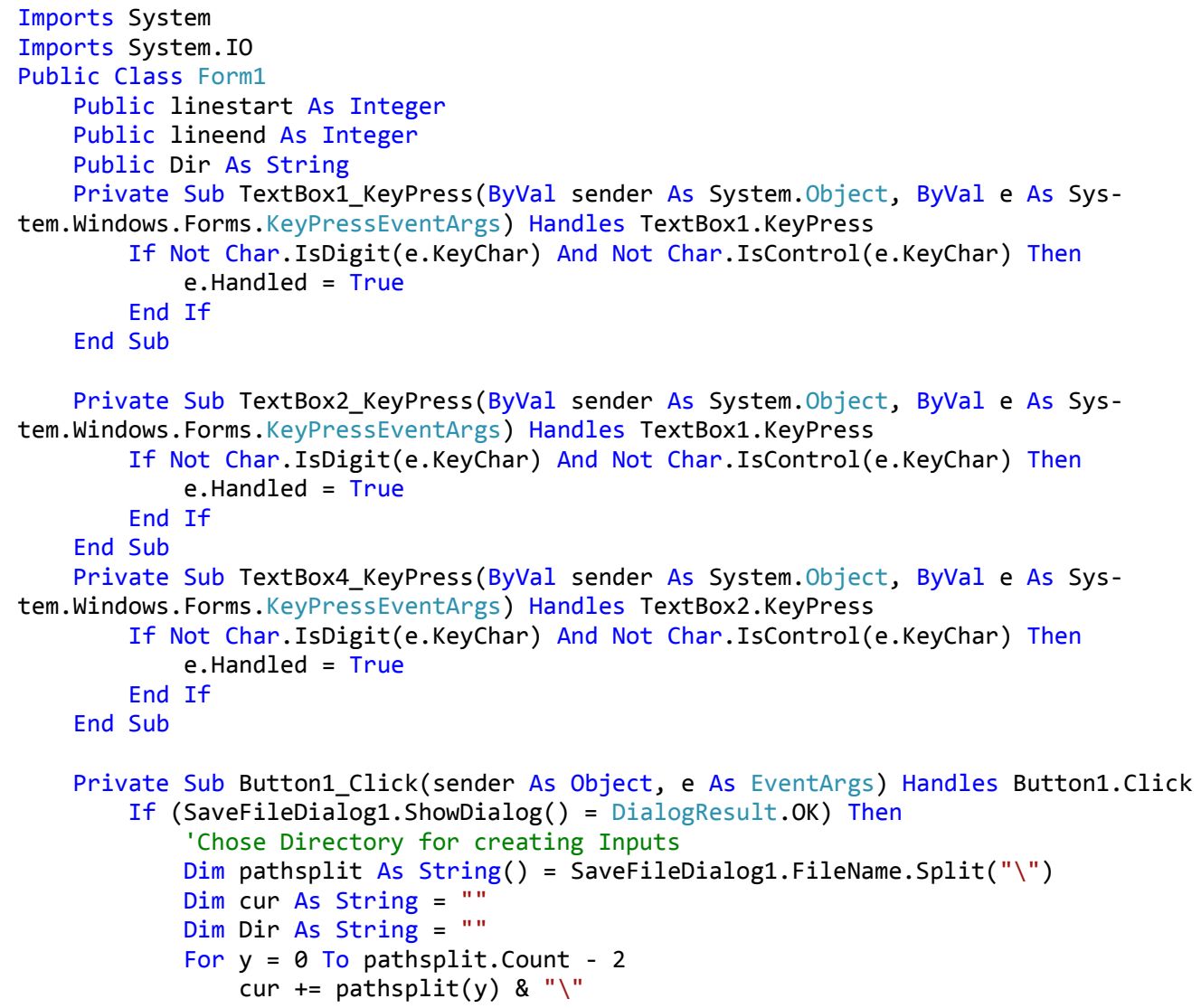


Next

Dir $=$ cur

cur $=" 1$

'to differentiate between $g+$ and $g-g$ and $b$ are used

Dim Conformers $(3 \wedge$ (Convert.ToInt32(TextBox1.Text)) - 1) As String

Dim kopie(2) As String

$\operatorname{kopie}(\theta)=" t "$

$\operatorname{kopie}(1)=" \mathrm{~g} "$

$\operatorname{kopie}(2)=" b "$

Dim stepone(3) As String

stepone $(\theta)=$ " $t "$

stepone $(1)=" g "$

stepone $(2)=" b "$

'stepwise creation of conformational tree

For $j=1$ To Convert. ToInt32(TextBox1. Text) - 1

ReDim Conformers $(3 \wedge(j+1)-1)$

For $x=0$ To $3 \wedge j-1$

Conformers $(3 * x)=\operatorname{kopie}(x)+\operatorname{stepone}(\theta)$

Conformers $(3 * x+1)=\operatorname{kopie}(x)+\operatorname{stepone}(1)$

Next

Conformers $(3 * x+2)=\operatorname{kopie}(x)+\operatorname{stepone}(2)$

$\operatorname{ReDim} \operatorname{kopie}(3 \wedge(j+1)-1)$

Next

Array.Copy(Conformers, kopie, Conformers.Length)

'delete enantiomers

Dim vertauscher As String = " "

For $\mathrm{x}=0$ To Conformers. Length - 1

vertauscher $=$ Conformers $(x)$

Dim austausch As String = ""

For $i=0$ To vertauscher. Length - 1

If vertauscher $(i)=$ " $t$ " Then austausch $+=" t "$

ElseIf vertauscher $(i)=$ "g" Then austausch $+=" b "$

ElseIf vertauscher $(i)=$ "b" Then austausch $+=$ "g"

Next

End If

For $\mathrm{a}=0$ To Conformers. Length -1

If Conformers(a) $=$ austausch And (Conformers(a).Contains ("g") Or Conform-

ers (a).Contains("b")) Then

Conformers $(a)=$ "marked"

Next

End If

Next

Dim konfi As ArrayList $=$ New ArrayList (Conformers)

For $x=$ konfi.Count -1 To 0 Step -1

If $\operatorname{konfi}(x)=$ "marked" Then

konfi.RemoveAt $(x)$

Next

End If

'delete rotational isomers

For $\mathrm{x}=0$ To konfi.Count -1

vertauscher $=" "$

vertauscher $=\operatorname{konfi}(\mathrm{x})$

Dim rueckwaerts As String = "

For $i=$ vertauscher. Length -1 To 0 Step -1

If vertauscher $(i)=$ " $t$ " Then rueckwaerts $+=$ " $t "$

ElseIf vertauscher $(i)=$ "g" Then rueckwaerts $+=" \mathrm{~g} "$

ElseIf vertauscher $(i)=$ "b" Then rueckwaerts $+=" b "$

Next End If

If rueckwaerts $=$ vertauscher Then

rueckwaerts $=$ "disregard"

End If

For $a=0$ To konfi.Count -1

If $\operatorname{konfi}(a)=$ rueckwaerts And $(\operatorname{konfi}(a)$.Contains $(" g ")$ Or kon-

fi(a). Contains("b")) Then

$\operatorname{konfi}(a)=$ "marked"

Next

End If 
Next

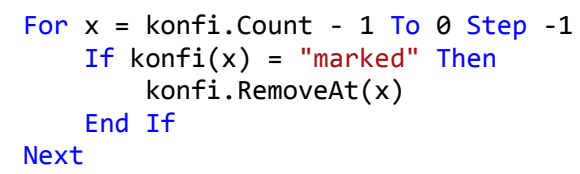

'delete conformers that have equal sequences after rotation and mirroring

For $\mathrm{x}=0$ To konfi.Count -1

vertauscher $=" "$

vertauscher $=\operatorname{konfi}(\mathrm{x})$

Dim rueckwaerts As String = " "

For $i=$ vertauscher. Length -1 To 0 Step -1

If vertauscher $(i)=$ " $t$ " Then

rueckwaerts $+=$ " $t "$

ElseIf vertauscher $(i)=" g "$ Then rueckwaerts $+=$ " $b$ "

ElseIf vertauscher $(i)=$ "b" Then End If rueckwaerts $+=$ "g"

Next

If rueckwaerts $=$ vertauscher Then

End If

rueckwaerts = "disregard"

For $a=0$ To konfi. Count - 1

If $\operatorname{konfi}(a)=$ rueckwaerts And $(\operatorname{konfi}(a)$. Contains $(" g ")$ Or kon-

$f i(a)$. Contains("b")) Then

End If

Next

Next

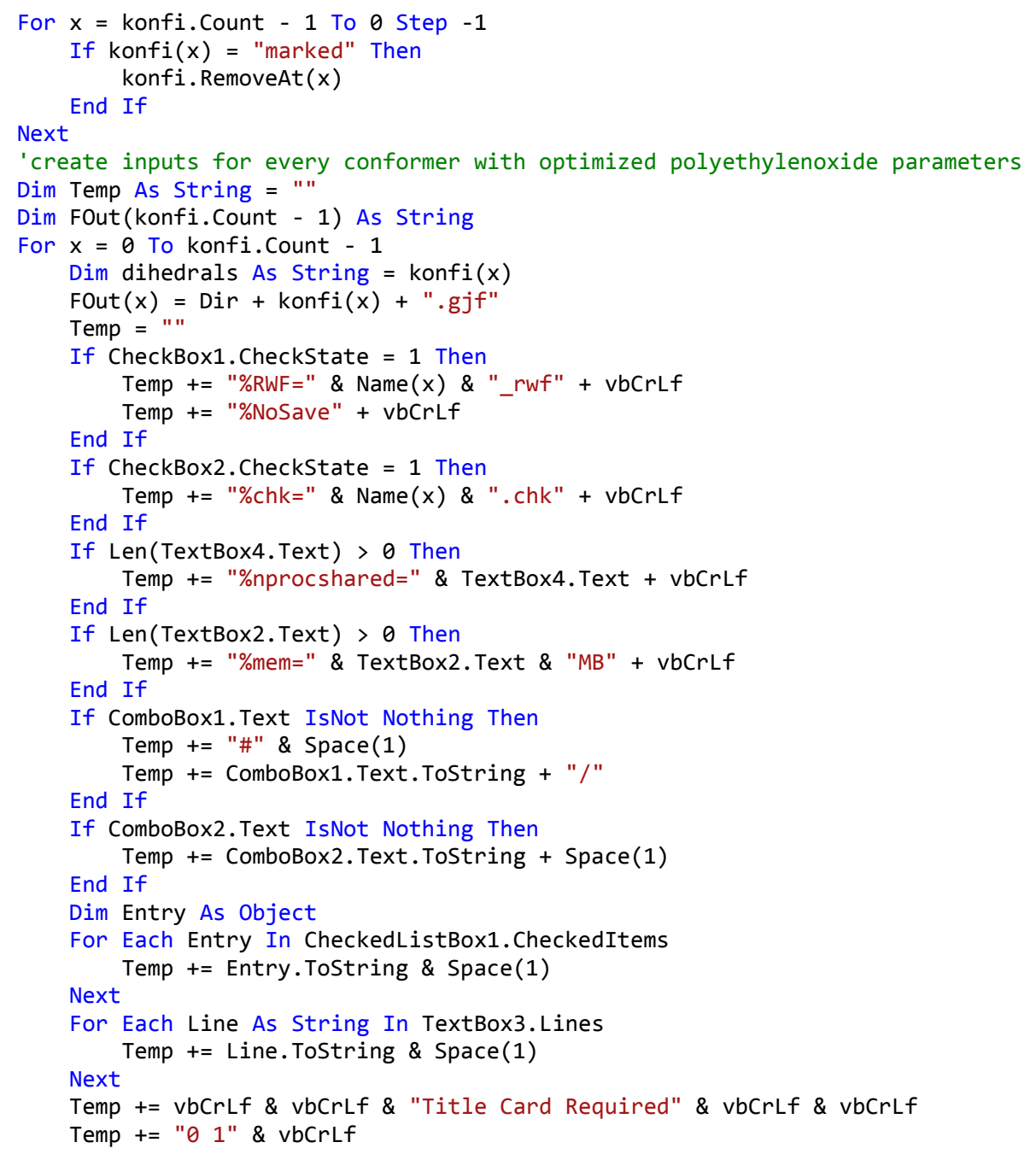


'Set correct dihedrals for the conformer

Dim dvier As String = ""

Dim dfuenf As String $=" "$

Dim dsechs As String = "

Dim dsieben As String $="$ "

Dim dacht As String = " "

Dim dneun As String = " "

Dim dzehn As String = ""

Dim delf As String = " "

Dim dzwoelf As String = " "

Dim ddreizehn As String = "

Dim dvierzehn As String = ""

Dim dfuenfzehn As String = " "

Dim dsechszehn As String = "

Dim dsiebzehn As String = ""

If dihedrals $(\theta)=$ " $t$ " Then

dvier $=" 60.0 "$

dfuenf $="-60 \cdot 0^{\prime \prime}$

dsechs $=" 180.0 "$

ElseIf dihedrals $(\theta)=$ "g" Then

dvier $="-42.0 "$

dfuenf $="-160 \cdot 0 "$

dsechs $=" 80.0 "$

ElseIf dihedrals $(\theta)=$ "b" Then

dvier $=" 160.0 "$

dfuenf $=" 42.0 "$

dsechs $="-80.0 "$

End If

If dihedrals $(1)=$ " $t$ " Then

dsieben $=" 60.0 "$

dacht $="-60 . \theta^{\prime \prime}$

dneun $=" 180.0 "$

ElseIf dihedrals(1) = "g" Then

dsieben $="-46.0 "$

dacht $="-164.0 "$

dneun $=" 75.0 "$

ElseIf dihedrals(1) = "b" Then

dsieben $=" 160.0 "$

dacht $=$ "42.0"

dneun $="-75.0 "$

End If

If dihedrals(2) = " $t$ " Then dzehn $=" 180.0 "$

ElseIf dihedrals(2) = "g" Then dzehn $=$ "75.0"

ElseIf dihedrals(2) = "b" Then dzehn $="-75.0 "$

End If

If dihedrals(3) $=$ " $t$ " Then delf $=$ "60.0"

dzwoelf $="-60.0 "$ ddreizehn = "180.0"

ElseIf dihedrals $(3)=$ "g" Then delf $=$ "-50.0"

dzwoelf $="-168.0 "$ ddreizehn $=" 75.0 "$

ElseIf dihedrals $(3)=$ "b" Then delf $=" 163.0 "$

dzwoelf = "47.0"

ddreizehn $="-75.0 "$

End If

If dihedrals(4) = " $t$ " Then

dvierzehn $=" 60.0 "$

dfuenfzehn $="-60 \cdot \theta^{\prime \prime}$

dsechszehn $=" 180.0 "$

ElseIf dihedrals(4) = "g" Then

dvierzehn $="-50.0 "$

dfuenfzehn $="-168.0 "$

dsechszehn $=" 75.0 "$

ElseIf dihedrals(4) = "b" Then

dvierzehn = "163.0"

dfuenfzehn = "47.0"

dsechszehn $="-75.0 "$

End If

If dihedrals(5) = " $t$ " Then 
dsiebzehn $=" 180.0 "$

ElseIf dihedrals(5) = "g" Then dsiebzehn $=" 75.0 "$

ElseIf dihedrals(5) = "b" Then dsiebzehn $="-75.0 "$ End If

'Build z-matrix

Temp $+=" H "+$ vbCrLf

Temp += "C" + " " + "1" + " " + "B1" + vbCrLf

Temp += "H" + " + + "2" + " " + "B2" + " " + "1" + " " + "A1" + vbCrLf

Temp += "H" + " + "2" + " + "B3" + " + "1" + " + +A2" + " + + "3" + " +

"D1" + " " + "0" + vbCrLf

Temp += "O" + " + "2" + " + "B4" + " + + $1 "$ + " " + $\mathrm{A3}$ + " " + "3" + " +

"D2" + " " + "0" + vbCrLf

Temp += "C" + " + "5" + " + "B5" + " + "2" + " + "A4" + " + + "1" + " +

"D3" + " + + "0" + vbCrLf

Temp += "H" + " + + "6" + " " + "B6" + " + + "5" + " + + $\mathrm{A5}$ + " " + "2" + " " +

"D4" + " + + "0" + vbCrLf

Temp += "H" + " + + "6" + " " + "B7" + " + + "5" + " + + $\mathrm{A6}$ + " " + "2" + " + +

"D5" + " + " $0 "+$ vbCrLf

Temp += "C" + " " + "6" + " " + "B8" + " " + "5" + " " + "A7" + " " + "2" + " " +

"D6" + " + + "0" + vbCrLf

Temp += "H" + " " + "9" + " " + "B9" + " " + "6" + " " + "A8" + " " + "5" + " " +

"D7" + " + + "0" + vbCrLf

Temp += "H" + " " + "9" + " " + "B10" + " " + "6" + " " + "A9" + " " + "5" + " " +

"D8" + " " + "0" + vbCrLf

Temp += "0" + " + "9" + " + "B11" + " + "6" + " + "A10" + " + + "5" + " +

"D9" + " " + "0" + vbCrLf

Temp += "C" + " " + "12" + " " + "B12" + " " + "9" + " " + "A11" + " " + "6" + " "

+ "D10" + " + + "0" + vbCrLf

Temp += "H" + " " + "13" + " " + "B13" + " " + "12" + " + + $\mathrm{A} 12 "$ + " " + "9" + " "

+ "D11" + " " + "0" + vbCrLf

Temp += "H" + " " + "13" + " " + "B14" + " " + "12" + " " + "A13" + " " + "9" + "

+ "D12" + " " + "0" + vbCrLf

Temp += "C" + " " + "13" + " " + "B15" + " " + "12" + " " + "A14" + " " + "9" + " "

+ "D13" + " + + "0" + vbCrLf

Temp += "H" + " " + "16" + " " + "B16" + " " + "13" + " " + "A15" + " " + "12" + "

" + "D14" + " " + "0" + vbCrLf

Temp += "H" + " + "16" + " " + "B17" + " " + "13" + " " + "A16" + " " + "12" + "

$"+$ + D15" + " " + "0" + vbCrLf

Temp + = "0" + " " + "16" + " " + "B18" + " " + "13" + " " + "A17" + " " + "12" + "

$"+$ + D16" + " + + "0" + vbCrLf

Temp += "C" + " + + "19" + " " + "B19" + " " + "16" + " " + "A18" + " " + "13" + "

$"+$ "D17" + " " + "0" + vbCrLf

Temp += "H" + " " + "20" + " " + "B20" + " " + "19" + " " + "A19" + " " + "16" + "

" + "D18" + " " + "0" + vbCrLf

Temp += "H" + " " + "20" + " " + "B21" + " " + "19" + " " + "A20" + " " + "16" + "

" + "D19" + " + + "0" + vbCrLf

Temp += "H" + " + + "20" + " " + "B22" + " " + "19" + " " + "A21" + " " + "16" + "

$"+$ + D20" + " " + " $\theta "+$ vbCrLf + vbCrLf

Temp += "B1" + " " + "1.09678257" + vbCrLf + "B2" + " " + "1.0934579" + vbCrLf +

"B3" + " " + "1.09163193" + vbCrLf + "B4" + " " + "1.41320089" + vbCrLf + "B5" + " " + "1.4129909"

+ vbCrLf + "B6" + " " + "1.09595536" + vbCrLf + "B7" + " " + "1.09747742" + vbCrLf + "B8" + " " +

$" 1.51651879 "$ + vbCrLf + "B9" + " " + "1.09783279" + vbCrLf + "B10" + " " + "1.09685647" + vbCrLf +

"B11" + " " + "1.41436405" + vbCrLf + "B12" + " " + "1.41366661" + vbCrLf + "B13" + " " +

$" 1.09617968 "$ + vbCrLf + "B14" + " " + "1.09731312" + vbCrLf + "B15" + " " + "1.51783602" + vbCrLf +

"B16" + " + " $1.09735206 "$ + vbCrLf + "B17" + " " + "1.09676657" + vbCrLf + "B18" + " " +

$" 1.41239649 "$ + vbCrLf + "B19" + " " + "1.4115081" + vbCrLf + "B20" + " " + "1.09732362" + vbCrLf +

"B21" + " " + "1.0896445" + vbCrLf + "B22" + " " + "1.09603445" + vbCrLf Temp += "A1" + " " + "108.734363" + vbCrLf + "A2" + " " + "108.816312" + vbCrLf +

"A3" + " " + "111.434995" + vbCrLf + "A4" + " " + "113.922565" + vbCrLf + "A5" + " " + "108.650938"

+ vbCrLf + "A6" + " " + "109.950758" + vbCrLf + "A7" + " " + "111.703345" + vbCrLf + "A8" + " " +

"109.521194" + vbCrLf + "A9" + " " + "109.279932" + vbCrLf + "A10" + " " + "110.221446" + vbCrLf +

"A11" + " " + "114.277694" + vbCrLf + "A12" + " " + "109.536914" + vbCrLf + "A13" + " " +

"110.275554" + vbCrLf + "A14" + " " + "110.018055" + vbCrLf + "A15" + " " + "109.665473" + vbCrLf +

"A16" + " " + "109.292313" + vbCrLf + "A17" + " " + "110.527195" + vbCrLf + "A18" + " " +

"113.558148" + vbCrLf + "A19" + " " + "111.475442" + vbCrLf + "A20" + " " + "107.52378" + vbCrLf +

"A21" + " + + "111.207255" + vbCrLf

Temp += "D1" + " " + "118.0000000" + vbCrLf + "D2" + " " + "-120.00000000" + vbCrLf

+ "D3" + " " + "60.00000000" + vbCrLf + "D4" + " " + dvier + vbCrLf + "D5" + " " + dfuenf + vbCrLf

+ "D6" + " " + dsechs + vbCrLf + "D7" + " " + dsieben + vbCrLf + "D8" + " " + dacht + vbCrLf + "D9" + " " + dneun + vbCrLf + "D10" + " " + dzehn + vbCrLf + "D11" + " " + delf + vbCrLf + "D12" + " " + dzwoelf + vbCrLf + "D13" + " " + ddreizehn + vbCrLf + "D14" + " " + dvierzehn + vbCrLf + "D15" + "

$"$ + dfuenfzehn + vbCrLf + "D16" + " " + dsechszehn + vbCrLf + "D17" + " " + dsiebzehn + vbCrLf + 
"D18" + " + + "-60.00000" + vbCrLf + "D19" + " + + "180.000000" + vbCrLf + "D20" + " +

"60.000000" + vbCrLf + vbCrLf + vbCrLf + vbCrLf

Dim OutNum As Integer $=$ FreeFile ()

FileOpen(OutNum, FOut(x), OpenMode.Output)

PrintLine(OutNum, Temp)

Next

Fileclose(OutNum)

MessageBox.Show("DONE")

Else

MessageBox.Show("Error")

End Sub End If

Private Sub Button2 Click(sender As Object, e As EventArgs) Handles Button2.Click

check log files for variation (during optimization) of input dihedral sequence; code is optimized for checking triglyme log files

'Import

OpenFileDialog1.Filter = "log Files |*.log"

If (OpenFileDialog1.ShowDialog( ) = DialogResult.OK) Then

Else

MessageBox.Show(OpenFileDialog1.FileNames.Length \& "Files loaded.")

End If

Dim amount As Integer = OpenFileDialog1.FileNames.Length -1

Dim startkonfis(amount) As String

Dim endkonfis(amount) As String

Dim FName(amount) As String

Dim cur As String = "

get filepath and filename (conformer name)

For $\mathrm{x}=0$ To OpenFileDialog1.FileNames.Length - 1

FName $(x)=$ OpenFileDialog1.FileNames $(x)$

Dim pathsplit As String() = FName(x).Split("\")

For $\mathrm{y}=0$ To pathsplit. Count - 2

Next cur $+=$ pathsplit $(y) \&$ " \"

Dir = cur

cur = ""

Dim splitti As String() = FName(x).Split("\")

Dim part As String ()$=\operatorname{splitti}($ splitti.Count - 1).Split(".")

$\operatorname{startkonfis}(x)=\operatorname{part}(\theta)$

'check and display if jobs were terminated erroneous

Using reader As New IO.StreamReader(OpenFileDialog1.FileNames (x))

While Not reader. EndofStream

Dim line As String = reader . ReadLine ()

If line.Contains("Error termination") Then

MessageBox. Show(startkonfis $(x)$ )

Exit while

End If

End While

Next

End Using

'search for dihedrals in optimized Structures

For $x=0$ To OpenFileDialog1.FileNames. Length - 1

Dim image As String = ""

Dim lines() As String = I0.File.ReadAllLines(OpenFileDialog1.FileNames (x))

Dim linestart As Integer

Dim lineend As Integer

$\mathrm{Dim} i$ As Integer $=0$

Do Until lines(i).Contains("Stationary point found.") $i+=1$

Loop

linestart $=i$

Do Until lines(i).Contains("GradGradGrad")

$$
i+=1
$$

Loop

lineend $=i$

Dim Deins As String = ""

Dim Dzwei As String = ""

Dim Ddrei As String = ""

Dim Dvier As String = ""

Dim Dfuenf As String = ""

Dim Dsechs As String = " "

Dim Dsieben As String = "

Dim Dacht As String = "" 


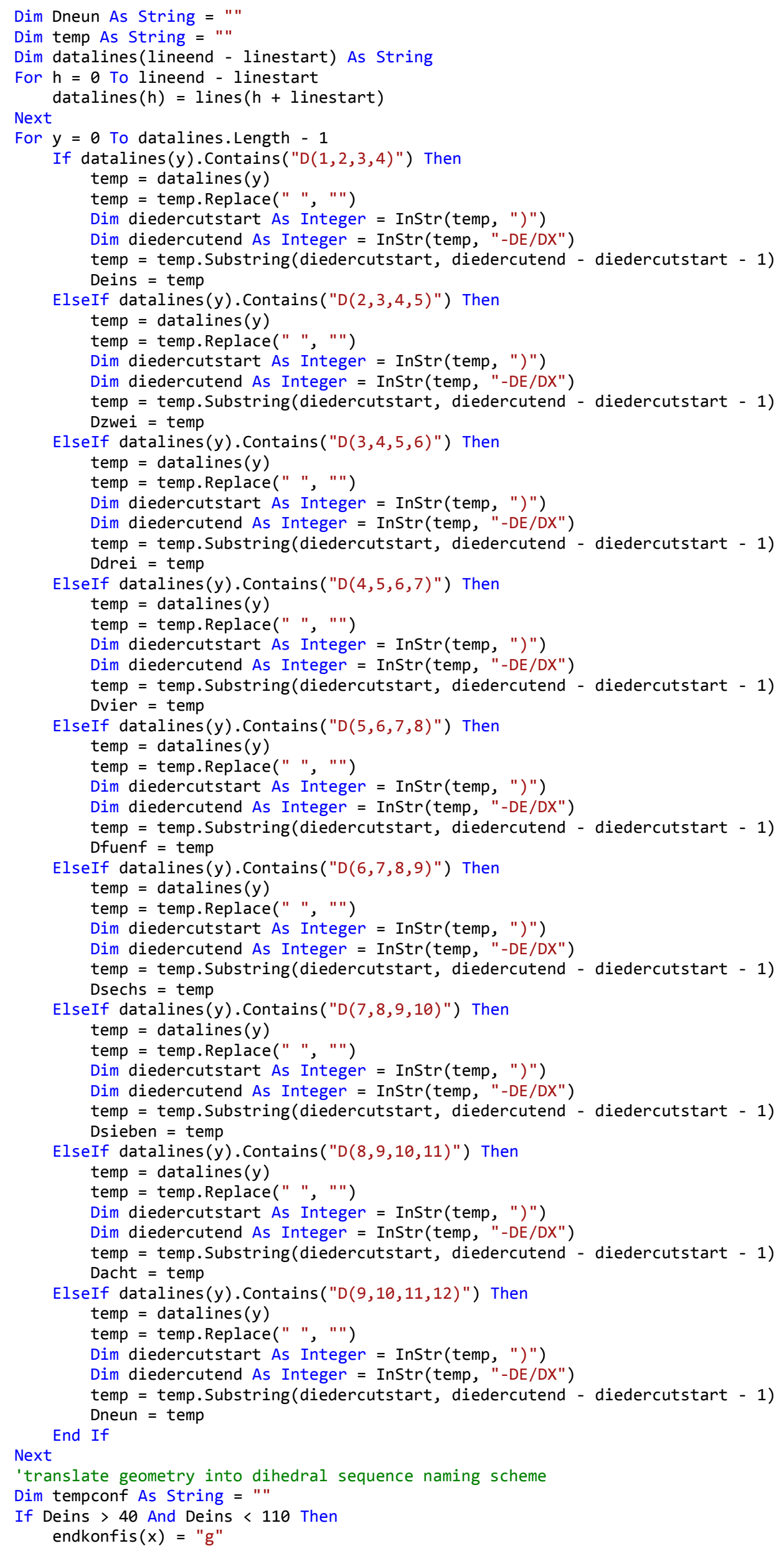


ElseIf Deins > 150 And Deins < 210 Then endkonfis $(x)=$ " $t "$

ElseIf Deins > -210 And Deins $<-150$ Then endkonfis $(x)=$ " $t "$

ElseIf Deins > -100 And Deins < -40 Then endkonfis $(x)=$ "b"

End If

If Dzwei > 40 And Dzwei $<110$ Then endkonfis $(x)+=$ "g"

ElseIf Dzwei > 150 And Dzwei < 210 Then endkonfis $(x)+=$ " $t$ "

ElseIf Dzwei > -210 And Dzwei < -150 Then endkonfis $(x)+=$ " $t$ "

ElseIf Dzwei > -100 And Dzwei $<-40$ Then endkonfis $(x)+=" b "$

End If

If Ddrei > 40 And Ddrei < 110 Then endkonfis $(x)+=" g "$

ElseIf Ddrei > 150 And Ddrei < 210 Then endkonfis $(x)+=$ " $t "$

ElseIf Ddrei > -210 And Ddrei < -150 Then endkonfis $(x)+=$ " $t "$

ElseIf Ddrei > -120 And Ddrei < -40 Then endkonfis $(x)+=" b "$

End If

If Dvier > 40 And Dvier < 110 Then endkonfis $(x)+=$ "g"

ElseIf Dvier > 150 And Dvier < 210 Then endkonfis $(x)+=$ " $t "$

ElseIf Dvier > -210 And Dvier < -150 Then endkonfis $(x)+=$ " $t$ "

ElseIf Dvier > -100 And Dvier $<-40$ Then endkonfis $(x)+=" b "$

End If

If Dfuenf > 40 And Dfuenf $<110$ Then endkonfis $(x)+=$ "g"

ElseIf Dfuenf > 150 And Dfuenf < 210 Then endkonfis $(x)+=$ " $t "$

ElseIf Dfuenf $>-210$ And Dfuenf $<-150$ Then endkonfis $(x)+=$ " $t$ "

ElseIf Dfuenf $>-100$ And Dfuenf $<-40$ Then endkonfis $(x)+=" b "$

End If

If Dsechs > 40 And Dsechs < 110 Then endkonfis $(x)+=$ "g"

ElseIf Dsechs > 150 And Dsechs < 210 Then endkonfis $(x)+=$ " $t "$

ElseIf Dsechs $>-210$ And Dsechs $<-150$ Then endkonfis $(x)+=$ " $t$ "

ElseIf Dsechs > -100 And Dsechs $<-40$ Then endkonfis $(x)+=" b "$

End If

If Dsieben > 40 And Dsieben < 110 Then endkonfis $(x)+=$ "g"

ElseIf Dsieben > 150 And Dsieben < 210 Then endkonfis $(x)+=$ " $t$ "

ElseIf Dsieben > -210 And Dsieben < -150 Then endkonfis $(x)+=$ " $t "$

ElseIf Dsieben > -100 And Dsieben $<-40$ Then endkonfis $(x)+=" b "$

End If

If Dacht $>40$ And Dacht $<110$ Then endkonfis $(x)+=$ "g"

ElseIf Dacht > 150 And Dacht $<210$ Then endkonfis $(x)+=$ " $t$ "

ElseIf Dacht $>-210$ And Dacht $<-150$ Then endkonfis $(x)+=$ " $t$ "

ElseIf Dacht > -100 And Dacht $<-40$ Then endkonfis $(x)+=$ "b"

End If

If Dneun > 40 And Dneun $<110$ Then endkonfis $(x)+=" g "$

ElseIf Dneun > 150 And Dneun < 210 Then endkonfis $(x)+=$ " $t "$

ElseIf Dneun > -200 And Dneun < -150 Then endkonfis $(x)+=$ " $t$ " 


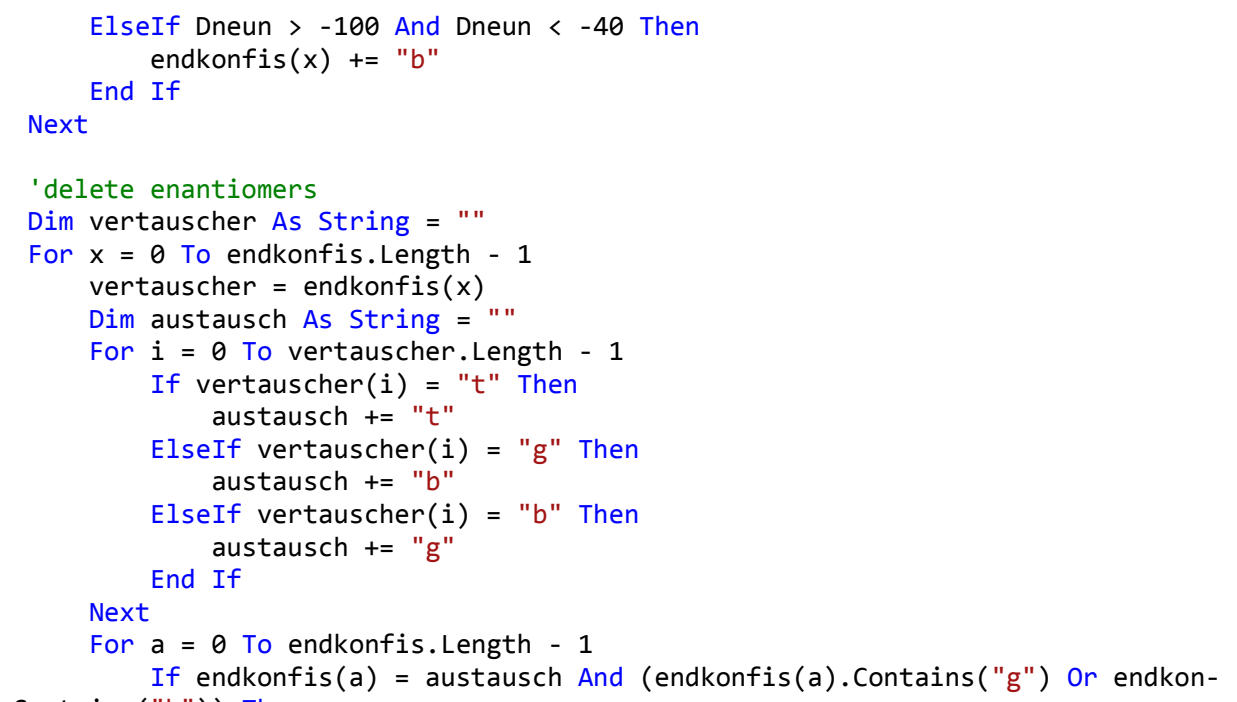

fis (a).Contains ("b")) Then

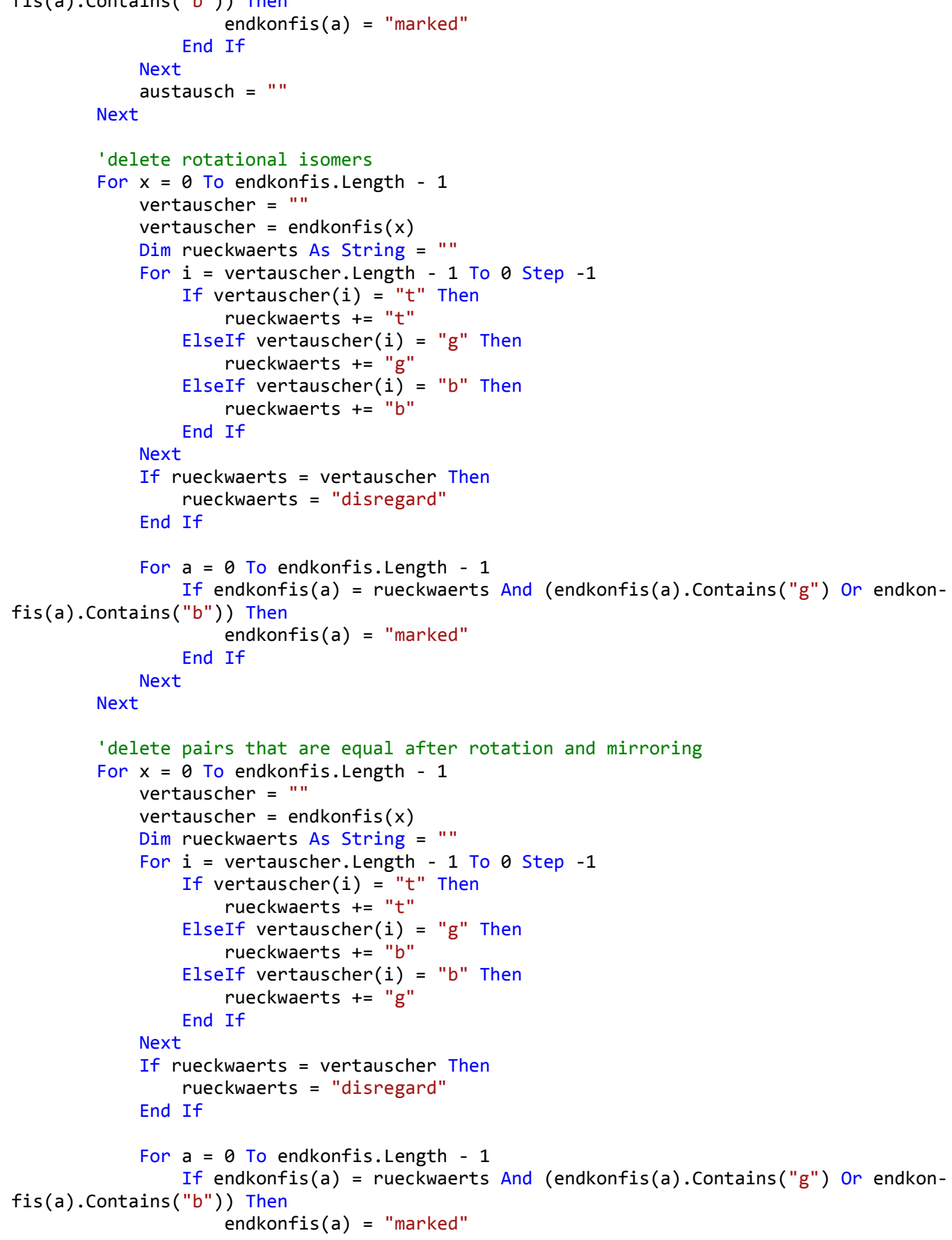




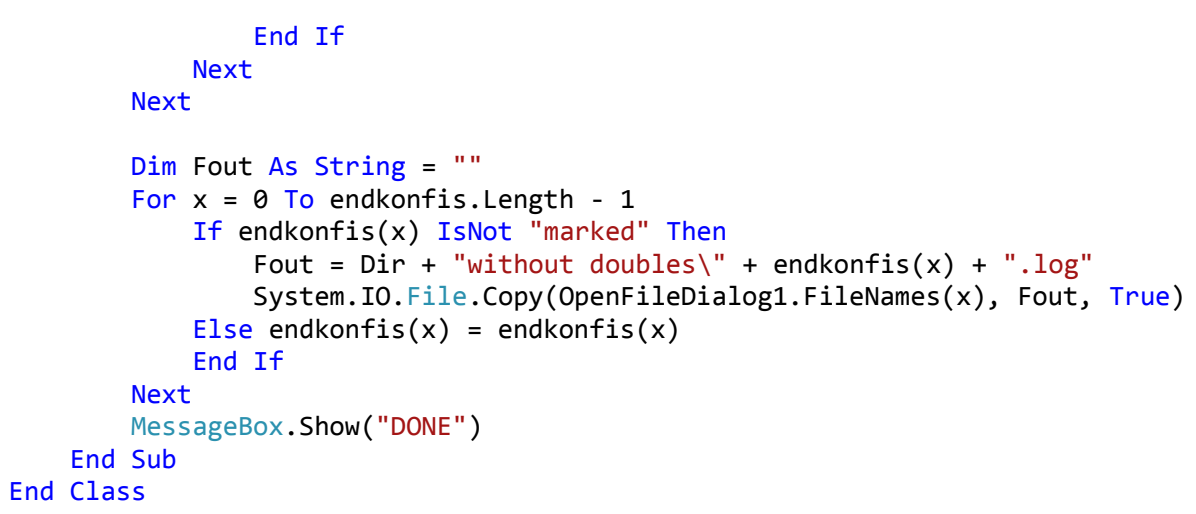

\section{A.11 - Log File Analyzer}

Quantum chemical calculations were used throughout this work to support the experimental results. Typically, calculations on numerous conformers of the same molecule were carried out in Gaussian 09. In order to speed up the analysis of the rather poorly formatted log files a selfmade program "Log File Analyzer" was used. It allows comparing energies of all loaded log files and outputs an energy table that is sorted based on increasing electronic energies if vibrational zero-point energies were not computed otherwise it is sorted by ascending zero-point corrected energies. Furthermore, the program can extract rotational constants, dipole moments, vibrational frequencies or Raman activites of all loaded log files via user request. Additionally, it allows creating new Gaussian 09 input files that use the last geometries of the loaded log files. Some frequently used input parameters, options, functionals and basis sets are selectable for user convenience. The graphical user interface (GUI) of the program is shown in Figure A.11.1. The source code of the program which was written in the Visual Basic language is posted subsequently.

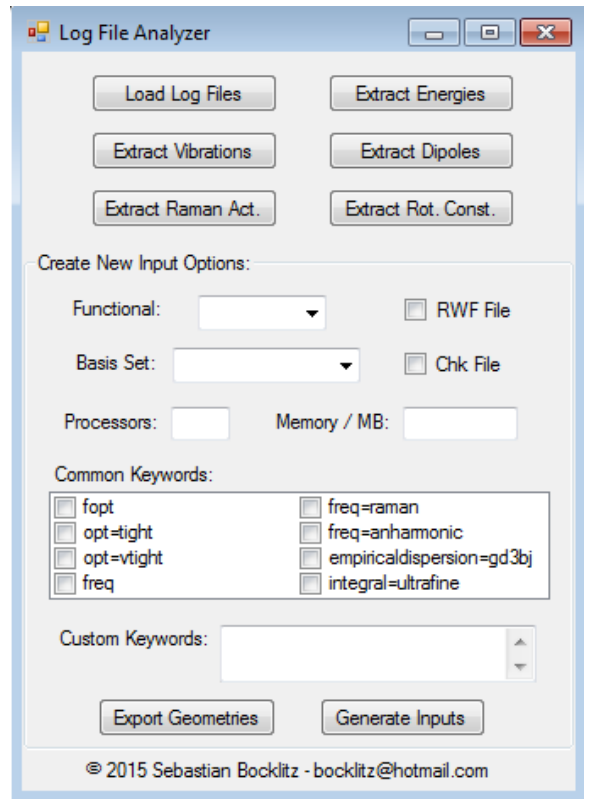

Figure A.11.1: Graphical user interface of the Log File Analyzer. 
Public Class Form 1

' Log File Analyzer 1.1 by Sebastian Bocklitz @ 2015

Public Fout As String

Public Energy As Double

Public zpve As Double

Public firstfreq As Double

Public gibbs As Double

Public lastEnergy As Double

Public linestart As Integer

Public lineend As Integer

Public lineendalt As Integer

Public lineno As Integer

Public laserwav As String

Public outlaser As String

Public Temperature As String

Private Sub Button1_Click(sender As Object, e As EventArgs) Handles Button1.Click OpenFileDialog1.Filter = "log Files |*.log;*.out"

If (OpenFileDialog1.ShowDialog ()$=$ DialogResult.OK) Then Else

MessageBox.Show(OpenFileDialog1.FileNames.Length \& "Files loaded.")

MessageBox.Show("Error: No Files selected.") End If

End Sub

Private Sub TextBox1_KeyPress(ByVal sender As System.Object, ByVal e As System.Windows.Forms.KeyPressEventArgs) Handles TextBox1.KeyPress

If Not Char.IsDigit(e.KeyChar) And Not Char.IsControl(e.KeyChar) Then End If e. Handled = True

End Sub

Private Sub TextBox2_KeyPress(ByVal sender As System.Object, ByVal e As System.Windows. Forms. KeyPressEventArgs) Handles TextBox2.KeyPress If Not Char.IsDigit(e.KeyChar) And Not Char.IsControl(e.KeyChar) Then e. Handled $=$ True

End Sub End If

Private Sub Button3 Click(sender As Object, e As EventArgs) Handles Button3.Click Dim amount As Integer = OpenFileDialog1.FileNames.Length - 1

Dim Energies(amount) As String

Dim FName(amount) As String

Dim cur As String = ""

Dim Dir As String

Dim Cache(amount) As String

Dim Cachezwei(amount) As String

Dim Diff(amount) As String

Dim gibbsdiff(amount) As String

For $\mathrm{x}=0$ To OpenFileDialog1.FileNames.Length - 1

FName $(x)=$ OpenFileDialog1.FileNames $(x)$

Dim pathsplit As String() = FName(x).Split("\")

For $\mathrm{y}=0$ To pathsplit. Count -2

Next cur $+=$ pathsplit $(y) \&$ " $\backslash "$

Dir = cur

cur = " "

Dim Name(amount) As String

Dim splitti As String() = FName(x).Split(" $\backslash ")$

Dim kaunt As Integer = splitti.Count - 1

Dim part As String( $)=\operatorname{splitti}($ kaunt).Split(".")

$\operatorname{Name}(\mathrm{x})=\operatorname{part}(0)$

Fout = Dir + "Energies.dat"

Dim TempL As String = ""

Dim TempZero As String = " "

Dim GibbsTemp As String = ""

Dim freqtemp As String = "

Using reader As New IO.StreamReader(OpenFileDialog1.FileNames(x))

While Not reader. EndofStream

Dim line As String = reader . ReadLine ()

If line.Contains("E(RB3LYP)") Then

TempL += line + vbCrLf

ElseIf line.Contains("E2(B2PLYP)") Then TempL $+=$ line + vbCrLf

ElseIf line.Contains("E(RB97") Then TempL $+=$ line + vbCrLf

ElseIf line.Contains("EUMP2") Then TempL += line + vbCrLf 


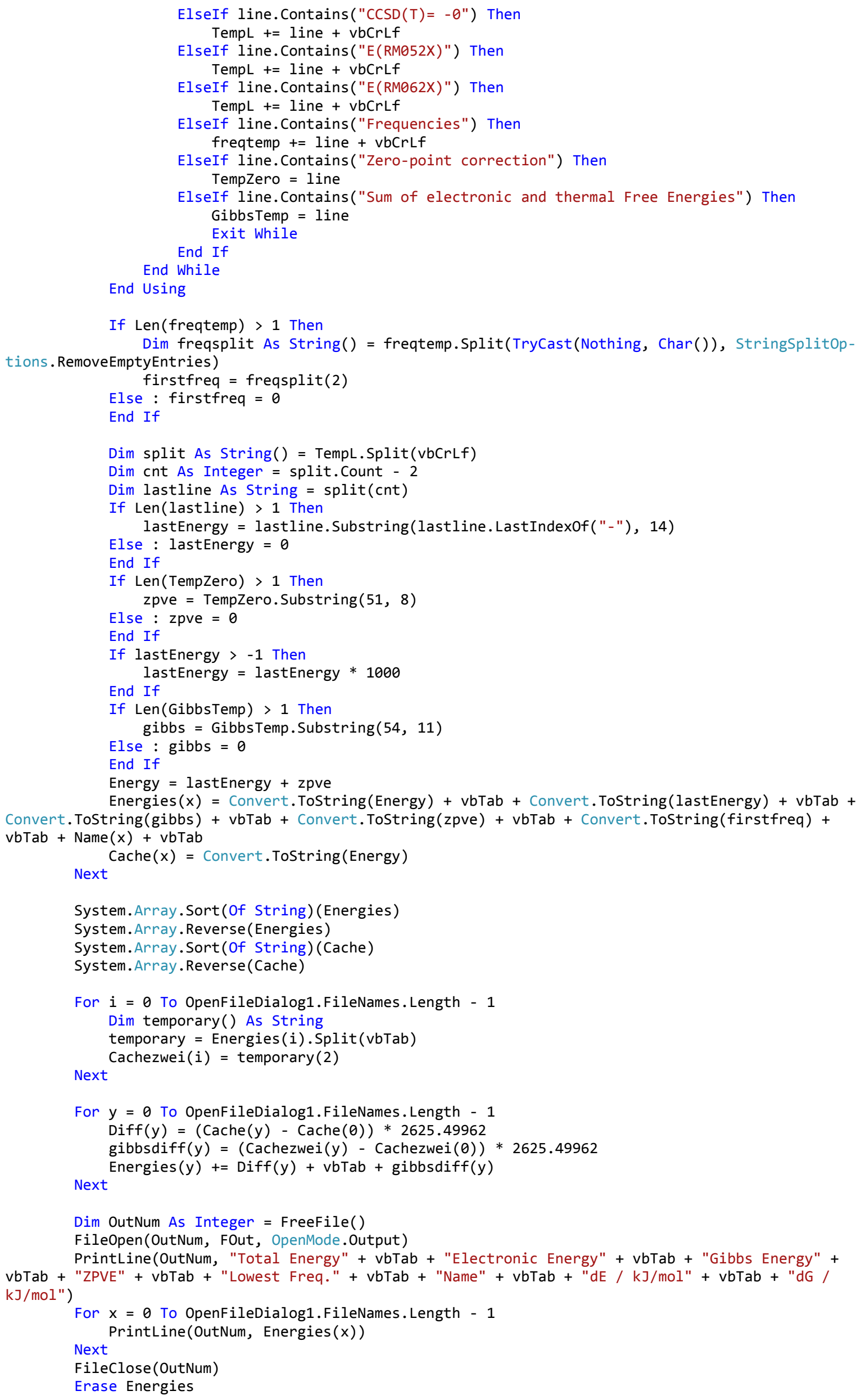


If zpve $=0$ Then

MessageBox.Show("No Zero-point vibrational energies were found.") End If

If lastEnergy $=0$ Then

MessageBox.Show("No electronic Energies were found.")

Else : MessageBox.Show(OpenFileDialog1.FileNames.Length \& " Energies were analyzed." + vbCrLf + "Energy Table has been created.") End If

End Sub

Private Sub Button2_Click(sender As Object, e As EventArgs) Handles Button2.Click

Dim amount As Integer = OpenFileDialog1.FileNames. Length -1

Dim FName(amount) As String

Dim cur As String = " "

Dim Dir As String

Dim Cache(amount) As String

Dim Diff(amount) As String

Dim Temp As String = " "

For $x=0$ To OpenFileDialog1.FileNames.Length - 1

$\mathrm{FName}(\mathrm{x})=$ OpenFileDialog1.FileNames $(\mathrm{x})$

Dim pathsplit As String ()$=$ FName $(x)$.Split (" $\backslash ")$

For $\mathrm{y}=0$ To pathsplit. Count - 2

Next cur $+=$ pathsplit $(y) \& " \backslash "$

Dir $=$ cur

cur $=" "$

Dim Name(amount) As String

Dim FOut(amount) As String

Dim splitti As String ()$=$ FName(x).Split(" $(")$

Dim kaunt As Integer = splitti. Count -1

Dim part As String ()$=$ splitti(kaunt).Split $(" . ")$

$\operatorname{Name}(x)=\operatorname{part}(\theta)$

$\operatorname{FOut}(x)=\operatorname{Dir}+\operatorname{Name}(x)+" \cdot g j f "$

Temp $="$ "

If CheckBox1. CheckState $=1$ Then

Temp $+=$ "RWF=" \& Name(x) \& "_rwf" + vbCrLf

Temp $+=$ "NoSave" + vbCrLf

End If

If CheckBox2. CheckState $=1$ Then

End If

Temp $+=$ \%chk=" \& Name $(x) \& "$. chk" + vbCrLf

If Len(TextBox1.Text) $>0$ Then

End If

Temp $+=$ "\%nprocshared=" \& TextBox1.Text + vbCrLf

If Len(TextBox2. Text) $>0$ Then

End If

Temp $+=$ \%mem=" \& TextBox2. Text \& "MB" + vbCrLf

If ComboBox1. Text IsNot Nothing Then

Temp $+=$ "\#" \& Space(1)

End If

Temp $+=$ ComboBox1. Text.ToString $+" / "$

If ComboBox2. Text IsNot Nothing Then

Temp $+=$ ComboBox2.Text.ToString + Space(1)

End If

Dim Entry As Object

For Each Entry In CheckedListBox1. CheckedItems

Next

Temp $+=$ Entry.ToString \& Space(1)

For Each Line As String In TextBox3.Lines

Next

Temp $+=$ Line. ToString \& Space(1)

Temp $+=$ vbCrLf \& vbCrLf \& "Title Card Required" \& vbCrLf \& vbCrLf

Temp $+=" 01 "$

Dim TempL As String $=" "$

IO.File.ReadLines(OpenFileDialog1.FileNames $(x)$ )

Dim lines () As String $=$ IO. File. ReadAllLines (OpenFileDialog1.FileNames $(x)$ )

For $\mathrm{h}=0$ To lines. Length - 1

Dim check As Integer $=$ InStr(lines(h), "Standard orientation")

If check $>0$ Then

linestart $=h$

End If

Next

For $j=0$ To lines.Length -1 


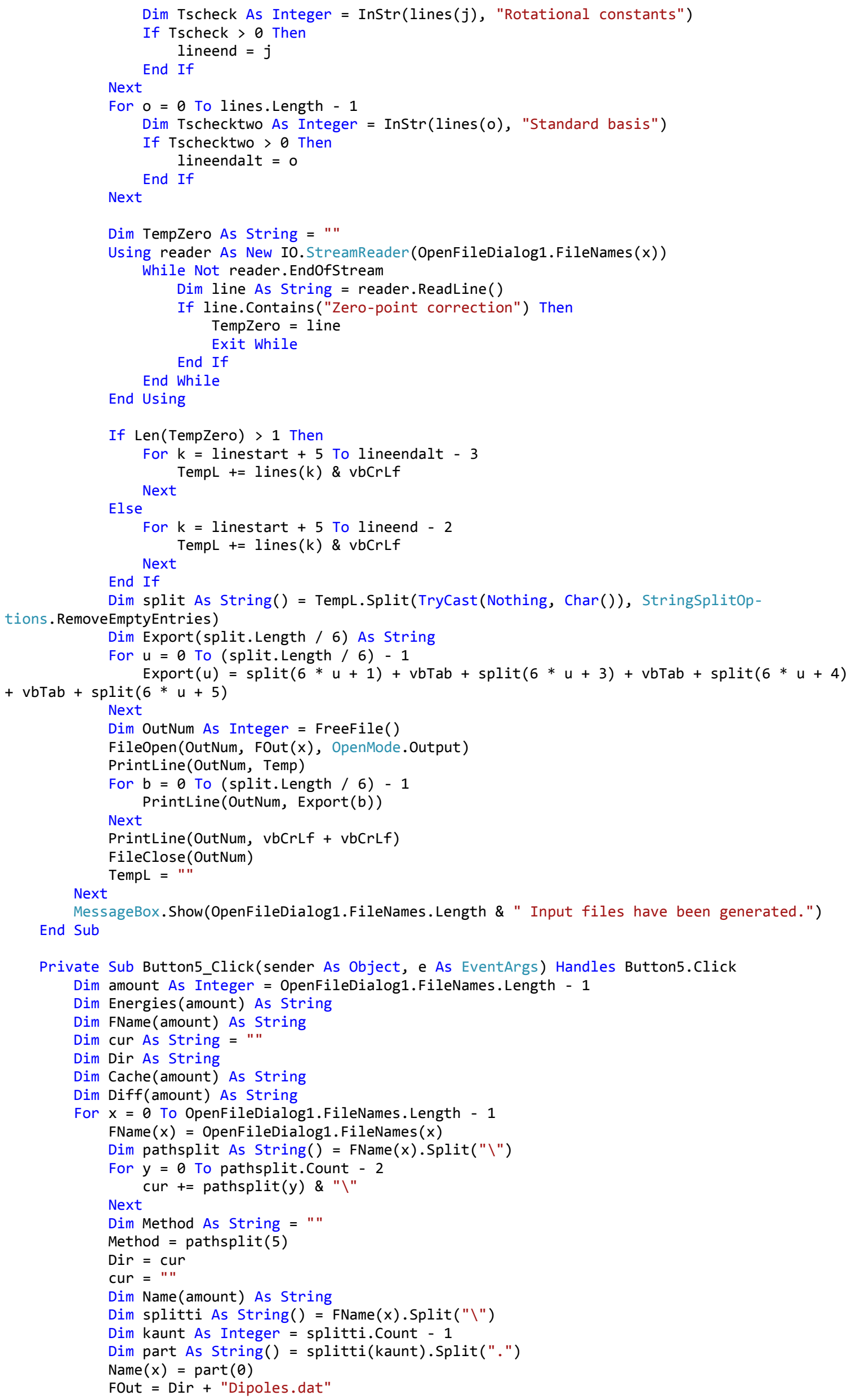


Dim TempL As String = ""

I0.File.ReadLines (OpenFileDialog1.FileNames $(x)$ )

Dim lines () As String $=$ IO.File.ReadAllLines(OpenFileDialog1.FileNames $(x)$ )

For $\mathrm{h}=0$ To lines.Length -1

Dim check As Integer = InStr (lines $(\mathrm{h})$, "Dipole moment")

If check $>0$ Then

lineno $=h$

End If

Next

TempL $=$ lines $($ lineno +1$)$

Dim split As String() = TempL.Split(TryCast(Nothing, Char()), StringSplitop-

tions. RemoveEmptyEntries)

Dim Export As String = " "

For $\mathrm{u}=0$ To 3

Next

Export $+=\operatorname{split}(2 * u+1)+\operatorname{vbTab}$

Next

$\operatorname{Energies}(x)=\operatorname{Name}(x)+\operatorname{vbTab}+\operatorname{TempL}$

Dim OutNum As Integer = FreeFile()

Fileopen(OutNum, FOut, OpenMode.Output)

"Total/D")

PrintLine(OutNum, "Conformer" + vbTab + "X/D" + vbTab + "Y/D" + vbTab + "Z/D" + vbTab +

For $x=0$ To OpenFileDialog1.FileNames.Length -1

Next

PrintLine(OutNum, Energies $(x)$ )

Fileclose(OutNum)

MessageBox.Show(OpenFileDialog1.FileNames.Length * 3 \& " Dipole components were analyzed."

+ vbCrLf + "Table With Dipoles has been created.")

Erase Energies

End Sub

Private Sub Button4_Click(sender As Object, e As EventArgs) Handles Button4.Click

Dim amount As Integer = OpenFileDialog1.FileNames.Length -1

Dim Frequencies As String = "

Dim FName(amount) As String

Dim cur As String = " "

Dim Dir As String

Dim Cache(amount) As String

Dim Diff(amount) As String

For $\mathrm{x}=0$ To OpenFileDialog1.FileNames.Length - 1

$\mathrm{FName}(\mathrm{x})=$ OpenFileDialog1.FileNames $(\mathrm{x})$

Dim pathsplit As String() = FName $(x)$.Split(" $\backslash ")$

For $y=0$ To pathsplit. Count - 2

Next cur $+=$ pathsplit $(\mathrm{y}) \&$ \&"

Dim Method As String = " "

Method = pathsplit(5)

Dir $=$ cur

cur $="$ "

Dim Name(amount) As String

Dim splitti As String() = FName(x).Split(" $\backslash ")$

Dim kaunt As Integer = splitti. Count -1

Dim part As String ()$=\operatorname{splitti}($ kaunt $)$.Split (".")

$\operatorname{Name}(x)=\operatorname{part}(\theta)$

FOut $=$ Dir + Name $(x)+$ "_Frequencies.dat"

Dim TempL As String =

Dim Symmetry As String = ""

Dim Intensity As String = " "

IO.File. ReadLines (OpenFileDialog1.FileNames $(x)$ )

Dim lines () As String $=$ IO.File.ReadAllLines (OpenFileDialog1. FileNames $(x)$ )

For $\mathrm{h}=0$ To lines.Length -1

Dim check As Integer $=\operatorname{InStr}(\operatorname{lines}(\mathrm{h})$, "Frequencies")

If check $>0$ Then

TempL $+=\operatorname{lines}(h)+v b C r L f$

End If

Symmetry $+=\operatorname{lines}(h-1)+\operatorname{vbCrLf}$

Dim checktwo As Integer = InStr(lines(h), "IR Inten")

If checktwo $>0$ Then

Intensity $+=\operatorname{lines}(h)+\operatorname{vbCrLf}$

Next

End If 
Dim symsplit As String() = Symmetry.Split(TryCast(Nothing, Char()), StringSplitoptions.RemoveEmptyEntries)

Dim freqsplit As String() = TempL.Split(TryCast(Nothing, Char()), StringSplitoptions.RemoveEmptyEntries)

Dim intsplit As String() = Intensity.Split(TryCast(Nothing, Char()), StringSplitoptions.RemoveEmptyEntries)

Dim ExportSym(symsplit.Length - 1) As String

Dim ExportVib(symsplit.Length - 1) As String

Dim ExportInt(symsplit.Length - 1) As String

Dim vibno As Integer

Dim intno As Integer

For $u=0$ To symsplit. Length - 1

$\operatorname{ExportSym}(u)=\operatorname{symsplit}(u)$

vibno $=2 * \operatorname{Int}(u / 3)+2+(u)$

$\operatorname{ExportVib}(u)=$ freqsplit (vibno)

intno $=3 * \operatorname{Int}(u / 3)+3+(u)$

Next

$\operatorname{ExportInt}(u)=\operatorname{intsplit}($ intno)

Dim OutNum As Integer $=$ FreeFile ()

FileOpen(OutNum, FOut, OpenMode.Output)

ty $/ \mathrm{km} / \mathrm{mol} "$ )

PrintLine(OutNum, "Symmetry" + vbTab + "Frequency/cm-1" + vbTab + "IR Intensi-

For $y=0$ To ExportSym. Length - 1

PrintLine(OutNum, ExportSym $(y)+v b T a b+\operatorname{ExportVib}(y)+\operatorname{vbTab}+\operatorname{ExportInt}(y))$ Next

Next

Fileclose(OutNum)

MessageBox.Show(OpenFileDialog1.FileNames.Length \& " Files have been created.")

End Sub

Private Sub Button7_Click(sender As Object, e As EventArgs) Handles Button7.Click

Dim amount As Integer = OpenFileDialog1.FileNames. Length -1

Dim Energies(amount) As String

Dim FName(amount) As String

Dim cur As String = " "

Dim Dir As String

Dim Cache(amount) As String

Dim Diff(amount) As String

For $\mathrm{x}=0$ To OpenFileDialog1.FileNames.Length - 1

FName $(x)=$ OpenFileDialog1.FileNames $(x)$

Dim pathsplit As String() = FName(x).Split ("\")

For $\mathrm{y}=0$ To pathsplit. Count - 2

Next cur $+=$ pathsplit $(y) \&$ " $\backslash "$

Dim Method As String = " "

Method = pathsplit(5)

Dir $=$ cur

cur $=" n$

Dim Name(amount) As String

Dim splitti As String() = FName(x).Split(" $(")$

Dim kaunt As Integer = splitti.Count - 1

Dim part As String ()$=$ splitti(kaunt).Split (".")

$\operatorname{Name}(x)=\operatorname{part}(\theta)$

FOut $=$ Dir + "Rotconstants. dat"

Dim TempL As String = ""

I0.File.ReadLines(OpenFileDialog1.FileNames $(x)$ )

Dim lines() As String = IO.File. ReadAllLines(OpenFileDialog1.FileNames $(x)$ )

For $\mathrm{h}=0$ To lines. Length -1

Dim check As Integer $=\operatorname{InStr}(\operatorname{lines}(\mathrm{h})$, "Rotational constant")

If check $>0$ Then

lineno $=h$

Next

End If

TempL $=$ lines(lineno)

Dim split As String ()$=$ TempL.Split(TryCast(Nothing, Char()), StringSplitop-

tions. RemoveEmptyEntries)

Dim Export As String = " "

For $\mathrm{u}=0$ To 2

Next

Export $+=\operatorname{split}(u+3)+$ vbTab

Next

$\operatorname{Energies}(x)=\operatorname{Name}(x)+v b T a b+$ Export 


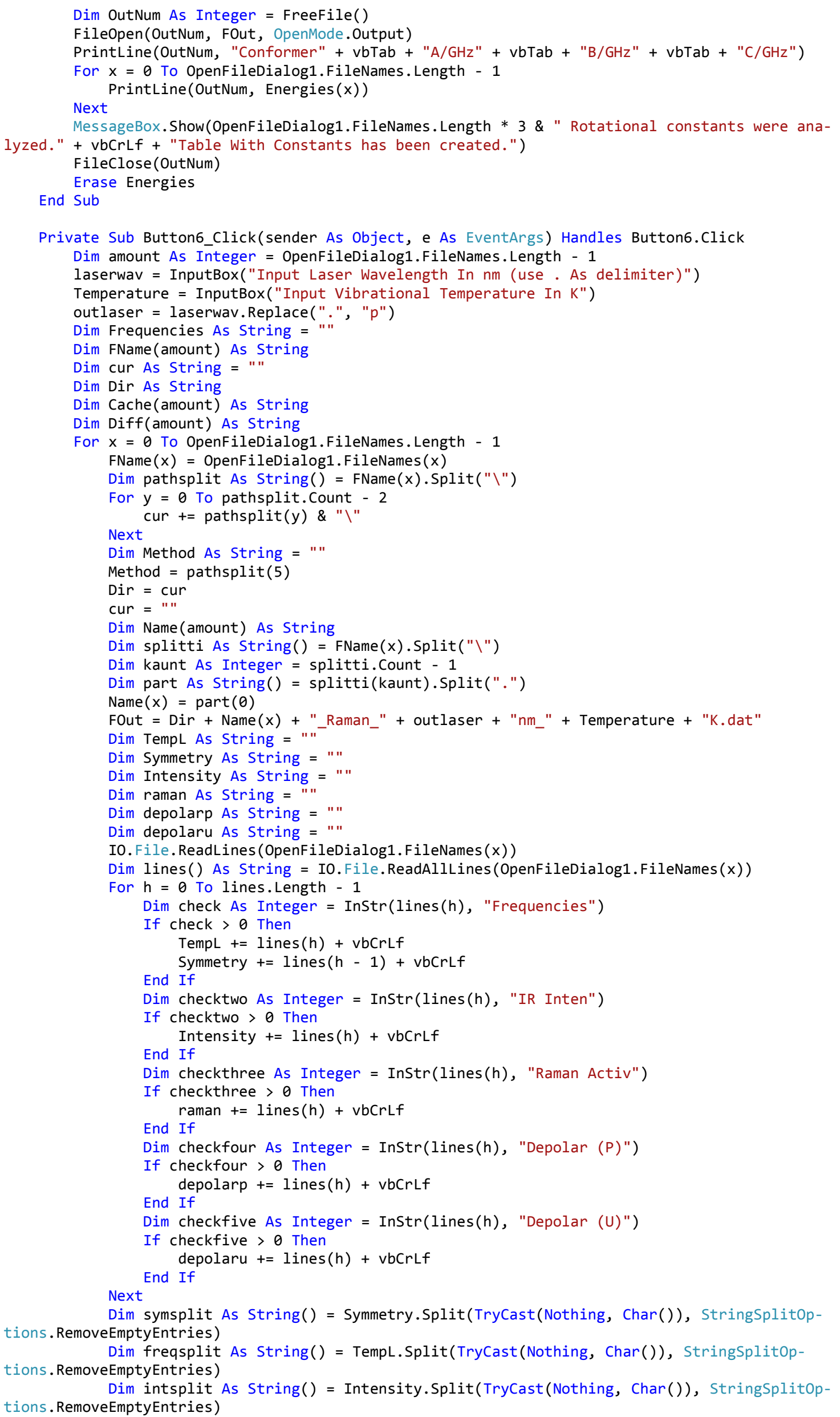


Dim ramansplit As String() = raman.Split(TryCast(Nothing, Char()), StringSplitoptions.RemoveEmptyEntries)

Dim depolarpsplit As String ()$=$ depolarp.Split(TryCast(Nothing, Char()), StringSplitoptions.RemoveEmptyEntries)

Dim depolarusplit As String ()$=$ depolaru.Split(TryCast(Nothing, Char()), StringSplitoptions. RemoveEmptyEntries)

Dim ExportSym(symsplit.Length - 1) As String

Dim ExportVib(symsplit.Length - 1) As String

Dim ExportInt(symsplit.Length - 1) As String

Dim Exportraman(symsplit. Length - 1) As String

Dim Exportdepolarp(symsplit.Length - 1) As String

Dim Exportdepolaru(symsplit.Length - 1) As String

Dim Exportramanscatt(symsplit.Length - 1) As String

Dim gamma(symsplit. Length - 1) As String

Dim alpha(symsplit.Length - 1) As String

Dim ramanangepasst(symsplit.Length - 1) As String

Dim ramanscattangepasst(symsplit.Length - 1) As String

Dim vibno As Integer

Dim intno As Integer

For $u=0$ To symsplit. Length -1

$\operatorname{ExportSym}(u)=\operatorname{symsplit}(u)$

vibno $=2 * \operatorname{Int}(u / 3)+2+(u)$

$\operatorname{ExportVib}(u)=$ freqsplit (vibno)

intno $=3 * \operatorname{Int}(u / 3)+3+(u)$

Exportint $(u)=$ intsplit(intno)

Exportraman $(u)=\operatorname{ramansplit}($ intno)

Exportdepolarp $(u)=$ depolarpsplit(intno)

Exportdepolaru(u) = depolarusplit(intno)

Exportramanscatt $(\mathrm{u})=2 *($ Math.PI $) \wedge 2 * 6.62607004 * 10 \wedge(-34) / 45 / 299792458$

/ ExportVib(u) / $100 *(($ laserwav $* 10 \wedge(-9)) \wedge(-1)-(\operatorname{ExportVib}(\mathrm{u}) * 100)) \wedge 3 *$ (Exportraman(u)

$* 10 \wedge(-40)) *(($ laserwav * $10 \wedge(-9)) \wedge(-1)) /(1.660538921 * 10 \wedge(-27)) *$ ((1 - Math. $\operatorname{Exp}(-$

$6.62607004 * 10 \wedge(-34) * 299792458 * \operatorname{ExportVib}(\mathrm{u}) * 100 /(1.38064852 * 10 \wedge(-23) *$

Temperature))) $\wedge(-1))$

$\operatorname{gamma}(u)=(\operatorname{Exportraman}(u)) *(\operatorname{Exportdepolarp}(u)) /(3 * \operatorname{Exportdepolarp}(u)+3)$

$\operatorname{alpha}(u)=\operatorname{Exportraman}(u) / 45-(7 * \operatorname{gamma}(u) / 45)$

ramanangepasst $(u)=(((45 * \operatorname{alpha}(u)+4 * \operatorname{gamma}(u)) / 45)+\operatorname{gamma}(u) /(15 *(1.5$

$+2 * 10 \wedge(-4) *$ ExportVib(u)) )) *45

ramanscattangepasst $(\mathrm{u})=2 *($ Math.PI $) \wedge 2 * 6.62607004 * 10 \wedge(-34) / 45 /$

299792458 / ExportVib(u) / $100 *(($ laserwav $* 10 \wedge(-9)) \wedge(-1)-(\operatorname{ExportVib}(\mathrm{u}) * 100)) \wedge 3 *$

$($ ramanangepasst $(u) * 10 \wedge(-40)) *(($ laserwav $* 10 \wedge(-9)) \wedge(-1)) /(1.660538921 * 10 \wedge(-27)) *$

$((1-$ Math. $\operatorname{Exp}(-6.62607004 * 10 \wedge(-34) * 299792458 * \operatorname{ExportVib}(\mathrm{u}) * 100 /(1.38064852 * 10 \wedge(-23)$

$*$ Temperature $)) \wedge(-1))$

Next

Dim OutNum As Integer = FreeFile ()

FileOpen(OutNum, FOut, OpenMode.Output)

PrintLine(OutNum, "Symmetry" + vbTab + "Frequency/cm-1" + vbTab + "IR Intensity/km/mol"

+ vbTab + "Depol.Ratio(plane)" + vbTab + "Depol.Ratio(unpol.)" + vbTab + "Raman Activity/A^4/amu" + $\mathrm{vbTab}+$ + Raman Scat.Cross Section/m^2/sr" + vbTab + "Raman Activity/A^4/amu adjusted For McPherson 2501 - N.Lüttschwager data" + vbTab + "Raman Scat.Cross Section/m^2/sr adjusted For McPherson 2501 - N. Lüttschwager data")

For $y=0$ To ExportSym. Length -1

PrintLine(OutNum, ExportSym $(y)+v b T a b+\operatorname{ExportVib}(y)+$ vbTab + ExportInt $(y)+v b T-$ $a b+$ Exportdepolarp $(y)+$ vbTab + Exportdepolaru(y) + vbTab + Exportraman $(y)+v b T a b+E x p o r t r a m a n s-$ $\operatorname{catt}(y)+v b T a b+\operatorname{ramanangepasst}(y)+\operatorname{vbTab}+\operatorname{ramanscattangepasst}(y))$

Next

Next

Fileclose(OutNum) End Sub

MessageBox.Show(OpenFileDialog1.FileNames.Length \& " Files have been created.")

Private Sub Button8_Click(sender As Object, e As EventArgs) Handles Button8.Click

Dim amount As Integer = OpenFileDialog1. FileNames.Length -1

Dim FName(amount) As String

Dim cur As String = ""

Dim Dir As String

Dim Cache(amount) As String

Dim Diff(amount) As String

Dim Temp As String = " "

For $x=0$ To OpenFileDialog1.FileNames.Length - 1

FName $(x)=$ OpenFileDialog1.FileNames $(x)$

Dim pathsplit As String() = FName(x).Split ("\")

For $\mathrm{y}=0$ To pathsplit. Count - 2

Next

cur $+=$ pathsplit $(y) \&$ \& $\backslash "$

Dir = cur 
cur = " "

Dim Name(amount) As String

Dim Fout(amount) As String

Dim splitti As String() = FName(x).Split("\")

Dim kaunt As Integer = splitti.Count - 1

Dim part As String ()$=$ splitti(kaunt).Split(".")

Name $(x)=\operatorname{part}(\theta)$

FOut $(x)=\operatorname{Dir}+\operatorname{Name}(x)+" \cdot x y z "$

Temp $=" "$

Dim TempL As String = " "

I0.File.ReadLines(OpenFileDialog1.FileNames (x))

Dim lines() As String = IO.File.ReadAllLines(OpenFileDialog1.FileNames $(x)$ )

For $\mathrm{h}=0$ To lines. Length -1

Dim check As Integer $=\operatorname{InStr}(\operatorname{lines}(\mathrm{h})$, "Standard orientation")

If check $>0$ Then

linestart $=\mathrm{h}$

Next

End If

For $j=0$ To lines. Length -1

Dim Tscheck As Integer $=\operatorname{InStr}(\operatorname{lines}(j)$, "Rotational constants")

If Tscheck $>0$ Then

lineend $=\mathrm{j}$

Next

End If

For $0=0$ To lines. Length -1

Dim Tschecktwo As Integer $=\operatorname{InStr}(\operatorname{lines}(0)$, "Standard basis")

If Tschecktwo $>0$ Then

lineendalt $=0$

Next

End If

Dim TempZero As String = ""

Using reader As New IO.StreamReader(OpenFileDialog1.FileNames $(x)$ ) While Not reader. EndOfStream

Dim line As String = reader. ReadLine ()

If line.Contains("Zero-point correction") Then

TempZero = line

End If

Exit While

End While

End Using

If Len(TempZero) > 1 Then

For $\mathrm{k}=$ linestart +5 To lineendalt -3

Next TempL $+=\operatorname{lines}(k) \&$ vbCrLf

Else

For $\mathrm{k}=$ linestart +5 To lineend -2

TempL $+=\operatorname{lines}(k) \& \operatorname{vbcrLf}$

End If

Dim split As String ()$=$ TempL.Split(TryCast(Nothing, Char ()), StringSplitop-

tions.RemoveEmptyEntries)

Dim Export(split.Length / 6) As String

For $\mathrm{u}=0$ To (split. Length / 6) - 1

If $\operatorname{split}(6 * u+1)=" 1 "$ Then

$\operatorname{split}(6 * \mathrm{u}+1)=" \mathrm{H}^{\prime}$

End If

If $\operatorname{split}(6 * u+1)=" 2 "$ Then

$\operatorname{split}(6 * u+1)=" \mathrm{He} "$

End If

If $\operatorname{split}(6 * u+1)=" 3 "$ Then

$\operatorname{split}(6 * u+1)=$ "Li"

End If

If $\operatorname{split}(6 * u+1)=" 4 "$ Then $\operatorname{split}(6 * u+1)=$ "Be"

End If

If $\operatorname{split}(6 * u+1)=" 5 "$ Then $\operatorname{split}(6 * u+1)=" B "$

End If

If $\operatorname{split}(6 * u+1)=" 6 "$ Then

$\operatorname{split}(6 * u+1)=" C "$

End If

If $\operatorname{split}(6 * u+1)=" 7 "$ Then $\operatorname{split}(6 * \mathrm{u}+1)=" \mathrm{~N} "$ 


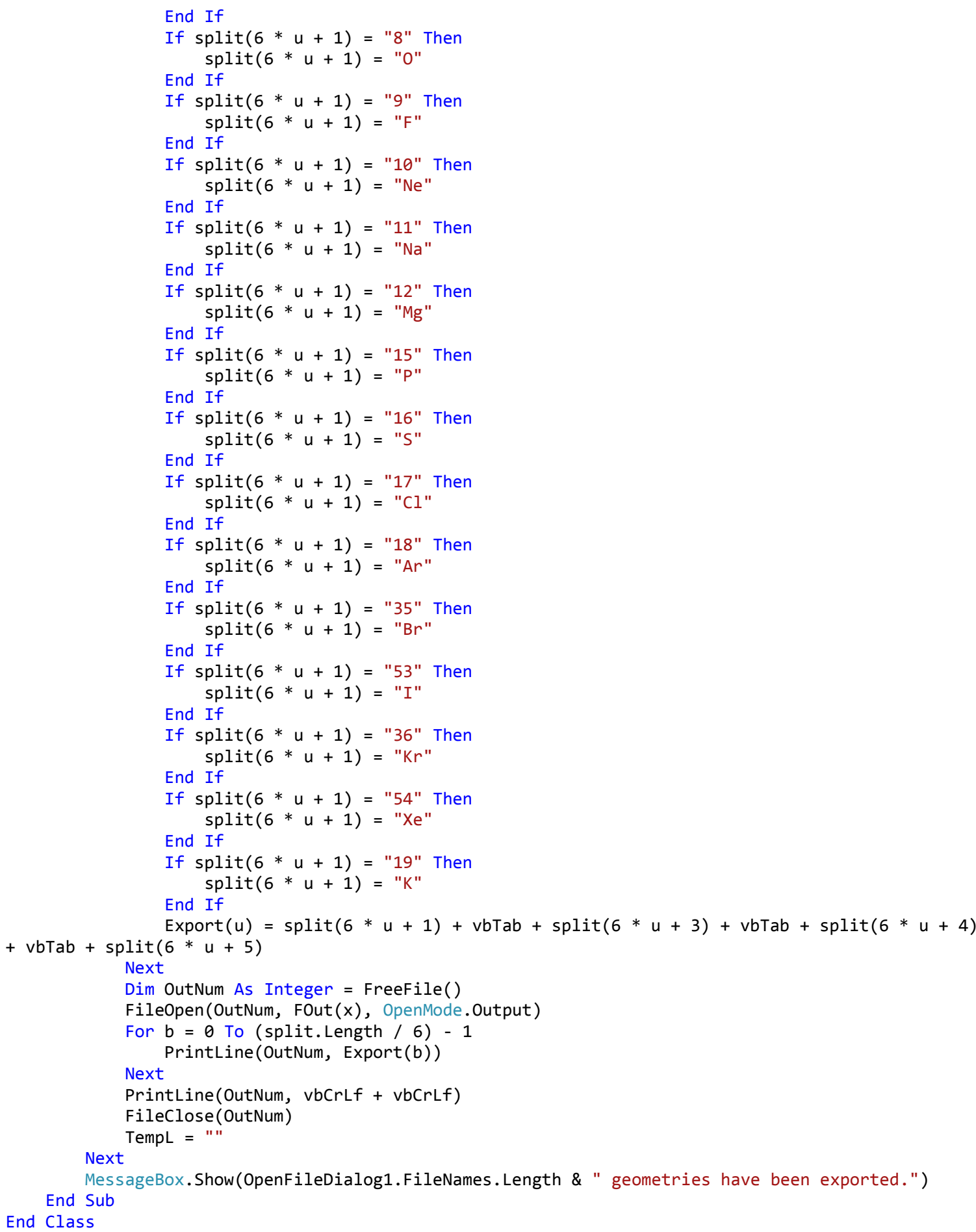


- References

[1] C.M. Dobson, Protein folding and misfolding, Nature 2003, 426, 884-890.

[2] G. Ashraf, N. Greig, T. Khan, I. Hassan, S. Tabrez, S. Shakil, I. Sheikh, S. Zaidi, M. Akram, N. Jabir, C. Firoz, A. Naeem, I. Alhazza, G. Damanhouri, M. Kamal, Protein Misfolding and Aggregation in Alzheimer's Disease and Type 2 Diabetes Mellitus, CNSNDDT 2014, 13, 1280-1293.

[3] E. Frieden, Non-covalent interactions: Key to biological flexibility and specificity, J. Chem. Educ. 1975, 52, 754.

[4] J. Cerný, P. Hobza, Non-covalent interactions in biomacromolecules, Phys. Chem. Chem. Phys. 2007, 9, 5291-5303.

[5] R.E. Smalley, L. Wharton, D.H. Levy, Molecular optical spectroscopy with supersonic beams and jets, Acc. Chem. Res. 1977, 10, 139-145.

[6] R.S. Ruoff, T.D. Klots, T. Emilsson, H.S. Gutowsky, Relaxation of conformers and isomers in seeded supersonic jets of inert gases, J. Chem. Phys. 1990, 93, 31423150.

[7] S. Grimme, J. Antony, S. Ehrlich, H. Krieg, A consistent and accurate ab initio parametrization of density functional dispersion correction (DFT-D) for the 94 elements H-Pu, J. Chem. Phys. 2010, 132, 154104.

[8] S. Tang, H. Zhao, Glymes as Versatile Solvents for Chemical Reactions and Processes: from the Laboratory to Industry, RSC advances 2014, 4, 11251-11287.

[9] K. Halake, M. Birajdar, B.S. Kim, H. Bae, C. Lee, Y.J. Kim, S. Kim, H.J. Kim, S. Ahn, S.Y. An, J. Lee, Recent application developments of water-soluble synthetic polymers, J. Ind. Eng. Chem. 2014, 20, 3913-3918.

[10] R.G. Snyder, The structure of chain molecules in the liquid state: Low-frequency Raman spectra of $n$-alkanes and perfluoro- $n$-alkanes, J. Chem. Phys. 1982, 76, 3921-3927.

[11] N. Goutev, K. Ohno, H. Matsuura, Raman Spectroscopic Study on the Conformation of 1,2-Dimethoxyethane in the Liquid Phase and in Aqueous Solutions, $J$. Phys. Chem. A 2000, 104, 9226-9232.

[12] M. Georgiev, T. Popova, Z. Nickolov, N. Goutev, G. Georgiev, H. Matsuura, Raman spectroscopic study of the hydration of short-chain poly(oxyethylene)s $C_{1} E_{n} C_{1}$ (n=1-4), Open Chemistry 2004, 2.

[13] R. Wada, K. Fujimoto, M. Kato, Why is poly(oxyethylene) soluble in water? Evidence from the thermodynamic profile of the conformational equilibria of 1,2dimethoxyethane and dimethoxymethane revealed by Raman spectroscopy, $J$. Phys. Chem. B 2014, 118, 12223-12231.

[14] R.F. Schaufele, T. Shimanouchi, Longitudinal Acoustical Vibrations of Finite Polymethylene Chains, J. Chem. Phys. 1967, 47, 3605-3610.

[15] K. Song, S. Krimm, Elastic modulus of poly(ethylene oxide) determined from the Raman longitudinal acoustic mode, J. Polym. Sci. B Polym. Phys. 1990, 28, 63-69.

[16] R.G. Snyder, H.L. Strauss, R. Alamo, L. Mandelkern, Chain-length dependence of interlayer interaction in crystalline $n$-alkanes from Raman longitudinal acoustic mode measurements, J. Chem. Phys. 1994, 100, 5422-5431. 
[17] T.N. Wassermann, J. Thelemann, P. Zielke, M.A. Suhm, The stiffness of a fully stretched polyethylene chain: A Raman jet spectroscopy extrapolation, J. Chem. Phys. 2009, 131, 161108.

[18] N.O.B. Lüttschwager, M.A. Suhm, Stretching and folding of 2-nanometer hydrocarbon rods, Soft matter 2014, 10, 4885-4901.

[19] N.O.B. Lüttschwager, Raman Spectroscopy of Conformational Rearrangements at Low Temperatures: Folding and Stretching of Alkanes in Supersonic Jets, Springer International Publishing, Chum, 2014.

[20] M.D. Morse, Supersonic Beam Sources, in: F.B. Dunning (Ed.), Atomic, molecular and optical physics: Atoms and molecules, Acad. Press, San Diego, Calif., 1996, pp. 21-47.

[21] A. Kantrowitz, J. Grey, A High Intensity Source for the Molecular Beam. Part I. Theoretical, Rev. Sci. Instrum. 1951, 22, 328-332.

[22] M. Herman, R. Georges, M. Hepp, D. Hurtmans, High resolution Fourier transform spectroscopy of jet-cooled molecules, Int. Rev. Phys. Chem. 2000, 19, 277-325.

[23] R. Campargue, Progress in overexpanded supersonic jets and skimmed molecular beams in free-jet zones of silence, J. Phys. Chem. 1984, 88, 4466-4474.

[24] R.E. Smalley, B.L. Ramakrishna, D.H. Levy, L. Wharton, Laser spectroscopy of supersonic molecular beams: Application to the $\mathrm{NO}_{2}$ spectrum, J. Chem. Phys. 1974, 61, 4363-4364.

[25] K. Otto, Raman-Spektroskopie kleiner Moleküle und Molekülaggregate im Überschallstrahl nach thermischer Anregung. e-Dissertation, available at http://hdl.handle.net/11858/00-1735-0000-0022-6045-7.

[26] T.N. Wassermann, M.A. Suhm, Ethanol monomers and dimers revisited: a Raman study of conformational preferences and argon nanocoating effects, J. Phys. Chem. A 2010, 114, 8223-8233.

[27] P. Felder, H.H. Günthard, Conformational interconversions in supersonic jets: Matrix IR spectroscopy and model calculations, Chem. Phys. 1982, 71, 9-25.

[28] M. Heger, K.E. Otto, R.A. Mata, M.A. Suhm, Bracketing subtle conformational energy differences between self-solvated and stretched trifluoropropanol, Phys. Chem. Chem. Phys. 2015, 17, 9899-9909.

[29] J.M. Hayes, Analytical spectroscopy in supersonic expansions, Chem. Rev. 1987, 87, 745-760.

[30] W.Y. Sohn, J.S. Kang, S.Y. Lee, H. Kang, Fluorescence excitation spectrum and solvent-assisted conformational isomerization (SACI) of jet-cooled acetaminophen, Chem. Phys. Lett. 2013, 581, 36-41.

[31] M.A. Suhm, F. Kollipost, Femtisecond single-mole infrared spectroscopy of molecular clusters, Phys. Chem. Chem. Phys. 2013, 15, 10702-10721.

[32] J.B. Anderson, Separation of gas mixtures in free jets, AIChE J. 1967, 13, 11881192.

[33] J.B. Anderson, P. Davidovits, Isotope separation in a "seeded beam", Science 1975, 187, 642-644. 
[34] T.S. Zwier, Laser Spectroscopy of Jet-Cooled Biomolecules and Their WaterContaining Clusters: Water Bridges and Molecular Conformation, J. Phys. Chem. A 2001, 8827-8839.

[35] A. Bach, S. Coussan, A. Müller, S. Leutwyler, Water-chain clusters: Vibronic spectra of 7-hydroxyquinoline-(H20)2, J. Chem. Phys. 2000, 112, 1192-1203.

[36] R.H. Page, Y.R. Shen, Y.T. Lee, Local modes of benzene and benzene dimer, studied by infrared-ultraviolet double resonance in a supersonic beam, J. Chem. Phys. 1988, 88, 4621-4636.

[37] R.H. Page, Y.R. Shen, Y.T. Lee, Infrared-ultraviolet double resonance studies of benzene molecules in a supersonic beam, J. Chem. Phys. 1988, 88, 5362-5376.

[38] T. Walther, H. Bitto, T.K. Minton, J.R. Huber, UV-IR double-resonance spectroscopy of jet-cooled propynal detected by the fluorescence dip method, Chem. Phys. Lett. 1994, 231, 64-69.

[39] M. J. Frisch, G. W. Trucks, H. B. Schlegel, G. E. Scuseria, M. A. Robb, J. R. Cheeseman, G. Scalmani, V. Barone, G. A. Petersson, H. Nakatsuji, X. Li, M. Caricato, A. V. Marenich, J. Bloino, B. G. Janesko, R. Gomperts, B. Mennucci, H. P. Hratchian, J. V. Ortiz, A. F. Izmaylov, J. L. Sonnenberg, D. Williams-Young, F. Ding, F. Lipparini, F. Egidi, J. Goings, B. Peng, A. Petrone, T. Henderson, D. Ranasinghe, V. G. Zakrzewski, J. Gao, N. Rega, G. Zheng, W. Liang, M. Hada, M. Ehara, K. Toyota, R. Fukuda, J. Hasegawa, M. Ishida, T. Nakajima, Y. Honda, O. Kitao, H. Nakai, T. Vreven, K. Throssell, J. A. Montgomery, Jr., J. E. Peralta, F. Ogliaro, M. J. Bearpark, J. J. Heyd, E. N. Brothers, K. N. Kudin, V. N. Staroverov, T. A. Keith, R. Kobayashi, J. Normand, K. Raghavachari, A. P. Rendell, J. C. Burant, S. S. Iyengar, J. Tomasi, M. Cossi, J. M. Millam, M. Klene, C. Adamo, R. Cammi, J. W. Ochterski, R. L. Martin, K. Morokuma, O. Farkas, J. B. Foresman, and D. J. Fox, Gaussian 09, Revision D.01, Gaussian, Inc. 2009.

[40] M. J. Frisch, G. W. Trucks, H. B. Schlegel, G. E. Scuseria, M. A. Robb, J. R. Cheeseman, G. Scalmani, V. Barone, G. A. Petersson, H. Nakatsuji, X. Li, M. Caricato, A. V. Marenich, J. Bloino, B. G. Janesko, R. Gomperts, B. Mennucci, H. P. Hratchian, J. V. Ortiz, A. F. Izmaylov, J. L. Sonnenberg, D. Williams-Young, F. Ding, F. Lipparini, F. Egidi, J. Goings, B. Peng, A. Petrone, T. Henderson, D. Ranasinghe, V. G. Zakrzewski, J. Gao, N. Rega, G. Zheng, W. Liang, M. Hada, M. Ehara, K. Toyota, R. Fukuda, J. Hasegawa, M. Ishida, T. Nakajima, Y. Honda, O. Kitao, H. Nakai, T. Vreven, K. Throssell, J. A. Montgomery, Jr., J. E. Peralta, F. Ogliaro, M. J. Bearpark, J. J. Heyd, E. N. Brothers, K. N. Kudin, V. N. Staroverov, T. A. Keith, R. Kobayashi, J. Normand, K. Raghavachari, A. P. Rendell, J. C. Burant, S. S. Iyengar, J. Tomasi, M. Cossi, J. M. Millam, M. Klene, C. Adamo, R. Cammi, J. W. Ochterski, R. L. Martin, K. Morokuma, O. Farkas, J. B. Foresman, and D. J. Fox, Gaussian 09, Revision E.01, Gaussian, Inc. 2013.

[41] S. Grimme, S. Ehrlich, L. Goerigk, Effect of the damping function in dispersion corrected density functional theory, J. Comput. Chem. 2011, 32, 1456-1465.

[42] a development of University of Karlsruhe and Forschungszentrum Karlsruhe GmbH, TURBOMOLE V6.4 2012. 
[43] a development of University of Karlsruhe and Forschungszentrum Karlsruhe GmbH, TURBOMOLE V7.0 2015.

[44] T.A. Halgren, Merck molecular force field. I. Basis, form, scope, parameterization, and performance of MMFF94, J. Comput. Chem. 1996, 17, 490-519.

[45] Wavefunction Inc., Spartan'08 v.1.2.0 1991-2013.

[46] A.D. Becke, Density-functional thermochemistry. III. The role of exact exchange, J. Chem. Phys. 1993, 98, 5648-5652.

[47] T. Schwabe, S. Grimme, Double-hybrid density functionals with long-range dispersion corrections: higher accuracy and extended applicability, Phys. Chem. Chem. Phys. 2007, 9, 3397-3406.

[48] S. Grimme, Semiempirical hybrid density functional with perturbative secondorder correlation, J. Chem. Phys. 2006, 124, 34108.

[49] L. Goerigk, S. Grimme, Double-hybrid density functionals, WIREs Comput. Mol. Sci. 2014, 4, 576-600.

[50] L. Goerigk, S. Grimme, A thorough benchmark of density functional methods for general main group thermochemistry, kinetics, and noncovalent interactions, Phys. Chem. Chem. Phys. 2011, 13, 6670-6688.

[51] C. Møller, M.S. Plesset, Note on an Approximation Treatment for Many-Electron Systems, Phys. Rev. 1934, 46, 618-622.

[52] J.A. Pople, R. Krishnan, H.B. Schlegel, J.S. Binkley, Electron correlation theories and their application to the study of simple reaction potential surfaces, Int. J. Quantum Chem. 1978, 14, 545-560.

[53] R.J. Bartlett, G.D. Purvis, Many-body perturbation theory, coupled-pair manyelectron theory, and the importance of quadruple excitations for the correlation problem, Int. J. Quantum Chem. 1978, 14, 561-581.

[54] J.A. Pople, M. Head-Gordon, K. Raghavachari, Quadratic configuration interaction. A general technique for determining electron correlation energies, J. Chem. Phys. 1987, 87, 5968-5975.

[55] J. Řezáč, P. Hobza, Describing Noncovalent Interactions beyond the Common Approximations: How Accurate Is the "Gold Standard," CCSD(T) at the Complete Basis Set Limit?, J. Chem. Theory Comput. 2013, 9, 2151-2155.

[56] F. Weigend, M. Häser, RI-MP2: First derivatives and global consistency, Theor. Chem. Acc. 1997, 97, 331-340.

[57] F. Weigend, M. Häser, H. Patzelt, R. Ahlrichs, RI-MP2: Optimized auxiliary basis sets and demonstration of efficiency, Chem. Phys. Lett. 1998, 294, 143-152.

[58] F. Weigend, A. Köhn, C. Hättig, Efficient use of the correlation consistent basis sets in resolution of the identity MP2 calculations, J. Chem. Phys. 2002, 116, 3175-3183.

[59] C. Hättig, F. Weigend, CC2 excitation energy calculations on large molecules using the resolution of the identity approximation, J. Chem. Phys. 2000, 113, 5154.

[60] C. Hättig, K. Hald, Implementation of RI-CC2 triplet excitation energies with an application to trans-azobenzene, Phys. Chem. Chem. Phys. 2002, 4, 2111-2118. 
[61]F. Weigend, R. Ahlrichs, Balanced basis sets of split valence, triple zeta valence and quadruple zeta valence quality for $\mathrm{H}$ to $\mathrm{Rn}$ : Design and assessment of accuracy, Phys. Chem. Chem. Phys. 2005, 7, 3297-3305.

[62] T.H. Dunning, Gaussian basis sets for use in correlated molecular calculations. I. The atoms boron through neon and hydrogen, J. Chem. Phys. 1989, 90, 10071023.

[63] R.A. Kendall, T.H. Dunning, R.J. Harrison, Electron affinities of the first-row atoms revisited. Systematic basis sets and wave functions, J. Chem. Phys. 1992, 96, 6796-6806.

[64] D.E. Woon, T.H. Dunning, Gaussian basis sets for use in correlated molecular calculations. III. The atoms aluminum through argon, J. Chem. Phys. 1993, 98, 13581371.

[65] H.B. Schlegel, Optimization of equilibrium geometries and transition structures, $J$. Comput. Chem. 1982, 3, 214-218.

[66] V. Barone, Anharmonic vibrational properties by a fully automated second-order perturbative approach, J. Chem. Phys. 2005, 122, 14108.

[67] V. Barone, M. Biczysko, J. Bloino, M. Borkowska-Panek, I. Carnimeo, P. Panek, Toward anharmonic computations of vibrational spectra for large molecular systems, Int. J. Quantum Chem. 2012, 112, 2185-2200.

[68] T. Wassermann, Umgebungseinflüsse auf die C-C- und C-O-Torsionsdynamik in Molekülen und Molekülaggregaten: Schwingungsspektroskopie bei tiefen Temperaturen, Logos, Berlin, 2010.

[69] Private Communication, Technical Support, Gaussian Inc. 2015.

[70] C.V. Raman, K.S. Krishnan, A New Type of Secondary Radiation, Nature 1928, 121, 501-502.

[71] P. Zielke, Ramanstreuung am Überschallstrahl: Wasserstoffbrückendynamik aus neuer Perspektive. Dissertation, Cuvillier, Göttingen, 2007.

[72] Zhifeng Xue, Raman Spectroscopy of Carboxylic Acid and Water Aggregates, Logos Verlag Berlin, 2014.

[73] T. Forsting, Dissertation, expected 2018.

[74] L.H. Spangler, R. van Zee, T.S. Zwier, Assignment of the low-frequency modes in trans-stilbene: Evidence for planarity in the isolated molecule, J. Phys. Chem. 1987, 91, 2782-2786.

[75] F.A. Ensminger, J. Plassard, T.S. Zwier, S. Hardinger, Mode-selective photoisomerization in 5-hydroxytropolone. I. Experiment, J. Chem. Phys. 1995, 102, 52465259.

[76] N.R. Pillsbury, J.A. Stearns, C.W. Müller, D.F. Plusquellic, T.S. Zwier, State-specific studies of internal mixing in a prototypical flexible bichromophore: Diphenylmethane, J. Chem. Phys. 2008, 129, 114301.

[77] R.E. Miller, Infrared laser photodissociation and spectroscopy of van der Waals molecules, J. Phys. Chem. 1986, 90, 3301-3313.

[78] S. Oswald, M.A. Suhm, Experimental Reference Data for Hexafluorinated Propanol by Exploring an Unusual Intermolecular Torsional Balance, Angew. Chem. Int. Ed. 2017. 
[79] D.P. Tabor, D.M. Hewett, S. Bocklitz, J.A. Korn, A.J. Tomaine, A.K. Ghosh, T.S. Zwier, E.L. Sibert, Anharmonic modeling of the conformation-specific IR spectra of ethyl, n-propyl, and n-butylbenzene, J. Chem. Phys. 2016, 144, 224310.

[80] D.M. Hewett, S. Bocklitz, D.P. Tabor, E.L. Sibert III, M.A. Suhm, T.S. Zwier, Identifying the first folded alkylbenzene via ultraviolet, infrared, and Raman spectroscopy of pentylbenzene through decylbenzene, Chem. Sci. 2017, 8, 5305-5318.

[81] F. Battin-Leclerc, Detailed chemical kinetic models for the low-temperature combustion of hydrocarbons with application to gasoline and diesel fuel surrogates, Prog. Energy Combust. Sci. 2008, 34, 440-498.

[82] M.S. Eschner, T.M. Gröger, T. Horvath, M. Gonin, R. Zimmermann, Quasisimultaneous acquisition of hard electron ionization and soft single-photon ionization mass spectra during GC/MS analysis by rapid switching between both ionization methods: analytical concept, setup, and application on diesel fuel, Anal. Chem. 2011, 83, 3865-3872.

[83] M.S. Eschner, W. Welthagen, T.M. Gröger, M. Gonin, K. Fuhrer, R. Zimmermann, Comprehensive multidimensional separation methods by hyphenation of singlephoton ionization time-of-flight mass spectrometry (SPI-TOF-MS) with GC and GCxGC, Anal. Bioanal. Chem. 2010, 398, 1435-1445.

[84] R.C. Striebich, L.M. Shafer, R.K. Adams, Z.J. West, M.J. DeWitt, S. Zabarnick, Hydrocarbon Group-Type Analysis of Petroleum-Derived and Synthetic Fuels Using Two-Dimensional Gas Chromatography, Energy Fuels 2014, 28, 5696-5706.

[85] D. Darcy, H. Nakamura, C.J. Tobin, M. Mehl, W.K. Metcalfe, W.J. Pitz, C.K. Westbrook, H.J. Curran, An experimental and modeling study of surrogate mixtures of n-propyl- and n-butylbenzene in n-heptane to simulate n-decylbenzene ignition, Combust. Flame 2014, 161, 1460-1473.

[86] P. Hobza, Z. Havlas, Blue-Shifting Hydrogen Bonds, Chem. Rev. 2000, 100, 42534264.

[87] S. Karthikeyan, V. Ramanathan, B.K. Mishra, Influence of the substituents on the CH... interaction: benzene-methane complex, J. Phys. Chem. A 2013, 117, 66876694.

[88] L.L. Thomas, T.J. Christakis, W.L. Jorgensen, Conformation of alkanes in the gas phase and pure liquids, J. Phys. Chem. B 2006, 110, 21198-21204.

[89] R.M. Balabin, Enthalpy difference between conformations of normal alkanes: Effects of basis set and chain length on intramolecular basis set superposition error, Mol. Phys. 2011, 109, 943-953.

[90] J.M.L. Martin, What can we learn about dispersion from the conformer surface of n-pentane?, J. Phys. Chem. A 2013, 117, 3118-3132.

[91] J.M. Goodman, What Is the Longest Unbranched Alkane with a Linear Global Minimum Conformation?, J. Chem. Inf. Comput. Sci. 1997, 37, 876-878.

[92] A. Fujii, H. Hayashi, J.W. Park, T. Kazama, N. Mikami, S. Tsuzuki, Experimental and theoretical determination of the accurate $\mathrm{CH} / \pi$ interaction energies in benzenealkane clusters: correlation between interaction energy and polarizability, Phys. Chem. Chem. Phys. 2011, 13, 14131-14141. 
[93] A. Goursot, T. Mineva, R. Kevorkyants, D. Talbi, Interaction between n-Alkane Chains: Applicability of the Empirically Corrected Density Functional Theory for Van der Waals Complexes, J. Chem. Theory Comput. 2007, 3, 755-763.

[94] G. Tasi, F. Mizukami, I. Pálinkó, J. Csontos, W. Győrffy, P. Nair, K. Maeda, M. Toba, S.-i. Niwa, Y. Kiyozumi, I. Kiricsi, Enumeration of the Conformers of Unbranched Aliphatic Alkanes, J. Phys. Chem. A 1998, 102, 7698-7703.

[95] S. Bocklitz, M.A. Suhm, Constraining the Conformational Landscape of a Polyether Building Block by Raman Jet Spectroscopy, Z. Phys. Chem. 2015, 229.

[96] J. A. Dickinson, P. W. Joireman, R. T. Kroemer, E. G. Robertson, J. P. Simons, Conformationally induced transition moment rotations in the $\mathrm{S} 1 \leftarrow \mathrm{S} 0$ electronic spectra of n-propylbenzene and n-butylbenzene, Faraday Trans. 1997, 93, 1467.

[97] J.B. Klauda, B.R. Brooks, A.D. MacKerell, R.M. Venable, R.W. Pastor, An ab initio study on the torsional surface of alkanes and its effect on molecular simulations of alkanes and a DPPC bilayer, J. Phys. Chem. B 2005, 109, 5300-5311.

[98] N.O.B. Lüttschwager, T.N. Wassermann, R.A. Mata, M.A. Suhm, The last globally stable extended alkane, Angew. Chem. Int. Ed. 2013, 52, 463-466.

[99] U. Olsher, F. Frolow, G. Shoham, G.S. Heo, R.A. Bartsch, Alkali-metal and alkalineearth cation and proton selectivities of dibenzo-14-crown-4 and its derivatives in polymeric membranes, Anal. Chem. 2002, 61, 1618-1621.

[100] N.A. Macleod, J.P. Simons, Conformation, structure and molecular solvation: A spectroscopic and computational study of 2-phenoxy ethanol and its singly and multiply hydrated clusters, Chem. Phys. 2002, 283, 221-236.

[101] C.G. Eisenhardt, G. Pietraperzia, M. Becucci, The high resolution spectrum of the S1 $\leftarrow$ S0 transition of anisole, Phys. Chem. Chem. Phys. 2001, 3, 1407-1410.

[102] E.G. Buchanan, E.L.3. Sibert, T.S. Zwier, Ground state conformational preferences and $\mathrm{CH}$ stretch-bend coupling in a model alkoxy chain: 1,2diphenoxyethane, J. Phys. Chem. A 2013, 117, 2800-2811.

[103] E.G. Buchanan, J.R. Gord, T.S. Zwier, Solvent Effects on Vibronic Coupling in a Flexible Bichromophore: Electronic Localization and Energy Transfer induced by a Single Water Molecule, J. Phys. Chem. Lett. 2013, 4, 1644-1648.

[104] C. Guanquan, J. Bevan Ott, J. Rex Goates, (Solid + liquid) phase equilibria and solid-compound formation in 1,2-dimethoxyethane + tetrachloromethane, + trichlorofluoromethane, and + trichloromethane, J. Chem. Thermodyn. 1986, 18, 3137.

[105] K. Aizawa, M. Kato, Vapor-liquid equilibrium determination by total pressure measurements for three binary systems made of 1,2-dimethoxyethane with toluene, methylcyclohexane, or (trifluoromethyl)benzene, J. Chem. Eng. Data 1991, $36,159-161$.

[106] Clayton, George D., Clayton, Florence E., Patty's Industrial Hygiene and Toxicology, 4th ed., 1994.

[107] P.R. Blakemore, W.J. Cole, P.J. Kocieński, A. Morley, A Stereoselective Synthesis of trans -1,2-Disubstituted Alkenes Based on the Condensation of Aldehydes with Metallated 1-Phenyl-1 H -tetrazol-5-yl Sulfones, Synlett 1998, 1998, 26-28. 
[108] T. Sugihara, M. Yamaguchi, The Catalytic Pauson-Khand Reaction Promoted by A Small Amount of 1,2-Dimethoxyethane or Water, Synlett 1998, 1998, 13841386.

[109] Y. Chen, S. Du, C. Huang, G.A. Solan, X. Hao, W.-H. Sun, Balancing high thermal stability with high activity in diaryliminoacenaphthene-nickel(II) catalysts for ethylene polymerization, J. Polym. Sci. Part A: Polym. Chem. 2017, 55, 1971-1983.

[110] Y.-C. Lu, D.G. Kwabi, K.P.C. Yao, J.R. Harding, J. Zhou, L. Zuin, Y. Shao-Horn, The discharge rate capability of rechargeable Li- $\mathrm{O}_{2}$ batteries, Energy Environ. Sci. 2011, 4, 2999.

[111] S. He, K.V. Nielson, J. Luo, T.L. Liu, Recent advances on $\mathrm{MgCl} 2$ based electrolytes for rechargeable Mg batteries, Energy Storage Materials 2017, 8, 184-188.

[112] J.O. Besenhard, M. Winter, J. Yang, W. Biberacher, Filming mechanism of lithium-carbon anodes in organic and inorganic electrolytes, J. Power Sources 1995, 54, 228-231.

[113] R.E. Doe, R. Han, J. Hwang, A.J. Gmitter, I. Shterenberg, H.D. Yoo, N. Pour, D. Aurbach, Novel, electrolyte solutions comprising fully inorganic salts with high anodic stability for rechargeable magnesium batteries, Chem. Commun. 2014, 50, 243-245.

[114] J.J. Lee, M. Albrecht, C.A. Rice, M.A. Suhm, A. Stamm, M. Zimmer, M. Gerhards, Adaptive aggregation of peptide model systems, J. Phys. Chem. A 2013, 117, 7050-7063.

[115] R.G. Snyder, G. Zerbi, Vibrational analysis of ten simple aliphatic ethers: Spectra, assignments, valence force field and molecular conformations, Spectrochim. Acta Mol. Biomol. Spectrosc. 1967, 23, 391-437.

[116] Y. Ogawa, M. Ohta, M. Sakakibara, H. Matsuura, I. Harada, T. Shimanouchi, Vibration Spectra and Rotational Isomerism of Chain Molecules. V. 2,5-

Dioxahexane, 2,5-Dithiahexane, and 2-0xa-5-thiahexane, BCSJ 1977, 50, 650660.

[117] G.D. Smith, R.L. Jaffe, D.Y. Yoon, Conformations of 1,2-Dimethoxyethane in the Gas and Liquid Phases from Molecular Dynamics Simulations, J. Am. Chem. Soc. 1995, 117, 530-531.

[118] S. Masatoki, M. Takamura, H. Matsuura, K. Kamogawa, T. Kitagawa, Raman Spectroscopic Observations of Anomalous Conformational Behavior of Short Poly(oxyethylene) Chains in Water, Chem. Lett. 1995, 24, 991-992.

[119] H. Matsuura, T. Sagawa, Anomalous conformational behavior of short poly(oxyethylene) chains in water: An FT-IR spectroscopic study, in: Structure, Fluctuation, and relaxtion in solutions, Proceedings of the Yamada Conference XXXXII, Elsevier, 1995, pp. 313-316.

[120] R. Begum, a. Hiroatsu Matsuura, Conformational properties of short poly(oxyethylene) chains in water studied by IR spectroscopy, Faraday Trans. 1997, 93, 3839-3848.

[121] D. Bedrov, M. Pekny, G.D. Smith, Quantum-Chemistry-Based Force Field for 1,2-Dimethoxyethane and Poly(ethylene oxide) in Aqueous Solution, J. Phys. Chem. B 1998, 102, 996-1001. 
[122] J. Fischer, D. Paschek, A. Geiger, G. Sadowski, Modeling of aqueous poly(oxyethylene) solutions: 1 . Atomistic simulations, J. Phys. Chem. B 2008, 112, 2388-2398.

[123] M. Kobayashi, M. Takahashi, H. Sato, Conformational analysis for hydrated ethylene oxide oligomer models by quantum chemical calculations, Polym. Bull. 2009, 63, 299-312.

[124] D. Rigby, H. Sun, B.E. Eichinger, Computer simulations of poly(ethylene oxide): Force field, pvt diagram and cyclization behaviour, Polym. Int. 1997, 44, 311-330.

[125] K. Tasaki, A. Abe, NMR Studies and Conformational Energy Calculations of 1,2Dimethoxyethane and Poly(oxyethylene), Polym. J. 1985, 17, 641-655.

[126] S. Aparicio, R. Alcalde, J.L. Trenzado, M.N. Caro, M. Atilhan, Study of dimethoxyethane/ethanol solutions, J. Phys. Chem. B 2011, 115, 8864-8874.

[127] E.E. Astrup, L. Pataki, R. Steudel, An Investigation of the Molecular Structure and Conformation of 1,2-Dimethoxyethane, $\mathrm{CH}_{3}-\mathrm{O}-\mathrm{CH}_{2}-\mathrm{CH}_{2}-\mathrm{O}-\mathrm{CH}_{3}$, in the Gas Phase, Acta Chem. Scand. 1979, 33a, 655-664.

[128] K. Inomata, A. Abe, Conformation of 1,2-dimethoxyethane in the gas phase: A rotational isomeric state simulation of NMR vicinal coupling constants, J. Phys. Chem. 1992, 96, 7934-7937.

[129] H. Yoshida, T. Tanaka, H. Matsuura, Conformational Stability of 1,2-

Dimethoxyethane in the Gas Phase Studied by Infrared Spectroscopy: Importance

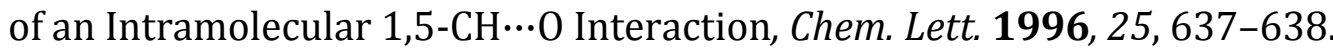

[130] O. Takahashi, Y. Kohno, M. Nishio, Relevance of weak hydrogen bonds in the conformation of organic compounds and bioconjugates: evidence from recent experimental data and high-level ab initio MO calculations, Chem. Rev. 2010, 110, 6049-6076.

[131] H. Yoshida, I. Kaneko, H. Matsuura, Y. Ogawa, M. Tasumi, Importance of an intramolecular 1,5-CH...O interaction and intermolecular interactions as factors determining conformational equilibria in 1,2-dimethoxyethane: A matrix-isolation infrared spectroscopic study, Chem. Phys. Lett. 1992, 196, 601-606.

[132] W. Li, A. Vigorito, C. Calabrese, L. Evangelisti, L.B. Favero, A. Maris, S. Melandri, The microwave spectroscopy study of 1,2-dimethoxyethane, J. Mol. Spectrosc. 2017, 337, 3-8.

[133] M. Barzaghi, A. Gamba, G. Morosi, The conformational structure of 1,2dimethoxy-ethane in the gas phase, Comput. Theor. Chem. 1988, 170, 69-74.

[134] S. Tsuzuki, T. Uchimaru, K. Tanabe, T. Hirano, Conformational analysis of 1,2dimethoxyethane by ab initio molecular orbital and molecular mechanics calculations: Stabilization of the TGG' rotamer by the $1,5 \mathrm{CH}_{3} / 0$ nonbonding attractive interaction, J. Phys. Chem. 1993, 97, 1346-1350.

[135] F. Mueller-Plathe, W.F. van Gunsteren, Can Simple Quantum-Chemical Continuum Models Explain the Gauche Effect in Poly(ethylene oxide)?, Macromolecules 1994, 27, 6040-6045.

[136] H. Yoshida, H. Matsuura, Density Functional Study of the Conformations and Vibrations of 1,2-Dimethoxyethane, J. Phys. Chem. A 1998, 102, 2691-2699. 
[137] M.A. Murcko, R.A. DiPaola, Ab initio molecular orbital conformational analysis of prototypical organic systems. 1. Ethylene glycol and 1,2-dimethoxyethane, J. Am. Chem. Soc. 1992, 114, 10010-10018.

[138] R.L. Jaffe, G.D. Smith, D.Y. Yoon, Conformation of 1,2-dimethoxyethane from ab initio electronic structure calculations, J. Phys. Chem. 1993, 97, 12745-12751.

[139] P.M. Anderson, M.R. Wilson, Developing a force field for simulation of poly(ethylene oxide) based upon ab initio calculations of 1,2-dimethoxyethane, Mol. Phys. 2005, 103, 89-97.

[140] Y.-K. Han, K.H. Kim, Son Sang-Kil, Y.S. Lee, Effects of Intramolecular Basis Set Superpositon Error on Conformational Energy Difference of 1,2-Difluoroethane and 1.2-Dimethoxyethane, Bull. Korean Chem. Soc. 2002, 23, 1267-1271.

[141] S.A. Wahab, T. Harada, T. Matsubara, M. Aida, Quantum chemical study of the interaction of the short-chain poly(oxyethylene)s $\mathrm{CH} 3\left(\mathrm{OCH}_{2} \mathrm{CH}_{2}\right)_{\mathrm{m}} \mathrm{OCH}_{3}\left(\mathrm{C}_{1} \mathrm{E}_{\mathrm{m}} \mathrm{C}_{1}\right.$; $\mathrm{m}=1$ and 2) with a water molecule in the gas phase and in solutions, J. Phys. Chem. A 2006, 110, 1052-1059.

[142] O. Engkvist, G. Karlström, Monte Carlo Simulation Study of Short Poly(ethylene oxide) Chains at Different Concentrations, J. Phys. Chem. B 1997, 101, 1631-1633.

[143] P. Bultinck, A. Goeminne, D. van de Vondel, Ab initio and molecular mechanics study of 1,2-dimethoxyethane and 12-crown-4, Comput. Theor. Chem. 1999, 467, 211-222.

[144] M. Pitoňák, T. Janowski, P. Neogrády, P. Pulay, P. Hobza, Convergence of the CCSD(T) Correction Term for the Stacked Complex Methyl Adenine-Methyl Thymine: Comparison with Lower-Cost Alternatives, J. Chem. Theory Comput. 2009, 5, 1761-1766.

[145] V. Majer, V. Svoboda, Enthalpies of vaporization of organic compounds: A crit. review and data compilation, Blackwell Scient. Publ, Oxford, 1985.

[146] W.V. Steele, R.D. Chirico, S.E. Knipmeyer, A. Nguyen, N.K. Smith, Thermodynamic Properties and Ideal-Gas Enthalpies of Formation for Butyl Vinyl Ether, 1,2-Dimethoxyethane, Methyl Glycolate, Bicyclo[2.2.1]hept-2-ene, 5Vinylbicyclo[2.2.1]hept-2-ene, trans -Azobenzene, Butyl Acrylate, Di- tert -butyl Ether, and Hexane-1,6-diol, J. Chem. Eng. Data 1996, 41, 1285-1302.

[147] K. Machida, T. Miyazawa, Infrared and Raman spectra of polyethyleneglycol dimethylethers in the liquid state, Spectrochim. Acta 1964, 20, 1865-1873.

[148] M.F. Fox, E. Powell, J.W. Stafford, M.J. Blandamer, Spectroscopic and viscosimetric studies of aqueous polyethylene oxide solutions, Chem. Commun. 1968, 1022.

[149] M.Z. Jora, M.V.C. Cardoso, E. Sabadini, Dynamical aspects of waterpoly(ethylene glycol) solutions studied by 1 H NMR, J. Mol. Liq. 2016, 222, $94-$ 100.

[150] S.A. Wahab, H. Matsuura, Raman spectroscopic study of conformational properties of a short-chain poly(oxyethylene) (C1E3C1) in polar solvents at different temperatures, J. Mol. Struct. 2002, 606, 35-43. 
[151] S.A. Wahab, H. Matsuura, IR spectroscopic study of conformational properties of a short-chain poly(oxyethylene) $\left(\mathrm{C}_{1} \mathrm{E}_{3} \mathrm{C}_{1}\right)$ in binary mixtures with liquids of different hydrogen-bonding abilities, Phys. Chem. Chem. Phys. 2001, 3, 4689-4695.

[152] P.S. Walsh, E.G. Buchanan, J.R. Gord, T.S. Zwier, Binding water to a PEG-linked flexible bichromophore: IR spectra of diphenoxyethane- $\left(\mathrm{H}_{2} \mathrm{O}\right)_{n}$ clusters, $\mathrm{n}=2-4$, $\mathrm{J}$. Chem. Phys. 2015, 142, 154303.

[153] J.R. Lane, CCSDTQ Optimized Geometry of Water Dimer, J. Chem. Theory Comput. 2013, 9, 316-323.

[154] R.H. Beaumont, B. Clegg, G. Gee, J.B.M. Herbert, D.J. Marks, R.C. Roberts, D. Sims, Heat capacities of propylene oxide and of some polymers of ethylene and propylene oxides, Polymer 1966, 7, 401-417.

[155] D.R. Lide, G.W.A. Milne, R.C. Weast, J.G. Grasselli (Eds.), Handbook of data on organic compounds, 2nd ed., CRC Press, Boca Raton, 1989.

[156] C.D. Driscoll, R. Valentine, R.E. Staples, N.C. Chromey, G.L. Kennedy JR, Developmental toxicity of diglyme by inhalation in the rat, Drug. Chem. Toxicol. 1998, 21, 119-136.

[157] R. Valentine, A.J. O'Neill, K.P. Lee, G.L. Kennedy, Subchronic inhalation toxicity of diglyme, Food Chem. Toxicol. 1999, 37, 75-86.

[158] Britt E. Erickson, Nine Chemicals Added To High-Concern List, Chem. Eng. News 2014, 35, 38.

[159] European Chemicals Agency, Authorisation List, available at https://echa.europa.eu/de/addressing-chemicals-ofconcern/authorisation/recommendation-for-inclusion-in-the-authorisationlist/authorisation-list/-/dislist/details/0b0236e1807e00e8 (accessed on February 16, 2017).

[160] H. C. Brown, S. U. Kulkarni, Organoboranes, J. Organomet. Chem. 1981, 218, 299-307.

[161] P.J. Maddocks, A. Pelter, K. Rowe, K. Smith, C. Subrahmanyam, Reactions of dialkylbromoboranes with alkali metal hydrides in the presence of alkenes. Synthesis of 'mixed' trialkylboranes (R A2 R B B) in solution, J. Chem. Soc., Perkin Trans. 1 1981, 653-656.

[162] S.L. Zultanski, G.C. Fu, Nickel-catalyzed carbon-carbon bond-forming reactions of unactivated tertiary alkyl halides: Suzuki arylations, J. Am. Chem. Soc. 2013, $135,624-627$.

[163] W. Xu, J. Hu, M.H. Engelhard, S.A. Towne, J.S. Hardy, J. Xiao, J. Feng, M.Y. Hu, J. Zhang, F. Ding, M.E. Gross, J.-G. Zhang, The stability of organic solvents and carbon electrode in nonaqueous $\mathrm{Li}_{-} \mathrm{O}_{2}$ batteries, J. Power Sources 2012, 215, 240247.

[164] C. Zhang, K. Ueno, A. Yamazaki, K. Yoshida, H. Moon, T. Mandai, Y. Umebayashi, K. Dokko, M. Watanabe, Chelate effects in glyme/lithium bis(trifluoromethanesulfonyl)amide solvate ionic liquids. I. Stability of solvate cations and correlation with electrolyte properties, J. Phys. Chem. B 2014, 118, 5144-5153. 
[165] C. Zhang, A. Yamazaki, J. Murai, J.-W. Park, T. Mandai, K. Ueno, K. Dokko, M. Watanabe, Chelate Effects in Glyme/Lithium Bis(trifluoromethanesulfonyl)amide Solvate Ionic Liquids, Part 2: Importance of Solvate-Structure Stability for Electrolytes of Lithium Batteries, J. Phys. Chem. C 2014, 118, 17362-17373.

[166] C.P. Rhodes, R. Frech, Local Structures in Crystalline and Amorphous Phases of Diglyme- $\mathrm{LiCF}_{3} \mathrm{SO}_{3}$ and Poly(ethylene oxide) $-\mathrm{LiCF}_{3} \mathrm{SO}_{3}$ Systems: Implications for the Mechanism of Ionic Transport, Macromolecules 2001, 34, 2660-2666.

[167] I. Geoffroy, P. Willmann, K. Mesfar, B. Carré, D. Lemordant, Electrolytic characteristics of ethylene carbonate-diglyme-based electrolytes for lithium batteries, Electrochim. Acta 2000, 45, 2019-2027.

[168] A. Sutjianto, L.A. Curtiss, $\mathrm{Li}^{+}-$Diglyme Complexes: Barriers to Lithium Cation Migration, J. Phys. Chem. A 1998, 102, 968-974.

[169] N. Sa, B. Pan, A. Saha-Shah, A.A. Hubaud, J.T. Vaughey, L.A. Baker, C. Liao, A.K. Burrell, Role of Chloride for a Simple, Non-Grignard Mg Electrolyte in EtherBased Solvents, ACS Appl. Mater. Interfaces 2016, 8, 16002-16008.

[170] A. Baskin, D. Prendergast, Exploration of the Detailed Conditions for Reductive Stability of Mg(TFSI) 2 in Diglyme: Implications for Multivalent Electrolytes, J. Phys. Chem. C 2016, 120, 3583-3594.

[171] Kitada Atsushi, K. Nakamura, K. Fukami, K. Murase, $\mathrm{AlCl}_{3}$-dissolved Diglyme as Electrolyte for Room-Temperature Aluminum Electrodeposition, Electrochem. 2014, 82, 946-948.

[172] G. Kearley, Structure, vibrational-dynamics and first-principles study of diglyme as a model system for poly(ethyleneoxide), Solid State Ionics 2002, 147, 237-242.

[173] N.R. Dhumal, S.P. Gejji, Molecular interactions and vibrations in $\mathrm{CH}_{3}\left(\mathrm{OCH}_{2} \mathrm{CH}_{2}\right)_{2} \mathrm{OCH}_{3}-\mathrm{M}^{+}-\mathrm{X}-\left(\mathrm{M}=\mathrm{Li}, \mathrm{Na}, \mathrm{K}\right.$ and $\left.\mathrm{X}=\mathrm{PF}_{6}, \mathrm{AsF}_{6}, \mathrm{SbF}_{6}\right)$ : An ab initio study, Comput. Theor. Chem. 2008, 859, 86-92.

[174] W.S. Compel, O.A. Wong, X. Chen, C. Yi, R. Geiss, H. Hakkinen, K.L. Knappenberger JR, C.J. Ackerson, Dynamic Diglyme-Mediated Self-Assembly of Gold Nanoclusters, ACS nano 2015, 9, 11690-11698.

[175] Y. Ren, Z. Huang, H. Miao, D. Jiang, K. Zeng, B. Liu, X. Wang, Effect of the Addition of Diglyme in Diesel Fuel on Combustion and Emissions in a Compression-Ignition Engine, Energy Fuels 2007, 21, 2573-2583.

[176] Y. Di, C.S. Cheung, Z. Huang, Experimental investigation of particulate emissions from a diesel engine fueled with ultralow-sulfur diesel fuel blended with diglyme, Atmos. Environ. 2010, 44, 55-63.

[177] H. Matsuura, T. Miyazawa, Infrared spectra of poly(ethylene glycol) dimethyl ethers in the crystalline state, Spectrochim. Acta 1973, 771-779.

[178] H. Matsuura, K. Fukuhara, H. Tamaoki, Raman spectra of perdeuterated ethylene glycol dimethyl ether and diethylene glycol dimethyl ether and the molecular force field of oxyethylene compounds, J. Mol. Struct. 1987, 156, 293-301.

[179] P. Johansson, J. Grondin, J.-C. Lassegues, Structural and vibrational properties of diglyme and longer glymes, J. Phys. Chem. A 2010, 114, 10700-10705. 
[180] A. Sutjianto, L.A. Curtiss, Theoretical study of the potential energy surface of diglyme, Chem. Phys. Lett. 1997, 264, 127-133.

[181] S.P. Gejji, J. Tegenfeldt, J. Lindgren, Conformational analysis of poly(ethylene oxide) oligomers: Diglyme, Chem. Phys. Lett. 1994, 226, 427-432.

[182] H. Matsuura, K. Fukuhara, Vibrational spectroscopic studies of conformation of poly(oxyethylene). II. Conformation-spectrum correlations, J. Polym. Sci. B Polym. Phys. 1986, 24, 1383-1400.

[183] S.P. Gejji, S.R. Gadre, V.J. Barge, Theoretical investigations on structure, electrostatic potentials and vibrational frequencies of diglyme and $\mathrm{Li}^{+}-($diglyme) conformers, Chem. Phys. Lett. 2001, 344, 527-535.

[184] M. Quack, On the measurement of the parity violating energy difference between enantiomers, Chem. Phys. Lett. 1986, 132, 147-153.

[185] NIST Chemistry Webbook, diglyme phase change data, available at http://webbook.nist.gov/cgi/cbook.cgi?ID=C111966\&Units=SI\&Mask=4\#Therm o-Phase (accessed on February 13, 2017).

[186] R.W. Larsen, P. Zielke, M.A. Suhm, Hydrogen-bonded OH stretching modes of methanol clusters: a combined IR and Raman isotopomer study, J. Chem. Phys. 2007, 126, 194307.

[187] M. Nedic, T.N. Wassermann, R.W. Larsen, M.A. Suhm, A combined Raman- and infrared jet study of mixed methanol-water and ethanol-water clusters, Phys. Chem. Chem. Phys. 2011, 13, 14050-14063.

[188] D. Li, W. Fang, W. Xie, Y. Xing, Y. Guo, R. Lin, Measurements on Vapor Pressure and Thermal Conductivity for Pseudo-binary Systems of a Hydrocarbon Fuel with Ethylene and Diethylene Glycol Dimethyl Ethers, Energy Fuels 2009, 23, 794798.

[189] P.J. Carvalho, C.H.G. Fonseca, M.-L.C.J. Moita, Â.F.S. Santos, J.A.P. Coutinho, Thermophysical Properties of Glycols and Glymes, J. Chem. Eng. Data 2015, 60, 3721-3737.

[190] H. Tadokoro, Y. Chatani, T. Yoshihara, S. Tahara, S. Murahashi, Structural Studies on Polyethers, [-(CH2)m-0-]n - Molecular Structure of Polyethylene Oxide, Makromol. Chem. 1964, 73, 109-127.

[191] Y. Takahashi, H. Tadokoro, Structural Studies of Polyethers, (-(CH 2 )m-0-) n X. Crystal Structure of Poly(ethylene oxide), Macromolecules 1973, 6, 672-675.

[192] A.C. French, A.L. Thompson, B.G. Davis, High-purity discrete PEG-oligomer crystals allow structural insight, Angew. Chem. Int. Ed. 2009, 48, 1248-1252.

[193] J. Buckingham, S.M. Donaghy (Eds.), Dictionary of organic compounds, 5th ed., Chapman and Hall, New York, 1982.

[194] R.G. Snyder, S.L. Wunder, Long-range conformational structure and lowfrequency isotropic Raman spectra of some highly disordered chain molecules, Macromolecules 1986, 19, 496-498.

[195] S. Shashkov, The disorder-longitudinal acoustic mode of oligo (ethylene glycol)-lithium trifluoromethane sulfonate solutions as studied by FT Raman spectroscopy, Solid State Ionics 1996, 90, 261-268. 
[196] Han L., Peng L., Cai L.-Y., Zheng X.-M., Zhang F.-S., CH2 Scissor and Twist Vibrations of Liquid Polyethylene Glycol - Raman Spectra and Density Functional Theory Calculations, Acta Phys. -Chim. Sin. 2017, 1043-1050.

[197] T. Shimanouchi, Local and overall vibrations of polymer chains, Pure Appl. Chem. 1973, 36.

[198] S.J. Daunt, H.F. Shurvell, The gas phase infrared band contours of s-triazine and s-triazine- $d_{3}$, J. Mol. Spectrosc. 1976, 62, 373-395.

[199] H. Matsuura, T. Miyazawa, Young's modulus of poly(ethylene glycol), J. Polym. Sci. B Polym. Lett. 1969, 7, 65-66.

[200] M.A. Al-Nasassrah, F. Podczeck, J.M. Newton, The effect of an increase in chain length on the mechanical properties of polyethylene glycols, Eur. J. Pharm. Biopharm. 1998, 46, 31-38.

[201] I. Sakurada, T. Ito, K. Nakamae, Elastic moduli of the crystal lattices of polymers, J. polym. sci., C Polym. symp. 1967, 15, 75-91.

[202] L.M. Bellan, J. Kameoka, H.G. Craighead, Measurement of the Young's moduli of individual polyethylene oxide and glass nanofibres, Nanotechnology 2005, 16, 1095-1099.

[203] I.W. Levin, R.A.R. Pearce, R.C. Spiker, Vibrational Raman spectra and intramolecular potential function of solid solutions of dimethyl ether- $d_{0}$ and dimethyl ether- $d_{6}$, J. Chem. Phys. 1978, 68, 3471-3480.

[204] R.A.R. Pearce, I.W. Levin, Normal coordinate treatment for propane: Force constant selection through a backward elimination regression-refinement procedure, J. Chem. Phys. 1979, 70, 370.

[205] A.T. Bruni, V.B.P. Leite, M.M.C. Ferreira, Conformational analysis: a new approach by means of chemometrics, J. Comput. Chem. 2002, 23, 222-236.

[206] T. Scharge, C. Cezard, P. Zielke, A. Schutz, C. Emmeluth, M.A. Suhm, A peptide co-solvent under scrutiny: self-aggregation of 2,2,2-trifluoroethanol, Phys. Chem. Chem. Phys. 2007, 9, 4472-4490.

[207] S. Oswald, M.A. Suhm, Experimental Reference Data for Hexafluorinated Propanol by Exploring an Unusual Intermolecular Torsional Balance, Angew. Chem. Int. Ed. 2017, 56. 